\title{
SINGULAR INTEGRAL OPERATORS AND ELLIPTIC BOUNDARY-VALUE PROBLEMS. PART I
}

\author{
A. P. Soldatov
}

UDC 517.968

\begin{abstract}
The monograph consists of three parts. Part I is presented here. In this monograph, we develop a new approach (mainly based on papers of the author). Many results are published for the first time here.

Chapter 1 is introductory. It provides the necessary background from functional analysis (for completeness). In this monograph, we mostly use weighted Hölder spaces; they are considered in Chap. 2. Chapter 3 plays the key role: in weighted Hölder spaces, we consider estimates of integral operators with homogeneous difference kernels, covering potential-type integrals and singular integrals as well as Cauchy-type integrals and double layer potentials. In Chap. 4, similar estimates in weighted Lebesgue spaces are proved.

Integrals with homogeneous difference kernels will play an important role in Part III of the monograph, which will be devoted to elliptic boundary-value problems. They naturally arise in integral representations of solutions of first-order elliptic systems in terms of fundamental matrices or their parametrices. The investigation of boundary-value problems for second-order and higher-order elliptic equations or systems is reduced to first-order elliptic systems.
\end{abstract}

\section{CONTENTS}

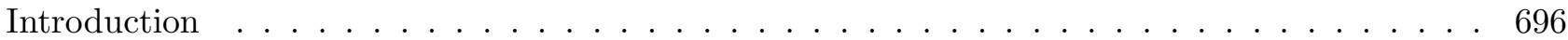

Chapter 1. Banach Spaces and Algebras . . . . . . . . . . . . . . . . . . . . 697

1.1. Banach Spaces . . . . . . . . . . . . . . . . . . . . . . . 697

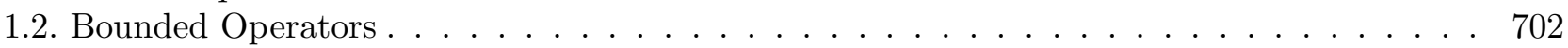

1.3. Fredholm Operators . . . . . . . . . . . . . . . . . . . . . 708

1.4. Banach Algebras . . . . . . . . . . . . . . . . . . . . . . . 715

1.5. Spectrum and Resolvent . . . . . . . . . . . . . . . . . . . . . . . . 722

1.6. Number Matrices . . . . . . . . . . . . . . . . . . . . . . . . . . 726

1.7. Semi-Almost Periodic Functions ． . . . . . . . . . . . . . . . . . . . . . . 729

1.8. Lebesgue Integrals and Generalized Functions . . . . . . . . . . . . . . . . . . . . 734

1.9. Second-Kind Fredholm Equations . . . . . . . . . . . . . . . . . . . . . . . . 741

Chapter 2. Hölder Spaces . . . . . . . . . . . . . . . . . . . . . . . . . . . . 745

2.1. Hölder Condition . . . . . . . . . . . . . . . . . . . . . . . . . . . . . . . 745

2.2. Hölder Spaces $C^{\mu}(G) \ldots \ldots \ldots \ldots$. . . . . . . . . . . . . . 750

2.3. Lipschitz Maps and Domains . . . . . . . . . . . . . . . . . . . . . . . 755

2.4. Smooth Surfaces . . . . . . . . . . . . . . . . . . . . . . . . 759

2.5. Smooth and Piecewise-Smooth Curves . . . . . . . . . . . . . . . . . . 764

2.6. The Space $C_{*}^{\mu}(G)$ on the Riemann Sphere . . . . . . . . . . . . . . . . . . . . 770

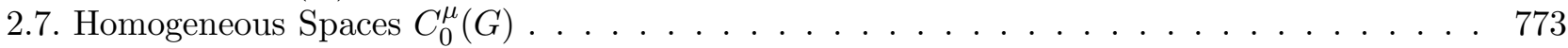

2.8. Weight Spaces $C_{\lambda}^{\mu}(G, F) \quad \ldots \ldots \ldots \ldots \ldots \ldots \ldots \ldots \ldots$

2.9. Hölder Spaces of Differentiable Functions ． . . . . . . . . . . . . . . . . . . . . . . 781

2.10. Modified Spaces $C_{(\lambda)}^{n, \mu} \ldots \ldots \ldots \ldots \ldots \ldots \ldots \ldots \ldots 78 \ldots \ldots \ldots$

Translated from Sovremennaya Matematika. Fundamental'nye Napravleniya (Contemporary Mathematics. Fundamental Directions), Vol. 63, No. 1, Functional Analysis, 2017. 
Chapter 3. Integrals with Homogeneous Difference Kernels . . . . . . . . . . . . . . . . . . 793

3.1. Homogeneous Functions ． . . . . . . . . . . . . . . . . . . . . 793

3.2. Integrals with Weak Singularities ． . . . . . . . . . . . . . . . . 797

3.3. The Notion of Singular Integrals . . . . . . . . . . . . . . . . . . . . . 800

3.4. $C^{\mu}$-Estimates of Singular Integrals . . . . . . . . . . . . . . . . . . . 805

3.5. Estimates up to the Boundary . . . . . . . . . . . . . . . . . . 808

3.6. Generalized Cauchy-Type Integrals . . . . . . . . . . . . . . . . . . . . . . . . 814

3.7. Relations for Boundary Values . . . . . . . . . . . . . . . . . . . . . . 820

3.8. Line Cauchy-Type Integral ． . . . . . . . . . . . . . . . . . . . . . . 823

3.9. Line Singular Integrals . . . . . . . . . . . . . . . . . . . . . . . . . . . . . 829

3.10. Weight $C^{\mu}$-Estimates of Cauchy-Type Integrals $\ldots \ldots \ldots \ldots$. . . . . . . . 835

3.11. Weight $C^{\mu}$-Estimates of Singular Integrals . . . . . . . . . . . . . . . . . . . . . 841

Chapter 4. Lebesgue Spaces . . . . . . . . . . . . . . . . . . . . . . . . . 844

4.1. Spaces $L^{p}$ and $L_{\lambda}^{p} \ldots \ldots \ldots \ldots \ldots \ldots \ldots \ldots \ldots \ldots \ldots \ldots \ldots$

4.2. Convolution of Functions . . . . . . . . . . . . . . . . . . . . . 848

4.3. Fourier Transformation . . . . . . . . . . . . . . . . . . . . 854

4.4. Convolution-Type Operators on the Real Line . . . . . . . . . . . . . . . . . . . . . . . 859

4.5. Multiplicative Convolutions on the Semiaxis . . . . . . . . . . . . . . . . . . . 864

4.6. $L^{p}$-Estimates of Integrals with Weak Singularities . . . . . . . . . . . . . . . . . . 870

4.7. $L^{p}$-Estimates of Singular Integrals $\ldots \ldots \ldots \ldots$. . . . . . . . . . . . . 873

4.8. Singular Cauchy Integrals with $L^{p}$-Density $\ldots \ldots \ldots \ldots \ldots$. . . . . . . . 878

4.9. Cauchy-Type Generalized Integrals with $L^{p}$-Density $\ldots \ldots \ldots \ldots \ldots$. . . . . . . 883

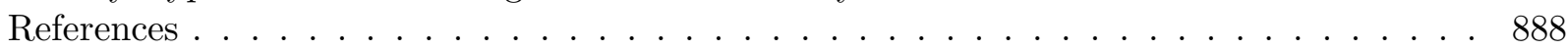

\section{Introduction}

The theory of one-dimensional singular integral equations appeared in works of D. Hilbert and H. Poincaré at the end of the XIXth century. Foundations of this theory were created by F. Noether and T. Carleman. Since the beginning of the 1930s, this theory is substantially developed by Soviet mathematicians. The main investigation method for singular equations and boundary-value problems for analytic functions is the technique of Cauchy-type integrals; its final form (in a way) is presented in the well-known monographs [45] and [17].

Further, various directions of the theory of singular integral equations and boundary-value problems were developed; for example, the requirements were weakened for the class of desired functions (the $L p$-theory) and for coefficients of the equations and boundary-value problems or these coefficients are replaced by more general function operators with translations. Ideas and methods of functional analysis are broadly applied in investigations of singular equations. A close relation of the specified equations with the Wiener-Hopf equations is found (see [7, 24, 39, 44, 54, 78]).

The present monograph is specified by a new approach; in a substantial part, it is based on works of the author. Many results are published for the first time. The monograph consists of three parts; the first part is the content of the present volume. Chapter 1 is an introduction. It contains necessary preliminary data from the functional analysis (to make the explanation as close as possible). In further chapters, we mainly deal with weight Hölder spaces $C^{\mu}$; Chap. 2 is devoted to such spaces. Chapter 3 is especially important: it provides necessary estimates of integral operators in Hölder spaces with homogeneous-difference kernels, covering potential-type integrals, singular integrals, Cauchy-type integrals, and double-layer potentials. In the last chapter, similar estimates in weight Lebesgue $L^{p_{-}}$ spaces are considered. 
Integrals with homogeneous-difference kernels are important for the third part of the monograph, devoted to elliptic boundary-value problems. They naturally arise in integral representations of solutions of first-order elliptic systems in terms of fundamental matrices or their parametrices. The investigation of boundary-value problems for elliptic equations and system of order two and higher orders is reduced to the investigation of first-order elliptic systems. Note that a similar direct approach is undertaken in works of Fichera and his disciples (see $[15,16]$ ) directly for high-order elliptic systems (analogs of simple-layer potentials are used).

The second part of the monograph contains the technique of singular integrodifferential equations. The approach explained in [65, 67-69] is developed: an operator algebra containing the singular Cauchy operator and integral operators with fixed singularities at singular point of a piecewise-smooth curve is investigated. Elements of this algebra arise in a natural way in the study of elliptic boundaryvalue problems in planar domains with piecewise-smooth boundaries (including nonlocal boundaryvalue problems and problems on stratified sets) and in applications to mixed-type elliptic-hyperbolic equations.

\section{CHAPter 1}

\section{BANACH SPACES AND ALGEBRAS}

\subsection{Banach Spaces}

The majority of the content of the present chapter is well known: it can be found in any guidebook on functional analysis (see, e.g., [56, 79]). To make our explanation closed whenever it is possible, we provide a brief proof for any assertion. Theorems without proofs are supplied by separate comments.

Let us describe main notions related to normed vector spaces and bounded linear operators. Unless the opposite is stated, these spaces are considered over the field $\mathbb{C}$ of complex numbers. First, we recall general notions regarding vector spaces.

A subset $X_{1} \subseteq X$ is called a subspace of a vector space $X$ if $\lambda_{1} x_{1}+\lambda_{2} x_{2} \in X_{1}$ for any $x_{j}$ from $X_{1}$ and any complex $\lambda_{j}$. The intersection $X_{1} \cap X_{2}$ and the sum $X_{1}+X_{2}=\left\{x_{1}+x_{2}, x_{j} \in X_{j}\right\}$ of any subspaces $X_{1}$ and $X_{2}$ are also subspaces. If the relation $x_{1}+x_{2}=0$ implies the relation $x_{1}=x_{2}=0$ provided that $x_{j} \in X_{j}$, then we say that the sum $X_{1}+X_{2}$ is direct and denote it by $X_{1} \oplus X_{2}$. If it coincides with $X$, then we say that $X$ is expanded into the directed sum of the subspaces $X_{1}$ and $X_{2}$; also, the notation $X_{2}=X \ominus X_{1}$ is used in such a case. For any pair $X_{1}$ and $X_{2}$ of spaces, the direct product $X_{1} \times X_{2}$ is a vector space with respect to the coordinate-wise linear operations $\lambda\left(x_{1}, x_{2}\right)=\left(\lambda x_{1}, \lambda x_{2}\right)$ and $\left(x_{1}, x_{2}\right)+\left(y_{1}, y_{2}\right)=\left(x_{1}+y_{1}, x_{2}+y_{2}\right)$. The direct product $X_{1} \times \ldots \times X_{n}$ has a similar sense. For any subspace $Y \subseteq X$, one can introduce the quotient space $X / Y$. Its elements are cosets $\tilde{x}=\{x+y, y \in Y\}$ and its linear operations are $\lambda \tilde{x}=\widetilde{\lambda x}$ and $\tilde{x}+\tilde{y}=\widetilde{x+y}$.

If $e_{j} \in X$ and $\lambda_{j} \in \mathbb{C}, j=1, \ldots, n$, then the vector $x=\lambda_{1} e_{1}+\ldots+\lambda_{n} e_{n}$ is called a linear combination of vectors $e_{j}$. The set of all such vectors forms a subspace of $X$, denoted by $\left[e_{1}, \ldots, e_{n}\right]$. We say that the vector system $\left\{e_{1}, \ldots, e_{n}\right\}$ is linearly independent if the relation $\lambda_{1} e_{1}+\ldots+\lambda_{n} e_{n}=0$ implies the relation $\lambda_{1}=\ldots=\lambda_{n}=0$. We say that a subspace $X_{0} \subseteq X$ is finite-dimensional if there exists a linearly independent system $\left\{e_{1}, \ldots, e_{n}\right\}$ such that $X_{0}=\left[e_{1}, \ldots, e_{n}\right]$. If this holds, then $\left\{e_{1}, \ldots, e_{n}\right\}$ is called a base of $X_{0}$. The number $n$ of elements of this base depends only on $X_{0}$, is called the dimension of the space $X_{0}$, and is denoted by $\operatorname{dim} X_{0}$. For infinite-dimensional spaces $X$, we write $\operatorname{dim} X=\infty$.

Let a subspace $X_{1} \subseteq X$ be such that the quotient space $X / X_{1}$ is infinite-dimensional. Then the dimension $\operatorname{dim}\left(X / X_{1}\right)$ is called the codimension of $X_{1}$ and is denoted by codim $X_{1}$. In this case, we say that the vector space $X$ is a finite-dimensional extension of its subspace $X_{1}$ and $X_{1}$ is a finite-dimensional contraction of $X$. If vectors $\tilde{e}_{i}$ form a base in $X / X_{1}$, then it is obvious that 
the vectors $e_{i} \in X, i=1, \ldots, n$, are linearly independent and $X$ is expanded into the direct sum $X=X_{1} \oplus\left[e_{1}, \ldots, e_{n}\right]$.

A nonnegative function $x \rightarrow|x|$ defined on a vector space $X$ is called a norm if

(1) the relation $|x|=0$ is equivalent to the relation $x=0$;

(2) the relation $|\lambda x|=|\lambda||x|$ holds for any complex $\lambda \in \mathbb{C}$;

(3) the inequality $|x+y| \leq|x|+|y|$ holds.

The last condition is called the triangle inequality.

It follows from (1)-(3) that $X$ is a metric space with respect to the metric $d(x, y)=|x-y|$. Respectively, all the notions of metric spaces are transferred to $X$. The set $B(a, r)=\{x \in X|| x-a \mid<$ $r\}$ is called the ball of radius $r$ centered at $a$. We say that the sequence $x_{n} \in X, n=1, \ldots$, converges in $X$ if there exists $x$ in $X$ such that for any ball centered at $x$, only a finite amount of elements of the sequence are located outside this ball. This requirement is equivalent to the requirement that $\left|x_{n}-x\right|_{X} \rightarrow 0$ as $n \rightarrow \infty$. The vector $x$ is called the limit of the sequence and this is denoted as follows: $x=\lim _{n \rightarrow \infty} x_{n}$ (the notation $x_{n} \rightarrow x$ in $X$ as $n \rightarrow \infty$ is also used). It is clear that converging sequences are bounded, i.e., their norms satisfy the inequality $\left|x_{n}\right| \leq C$, where the positive constant $C$ is independent of $n$.

We say that a sequence $x_{n}$ is a fundamental sequence (or a Cauchy sequence) if $\left|x_{n}-x_{m}\right| \rightarrow 0$ as $n, m \rightarrow \infty$, i.e., for any positive $\varepsilon$ there exists a number $N$ such that $\left|x_{n}-x_{m}\right| \leq \varepsilon$ provided that $n \geq N$ and $m \geq N$. A normed space $X$ is complete if any Cauchy sequence of its elements has a limit. Complete normed spaces are called Banach spaces.

Frequently, it is convenient to verify the completeness property by means of series. We say that a series $\sum_{k=1}^{\infty} x_{k}$ converges in $X$ if the sequence of its partial sums $s_{n}=x_{1}+\ldots+x_{n}$ converges to an element $s$ of $X$. In this case, the element $s$ is called the sum of the series. We recall the following Bergh-Löfström completeness criterion (see [2]).

Lemma 1.1.1. A normed vector space $X$ is complete if and only if the condition

$$
\sum_{k=1}^{\infty}\left|x_{k}\right|<\infty
$$

implies the convergence of the series $\sum x_{k}$ in $X$.

Proof. Assigning $s_{n}=x_{1}+\ldots+x_{n}$, we obtain that $\left|s_{n}-s_{m}\right| \leq\left|x_{m+1}\right|+\ldots+\left|x_{n}\right|$ provided that $n \geq m$ (by virtue of the triangle inequality).

Therefore, the sequence $s_{n}$ is fundamental by virtue of (1.1.1). In particular, the completeness of $X$ implies the convergence of the series $\sum x_{k}$.

Conversely, let the condition of the lemma be satisfied and a Cauchy sequence $y_{n}$ be given in $X$. It suffices to verify that there exists its subsequence $y_{n_{k}}$ such that it converges in $X$. To select this subsequence, we impose the condition

$$
\left|y_{n_{k+1}}-y_{n_{k}}\right| \leq \frac{1}{2^{k}}
$$

Then the sequence $x_{k}=y_{n_{k+1}}-y_{n_{k}}$ satisfies the condition (1.1.1). Since partial sums of the series $\sum x_{k}$ coincide with $y_{n_{k}}$, it follows that the last sequence converges and, therefore, $X$ is complete.

Let $X$ be a normed space and $Y$ be its closed subspace. For elements $\tilde{x}=\{x+y, y \in Y\}$ of the quotient space $X / Y$, we assign

$$
|\tilde{x}|=\inf _{y \in Y}|x+y|_{X}
$$

It is easy to see that this relation defines a norm in $X / Y$. Using Lemma 1.1.1, one can easily show that the completeness of $X$ implies the completeness of the quotient space $X / Y$. If the codimension of a closed subspaces id finite, then we say that it is a finite-codimensional subspace. 
hat

Norms $|\cdot|$ and $\mid \cdot],\left.\right|^{\prime}$ defined on $X$ are said to be equivalent if the exists a positive constant $C$ such

$$
|x|^{\prime} \leq C|x|, \quad|x| \leq C|x|^{\prime}
$$

for any $x$ from $X$. If this holds, then the convergence in $X$ with respect to one of these norms coincides with the convergence with respect to the other one. In particular, if $X$ is complete with respect to one of these norms, then it is also complete with respect to the other.

\section{Theorem 1.1.1.}

(a) Any finite-dimensional normed space is complete and any two its norms are equivalent.

(b) Let a normed space $X$ be a finite-dimensional extension of a Banach space $Y$ and their norms coincide on $Y$. Then $X$ is a Banach space and all its norms possessing this property coincide.

(c) Let $X$ be a Banach space and $Y$ be a finite-codimensional subspace. Then any subspace $X_{1} \supseteq Y$ possesses this property and $\operatorname{codim} X_{1}=\operatorname{codim} Y-\operatorname{dim}\left(X_{1} / Y\right)$.

Proof. (a) Consider a basis $e_{1}, \ldots, e_{n}$ of a finite-dimensional normed space $X$ such that any element $x$ from $X$ is uniquely represented in the form $x=\xi_{1} e_{1}+\ldots+\xi_{n} e_{n}$, where $\xi_{i} \in \mathbb{C}(i=1, \ldots, n)$. It suffices to verify that the original norm in $X$ is equivalent to the norm

$$
|x|^{\prime}=\max _{1 \leq i \leq n}\left|\xi_{i}\right| \text {. }
$$

The following inequality from (1.1.3) is obvious for the norms considered:

$$
|x| \leq \sum_{1}^{n}\left|\xi_{i}\right|\left|e_{i}\right| \leq\left(\sum_{1}^{n}\left|e_{i}\right|\right)|x|^{\prime} .
$$

To prove the opposite inequality, consider the positive function $f(\xi)=\left|\xi_{1} e_{1}+\ldots+\xi_{n} e_{n}\right|$ on the compact set $K=\left\{\xi \in \mathbb{C}^{n}, \max _{i}\left|\xi_{i}\right|=1\right\}$. Obviously, it is continuous on $K$ and, therefore, achieves its positive minimum $m$. Therefore, $|x| \geq m$ for any $x$ from $X$ such that $|x|^{\prime}=1$. Changing $x$ for $x /|x|^{\prime}$ with an arbitrary $x \in X$, we arrive at the second inequality of (1.1.3).

(b) Let $X=Y \oplus Z$ and $e_{1}, \ldots, e_{n}$ be a basis of $Z$. Then any element $x \in X$ can be uniquely represented in the form

$$
x=y+z(\xi), \quad z(\xi)=\xi_{1} e_{1}+\ldots \xi_{n} e_{n},
$$

where $y \in Y$ and $\xi \in \mathbb{C}^{n}$. For definiteness, select the norm in $\mathbb{C}^{n}$ by the relation $|\xi|=\max _{i}\left|\xi_{i}\right|$. Let us prove that the vector $\xi$ from $\mathbb{C}^{n}$ in the expansion (1.1.4) satisfies the estimate

$$
|\xi| \leq C|x|,
$$

where the positive constant $C$ is independent of $x$.

Indeed, if no such estimate takes place, then there exists a sequence $x^{k}$ from $X$ such that $\left|x^{k}\right|=1$ and $\left|\xi^{k}\right| \rightarrow+\infty$. Denoting $x^{k} /\left|\xi^{k}\right|$ by $x^{k}$ again, we obtain the relation $x^{k}=y^{k}+z\left(\xi^{k}\right)$, where $x^{k} \rightarrow 0$ and $\left|\xi^{k}\right|=1$ for any $k$. Due to the Bolzano-Weierstrass theorem, there exists a subsequence $\xi^{k_{s}}$ of the sequence $\xi^{k}$ such that $\xi^{k_{s}}$ converges to a vector $\xi$ from $\mathbb{C}^{n},|\xi|=1$. Therefore, $y^{k_{s}}=x^{k_{s}}-z\left(\xi^{k_{s}}\right) \rightarrow$ $-z(\xi)$ as $s \rightarrow \infty$. On the other hand, by virtue of the completeness of the space $Y$, the vector $y=-z(\xi)$ belongs to $Y$, which contradicts the uniqueness of the expansion (1.1.4).

Based on the expansion (1.1.4), introduce the norm $|x|^{\prime}=|y|+|\xi|$ in the normed space $X$. The space $X$ is isomorphic to $Y \times \mathbb{C}^{n}$ with respect to this norm. Hence, $X$ is a Banach space. It suffices to verify that the norms $|x|$ and $|x|^{\prime}$ are equivalent, i.e., the inequalities (1.1.3) hold. It is obvious that

$$
|x| \leq(1+M)|x|^{\prime}, M=\left|e_{1}\right|+\ldots+\left|e_{n}\right| .
$$

On the other hand, it follows from (1.1.5) that both terms of the expansion (1.1.4) satisfy the inequalities

$$
|z(\xi)| \leq M C|x|, \quad|y| \leq(1+M C)|x|,
$$

which leads to the opposite inequality in (1.1.3). 
(c) The property of the closeness of $X_{1}$ follows from (b): we replace $X$ by $X_{1}$. Assigning $Z_{1}=X_{1} \ominus Y$ and $Z_{2}=X \ominus X_{1}$, we obtain the expansion $X=Y \oplus Z$ with the finite-dimensional space $Z=Z_{1} \oplus Z_{2}$, which implies the dimensional relation of the lemma.

In the sequel, the norm in $X$ is selected up to an equivalent one. For example, in the direct-product Banach space $X=X_{1} \times \ldots \times X_{n}$, the relations $|x|=\max _{i}\left|x_{i}\right|_{X_{i}}$ and $|x|^{\prime}=\sum_{i}\left|x_{i}\right|_{X_{i}}$ define equivalent norms. In further sections, $X$ is assigned to be various function spaces, e.g., Lebesgue $L^{p}$-spaces or Hölder $C^{\mu}$-spaces. In this case, elements of the direct product $X^{n}=X \times \ldots \times X$ can be treated as $n$-vector-functions.

In the sequel, we use the following notation. If a Banach space $X$ of scalar functions is given, then the symbol $X$ denotes the direct-product space $X^{n}$ equipped with the norm $|x|=\max \left|x_{i}\right|_{X}$ (or any other equivalent norm). If such a notation causes a confusion, then we use the accurate notation $X^{n}$.

According to Theorem 1.1.1, any normed finite-dimensional space is a Banach space. Combining this fact with the Bolzano-Weierstrass theorem (used to prove Theorem 1.1.1), one can conclude that the unit ball of any normed finite-dimensional space is compact. The following Riesz theorem shows that the said property entirely characterizes finite-dimensional spaces.

Theorem 1.1.2. Let a normed space $X$ possess the Bolzano-Weierstrass property, i.e., any bounded sequence contains a Cauchy subsequence. Then this space is finite-dimensional.

Proof. The following property of normed spaces is used. If a subspace $X_{0} \subseteq X$ is closed, then there exists a vector $e$ from $X$ such that

$$
|e|=1 ; \quad|e-x| \geq 1 / 2, \quad x \in X_{0} .
$$

Indeed, let $a \in X$ and $a \notin X_{0}$. By virtue of the closeness of $X_{0}$, there exists a positive $r$ such that the ball $B(a, r)$ of radius $r$ centered at $a$ does not intersect $X_{0}$. Let $r$ be selected such that the similar ball of radius $2 r$ intersects $X_{0}$, i.e., there exists $b$ from $X_{0}$ such that $|a-b| \leq 2 r$. Then $|e-x|=|a-b|^{-1}|a-b-| a-b|x| \geq|a-b|^{-1} r \geq 1 / 2$ for any $x$ from $X_{0}$, where $e=(a-b) /|a-b|$, which proves (1.1.6).

Now, assume that $\operatorname{dim} X=\infty$. Then $X$ contains an increasing sequence of finite-dimensional subspaces $X_{1} \subseteq X_{2} \subseteq \ldots$ and it follows from (1.1.6) that there exists a sequence of unit vectors $e_{k}$ from $X_{k}$ such that

$$
\left|e_{n}-e_{m}\right| \geq 1 / 2, \quad n \neq m .
$$

By assumption, there exists a Cauchy subsequence $e_{n_{k}}$, which is impossible due to (1.1.7).

In the sequel, we consider families $\left(X_{i}, i \in I\right)$ of Banach spaces contained in a vector space. The specified property is satisfied if this family is a lattice with respect to embedding, i.e., for any finite subset $I_{0} \subseteq I$ there exist $k^{0}$ and $k^{1}$ from $I$ such that

$$
X_{k^{0}} \subseteq X_{i} \subseteq X_{k^{1}}, \quad i \in I_{0} .
$$

In particular, the vector space $X=\bigcup_{i} X_{i}$ consisting of all finite sums $\sum x_{i}$ with elements $x_{i}$ from $X_{i}$ is well defined. Consider the case $I_{0}=\{1,2\}$ of a pair of spaces. It is easy tom see that $X_{1} \cap X_{2}$ is a Banach space with respect to the norm

$$
|x|=\max \left(|x|_{X_{1}},|x|_{X_{2}}\right) .
$$

Under a natural assumption, a similar result for $X_{1}+X_{2}$ holds.

Lemma 1.1.2. Let Banach spaces $X_{j}$ be embedded into a separable topological space $X$. Then the relation

$$
|x|=\inf _{x=x_{1}+x_{2}}\left(\left|x_{1}\right|_{X_{1}}+\left|x_{2}\right|_{X_{2}}\right), \quad x_{i} \in X_{i},
$$

defines a norm in $X_{1}+X_{2}$ such that it is a Banach space with respect to this norm. 
Proof. It easy to check whether the corresponding norm axioms are satisfied for (1.1.9). For example, if $|x|=0$, then, by definition, there exist sequences $x_{j n}$ from $X_{j}$ such that they converge to zero and $x=x_{1 n}+x_{2 n}$ for any $n$. Since $X_{1}$ and $X_{2}$ are embedded into a separable topological space, it follows that $x=0$. The triangle inequality is verified in the standard way. Let $x=x_{1}+x_{2}$ and $y=y_{1}+y_{2}$, where $x_{i}, y_{i} \in X_{i}$. Fix $x_{i}$ and consider the inequality

$$
|x+y|-\left|x_{1}\right|_{1}-\left|x_{2}\right|_{2} \leq\left|y_{1}\right|_{1}+\left|y_{2}\right|_{2},
$$

where $\mid \cdot{ }_{i}$ denotes the norm in $X_{i}$. Then, from the definition of (1.1.8), we have the inequality

$$
|x+y|-\left|x_{1}\right|_{1}-\left|x_{2}\right|_{2} \leq\left|y_{1}+y_{2}\right| \text {. }
$$

Transporting terms with $\left|x_{j}\right|_{j}$ to the right-hand side and repeating this procedure, we obtain the inequality $|x+y| \leq|x|+|y|$.

Thus, the relation (1.1.9) defines a norm. Using Lemma 1.1.1, we easily show that the space $X_{1}+X_{2}$ is complete with respect to this norm.

We say that a subset $K$ of a Banach space is relatively compact if any sequence of its elements contains a converging subsequence; if a relatively compact subset $K$ of a Banach space is closed, i.e., the limit of any converging subsequence belong to $K$, then we say that $K$ is compact. Let us provide a classical compactness criterion for subsets of the space $X=C(Q)$ consisting of functions defined and continuous a metric compact set $Q$. It is well known that any function $f$ continuous on a compact set $Q$ is bounded and uniformly continuous. The first property means the existence of a positive constant $M$ such that $|f(x)| \leq M, x \in Q$, while the second one is as follows: for any positive $\varepsilon$ there exists a positive $\delta$ such that $|f(x)-f(y)| \leq \varepsilon$ provided that $d(x, y) \leq \delta$, where $d(x, y)$ is the metric on $Q$. If there exists a set $K \subseteq C(Q)$ such that the above properties are satisfied uniformly for all functions $f$ from $K$, then we say that the set of these functions is uniformly bounded and equicontinuous. The norm of the vector space $C(X)$ is defined by the relation

$$
|f|=\max _{x \in Q}|f(x)| .
$$

This is a Banach space with respect to the above norm. The following well-known theorem (see, e.g., [56]) provides a compactness criterion for subsets of this space.

Theorem (Arzela-Ascoli). A set $K \subseteq C(Q)$ is relatively compact if and only if it is uniformly bounded and equicontinuous.

To conclude, consider the case where the compact set $Q$ is a piecewise-smooth curve $\Gamma$ on the complex plane $\mathbb{C}$. Recall that this curve as a union a finite set of smooth arcs such that only their ends might be points of their pairwise intersections. If connected components of $\Gamma$ are homeomorphic to a circle, we say that $\Gamma$ is a piecewise-smooth contour (it might be simple or composite regarding the amount of these components).

Theorem (Walsh).

(a) Let $\Gamma$ be a piecewise-smooth curve on the complex plane. Then the set of rational functions such that their poles lie outside $\Gamma$ is dense in $C(\Gamma)$.

(b) Let a finite domain $D$ of the plane be bounded by a simple piecewise-smooth contour and $A(\bar{D})$ be a closed subspace of $C(\bar{D})$, consisting of functions analytic in $D$. Then the set of polynomials is dense in $A(\bar{D})$.

(c) Let (b) be satisfied, $z_{0} \in D$, and $u_{n}(z)=\left(z-z_{0}\right)^{n}, n=0, \pm 1, \ldots$ Then the set of all rational functions represented by finite sums

$$
R(z)=\sum c_{n} u_{n}(z), \quad c_{n} \in \mathbb{C}
$$

is dense in $C(\Gamma)$. 
Note that the third assertion of the theorem follows from the first and second ones. Indeed, any rational function $R(z)$ such that its poles lie outside $\Gamma$ can be represented by a sum $R_{1}+R_{2}$, where the poles of $R_{1}$ lie outside $\bar{D}$. Then, by virtue of (b), the function $R_{1}$ can be approximated by polynomials, i.e., by finite sums of (1.1.10) with respect to nonnegative integers $n$. To prove the corresponding assertion for $R_{2}$, apply the transformation $z \rightarrow 1 /\left(z-z_{0}\right)$ first; then the same arguing is used.

\subsection{Bounded Operators}

We say that a linear operator $N$ acting from a Banach space $X$ to a Banach space $Y$ is bounded if there exists a positive constant $C$ such that

$$
|N x|_{Y} \leq C|x|_{X}, \quad x \in X .
$$

In the same way, the notion of the boundedness is introduced for bilinear maps $B: X_{1} \times X_{2} \rightarrow Y$ by means of the inequality

$$
\left|B\left(x_{1}, x_{2}\right)\right|_{Y} \leq C\left|x_{1}\right|_{X_{1}}\left|x_{2}\right|_{X_{2}}, \quad x_{i} \in X_{i}
$$

Both for linear and bilinear maps, the boundedness is equivalent to the continuity.

The class $N: X \rightarrow Y$ of all bounded operators is a vector space denoted by $\mathcal{L}(X, Y)$. For $X=Y$, the brief notation $\mathcal{L}(X)=\mathcal{L}(X, Y)$ is used. The least constant $C$ in (1.2.1) is equal to

$$
|N|_{\mathcal{L}}=\sup _{|x| \leq 1}|N x|_{Y}
$$

i.e., to the norm in the vector space $\mathcal{L}(X, Y)$. Note that the composition $M N$ of bounded operators $M$ and $N$ is a bounded operator and

$$
|M N|_{\mathcal{L}} \leq|M|_{\mathcal{L}}|N|_{\mathcal{L}}
$$

Lemma 1.2.1. Let a space $Y$ be complete, the sequence of operators $N_{k}$ be bounded in $\mathcal{L}(X, Y)$, and there exist a dense subspace $X_{0} \subseteq X$ such that the limit

$$
\lim _{k \rightarrow \infty} N_{k} x=N x
$$

with respect to the norm $Y$ exists for any $x$ from $X_{0}$. Then the specified limit exists for any $x$ from $X$ and the operator $N$ belongs to $\mathcal{L}(X, Y)$. In particular, the normed space $\mathcal{L}(X, Y)$ is complete.

Proof. By assumption, the inequality

$$
\left|N_{k} x\right|_{Y} \leq C|x|_{X}
$$

holds and the positive constant $C$ is independent of $k$. Since (1.2.3) holds for any $x$ from $X_{0}$, it follows that the said inequality is extended to $N$. Hence, taking into account the fact that $X_{0}$ is dense in $X$, we conclude that the operator $N$ can be extended as an element of $\mathcal{L}(X, Y)$; we denote this element by $N$.

For given positive $\varepsilon$ and $x$ from $X$, select $x_{0}$ from $X_{0}$ and a number $n$ to satisfy the conditions $\left|x-x_{0}\right| \leq \varepsilon$ and $\left|\left(N_{k}-N\right) x_{0}\right| \leq \varepsilon, k \geq n$. Then, taking into account (1.2.4), we see that the following inequality holds provided that $k \geq n$ :

$$
\left|\left(N_{k}-N\right) x\right| \leq\left|N_{k}\left(x-x_{0}\right)\right|+\left|N\left(x-x_{0}\right)\right|+\left|\left(N_{k}-N\right) x_{0}\right| \leq(2 C+1) \varepsilon
$$

this means that (1.2.3) holds for any $x$.

The second assertion of the lemma follows from the first one. Let $N_{k}$ from $\mathcal{L}(X, Y)$ be a Cauchy sequence. Then this sequence is bounded in $\mathcal{L}(X, Y)$. By virtue of the obvious inequality

$$
\left|\left(N_{m}-N_{n}\right) x\right| \leq\left|N_{m}-N_{n}\right| \mathcal{L}|x|
$$

and the completeness of $Y$, limit (1.2.3) exists for any $x$. It remains to verify that $N_{k} \rightarrow N$ in $\mathcal{L}(X, Y)$.

For a given positive $\varepsilon$, select a number $n_{0}$ such that the inequality $\left|N_{m}-N_{n}\right|_{\mathcal{L}} \leq \varepsilon$ holds provided that $n \geq n_{0}$ and $m \geq n_{0}$. Then (1.2.5) passes into the inequality $\left|\left(N_{m}-N_{n}\right) x\right| \leq \varepsilon|x|, n, m \geq n_{0}$. 
Pass to the limit as $n \rightarrow \infty$ in this inequality, we obtain that $\left|N_{n}-N\right|_{\mathcal{L}} \leq \varepsilon, n \geq n_{0}$. Hence, the space $\mathcal{L}(X, Y)$ is complete.

In the sequel, unless the opposite is stated, we assume that any considered normed space is a Banach space and the term "operator" is treated as "bounded linear operator."

If $N \in \mathcal{L}(X, Y)$, then the subspace ker $N=\{x \mid N x=0\}$ called the kernel of the operator $N$ is closed. However, the closeness of its image $\operatorname{Im} N=\{N x, x \in X\}$ in $Y$ is not guaranteed. Operators $N: X \rightarrow Y$ with zero kernels (i.e., one-to-one operators) are called embeddings of Banach spaces. For example, we say that a family of Banach spaces $X_{i}, i \in I$, where $I \subseteq \mathbb{R}$, monotonously increases with respect to $i$ (in the sense of embeddings) if $X_{i} \subseteq X_{j}$ for $i \leq j$ and the identical embedding operator $X_{i} \rightarrow X_{j}$ is bounded, i.e.,

$$
|x|_{X_{j}} \leq C|x|_{X_{i}}, \quad i \leq j .
$$

For a family of spaces forming a lattice, embeddings (1.1.8) are treated in the same sense.

We say that an operator $N$ from $\mathcal{L}(X, Y)$ is invertible if $\operatorname{ker} N=0, \operatorname{Im} N=Y$, and the inverse linear map $N^{-1}$ belongs to $\mathcal{L}(Y, X)$. In other words, invertible operators realize isomorphisms of Banach spaces.

Obviously, the product of two invertible operators is also an invertible operator.

Theorem 1.2.1. The set $G(X, Y)$ of invertible operators from $X$ to $Y$ is open in $\mathcal{L}(X, Y)$ and the map $N \rightarrow N^{-1}$ continuously takes $G(X, Y)$ to $G(Y, X)$.

Proof. The following assertion is valid: if $A \in \mathcal{L}(X)$ and $|A|_{\mathcal{L}} \leq q<1$, then the operator $1-A$ is invertible and its inverse operator is determined by the converging series

$$
(1-A)^{-1}=\sum_{n=0}^{\infty} A^{n} .
$$

Indeed, by virtue of (1.2.2), we have the estimate $\left|A^{n}\right|_{\mathcal{L}} \leq|A|_{\mathcal{L}}^{n} \leq q^{n}$. Taking into account the completeness of $\mathcal{L}(X, Y)$, we conclude that the series at the right-hand side of (1.2.6) converges. Let $B$ from $\mathcal{L}(X)$ be its sum. Then

$$
(1-A) B=\sum_{n=0}^{\infty} A^{n}-\sum_{n=0}^{\infty} A^{n+1}=1 .
$$

The relation $B(1-A)=1$ is verified in the same way. Hence, $1-A$ is invertible and $(1-A)^{-1}=B$.

For $|A|_{\mathcal{L}} \leq q<1$, it follows from (1.2.6) that

$$
\left|(1-A)^{-1}-1\right|_{\mathcal{L}} \leq(1-q)^{-1}|A|_{\mathcal{L}}
$$

and, therefore, $(1-A)^{-1} \rightarrow 1$ as $|A|_{\mathcal{L}} \rightarrow 0$.

Now, let an operator $N$ from $G(X, Y)$ and $B$ from $\mathcal{L}(X, Y)$ satisfy the condition $\left|N^{-1}\right|_{\mathcal{L}}|B|_{\mathcal{L}}<1$. Then $N^{-1} B \in \mathcal{L}(X)$ and $\left|N^{-1} B\right|_{\mathcal{L}}<1$. Therefore, by virtue of the previous assertion, $N-B=$ $N\left(1-N^{-1} B\right) \in G(X, Y)$. Hence, the set $G(X, Y)$ is open.

If $B \rightarrow 0$ in $\mathcal{L}(X, Y)$, then, as we prove above, $\left[1-\left(N^{-1} B\right)\right]^{-1} \rightarrow 1$ in $\mathcal{L}(X)$ and, therefore, $(N-B)^{-1} \rightarrow N^{-1}$ in $\mathcal{L}(X, Y)$.

The next basic theorem (see [56]) shows that the boundedness requirement for the inverse operator $N^{-1}$ can be taken off the invertibility definition for $N$.

Theorem (The Banach open maps theorem). Let $N \in \mathcal{L}(X, Y)$ and $\operatorname{Im} N=Y$. Then the linear map $N$ is bounded in the following sense: for any open set $G \subseteq X$, the image $N(G)$ is open in $Y$. In particular, if $\operatorname{ker} N=0$, then the inverse map $N^{-1}$ continuously takes $Y$ into $X$, i.e., the operator $N$ is invertible. 
For example, the canonical quotient map $x \rightarrow \tilde{x}=x+X_{0}$ from $X$ to the Banach quotient space $X / X_{0}$, where $X_{0}$ is a closed subspace of $X$, is an example of an operator "onto" for this theorem. Indeed, by the definition of (1.1.3), the norm satisfies the inequality $|\tilde{x}| \leq|x|$. Hence, the map $x \rightarrow \tilde{x}$ is bounded. It is clear that its image coincides with $X / X_{0}$. Hence, due to the Banach theorem, this map is open.

Another example of an operator "onto" is the expansion $X$ into the direct sum of closed subspaces $X_{i}$ :

$$
X=X_{1} \oplus \ldots \oplus X_{n}
$$

Due to the Banach theorem, the linear map $\left(x_{1}, \ldots, x_{n}\right) \rightarrow x=x_{1}+\ldots x_{n}$ boundedly takes $X_{1} \times \ldots \times$ $X_{n}$ into $X$ and its image is $X$. Hence, this operator is invertible and the norm in $X$ is equivalent to the norm of the space $X_{1} \times \ldots \times X_{n}$.

In particular, the operators $P_{i} x=x_{i}$, where $x=x_{1}+\ldots+x_{n}, x_{i} \in X_{i}$, are bounded in $X$. Obviously, they possess the property

$$
P_{i} P_{j}=\delta_{i j} P_{i}, \quad P_{1}+\ldots+P_{n}=1,
$$

where $\delta_{i j}$ denotes the Kronecker symbol, i.e., $\delta_{i j}=0$ if $i \neq j$ and $\delta_{i i}=1$.

If an operator $P$ from $\mathcal{L}(X)$ is such that $P^{2}=P$, then it is called a projector. If $P$ is a projector, then $Q=1-P$ is also a projector. For any projector, its image $X_{1}=\operatorname{Im} P$ is a closed subspace and $X=X_{1} \oplus X_{2}$ and $X_{2}=\operatorname{Im} Q=\operatorname{ker} P$. In the same way, the expansion (1.2.8) of the identity operator into a sum of projectors is equivalent to the expansion (1.2.7).

We say that a closed subspace $X_{1} \subseteq X$ is complemented if there exists a closed subspace $X_{2} \subseteq X$ such that $X=X_{1} \oplus X_{2}$. As we note above, this is equivalent to the existence of a projector $P$ from $\mathcal{L}(X)$ such that $\operatorname{Im} P=X_{1}$. Obviously, any closed subspace $X_{1} \subseteq X$ of a finite codimension is complemented. Below, we show that finite-dimensional subspaces are also complemented.

If a space $Y$ is represented by a direct sum $Y_{1} \oplus \ldots \oplus Y_{m}$ of closed subspaces, then each operator $N$ from $\mathcal{L}(X, Y)$ can be identified with an operator $m \times n$-matrix $\left(N_{i j}\right), N_{i j} \in \mathcal{L}\left(X_{j}, Y_{i}\right)$, acting as follows:

$$
(N x)_{i}=\sum_{j=1}^{n} N_{i j} x_{j}, \quad i=1, \ldots, m .
$$

The notion of one-side invertible operators is closely related to the notion of invertible ones. Let bounded operators $N: X \rightarrow Y$ and $R: Y \rightarrow X$ be such that their product $N R$ is the identity operator. Then we say that the operator $N$ is invertible from the right, the operator $R$ is invertible from the left, $R$ is the right inverse operator for $N$, and $N$ is the left inverse operator for $R$. It is easy to characterize the classes of such operators completely.

\section{Theorem 1.2.2.}

(a) An operator $N$ from $\mathcal{L}(X, Y)$ is invertible from the right if and only if $\operatorname{Im} N=Y$ and $\operatorname{ker} N$ is complemented in $X$. The set of all such operators is open in $\mathcal{L}(X, Y)$.

(b) An operator $R$ from $\mathcal{L}(Y, X)$ is invertible from the left if and only if $\operatorname{Im} R$ is complemented in $X$ and $\operatorname{ker} R=0$. The set of all such operators is open in $\mathcal{L}(Y, X)$.

Proof. If $N R=1$, then $\operatorname{Im} N=Y$ and $\operatorname{ker} R=0$. Since $R N R N=R N$, it follows that the operator $P=R N$ from $\mathcal{L}(X)$ is a projector such that $\operatorname{ker} P=\operatorname{ker} N$ and $\operatorname{Im} P=\operatorname{Im} R$. In particular, $X=\operatorname{ker} N \oplus \operatorname{Im} R$, i.e., the kernel ker $N$ and the image $\operatorname{Im} R$ are complemented in $X$. This proves the first assertion of (a) and the first assertion of (b).

Further, let an operator $N$ from $\mathcal{L}(X, Y)$ be such that $\operatorname{Im} N=Y$ and ker $N$ is complemented in $X$. Then the restriction $N_{1}$ of the operator $N$ to the closed subspace $X_{1}=X \ominus \operatorname{ker} N$ is an invertible operator in $\mathcal{L}\left(X_{1}, Y\right)$ and the inverse operator $R=N_{1}^{-1}$ treated as an element of $\mathcal{L}(Y, X)$ is right inverse for $N$.

The corresponding assertion of (b) is proved in the same way. Let the image $X_{1}=\operatorname{Im} R$ of an operator $R$ from $\mathcal{L}(Y, X)$ be complemented in $X$ and ker $R=0$. Then $R$ admits a left inverse operator 
$N_{1}$ from $\mathcal{L}\left(X_{1}, Y\right)$. If an operator $P$ from $\mathcal{L}(X)$ is a projector of $X$ onto $X_{1}$, then the operator $N=N_{1} P$ is a left inverse for $R$.

The last assertion of (a) and the last assertion of (b) are proved in the same way as Theorem 1.2.1.

If $Y=\mathbb{C}$, then elements of the space $\mathcal{L}(X, \mathbb{C})$ are called linear functionals, while the space $\mathcal{L}(X, \mathbb{C})$ itself is called the adjoint space to $X$ and is denoted by $X^{*}$. Due to the known Hahn-Banach theorem (see [56]), any functional $x_{0}^{*}$ from $X_{0}^{*}$, where $X_{0}$ is a closed subspace of $X$, can be extended as a bounded functional $x^{*}$ from $X^{*}$ such that the norm of $x^{*}$ is equal to the norm of $x_{0}^{*}$. For our purposes, the following particular case of this theorem is sufficient.

Theorem (H. Hahn, S. Banach). For any closed subspace $X_{0} \subseteq X$ and any vector $x_{0}$ located outside $X_{0}$ there exists a bounded linear functional $x^{*}$ from $X^{*}$ such that its its norm is equal to one, $x^{*}\left(x_{0}\right)=$ $\left|x_{0}\right|$, and $x^{*}(x)=0, x \in X_{0}$.

Note that the second part of the theorem follows from the first part applied to the quotient space $X / X_{0}$.

In particular, this theorem implies that the norm of any vector $x$ is equal to

$$
|x|=\sup _{\left|x^{*}\right| \leq 1}\left|x^{*}(x)\right| \text {. }
$$

In other words, if $x$ is treated as a linear functional on $X^{*}$ with respect to the bilinear form

$$
\left(x, x^{*}\right)=x^{*}(x),
$$

then the norm of this functional coincides with the norm $|x|$. Thus, we obtain a canonical isometric embedding $X \subseteq\left(X^{*}\right)^{*}$. We say that a space $X$ is reflexive, if the image of this embedding coincides with $\left(X^{*}\right)^{*}$.

We say that a vector $x$ from $X$ and a vector $x^{*}$ from $X^{*}$ are orthogonal with respect to form (1.2.9) and denote this by the symbol $x \perp x^{*}$ if $\left(x, x^{*}\right)=0$. If $Y \subseteq X^{*}$, then the notation $x \perp Y$ means that $\left(x, x^{*}\right)=0$ for any $x^{*}$ from $Y$. The notation $x^{*} \perp Y$ means the same for $Y \subseteq X$. The set $\{x \in X, x \perp Y\}$ is a closed subspace of $X$, it is denoted by $Y^{\perp}$, and it is called the orthogonal complement of $Y$. The orthogonal complement $Y^{\perp} \subseteq X^{*}$ for $Y \subseteq X$ is defined in the same way.

It follows from the Hahn-Banach theorem that the following relation holds for any closed subspace $Y \subseteq X$ :

$$
\left(Y^{\perp}\right)^{\perp}=Y, \quad Y \subseteq X
$$

Really, all elements of $Y$ are orthogonal to $Y^{\perp}$ by definition. Therefore, $Y \subseteq\left(Y^{\perp}\right)^{\perp}$. If this inclusion is not an equality and $x_{0} \in\left(Y^{\perp}\right)^{\perp}, x_{0} \notin Y$, then, due to the Hahn-Banach theorem, there exists a functional $x^{*}$ from $X^{*}$ such that $x^{*}\left(x_{0}\right) \neq 0$ and $x^{*}$ vanishes on $Y$. In other words, $\left(x_{0}, x^{*}\right) \neq 0$ and $x^{*} \in Y^{\perp}$. However, this contradicts the inclusion $x_{0} \in\left(Y^{\perp}\right)^{\perp}$.

If the subspace $Y \subseteq X$ is closed, then the composition of any linear functional $f$ from $(X / Y)^{*}$ with the canonical map $x \rightarrow \tilde{x}$ defines an element $x^{*}(x)=f(\tilde{x})$ of the space $X^{*}$, vanishing on $Y$, i.e., an element $x^{*}$ from $Y^{\perp}$. The inverse assertion is also valid: any element $x^{*}$ of $Y^{\perp}$ can be represented in the specified way. Thus, the vector spaces $(X / Y)^{*}$ and $Y^{\perp}$ are isomorphic and therefore, their dimensions are equal. Hence, codim $Y=\operatorname{dim} Y^{\perp}$. Assigning $Z=Y^{\perp}$ and taking into account (1.2.10), we obtain the dual relation $\operatorname{dim} Z=\operatorname{codim} Z^{\perp}$ for the finite-dimensional space $Z \subseteq X^{*}$.

Also, note that the following relation holds for any two finite-dimensional subspaces $Z_{j} \subseteq X^{*}$, $j=1,2$ :

$$
Y_{1} \cap Y_{2}=\left(Z_{1}+Z_{2}\right)^{\perp}, \quad Y_{j}=Z_{j}^{\perp} .
$$

Indeed, since $Z_{j} \subseteq Z_{1}+Z_{2}$, we have the relation $\left(Z_{1}+Z_{2}\right)^{\perp} \subseteq Y_{j}$ and, therefore, $\left(Z_{1}+Z_{2}\right)^{\perp} \subseteq Y_{1} \cap Y_{2}$. Conversely, the subspace $Y_{1} \cap Y_{2}$ is orthogonal to $Z_{j}, j=1,2$, and, therefore, is orthogonal to $Z_{1}+Z_{2}$; hence, $Y_{1} \cap Y_{2} \subseteq\left(Z_{1}+Z_{2}\right)^{\perp}$. 
Relation (1.2.11) shows that the intersection of any two finite-codimensional subspaces is also a finite-codimensional subspace.

We say that vector systems $e_{1}, \ldots, e_{n}$ from $X$ and $e_{1}^{*}, \ldots, e_{n}^{*}$ from $X^{*}$ are biorthogonal if $\left(e_{i}, e_{j}^{*}\right)=\delta_{i j}$, where $\delta_{i j}$ denotes the Kronecker symbol. Obviously, each of these two systems is linearly independent. This immediately follows from the definition of the fact that the operator $P$ acting according to the relation

$$
P x=\sum_{1}^{n}\left(x, e_{i}^{*}\right) e_{i}
$$

is a projector such that its image is the subspace spanned by $e_{1}, \ldots, e_{n}$, while its kernel ker $P$ coincides with the orthogonal complement to the subspace spanned by $e_{1}^{*}, \ldots, e_{n}^{*}$.

Lemma 1.2.2. Let subspaces $X_{0} \subseteq X$ and $X_{1} \subseteq X^{*}$ be such that $X_{0}$ is finite-dimensional and $X_{0}$ does not contain nonzero elements orthogonal to $X_{1}$. Then, for any base of $X_{0}$, the subspace $X_{1}$ contains a vector system biorthogonal to this base. A similar assertion is valid in the case where $X_{0} \subseteq X^{*}$ and $X_{1} \subseteq X$.

In particular, any finite-dimensional space $X_{0}$ is complemented, i.e., there exists a projector $P$ such that $\operatorname{Im} P=X_{0}$. If a closed subspace $Y \subseteq X$ is such that $X_{0} \cap Y=0$, then it is possible to select $P$ such that the condition $\operatorname{ker} P \supseteq Y$ is satisfied.

Proof. In $X_{0}$, select a base $e_{1}, \ldots, e_{n}$ and consider the linear operator $T$ from $\mathcal{L}\left(X_{1}, \mathbb{C}^{n}\right)$ acting according to the relation $\left(T x^{*}\right)_{i}=\left(e_{i}, x^{*}\right)$. It is an operator acting "onto," i.e., $\operatorname{Im} T=\mathbb{C}^{n}$. If, to the contrary, there exists a nonzero vector $\eta=\left(\eta_{1}, \ldots, \eta_{n}\right)$ from $\mathbb{C}^{n}$ such that $\sum_{i} \eta_{i}\left(T x^{*}\right)_{i}=0$ for any $x^{*}$ from $X_{1}$, then, for the vector $x=\sum_{i} \eta_{i} e_{i}$, the relation $\left(x, x^{*}\right)=0, x^{*} \in X_{1}$, holds. In other words, the nonzero element $x$ from $X_{0}$ is orthogonal to $X_{1}$, which contradicts the assumption of the lemma. Thus, $\operatorname{Im} T=\mathbb{C}^{n}$ and there exist $e_{i}^{*}$ from $X_{1}$ such that $T e_{1}^{*}=(1,0, \ldots, 0), \ldots, T e_{n}^{*}=(0,0, \ldots, 1)$. This means that $\left(e_{i}, e_{j}^{*}\right)=\delta_{i j}$. The case where $X_{0} \subseteq X^{*}$ and $X_{1} \subseteq X$ is considered in the same way.

The last assertion of the lemma follows from the first one provided that $X_{1}$ is the subspace $Y^{\perp}$, which satisfies the assumption of the first part of the lemma by virtue of (1.2.10).

If $N \in \mathcal{L}(X, Y)$, then the orthogonal complement $(\operatorname{Im} N)^{\perp} \subseteq Y^{*}$ of its image is called its cokernel and is denoted by coker $N$. By virtue of (1.2.10), we have the relation

$$
\overline{\operatorname{Im} N}=(\operatorname{coker} N)^{\perp} \text {. }
$$

This means that if the image $\operatorname{Im} N$ of the operator $N$ is closed, then the solvability of the equation $N x=y$ is equivalent to the orthogonality of the right-hand side $y$ to the cokernel of the operator $N$.

It is not guaranteed that the adjoint space $Y^{*}$ and canonical form (1.2.9) related to it can be described for any particular case. Instead, it is more convenient to consider a Banach space $Y^{\prime}$ and a bounded bilinear form $\left\langle y, y^{\prime}\right\rangle$ on $Y \times Y^{\prime}$. This form is assumed to be nondegenerate in the following sense: $y=0$ if $\left\langle y, y^{\prime}\right\rangle=0$ for any $y^{\prime}$ from $Y^{\prime}$ and $y^{\prime}=0$ if $\left\langle y, y^{\prime}\right\rangle=0$ for any $y$ from $Y$. The triple $\left(Y, Y^{\prime},\langle\rangle,\right)$ is called the duality structure such that the space $Y$ is endowed with. It follows from the Hahn-Banach theorem that the form (1.2.9) is nondegenerate and, therefore, the triple $\left(X, X^{*},(),\right)$ defines the so-called canonical duality structure.

The notion of the orthogonality and orthogonal complement $Z^{\perp}$ with respect to the form $\left\langle y, y^{\prime}\right\rangle$ is defined as above. Since the form $\langle\cdot, \cdot\rangle$ is nondegenerate, it follows that any element $y^{\prime}$ of $Y^{\prime}$ can be identified with the linear functional $y \rightarrow\left\langle y, y^{\prime}\right\rangle$ of the adjoint space $Y^{*}$. Thus, we have the canonical embeddings $Y^{\prime} \subseteq Y^{*}$ and $Y \subseteq\left(Y^{\prime}\right)^{*}$. This implies that if $Y$ or $Y^{\prime}$ is finite-dimensional, then another space is also finite-dimensional and their dimensions coincide.

Indeed, assume that, e.g., $Y$ is finite-dimensional. Then, by virtue of the embedding $Y^{\prime} \subseteq Y^{*}$, the space $Y^{\prime}$ is finite-dimensional and $\operatorname{dim} Y^{\prime} \leq \operatorname{dim} Y^{*}=\operatorname{dim} Y$. In the same way, the embedding $Y \subseteq\left(Y^{\prime}\right)^{*}$ yields the opposite inequality for the dimensions. 
If the cokernel coker $N$ is contained in $Y^{\prime}$ with respect to the embedding $Y^{\prime} \subseteq Y^{*}$, then the orthogonality in the relation (1.2.13) can be treated as the orthogonality with respect to the bilinear form $\left\langle y, y^{\prime}\right\rangle$.

To any operator $N \in \mathcal{L}(X, Y)$, one can assign a linear operator $N^{*}: Y^{*} \rightarrow X^{*}$ mapping any linear functional $y^{*} \in Y^{*}$ to the functional $x^{*}=N^{*} y^{*}$ from $X^{*}$ according to the relation $\left(N^{*} y^{*}\right)(x)=y^{*}(N x)$, $x \in X$. We say that this operator is adjoint to $N$. Since $\left|N^{*} y^{*}\right| \leq|N|_{\mathcal{L}}\left|y^{*}\right|$, where $|\cdot . \cdot|$ are the norms in the adjoint spaces, it follows that the operator $N^{*}$ belongs to $\mathcal{L}\left(Y^{*}, X^{*}\right)$ and its norm does not exceed $|N|_{\mathcal{L}}$. Using the the Hahn-Banach theorem, one can verify that these norms coincide.

From the definition of adjoint operators, it follows that the cokernel coker $N$ coincides with the kernel ker $N^{*}$ of the adjoint operator. Hence, in (1.2.13), one can replace coker $N$ by $\operatorname{ker} N^{*}$. In particular, if the image $\operatorname{Im} N$ is finite-codimensional, then its codimension coincides with the dimension of the kernel ker $N^{*}$.

If $N \in \mathcal{L}(X, Y)$ and $\operatorname{Im} N$ is finite-dimensional, then we say that $N$ is finite-dimensional; denote the class of such operators by $\mathcal{T}_{0}(X, Y)$. Obviously, the composition of two operators is finite-dimensional if at least one of them is finite-dimensional. In general, the subspace $\mathcal{T}_{0}$ of finite-dimensional operators is not closed in $\mathcal{L}$. The subspace of compact (completely continuous) operators defined by $\mathcal{T}(X, Y)$ is preferable in this sense. We say that an operator $N$ from $\mathcal{L}(X, Y)$ is compact if for any bounded sequence $\left\{x_{n}\right\}$ from $X$ there exists a converging subsequence of the sequence $\left\{N x_{n}\right\}$ from $Y$. An equivalent definition is as follows: an operator $N$ from $\mathcal{L}(X, Y)$ is compact if the image $N(B)$ of the unit ball $B \subseteq X$ is relatively compact in $Y$.

From the definition, we see that the composition of two bounded operators is compact if one of them is compact. Also, it is clear that $\mathcal{T}_{0} \subseteq \mathcal{T}$, i.e., any finite-dimensional operator is compact.

Theorem 1.2.3. The subspace $\mathcal{T}(X, Y)$ of compact operators is closed in $\mathcal{L}(X, Y)$ and the belonging of $N$ to $\mathcal{T}(X, Y)$ implies that $N^{*} \in \mathcal{T}\left(Y^{*}, X^{*}\right)$.

Proof. Let a sequence of operators $N_{s}$ from $\mathcal{T}(X, Y)$ converge to $N$ from $\mathcal{L}(X, Y)$ with respect to the operator norm. Take a sequence $x_{k}$ from $X,\left|x_{k}\right| \leq 1$, and consider its subsequence $x_{k, 1}, k=1, \ldots$, such that $N_{1} x_{k, 1}$ converges to $Y$. Let a sequence $x_{k, s}$ from $X$ be a subsequence $x_{k, s-1}$ for positive integers $s$ exceeding two, where $N_{s} x_{k, s}, k=1, \ldots$, converges in $Y$. Then the subsequence $N x_{k k}$ also converges.

Indeed, fix a positive $\varepsilon$ and select $s$ to satisfy the condition $\left|N_{s}-N\right|_{\mathcal{L}} \leq \varepsilon$. Then

$$
\left|\left(N_{s}-N\right) x_{k r}\right| \leq \varepsilon .
$$

Let $n \geq s$ and

$$
\left|N_{s}\left(x_{k s}-x_{r s}\right)\right| \leq \varepsilon, \quad k, r \geq n .
$$

Then the following relation holds provided that $k \geq n$ and $r \geq n$ :

$$
\left|N\left(x_{k k}-x_{r r}\right)\right|=\left|\left(N-N_{s}\right)\left(x_{k k}-x_{r r}\right)+N_{s}\left(x_{k k}-x_{r r}\right)\right| \leq 3 \varepsilon .
$$

This means the convergence of the sequence $N x_{k k}$. Hence, the operator $N$ is compact and, therefore, the subspace $\mathcal{T}(X, Y)$ is closed.

Pass to the second assertion of the theorem. Let $N \in \mathcal{T}(X, Y), y_{k}^{*} \in Y^{*}$, and $\left|y_{k}^{*}\right| \leq 1$. We must verify that the sequence $x_{k}^{*}(x)=y_{k}^{*}(N x)$ contains a subsequence converging in $X^{*}$. Let $B$ be the unit ball in $X$ and $Q=\overline{N(B)}$. Then the set $Q$ is compact with respect to the metric of the Banach space $Y$ and the sequence of continuous functions $f_{k}(y)=y_{k}^{*}(y), y \in Q$, is uniformly bounded. Also, the family $\left\{f_{k}\right\}$ is equicontinuous since

$$
\left|f_{k}(y)-f_{k}\left(y^{\prime}\right)\right|=\left|y_{k}^{*}\left(y-y^{\prime}\right)\right| \leq\left|y-y^{\prime}\right|, \quad y, y^{\prime} \in Q .
$$

Hence, due to the Arzela-Ascoli theorem, the sequence $\left\{f_{k}\right\}$ contains a subsequence $\left\{f_{k_{i}}\right\}$ uniformly converging to a continuous function on $Q$. Then it is easy to see that $\left\{x_{k_{i}}^{*}\right\}$ also converges in $X^{*}$. 
The notions of bounded and compact operators can be extended to the case of families of Banach spaces. Let families $\left(X_{i}, i \in I\right)$ and $\left(Y_{i}, i \in I\right)$ of Banach spaces form lattices (in the sense of embeddings (1.1.8) of Banach spaces) such that the inclusion $X_{i} \subseteq X_{j}$ implies the inclusion $Y_{i} \subseteq Y_{j}$. We say that a linear operator $N: \bigcup_{i \in I} X_{i} \rightarrow \bigcup_{i \in I} Y_{i}$ is bounded in these families if the restriction $N_{i}=N \mid X_{i}$ is bounded by $X_{i} \rightarrow Y_{i}$ for any $i$ from $I$. The space of such operators is denoted by $\mathcal{L}\left(X_{i}, Y_{i} ; i \in I\right)$. If $X_{i}=Y_{i}$, then the notation $\mathcal{L}\left(X_{i} ; i \in I\right)$ is used. If the family $\left(X_{1}, X_{2}\right)$ consists of two elements, then the last notation is reduced to $\mathcal{L}\left(X_{1} ; X_{2}\right)$, which differs from the notation of the space $\mathcal{L}\left(X_{1}, X_{2}\right)$ of bounded operators from $X_{1}$ to $X_{2}$.

For compact operators, similar definitions can also be introduced; in the notation, the symbol $\mathcal{L}$ is replaced by the symbol $\mathcal{T}$.

Note that the embedding $Y_{i} \subseteq Y_{j}$ implies the embedding $Y_{j}^{*} \subseteq Y_{i}^{*}$. Respectively, the operator $N_{j}^{*}$ coincides with the restriction of $N_{i}^{*}$ to $X_{j}^{*}$. Therefore, the family of adjoint spaces $\left(X_{i}^{*}, i \in I\right)$ also forms a lattice, and the adjoint operator $N^{*}$ from $\mathcal{L}\left(Y_{i}^{*}, X_{i}^{*} ; i \in I\right)$ is defined. Note that elements $y^{*}$ of $\bigcap_{i} Y_{i}^{*}$ can be treated as linear functionals over the vector space $\bigcup_{i} Y_{i}$. Obviously, the kernel $\operatorname{ker} N \stackrel{i}{=}=\bigcup_{i} \operatorname{ker} N_{i}$ of the operator $N$ from $\mathcal{L}\left(X_{i}, Y_{i} ; i \in I\right)$ is contained in $\bigcup_{i} X_{i}$ and its cokernel coker $N={ }^{i} \operatorname{ker} N^{*}$ is contained in $\bigcup_{i} Y_{i}^{*}$. If coker $N \subseteq \bigcap_{i} Y_{i}^{*}$, then the following relations similar to (1.2.10) hold:

$$
\overline{\operatorname{Im} N_{i}}=Y_{i} \cap(\operatorname{coker} N)^{\perp}, \quad i \in I .
$$

\subsection{Fredholm Operators}

We say that $N$ from $\mathcal{L}(X, Y)$ is a Fredholm operator if its kernel ker $N$ and its cokernel are finitedimensional and

$$
\operatorname{Im} N=(\operatorname{coker} N)^{\perp} .
$$

In other words, $N$ is a Fredholm operator if its kernel ker $N$ is finite-dimensional and its image $\operatorname{Im} N$ is finite-codimensional. For brevity, the dimensions $\operatorname{dim}(\operatorname{ker} N)$ and $\operatorname{codim}(\operatorname{Im} N)=\operatorname{dim}(\operatorname{coker} N)$ are denoted by $\operatorname{dim} N$ and $\operatorname{codim} N$ respectively. The integer ind $N=\operatorname{dim} N-\operatorname{codim} N$ is called the index of the operator $N$. In $[29,32,48]$, the theory of Fredholm operators is explained in detail.

If $N$ is a Fredholm operator, then the spaces $X$ and $Y$ can be expanded into the direct sums

$$
X=X_{(0)} \oplus X_{(1)}, X_{(0)}=\operatorname{ker} N, \quad Y=Y_{(0)} \oplus Y_{(1)}, Y_{(1)}=\operatorname{Im} N
$$

These expansions are defined not uniquely. However, any such finite-dimensional space $Y_{(0)}$ is called a coimage of the operator $N$. Obviously, the number $\operatorname{dim} Y_{(0)}=\operatorname{codim} N$ coincides with the dimension of the cokernel of the operator $N$, i.e., with the dimension $\operatorname{dim} N^{*}$ of the kernel of the adjoint operator. Therefore, ind $N=\operatorname{dim} N-\operatorname{dim} N^{*}$ and (1.3.1) can be represented as follows:

$$
\operatorname{Im} N=\left(\operatorname{ker} N^{*}\right)^{\perp} .
$$

Thus, the following three assertions (the Fredholm alternative) hold for the equation $N x=y$.

(i) The homogeneous equation $N x=0$ has a finite set of $n$ linearly independent solutions $x_{1}, \ldots, x_{n}$.

(ii) The homogeneous adjoint equation $N^{*} y^{*}=0$ has a finite set of $m$ linearly independent solutions $y_{1}^{*}, \ldots, y_{m}^{*}$ from $Y^{*}$ and the heterogeneous equation is solved if and only if $\left(y, y_{i}^{*}\right)=0,1 \leq i \leq m$.

(iii) The difference $n-m$ coincides with the index ind $N$ of the operator $N$.

From expansions (1.3.2), it follows that the operator $N$ maps $X_{(1)}$ onto $Y_{(1)}$ bijectively. Hence, due to the Banach theorem, the restriction $N_{1}=N \mid X_{1}$ treated as an operator from $X_{(1)}$ to $Y_{(1)}$ is invertible. Consider the operator $N^{(-1)}$ from $\mathcal{L}(Y, X)$ such that $\operatorname{ker} N^{(-1)}=Y_{(0)}$ and the restriction $N_{(1)}^{(-1)}=N^{(-1)} \mid Y_{(1)}$ coincides with $N_{1}^{-1}$. Obviously, this is a Fredholm operator and the relations

$$
\text { ind } N^{(-1)}=-\operatorname{ind} N, \quad N^{(-1)} N=1+P_{0}, \quad N N^{(-1)}=1+Q_{0},
$$


where $P_{0}$ and $Q_{0}$ and the projectors from $X$ to $X_{(0)}$ and from $Y$ to $Y_{(0)}$ defined by the relations (1.3.2), hold.

If $X$ and $Y$ are finite-dimensional, then it is obvious that any $N$ from $\mathcal{L}(X, Y)$ is a Fredholm operator. Since the dimensions of the spaces $X_{(1)}$ and $Y_{(1)}$ in expansions (1.3.2) are equal in this case, it follows that ind $N=\operatorname{dim} X-\operatorname{dim} Y$.

For example, if an operator is invertible from the right and its kernel is finite-dimensional, then it is a Fredholm operator; its index is equal to the dimension $\operatorname{dim} N$ of its kernel. In the same way, if an operator $R$ from $\mathcal{L}(Y, X)$ is invertible from the left and its image is finite-codimensional, then it is a Fredholm operator; its index is equal to ind $R=-\operatorname{codim} R$.

Simple properties of Fredholm operators provided by the next lemma immediately follow from the definitions.

\section{Lemma 1.3.1.}

(a) If $N_{1} N$ and $N N_{2}$ are Fredholm operators, then $N$ is also a Fredholm operator.

(b) Let the kernel of the operator $N$ from $\mathcal{L}(X, Y)$ be finite-dimensional and a finite-codimensional subspace $X_{1} \subseteq X$ be such that $X_{1} \cap \operatorname{ker} N=0$. Then $N$ is a Fredholm operator if and only if the subspace $Y_{1}=N\left(X_{1}\right)$ is finite-codimensional and the restriction $N_{1}=N \mid X_{1}$ treated as an operator from $X_{1}$ to $Y_{1}$ is invertible. If this holds, then ind $N=\operatorname{codim} X_{1}-\operatorname{codim} Y_{1}$.

(c) If $T \in \mathcal{T}_{0}(X)$, then $N=1+T$ is a Fredholm operator and its index is equal to zero.

Proof. (a) The relations ker $N \subseteq \operatorname{ker}\left(N_{1} N\right)$ and $\operatorname{Im} N \supseteq \operatorname{Im}\left(N N_{2}\right)$ are obvious. The former relation means that the kernel ker $N$ is finite-dimensional. Due to Theorem 1.1.1(c), the latter relation implies that the image $\operatorname{Im} N$ is finite-codimensional. Hence, $N$ is a Fredholm operator.

(b) If $Y_{1}$ is finite-codimensional, then, due to Theorem 1.1.1(c), the subspace $\operatorname{Im} N \supseteq Y_{1}$ also possesses this property. Then $N$ is a Fredholm operator.

Conversely, let $N$ be a Fredholm operator. By assumption, there exists an expansion $X=X_{1} \oplus X_{0}$, $X_{0} \supseteq \operatorname{ker} N$. Using the representation $X_{0}=X_{0}^{\prime} \oplus \operatorname{ker} N$, assign $X_{(1)}=X_{1} \oplus X_{0}^{\prime}$ in expansions (1.3.2). As we note above, the operator $N_{1}=N \mid X_{(1)}$ acting from $X_{(1)}$ to $Y_{(1)}$ is invertible. Then the image $\operatorname{Im} N=Y_{(1)}$ is expanded into the direct sum $Y_{1} \oplus Y_{0}^{\prime}$, where $Y_{1}=N\left(X_{1}\right), Y_{0}^{\prime}=N\left(X_{0}^{\prime}\right)$, and the dimensions of the finite-codimensional spaces $X_{0}^{\prime}$ and $Y_{0}^{\prime}$ are equal. Thus, the subspace $Y_{1}$ is finite-codimensional, the operator $N_{\mid} X_{1}$ treated as an operator from $X_{1} \rightarrow Y_{1}$ is invertible, $\operatorname{codim} X_{1}=\operatorname{dim} X_{0}^{\prime}+\operatorname{dim} N$, and $\operatorname{codim} Y_{1}=\operatorname{dim} Y_{0}^{\prime}+\operatorname{codim} N$. Subtracting the latter relation from the former, we obtain the relation for the index.

(c) Obviously, the kernel $X_{1}=\operatorname{ker} T$ of the finite-dimensional operator $T$ is finite-codimensional and the operator $N=1+T$ on this subspace is the identity operator. Since $X_{1} \cap \operatorname{ker} N=0$, it remains to use assertion (b) of the lemma, assigning $Y_{1}=X_{1}$.

The following classical result generalizes assertion (c) of Lemma 1.3.1.

Theorem (Riesz-Schauder). If $T \in \mathcal{T}(X)$, then $N=1+T$ is a Fredholm operator and its index is equal to zero.

Proof. In the normed space ker $N$, the identity operator 1 coincides with the compact operator $-T$. Thus, this space possesses the Bolzano-Weierstrass property. Hence, this is finite-dimensional due to Theorem 1.1.2. According to Theorem 1.2.2, the kernel ker $N^{*}$ is also finite-dimensional. Therefore, taking into account (1.2.13), it remains to verify that the subspace $\operatorname{Im} N$ is closed in $X$.

Consider the expansion $X=X_{1} \oplus \operatorname{ker} N$. It is obvious that $N\left(X_{1}\right)=\operatorname{Im} N$. Consider a sequence $x_{n}=z_{n}+T z_{n}, z_{n} \in X_{1}$ converging to an element $x$ of $X$. If the sequence $z_{n}$ is bounded, then, due to the compactness of $T$, there exists a converging subsequence $T z_{n_{k}}$. Then $z_{n_{k}}=x_{n_{k}}-T z_{n_{k}}$ converges to $z$ from $X_{1}$ and, therefore, $x=z+T z \in \operatorname{Im}(1+T)$. Assume that the sequence $z_{n}$ is unbounded. Passing to its subsequence, one can assume that $\left|z_{n}\right| \rightarrow \infty$. Then $z_{n}^{\prime}+T z_{n}^{\prime} \rightarrow 0, z_{n}^{\prime}=z_{n} /\left|z_{n}\right|$. Arguing as above, we find a sequence $z_{n_{k}}^{\prime}$ converging to $z^{\prime}$ from $X_{1},\left|z^{\prime}\right|=1$. Then, passing to the limit, we 
obtain that $z^{\prime}+T z^{\prime}=0$, which contradicts the definition of $X_{1}$. Thus, the space $\operatorname{Im} N$ is closed and $N=1+T$ is a Fredholm operator.

To prove that its index is equal to zero, see assertion (d) of the next theorem.

\section{Theorem 1.3.1.}

(a) If $N$ from $\mathcal{L}(X, Y)$ and $M$ from $\mathcal{L}(Y, Z)$ are Fredholm operators, then their composition is also a Fredholm operator and

$$
\text { ind } M N=\text { ind } M+\text { ind } N \text {. }
$$

(b) Let operators $N$ from $\mathcal{L}(X, Y)$ and $R$ from $\mathcal{L}(Y, X)$ be such that

$$
R N-1 \in \mathcal{T}(X), \quad N R-1 \in \mathcal{T}(Y) .
$$

Then $N$ and $R$ are Fredholm operators.

(c) The set of Fredholm operators is open in $\mathcal{L}(X, Y)$ and the integer-valued function ind is constant on connected components of this set.

(d) If $N$ from $\mathcal{L}(X, Y)$ is a Fredholm operator and $T \in \mathcal{T}(X, Y)$, then $N+T$ is a Fredholm operator and $\operatorname{ind}(N+T)=\operatorname{ind} N$.

Proof. (a) Since $\operatorname{ker}(M N)=N^{-1}(\operatorname{ker} M)$, it follows that the kernel of the operator $M N$ is finite-dimensional. Select a finite-codimensional subspace $X_{1}$ to satisfy the condition $X_{1} \cap \operatorname{ker}(M N)=0$. Then, by virtue of Lemma 1.3.1(b), the subspace $Y_{1}=N\left(X_{1}\right)$ is finite-dimensional. Also, it is obvious that $Y_{1} \cap \operatorname{ker} M=0$. Therefore, arguing in the same way, we conclude that the subspace $Z_{1}=M\left(Y_{1}\right)$ is finite-codimensional. Hence, $M N$ is a Fredholm operator and ind $M N=\operatorname{codim} X_{1}-\operatorname{codim} Z_{1}$. In the same way, we obtain that ind $N=\operatorname{codim} X_{1}-\operatorname{codim} Y_{1}$ and ind $M=\operatorname{codim} Y_{1}-\operatorname{codim} Z_{1}$, which leads to the relation (1.3.5).

(b) This relation immediately follows from Lemma 1.3.1(a) and the first part of the Riesz-Schauder theorem.

(c) Let $N$ from $\mathcal{L}(X, Y)$ be a Fredholm operator. Denote $N^{(-1)}$ from (1.3.4) by $R$ and assume that it belongs to $\mathcal{L}(Y, X)$. As we note above, this is a Fredholm operator and its index is opposite to ind $N$. Let $B \in \mathcal{L}(X, Y)$ and

$$
|B|_{\mathcal{L}}<\frac{1}{|R|_{\mathcal{L}}}
$$

It suffices to verify that $N+B$ is a Fredholm operator and $\operatorname{ind}(N+B)=\operatorname{ind} N$.

It is obvious that $|B R|_{\mathcal{L}} \leq|B|_{\mathcal{L}}|R|_{\mathcal{L}}<1$. As we found, proving Theorem 1.2.1, this implies the invertibility of the operator $1+B R$ in $\mathcal{L}(Y)$. In the same way, the operator $1+R B$ is invertible in $\mathcal{L}(X)$. Therefore, from (1.3.4), we obtain that

$$
R(N+B)=1+R B+P_{0}=(1+R B)\left(1+T_{1}\right), \quad T_{1}=\left[1+(1+R B)^{-1} P_{0}\right]
$$

and

$$
(N+B) R=1+B R+Q_{0}=(1+B R)\left(1+T_{2}\right), \quad T_{2}=\left[1+(1+B T)^{-1} Q_{0}\right],
$$

where $T_{j}$ are finite-dimensional operators. By virtue of (b) and Lemma 1.3.1(c), this implies that $N+B$ is a Fredholm operator; taking into account (1.3.5), we conclude that ind $(N+B)+\operatorname{ind} R=0$. It remains to recall that ind $R=-$ ind $N$.

(d) Consider the operator $R=N^{(-1)}$ from $\mathcal{L}(Y, X)$ involved in the relation (1.3.4). By virtue of (1.3.6), there exist compact operators $T_{1}$ and $T_{2}$ such that $R(N+T)=1+T_{1}$ and $(N+T) R=1+T_{2}$. Thus, it follows from (b) that $N+T$ is a Fredholm operator. For any $\lambda$ from $[0,1]$, the operator $N+\lambda T$ also possesses the Fredholm property. By virtue of (c), its index is independent of $\lambda$. Hence, $\operatorname{ind}(N+T)=\operatorname{ind} N$.

From this theorem, it follows that Fredholm operators map finite-codimensional spaces into finitecodimensional ones. Indeed, if $N$ from $\mathcal{L}(X, Y)$ is a Fredholm operator and a subspace $X_{1} \subseteq X$ is finite-codimensional, then $N\left(X_{1}\right)$ coincides with the image of the operator $N P$, where $P$ projects $X$ 
onto $X_{1}$. Since $1-P$ is a finite-dimensional operator, it follows that $P$ is a Fredholm operator and, therefore, $N P$ possesses the same property.

If operators $N$ and $R$ possess the property (1.3.6), then we say that they are invertible modulo $\mathcal{T}$. The operator $R$ is called a regularizer of the operator $N$. From (1.3.4) and Theorem 1.3.1(b), it follows that $N$ is a Fredholm operator if and only if it is invertible modulo $\mathcal{T}$.

The notion of the one-side invertibility modulo $\mathcal{T}$ can also be introduced. However, the invertibility of on operator $N$ from the left and from the right modulo $\mathcal{T}$ (i.e., the existence of its left- and righthand regularizers) implies that these regularizers coincide (modulo $\mathcal{T}$ ). Indeed, if $R_{1} N \sim 1, N R_{2} \sim 1$, where $\sim$ denotes the equality modulo $\mathcal{T}$, then $R_{2} \sim R_{1} N R_{2} \sim R_{1}$.

An operator $N$ from $\mathcal{L}(X, Y)$ is called a finite-dimensional extension of an operator $N_{1}$ from $\mathcal{L}\left(X_{1}, Y\right)$ if $X_{1} \subseteq X$ is a finite-dimensional subspace and $N_{1} x=N x$ provided that $x \in X_{1}$. In such a case, the operator $N_{1}$ is called a finite-dimensional restriction of the operator $N$. These operators are Fredholm equivalent and their indices satisfy the relation

$$
\text { ind } N=\operatorname{ind} N_{1}+\operatorname{codim} X_{1} \text {. }
$$

Indeed, let $X=X_{1} \oplus X_{0}$ and the operator $\widetilde{N}$ be the extension of $N_{1}$, satisfying the condition $\tilde{N} \mid X_{0}=0$. Then the relation (1.3.7) with respect to $N_{1}$ and $\widetilde{N}$ is obvious. On the other hand, the difference $N-\widetilde{N}$ is a finite-dimensional operator and it remains to use Theorem 1.3.1(d).

If $N \in \mathcal{L}(X, Y)$, then any operator $\tilde{N}$ from $\mathcal{L}\left(X \times \mathbb{C}^{m}, Y \times \mathbb{C}^{n}\right)$ such that $\tilde{N}_{11}=N$ in its canonical representation by a $2 \times 2$-matrix is called a finite-dimensional perturbation of the operator $N$. By virtue of Theorem 1.3.1(d), the operators $N$ and $\tilde{N}$ are Fredholm equivalent and ind $\tilde{N}=$ ind $N+m-n$.

Indeed, the difference between $\tilde{N}$ and

$$
\tilde{N}^{0}=\left(\begin{array}{cc}
N & 0 \\
0 & 0
\end{array}\right) \in \mathcal{L}\left(X \times \mathbb{C}^{m}, Y \times \mathbb{C}^{n}\right)
$$

is a finite-dimensional term and the latter is obviously Fredholm equivalent to $N$.

Also, the theorem implies that if the product $N_{1} N_{2}$ of two operators and one factor is a Fredholm operator, then the second factor is also a Fredholm operator. Due to the same reason, if there exists a positive integer $k$ such that $N^{k}$ is a Fredholm operator, then $N$ is also a Fredholm operator. The next lemma provides one more corollary of such a kind.

Lemma 1.3.2. Let $X=X_{1} \times \cdots \times X_{n}$ and an operator $N$ from $\mathcal{L}(X)$ be represented by an $n \times n$ matrix $\left(N_{i j}\right), N_{i j} \in \mathcal{L}\left(X_{j}, X_{i}\right)$ triangle modulo $\mathcal{T}$ (e.g., low-triangle, i.e., $N_{i j} \in \mathcal{T}$ for $\left.i<j\right)$. Then, if the diagonal elements $N_{i i} \in \mathcal{L}\left(X_{i}\right)$ possess the Fredholm property, then $N$ is a Fredholm operator,

$$
\text { ind } N=\sum_{1}^{n} \operatorname{ind} N_{i i} \text {, }
$$

and the regularizer $R=\left(R_{i j}\right)$ of the operator $N$ is also low-triangle modulo $\mathcal{T}$.

If the matrix $N$ is diagonal modulo $\mathcal{T}$, then the Fredholm property of $N$ implies the Fredholm property of all its diagonal elements $N_{i i}$.

Proof. It suffices to prove the lemma for $n=2$. In the general case, it suffices to represent $X$ as $X_{1} \times\left(X_{2} \times \cdots \times X_{n}\right)$ and use induction with respect to $n$. Let

$$
N=\left(\begin{array}{ll}
N_{11} & N_{12} \\
N_{21} & N_{22}
\end{array}\right)
$$

where $N_{i i}$ possess the Fredholm property and $N_{12} \in \mathcal{T}$. If $R_{i}$ a regularizer of $N_{i i}$, then

$$
\left(\begin{array}{cc}
R_{1} & 0 \\
0 & R_{2}
\end{array}\right) N \sim\left(\begin{array}{cc}
1 & 0 \\
R_{2} N_{21} & 1
\end{array}\right)
$$

where $\sim$ denotes the equality modulo $\mathcal{T}$. The operator on the right-hand side of this relation is invertible and the inverse one has the same form, where $R_{2} N_{21}$ is replaced by $-R_{2} N_{21}$. Therefore, $N$ 
is a Fredholm operator and ind $N=-$ ind $R_{1}-$ ind $R_{2}=$ ind $N_{11}+$ ind $N_{22}$. This completes the proof of the first part of the lemma.

Now, let $N$ be a Fredholm operator and

$$
N \sim\left(\begin{array}{cc}
N_{11} & 0 \\
0 & N_{22}
\end{array}\right)
$$

Then its regularizer $R=\left(R_{i j}\right)$ satisfies the relations $N R \sim\left(N_{i i} R_{i j}\right) \sim 1$ and $R N \sim\left(R_{i j} N_{j j}\right) \sim 1$, which implies that $N_{i i} R_{i i} \sim R_{i i} N_{i i} \sim 1$. Hence, $N_{i i}$ are Fredholm operators.

For a Fredholm operator $N$, let us find conditions providing the existence of a Banach space $Y^{\prime}$ embedded into $Y^{*}$ such that the cokernel coker $N$ is contained in $Y^{\prime}$. Let Banach spaces $X$ and $Y$ be endowed with the duality structures $\left(X, X^{\prime},\langle\rangle,\right)$ and $\left(Y, Y^{\prime},\langle\rangle,\right)$ respectively (in the sense of the definition from Sec. 1.2) such that the embeddings $X^{\prime} \subseteq X^{*}$ and $Y^{\prime} \subseteq Y^{*}$ take place. Following [45], we say that an operator $N$ from $\mathcal{L}(X, Y)$ admits an associated operator $N^{\prime}$ from $\mathcal{L}\left(Y^{\prime}, X^{\prime}\right)$ if

$$
\langle N x, y\rangle=\left\langle x, N^{\prime} y\right\rangle
$$

identically with respect to $x$ from $X$ and $y$ from $Y^{\prime}$. If $N^{\prime}$ exists, then it is uniquely defined by $N$ since the forms are nondegenerate. It is clear that if $N_{1}$ and $N_{2}$ admit associated operators, then their product $N_{1} N_{2}$ also admits an associated operator and $\left(N_{1} N_{2}\right)^{\prime}=N_{2}^{\prime} N_{1}^{\prime}$. Obviously, an operator $N$ admits an associated operator $N^{\prime}$ with respect to the canonical embeddings $X^{\prime} \subseteq X^{*}$ and $Y^{\prime} \subseteq Y^{*}$ if and only if the adjoint operator $N^{*}$ is bounded in the space pair $Y^{*} \rightarrow X^{*}$ and $Y^{\prime} \rightarrow X^{\prime}$ in the sense of the definition from Sec. 1.2 and $N^{\prime}=N^{*} \mid Y^{\prime}$.

We say that $N$ is an associatedly Fredholm operator if it admits an associated operator $N^{\prime}$, the operators $N$ and $N^{\prime}$ possess the Fredholm property, and the relations

$$
\operatorname{coker} N=\operatorname{ker} N^{\prime}, \quad \operatorname{coker} N^{\prime}=\operatorname{ker} N
$$

hold. It is clear that if these relations hold, then the indices of the operators $N$ and $N^{\prime}$ are opposite. Actually the last property describes associatedly Fredholm operators completely.

\section{Theorem 1.3.2.}

(a) An operator $N$ from $\mathcal{L}(X, Y)$ is an associatedly Fredholm operator if and only if both $N$ and $N^{\prime}$ are Fredholm operators and their indices are opposite.

(b) Let operators $N$ from $\mathcal{L}(X, Y)$ and $R$ from $\mathcal{L}(Y, X)$ satisfy conditions (a), $R$ be a regularizer for $N$, and $R^{\prime}$ be a regularizer for $N^{\prime}$. Then both $N$ and $R$ are associatedly Fredholm operators.

Proof. (a) Let $N$ from $\mathcal{L}(X, Y)$ be a Fredholm operator admitting an associated operator $N^{\prime}$ from $\mathcal{L}\left(Y^{\prime}, X^{\prime}\right)$ and let $N^{\prime}$ be also a Fredholm operator. Then

$$
\text { ind } N+\operatorname{ind} N^{\prime} \leq 0 \text {. }
$$

Indeed, by virtue of (1.3.8), we have the relations

$$
\operatorname{ker} N^{\prime} \subseteq \operatorname{coker} N, \quad \operatorname{ker} N \subseteq \operatorname{coker} N^{\prime}
$$

that imply

$$
\operatorname{dim} N^{\prime} \leq \operatorname{codim} N, \quad \operatorname{dim} N \leq \operatorname{codim} N^{\prime} .
$$

These inequalities lead to (1.3.9).

Since the bilinear forms defining the dualities are nondegenerate, it follows that the equality in (1.3.10) leads to the equalities in (1.3.11) and, therefore, to the relations (1.3.9).

(b) By assumption, ind $N+$ ind $R=$ ind $N^{\prime}+$ ind $R^{\prime}=0$. On the other hand, $R$ satisfies an inequality similar to (1.3.10), which is possible only if ind $N=-\operatorname{ind} N^{\prime}$ and ind $R=-\operatorname{ind} R^{\prime}$. Therefore, it remains to apply assertion (a) of the theorem to these operators. 
Consider the canonical dualities $\left(X, X^{*}\right)$ and $\left(Y, Y^{*}\right)$ with the corresponding bilinear forms given by (1.2.9). If $N$ from $\mathcal{L}(X, Y)$ is a Fredholm operator and $R$ from $\mathcal{L}(Y, X)$ is its regularizer, then, by virtue of Theorems 1.2.3 and 1.3.1, the operator $R^{*}$ is a regularizer for $N^{*}$. Therefore, due to Theorem 1.3.2(b), the indices of the operators $N$ and $N^{*}$ are opposite:

$$
\text { ind } N^{*}=- \text { ind } N \text {. }
$$

The notion of the Fredholm property can also be introduced for operators acting in a family of Banach spaces. Using the notation of Sec. 1.2, assume that $N \in \mathcal{L}\left(X_{i}, Y_{i} ; i \in I\right)$. We say that it is a Fredholm operator if its kernel $\operatorname{ker} N$ is finite-dimensional and is contained $\operatorname{in} \bigcap_{i} X_{i}$, its cokernel coker $N$ is finite-codimensional and is contained in $\bigcap_{i} Y_{i}^{*}$, and the following relations hold:

$$
N\left(X_{i}\right)=Y_{i} \cap(\operatorname{coker} N)^{\perp}, \quad i \in I .
$$

The difference ind $N=\operatorname{dim} N-\operatorname{codim} N$ is called the index of the operator $N$.

It immediately follows from this definition that $\operatorname{ker} N_{i}=\operatorname{ker} N$ and coker $N_{i}=\operatorname{coker} N, i \in I$. In particular, the relation ind $N_{i}=$ ind $N$ holds for any $i$ from $I$. The inverse assertion also holds: if $N_{i}$ is a Fredholm operator for any $i$ from $I$, while the kernel ker $N_{i}$ and the cokernel coker $N_{i}$ do not depend on $i$, then $N$ is a Fredholm operator. The next two corollaries are almost obvious.

Lemma 1.3.3. Let $N$ from $\mathcal{L}\left(X_{i}, Y_{i} ; i \in I\right)$ be a Fredholm operator. Then, for any $X_{i}$ from $X_{j}$, any solution $x$ from $X_{j}$ of the equation $N x=y$ with the right-hand side $y$ from $X_{i}$ also belongs to $X_{i}$. If the assumption of the lemma is satisfied and the form $\left(y, y^{*}\right)=y^{*}(y)$ is nondegenerate on the product $\left(\bigcup_{i} Y_{i}\right) \times\left(\bigcap_{i} Y_{i}^{*}\right)$, then there exists a finite-dimensional subspace $Z \subseteq \bigcap_{i} Y_{i}$ of dimension codim $N$ such that

$$
Y_{i}=Z \oplus \operatorname{Im} N_{i}, \quad i \in I .
$$

Proof. Let $X_{i} \subseteq X_{j}, x \in X_{j}$, and $N x=y \in Y_{i}$. Then $y \perp$ coker $N$ and, by virtue of (1.3.13), there exists a vector $x_{1} \in X_{i}$ such that $N x_{1}=y$. Then $x_{0}=x-x_{1} \in \operatorname{ker} N \subseteq \bigcap_{i} X_{i}$ and, therefore, $x=x_{0}+x_{1} \in X_{i}$.

Let the assumptions of the second part of the lemma be satisfied and the vectors $y_{1}^{*}, \ldots, y_{n}^{*}$ form a base of coker $N$. Then, similarly to Lemma 1.2.2, it is easy to prove that there exist vectors $z_{1}, \ldots, z_{n}$ from $\bigcap_{i} X_{i}$ such that they are biorthogonal to this base. Let the space $Z$ be spanned by these vectors. If there exists $i$ such that a vector $z=\lambda_{1} x_{1}+\ldots+\lambda_{n} x_{n}$ belongs to the image of the operator $N_{i}$, then it is orthogonal to all $z_{1}, \ldots, z_{n}$, which is possible only in the case where $\lambda_{1}=\ldots=\lambda_{n}=0$. Thus, $Z \cap \operatorname{Im} N_{i}=0$; combining this with the relation $\operatorname{dim} Z=\operatorname{codim} N_{i}$, we arrive at the relation (1.3.14).

The following simple criterion of the Fredholm property for operators acting in a family of Banach spaces allows one to extend Theorem 1.3.1 to these operators.

\section{Theorem 1.3.3.}

(a) An operator $N$ from $\mathcal{L}\left(X_{i}, Y_{i} ; i \in I\right)$ possesses the Fredholm property if and only if $N_{i}=N \mid X_{i}$ from $\mathcal{L}\left(X_{i}, Y_{i}\right)$ possesses the Fredholm property for any $i$ and ind $N_{i}$ is independent of $i$. In particular, if $K \in \mathcal{T}\left(X_{i} ; i \in I\right)$, then $1+K$ is a Fredholm operator and its index is equal to zero.

(b) If $N$ from $\mathcal{L}\left(X_{i}, Y_{i} ; i \in I\right)$ and $M$ from $\mathcal{L}\left(Y_{i}, Z_{i} ; i \in I\right)$ are Fredholm operators, then $M N$ is also a Fredholm operator and $\operatorname{ind}(M N)=$ ind $N+$ ind $M$.

(c) If $N$ from $\mathcal{L}\left(X_{i}, Y_{i} ; i \in I\right)$ is a Fredholm operator and $T \in \mathcal{T}\left(X_{i}, Y_{i} ; i \in I\right)$, then $N+T$ is a Fredholm operator and $\operatorname{ind}(N+T)=$ ind $N$.

(d) Let $N \in \mathcal{L}\left(X_{i}, Y_{i} ; i \in I\right), R \in \mathcal{L}\left(Y_{i}, X_{i} ; i \in I\right)$, and $R N$ and $N R$ be Fredholm operators. Then $N$ and $R$ are also Fredholm operators. In particular, if

$$
R N-1 \in \mathcal{T}\left(X_{i} ; i \in I\right), \quad N R-1 \in \mathcal{T}\left(Y_{i} ; i \in I\right),
$$


then $N$ and $R$ are Fredholm operators and their indices are opposite.

Proof. (a) Let $N_{i}$ be a Fredholm operator for any $i$ and their indices do not depend on $i$. It suffices to verify that the kernels and cokernels of the operators $N_{i}$ do not depend on $i$, i.e.,

$$
\operatorname{ker} N_{i}=\operatorname{ker} N_{j}, \quad \operatorname{coker} N_{i}=\operatorname{coker} N_{j}
$$

for any pair $i, j$ from $I$. Due to (1.1.8), without loss of generality, one can assume that $X_{i} \subseteq X_{j}$. Then $Y_{i} \subseteq Y_{j}$ and $Y_{j}^{*} \subseteq Y_{i}^{*}$. Therefore,

$$
\text { ker } N_{i} \subseteq \operatorname{ker} N_{j}, \quad \text { coker } N_{j} \subseteq \operatorname{coker} N_{i} .
$$

In particular, both $s=\operatorname{dim} N_{j}-\operatorname{dim} N_{i}$ and $s^{\prime}=\operatorname{codim} N_{i}-\operatorname{codim} N_{j}$ are nonnegative. However, $0=$ ind $N_{j}-$ ind $N_{i}=s+s^{\prime}$ by assumption. Hence, $s=s^{\prime}=0$, which leads to the relations (1.3.15).

The proof of the inverse implication is obvious.

(b)-(d) All the assertions are intermediate corollaries of (a) Theorem 1.2.1.

Note that Theorems 1.3.2-1.3.3 occur (in various forms) in many papers (see, e.g., [8, 60]).

All the above considerations refer to spaces over the the scalar field $\mathbb{C}$. The Fredholm property can also be introduced for $\mathbb{R}$-linear operators. Note that $\mathbb{C}$-linear Fredholm operators can also be treated as $\mathbb{R}$-linear operators, but one should take into account the fact that dimensions are doubled under the passing from $\mathbb{C}$ to $\mathbb{R}$.

A typical situation arise if a Banach space $X$ is endowed with a $\mathbb{R}$-linear operator $J \in \mathcal{L}(X)$ such that

$$
J^{2}=1, \quad J(i x)=-i J x, x \in X
$$

where $i$ from $\mathbb{C}$ is the imaginary unity. For example, if elements of $X$ are complex functions $\varphi(t), t \in E$, then the complex-conjugation operator $J: \varphi(t) \rightarrow \overline{\varphi(t)}$ satisfies the conditions (1.3.16). For this reason, in the general case $J$ is also called the complex-conjugation operator.

Elements $x$ of $X$ such that $J x=x$ are called real elements. They form a closed subspace (over the field $\mathbb{R}$ ) in $X$; this subspace is denoted by $X_{\mathbb{R}}$. The space $X$ is expanded into the direct sum $X_{\mathbb{R}} \oplus i X_{\mathbb{R}}$ over the field $\mathbb{R}$. Therefore, the pair $(X, J)$ is called a complex structure such that the space $X$ is endowed with.

In the sequel, the complex-conjugation operator for various complex structures is denoted by the same symbol $J$ unless this causes a confusion.

Let Banach spaces $X$ and $Y$ be endowed with a complex structure. Then a complex structure can also be introduced in the space $\mathcal{L}(X, Y)$ of bounded $\mathbb{C}$-linear operators. In this complex structure, the map $N \rightarrow J N J$ serves as the complex-conjugation operator (recall that the corresponding factors $J$ act in the spaces $X$ and $Y$ in such a case). Obviously, the necessary conditions given by (1.3.16) are satisfied for this map. This map is denoted as follows:

$$
\bar{N}=J N J \text {. }
$$

In particular, the operator $N$ is real with respect to this structure if $\bar{N}=N$ or, which is equivalent, the operators $N$ and $J$ commute:

$$
N J=J N \text {. }
$$

In this case, its restriction to $X_{\mathbb{R}}$ defines an $\mathbb{R}$-linear operator from $X_{\mathbb{R}}$ to $Y_{\mathbb{R}}$ denoted by $N_{\mathbb{R}}$.

\section{Theorem 1.3.4.}

(a) Let a $\mathbb{C}$-linear operator $N$ from $\mathcal{L}(X, Y)$ be real with respect to complex structures in spaces $X$ and $Y$. Then it is Fredholm equivalent with an operator $N_{\mathbb{R}}$ from $\mathcal{L}\left(X_{\mathbb{R}}, Y_{\mathbb{R}}\right)$ and their indices (over the corresponding fields) coincide and $\operatorname{ker} N_{\mathbb{R}}$ consists of real elements of the kernel $\operatorname{ker} N$.

(b) Let $\mathbb{C}$-linear operators $N_{1}$ and $N_{2}$ belong to $\mathcal{L}(X)$ and

$$
N_{*}=\left(\frac{N_{1}}{N_{2}} \frac{N_{2}}{N_{1}}\right) \in \mathcal{L}(X \times X) .
$$


Then the $\mathbb{R}$-linear operator $N=N_{1}+N_{2} J$ is Fredholm equivalent with the $\mathbb{C}$-linear operator $N_{*}$ and their indices coincide.

Proof. (a) With respect to the canonical expansions $X=X_{\mathbb{R}} \oplus i X_{\mathbb{R}} \simeq X_{\mathbb{R}}^{2}$, the operator $N$ is represented as a $2 \times 2$-matrix with elements $N_{i j}$ from $\mathcal{L}\left(X_{\mathbb{R}}, Y_{\mathbb{R}}\right)$. An analogous representation is valid for $Y$. If $N$ is real, then, by virtue of (1.3.18), this matrix is diagonal and $N_{11}=N_{22}=N_{\mathbb{R}}$. Therefore, it remains to use Lemma 1.3.2.

(b) The matrix

$$
J_{*}=\left(\begin{array}{ll}
0 & J \\
J & 0
\end{array}\right)
$$

defines a complex structure in the space $X^{2}$ such that the operator $N_{*}$ is real with respect to it, i.e., $N_{*} J_{*}=J_{*} N_{*}$. Obviously, $\left(X^{2}\right)_{\mathbb{R}}$ consists of elements $(x, \bar{x})$, where $x \in X$, and the $\mathbb{R}$-linear operator $L x=(x, \bar{x})$ isomorphically maps $X$ onto $\left(X^{2}\right)_{\mathbb{R}}$. Since

$$
L\left(N_{1}+N_{2} J\right)=N_{*} L,
$$

the operators $N=N_{1}+N_{2} J$ and $\left(N_{*}\right)_{\mathbb{R}}$ are Fredholm equivalent and their indices coincide, it follows that it remains to apply assertion (a) of the theorem to $\left(N_{*}\right)_{\mathbb{R}}$.

\subsection{Banach Algebras}

Let $A$ be a Banach space. Let $(x, y) \rightarrow x y$ be a bounded bilinear map from $A \times A$ to $A$, satisfying the associativity condition $x(y z)=(x y) z$. Then the space $A$ together with this bilinear map treated as the multiplying operation is called a Banach algebra. In particular, $A$ is a ring with respect to the addition and multiplication. We say that an algebra $A$ is commutative if $x y=y x$ for all $x$ and $y$ from A.

Due to Sec. 1.1, the boundedness condition for the multiplying operation is equivalent to the estimate

$$
|x y| \leq C|x||y|
$$

where $|\cdot|$ denotes the norm in $A$ and the positive constant $C$ is independent of $x$ and $y$ from $A$.

By virtue of (1.2.2), the Banach space $\mathcal{L}(X)$ of all operators bounded in $X$ is an example of a Banach algebra. Another example is the algebra $A=C(K)$ of all functions continuous and bounded on a topological space $K$, endowed with the pointwise operations and sup-norm. Based on Banach algebras $A_{1}, \ldots, A_{n}$, one can introduce the Banach algebra $A_{1} \times \ldots \times A_{n}$ of the direct product with component-wise operations.

One more example is the Banach algebra $A^{n \times n}$ consisting of $n \times n$-matrices $a=\left\{a_{i j}\right\}_{1}^{n}$ with elements $a_{i j}$ from $A$, endowed with the standard matrix multiplication operation

$$
(a b)_{i j}=\sum_{k=1}^{n} a_{i k} b_{k j} .
$$

An example of such an algebra is the algebra $C^{n \times n}(K)$ of continuous and bounded $n \times n$-matrixfunctions on $K$. We denote Banach algebras $A^{n \times n}$ of matrix-functions by the same symbol $A$ as scalar functions unless this causes a confusion.

In a natural way, operation (1.4.2) is extended to rectangular matrices $a$ from $A^{m \times n}$ and $b$ from $A^{n \times l}$; its result is the matrix $a b$ from $A^{m \times l}$. If decompositions $m=m_{1}+\ldots+m_{k}$ and $n=n_{1}+\ldots+n_{s}$ are given, then the matrix $a$ from $A^{m \times n}$ can be represented in the block form

$$
a=\left(\begin{array}{ccc}
a_{(11)} & \ldots & a_{(1 s)} \\
\ldots \ldots & \ldots & \ldots \\
a_{(k 1)} & \ldots & a_{(k s)}
\end{array}\right)
$$


with elements $a_{(i j)}$ from $A^{m_{i} \times n_{j}}$. If $k=1$ or $s=1$, then the corresponding block row and column are represented as follows:

$$
a=\left(\begin{array}{ccc}
n_{1} & \cdots & n_{s} \\
a_{(1)} & \cdots & a_{(s)}
\end{array}\right), \quad a=\downarrow\left(\begin{array}{ccc}
m_{1} & \cdots & m_{k} \\
a_{(1)} & \cdots & a_{(k)}
\end{array}\right) .
$$

In the general case provided by (1.4.3), the orders can be shown in a similar way. If (1.4.3) is a square matrix, i.e., $k=s$, and its non-diagonal block elements are equal to 0 , then we say that it is a block-diagonal matrix and represent it as follows: $a=\operatorname{diag}\left(a_{11}, \ldots, a_{k k}\right)$.

Consider the following general notions related to a Banach algebra $A$. An element $e$ of $A$ is called the unit if $x e=e x=x$ for any $x$ from $A$. If the unit of an algebra exists, then it is unique. In the sequel, it is denoted by 1 (as in the case of the zero vector 0 from $A$, the distinction of this notation from the field $\mathbb{C}$ of scalar values is clear from the context). If we must mark the dependence of 1 on $A$, then the notation $1=1_{A}$ is used. For example, the identical matrix $\left(1_{A} \delta_{i j}\right)$, where $\delta_{i j}$ is the Kronecker symbol, is the unit of the algebra $A^{n \times n}$.

In any Banach algebra $A$ with unity, one can introduce the equivalent norm $\|\left.\right|^{\prime}$ such that the constant $C$ in the estimate (1.4.1) is equal to 1, i.e., $|x y|^{\prime} \leq|x|^{\prime}|y|^{\prime}$ for any $x \in A$ and any $y \in A$.

To prove this, to any element $x$ of $A$, we put in correspondence the operator $L(x)$, acting as follows: $L(x) z=x z, z \in A$. By virtue of (1.4.1), this operator is bounded in $A$. Since $L(x y)=L(x) L(y)$, it follows that the norm defined by the relation $|x|^{\prime}=|L(x)|_{\mathcal{L}}$ in $A$ possesses the specified property. Inequality (1.4.1) represented in the form $|L(x) y| \leq C|x||y|$ means that $|x|^{\prime} \leq C|x|$. On the other hand, the relation $x=L(x) 1_{A}$ implies the opposite estimate $|x| \leq\left|1_{A}\right||x|^{\prime}$.

We say that a subspace $A_{0} \subseteq A$ is a subalgebra of $A$ if $x y \in A_{0}$ for all $x$ and $y$ from $A_{0}$. Any subalgebra $A_{0}$ either contains the unit 1 or does not contain it. It is clear that the closure $\bar{A}_{0}$ is also a subalgebra.

We say that a subalgebra $J \subseteq A$ is a (two-sided) ideal of $A$ if $x y \in J$ provided that $x$ or $y$ belongs to $J$. If an ideal $J$ is proper, i.e., it does not coincide with $A$, then it is obvious that $1 \notin J$. Obviously, the closure of any ideal is an ideal.

A bounded linear operator $\psi: A \rightarrow B$ is called a homomorphism of Banach algebras if

$$
\psi(x y)=(\psi x)(\psi y), \quad x, y \in A
$$

and $\psi\left(1_{A}\right)=1_{B}$ provided that both units exist. It is clear that the kernel ker $\psi$ is a closed ideal of $A$.

We say that a Banach space $X$ is an $A$-module if a bounded bilinear map $A \times X$ called the multiplication and denoted by $(a, x) \rightarrow a x$ is given such that the associativity condition $a(b x)=(a b) x, x \in X$, is satisfied and $1 x=x$ provided that the algebra $A$ contains contains the unit. Obviously, this bilinear map defines the homomorphism $R: A \rightarrow \mathcal{L}(X)$ of Banach algebras according to the rule $R(a) x=a x$. The inverse assertion also holds: the existence of such a homomorphism determines an $A$-module structure in $X$.

For example, any space $X$ is an $\mathcal{L}(X)$-module with the corresponding multiplication operation. The homomorphism $R$ corresponding to this module is the identical map. Another example is the Banach space $A^{n}$ of the direct product: it is an $A^{n \times n}$-module. The Banach algebra itself is an $A$-module with respect to the multiplication; earlier, this is used to construct an equivalent norm related to (1.4.1).

Consider an arbitrary Banach algebra $A$ with unit. We say that its element $x$ is invertible if there exists $y$ from $A$ such that $x y=y x=1$. For any $x$, the element $y$ with such properties is determined uniquely; it is denoted by $x^{-1}$ and is called the element inverse to the element $x$.

A systematic explanation of the theory of Banach algebras can be found in [56]. Recall that the sum $\sum_{n \geq 0} a_{n}$ with elements $a_{n}$ from $A$ is called absolutely converging if $\sum\left|a_{n}\right|<\infty$. By virtue of the completeness of the Banach space $A$, the original series converges indeed. 
Lemma 1.4.1. If $|x| \leq(2 C)^{-1}$, where $C$ is the constant from (1.4.1) and $C \geq 1$, then the element $1-x$ is invertible and its inverse is defined by the absolutely converging series

$$
(1-x)^{-1}=\sum_{n \geq 0} x^{n}
$$

with the norm estimate

$$
\left|(1-x)^{-1}-1\right| \leq(C|e|+1)|x|,
$$

where $e=1$ is the unit element of the algebra.

Proof. For $|x| \leq(2 C)^{-1}$, we have the inequality

$$
\left|x^{n}\right| \leq C^{n-1}|x|^{n} \leq C^{-1} 2^{-n}, \quad n \geq 1 .
$$

Thus, the series at the right-hand side of (1.4.6) absolutely converges and the norm of its sum $y$ does not exceed

$$
|y| \leq|e|+\frac{1}{C} \sum_{n \geq 1} \frac{1}{2^{n}}=|e|+\frac{1}{C} .
$$

The fact that $y$ is the inverse element for $1-x$ follows from the termwise multiplication of this series by $1-x$. In particular, $1=y-x y$ and $|y-1| \leq C|x||y| \leq(C|e|+1)|x|$.

Denote the set of all elements invertible in $A$ by $G(A)$; it is a group with respect to the multiplication. The connected component of this set, containing the unit 1 , is denoted by $G_{0}(A)$ and is called the unit component of the group $G(A)$.

\section{Theorem 1.4.1.}

(a) The set $G(A)$ is open and the map $x \rightarrow x^{-1}$ of this set into itself is continuous.

(b) The unit component $G_{0}(A)$ is open in $A$ and is an invariant subgroup of the group $G(A)$.

(c) Let $V \subseteq G_{0}(A)$ be a neighborhood of the unit 1 . Then any $x$ from $G_{0}(A)$ is representable as a finite product of elements of $V \cup V^{-1}$, where $V^{-1}=\left\{x^{-1}, x \in V\right\}$.

Proof. (a) Let $x_{0} \in G(A)$ and $|y| \leq\left(2 C^{2}\left|x_{0}\right|\right)^{-1}$. Then $x_{0}^{-1}-y=x_{0}^{-1}\left(1-x_{0} y\right)$ and, due to Lemma 1.4.1, the element $\left(1-x_{0} y\right)$ is invertible. Hence, the element $x_{0}^{-1}-y$ is also invertible. Therefore, the set $G(A)$ is open. Due to the same reasons, $x_{0}^{-1}-y \rightarrow 0$ as $y \rightarrow 0$ in $A$; hence, the map $x \rightarrow x^{-1}$ is continuous.

(b) In any Banach space, any connected open set $D$ is linearly connected. This means that for any points $x_{0}$ and $x_{1}$ from $D$ there exists a linear map $x(t), 0 \leq t \leq 1$, valued in $D$ such that $x(0)=x_{0}$ and $x(1)=x_{1}$. In other words, one can connect $x_{0}$ and $x_{1}$ by a path in $D$.

Getting back to the open set $G(A)$ of elements invertible in $A$, consider its connected component $G_{0}(A)$ containing 1 . As we note above, the open connected set $G_{0}(A)$ is linearly connected. In particular, any its point $x$ can be connected with 1 by a path, i.e., there exists a continuous map $x(t)$, $0 \leq t \leq 1$, valued in $G(A)$ such that $x(0)=1$ and $x(1)=x$. Since the functions $x(t)$ and $x^{-1}(t)$, $0 \leq t \leq 1$, are continuous, it follows that the inverse element $x^{-1}$ belongs to $G_{0}(A)$. Due to the same reasons, $a^{-1} x a \in G_{0}(A)$ for any a from $G(A)$. In the same way, we prove that $x y \in G_{0}(A)$ for all $x$ and $y$ from $G_{0}(A)$. Thus, $G_{0}(A)$ is an invariant semigroup of $G(A)$ and is a connected topological group.

(c) Let $D$ consists of all finite products of elements considered in the theorem. Obviously, $D$ is an open subset of $G_{0}(A)$, containing $V$. Let us prove that it is relatively closed in $G_{0}(A)$, i.e., any limit point $x_{0}$ of $\bar{D} \cap G_{0}(A)$ belongs to $D$.

Indeed, by assumption, for any given positive $\varepsilon$ there exists $x$ from $D$ such that $x_{0}=x+y$ and $|y|<\varepsilon$. Since $V$ is a neighborhood of 1 , it follows that $1-x_{0}^{-1} y \in V$ provided that $\varepsilon$ is sufficiently small. Hence, $x_{0}=x\left(1-x_{0}^{-1} y\right)^{-1} \in D$. Thus, the set $D$ is both open and closed in $G_{0}(A)$; since the latter one is connected, this implies that $D=G_{0}(A)$. 
Due to (1.4.5), the homomorphism $\psi: A \rightarrow B$ of Banach algebras maps invertible elements to invertible ones. In the same way, $\psi x \in G_{0}(B)$ if $x \in G_{0}(A)$. The next theorem shows that both these properties are converted simultaneously.

Theorem 1.4.2. Let $\psi: A \rightarrow B$ be a homomorphism of Banach algebras such that its image $\psi(A)$ is dense in $B$ and the invertibility of $x$ in $A$ is equivalent to the invertibility of $\psi x$ in $B$. Then $x \in G_{0}(A)$ if and only if $\psi x \in G_{0}(B)$, i.e.,

$$
\psi^{-1}\left[G_{0}(B)\right]=G_{0}(A)
$$

Proof. Consider the sets

$$
U_{A}=\{x \in A \mid \lambda-x \in G(A) \quad \text { for } \quad|\lambda| \geq 1\}, \quad V_{A}=\left\{1-x \mid x \in U_{A}\right\} .
$$

Due to Lemma 1.4.1, the set $U_{A}$ contains the ball $\left\{|x| \leq(2 C)^{-1}\right\}$ and, therefore, is a neighborhood of the origin since for any its point $x$, it contains the segment $[0, x]=\{t x \mid 0 \leq t \leq 1\}$. Hence, the set $V_{A}$ is connected, is contained in $G(A)$, and is a neighborhood of the unit. By virtue of the connectedness, it is contained in $G_{0}(A)$ and can be taken as a neighborhood from Theorem 1.4.1(c).

Let $U_{B}$ and $V_{B}$ be defined with respect to $B$ similarly to (1.4.8). From the definition given by (1.4.8), we see that

$$
\psi^{-1}\left(V_{B}\right)=V_{A} .
$$

Then, using Theorem 1.4.1(c), we easily derive (1.4.7). Let $\psi a \in G_{0}(B)$ such that $\psi a=b_{1} \cdots b_{n}$, where each $b_{j}$ belongs to $V_{B} \cup V_{B}^{-1}$. Since the image $\psi(A)=\operatorname{Im} \psi$ is dense in $B$ and the set $V_{B} \cup V_{B}^{-1}$ is open, it follows that there exist $a_{j}$ from $A$ such that $\psi a_{j} \in V_{B} \cup V_{B}^{-1}$ and $\left(b_{1} \cdots b_{n}\right)^{-1} \psi\left(a_{1} \cdots a_{n}\right) \in V_{B}$. By virtue of (1.4.9), the elements $a_{j}$ belong to $V_{A} \cup V_{A}^{-1}$, i.e., $\psi\left(a_{1} \cdots a_{n}\right)=(\psi a) \cdot b, b \in V_{B}$. Use (1.4.9) again. We obtain that $\left(a_{1} \cdots a_{n}\right) a^{-1} \in V_{A}$. Hence, $a \in G_{0}(A)$, which completes the proof of (1.4.7).

If $\psi$ is the embedding $A \subseteq B$, then we deal with a special case. If the invertibility condition for a subalgebra $A$ coincides with the invertibility in $B$, then $A$ is called a filled subalgebra (see [49]).

In the general case, the algebra $C=C^{n \times n}(K)$ of all matrix-functions $x(t)$ continuous on a compact set $K$ can be taken as $B$. Obviously, the invertibility condition for $x(t)$ is as follows: $\operatorname{det} x(t) \neq 0$, $t \in K$. The belonging of $x(t)$ to the group $G_{0}$ is determined by the possibility to select a branch of ln $\operatorname{det} x(t)$, continuous on $K$.

Theorem 1.4.2 belongs to the author (see [59]). It describes $G_{0}(A)$ in the case where a similar description of $G_{0}(B)$ is already known. For example, the algebra $C=C^{n \times n}(K)$ of all matrix-functions $x(t)$ continuous on a compact set $K$ can be taken as $B$. Obviously, the invertibility condition for $x(t)$ is as follows: $\operatorname{det} x(t) \neq 0, t \in K$. The belonging of $x(t)$ to the group $G_{0}$ is determined by the possibility to select a branch of $\ln \operatorname{det} x(t)$, continuous on $K$. The following two important cases are well known.

(1) If the compact set $K$ is simply connected, then the group $G(C)$ is connected and, therefore, coincides with $G_{0}(C)$.

(2) If the compact set $K$ is homeomorphic to a circle, then the following integer-valued function can be introduced on the group $G(C)$ of invertible elements:

$$
\operatorname{Ind}_{K} x=\left.\frac{1}{2 \pi i} \ln \operatorname{det} x(t)\right|_{K}
$$

the right-hand side is the increment of the continuous branch of $\ln \operatorname{det} x$ on the contour $K$ along a selected direction. In the framework of this notation, the unit component $G_{0}(C)$ is determined by the condition Ind $x=0$.

Now, it is convenient to introduce the following notion. A continuous complex-valued function function $\chi$ on the group $G(A)$ is called a character if it possesses the group properties

$$
\chi(x y)=\chi(x)+\chi(y), \quad \chi(1)=0 .
$$


Integer-valued character on $G(A)$ are especially important: by virtue of their continuity, such a character preserves a constant value on any connected component of the set $G(A)$ and, therefore, can be identified with a homomorphism $\tilde{\chi}$ mapping the quotient group $G / G_{0}$ into an additive group $\mathbb{Z}$. For example, the function (1.4.10) is an integer-valued character for the algebra $C$ of matrix-functions continuous on the circle $K$. For it, the properties (1.4.11) are verified immediately. If the compact set $K$ is a segment of a line, then the similar function (1.4.10) is a complex-valued character. In both cases, the function Ind is called the Cauchy index.

The next theorem provides another typical example of an integer-valued character. First, we clarify the terminology. Let $J$ be a closed two-sided ideal of $A$. Then the multiplication operation $(x+J)(y+$ $J)=x y+J$ is well defined in the Banach quotient space $A / J$ such that this space is a Banach algebra with respect to this operation. It is convenient to express all the corresponding notions in $A / J$ in terms of elements of the algebra $A$ "modulo $J$ ". For example, the assertion " $x=y$ modulo $J$ " or $x \sim y$ means that $x-y \in J$. In the same way, an element $x$ is invertible in $A$ modulo $J$ if $x+J$ is invertible in $A / J$, i.e., if there exists $y$ from $A$ such that $x y \sim y x \sim 1$. Here, it is natural to say that the element $y$ is inverse modulo $J$ for $x$. In particular, the set of all such elements is open in $A$ since it is the preimage of the open set $G(A / J)$ under the quotient map $x \rightarrow x+J$.

For example, according to Theorem 1.2.2, the subspace $\mathcal{T}(X)$ of compact operators is a closed ideal of the Banach algebra $\mathcal{L}(X)$. In the above notation, Theorem 1.3.2 can be worded as follows.

\section{Theorem 1.4.3.}

(a) The quotient map $N \rightarrow N+\mathcal{T}$ takes the class $N \in \mathcal{L}(X)$ of Fredholm operators into the group $G(\mathcal{L} / \mathcal{T})$ of elements invertible in the Banach algebra $\mathcal{L}(X) / \mathcal{T}(X)$ and induces an integer-valued character $\widetilde{\operatorname{ind}}(N+\mathcal{T})=\operatorname{ind} N$ on this algebra.

(b) Let a bounded linear map $L$ of a Banach algebra $A$ in $\mathcal{L}(X)$ be such that $L 1 \sim 1$ and $(L x) L y \sim$ $L(x y)$ modulo $\mathcal{T}(X)$ for all $x$ and $y$ from $A$. Then, if $x \in G(A)$, then $L x$ is a Fredholm operator and the function $\mathrm{ind} x=$ ind $L x$ is an integer-valued character on $G(A)$.

Proof. The first assertion in (a) is a corollary from assertion (d), while the second one follows from assertions (a)-(c) of Theorem 1.3.2. Pass to the part (b) of the theorem. By assumption, $L$ induces the homomorphism $\tilde{L}: A \rightarrow \mathcal{L} / \mathcal{T}$ of Banach algebras. Thus, it remains to use (a).

Consider linear bounded functionals $x^{*}$ on a Banach algebra $A$, possessing the multiplicative property (1.4.5). In other words, we consider functionals realizing homomorphisms of Banach algebras $A$ to $\mathbb{C}$. The set of all such multiplicative functionals is denoted by $M(A) \subseteq A^{*}$. Let us verify that this set is contained in the ball $\left|x^{*}\right| \leq C$ of the adjoint Banach space $A^{*}$, where $C$ is the positive constant from (1.4.1).

For brevity, denote the norm of the multiplicative functional $x^{*}$ from $M(A)$ by $q$. If $x \in A$, then $\left|x^{2}\right| \leq C|x|^{2}$ by virtue of (1.4.1). Therefore, taking into account the multiplicativity of the functional, we have the inequality $\left|x^{*}(x)\right|^{2}=\left|x^{*}\left(x^{2}\right)\right| \leq C q|x|^{2}$, whence $\left|x^{*}(x)\right| \leq \sqrt{C q}|x|$ for all $x$ from $A$. Hence, $q \leq \sqrt{C q}$ and, therefore, $q \leq C$.

Obviously, the kernel ker $x^{*}$ of any multiplicative functional is both an ideal and a closed subspace of codimension 1 . Such ideals are called maximal. The inverse assertion also holds: any maximal ideal is the kernel of a multiplicative functional $x^{*}$ from $M(A)$. The set $M(A)$ is especially important for commutative Banach algebras $A$.

Theorem (Gel'fand). An element a of a commutative Banach algebra $A$ with unit is invertible if and only if $x^{*}(a) \neq 0$ for any $x^{*}$ from $M(A)$.

Proof. The scheme of the proof is as follows. If $a$ is invertible, then, by virtue of (1.4.2), the relation $a a^{-1}=1$ implies the relation $x^{*}(a) x^{*}\left(a^{-1}\right)=1$ and, therefore, $x^{*}(a) \neq 0, x^{*} \in M(A)$.

Conversely, let $a$ be not invertible in $A$. Then the set $J=\{x a, x \in A\}$ is a proper ideal of $A$. Arguing as in the proof of the Hahn-Banach theorem, one can prove that there exists a maximal ideal 
$J_{0} \supseteq J$. If $x^{*}$ from $M(A)$ is determined by the kernel ker $x^{*}=J_{0}$, then $x^{*}(x)=0, x \in J$, and, in particular, $x^{*}(a)=0$.

in [20], more detailed explanation of the theory of commutative Banach algebras is provided. Due to the Banach-Alaoglu theorem (see [56]), the unit ball $B$ in the adjoint space is weakly compact. The set $M(A)$ of multiplicative functionals is a closed subset of $B$ with respect to this topology. Hence, it is a compact set. By virtue of the one-to-one correspondence between multiplicative functionals $x^{*}$ from $M(A)$ and their kernels $\operatorname{ker} x^{*}$, the set $M(A)$ is called the compact of maximal ideals.

An analog of Theorem 1.4.2 for commutative Banach algebras is the Arens-Royden theorem (see [18]) stating that the Gel'fand transformation induces an isomorphism of the quotient groups $G(A) / G_{0}(A)$ and $G(C) / G_{0}(C)$, where $C=C(K)$, while $K$ is the compact of maximal ideals.

An element $e$ of a Banach algebra $A$ is said to be involute if $e^{2}=1$. Obviously, it coincides with the inverse element $e^{-1}=e$ and determines an isomorphism $x \rightarrow x^{\diamond}=e x e$ of the algebra $A$ into itself, possessing the involute property $\left(x^{\diamond}\right)^{\diamond}=x$.

Let there exist a subalgebra $\mathcal{A} \subseteq A$ such that the specified transform is invariant on this subalgebra. Then elements of the kind $x=a+$ be form an algebra denoted by $\mathcal{A}_{e}$.

Lemma 1.4.2. An element $x=a+$ be and the element $x_{1}=a-$ be associated with it are invertible in the algebra $\mathcal{A}_{e}$ if and only if the $2 \times 2$-matrix

$$
x=\left(\begin{array}{cc}
a & b \\
b^{\diamond} & a^{\diamond}
\end{array}\right)
$$

is invertible in $\mathcal{A}$.

Recall that we also use the symbol $\mathcal{A}$ to denote the matrix algebra with elements from $\mathcal{A}$.

Proof. Denote the class of matrices of kind (1.4.12) by $\tilde{\mathcal{A}}$. It is easy to see that a matrix $x=\left(x_{i j}\right)_{1}^{2}$ from $A$ belongs to $\tilde{\mathcal{A}}$ if and only if it commutes with the involute matrix

$$
\tilde{e}=\left(\begin{array}{ll}
0 & e \\
e & 0
\end{array}\right) .
$$

Therefore, the invertibility of the matrix $x$ in the algebra $\tilde{\mathcal{A}}$ is equivalent to its invertibility in $\mathcal{A}$.

Now, we use the matrix relation

$$
\left(\begin{array}{ll}
a & b \\
\hat{b} & \hat{a}
\end{array}\right)\left(\begin{array}{cc}
1 & 1 \\
e & -e
\end{array}\right)=\left(\begin{array}{cc}
1 & 1 \\
e & -e
\end{array}\right)\left(\begin{array}{cc}
a+b e & 0 \\
0 & a-b e
\end{array}\right) .
$$

The matrix from this relation is invertible:

$$
\left(\begin{array}{cc}
1 & 1 \\
e & -e
\end{array}\right)^{-1}=\frac{1}{2}\left(\begin{array}{cc}
1 & e \\
1 & -e
\end{array}\right) .
$$

Therefore, this matrix relation above shows that the invertibility of $a \pm b e$ in the algebra $\mathcal{A}_{e}$ is equivalent to the invertibility of matrix (1.4.12) in the algebra $\tilde{\mathcal{A}}$, and, therefore, in $\mathcal{A}$.

An algebra $\mathcal{A}$ invariant with respect to the involution $x \rightarrow x^{\diamond}=$ exe can be constructed as follows. Let an involution $s$ be linked with $e$ by the relation $e s=-s e$. Then the class $\mathcal{A}$ of all elements commuting with $s$ forms a subalgebra satisfying the specified requirement. Indeed, if $a \in \mathcal{A}$, i.e., $a s=s a$, then $a^{\diamond} s=-e a s e=-e s a e=s a^{\diamond}$. Note that an element of the kind $2 x=a(1+s)+b(1-s)$ is invertible in $\mathcal{A}$ if both $a$ and $b$ are invertible, and $2 x^{-1}=a^{-1}(1+s)+b^{-1}(1-s)$ in such a case.

To conclude, we introduce analytic complex-variable functions valued in a Banach algebra $A$. Let a function $F(z)$ valued in $A$ be defined and continuous in a domain $D \subseteq \mathbb{C}$, where domains are treated as open sets (no connectedness is required). In particular, restrictions of $F$ to connected components 
of this set have no relation to each other. We say that the function $F(z)$ is analytic in this domain if the limit

$$
\lim _{z \rightarrow z_{0}}\left(z-z_{0}\right)^{-1}\left[F(z)-F\left(z_{0}\right)\right]=F^{\prime}\left(z_{0}\right)
$$

exists at any point $z_{0}$ of $D$. Respectively, the function $F^{\prime}(z), z \in D$, is called the derivative of $F(z)$.

All properties of classical analytic functions based on the Cauchy integral relation are easily extended for functions valued in $A$. First, we must introduce integrals of continuous functions $F$ valued in Banach spaces. Using the Riemann sums, one can define such an integral in the same way as in the scalar case. In the same way, one can introduce the line integral

$$
\int_{\Gamma} F(z) d z=\int_{\Gamma} F(z)(d x+i d y)
$$

of a continuous functions $F(z)$ defined on an oriented piecewise-smooth contour $\Gamma$ of the complex plane of the variable $z=x+i y$.

This integral satisfies the estimate

$$
\left|\int_{\Gamma} F(z) d z\right| \leq \int_{\Gamma}|F(z)| d s_{z} \leq L \max _{\Gamma}|F(z)|,
$$

where $|F(z)|$ denotes the norm in $A$, while $L$ is the length of the contour $\Gamma$. Also, for any continuous linear functional $x^{*}$ from $A^{*}$, the following relation holds:

$$
x^{*}\left(\int_{\Gamma} F(z) d z\right)=\int_{\Gamma} x^{*}[F(z)] d z .
$$

To prove these relations, we note that they are obvious for the Riemann sums; therefore, according to the definition of the integral, it suffices to pass to the limit.

Theorem (Cauchy). Let a function $F(z)$ valued in a Banach algebra $A$ be analytic in a finite domain $D$ bounded by a piecewise-smooth contour $\Gamma$ and continuous in $\bar{D}$. Let the contour $\Gamma$ be oriented positively with respect to $D$, i.e., the domain $D$ is located to the left with respect to such an orientation. Then

$$
\int_{\Gamma} F(z) d z=0
$$

and the Cauchy relation

$$
F\left(z_{0}\right)=\frac{1}{2 \pi i} \int_{\Gamma}\left(z-z_{0}\right)^{-1} F(z) d z, \quad z_{0} \in D .
$$

holds.

Note that, in general, the contour $\Gamma$ consists of several connected components. We say that it envelopes a compact set $K$ of a complex plane if $\Gamma$ is the boundary of a finite domain containing $K$ and the contour is oriented positively with respect to the specified domain.

Proof. From the above definition of the analyticity, we see that if $x^{*} \in A^{*}$, then the scalar function $x^{*}[F(z)]$ is analytic in the domain $D$. Therefore, the Cauchy theorem is applicable to this function:

$$
\int_{\Gamma} x^{*}[F(z)] d z=0 .
$$


Taking into account (1.4.14), this yields that

$$
x^{*}\left[\int_{\Gamma} F(z) d z\right]=0 .
$$

Since this relation holds for any $x^{*}$ from $A^{*}$, the element of the algebra $A$, defined by the integral in the square brackets, is equal to zero. The proof of the Cauchy relation is similar.

\subsection{Spectrum and Resolvent}

To any element $a$ of a Banach algebra $A$ with unit, assign the set of points $\lambda$ of the complex plane $\mathbb{C}$ such that the element $\lambda-a$ is invertible in $A$. This set is called the resolvent set of $A$ and is the domain of the function $R(\lambda)=(\lambda-a)^{-1}$ valued in $A$ and called the resolvent of the element $a$. The complement

$$
\sigma(a)=\{\lambda \in \mathbb{C} \mid \lambda-a \notin G(A)\}
$$

of this domain is called the spectrum of the element $a$. Due to Theorem 1.4.1, the resolvent is a continuous function. From Lemma 1.4.1, we see that if $|\lambda| \geq 2 C|a|$, then the element $\lambda-a$ is invertible and, therefore, the specified points $\lambda$ belong to the resolvent set and the function $R(\lambda)$ is expanded into the uniformly and absolutely converging series

$$
R(\lambda)=\frac{1}{\lambda}\left(1-\frac{a}{\lambda}\right)^{-1}=\sum_{n=0}^{\infty} \frac{a^{n}}{\lambda^{n+1}}, \quad|\lambda| \geq 2 C|a| .
$$

In particular, the spectrum $\sigma(a)$ is a bounded set of the plane.

By definition, the resolvent set is the preimage of an open set $G(A)$ under the continuous map $\lambda \rightarrow \lambda-a$ and, therefore, is open on the plane. Respectively, the spectrum $\sigma(a)$ is closed; taking into account its boundedness, we conclude that it is a compact set.

By virtue of the obvious identity $R(\lambda)-R\left(\lambda_{0}\right)=-\left(\lambda-\lambda_{0}\right) R(\lambda) R\left(\lambda_{0}\right)$, the following relation holds:

$$
\lim _{\lambda \rightarrow \lambda_{0}}\left(\lambda-\lambda_{0}\right)^{-1}\left[R(\lambda)-R\left(\lambda_{0}\right)\right]=-\left[R\left(\lambda_{0}\right)\right]^{2} .
$$

Hence, the resolvent $R(\lambda)=(\lambda-a)^{-1}$ treated as a function valued in $A$ is analytic in the open set $\mathbb{C} \backslash \sigma(a)$.

Theorem 1.5.1. For any nonnegative integer $m$, the relation

$$
a^{m}=\frac{1}{2 \pi i} \int_{|z|=r} z^{m} R(z) d z,
$$

where the positive $r$ is sufficiently large and the circle is oriented counterclockwise, holds.

In particular, the spectrum $\sigma(a)$ is always nonempty and continuously depends on a in the following sense: for any open set $G \subseteq \mathbb{C}$, the set $\sigma_{G}=\{a \in A \mid \sigma(a) \subseteq G\}$ is open in $A$.

Proof. As we note above, series (1.5.1) holds absolutely and uniformly on the circle $|z|=r$ provided that $r \geq 2 C|a|$. Multiplying this series by $z^{m}$ and integrating termwise, we obtain the expansion

$$
\frac{1}{2 \pi i} \int_{|z|=r} z^{m} R(z) d z=\sum_{n \geq 0} \frac{a^{n}}{2 \pi i} \int_{|z|=r} z^{m-n-1} d z
$$

immediately implying (1.5.2).

If $\sigma(a)=\varnothing$, then the function $R(\lambda)$ is analytic on the whole complex plane. Then, due to the Cauchy theorem, all integrals on the right-hand side of (1.5.2) are equal to zero. However, if $m=0$, then this contradicts the fact that the left-hand side of the inequality is equal to zero 1 .

Further, let a set $G \subseteq \mathbb{C}$ be open and a compact set $K \subseteq \mathbb{C}$ do not intersect $G$. Since the resolvent $R(\lambda)$ is continuous on $\bar{K}$, it follows that there exists a positive constant $M$ such that $\left|(\lambda-a)^{-1}\right| \leq M$, 
$\lambda \in K$. Let $|y| \leq 1 / 2 M C^{2}$, where $C$ is the constant from (1.4.1). Then $\left|(\lambda-a)^{-1} y\right| \leq M C|y| \leq 1 / 2 C$ and, due to Lemma 1.4.1, the element $1-(\lambda-a)^{-1} y$ belongs to $G(A)$ for any $\lambda$ from $K$. Hence, the same is also valid with respect to $\lambda-a-y=(\lambda-a)\left[1-(\lambda-a)^{-1} y\right]$ since the sets $K$ and $\sigma(a+y)$ do not intersect. Since the compact set $K$ outside $G$ is selected arbitrarily, it follows that the set $\sigma_{G}=\{a \in A \mid \sigma(a) \subseteq G\}$ is open.

To any element $a$ of $A$, assign the nonnegative number

$$
\operatorname{spr} a=\max _{\nu \in \sigma(a)}|\nu|
$$

called its spectral radius. In other words, the spectral radius is the radius of the least disc $|z| \leq \rho$ containing the spectrum $\sigma(a)$.

Lemma 1.5.1. For any $r$ exceeding $\operatorname{spr} a$, there exists a positive constant $M$ such that

$$
\left|a^{n}\right| \leq M r^{n}, \quad n=0,1, \ldots
$$

Conversely, if such an estimate holds, then $r \geq \operatorname{spr} a$.

Proof. Let $r>\operatorname{spr} a$. By virtue of the Cauchy theorem, the relation (1.5.2), which is already found for $r \geq 2 C|a|$, also holds for the considered values of $r$. Therefore, taking into account (1.4.13), we see that the relation (1.5.2) implies the estimate (1.5.3) with the constant

$$
M=\frac{1}{2 \pi} \int_{|z|=r}|R(z)| d s_{z} .
$$

Conversely, let the estimate (1.5.3) be satisfied and $|\lambda|>r$. Then the series

$$
b=\sum_{n \geq 0} \frac{a^{n}}{\lambda^{n}}
$$

absolutely converges and, similarly to Lemma 1.4.1, we verify that its sum $b$ satisfies the relation

$$
b\left(1-\frac{a}{\lambda}\right)=\left(1-\frac{a}{\lambda}\right) b=1 .
$$

Hence, the element $\lambda-a$ is invertible and the spectrum $\sigma(a)$ lies in the disc $|\nu| \leq r$.

From Lemma 1.5.1 and the definition of upper limits, it follows that

$$
\operatorname{spr} a=\limsup _{n \rightarrow \infty}\left|a^{n}\right|^{1 / n} .
$$

Note that the limit on the right-hand side of this relation is independent of the choice of the equivalent norm in the Banach algebra $A$, which is consistent with the definition of the spectral radius. It is possible to show that the upper limit in (1.5.4) can be replaced by the standard limit.

The case where $\operatorname{spr} a=0$ or, which is equivalent, $\sigma(a)=\{0\}$, is considered separately. In this case, (1.5.4) implies that $\left|a^{n}\right|^{1 / n} \rightarrow 0$ as $n \rightarrow \infty$. Elements $a$ possessing this property are called quasinilpotent. Recall that an element $a$ is called nilpotent if there exists a positive integer $n$ such that $a^{n}=0$. The least positive integer $n$ possessing this property is called the order (of the nilpotency) of the element $a$.

Let a scalar function $f(z)$ be analytic in a neighborhood of $\sigma(a)$. Select a smooth contour $\Gamma \subseteq D$ such that it envelopes the spectrum $\sigma(a)$ and is positively oriented with respect to it. Then the Cauchy relation

$$
f(\nu)=\frac{1}{2 \pi i} \int_{\Gamma} f(z)(z-\nu)^{-1} d z, \nu \in \sigma(a)
$$

holds. 
Define the value of $f(z)$ of an element $a$ from $A$ as the integral

$$
f(a)=\frac{1}{2 \pi i} \int_{\Gamma} f(z)(z-a)^{-1} d z
$$

corresponding to the formal change of $\nu$ by $a$ in the Cauchy relation. By virtue of the Cauchy theorem, this definition is independent of the choice of the contour $\Gamma$. Note that, for $f(z)=z^{m}, m=0,1, \ldots$, this definition is consistent with the relation (1.5.2), where the contour of the integrating can be replaced by $\Gamma$ due to the Cauchy theorem.

Definition (1.5.5) and the property (1.4.14) of the integral imply the estimate

$$
|f(a)| \leq \frac{L}{2 \pi} \max _{z \in \Gamma}\left|f(z)(z-a)^{-1}\right|
$$

of the norm of the element $f(a)$, where $L$ denotes the length of the contour $\Gamma$.

If an element $b$ of $A$ is invertible, then the similarity transformation $x \rightarrow b^{-1} x b$ commutes with operation (1.5.5):

$$
b^{-1}[f(a)] b=f\left(b^{-1} a b\right) .
$$

This immediately follows from the obvious relation $b^{-1}(z-a)^{-1} b=\left[z-\left(b^{-1} a b\right)\right]^{-1}$.

If the function $f$ is analytic in a neighborhood of the disc $|z| \leq \operatorname{spr} a$, the definition given by (1.5.5) can be refined. In this case, there exists $r$ exceeding spr $a$ such that the said function is expanded in the uniformly converging power series

$$
f(z)=\sum_{k=0}^{\infty} \alpha_{k} z^{k}, \quad|z| \leq r .
$$

Let us prove that

$$
f(a)=\sum_{k=0}^{\infty} \alpha_{k} a^{k} .
$$

By virtue of the estimate (1.5.3), the series at the right-hand side of (1.5.8) converges absolutely. If (1.5.7) is a finite sum, then the relation (1.5.8) follows from (1.5.2) and the Cauchy theorem (see above). In the general case, the sequence $f_{n}(z)=\alpha_{0}+\alpha_{1} z+\ldots+\alpha_{n} z^{n}$ of polynomials converges to $f(z)$ uniformly on the circle $|\lambda|=r$. Hence, applying the estimate (1.5.6) to the difference $f-f_{n}$, we conclude that $f_{n}(a) \rightarrow f(a)$ as $n \rightarrow \infty$. Finally, passing to the limit, we obtain the validity of (1.5.8) in the general case.

Functions of elements of a Banach algebra are related (in a natural way) with the multiplication and superposition operations for functions.

Theorem 1.5.2. If functions $f(z)$ and $g(z)$ are analytic in a neighborhood of $\sigma(a)$, then

$$
f(a) g(a)=(f g)(a) .
$$

The spectrum $\sigma[f(a)]$ coincides with the set $f(\sigma)=\{f(\nu) \mid \nu \in \sigma\}$. If a function $h(z)$ is analytic in a neighborhood of the disc $|z| \leq \operatorname{spr}[f(a)]$, then

$$
h[f(a)]=(h \circ f)(a), \quad(h \circ f)(z)=h[f(z)] .
$$

Proof. Similarly to (1.5.5), we have the relation

$$
g(a)=\frac{1}{2 \pi i} \int_{\Gamma_{1}} g\left(z_{1}\right)\left(z_{1}-a\right)^{-1} d z_{1},
$$


where the contour $\Gamma_{1} \subseteq D$ is such that $\Gamma$ lies strictly inside it. Then the product of integrals can be represented by the iterated integral

$$
f(a) g(a)=\frac{1}{(2 \pi i)^{2}} \int_{\Gamma} \int_{\Gamma_{1}} f(z) g\left(z_{1}\right)(z-a)^{-1}\left(z_{1}-a\right)^{-1} d z d z_{1} .
$$

Using the obvious identity

$$
(z-a)^{-1}\left(z_{1}-a\right)^{-1}=-\left(z-z_{1}\right)^{-1}\left[(z-a)^{-1}-\left(z_{1}-a\right)^{-1}\right]
$$

we represent the integral from the right-hand side of the previous inequality as follows:

$$
\begin{gathered}
f(a) g(a)=-\frac{1}{(2 \pi i)^{2}} \int_{\Gamma} \int_{\Gamma_{1}} f(z) g\left(z_{1}\right)\left(z-z_{1}\right)^{-1}(z-a)^{-1} d z d z_{1} \\
+\frac{1}{(2 \pi i)^{2}} \int_{\Gamma} \int_{\Gamma_{1}} f(z) g\left(z_{1}\right)\left(z-z_{1}\right)^{-1}\left(z_{1}-a\right)^{-1} d z d z_{1} .
\end{gathered}
$$

Since

$$
\frac{1}{2 \pi i} \int_{\Gamma_{1}} g\left(z_{1}\right)\left(z-z_{1}\right)^{-1} d z_{1}=-g(z), \quad \frac{1}{2 \pi i} \int_{\Gamma} f(z)\left(z-z_{1}\right)^{-1} d z=0
$$

(due to the Cauchy theorem), it follows that (1.5.9) is valid.

If there exists $\nu$ from $\sigma=\sigma(a)$ such that $f(\nu)=0$, then $f(z)=(z-\nu) g(z)$, where $g(z)$ is analytic in $D$. Then, due to (1.5.9), we have the relation $f(a)=(a-\nu) g(a)=g(a)(a-\nu)$. This means that $f(a)$ is not an invertible element since $a-\nu$ and $g(a)$ would be invertible in this case, while their invertibility does not hold due to the choice of $\nu$. Thus, $f(\sigma)$ is a subset of $\sigma[f(a)]$. Conversely, if $\nu \notin f(\sigma)$, then $\nu-f(\lambda) \neq 0, \lambda \in \sigma$, and, therefore, the function $g(\lambda)=[\nu-f(\lambda)]^{-1}$ is analytic in a neighborhood of $\sigma$. Due to (1.5.9), this implies that $\nu \notin \sigma[f(a)]$. If $h(z)=z^{k}$, then the relation (1.5.10) follows from (1.5.9). Therefore, it is also valid for polynomials $h(z)=p(z)$. In the general case, as for the proof of (1.5.8), it remains to pass to the limit.

It follows from Theorem 1.5.2 that the exponents

$$
\exp a=\sum_{n \geq 0} \frac{a^{n}}{n !}
$$

form a neighborhood of 1 . Indeed, let $x=1+y$ and $\operatorname{spr} y<1$. Then, by virtue of the said theorem,

$$
x=\exp a, \quad a=\ln x=-\sum_{n \geq 1} \frac{(-y)^{n}}{n} .
$$

Obviously, exponents $\exp a$ belong to the unit component $G_{0}(A)$ of the group $G(A)$ since the function $x(t)=\exp (t a)$ is continuous with respect to $t$ from $[0,1]$ and $x(0)=1$. In particular, due to Theorem 1.4.1(c), any element $x$ from $G_{0}(A)$ can be represented as a finite product of exponents: $x=\exp a_{1} \exp a_{2} \cdots \exp a_{n}$.

If the spectrum $\sigma(a)$ consists of the only point $\nu$ from $\mathbb{C}$, i.e., the element $\nu-a$ is quasinilpotent, then

$$
f(a)=\sum_{n=0}^{\infty} \frac{f^{(n)}(\nu)}{n !}(a-\nu)^{n}, \quad \sigma(a)=\{\nu\} .
$$

If the element $a-\nu$ is nilpotent and its order is equal to $m$, then the above series is a finite sum: it is finished at $n=m$. In this case, we have the following expansion for the resolvent $R(z)=(z-a)^{-1}=$ $[(z-\nu)-(\nu-a)]^{-1}$ :

$$
(z-a)^{-1}=\sum_{n=0}^{m-1}(z-\nu)^{-n-1}(a-\nu)^{n}
$$


thus, it has an $m$-order pole at the point $z=\nu$.

Consider the Banach algebra $A=\mathcal{L}(X)$ of bounded linear operators acting in a Banach space $X$. By definition, a point $\lambda$ belongs to the spectrum $\sigma(N)$ of an operator $N$ from $\mathcal{L}(X)$ if the operator $\lambda-N$ treated as an element of $\mathcal{L}$ is not invertible or, which is equivalent, the said operator has no bounded inverse operator. Due to the Banach theorem from Sec. 1.2, this is possible if either the kernel $\operatorname{ker} N$ is different from zero or $\operatorname{ker} N=0$, but the image $\operatorname{Im} N$ does not coincide with the whole space $X$. In the former case, points $\lambda$ of the spectrum are called eigenvalues of the operator $N$. Respectively, nonzero vectors of the kernel ker $N$, i.e., solutions $x$ of the homogeneous equation $\lambda x-N x=0$, belonging to $X$, are called eigenvectors corresponding to the given eigenvalue.

\subsection{Number Matrices}

Consider the Banach algebra $\mathbb{C}^{n \times n}$ of number matrices such that its elements are denoted by capital Latin letters $A=\left(A_{i j}\right)_{1}^{n}, B, \ldots$ Also, it is convenient to treat the matrix $A$ as a linear transformation, i.e., a linear operator $A$ from $\mathcal{L}\left(\mathbb{C}^{n}\right)$, mapping any vector $x$ from $\mathbb{C}^{n}$ to the vector with coordinates $(A x)_{i}=A_{i 1} x_{1}+\ldots+A_{i n} x_{n}, i=1, \ldots, n$. If the columns of the matrix are denoted by $A_{(j)}=\left(A_{1 j}, \ldots, A_{n j}\right)$ and are treated as elements of $\mathbb{C}^{n}$, then the matrix product is expressed as follows:

$$
(A B)_{(j)}=A B_{(j)}=B_{1 j} A_{(1)}+\ldots+B_{n j} A_{(n)} .
$$

The column $A_{(j)}$ is the linear combination $A e_{j}=A_{1 j} e_{1}+\ldots+A_{n j} e_{n}$, where $e_{1}=(1,0, \ldots, 0), \ldots$, $e_{n}=(0, \ldots, 0,1)$ is the standard base. Such a linear combination is obtained for any base $b_{1}, \ldots, b_{n}$ of the space $\mathbb{C}^{n}$ if we assign

$$
A b_{j}=J_{1 j} b_{1}+\ldots+J_{n j} b_{n}, \quad 1 \leq j \leq n,
$$

where $J_{i k} \in \mathbb{C}$. The matrix $J=\left(J_{i k}\right)_{1}^{n}$ is called the matrix of the operator $A$ with respect to the base $b_{1}, \ldots, b_{n}$. If the matrix $B$ is formed by columns $b_{j}$, i.e., $B_{(j)}=b_{j}$, then, by virtue of (1.6.1), the relation (1.6.2) can be represented by the matrix relation $A B=B J$, i.e., the matrix $J=B^{-1} A B$ is homothetic to $A$. The same can be done for subspaces $X \subseteq \mathbb{C}^{n}$ invariant with respect to $A$. In this case, we have a rectangular matrix $B$.

Lemma 1.6.1. Let an l-dimensional subspace $X$ of $\mathbb{C}^{n}$ be invariant with respect to the matrix $A$ from $\mathbb{C}^{n \times n}$ and columns of a matrix $B$ from $\mathbb{C}^{n \times l}$ form a base of $X$. Then there exists a unique matrix $J$ from $\mathbb{C}^{l \times l}$ such that $A B=B J$. If $\tilde{B}, \tilde{J}$ is another matrix pair possessing this property, then there exists an invertible matrix $D$ from $\mathbb{C}^{l \times l}$ such that $\tilde{B}=B D$ and $\tilde{J}=D^{-1} J D$.

Proof. By assumption, there exist $J_{i k}$ from $\mathbb{C}$ such that

$$
A B_{(j)}=J_{1 j} B_{(1)}+\ldots+J_{l j} B_{(l)}, \quad 1 \leq j \leq l .
$$

As above, this relation can be represented by the matrix relation $A B=B J$. If $\tilde{B}, \tilde{J}$ is another matrix pair possessing this property, then there exist $D_{i k}$ from $\mathbb{C}$ such that the matrix $D=\left(D_{i k}\right)_{1}^{l}$ is invertible and

$$
\tilde{B}_{(j)}=D_{1 j} B_{(1)}+\ldots+D_{l j} B_{(l)} .
$$

By virtue of (1.6.1), this relation can be represented in the form $\tilde{B}=B D$. Substituting this relation to the relation $A \tilde{B}=\tilde{B} \tilde{J}$, we obtain the relation $A B=B J_{1}$ with the matrix $J_{1}=D \tilde{J} D^{-1}$. It remains to note that this implies that $J_{1}=J$ by virtue of the uniqueness.

As above, it is natural to call $J$ the matrix of the operator $A$ in in the invariant subspace $X$ (with respect to a base). If the whole space $\mathbb{C}^{n}$ is expanded into a direct sum of invariant subspaces $X_{k}$, $k=1, \ldots, m$, and the matrices $B_{k}$ and $J_{k}$ are constructed by $X_{k}$ as in the lemma, then the following relation is valid for the invertible matrix $B=\left(B_{1}, \ldots, B_{m}\right)$ from $\mathbb{C}^{n \times n}$ :

$$
B^{-1} A B=\operatorname{diag}\left(J_{1}, \ldots, J_{m}\right) .
$$


In other words, we find an appropriate matrix $B$ to reduce the matrix $A$ to a block-diagonal form.

An important example of invariant subspaces is related to the spectrum $\sigma(A)$ of the matrix $A$. This spectrum consists of different roots $\nu_{1}, \ldots, \nu_{m}$ of the characteristic polynomial $\chi(z)=\operatorname{det}(z-A)$, called eigenvalues of the matrix $A$. In the expansion

$$
\chi(z)=\left(z-\nu_{1}\right)^{k_{1}} \ldots\left(z-\nu_{m}\right)^{k_{m}}, \quad n=k_{1}+\ldots+k_{m},
$$

of this polynomial into factors, the power $k_{j}$ is the multiplicity of the eigenvalue $\nu_{j}$. The resolvent $(z-A)^{-1}$ is a matrix polynomial divided by the characteristic polynomial $\chi$. Therefore, the function $R(z)=(z-a)^{-1}$ has a pole at the point $\nu_{j}$; its order $r_{j}$ does not exceed the multiplicity $k_{j}$ and is called the order of the eigenvalue $\nu_{j}$. If the polynomial $\chi_{0}(z)$ is obtained from (1.6.4) by means of the replacement of $k_{j}$ by $r_{j}$, then the singularities $\nu_{j}$ of the matrix-function $\chi_{0}(z)(z-A)^{-1}$ are removable and, therefore, it is a polynomial. In particular, the integral (1.5.5) vanishes for $f=\chi_{0}$, i.e., $\chi_{0}(A)=0$. The polynomial $\chi_{0}(z)$ is called the minimal polynomial of the matrix $A$. The same argument yields that $\chi(A)=0$, which is the assertion of the known Hamilton-Cayley theorem. For any function $f(z)$ analytic in a neighborhood of the spectrum $\sigma(A)$ of the matrix $A$ and any polynomial $p(z)$ such that $f(z)-p(z)=O(1)\left(z-\nu_{j}\right)^{r_{j}}$ in a neighborhood of $\nu_{j}, j=1, \ldots, m$, we have the relation $f(A)=p(A)$. This yields a practical method to compute the matrix $f(A)$.

Consider a function $p_{j}(z)$ such that it is equal to 1 in a neighborhood of $\nu_{j}$ and to 0 in neighborhoods of other points $\nu$ from $\sigma(a)$. Obviously, the relations $p_{i} p_{j}=\delta_{i j} p_{i}$, where $\delta$ is the Kronecker symbol, and $p_{1}+\ldots+p_{m}=1$, hold in a neighborhood of the spectrum. Due to Theorem 1.5.2, this implies that $P_{j}=p_{j}(A)$ are projectors possessing the similar property: $P_{i} P_{j}=\delta_{i j} P_{i}$ and $P_{1}+\ldots+P_{m}=1$. Hence, the space $\mathbb{C}^{n}$ is decomposed into the direct $\operatorname{sum} X_{1} \oplus \ldots \oplus X_{m}, X_{i}=\operatorname{Im} P_{i}$. Since $A P_{i}=P_{i} A$, it follows that the subspace $X_{i}$ is invariant with respect to the transformation $A$; it is called the eigensubspace corresponding to the eigenvalue $\nu_{j}$.

If $A_{j}$ from $\mathcal{L}\left(X_{j}\right)$ is the restriction of $A$ to $X_{j}$, then the operator $\nu-A_{j}$ is invertible provided that $\nu \neq \nu_{j}$ and $\left(\nu_{j}-A_{j}\right)^{r_{j}}=0$. Thus, for any $x$ from $X_{j}$ there exists a positive integer $r$ such that $r \leq r_{j},\left(\nu_{j}-A\right)^{r} x=0$, and $\left(\nu_{j}-A\right)^{r-1} x \neq 0$. Vectors $x$ from $\mathbb{C}^{n}$ possessing this property are called augmented vectors of the matrix $A$, corresponding to the eigenvalue $\nu_{j}$. Assigning $x_{1}=\left(\nu_{j}-A\right)^{r-1} x$, $x_{2}=\left(\nu_{j}-A\right)^{r-2} x, \ldots, x_{r}=x$, we obtain a chain of eigenvalues and augmented vectors linked by the relations

$$
(\nu-A) x_{1}=0,(\nu-A) x_{2}=x_{1}, \ldots,(\nu-A) x_{s}=x_{s-1},
$$

where $\nu=\nu_{j}$.

Sequentially selecting elements of the bases of the subspaces $X_{j}$ as columns of the matrix $B$, one can reduce the matrix $A$ to the block-diagonal form represented by (1.6.3). Here, the matrix $J_{j}$ is similar to the operator $A_{j}$ acting in the space $X_{j}$. In particular, the characteristic polynomial (1.6.4) coincides with the product of the analogous polynomials $\operatorname{det}\left(z-A_{j}\right)=\left(z-\nu_{j}\right)^{k_{j}}$ and $k_{j}=\operatorname{dim} X_{j}$.

Taking into account (1.5.7) and (1.5.11), from (1.6.3) we obtain the following relation for the computing of the function $f(A)$ of the matrix $A$ :

$$
B^{-1} f(A) B=\operatorname{diag}\left[f\left(J_{1}\right), \ldots, f\left(J_{m}\right)\right], f\left(J_{j}\right)=\sum_{k \geq 0} \frac{f^{(k)}\left(\nu_{j}\right)}{k !}\left(J_{j}-\nu_{j}\right)^{k}
$$

Here, the series is actually a finite sum; it terminates by the order $k=r_{j}$ of the eigenvalue $\nu_{j}$.

A simple example of $f(A)$ is provided by the so-called Jordan box $A=J$ : the elements $\nu$ are located at its main diagonal, the elements 1 are located at the next diagonal above the main one, and all other elements are equal to zero. Thus,

$$
J_{i j}=\nu \delta_{i j}+\delta_{i+1, j}
$$


where $\delta_{i j}$ denotes the Kronecker symbol. The simple verification confirms the relation $\left[(J-\nu)^{k}\right]_{i j}=$ $\delta_{i+k, j}$ for this matrix. Therefore, (1.5.11) leads to the following explicit relation:

$$
[f(J)]_{i j}= \begin{cases}0, & j-i<0, \\ f^{(j-i)}(\nu) /(j-i) !, & j-i \geq 0 .\end{cases}
$$

The block-diagonal matrix composed of Jordan $\nu$-boxes is called a (composite) box again. Respectively, matrices (1.6.7) are treated as simple boxes.

The next classical result is the main theorem of linear algebra (see, e.g., [40]).

Theorem 1.6.1 (Jordan). For any matrix $A$ from $\mathbb{C}^{n \times n}$ such that its spectrum $\sigma(A)$ consists of points $\nu_{1}, \ldots, \nu_{m}$ there exists an invertible matrix $B=\left(B_{1}, \ldots, B_{m}\right)$ from $\mathbb{C}^{n \times n}$, reducing $A$ to the blockdiagonal form (1.6.3), where $J_{j}$ is a composite Jordan $\nu_{j}$-box. The columns of the matrix $B_{j}$ are sequentially composed of chains of eigenvectors and augmented vectors corresponding to $\nu_{j}$ and the number of simple Jordan boxes of the same order, contained in $J_{j}$, is an invariant of the matrix A, i.e., is independent of the choice of $B$.

Proof. According to Lemma 1.6.1, it suffices to show that a base consisting of chains of eigenvectors and augmented vectors can be selected in the eigensubspace $X=X_{j}$ of the matrix $A$, corresponding to the eigenvalue $\nu=\nu_{j}$. Without loss of generality, one can assume that $\nu=0$ ( $A$ can be replaced by $A-\nu$ otherwise). Then $A^{r} X=0$ and $A^{r-1} X$ is a nonzero eigensubspace of $X$, where $r$ is the order of the eigenvalue $\nu=0$. Select a subspace $Y_{1}$ of $X$ such that

$$
A^{r-1} X=A^{r-1} Y_{1}, \quad Y_{1} \cap \operatorname{ker} A^{r-1}=0 .
$$

In particular, its dimension coincides with the dimension of the image $A^{r-1} X$,

$$
X=A^{r-1} Y_{1} \oplus X_{0}, \quad X_{0} \subseteq \operatorname{ker} A^{r-1} .
$$

To prove that $Y_{1}+A Y_{1}+\ldots+A^{r-1} Y_{1}$ is a direct sum of subspaces, assume that there exist $y_{j}$ from $Y_{1}$ such that $y_{1}+A y_{2}+\ldots+A^{r-1} y_{r}=0$. Apply the operator $A^{r-1}$ to this relation. We obtain that $A^{r-1} y_{1}=0$. Taking into account (1.6.9), we conclude that $y_{1}=0$. Thus, $A y_{2}+\ldots+A^{r-1} y_{r}=0$. Applying the operator $A^{r-2}$ to this relation, we obtain that $y_{2}=0$. Repeating this procedure, we verify that $y_{j}=0$ for any $j$. Thus, the specified sum is direct. Since the subspaces $A^{j} Y_{1}$ are contained in ker $A^{r-1}$ provided that $j \geq 1$, we take into account (1.6.10) to obtain the expansion

$$
X=Y_{1} \oplus \ldots \oplus A^{r-1} Y_{1} \oplus \tilde{X}, \quad \tilde{X} \subseteq \operatorname{ker} A^{r-1} .
$$

Applying the same argument to $\tilde{X}$ and repeating this procedure, we obtain the expansion

$$
X=\left(Y_{1} \oplus \ldots \oplus A^{r-1} Y_{1}\right) \oplus\left(Y_{2} \oplus \ldots \oplus A^{r-2} Y_{2}\right) \oplus \ldots \oplus\left(Y_{r-1} \oplus A Y_{r-1}\right) \oplus Y_{r}
$$

where

$$
Y_{j} \cap \operatorname{ker} A^{r-j}=0,1 \leq j \leq r-1, \quad Y_{1} \subseteq \operatorname{ker} A .
$$

Note that the above expansion can contain zero spaces $Y_{j}$ since it is possible that there exists $s$ exceeding 1 such that $\tilde{X} \subseteq \operatorname{ker} A^{r-s}$ in (1.6.11) and similar further relations.

Now, select a base $e_{k}^{j}, 1 \leq k \leq s_{j}$, in the space $Y_{j}$. Then, due to (1.6.12), the vectors $A^{i} e_{k}^{j}$, $0 \leq i \leq r-j-1$, form a chain of eigenvectors and augmented vectors, and all these chains form a base of the space $X$.

The matrix $J$ from the last theorem is called the Jordan form of the matrix $A$. Then the columns of the matrix $B$ have the following geometric interpretation.

Frequently, it is more convenient to consider an "enlarged" expansion instead of (1.6.3); the said expansion corresponds to the decomposition of the spectrum $\sigma(A)$ into the three sets $\sigma_{0}=\mathbb{R} \cap \sigma$ and $\sigma_{ \pm}=\{\nu \in \sigma \mid \pm \operatorname{Im} \nu>0\}$ on the plane, determined by the real axis $\mathbb{R}$. Let $X_{0}$ and $X_{ \pm}$ correspond to the direct sum of eigensubspaces $X_{j}$ corresponding to the values $\nu_{j}$ from $\sigma_{0}$ and $\nu_{j}$ 
from $\sigma_{ \pm}$respectively. Denote the dimensions of these spaces by $n_{0}$ and $n_{ \pm}$, respectively. Then $\mathbb{C}^{n}=X_{0} \oplus X_{+} \oplus X_{-}$. Respectively, the relation (1.6.3) takes the form

$$
B^{-1} A B=\operatorname{diag}\left(J_{0}, J_{+}, J_{-}\right), \quad \sigma\left(J_{0}\right)=\sigma_{0}, \quad \sigma\left(J_{ \pm}\right)=\sigma_{ \pm}
$$

with the matrix $B=\left(B_{0}, B_{+}, B_{-}\right)$, where $B_{0} \in \mathbb{C}^{n \times n_{0}}, J_{0} \in \mathbb{C}^{n_{0} \times n_{0}}$, and the matrices $B_{ \pm}$and $J_{ \pm}$ have the same sense with respect to $n_{ \pm}=\operatorname{dim} X_{ \pm}$.

If the matrix $A$ is real, then $n_{+}=n_{-}$and, therefore, the complex-conjugation operation $x \rightarrow \bar{x}$ is invariant on $X_{0}$ and maps $X_{+}$onto $X_{-}$. Then the bases in these spaces (they form columns of the matrices $B_{0}$ and $B_{ \pm}$) can be selected so that they satisfy the following conditions:

$$
B_{0} \in \mathbb{R}^{n \times n_{0}}, \quad J_{0} \in \mathbb{R}^{n_{0} \times n_{0}}, \quad B_{-}=\bar{B}_{+}, \quad J_{-}=\bar{J}_{+} .
$$

It might be more convenient to pass from $B_{-}$and $J_{-}$to complex-conjugated matrices in (1.6.13). Then the spectrum $\sigma\left(J_{-}\right)=\bar{\sigma}_{-}$is located in the upper half-plane and one must assign $J_{+}=J_{-}$for the real matrix $A$ in (1.6.14).

More detailed data about number matrices can be found in [19].

\subsection{Semi-Almost Periodic Functions}

Consider the Banach algebra $C$ of all continuous and bounded on $\mathbb{R}$ functions, endowed with the pointwise operations and the sup-norm

$$
|x|_{0}=\sup _{t}|x(t)|
$$

The subspace $C^{0}$ of all functions vanishing at $\infty$ is a closed ideal of this algebra. Obviously, the inequality

$$
\inf _{t}|x(t)|>0
$$

is a necessary and sufficient condition of the invertibility of $x$ in $C$. If $x(t)$ possesses the above property, then we call it a nondegenerate function.

We say that a function $x(t)$ from $C$ is almost periodic if for any positive $\varepsilon$ there exists a positive $l$ such that any interval of length $l$ contains a number $\tau$ such that

$$
|x(t+\tau)-x(t)| \leq \varepsilon, \quad t \in \mathbb{R} .
$$

In [38], a detailed explanation of the theory of almost periodic functions is provided. In particular, it is known that the class of all almost periodic functions forms a closed subalgebra in $C$ and trigonometric polynomials, i.e., finite sums of kind

$$
x(t)=\sum c_{k} e^{i a_{k} t}, \quad c_{k} \in \mathbb{C}, \quad a_{k} \in \mathbb{R},
$$

are dense in this class. One of the main results of the theory of almost periodic functions is as follows.

Theorem (on the argument of almost periodic functions). If an almost periodic function $x(t)$ is nondegenerate, then the continuous branch of its logarithm is representable in the form

$$
\ln x(t)=i a t+y(t)
$$

where $a \in \mathbb{R}$ and $y$ is an almost periodic function. In particular, the inverse function $x^{-1}$ is also almost periodic.

We say that a function $c$ from $C$ has (one-sided) mean values $m^{ \pm} x$ at $\pm \infty$ if there exist

$$
m^{+} x=\lim _{n \rightarrow \infty} \frac{1}{n} \int_{n}^{2 n} x(t) d t, \quad m^{-} x=\lim _{n \rightarrow \infty} \frac{1}{n} \int_{-2 n}^{-n} x(t) d t .
$$


Obviously, the class of functions possessing these mean values is a closed subspace of $C$. If both one-sided mean values coincide, then they are denoted by $m x$ and are called the mean value of the function $x$. It is obvious that

$$
m\left(e^{-i a t}\right)= \begin{cases}0, & a \neq 0, \\ 1, & a=0 .\end{cases}
$$

In particular, the mean value $m u$ exists for trigonometric polynomials (1.7.2) and its coefficients $c_{k}=m\left(e^{-a_{k} t} u\right)$ are defined uniquely for any given collection of $a_{j}$. Taking into account the density, we conclude that the mean value $m x$ exists for all almost periodic functions. Arguing in the same way, we conclude that if (1.7.2) is an absolutely converging series, then its coefficients $c_{k}$ are uniquely defined by $x$.

The class of all such functions is denoted by $W$. It is obvious that $W$ is a Banach algebra with respect to the norm

$$
|x|=\sum_{k}\left|c_{k}\right| .
$$

In [20], the commutative Banach algebra $W$ is studied in more detail. In particular, the theorem on the argument of almost periodic functions also holds for this algebra, i.e., if a function $x$ from $W$ is nondegenerate, then the function $y$ from the expansion (1.7.3) belongs to $W$.

We say that a function $x$ from $C$ is semi-almost periodic if there exist almost periodic functions $x^{ \pm}(t)$ such that

$$
x(t)-x^{ \pm}(t) \rightarrow 0 \text { as } t \rightarrow \pm \infty .
$$

From the almost-periodicity definition given by (1.7.1), it immediately follows that the functions $x^{ \pm}$ are uniquely defined by $x$ and the estimate

$$
\left|x^{ \pm}\right|_{0} \leq|x|_{0}
$$

holds for the sup-norms. The functions $x^{ \pm}$are called the one-sided (left- and right-hand) almost periodic components of $x$. The estimate (1.7.7) shows that the class of semi-almost periodic functions is a closed subalgebra of $C$ and the linear maps $x \rightarrow x^{ \pm}$are homomorphisms of algebras such that $m^{ \pm} x=m x^{ \pm}$.

If a function $x(t)$ admits limits $c^{ \pm}$from $\mathbb{C}$ as $t \rightarrow \pm \infty$, then it can be treated as a constant almost periodic function. In other words, this function is semi-almost periodic and its one-sided almost periodic components are equal to $c^{ \pm}$.

If a semi-almost periodic function $x(t)$ is nondegenerate, then, due to (1.7.6), there exists a positive integer $n$ such that

$$
\inf _{ \pm t \geq n}\left|x^{ \pm}(t)\right|>0
$$

Then, due to the almost-periodicity definition given by (1.7.2), we conclude that the functions $x^{ \pm}$are also nondegenerate. Hence, the inverse function $x^{-1}$ is semi-almost periodic and $\left(x^{-1}\right)^{ \pm}=\left(x^{ \pm}\right)^{-1}$. Since $x\left(x^{ \pm}\right)^{-1} \rightarrow 1$ as $t \rightarrow \pm \infty$, it follows from the argument theorem applied to $x^{ \pm}$that there exist $a^{ \pm}$from $\mathbb{R}$ and almost periodic functions $y^{ \pm}$such that

$$
\ln x(t)-i a^{ \pm} t-y^{ \pm}(t) \rightarrow 0 \text { as } t \rightarrow \pm \infty,
$$

where $\ln x(t)$ is the continuous branch of the logarithmic function. This relation can be treated as an analog of the said theorem for semi-almost periodic functions.

Note that the continuous branch $\ln x(t)$ of the logarithmic function is defined up to an additive term $2 \pi i k, k \in \mathbb{N}$, but the difference $y^{+}-y^{-}$is defined uniquely. The mean value of this difference divided by $2 \pi i$ is called the Cauchy index of the function $x$ and is denoted by

$$
\operatorname{Ind}_{\mathbb{R}} x=\frac{1}{2 \pi i} m\left(y^{+}-y^{-}\right) .
$$


If $x(t)$ has limits at $\pm \infty$, then the function $\ln x(t)$ possesses the same property and (1.7.9) coincides with the classical definition of the Cauchy index as the increment of the continuous branch $\ln x$ of the logarithmic function, i.e.,

$$
\operatorname{Ind}_{\mathbb{R}} x=\left.\frac{1}{2 \pi i} \ln x(t)\right|_{-\infty} ^{\infty}
$$

Obviously, the Cauchy index possesses the group property (1.4.11), i.e.,

$$
\operatorname{Ind}_{\mathbb{R}}\left(x_{1} x_{2}\right)=\operatorname{Ind}_{\mathbb{R}} x_{1}+\operatorname{Ind}_{\mathbb{R}} x_{2}, \quad \operatorname{Ind}_{\mathbb{R}} 1=0 .
$$

Being a complex function on the group of invertible elements of the algebra $C$, the said index continuously depends on $x$ with respect to the sup-norm. Indeed, if $\left|x_{n}-x\right|_{0} \rightarrow 0$, then $x_{n}=x\left(1+x_{n}^{0}\right)$, where $x_{n}^{0} \in C$ and $x_{n}^{0} \rightarrow 0$ with respect to the sup-norm. Respectively, in the relation (1.7.8) for $x_{n}$, the functions $y^{ \pm}$are to be replaced by $y^{ \pm}+\left[\ln \left(1+x_{n}^{0}\right)\right]^{ \pm}$. It remains to note that $\ln \left(1+x_{n}^{0}\right) \rightarrow 0$ with respect to the sup-norm as $n \rightarrow \infty$ and to use the estimate (1.7.7). Thus, according to (1.7.10), it is the Cauchy index in the sense of the definition from Sec. 1.3.

Note that the expansion (1.7.5) for the almost periodic components $x^{ \pm}$of the function $x$ has the form

$$
\ln x^{ \pm}(t)=i a^{ \pm} t+y^{( \pm)}(t),
$$

where $a^{ \pm}$are the constants from (1.7.8). The functions $y^{( \pm)}$differ from $y^{ \pm}$by the constant term $2 \pi i k^{ \pm}$, where $k^{ \pm}$are integers. Hence,

$$
\operatorname{Ind}_{\mathbb{R}} x=\frac{1}{2 \pi i} m\left[y^{(+)}-y^{(-)}\right]+\quad \text { is an integer. }
$$

Consider the case where the function $\ln x$ is bounded, i.e., $a^{+}=a^{-}=0$ in (1.7.8).

Theorem 1.7.1. Let a semi-almost periodic function $x(t)$ be nondegenerate and $\ln x$ be bounded. Then

$$
\operatorname{Ind}_{\mathbb{R}} x=\lim _{n \rightarrow \infty} \frac{1}{n} \int_{n}^{2 n}[(\ln x)(t)-(\ln x)(-t)] d t
$$

and the existence of the limit

$$
\lim _{n \rightarrow \infty} \int_{-n}^{n} \frac{(\ln x)(t) d t}{t-i}
$$

implies the relation Ind $x=0$.

Proof. Consider the almost periodic function $y(t)=y^{+}(t)-y^{-}(-t)$ in notation (1.7.8). Since $m y=$ $m\left(y^{+}-y^{-}\right)$, it follows from the definition that $\operatorname{Ind}_{\mathbb{R}} x=m y$. On the other hand, the almost periodic function $y$ satisfies the relation

$$
m y=\lim _{n \rightarrow \infty} \frac{1}{n} \int_{n}^{2 n} y(t) d t .
$$

Taking into account the fact that $y_{0}(t)=[(\ln x)(t)-(\ln x)(-t)]-y(t) \rightarrow 0$ as $t \rightarrow \infty$, we deduce (1.7.13).

Further, let limit (1.7.14) exist. Then

$$
\lim _{n \rightarrow \infty} \int_{-n}^{n}\left[\frac{(\ln x)(t)}{t-i}-\frac{(\ln x)(-t)}{t+i}\right] d t=0 .
$$


The last integral can be represented by the sum

$$
\int_{n}^{2 n} \frac{y(t) d t}{t-i}+\int_{n}^{2 n} \frac{y_{0}(t) d t}{t-i}+2 i \int_{n}^{2 n} \frac{(\ln x)(-t) d t}{t^{2}+1} .
$$

Since the last two terms tend to zero as $n \rightarrow \infty$, it follows that this property also holds for the first term:

$$
\lim _{n \rightarrow \infty} \int_{n}^{2 n} \frac{y(t) d t}{t-i}=0
$$

Denote the linear functional defined by the last integral by $I_{n} y$. It is obvious that

$$
\left|I_{n} y\right| \leq \max _{n \leq t \leq 2 n}|y(t)|
$$

If $y(t)=e^{i a t}$, where $a \neq 0$, then the integrating by parts yields the relation

$$
I_{n} y=\frac{1}{i a}\left[\left.e^{i a t}\right|_{n} ^{2 n}-\int_{n}^{2 n} \frac{e^{i a t} d t}{(t-i)^{2}}\right] .
$$

Hence, $I_{n} y \rightarrow 0$ as $n \rightarrow \infty$. Therefore, this fact is valid for trigonometric polynomials $y$ such that $m y=0$, i.e., for finite sums of kind (1.7.2) such that $a_{j} \neq 0$ for any $j$. Then, taking into account (1.7.16), we conclude that $I_{n} y \rightarrow 0$ as $n \rightarrow \infty$ for any almost periodic function $y$ such that $m y=0$.

Consider the almost periodic function $y$ from (1.7.15). Since $m(y-m y)=0$ (see the property mentioned above), the relation (1.7.15) implies the relation $m y=0$. It remains to recall that $m y=\operatorname{Ind} x$.

Note that the existence of limit (1.7.14) implies the existence of the similar limit

$$
F(z)=\lim _{n \rightarrow \infty} \int_{-n}^{n} \frac{(\ln x)(t) d t}{t-z}
$$

for any point $z$ such that $\operatorname{Im} z \neq 0$. The said limit defines a function $F(z)$ analytic on the complex plane outside $\mathbb{R}$. To prove this, it suffices to represent $F$ in the form

$$
F(z)=F_{0}(z)+\int_{\mathbb{R}} \frac{(\ln x)(t) d t}{t-i}, \quad F_{0}(z)=(z-i) \int_{\mathbb{R}} \frac{(\ln x)(t) d t}{(t-z)(t-i)},
$$

and to take into account the fact that if the function $\ln x$ is bounded, then the integral at the righthand side of the second relation is understood in the standard sense and defines a function analytic outside $\mathbb{R}$. The same argument yields that the point $i$ in the theorem can be replaced by any other point $z$ such that $\operatorname{Im} z \neq 0$. $\zeta$.

Consider an analog of the algebra $C$ in the band $\lambda_{1} \leq \operatorname{Re} \zeta \leq \lambda_{2}$ of the complex plane of the variable

This band is denoted by $\left[\lambda_{1}, \lambda_{2}\right]$ (the difference of this notation from a segment of the line is clear from the context). Let $C\left[\lambda_{1}, \lambda_{2}\right]$ denote the Banach algebra of all functions continuous and bounded in this band and analytic inside it (provided that $\lambda_{1}<\lambda_{2}$ ). All the above notions are extended for functions $x(\zeta)$ from $C\left[\lambda_{1} \lambda_{2}\right]$ in a natural way. For example, to define their almost periodicity, we replace (1.7.1) by the condition $|x(\zeta+i \tau)-x(\zeta)| \leq \varepsilon, \lambda_{1} \leq \operatorname{Re} \zeta \leq \lambda_{2}$. Trigonometric polynomials are replaced by linear combinations of the functions $e^{a_{k} \zeta}$ with real powers $a_{k}$. The expansion (1.7.3) in 
the argument theorem is changed for $\ln x(\zeta)=a \zeta+y(\zeta)$. The mean values $m^{ \pm} x$ of functions $x$ from $C\left[\lambda_{1}, \lambda_{2}\right]$ are defined similarly to (1.7.4):

$$
m^{+} x=\lim _{n \rightarrow \infty} \frac{1}{n} \int_{i n}^{2 i n} x(\zeta) d \zeta, \quad m^{-} x=\lim _{n \rightarrow \infty} \frac{1}{n} \int_{-2 i n}^{-i n} x(\zeta) d \zeta .
$$

If there exist $x( \pm \infty)=\lim x(\zeta)$ as $\operatorname{Im} \zeta \rightarrow \pm \infty$ uniformly in the band $\left[\lambda_{1}, \lambda_{2}\right]$, then the one-sided mean values $m^{ \pm} x$ exist and coincide with $x( \pm \infty)$. An example of such a function is $s(\zeta)=\tanh \zeta$ belonging to $C\left[\lambda_{1}, \lambda_{2}\right]$ provided that $\lambda_{1}<\lambda_{2}$ and such that $s( \pm \infty)= \pm 1$.

The semi-almost periodicity definition given by (1.7.7) is extended to functions $x$ from $C\left[\lambda_{1}, \lambda_{2}\right]$ in the same way. Assuming that $x(\zeta)$ is nondegenerate, one can introduce almost periodic functions $y^{ \pm}(\zeta)$ replacing (1.7.8) by the condition $x(\zeta)-x^{ \pm}(\zeta) \rightarrow 0$ as $\operatorname{Im} \zeta \rightarrow \pm \infty$. Using these functions, we can define the Cauchy index by the same relation (1.7.9). Obviously, the properties (1.7.10) and (1.7.12) of the Cauchy index are also valid in the case considered.

In the same way, an analog of Theorem 1.7.1 is still valid. According to the remark to this theorem, the role of the singular integral (1.7.14) is played by the integral

$$
\int_{\operatorname{Re} \zeta=\lambda} \frac{(\ln x)(\zeta) d \zeta}{\zeta-\zeta_{0}}=\lim _{n \rightarrow \infty} \int_{\lambda-i n}^{\lambda+i n} \frac{(\ln x)(\zeta) d \zeta}{\zeta-\zeta_{0}}
$$

where $\lambda_{1} \leq \lambda \leq \lambda_{2}$ and the point $\zeta_{0}$ lies outside the band $\left[\lambda_{1}, \lambda_{2}\right]$.

The Cauchy index is related to the involution of the of the complex conjugation $x \rightarrow \bar{x}$ in the algebra $C\left[\lambda_{1}, \lambda_{2}\right]$, defined by the relation

$$
\bar{x}(\zeta)=\overline{x(\bar{\zeta})}
$$

where the bar on the right-hand side denotes the complex conjugation. For $\bar{x}$, the analog of (1.7.8) is the limit relation $\ln \bar{x}(\zeta)-a^{ \pm} \zeta-\bar{y}^{\mp}(\zeta) \rightarrow 0$ as $\operatorname{Im} \zeta \rightarrow \pm \infty$. Taking into account the obvious relation $m \bar{y}=\overline{m y}$ for the mean values of almost periodic functions, we arrive at the relation

$$
\text { Ind } \bar{x}=-\overline{\operatorname{Ind} x}
$$

where the bar on the right-hand side denotes the complex conjugation.

In addition to scalar semi-almost periodic functions, matrix functions also broadly occur. As we note in Sec. 1.1, the space of $n \times n$-matrix-functions such that their elements belong to $C\left[\lambda_{1}, \lambda_{2}\right]$, is denoted by the same symbol. For a matrix-function, the nondegeneracy condition is treated as the nondegeneracy condition for its determinant $\operatorname{det} x$. The Cauchy index $\operatorname{Ind} x=\operatorname{Ind}(\operatorname{det} x)$ is treated in the same way. Since $\left(x_{1} x_{2}\right)^{ \pm}=x_{1}^{ \pm} x_{2}^{ \pm}$, it follows that the determinant $\operatorname{det} x^{ \pm} \operatorname{coincides}$ with $(\operatorname{det} x)^{ \pm}$. Therefore, (1.7.11)-(1.7.12) also hold for det $x$.

If the nondegeneracy condition is satisfied only for selected lines $\operatorname{Re} \zeta=\lambda, \lambda_{1} \leq \lambda \leq \lambda_{2}$, then, in general, increment (1.7.9) with respect to $\ln \operatorname{det} x(\lambda+i t)$ depends on $\lambda$.

Lemma 1.7.1. Let an $n \times n$-matrix-function $x(\zeta)$ be semi-almost periodic in a band $\lambda_{1} \leq \operatorname{Re} \lambda \leq \lambda_{2}$, its almost periodic components $x^{ \pm}$be nondegenerate, and $x(\zeta)$ be nondegenerate on the boundary lines $\operatorname{Re} \zeta=\lambda_{k}$ of this band. Then there exists a rational matrix-function $r(\zeta)$ from $C\left[\lambda_{1}, \lambda_{2}\right]$ vanishing at infinity and such that $x(\zeta)+r(\zeta)$ is nondegenerate in the considered band and the difference

$$
\text { Ind } x\left(\lambda_{2}+i t\right)-\operatorname{Ind} x\left(\lambda_{1}+i t\right)
$$

coincides with the number of zeroes of the function $\operatorname{det} x(\zeta)$ in the band $\lambda_{1}<\operatorname{Re} \zeta<\lambda_{2}$, counted with their multiplicities. 
Proof. Since $x(\zeta)-x^{ \pm}(\zeta) \rightarrow 0$ as $\operatorname{Im} \zeta \rightarrow \pm \infty$ and the functions $x^{ \pm}$are nondegenerate, it follows that there exists a positive integer $n$ such that

$$
\inf _{|\operatorname{Im} \zeta| \geq n}|x(\zeta)|>0
$$

Taking into account the nondegeneracy of $x\left(\lambda_{j}+i t\right)$, we conclude that the number $m$ of zeroes of $\operatorname{det} x$, mentioned in the lemma, is finite indeed. If $m=0$, i.e., there are no such zeroes, then $x(\zeta)$ is nondegenerate in the whole band.

Let $\zeta_{1}$ be a zero of the function $\operatorname{det} x$. Then $(\operatorname{det} x)\left(\zeta_{1}\right)=0$ and, therefore, there exists a nonzero vector $\xi$ from $\mathbb{C}^{n}$ such that $x\left(\zeta_{1}\right) \xi=0$. Consider the matrix $p$ from $\mathbb{C}^{n \times n}$, projecting (as a linear operator) $\mathbb{C}^{n}$ onto the one-dimensional space spanned by the vector $\xi$. Thus, $p^{2}=p$ and $x\left(\zeta_{1}\right) p=0$. According to Sec. 1.7, the matrix $p$ is similar to a diagonal matrix such that all its diagonal elements except for one are equal to zero. Therefore, for any nonzero $c$ from $\mathbb{C}$, we have the relations

$$
\operatorname{det}(c p+1-p)=c, \quad(c p+1-p)^{-1}=c^{-1} p+1-p .
$$

Fix $\lambda$ exceeding $\lambda_{2}$ and consider the matrix-function

$$
x_{1}(\zeta)=x(\zeta) r_{1}^{-1}(\zeta), \quad r_{1}(\zeta)=\frac{\zeta-\zeta_{0}}{\zeta-\lambda} p+1-p
$$

Obviously, the rational matrix-function

$$
r_{1}(\zeta)-1=\frac{\lambda-\zeta_{0}}{\zeta-\lambda} p
$$

belongs to $C^{0}\left[\lambda_{1}, \lambda_{2}\right]$. Taking into account (1.7.20) and the relation $x\left(\zeta_{1}\right) p=0$, we conclude that the function

$$
x_{1}(\zeta)=\left[x(\zeta)-x\left(\zeta_{1}\right)\right] r_{1}^{-1}+x\left(\zeta_{1}\right)(1-p)
$$

belongs to $C\left[\lambda_{1}, \lambda_{2}\right]$. The matrix-function $x_{1}(\zeta)$ satisfies all the assumptions of the lemma, but its determinant $\operatorname{det} x=\left(\zeta-\zeta_{1}\right)^{-1} \operatorname{det} x(\zeta)$ has one zero less than $\operatorname{det} x$. Continuing this procedure till the $m$ th step, one can expand the function $x(\zeta)$ into the product

$$
x(\zeta)=x_{m}(\zeta) r_{m}(\zeta)
$$

where $x_{m}$ is a nondegenerate function from $C\left[\lambda_{1}, \lambda_{2}\right]$, while the function $r_{m}(\zeta)$ is rational, has the only pole at the point $\zeta=\lambda$, and tends to 1 as $\zeta \rightarrow \infty$. Assigning $r=r_{m}-1$, we obtain the validity of the first assertion of the lemma.

By virtue of (1.7.10), to prove the second assertion of the lemma, it suffices to prove it for the second factor $r_{m}(\zeta)$ of (1.7.21). In this case, it follows from the known Rouché theorem on the increment of the logarithm of an analytic function along the boundary contour.

\subsection{Lebesgue Integrals and Generalized Functions}

Recall elements of the Lebesgue integral theory, skipping the definitions of a Lebesgue-measurable set $G \subseteq \mathbb{R}^{k}$, its Lebesgue measure mes $G$, measurable functions, and the Lebesgue integral. Consider the class $L(G)$ of functions summable on a measurable set $E \subseteq \mathbb{R}^{k}$. This class is a vector space, and the relation

$$
|f|_{L}=\int_{G}|f(x)| d x
$$

defines a norm in this class under the assumption that any two functions that differ on a zero-measure set are identified.

It is known that $L$ is a Banach space with respect to norm (1.8.1). This completeness property of $L$ is one of the main advantages of the Lebesgue integral. Another important property of the Lebesgue integral refers to the passage to the limit under the integral sign. 
Theorem (on majorized convergence, Lebesgue). Let $\left\{f_{n}(x)\right\}$ be a sequence of measurable functions such that $f_{n}(x) \rightarrow f(x)$ as $n \rightarrow \infty$ for almost all $x$ from $G$ and there exists a nonnegative summable function $\varphi$ such that $\left|f_{n}(x)\right| \leq \varphi(x)$ for any $n$. Then the limit function $f$ is summable and

$$
\lim _{n \rightarrow \infty} \int_{G} f_{n} d x=\int_{G} f d x .
$$

In particular, the denumerable additivity property

$$
\int_{G} f d x=\sum_{i=1}^{\infty} \int_{G_{i}} f d x, \quad G=\bigcup_{i=1}^{\infty} G_{i},
$$

where the sets $G_{i}$ are pairwise disjoint, is a corollary of the Lebesgue theorem. To prove this, it suffices to apply the said theorem to the sequence of functions $f_{n}(x)$ equal to $f(x)$ for $x$ from $G_{1} \cup \ldots \cup G_{n}$ and to zero otherwise. In this case, the function $|f(x)|$ plays the role of $\varphi$. Here, we take into account the finite-additivity property of the integral, following from its linearity.

Usually, the Banach space $L(G)$ is considered for open or closed sets $G$. Let $D$ be an open set and $C_{0}(D)$ denote the class of continuous functions with compact supports contained in $D$. This class is dense in $L(D)$ and

$$
|f|_{L}=\sup _{|\varphi|_{C} \leq 1}(f, \varphi), \quad(f, \varphi)=\int_{D} f(x) \varphi(x) d x,
$$

where the supremum is taken over functions $\varphi$ from $C_{0}(D)$.

Let $B(x, r)$ denote the ball of radius $r$ centered at $x$. Points $x$ satisfying the relation

$$
\lim _{\varepsilon \rightarrow 0} \frac{1}{\operatorname{mes} B(x, \varepsilon)} \int_{B(x, \varepsilon)}|f(y)-f(x)| d y=0
$$

are called Lebesgue points of the function $f$. Note that all continuity points of the function $f$ are its Lebesgue points a fortiori.

Theorem (on Lebesgue points). If $f$ is locally summable, then (1.8.3) holds for almost all $x$.

By definition, a function $\varphi$ from $C(D)$ is continuously differentiable on an open domain set $D$ if its partial derivatives $\partial \varphi / \partial x_{i}$ exist and are continuous at any point $x$ of $D$, i.è.,

$$
\varphi^{\prime}=\left(\frac{\partial \varphi}{\partial x_{1}}, \ldots, \frac{\partial \varphi}{\partial x_{k}}\right) \in C(D) .
$$

For an $m$-vector-function $\varphi$, this gradient is treated as the Jacobi $m \times k$-matrix $\mathcal{D} \varphi$ such that its columns are the partial derivatives $\partial \varphi / \partial x_{j}$.

Most frequently, the Lebesgue majorized convergence theorem is used in the following situation.

Theorem 1.8.1. Let a function $\varphi(x, y)$ be given on the product $G \times G$, summable with respect to $y$ for any $x$, and continuous with respect to $x$ from $G$ for almost all $y$, where $G$ is an open subset of $\mathbb{R}^{s}$. Let there exist a nonnegative function $f$ from $L(G)$ such that $|\varphi(x, y)| \leq f(y)$ for all $x$ from $G$ and almost all $y$ from $G$. Then the integral

$$
\psi(x)=\int_{G} \varphi(x, y) d y
$$

defines s function continuous in $G$. 
If the above is satisfied, the function $\varphi(x, y)$ is continuously differentiable with respect to $x$, and its partial derivatives $\partial \varphi / \partial x_{i}$ satisfy the same conditions as $\varphi$ itself, then $\psi(x)$ is continuously differentiable and its derivatives can be computed under the integral sign:

$$
\frac{\partial \psi}{\partial x_{i}}=\int_{G} \frac{\partial \varphi}{\partial x_{i}}(x, y) d y .
$$

Proof. The first assertion immediately follows from the Lebesgue theorem. To prove the second one, it suffices to verify that the partial derivative with respect to the variable $x_{i}$ exists and coincides with the function $\psi_{i}$ defined by the integral of the corresponding partial derivative of $\varphi$. Therefore, without loss of generality, one can assume that $s=1$ and $G$ is an open interval of the line. Fix $a$ from $G$ and select a sequence $\left\{x_{n}\right\}$ of points of $G$ such that $x_{n} \rightarrow a$. We must show that the difference

$$
\frac{\psi\left(x_{n}\right)-\psi(a)}{x_{n}-a}-\int_{G} \frac{\partial \varphi}{\partial x}(a, y) d y
$$

tends to zero as $n \rightarrow \infty$, but this follows from the majorized convergence theorem applied to the sequence of functions

$$
\varphi_{n}(y)=\frac{\varphi\left(x_{n}, y\right)-\varphi(a, y)}{x_{n}-a}-\frac{\partial \varphi}{\partial x}(a, y)
$$

The next results related to the change of the order of integration and the change of variables under the integral sign are well known.

Theorem (Fubini). Let a function $\varphi(x, y)$ be summable over the product $G \times G$, where $G \subseteq \mathbb{R}^{s}$. Then it is summable with respect to $y$ over $G$ for almost all $x$ from $G$, the integral with respect to $y$ defines a function summable over $G$, and

$$
\int_{G}\left[\int_{G} \varphi(x, y) d y\right] d x=\int_{G \times G} \varphi(x, y) d x d y .
$$

If the function $\varphi$ is nonnegative, then the inverse assertion also holds: the existence of the iterated integral at the left-hand side of this inequality implies the summability of the function $\varphi$ over $G \times G$.

Theorem (on change of variables). Let $D$ be an open subset of $\mathbb{R}^{k}$ and a $k$-vector-function $\alpha(y)$ from $C^{1}(D)$ realize its homeomorphism onto $G=\alpha(D)$. Then, for any function $f(x)$ from $L(G)$, the function $f[\alpha(y)]|(\operatorname{det} \mathcal{D} \alpha)(y)|$, where $\mathcal{D} \alpha=\left(\partial \alpha_{i} / \partial y_{j}\right)_{1}^{k}$, denotes the Jacobi matrix, is summable on $D$ and the relation

$$
\int_{G} f(x) d x=\int_{D} f[\alpha(y)]|\operatorname{det}(\mathcal{D} \alpha)(y)| d y
$$

holds.

On smooth surfaces, the Lebesgue integral with similar properties can be defined with respect to the $(k-1)$-dimensional Lebesgue measure. For example, consider the unit sphere $\Omega$ consisting of points $y$ from $\mathbb{R}^{k}$ such that $|y|=1$. The transformation $(r, y) \rightarrow r y$ maps $\left[r_{1}, r_{2}\right] \times \Omega$ to the spherical layer $r_{1} \leq|x| \leq r_{2}$ and the previous two theorems for this transformation yield the relation

$$
\int_{r_{1} \leq|x| \leq r_{2}} \varphi(x) d x=\int_{r_{1}}^{r_{2}} r^{k-1} d r \int_{\Omega} \varphi(r y) d_{k-1} y .
$$


In particular, the function $\varphi(x)=|x|^{-\alpha}$ is summable in the ball $|x|<R$ for $\alpha<k$ and is summable in its complement for $|\alpha|>k$ :

$$
\int_{|x|<R}|x|^{-\alpha} d x=\frac{\operatorname{mes} \Omega}{k-\alpha} R^{k-\alpha}, \quad \alpha<k, \quad \int_{|x|>R}|x|^{-\alpha} d x=\frac{\operatorname{mes} \Omega}{\alpha-k} R^{\alpha-k}, \quad \alpha>k .
$$

Here mes $\Omega$ denotes the area of the $(k-1)$-dimensional unit sphere $\Omega$.

A similar relation is also valid for the function $\varphi(x)=(\ln |x|)^{n}|x|^{-\alpha}$.

The following Green formula (of integrating by parts) is closely related to the integrating over smooth surfaces.

Theorem (the Green formula). Let functions $\varphi_{j}$ from $C(\bar{D}), 1 \leq j \leq k$, be continuously differentiable in a domain $D$ bounded by a smooth surface $\Gamma$ and their partial derivatives $\partial \varphi_{j} / \partial x_{j}$ be summable in this domain. Then

$$
\int_{D}\left(\sum_{1}^{k} \frac{\partial \varphi}{\partial x_{j}}\right) d x=\int_{\Gamma}\left(\sum_{1}^{k} \varphi_{j}(y) n_{j}(y)\right) d_{k-1} y,
$$

where the vector $n(y)=\left(n_{1}, \ldots, n_{k}\right)$ denotes the unit outer normal to the surface $\Gamma$ at the point $y$.

Denote by $C^{n}(D)$ the class of all functions $\varphi(x)$ continuously differentiable $n$ times on an open set $D$, i.e., functions such that all their partial derivatives

$$
\frac{\partial^{\alpha} \varphi}{\partial x^{\alpha}}=\frac{\partial^{\alpha_{1}+\ldots+\alpha_{k}} \varphi}{\partial x^{\alpha_{1}} \ldots \partial x^{\alpha_{k}}}
$$

where $|\alpha|=\alpha_{1}+\ldots+\alpha_{k} \leq n$, exist and are continuous at each point of the set $D$. Here the ordered collection $\alpha=\left(\alpha_{1}, \ldots, \alpha_{k}\right)$ of nonnegative integers is called a multi-index of length $|\alpha|$.

The class $C^{\infty}(D)$ of infinitely differentiable functions is defined as the intersection of classes $C^{n}$ with respect to all $n$. The symbol $C_{0}^{\infty}(D)$ denotes the class of infinitely differentiable functions $\varphi$ such that each one vanishes outside a compact set contained in $D$. Obviously, if $\varphi \in C_{0}^{\infty}(D)$, then the intersection of all compact sets such that $\varphi=0$ outside each one is also a compact set. This compact set is called the support of the function $\varphi$ and is denoted by $\operatorname{supp} \varphi$.

A broad class of infinitely differentiable functions can be obtained by means of averaging kernels. Let a nonnegative function $h(y)$ from $C_{0}^{\infty}\left(\mathbb{R}^{k}\right)$ be such that

$$
h(y)=0 \quad \text { for } \quad|y| \geq 1 \text { and } \int_{|y| \leq 1} h(y) d y=1 .
$$

Such a choice is always possible. For example, one can assign $h(y)=c e^{1 /\left(1-|y|^{2}\right)}$ for $|y|<1$, where $c$ is the appropriate positive constant.

Assuming that a function $\varphi(x)$ is locally summable over $\mathbb{R}^{k}$ (i.e., is summable over any compact set $K$ ), consider the family of functions

$$
\left(T_{\varepsilon} \varphi\right)(x)=\frac{1}{\varepsilon^{k}} \int_{\mathbb{R}^{k}} h\left(\frac{x-y}{\varepsilon}\right) \varphi(y) d y, \quad 0<\varepsilon<1 .
$$

Obviously, the last integral exists since, by virtue of (1.8.8), the integration domain is the ball $B(x, \varepsilon)=$ $\{y,|y-x| \leq \varepsilon\}$. If $x$ varies in the ball $|x|<R$, then, arguing in the same way, one can integrate over the ball $|y|<R+1$. Hence, due to Theorem 1.8.1, all functions $T_{\varepsilon} \varphi$ belong to the class $C^{\infty}\left(\mathbb{R}^{k}\right)$. It is clear that if the function $\varphi$ vanishes outside a compact set, i.e., has a compact support, then functions $T_{\varepsilon} \varphi$ possess the same property, i.e., belong to $C_{0}^{\infty}\left(\mathbb{R}^{k}\right)$.

Family (1.8.9) is used to approximate the function $\varphi$.

Lemma 1.8.1. Let a function $\varphi(x)$ be bounded and uniformly continuous on $\mathbb{R}^{k}$. Then $T_{\varepsilon} \varphi \rightarrow \varphi$ with respect to the sup-norm as $\varepsilon \rightarrow 0$. 
Proof. Since the function $\varphi$ is uniformly continuous on $\mathbb{R}^{k}$, it follows that

$$
\omega(\varepsilon)=\sup _{|x-y| \leq \varepsilon}|\varphi(x)-\varphi(y)| \rightarrow 0 \quad \text { as } \quad \varepsilon \rightarrow 0 .
$$

Using the substitution $x-y=\varepsilon z$, represent (1.8.9) as follows:

$$
\left(T_{\varepsilon} \varphi\right)(x)=\int_{\mathbb{R}^{k}} h(z) \varphi(x-\varepsilon z) d z .
$$

Taking into account (1.8.8), we derive the relation

$$
\left(T_{\varepsilon} \varphi-\varphi\right)(x)=\int_{|y| \leq 1} h(y)[\varphi(x-\varepsilon y)-\varphi(x)] d y,
$$

which yields the estimate

$$
\left|T_{\varepsilon} \varphi-\varphi\right|_{0} \leq \omega(\varepsilon) \int_{|y| \leq 1} h(y) d y=\omega(\varepsilon) .
$$

This estimate shows that $T_{\varepsilon} \varphi \rightarrow \varphi$ with respect to the sup-norm as $\varepsilon \rightarrow 0$.

Let a domain $D$ contain a compact set $K$ such that the distance

$$
r=\inf _{x \in K, y \in \partial D}|x-y|
$$

between $K$ and the boundary of this domain is positive. Then, if a summable function $\varphi$ is equal to zero outside $K$, then the function $T_{\varepsilon} \varphi$ belongs to the class $C_{0}^{\infty}(D)$ provided that $\varepsilon \leq \delta<r$. Indeed, for any point $x$, introduce the distance

$$
d(x, K)=\inf _{y \in K}|x-y|
$$

between $x$ and $K$. This function is continuous. Hence, $K_{1}=\{x, d(x, K) \leq \delta\}$ is a compact set contained in $D$. If $x \notin K_{1}$, then the ball $B(x, \varepsilon)$ does not intersect $K$. Hence, $\varphi=0$ on this ball and, respectively, $\left(T_{\varepsilon} \varphi\right)(x)=0$. Thus, the function $T_{\varepsilon} \varphi$ from $C^{\infty}(D)$ is equal to zero outside the compact subset $K_{1}$ of $D$, i.e., it belongs to $C_{0}^{\infty}(D)$.

In the same way, if $\varphi=1$ on the compact set $K_{1}$ and $\varphi=0$ outside this set, then, if $\varepsilon$ is sufficiently small, then the nonnegative function $\chi=T_{\varepsilon} \varphi$ belongs to $C_{0}^{\infty}(D)$ and is identically equal to 1 on the compact set $K$. Functions of this kind are called cut-off functions. Their value is as follows: for any function $\varphi$ from $C^{\infty}(D)$, the product $\chi \varphi$ belongs to $C_{0}^{\infty}(D)$.

Combining the above reasoning with Lemma 1.8.1, we see that the supremum at (1.8.2) can be taken over $\varphi$ from $C_{0}^{\infty}(D)$. Indeed, due to the specified lemma, for any function $\varphi$ from $C_{0}(D)$, the sequence $T_{\varepsilon} \varphi$ belongs to $C_{0}^{\infty}(D)$ (provided that $\varepsilon$ are sufficiently small) and uniformly converges to $\varphi$ as $\varepsilon \rightarrow 0$.

Also, cut-off functions lead to the so-called partition of unity.

Lemma 1.8.2. Let the union of open sets $V_{1}, \ldots, V_{m}$ contain a compact set $K$. The there exist nonnegative functions $\chi_{j}$ from $C_{0}^{\infty}\left(V_{j}\right)$ such that their sum is identically equal to 1 on $\mathrm{K}$.

Proof. Since the compact set $K_{j}=\overline{V_{j} \cap K}$ is contained in $V_{j}$, it follows that there exists a cut-off function $\varphi_{j}$ from $C_{0}^{\infty}\left(V_{j}\right)$ identically equal to 1 on $K_{j}$. Therefore, the nonnegative function $\varphi=$ $\varphi_{1}+\ldots+\varphi_{m}$ from $C_{0}^{\infty}\left(\mathbb{R}^{k}\right)$ is greater than or equal to 1 at any point $x$ of $K$. Hence, there exists an open set $V \supseteq K$ such that $\varphi(x) \geq 1 / 2$ for any $x$ from $V$. Therefore, $1 / \varphi$ belongs to $C^{\infty}(V)$ and the functions $\chi_{j}=\varphi_{j} / \varphi$ satisfy the claims of the lemma. 
Introduce the notion of generalized functions in a domain $D$. To do this, define the following notion of the convergence in the class $C_{0}^{\infty}(D)$. The sequence $\varphi_{k}$ tends to $\varphi$ in this class as $k \rightarrow \infty$ if there exists a compact set $K \subseteq D$ such that, for sufficiently large $k$, the supports of the functions $\varphi_{k}$ and the function $\varphi$ are contained in $K$ and all partial derivatives satisfy the limit relations

$$
\frac{\partial^{\alpha} \varphi_{k}}{\partial x^{\alpha}} \rightarrow \frac{\partial^{\alpha} \varphi}{\partial x^{\alpha}}
$$

as $k \rightarrow \infty$ uniformly on $K$.

Linear functionals $u(\varphi)$ over the class $C_{0}^{\infty}(D)$ continuous with respect to this convergence are called generalized functions. The class of such functionals is a vector space. It is denoted by $\left(C_{0}^{\infty}\right)^{\prime}(D)$.

If a function $f$ is locally summable on $D$, i.e., is summable on any compact subset $K$ of $D$, then the relation

$$
\tilde{f}(\varphi)=\int_{D} f(y) \varphi(y) d y
$$

defines a linear functional $\tilde{f}$ over the class $C_{0}^{\infty}(D)$. Obviously, it is continuous with respect to the introduced convergence, i.e., it is a generalized function. As we note above, the supremum at (1.8.2) can be taken over all $\varphi$ from $C_{0}^{\infty}(D)$. Therefore, if $\tilde{f}=0$, i.e., $\tilde{f}(\varphi)=0$ for all $\varphi$ from $C_{0}^{\infty}(D)$, then $f=0$ almost everywhere. Thus, the generalized function $\tilde{f}$ is uniquely defined by $f$ and it can be identified with $f$. In the sequel, such an identifying is assumed. Generalized function of such kind are called regular.

If $f \in C^{\infty}(D)$ and $u \in\left(C_{0}^{\infty}\right)^{\prime}(D)$, then the linear functional $\tilde{u}(\varphi)=u(f \varphi)$ is also a generalized function. It is denoted by $\tilde{u}=f u$ and is called the product of $f$ and $u$. Obviously, the convergence $\varphi_{n} \rightarrow \varphi$ in $C_{0}^{\infty}$ also implies a similar property for partial derivatives. Therefore, the differentiation operation in the class of generalized functions can be introduced as follows:

$$
\frac{\partial u}{\partial x_{j}}(\varphi)=-u\left(\frac{\partial \varphi}{\partial x_{j}}\right)
$$

For regular generalized functions $u$ from $C^{1}(D)$, this operation corresponds to the classical differentiation. Indeed, let $\varphi \in C_{0}^{\infty}(D)$. Select a domain $D_{0}$ with a smooth boundary such that $\bar{D}_{0} \subseteq D$ and $\varphi \in C_{0}^{\infty}\left(D_{0}\right)$. Then, from the Gauss-Ostrogradskii relation, it follows that

$$
\int_{D} \frac{\partial u}{\partial x_{j}} \varphi d y=-\int_{D} u \frac{\partial \varphi}{\partial x_{j}} d y
$$

since the integrals over $\partial D_{0}$ vanish. According to the definition of regular generalized functions, given by (1.8.11), this relation corresponds to (1.8.12). Partial derivatives $\partial^{\alpha} u / \partial x^{\alpha}$ of any order $|\alpha|=\alpha_{1}+\ldots \alpha_{k}$ are defined in the same way.

In the vector space $\left(C_{0}^{\infty}\right)^{\prime}(D)$, introduce the notion of the pointwise convergence as follows: $u_{n} \rightarrow u$ if $u_{n}(\varphi) \rightarrow u(\varphi)$ as $n \rightarrow \infty$ for any $\varphi$ from $C_{0}^{\infty}(D)$.

Theorem (on the completeness of the space of generalized functions). Let $u_{n} \in\left(C_{0}^{\infty}\right)^{\prime}(D), n=1,2 \ldots$, and the limit $u(\varphi)=\lim u_{n}(\varphi)$ exists for any $\varphi$ from $C_{0}^{\infty}(D)$. Then $u \in\left(C_{0}^{\infty}\right)^{\prime}(D)$.

Assume that there exists an open subset $D_{0}$ of $D$ such that a generalized function $u$ vanishes on any function $\varphi$ from $C_{0}^{\infty}\left(D_{0}\right)$. Then we say that $u=0$ on $D_{0}$. The complement to the union of all open sets such that $u=0$ on such a set defines the support $\operatorname{supp} u$ of the generalized function $u$. An example of a generalized function with a compact support is the $\delta$-function $\delta=\delta_{a}$ concentrated at a point $a$ from $D$. This is defined by the relation

$$
\delta_{a}(\varphi)=\varphi(a), \quad \varphi \in C_{0}^{\infty} .
$$

Its support consists of the unique point $\{a\}$. 
Being a linear functional, a generalized function $u$ with a compact support can be defined on the whole class $C^{\infty}(D)$ in a natural way. Indeed, consider a cut-off function $\chi$ from $C_{0}^{\infty}(D)$ equal to 1 on $\operatorname{supp} u$. Then $\chi u=u$ and one can assign $u(\varphi)=u(\chi \varphi), \varphi \in C^{\infty}(D)$. Thus, it is reasonable to denote the class of generalized functions with compact supports by $\left(C^{\infty}\right)^{\prime}(D)$. According to the next lemma, the action of a generalized function with a compact support on functions depending on parameter commutes with the differentiating and integrating with respect to this parameter.

Lemma 1.8.3. Let $u$ be a generalized function with compact support $\operatorname{supp} u \subseteq D$, a domain $G$ be a subset of $\mathbb{R}^{s}$, and a function $\varphi(x, t)$ belong to $C^{\infty}(G \times D)$. Then the function $\psi(x)=u_{t}[\varphi(x, t)]$ belongs to $C^{\infty}(G)$,

$$
\frac{\partial \psi}{\partial x_{i}}=u_{t}\left(\frac{\partial \varphi}{\partial x_{i}}\right), \quad i=1, \ldots, s
$$

and

$$
\int_{Q} \psi(x) d x=u\left[\int_{Q} \varphi(x, t) d x\right]
$$

for any compact subset $Q$ of $G$.

Here the symbol $u_{t}$ means that $u$ acts on a function with respect to the variable $t$.

Proof. For simplicity, we consider only the one-dimensional case where $G$ is and interval of $\mathbb{R}$. Let a cut-off function $\chi$ from $C_{0}^{\infty}(D)$ be equal to 1 on the support $\operatorname{supp} u$. If $x \in G$ and $x_{n} \rightarrow x$, then $\chi(t) \varphi\left(x_{n}, t\right) \rightarrow \chi(t) \varphi(x, t)$ in $C_{0}^{\infty}(D)$. Then the function $\psi$ is continuous at the point $x$. In the same way, we have the following limit relation:

$$
\chi(t) \frac{\varphi(x+\varepsilon, t)-\varphi(x, t)}{\varepsilon} \rightarrow \chi(t) \frac{\partial \varphi}{\partial x}
$$

in $C_{0}^{\infty}(D)$ as $\varepsilon \rightarrow 0$. Therefore, we have the limit relation

$$
\frac{\psi(x+\varepsilon)-\psi(x)}{\varepsilon}=u_{t}\left[\frac{\varphi(x+\varepsilon, t)-\varphi(x, t)}{\varepsilon}\right] \rightarrow u\left(\frac{\partial \varphi}{\partial x}\right)
$$

implying (1.8.14).

Further, let $Q=[a, b] \subseteq G$. Decompose this segment into $n$ equal parts by points $x_{0}=a$, $x_{1}, \ldots, x_{n}=b$ and consider the Riemann sum

$$
S_{n} \psi=\frac{1}{n} \sum_{1}^{n} \psi\left(x_{k}\right) .
$$

Obviously, if $n \rightarrow \infty$, then

$$
\chi(t)\left(S_{n} \varphi\right)(t)=\frac{1}{n} \sum_{1}^{n} \chi(t) \varphi\left(x_{k}, t\right) \rightarrow \int_{Q} \chi(t) \varphi(x, t) d x
$$

in $C_{0}^{\infty}(D)$. Then

$$
S_{n} \psi=u\left[\left(S_{n} \varphi\right)(t)\right] \rightarrow u\left[\int_{Q} \varphi(x, t) d x\right]
$$

as $n \rightarrow \infty$, which leads to the relation (1.8.15). 


\subsection{Second-Kind Fredholm Equations}

Illustrate properties of Fredholm operators on the example of second-kind integral equations. Assuming that $G$ is a compact subset of $\mathbb{R}^{k}$ and $q(x, y) \in C(G \times G)$, consider the integral operator

$$
(T \varphi)(x)=\int_{G} \frac{q(x, y)}{|x-y|^{\alpha}} \varphi(y) d y, \quad x \in G,
$$

where $0<\alpha<k$. By virtue of (1.8.6), this integral exists for any bounded measurable function $\varphi$. It is obvious that, without loss of generality, one can assume that

$$
k / 2<\alpha<k .
$$

If there exists a (sufficiently small) $\delta$ such that $q(x, y)=0$ for $|x-y| \leq \delta$, then the kernel $q(x, y)|x-y|^{-\alpha}$ of the integral operator is continuous on $G \times G$ and the operator $T$ is compact in the space $C(G)$, i.e., belongs to $\mathcal{T}(C)$. This fact easily follows from the Arzela-Ascoli theorem since, for any sequence $\varphi_{n}$ bounded in $C(G)$, the function sequence $\left(T \varphi_{n}\right)(x)$ is uniformly bounded and equicontinuous.

Actually, this also holds in the general case.

Lemma 1.9.1. For any function $q$ from $C(G \times G)$, the operator $T(q)$ is compact int the space $C(G)$.

Proof. Let $|\cdot|_{0}$ denote the sup-norm of functions. Then, by virtue of (1.8.6), we have the estimate $|(T \varphi)(x)| \leq C|q|_{0}|\varphi|_{0}$ with the constant

$$
C=\sup _{x \in G} \int_{G}|x-y|^{-\alpha} d y .
$$

Thus, the following inequality holds:

$$
|T \varphi|_{0} \leq C|q|_{0}|\varphi|_{0}
$$

In the same way, one can verify that

$$
|T \varphi|_{0} \leq C \delta^{k-\alpha}|q|_{0}|\varphi|_{0}
$$

under the additional assumption that $q(x, y)=0$ provided that $|x-y| \geq \delta$.

Let a nonnegative continuous function $\chi_{n}(s)$ not exceed $1, \chi_{n}(s)=0$ for $|s| \leq 1 / 2 n$, and $\chi_{n}(s)=1$ for $|s| \geq 1 / n$. Then the operator $T\left(q_{n}\right)$, where $q_{n}(x, y)=\chi_{n}(|x-y|) q(x, y)$, is compact in the space $C(G)$. Applying (1.9.3) to the difference $T(q)-T\left(q_{n}\right)=T\left(q-q_{n}\right)$, we obtain the estimate

$$
\left|T\left(q-q_{n}\right) \varphi\right|_{0} \leq C n^{\alpha-k}|q|_{0}\left|\varphi_{0}\right| \text {. }
$$

It shows that the sequence of continuous functions $T\left(q_{n}\right) \varphi$ uniformly converges to $T(q) \varphi$. Then the last function is also continuous. Then, taking into account (1.9.3), we conclude that the operator $T(q)$ is bounded in the space $C$. Actually, the estimate (1.9.5) means that the sequence $T\left(q_{n}\right)$ of operators from $\mathcal{T}(C)$ converges to $T(q)$ with respect to the operator norm. Then, due to Theorem 1.2.3, we conclude that the operator $T(q)$ is also compact.

Combining Lemma 1.9.1 with the Riesz-Schauder theorem (see Sec. 1.3), we see that $1-T(q)$ is a Fredholm operator and its index is equal to zero. It is easy to see that the operator $T(q)$ admits an associated operator with respect to the bilinear form

$$
(\varphi, \psi)=\int_{G} \varphi(y) \psi(y) d y
$$

and the associated operator belongs to the same type:

$$
[T(q)]^{\prime}=T(\widetilde{q}), \quad \widetilde{q}(x, y)=q(y, x) .
$$

Hence, the associated operator $[1-T(q)]^{\prime}=1-T(\widetilde{q})$ is also a Fredholm operator of index zero and $N=1-T(q)$ is an associatedly Fredholm operator (due to Theorem 1.3.2). 
Thus, the following classical alternatives are valid for the second-kind Fredholm equation:

$$
\varphi(x)-[T(q) \varphi](x)=f(x), \quad x \in G .
$$

(1) the homogeneous equation corresponding to (1.9.6) has a finite set of linearly independent solutions $\varphi_{1}, \ldots, \varphi_{n}$ from $C(G)$;

(2) the homogeneous associated equation $\psi(x)-[T(\widetilde{q}) \psi](x)=0$ has the same amount of linearly independent solutions $\psi_{1}, \ldots, \psi_{n} \in C(G)$;

(3) the heterogeneous equation (1.9.6) is solvable if and only if $\left(f, \psi_{j}\right)=0,1 \leq j \leq n$.

If the kernel $\operatorname{ker}[1-T(q)]$ contains only zero element, then the operator $1-T(q)$ is invertible. Our main aim is to verify that the inverse operator has the same form, i.e., $[1-T(q)]^{-1}=1-T\left(q_{1}\right)$, where $q_{1} \in C(G \times G)$.

To do this, introduce the following bilinear operation $p * q$ in $C(G \times G)$ :

$$
(p * q)(x, y)=|x-y|^{\alpha} \int_{G} \frac{p(x, z) q(z, y)}{|x-z|^{\alpha}|y-z|^{\alpha}} d z, \quad x \neq y .
$$

This definition is motivated by the fact that the change of the order of the iterated integrating $T(p)[T(q) \varphi]$ leads to the relation

$$
T(p) T(q)=T(p * q) .
$$

First, we must verify that the result of operation (1.9.7) remains in the class $C(G \times G)$.

Lemma 1.9.2. Map (1.9.7) boundedly takes $C \times C$ into $C$ and the linear operator $R(p) q=p * q$ is compact in the space $C(G \times G)$ for any fixed $p$.

Proof. The scheme of the proof is the same as for Lemma 1.9.1. For $x \neq y$, we have the inequality

$$
|(p * q)(x, y)| \leq|p|_{0}|q|_{0} \int_{\mathbb{R}^{k}} \frac{|x-y|^{\alpha} d z}{|z-x|^{\alpha}|z-y|^{\alpha}},
$$

where the integral is treated as improper with respect to $z=x, z=y$, and $z=\infty$. By virtue of (1.8.6) and (1.9.2), this integral exists. The change $z=y+|x-y| z^{\prime}$ reduces it to the form

$$
|x-y|^{k-\alpha} \int_{\mathbb{R}^{k}} \frac{d z}{|z|^{\alpha}|z-e|^{\alpha}}, \quad e=\frac{x-y}{|x-y|} .
$$

Obviously,

$$
\frac{1}{|z|^{\alpha}|z-e|^{\alpha}} \leq 2^{\alpha} \begin{cases}|z|^{-\alpha}, & |z| \leq 1 / 2 \\ |z-e|^{-\alpha}, & 1 / 2 \leq|z| \leq 2, \\ |z|^{-2 \alpha}, & |z| \geq 1 / 2\end{cases}
$$

Therefore, taking into account (1.8.6), we conclude that the last integral is bounded uniformly with respect to $|e|$ satisfying the condition $|e|=1$. Thus, we have the estimate

$$
|p * q|_{0} \leq C|p|_{0}|q|_{0} .
$$

If $q(z, y) \equiv 0$ for $|z-y| \geq \delta$, then, in the same way, we obtain that

$$
|(p * q)(x, y)| \leq|x-y|^{k-\alpha}|p|_{0}|q|_{0} \int_{|x-y||z| \leq \delta} \frac{d z}{|z|^{\alpha}|z-e|^{\alpha}} .
$$

For $|x-y| \leq \sqrt{\delta}$, we have the obvious inequality

$$
|x-y|^{k-\alpha} \int_{|x-y||z| \leq \delta} \frac{d z}{|z|^{\alpha}|z-e|^{\alpha}} \leq \delta^{(k-\alpha) / 2} \int_{\mathbb{R}^{k}} \frac{d z}{|z|^{\alpha}|z-e|^{\alpha}} .
$$


If $|x-y| \geq \sqrt{\delta}$, then the ball $\{|z| \leq \delta /|x-y|\}$ is contained in the ball $\{|z| \leq \sqrt{\delta}$ and, therefore,

$$
|x-y|^{k-\alpha} \int_{|x-y||z| \leq \delta} \frac{d z}{|z|^{\alpha}|z-e|^{\alpha}} \leq R^{k-\alpha} \delta^{(k-\alpha) / 2},
$$

where $R$ is the diameter of $G$. This yields the estimate

$$
|p * q|_{0} \leq C|p|_{0}|q|_{0} \delta^{(k-\alpha) / 2} .
$$

In the same way, this estimate is proved in the case where $p(x, y)=0$ for $|x-y| \geq \delta$.

As in the proof of Lemma 1.9.1, define $p_{n}$ and $q_{n}$ by $p$ and $q$ respectively. Then take the relation

$$
p * q-p_{n} * q_{n}=\left(p-p_{n}\right) * q+p_{n} *\left(q-q_{n}\right)
$$

and apply the estimate (1.9.10) to the terms at its right-hand side. We obtain the inequality

$$
\left|p * q-p_{n} * q_{n}\right| \leq 2 C n^{(\alpha-k) / 2}|p|_{0}|q|_{0} .
$$

Hence, the sequence of functions $p_{n} * q_{n}$ from $C(G \times G)$ uniformly converges to $p * q$. Combining this result with (1.9.9), we conclude that the bilinear map $*$ boundedly takes $C \times C$ into $C$.

Represent the operator $R_{n} q=p_{n} * q$ in the form

$$
\left(R_{n} q\right)(x, y)=\int_{G} \frac{r_{n}(x, z) q(y, z)}{|y-z|^{\alpha}} d z,
$$

where $r_{n}(x, z)=p_{n}(x, z)|x-z|^{-\alpha}$ is a continuous function. If the variable $y$ is treated as a parameter, then this operator is expressed by (1.9.1), where the function $q(x, z)$ is replaced by the function $r_{n}(x, z)$. Hence, similarly to the proof of Lemma 1.9.1, we verify that $R_{n}$ is a compact operator in the space $C(G \times G)$. Similarly to (1.9.11), one can obtain the estimate

$$
\left|R\left(p_{n}\right) q-R(p) q\right|_{0} \leq C n^{(\alpha-k) / 2}|p|_{0}|q|_{0}
$$

implying that the sequence $R\left(p_{n}\right)$ converges to $R(p)$ with respect to the operator norm. Hence, $R(p)$ is a compact operator.

Now we present the main result.

Theorem 1.9.1. There exists a discrete (at most countable) set $\Lambda \subseteq \mathbb{C}$ such that the operator $1-\lambda T(q)$ is invertible for any $\lambda$ outside $\Lambda$ and

$$
[1-\lambda T(q)]^{-1}=1-T\left(r_{\lambda}\right)
$$

where the function $r_{z}(x, y)$ belongs to $C(G \times G)$, is analytic with respect to $z$ in the open set $\mathbb{C} \backslash \Lambda$, and admits poles at points $\lambda$ of $\Lambda$.

Based on the notion of analytic vector-functions valued in Banach spaces, introduced in Sec. 1.4, we complement the properties of Fredholm operators, listed in Sec. 1.3, by the following result.

Theorem 1.9.2. Let $X$ be a Banach space endowed with a duality with respect to a bilinear form (, ) on $X \times X$. Let an operator-function $N(\lambda)$ from $\mathcal{L}(X)$ be analytic with respect to $\lambda$ in the disc $|\lambda|<1$ and $N(0)$ be a Fredholm operator of index zero. Then there exist $r$ from $(0,1)$ and a positive integer $m$ such that the operator $N(\lambda)$ is invertible for $0<|\lambda|<r$ and the operator-function $\lambda^{m} N^{-1}(\lambda)$ is analytic in the disc $|\lambda|<r$.

Proof. By assumption, the space $X$ contains linearly independent vector systems $x_{1}, \ldots, x_{n}$ and $y_{1}, \ldots, y_{n}$ such that

$$
\operatorname{ker} N(0)=\left[x_{1}, \ldots, x_{n}\right], \quad X=\left[y_{1}, \ldots, y_{n}\right] \oplus \operatorname{Im} N(0)
$$


Let a system $\widetilde{x}_{1}, \ldots, \widetilde{x}_{n}$ be biorthogonal to $x_{1}, \ldots, x_{n}$. Consider the operator

$$
\widetilde{N}(\lambda) x=N(\lambda) x-\sum_{j=1}^{n}\left(x, \widetilde{x}_{j}\right) y_{j}, \quad x \in X .
$$

By virtue of (1.9.13), the relation $\widetilde{N}(0) x=0$ implies the relations $N(0) x=0$ and $\left(x, \widetilde{x}_{j}\right)=0,1 \leq$ $j \leq n$, which are equivalent to the relation $x=0$. Thus, $\widetilde{N}(0)$ is a Fredholm operator of index zero and its kernel contains only zero. Hence, it is invertible. Then there exists $r$ from $(0,1)$ such that the operator $\widetilde{N}(\lambda)$ is invertible provided that $|\lambda|<r$. It is clear that the operator-function $\widetilde{N}^{-1}(\lambda)$ is analytic in the disc $|\lambda|<r$. From (1.9.14), it follows that

$$
\widetilde{N}^{-1}(\lambda) N(\lambda) x=x-\sum_{j=1}^{n}\left(x, \widetilde{x}_{j}\right) \widetilde{y}_{j}(\lambda), \quad \widetilde{y}_{j}(\lambda)=\widetilde{N}^{-1}(\lambda) y_{j} .
$$

Consider the $n \times n$-matrix $A(\lambda)$ consisting of the elements $A_{i j}(\lambda)=\delta_{i j}-\left(\widetilde{x}_{i}, \widetilde{y}_{j}(\lambda)\right)$. Obviously, it is analytic in the disc $|\lambda|<r$. Let $m$ be the order of the zero of the function $\operatorname{det} A(\lambda)$ at the point $\lambda=0$ (if $\operatorname{det} A(0) \neq 0$, then we assign $m=0$ ). Select a (sufficiently small) positive $r$ such that $\operatorname{det} A(\lambda) \neq 0$ provided that $0<|\lambda|<r$. Then the function $B(\lambda)=\lambda^{m} A^{-1}(\lambda)$ is analytic in the disc $|\lambda|<r$. If $0<|\lambda|<r$, then the equation

$$
x-\sum_{j=1}^{n}\left(x, \tilde{x}_{j}\right) \tilde{y}_{j}(\lambda)=y
$$

is uniquely solvable. Indeed, multiplying it by $\tilde{x}_{i}$ scalarly, we obtain the system $A(\lambda) \xi=\eta$, where $\eta_{i}=\left(y, \tilde{x}_{i}\right)$, for the vector $\xi$ from $\mathbb{C}^{n}$, consisting of the elements $\xi_{i}=\left(x, \tilde{x}_{i}\right)$. Converting this system, we obtain the relation

$$
\left(x, \tilde{x}_{j}\right)=\lambda^{-m} \sum_{k=1}^{n} B_{j k}(\lambda)\left(y, \tilde{x}_{k}\right), \quad 1 \leq j \leq n .
$$

Thus, we obtain the following inversion formula for system (1.9.16):

$$
x=y+\lambda^{-m} \sum_{1 \leq j, k \leq n} B_{j k}(\lambda)\left(y, \tilde{x}_{k}\right) y_{j}(\lambda) .
$$

Denoting the right-hand side of this relation by $\lambda^{-m} M(\lambda) y$ and taking into account (1.9.15), we conclude that the operator $N(\lambda)$ is invertible and $N^{-1}(\lambda)=\lambda^{-m} M(\lambda) \widetilde{N}^{-1}(\lambda)$.

Proof of Theorem 1.9.1. Fix a complex $\lambda_{0}$ and apply Theorem 1.9.2 to the operator $N(z)=1-(z+$ $\left.\lambda_{0}\right) T(q)$. We obtain that there exist a positive $r$ and a nonnegative integer $m$ such that the operator $1-\lambda T(q)$ is invertible for $0<\left|\lambda-\lambda_{0}\right|<r$ and the operator-function $\left(\lambda-\lambda_{0}\right)^{m}[1-\lambda T(q)]^{-1}$ is analytic in the disc $\left|\lambda-\lambda_{0}\right|<r$.

By virtue of (1.9.8), the operator relation $[1-T(\lambda q)]\left[1-T\left(r_{\lambda}\right)\right]=1$ is equivalent to the relation

$$
\lambda q+r_{\lambda}=\lambda q * r_{\lambda} .
$$

Let us treat this relation as an equation with respect to $r_{\lambda}$. If $\widetilde{r}_{\lambda}$ is another solution of it, then

$$
[1-T(\lambda q)]\left[1-T\left(r_{\lambda}\right)\right]=[1-T(\lambda q)]\left[1-T\left(\widetilde{r}_{\lambda}\right)\right]=1 .
$$

Since the operator $1-T(\lambda q)$ is invertible for $\lambda \neq \lambda_{0}$, it follows that $z_{\lambda}=\widetilde{r}_{\lambda}$. Thus, Eq. admits at most one solution. In the notation of Lemma 1.9.2, it can be written in the form

$$
[1-\lambda R(q)] r_{\lambda}=-\lambda q,
$$

where $R(q)$ is a compact operator acting in the space $C(G \times G)$. Therefore, Eq. (1.9.17) is uniquely solvable. 
Thus, for any fixed complex $\lambda_{0}$ there exist a positive $r$ and a function $r_{\lambda}(x, y)$ from $C(G \times G)$ analytic with respect to $\lambda$ in the domain $0<\left|\lambda-\lambda_{0}\right|<r$ such that

$$
[1-\lambda T(q)]^{-1}=1-T\left(r_{\lambda}\right)
$$

and, at the point $\lambda=\lambda_{0}$, the function $r_{\lambda}(x, y)$ admits a pole of order not exceeding $m$.

Since the point $\lambda_{0}$ is selected arbitrarily, this implies the claim of the theorem.

in [55], second-kind Fredholm integral equations are explained in more detail.

\section{Chapter 2}

\section{HÖLDER SPACES}

\subsection{Hölder Condition}

Let $C(G)$ denote the class of all functions $\varphi(x)$ continuous on the set $G \subseteq \mathbb{R}^{k}$. If we add a requirement of the uniform continuity on $G$, then any such function $\varphi$ is extended as $\tilde{\varphi}$ from $C(\bar{G})$.

Indeed, if a sequence $x_{n}$ converges to $x$ from $\bar{G} \backslash G$, then, due to the uniform continuity on of $\varphi$, the number sequence $\varphi\left(x_{n}\right)$ is a Cauchy sequence. Therefore, there exists a $\operatorname{limit} \lim \varphi\left(x_{n}\right)$ independent of the choice of the sequence; denote this limit by $\tilde{\varphi}(x)$. Assigning $\tilde{\varphi}(x)=\varphi(x), x \in G$, we obtain the function $\tilde{\varphi}$; it is easy to see that it is continuous on $\bar{G}$.

Functions satisfying the Hölder condition form a more narrow class than the class of uniformly continuous functions. We say that a function $\varphi$ satisfies the Hölder condition with power $\mu$ from $(0,1)$ (if $\mu=1$, then we call this the Lipschitz condition) on a set $G$, if the estimate

$$
|\varphi(x)-\varphi(y)| \leq C|x-y|^{\mu}, \quad x, y \in G,
$$

where $C$ is a positive constant independent of $x$ and $y$, is satisfied. The least $C$ in this Hölder condition is equal to

$$
[\varphi]_{\mu}=\sup _{x, y \in G, x \neq y} \frac{|\varphi(x)-\varphi(y)|}{|x-y|^{\mu}} .
$$

This relation determines a seminorm, i.e., $[\cdot]_{\mu}$ satisfies all norm conditions (1)-(3) from Sec. 1.1 except for the first one: if $[\varphi]_{\mu}=0$, then the function $\varphi$ is constant on the set $G$. Notation (2.1.2) is also used in the case where $\mu=0$ : in this case, $[\varphi]_{0}$ is the least upper bound of $|\varphi(x)-\varphi(y)|$ and it characterizes the oscillation of the function $\varphi$ on the set $G$. To indicate the dependence of seminorm (2.1.2) on $G$, we use the notation $[\varphi]_{\mu, G}$.

From the definition given by (2.1.1), it is clear that the function $\varphi$ is uniformly continuous on the set $G$; therefore, it is extended to the closure $\bar{G}$ as a continuous function satisfying the same estimate. This is why the Hölder condition is usually considered for closed sets $G$ (without loss of generality).

Consider several examples. For $0 \leq s \leq 1$, the inequality $1-s^{\mu} \leq 1-s \leq(1-s)^{\mu}$ shows that $[\varphi]_{\mu}=1$, where $\varphi(t)=t^{\mu}$ on the positive semiaxis. In particular, the following double inequality holds:

$$
\left.|| x\right|^{\mu}-|y|^{\mu}|\leq||x|-|y|^{\mu} \leq|x-y|^{\mu} .
$$

Here, the second inequality follows from the triangle inequality; it means that the function $\varphi(x)=|x|$ satisfies the Lipschitz condition with constant $[\varphi]_{1}=1$. Another example of a function of such type is the distance $d(x, F)$ between a point $x$ and a set $F$ :

$$
d(x, F)=\inf _{z \in F}|x-z| .
$$

Let us prove that it satisfies the Lipschitz condition:

$$
|d(x, F)-d(y, F)| \leq|x-y| .
$$


Indeed, if $z \in F$, then $d(x) \leq|x-y|+|y-z|$ due to the triangle inequality and, therefore, $d(x) \leq$ $|x-y|+d(y)$. Since the points $x$ and $y$ can be swapped, it follows that (2.1.5) is valid.

Note that the function $\varphi(x)=d(x, F)$ vanishes at a closed set $\bar{F}$ and is positive outside it. It is easy to see that its seminorm $[\varphi]_{1}$ is equal to 1 .

Similarly to (2.1.4), one can introduce the distance between sets as follows:

$$
d(E, F)=\inf _{x \in E, y \in F}|x-y| .
$$

If these sets are closed and disjoint and one of them is bounded (i.e., compact), then $d(E, F)>0$.

The following simple estimates are valid for seminorms []$_{\mu}$ of products and superpositions of functions:

$$
\begin{aligned}
& \text { (a) }[\varphi \psi]_{\mu} \leq|\varphi|_{0}[\psi]_{\mu}+[\varphi]_{\mu}|\psi|_{0} ; \\
& \text { (b) }[f \circ \varphi]_{\mu, G} \leq[f]_{1, D}[\varphi]_{\mu, G}, \quad \varphi(G) \subseteq D ; \\
& \text { (c) }[\varphi \circ \alpha]_{\mu, \widetilde{G}} \leq[\varphi]_{\mu, G}[\alpha]_{1, \widetilde{G}}^{\mu}, \quad \alpha(\widetilde{G}) \subseteq G .
\end{aligned}
$$

Note that the Hölder condition can be also used for vector-functions $\varphi=\left(\varphi_{1}, \ldots, \varphi_{s}\right)$ valued in $\mathbb{R}^{s}$. In this case, $|\varphi(x)-\varphi(y)|$ on the left-hand side of (2.1.1) is replaced by a fixed norm of the space $\mathbb{R}^{s}$. Then $\varphi$ from $(2.1 .6)$ (b) can be treated as a vector-function valued in $D \subseteq \mathbb{R}^{s}$, while $\alpha$ from (2.1.6)(c) as a vector-function values in $G \subseteq \mathbb{R}^{k}$.

The interpolation property

$$
[\varphi]_{\mu} \leq[\varphi]_{0}^{1-\mu / \nu}[\varphi]_{\nu}^{\mu / \nu}, \quad 0 \leq \mu \leq \nu
$$

of the Hölder seminorm immediately follows from the relation

$$
\frac{|\varphi(x)-\varphi(y)|}{|x-y|^{\mu}}=|\varphi(x)-\varphi(y)|^{1-\mu / \nu}\left[\frac{|\varphi(x)-\varphi(y)|}{|x-y|^{\nu}}\right]^{\mu / \nu} .
$$

This inequality is well known; it is frequently used in the operator interpolation theory (see [33]).

A detailed explanation of properties of functions satisfying the Hölder condition is provided by [45]. Most frequently, these spaces occur in investigations of differential equations (see, e.g., [34, 36]). Below, we provide results not covered by the above books; regarding these results, it is not always possible to provide pertinent references.

Rather frequently, it suffices to verify the Hölder condition locally at neighborhoods of points. For example, let $G$ be bounded and for any point $a$ from $\bar{G}$ there exist a ball $B(a)$ centered at this point such that $\varphi$ satisfies the Hölder condition with power $\mu$ inside this ball. Then one can select a finite set of such balls $B\left(a_{j}\right), 1 \leq j \leq m$, covering $\bar{G}$. Then $\varphi$ satisfies the Hölder condition on the whole set (see the next theorem).

Let a neighborhood of $\infty$ be treated as a set containing the exterior of a ball.

Theorem 2.1.1. Let a closed set $\bar{G}$ be contained in the union of open sets $V_{1}, \ldots, V_{m}$ such that one of them is a neighborhood of $\infty$ if $G$ is unbounded. Then the estimate

$$
[\varphi]_{\mu, G} \leq C \max \left(|\varphi|_{0, G},[\varphi]_{\mu, G \cap V_{1}}, \ldots,[\varphi]_{\mu, G \cap V_{m}}\right),
$$

where $C$ is a positive constant independent of $\varphi$, holds.

Proof. There exists a positive $r$ such that for any pair $x, y$ of points from $G$ such that the distance between them does not exceed $r$ there exists $i$ such that the specified pair lies in $G_{i}$. Indeed, assuming (to the contrary) that no such $i$ exists, we see that there exist sequences $x_{n}$ and $y_{n}$ from $G$ such that $x_{n}-y_{n} \rightarrow 0$ as $n \rightarrow \infty$ and, for any $n$, the points $x_{n}$ and $y_{n}$ belong to two different sets $V_{i}$. Passing to subsequences, one can assume that either both sequences converge to a same point $a$ from $\bar{G}$ or $\left|x_{n}\right| \rightarrow \infty$. Let a set $V_{i}$ contain $a$ in the former case and be a neighborhood of $\infty$ in the latter one. Then, for both cases, both points $x_{n}$ and $y_{n}$ belong to a same set $V_{i}$ provided that $n$ is sufficiently large, which leads to a contradiction with our assumption. 
Thus, for any pair $x, y$ of points from $G$ such that the distance between them does not exceed $r$ there exists $i$ such that the said pair lies in $G_{i}$. Therefore,

$$
|\varphi(x)-\varphi(y)||x-y|^{-\mu} \leq \max \left([\varphi]_{\mu, G_{1}}, \ldots,[\varphi]_{\mu, G_{m}}\right)
$$

provided that $x \in G, y \in G$, and $|x-y| \leq r$. On the other hand, for $|x-y|>r$, we have an obvious inequality

$$
|\varphi(x)-\varphi(y)||x-y|^{-\mu} \leq r^{-\mu}[\varphi]_{0, G} .
$$

Then, in (2.1.8), one can assign $C=\max \left(1, r^{-\mu}\right)$.

In the general case, if $G$ is represented as the union of sets $G_{1}, \ldots, G_{m}$ and a function $\varphi$ defined on $G$ satisfies the Hölder condition on each set $G_{i}$, then a problem to find whether this condition is satisfied on the whole set $G$ arises. The following example shows that it is not always guaranteed.

Let $G=G_{0} \cup G_{1} \subseteq \mathbb{R}^{2}$, where $G_{0}$ is the segment $\left\{x_{2}=0,0 \leq x_{1} \leq 1\right\}$, while $G_{1}$ is the arc $\left\{x_{2}=x_{1}^{2}, 0 \leq x_{1} \leq 1\right\}$ of a parabola. On $G$, consider the function

$$
\varphi(x)= \begin{cases}0, & x \in G_{0}, \\ x_{1}, & x \in G_{1} .\end{cases}
$$

It is easy to see that this function satisfies the Lipschitz condition on $G_{1}$. On the other hand, for the points $x=(t, 0), y=\left(t, t^{2}\right)$ from $G$, the expression

$$
|\varphi(x)-\varphi(y)||x-y|^{-\mu}=t^{1-2 \mu}
$$

is bounded for $\mu>1 / 2$.

However, there are assumptions about the set $G$, guaranteeing the fulfillment of the Hölder condition on the whole set.

Any homeomorphic image $\gamma(I)$ of a segment $I$ of the line $\mathbb{R}$ is called a simple (or Jordan) arc $\Gamma$ from $\mathbb{R}^{k}$. The map $\gamma$ itself is called an arc parametrization; it determines the natural order of arc points, i.e., the orientation of the curve. We say that $\Gamma$ is a rectifiable arc if it admits a parametrization $\gamma$ such that it is a bounded-variation vector-function or, which is the same, there exists a positive constant $C$ such that

$$
\left|\gamma\left(t_{1}\right)-\gamma\left(t_{2}\right)\right|+\left|\gamma\left(t_{2}\right)-\gamma\left(t_{3}\right)\right|+\ldots+\left|\gamma\left(t_{n-1}\right)-\gamma\left(t_{n}\right)\right| \leq C
$$

for any collection $t_{1}, t_{2}, \ldots, t_{n}$ of the segment $I$ such that $t_{1}<t_{2}<\ldots<t_{n}$. From the geometrical viewpoint, the above sum is the length of a broken line inscribed in the curve $\Gamma$. For this reason, the upper bound of this sum is called the length of the arc $\Gamma$; notation $l(\Gamma)$.

We say that a connected set is linearly connected if any pair of its points can be connected by a Jordan arc. For example, domains are linearly connected. This definition can be strengthened. A set $G$ is called uniformly connected if there exists a positive constant $M$ such that any two points $x$ and $y$ from $G$ can be connected by a rectifiable arc $\Gamma_{x, y}$ from $G$ such that its length admits an estimate

$$
l\left(\Gamma_{x, y}\right) \leq M|x-y|, \quad x, y \in G .
$$

This definition depends on the constant $M$. For this reason, such sets are also called $M$-uniformly connected; another term is sets regular in the Whitney sense (see [28]).

An obvious example of a uniformly connected set is a convex set such that a segment can be taken instead of the $\operatorname{arc} \Gamma_{x, y}$, i.e., inequality (2.1.9) becomes the equality with $M=1$.

Theorem 2.1.2. Let an $M$-uniformly connected set $G$ be the union of its subsets $G_{1}, \ldots, G_{m}$. Then the estimate

$$
[\varphi]_{\mu, G} \leq(m-1) M^{\mu} \max \left([\varphi]_{\mu, G_{1}}, \ldots,[\varphi]_{\mu, G_{m}}\right)
$$

holds. 
Proof. Without loss of generality, one can assume the sets $G$ and $G_{1}, \ldots, G_{m}$ to be closed. Let $x \in G$, $y \in G$, and $\Gamma=\Gamma_{x, y}$ be the arc from (2.1.9). Then this arc contains points $x_{1}=x, x_{2}, \ldots, x_{n}=y$, $n<m$ (the order corresponds to its orientation), such that any two neighboring ones belong to a same set $G_{k}, k=\overline{1, m}$. This fact is easily proved by means of the induction with respect to $m$ as follows. For $m=1$, no proof is required. Assume that the assertion holds for $m-1$ sets $G_{k}$ and consider the case where the sets are $G_{1}, \ldots, G_{m}$. Without loss of generality, one can assume that the points $x$ and $y$ belong to different sets $G_{k}$; for definiteness, let $x \notin G_{m}$ and $y \in G_{m}$. Take a point $y^{\prime}$ from $\Gamma$ such that it is closest to $x$ among points of $G_{m}$; this is possible since $G_{m}$ is closed. Since the arc $\Gamma$ is connected, this point also belongs to the set $G_{1} \cup \ldots \cup G_{m-1}$. Then the arc $\Gamma^{\prime} \subseteq \Gamma$ with the ends $x, y^{\prime}$ is covered by $m-1$ sets $G_{1}, \ldots, G_{m-1}$ and it remains to use the inductive assumption.

Thus, let any two neighboring points of the $\operatorname{arc} \Gamma$, belonging to the finite sequence $x_{1}=x, x_{2}, \ldots$, $x_{n}=y, n \leq m-1$, belong to one set of $G_{1}, \ldots, G_{m}$. Then

$$
\begin{aligned}
& |\varphi(x)-\varphi(y)| \leq\left|\varphi\left(x_{1}\right)-\varphi\left(x_{2}\right)\right|+\ldots+\left|\varphi\left(x_{n-1}\right)-\varphi\left(x_{m}\right)\right| \\
\leq & \max \left(|\varphi|_{\mu, G_{1}}, \ldots,|\varphi|_{\mu, G_{m}}\right)\left(\left|x_{1}-x_{2}\right|^{\mu}+\ldots+\left|x_{n-1}-x_{n}\right|^{\mu}\right) .
\end{aligned}
$$

Taking into account the inequalities $\left|x_{i-1}-x_{i}\right| \leq l\left(\Gamma_{x, y}\right)$ and (2.1.9), we obtain the estimate (2.1.10).

To illustrate this theorem, consider the following case. Let $G$ be an arbitrary set and a function $\varphi$ from $C^{\mu}(\bar{G})$ vanish at $\partial G$. Then the function $\tilde{\varphi}$ extended by zero to $G=\mathbb{R}^{k}$ satisfies the assumption of the theorem with $G_{1}=G, G_{2}=\mathbb{R}^{k} \backslash G$, and $M=1$. Then $[\tilde{\varphi}]_{\mu}=[\varphi]_{\mu, G}$ due to (2.1.10).

From the proof of Theorem 2.1.1, we see that there exists a positive $r$ such that the seminorm

$$
[\varphi]_{\mu, G}^{\prime}=\sup _{x, y \in G,|x-y| \leq r} \frac{|\varphi(x)-\varphi(y)|}{|x-y|^{\mu}}
$$

is finite. The following estimate with respect to this seminorm is obvious:

$$
[\varphi]_{\mu} \leq \max \left(r^{-\mu}[\varphi]_{0},[\varphi]_{\mu}^{\prime}\right)
$$

If the set $G$ is connected, this estimate can be strengthened.

Theorem 2.1.3. If $G$ is a bounded connected set, then the estimate

$$
[\varphi]_{\mu} \leq C[\varphi]_{\mu}^{\prime},
$$

where $C$ is a positive constant depending only on $r$ and the diameter $R$ of the set $G$, holds in notation (2.1.11).

Proof. It suffices to estimate $[\varphi]_{0}$ via $[\varphi]_{\mu}^{\prime}$. Let a closed ball $B$ of radius $R$ contain the set $G$. This ball can be covered by the union of a finite set of open balls $B_{1}, \ldots, B_{m}$ of radius $r / 3$ each. Obviously, the least amount $m$ of such balls depends only on $r$ and $R$. Among these balls, take the ones such that their intersections with $G$ are nonempty; denote their union by $D$. Since $G$ is connected, it follows that the set $D$ is also connected. In particular, each two points $x$ and $y$ from $G$ can be connected by an arc $\Gamma$ such that $\Gamma \subseteq D$. Repeating the arguing from the proof of Theorem 2.1.2, one can select points $x_{1}=x, x_{2}, \ldots, x_{n}=y, n \leq m$, such that any two neighboring points $x_{k}$ and $x_{k+1}$ belong to a same set $B_{j_{k}}$ such that $B_{j_{k}} \subseteq D$. By assumption, for any $k$ between 1 and $n$ there exists a point $z_{k}$ from $B_{j_{k}} \cap G$ such that

$$
\left|z_{k}-z_{k+1}\right| \leq\left|z_{k}-x_{k}\right|+\left|x_{k}-x_{k+1}\right|+\left|z_{k+1}-x_{k+1}\right| \leq r .
$$

Assigning $z_{1}=x$ and $z_{n}=y$, we obtain that

$$
|\varphi(x)-\varphi(y)| \leq[\varphi]_{\mu}^{\prime} \sum_{k=1}^{n-1}\left|z_{k}-z_{k+1}\right|^{\mu} \leq n r^{\mu}[\varphi]_{\mu}^{\prime},
$$

which completes the proof of the theorem. 
The next lemma provides an important property of Hölder functions.

Lemma 2.1.1. Let a function $\psi(x, y)$ satisfy the Hölder condition with power $\nu$ on the set $G \times G$ and vanishes for $x=y$. Then, for $0<\mu<\nu$, the function $\psi_{0}(x, y)=|x-y|^{\mu-\nu} \psi(x, y)$ extended by zero for $x=y$ satisfies the Hölder condition with power $\mu$ and admits the estimate

$$
\left[\psi_{0}\right]_{\mu} \leq 6[\psi]_{\nu}
$$

Proof. First, we note that

$$
|\psi(x, y)|=|\psi(x, y)-\psi(x, x)| \leq[\psi]_{\nu}|x-y|^{\nu}
$$

and, therefore, $\psi(x, y) \rightarrow 0$ as $x-y \rightarrow 0$.

Fix $x_{0}$ from $G$ and consider functions $\varphi(x)=\psi\left(x, x_{0}\right)$ and $\varphi_{0}(x)=\psi_{0}\left(x, x_{0}\right)$ of one independent variable $x$. These functions are linked by the relation $\varphi_{0}(x)=\left|x-x_{0}\right|^{\mu-\nu} \varphi(x)$. Let us prove that

$$
\left[\varphi_{0}\right]_{\mu} \leq 3[\varphi]_{\nu}
$$

Obviously, it suffices to prove this estimate under the assumption that $x_{0}=0$ (otherwise, it suffices to apply the change $x^{\prime}=x-x_{0}$, leaving the seminorm $[\cdot]_{\mu}$ unchanged). Let $x \in G, y \in G$, and (for definiteness) $|y| \leq|x|$. For brevity, assign $\varepsilon=\nu-\mu$. Then

$$
\left|\varphi_{0}(x)-\varphi_{0}(y)\right| \leq|\varphi(x)-\varphi(y)||x|^{-\varepsilon}+\left.|\varphi(y)||| x\right|^{-\varepsilon}-|y|^{-\varepsilon} \mid .
$$

Using the inequality $|\varphi(y)| \leq[\varphi]_{\nu}|y|^{\mu+\varepsilon}$, we obtain that

$$
\frac{\left|\varphi_{0}(x)-\varphi_{0}(y)\right|}{|x-y|^{\mu}} \leq[\varphi]_{\nu} \Delta, \quad \Delta=\frac{|x-y|^{\varepsilon}}{|x|^{\varepsilon}}+\frac{\left(|x|^{\varepsilon}-|y|^{\varepsilon}\right)|y|^{\mu}}{|x-y|^{\mu}|x|^{\varepsilon}} .
$$

It is obvious that

$$
\Delta \leq \frac{(|x|+|y|)^{\varepsilon}}{|x|^{\varepsilon}}+\frac{\left(|x|^{\varepsilon}-|y|^{\varepsilon}\right)|y|^{\mu}}{(|x|-|y|)^{\mu}|x|^{\varepsilon}}=(1+t)^{\varepsilon}+t \frac{1-t^{\varepsilon}}{(1-t)^{\varepsilon}},
$$

where $t=|y| /|x| \leq 1$. Since $1-t^{\varepsilon} \leq 1-t \leq(1-t)^{\mu}$, it follows that $\Delta \leq 3$ and, respectively, the estimate (2.1.13) holds.

Using the estimate (2.1.12), we obtain the inequality

$$
\left|\psi_{0}(x, y)-\psi_{0}\left(x^{\prime}, y^{\prime}\right)\right| \leq\left|\psi_{0}(x, y)-\psi_{0}\left(x^{\prime}, y\right)\right|+\left|\psi_{0}\left(x^{\prime}, y\right)-\psi_{0}\left(x^{\prime}, y^{\prime}\right)\right|
$$

Applying (2.1.13) to the terms at the right-hand side, we obtain the inequality

$$
\left|\psi_{0}(x, y)-\psi_{0}\left(x^{\prime}, y^{\prime}\right)\right| \leq 3[\psi]_{\nu}\left(\left|x-x^{\prime}\right|^{\mu}+\left|y-y^{\prime}\right|^{\mu}\right) .
$$

Here, neither $\left|x-x^{\prime}\right|$ nor $\left|y-y^{\prime}\right|$ exceeds the distance between the points $(x, y)$ and $\left(x^{\prime}, y^{\prime}\right)$ in $\mathbb{R}^{k} \times \mathbb{R}^{k}$. This yields the estimate (2.1.12).

To conclude, we complement Theorem 2.1.2 as follows. Any set $K$ containing the ray $\{\tau+t(x-$ $\tau), t>0\}$ for any point $x$ of $K$ is called a cone with vertex $\tau$.

Lemma 2.1.2. Let $K_{1}$ and $K_{2}$ be closed cones in $\mathbb{R}^{k}$ such that the only their common point is their vertex $\tau$. Then the number

$$
r=\min \left[d\left(K_{1} \cap \Omega, K_{2}\right), d\left(K_{2} \cap \Omega, K_{1}\right)\right],
$$

where $\Omega$ denotes the unit sphere $\{\xi,|\xi|=1\}$ in $\mathbb{R}^{k}$, is positive and

$$
\left|x_{1}-x_{2}\right| \geq r\left(\left|x_{1}-\tau\right|+\left|x_{2}-\tau\right|\right), \quad x_{j} \in K_{j} .
$$

In particular, if a set $G$ is contained in $K_{1} \cup K_{2}$, then

$$
[\varphi]_{\mu, G} \leq r^{-\mu} \max \left(|\varphi|_{\mu, G \cap K_{1}},|\varphi|_{\mu, G \cap K_{2}}\right) .
$$


Proof. Without loss of generality, one can assume that $\tau=0$. Since $K_{1} \cap K_{2}=\{0\}$ by assumption, it follows that the intersection $K_{1} \cap \Omega$ is a compact set disjoint with $K_{2}$. Therefore, the distance $d\left(K_{1} \cap \Omega, K_{2}\right)$ between these sets is positive. Hence, the number $r$ is also positive. Thus, the inequalities $\left|x_{1}-x_{2}\right| \geq r|x|_{j}$ hold for all $x_{j}$ from $K_{j}, j=1,2$. Adding these inequalities, we obtain (2.1.14).

Let a set $G$ (without loss of generality, we assume that it is closed) be contained in $K_{1} \cup K_{2}$. Then the following inequality holds for any $x_{j}$ from $G \cap K_{j}, j=1,2$ :

$$
\left|\varphi\left(x_{1}\right)-\varphi\left(x_{2}\right)\right| \leq\left|\varphi\left(x_{1}\right)-\varphi(0)\right|+\left|\varphi(0)-\varphi\left(x_{2}\right)\right| \leq \max \left(|\varphi|_{\mu, G_{i}},|\varphi|_{\mu, G_{j}}\right)\left(\left|x_{1}\right|^{\mu}+\left|x_{2}\right|^{\mu}\right) .
$$

Taking into account the fact that $1+t^{\mu} \leq(1+t)^{\mu}$ provided that $t>0$, we combine the last double inequality with (2.1.14) to deduce that

$$
\left|\varphi\left(x_{1}\right)-\varphi\left(x_{2}\right)\right| \leq r^{-\mu} \max \left(|\varphi|_{\mu, G_{i}},|\varphi|_{\mu, G_{j}}\right)\left|x_{1}-x_{2}\right|^{\mu} .
$$

Obviously, a similar inequality holds in the case where $x_{1}$ and $x_{2}$ belong to $G \cap K_{j}$. Thus, we obtain the estimate (2.1.15).

\subsection{Hölder Spaces $C^{\mu}(G)$}

In $C(G)$, consider the class of all bounded continuous functions, denoted by $C^{0}(G)$. Obviously, this is a Banach space with respect to the sup-norm

$$
|\varphi|_{0}=\sup _{x \in G}|\varphi(x)| .
$$

If the set $G$ is compact, then the boundedness requirement for the functions can be omitted in the given definition and, respectively, the sup-norm can be replaced by $\max |\varphi(x)|$. However, if the set $G$ is not compact, then $C(G)$ and $C^{0}(G)$ are different classes. In the general case, no requirements are imposed on the set $G$; most frequently, it is assumed to be closed or open.

By $C^{\mu}(G), 0<\mu<1$, denote the space of all bounded functions $\varphi$ satisfying the Hölder condition with power $\mu$ on $G$. This space is endowed with the norm

$$
|\varphi|_{C^{\mu}}=|\varphi|_{0}+[\varphi]_{\mu}
$$

For $\mu=1$, the similar class of Lipschitz functions is denoted by $C^{0,1}(G)$. This class is different from the class $C^{1,0}(G)$ of continuously differentiable functions. A similar notation $C^{\mu}=C^{0, \mu}$ is also used in the case where $0 \leq \mu<1$. In particular, this notation is important for the definition of Hölder spaces of differentiable functions (see Sec. 2.7 below). In the sequel, unless the opposite is stated, it is assumed that $0<\mu \leq 1$.

Let us verify that $C^{0}, \mu$ is a Banach space for any such $\mu$. Let $\varphi_{n}$ be a fundamental sequence in $C^{0, \mu}(G)$, i.e., for any positive $\varepsilon$ there exists a number $N$ such that $\left|\varphi_{n}-\varphi_{m}\right|_{\mu} \leq \varepsilon$ provided that $m \geq N$ and $n \geq N$. Due to the completeness of the space $C^{0}$, this implies that there exists a function $\varphi$ from $C^{0}$ such that the said sequence converges to $\varphi$ with respect to the sup-norm. For any fixed $x$ and $y$ from $G$, the inequality $\left|\left(\varphi_{n}-\varphi_{m}\right)(x)-\left(\varphi_{n}-\varphi_{m}\right)(y)\right| \leq \varepsilon|x-y|^{\mu}$ holds provided that $m$ and $n$ are positive integers. In the last inequality, we pass to the limit as $m \rightarrow \infty$. We obtain a similar inequality for $\varphi_{n}-\varphi$. It shows that $\varphi$ belongs to $C^{0, \mu}(G)$ and $\left|\varphi_{n}-\varphi\right|_{\mu} \rightarrow 0$ as $n \rightarrow \infty$.

From inequality (2.1.6)(a), it follows that norm (2.1.2) possesses the property $|\varphi \psi|_{\mu} \leq|\varphi|_{\mu}|\psi|_{\mu}$, i.e., that the space $C^{\mu}$ is a Banach algebra with respect to multiplication. Assuming that elements of this algebra are complex-valued scalar functions, we can easily verify that the inequality $\inf _{x}|\varphi(x)|>0$ is a necessary and sufficient condition of their invertibility. This obviously follows from (2.1.6)(b). Relation (2.1.6)(c) means that the superposition $T(\alpha) \varphi=\varphi \circ \alpha$ is a linear bounded operator operator from $C^{\mu}(\bar{G})$ to $C^{\mu}(G)$ and its norm does not exceed $\left[\alpha^{\prime}\right]_{\mu}$.

In the interpolation inequality $(2.1 .7)$, change $[\varphi]_{0}$ and $[\varphi]_{\nu}$ for their maximums. We obtain the following estimate for Hölder norms:

$$
|\varphi|_{C^{\mu}} \leq 2|\varphi|_{C^{\nu}}, \quad \mu<\nu \leq 1 .
$$


Another application of this inequality is related to the approximation operator $T_{\varepsilon}$ introduced at Sec. 1.8. The claim of Lemma 1.8.1 about this operator can also be extended to the case of Hölder spaces.

Lemma 2.2.1. If $\varphi \in C^{\nu}\left(\mathbb{R}^{k}\right)$ and $0<\mu<\nu$, then $T_{\varepsilon} \varphi \rightarrow \varphi$ with respect to the norm of the space $C^{\mu}\left(\mathbb{R}^{k}\right)$ as $\varepsilon \rightarrow 0$.

Proof. Proving Lemma 1.8.1, we found the estimate for the sup-norm of the difference $\psi_{\varepsilon}=T_{\varepsilon} \varphi-\varphi$ : $\left|\psi_{\varepsilon}\right|_{0} \leq \omega(\varepsilon)$, where $\omega$ denotes the continuity modulus

$$
\omega(\varepsilon)=\sup _{|x-y| \leq \varepsilon}|\varphi(x)-\varphi(y)|
$$

of the function $\varphi$. If $\varphi \in C^{\nu}\left(\mathbb{R}^{k}\right)$, then it is obvious that $\omega(\varepsilon) \leq[\varphi]_{\nu} \varepsilon^{\nu}$. Hence, $\left[\psi_{\varepsilon}\right]_{0} \leq[\varphi]_{\nu} \varepsilon^{\nu}$. Due to (2.1.7), this implies the estimate

$$
\left[\psi_{\varepsilon}\right]_{\mu} \leq\left(2[\varphi]_{\nu} \varepsilon^{\nu}\right)^{1-\mu / \nu}[\varphi]_{\nu}^{\mu / \nu}=2 \varepsilon^{\nu-\mu}[\varphi]_{\nu}
$$

completing the proof of the lemma.

Inequality (2.2.3) shows that a the family of Banach space $\left(C^{\mu}\right)$ monotonously decreases with respect to the embedding. Then it is convenient to introduce the class

$$
C^{\mu+0}=\bigcup_{\varepsilon>0} C^{\mu+\varepsilon}, \quad 0 \leq \mu<1,
$$

of functions satisfying the Hölder condition with a power exceeding $\mu$. For $\mu=0$, this class is denoted by $C^{+0}$. In $[17,45]$, the class $C^{+0}$ is denoted by $H$.

The following important property of Hölder spaces is also a consequence of (2.1.7): if a function sequence $\varphi_{n}$ is bounded in $C^{\nu}$ and converges to a function $\varphi$ with respect to the sup-norm, then $\varphi \in C^{\nu}$ and $\varphi_{n} \rightarrow \varphi$ in $C^{\mu}$ for any $\mu$ such that $\mu<\nu$.

Indeed, by assumption, $\left[\varphi_{n}\right]_{\nu} \leq C$ for all $n$. Passing to the limit as $n \rightarrow \infty$ in the inequality $\left|\varphi_{n}(x)-\varphi_{n}(y)\right| \leq C|x-y|^{\nu}$, we obtain that $\varphi \in C^{\nu}$. Changing the function $\varphi$ for $\varphi-\varphi_{n}$ in (2.1.7), we obtain that $\left[\varphi-\varphi_{n}\right]_{\mu} \rightarrow 0$ as $n \rightarrow \infty$.

In particular, if $G$ is a bounded set, then, taking into account the Arzela-Ascoli theorem, we conclude that the embedding $C \nu(G) \subseteq C^{\mu}(G)$ is compact provided that $0<\nu<\nu \leq 1$. To prove that, we take into account the fact that one can assume that the set $G$ is closed and, therefore, compact.

It is convenient to use the distance function (2.1.4) to approximate functions vanishing on $F$ and $\infty$ by functions vanishing in neighborhoods of these sets. In the sequel, we treat neighborhoods of $F$ as sets of the kind $\{x, d(x, F)<r\}$, where $r$ is positive.

Theorem 2.2.1. Let $\varphi \in C^{\nu}(G)$ and $\varphi=0$ on a subset $F$ of $\bar{G}$. Let $\varphi(x) \rightarrow 0$ as $|x| \rightarrow+\infty$ in the case where the set $G$ is unbounded. Then there exists a sequence of functions $\varphi_{n}$ from $C^{\nu}(G)$ identically equal to zero in a neighborhood of $F$ and (in the case where the set $G$ is unbounded) in a neighborhood of $\infty$ such that it converges to $\varphi$ with respect to the norm of the space $C^{\mu}(G)$ provided that $0<\mu<\nu$.

Proof. Let a function $h(t)$ from $C^{0,1}(\mathbb{R})$ be equal to 1 for $|t| \leq 1$, be equal to zero for $|t|>2$, and be equal to $2-t$ for $1 \leq|t| \leq 2$. Obviously, $|h|_{0}=[h]_{1}=1$ for this function. Introduce the notation $d(x)=d(x, F)$ (for brevity) and consider the function

$$
\chi_{\varepsilon}(x)=h\left[\varepsilon^{-1} d(x)\right], \quad \varepsilon>0 .
$$

Obviously, this function is identically equal to one in the $\varepsilon$-neighborhood of the set $F$ and is identically equal to zero outside its $2 \varepsilon$-neighborhood. Taking into account (2.1.5)-(2.1.6), we have the following estimate:

$$
\left|\chi_{\varepsilon}\right|_{0} \leq 1, \quad\left[\chi_{\varepsilon}\right]_{1} \leq \varepsilon^{-1}
$$


Let us prove that

$$
\left[\chi_{\varepsilon} \varphi\right]_{\nu} \leq 5[\varphi]_{\nu}
$$

First, we note that, inverting the function $\varphi$ on $F$, we obtain the estimate

$$
|\varphi(x)| \leq[\varphi]_{\nu} d^{\nu}(x) \text {. }
$$

Indeed, if $z \in F$, then $|\varphi(x)|=|\varphi(x)-\varphi(z)| \leq[\varphi]_{\nu}|x-z|^{\nu}$. Since the point $z$ from $F$ is selected arbitrarily, this implies inequality (2.2.8).

Getting back to the proof of (2.2.7), consider the expression

$$
\Delta_{\varepsilon}=\frac{\left|\chi_{\varepsilon}(x) \varphi(x)-\chi_{\varepsilon}(y) \varphi(y)\right|}{|x-y|^{\nu}} .
$$

Obviously, it is equal to zero provided that $\min [d(x), d(y)] \geq 2 \varepsilon$. Therefore, estimating it from above, one can assume (without loss of generality) that $\min [d(x), d(y)] \leq 2 \varepsilon$.

The following two cases are possible: $2|x-y| \geq d(y)$ and $2|x-y| \leq d(y)$. Consider them separately. In the former case, we have $d(x) \leq|x-y|+d(y) \leq 3|x-y|$. Then, taking into account (2.2.8), we obtain the estimate

$$
\Delta_{\varepsilon} \leq|h|_{0}[\varphi]_{\nu}\left(\frac{[d(x)]^{\nu}}{|x-y|^{\nu}}+\frac{d(y)]^{\nu}}{|x-y|^{\nu}}\right) \leq|h|_{0}\left(3^{\nu}+2^{\nu}\right)[\varphi]_{\nu}
$$

Now, let $2|x-y| \leq d(y)$. In this case, we have the inequality

$$
\Delta_{\varepsilon} \leq\left|\chi_{\varepsilon}(x)\right| \frac{|\varphi(x)-\varphi(y)|}{|x-y|^{\nu}}+|\varphi(y)| \frac{\left|\chi_{\varepsilon}(x)-\chi_{\varepsilon}(y)\right|}{|x-y|^{\nu}} .
$$

Combining this with (2.2.6) and (2.2.8), we deduce the inequality

$$
\Delta_{\varepsilon} \leq|h|_{1}[\varphi]_{\nu}\left[1+\varepsilon^{-1} d^{\nu}(y)|x-y|^{1-\nu}\right] .
$$

From the inequalities $|d(x)-d(y)| \leq|x-y| \leq d(y) / 2$, it follows that $d(x) \leq 2 d(y)$ and $d(y) \leq 2 d(x)$. Taking into account the fact that $\min [d(x), d(y)] \leq 2 \varepsilon$, we deduce that $\max [d(x), d(y)] \leq 4 \varepsilon$. Then

$$
\Delta_{\varepsilon} \leq[\varphi]_{\nu}\left\{1+\varepsilon^{-1}[d(y)]^{\nu}[d(y) / 2]^{1-\nu}\right\} \leq 5[\varphi]_{\nu}
$$

Combining this with (2.2.9), we obtain that the estimate (2.2.7) holds.

Using notation (2.2.5), introduce the function sequence

$$
\varphi_{n}(x)=\left[1-\chi_{\varepsilon}(x)\right] h(\varepsilon|x|) \varphi(x), \quad \varepsilon=1 / n,
$$

Obviously, each of these functions is identically equal to zero in a neighborhood of $F$ and in a neighborhood of $\infty$. By virtue of (2.2.8), the $C^{\nu}(G)$-norms of these functions are bounded uniformly with respect $n=1,2, \ldots$ On the other hand, the difference $\varphi(x)-\varphi_{n}(x)$ is equal to zero provided that $d(x) \geq 2 / n$ and $|x| \leq n$. Since $\left|\varphi_{n}(x)\right| \leq|\varphi(x)|$, it follows that

$$
\left|\varphi-\varphi_{n}\right|_{0} \leq 2 \max _{d(x) \leq 2 / n}|\varphi(x)|+2 \max _{|x| \geq n}|\varphi(x)| .
$$

Taking into account (2.2.8), we conclude that each term at the right-hand side of the inequality tends to zero as $n \rightarrow \infty$. Hence, due to (2.1.7), the sequence $\varphi_{n}-\varphi$ tends to zero with respect to the norm of the space $C^{\mu}(G)$.

Now, we do not assume that the set $G$ is arbitrary. Instead, we consider the case where it is open and denote it by $D$. Recall that connected open sets are called domains. All domains can be decomposed into three classes with respect to the point at infinity: a domain is finite if it is bounded, i.e., lies in a finite part of the space $\mathbb{R}^{k}$, a domain is infinite if it is a neighborhood of $\infty$, i.e., contains the exterior of a ball, and a domain is semi-infinite if its boundary is not compact. 
Consider the class $C^{1}(D)$ of all functions continuously differentiable in the domain $D$. Recall that the gradient vector

$$
\varphi^{\prime}=\left(\frac{\partial \varphi}{\partial x_{1}}, \ldots, \frac{\partial \varphi}{\partial x_{k}}\right) \text { from } C(D)
$$

corresponds to any $\varphi$ from $C^{1}(D)$. If $\varphi$ is an $m$-vector-function, then this gradient is treated as the Jacobi $m \times k$-matrix $\mathcal{D} \varphi$ such that its columns are the partial derivatives $\partial \varphi / \partial x_{j}$. The notation $\mathcal{D} \varphi$ is also used in the case where $m=1$; in such a case, $\mathcal{D} \varphi$ is treated as a matrix-row. In the case of vector functions, the corresponding differential relations

$$
\mathcal{D}(\varphi \psi)=\psi \mathcal{D} \varphi+\varphi \mathcal{D} \psi, \quad \mathcal{D}(f \circ \varphi)=(\mathcal{D} f \circ \varphi) \mathcal{D} \varphi
$$

are treated in the sense of the multiplying of rectangular Jacobi matrices.

For example, consider the case where the domain of a scalar function $\varphi$ contains a segment $[a, b]$. Then the function $\varphi_{0}(t)=\varphi[t a+(1-t) b]$ of the variable $t$ from $[0,1]$ can be considered. Its derivative satisfies the relation

$$
\varphi_{0}^{\prime}(t)=\varphi^{\prime}[t a+(1-t) b](a-b),
$$

where the right-hand side is the scalar product of the vectors $\varphi^{\prime}$ and $a-b$.

The notation $C^{1,0}$ is used for the space of functions $\varphi$ from $C^{1}(D)$ such that $\varphi \in C^{0}(D)$ and $\varphi^{\prime} \in C^{0}(D)$. Obviously, this is a Banach space with respect to the norm $|\varphi|=|\varphi|_{0}+\left|\varphi^{\prime}\right|_{0}$. We define the class $C^{1}(\bar{D})$ as the set of functions $\varphi$ from $C(\bar{D})$ such that the derivative $\varphi^{\prime}$ of each one is extended as a function continuous on $\bar{D}$. Notation (2.2.11) is preserved for the limit values of the derivative at boundary points $x$ from $\partial D$. The corresponding space $C^{1,0}(\bar{D})$ of bounded functions has a similar sense. Finally, let $C^{1, \mu}(D), 0<\mu \leq 1$, denote the space of all functions $\varphi$ from $C^{1}(D)$ belonging to $C^{\mu}(D)$ together with all their first-order derivatives. This is a Banach space with respect to the norm

$$
|\varphi|=|\varphi|_{C^{\mu}}+\left|\varphi^{\prime}\right|_{C^{\mu}} .
$$

Let us investigate the relation between the space $C^{0,1}$ of functions satisfying the Lipschitz condition and the class $C^{1}$ of continuously differentiable functions.

Theorem 2.2.2. Let a function $\varphi$ be continuously differentiable in a domain $D$ and a finite domain $D_{0}$ together with its closure be contained in D. Then the estimate

$$
[\varphi]_{1, D_{0}} \leq C\left|\varphi^{\prime}\right|_{0, D}
$$

where $C$ is a positive constant depending only on the diameter of the domain $D_{0}$ and its distance to the boundary $\partial D$, holds.

If a rectifiable arc $\Gamma$ of length $l$ with ends $a$ and $b$ is contained in $D$, then

$$
|\varphi(a)-\varphi(b)| \leq \max _{x \in \Gamma}\left|\varphi^{\prime}(x)\right| l .
$$

In particular, if $D$ is a $M$-uniformly connected domain, then

$$
[\varphi]_{1, D} \leq M\left|\varphi^{\prime}\right|_{0, D}
$$

Proof. Let $2 r=d\left(D_{0}, \partial D\right)$ and the distance between the points $a$ and $b$ from $D_{0}$ not exceed $r$. Then the segment with ends $a$ and $b$ is contained in $D$ and the following relation holds due to (2.2.12):

$$
|\varphi(a)-\varphi(b)|=\left|\int_{0}^{1} \varphi_{0}^{\prime}(t) d t(a-b)\right| \leq\left|\varphi^{\prime}\right|_{0, D} .
$$

Therefore, the estimate (2.2.14) follows from Theorem 2.1.3. 
To prove the second claim of the theorem, we start from the assumption that $\Gamma$ is a broken line with tops $a=c_{0}, c_{1}, \ldots, c_{n}=b$. Then, similarly to the above arguing, we prove that

$$
|\varphi(a)-\varphi(b)| \leq \sum_{i=1}^{n}\left|\varphi\left(c_{i-1}\right)-\varphi\left(c_{i}\right)\right| \leq\left|\varphi^{\prime}\right|_{0} l(\Gamma) .
$$

Now, let $\Gamma$ be a rectifiable arc and $2 r$ be the distance between it and the boundary $\partial D$. Then, for $0<\varepsilon \leq r$, the set $G_{\varepsilon}=\{x \mid d(x, \Gamma) \leq \varepsilon\}$ is contained in $D$ and

$$
\lim _{\varepsilon \rightarrow 0}\left|\varphi^{\prime}\right|_{0, G_{\varepsilon}}=\left|\varphi^{\prime}\right|_{0, \Gamma} \text {. }
$$

On this arc, select points $a=c_{0}, c_{1}, \ldots, c_{n}=b$ are such that $\left|c_{i-1}-c_{i}\right| \leq r$ for any $i$. Then the broken line $\Gamma_{\varepsilon}$ with tops at these points is contained in $G_{\varepsilon}$ and, therefore, we have the inequality

$$
|\varphi(a)-\varphi(b)| \leq \max _{x \in G_{\varepsilon}}\left|\varphi^{\prime}(x)\right| l\left(\Gamma_{\varepsilon}\right) .
$$

Since $l\left(\Gamma_{\varepsilon}\right) \leq l(\Gamma)$, it follows that this inequality passes into (2.2.15) as $\varepsilon \rightarrow 0$.

Finally, if $D$ is a uniformly connected domain, $x \in D$, and $y \in D$, then, by the definition given by (2.1.9), the points $x$ and $y$ can be connected by a rectifiable arc $\Gamma \subseteq D$ of length $l$ such that $l \leq M|x-y|$. Due to (2.2.15), this implies the estimate (2.2.16).

The estimate (2.2.16) shows that the following embedding takes place for uniformly connected domains:

$$
C^{1,0}(D) \subseteq C^{0,1}(D)
$$

Theorem 2.2.2 is still valid if the domain $D$ is a neighborhood of $\infty$ and the subdomain $D_{0}$ is unbounded. Indeed, there exists a ball $B$ such that the domain $D$ contains its exterior $B^{\prime}$. Obviously, $B^{\prime}$ is a uniformly connected domain. Thus, there exists a ball $B_{0}$ such that the closed domain $\bar{D}_{0}$ can be covered by $B_{0}$ and $B^{\prime}$. The estimate $(2.2 .16)$ can be applied to each of these two sets. Thus, it remains to use Theorem 2.1.1.

The next useful assertion complements Theorem 2.2.2.

Lemma 2.2.2. Let a function $\varphi(x)$ be continuously differentiable in a cylinder $B=\left\{|\widetilde{x}|<r_{1},\left|x_{k}\right|<\right.$ $\left.r_{2}\right\} \subseteq \mathbb{R}^{k-1} \times \mathbb{R}$ of variables $x=\left(\widetilde{x}, x_{k}\right)$ and its gradient derivative $\varphi^{\prime}$ admit an estimate

$$
\left|\varphi^{\prime}(x)\right| \leq M x_{k}^{\mu-1}, \quad x \in B .
$$

Then $\varphi \in C^{\mu}(B)$ and $[\varphi]_{\mu} \leq C M$, where $C$ is a positive constant depending only on $\mu, r_{1}$, and $r_{2}$.

Proof. Let points $x$ and $y$ from $B$ be such that the inclination angle between the segment $[x, y]$ and the base $x_{k}=0$ of the cylinder is equal to $\pi / 4$. Then

$$
|x-y|=\sqrt{2}\left|x_{k}-y_{k}\right| \text {. }
$$

Then, taking into account the relation (2.2.13), we deduce the inequality

$$
|\varphi(x)-\varphi(y)| \leq M|x-y| \int_{0}^{1}\left[t x_{k}+(1-t) y_{k}\right]^{\mu-1} d t
$$

from the estimate (2.2.18). Using (2.1.3), we obtain the following estimate for the last integral:

$$
\int_{0}^{1}\left[t x_{k}+(1-t) y_{k}\right]^{\mu-1} d t=\frac{\left|x_{k}^{\mu}-y_{k}^{\mu}\right|}{\mu\left|x_{k}-y_{k}\right|} \leq \frac{1}{\mu\left|x_{k}-y_{k}\right|^{1-\mu}} .
$$

Combining this with (2.2.19), we deduce that

$$
|\varphi(x)-\varphi(y)| \leq C_{0} M|x-y|^{\mu}, \quad C_{0}=\sqrt{2}^{1-\mu} / \mu .
$$


Now, let points $x$ and $y$ be such that the segment $[x, y]$ be parallel or orthogonal to the cylinder base and a two-dimensional plane $P$ passing through these points be parallel to the base. Consider points $x^{\prime}$ and $y^{\prime}$ from $P$ such that the quadrangle formed by the points $x, y, x^{\prime}$, and $y^{\prime}$ is a rhombus with the opposite vertex pairs $x, y$ and $x^{\prime}, y^{\prime}$. Then, for

$$
|x-y|<\min \left(r_{1}, r_{2}\right),
$$

at least one of the points $x^{\prime}$ and $y^{\prime}$ belongs to $B$; let this be $x^{\prime}$. Since the inclination angle with respect to the cylinder base is equal to $\pi / 4$ for both segments $\left[x, x^{\prime}\right]$ and $\left[y, x^{\prime}\right]$ and $\left.|x-y|^{2}=\left|x-x^{\prime}\right|^{2}+\mid x^{\prime}-y\right]^{2}$, it follows from (2.2.20) that

$$
|\varphi(x)-\varphi(y)| \leq\left|\varphi(x)-\varphi\left(x^{\prime}\right)\right|+\left|\varphi\left(x^{\prime}\right)-\varphi(y)\right| \leq 2 C_{0} M|x-y|^{\mu} .
$$

Now, consider points $x=\left(\widetilde{x}, x_{k}\right)$ and $y=\left(\widetilde{y}, y_{k}\right)$ from $B$ and assign $z=\left(\widetilde{x}, y_{k}\right)$. The last point belongs to $B$ and, assuming that (2.2.21) is satisfied, one can apply the previous inequality to the point pairs $x, z$ and $y, z$. Since $|x-y|^{2}$ is still equal to $\left.|x-z|^{2}+\mid z-y\right]^{2}$, this yields the inequality

$$
|\varphi(x)-\varphi(y)| \leq 4 C_{0} M|x-y|^{\mu} .
$$

Finally, let $x$ and $y$ be arbitrary points of $B$. Obviously, they satisfy the inequality $|x-y|^{2}<4 r_{1}^{2}+r_{2}^{2}$. Select a positive integer $n$ satisfying the condition $\sqrt{4 r_{1}^{2}+r_{2}^{2}} \leq n \min \left(r_{1}, r_{2}\right)$ and divide the segment $[x, y]$ into $n$ equal parts. Then inequality $(2.2 .22)$ is applicable to neighboring dividing points. This yields the estimate

$$
|\varphi(x)-\varphi(y)| \leq 4 n C_{0} M|x-y|^{\mu}
$$

completing the proof of the lemma.

\subsection{Lipschitz Maps and Domains}

We say that a homeomorphic map $\widetilde{x}=\alpha(x)$ of a set $G \subseteq \mathbb{R}^{k}$ onto a set $\widetilde{G} \subseteq \mathbb{R}^{s}$ is a Lipschitz map if it and its inverse map satisfy the Lipschitz condition. In terms of the constant $M=\max \left([\alpha]_{1},\left[\alpha^{-1}\right]_{1}\right)$, this condition takes the form of the two-sided estimate

$$
M^{-1}|x-y| \leq|\alpha(x)-\alpha(y)| \leq M|x-y|, \quad x, y \in G .
$$

If this constant is to be indicated explicitly, then we say that $\alpha$ is an M-Lipschitz map.

According to the definition given by (2.1.4), the estimate (2.3.1) is extended to the distance from a point to a set: if $F \subseteq \bar{G}$, then

$$
M^{-1} d(x, F) \leq d[\alpha(x), \alpha(F)] \leq M d(x, F) .
$$

In the same way, if a map $B(a, r)=\{|x-a| \leq r\}$ is contained in $G$, then

$$
B[\alpha(a), r / M] \subseteq \alpha[B(a, r)] \subseteq B[\alpha(a), M r] .
$$

Indeed, if $|\alpha(x)-\alpha(a)| \leq r / M$, then, by virtue of (2.3.1), we have the inequalities $|x-a| / M \leq$ $|\alpha(x)-\alpha(a)| \leq r / M$ implying that $\alpha(x) \in \alpha[B(a, r)]$. The second inclusion of (2.3.3) is considered in the same way.

In the same way, we verify that the image $\widetilde{\Gamma}=\alpha(\Gamma)$ of a rectifiable arc $\Gamma$ is a rectifiable arc and

$$
M^{-1} l(\Gamma) \leq l(\widetilde{\Gamma}) \leq M l(\Gamma) .
$$

Indeed, by definition, $\Gamma$ admits a parametrization $\gamma: I \rightarrow \Gamma$, which is a bounded-variation vector-function. Then the vector-function $\gamma_{1}=\alpha \circ \gamma$ belongs to the same type. Really, due to (2.3.1), for any point collection $t_{1}, t_{2}, \ldots, t_{n}$ of the segment $I$ such that $t_{1}<t_{2}<\ldots<t_{n}$, the sum $\left|\gamma_{1}\left(t_{1}\right)-\gamma_{1}\left(t_{2}\right)\right|+$ $\ldots+\left|\gamma_{1}\left(t_{n-1}\right)-\gamma_{1}\left(t_{n}\right)\right|$ does not exceed

$$
M\left(\left|\gamma\left(t_{1}\right)-\gamma\left(t_{2}\right)\right|+\ldots+\left|\gamma\left(t_{n-1}\right)-\gamma\left(t_{n}\right)\right|\right) \leq M l(\Gamma) .
$$

Hence, the arc $\Gamma_{1}$ is rectifiable and $l\left(\Gamma_{1}\right) \leq M l(\Gamma)$. Since the $\operatorname{arcs} \Gamma$ and $\Gamma_{1}$ can be interchanged, it follows that (2.3.4). 
This implies that uniformly connected sets are invariant under Lipschitz transformations.

A bounded domain $D$ is called a Lipschitz domain if for any point $a$ from $\partial D$ there exist a neighborhood $V(a)$ and a Lipschitz map $\alpha$ from $V$ onto the ball $\widetilde{V}=\{|y|<r\}$ such that

$$
\alpha(V \cap D)=\left\{y \in \widetilde{V}, y_{k}>0\right\}, \quad \alpha(V \cap \partial D)=\left\{y \in \widetilde{V}, y_{k}=0\right\} .
$$

For example, if a Cartesian coordinate system exists in a neighborhood of any point $a$ from $\partial D$ such that its origin is the point $a$ and the boundary $D$ in this neighborhood is the graph $x_{k}=f\left(x^{\prime}\right)$, $x^{\prime}=\left(x_{1}, \ldots, x_{k-1}\right) \in G$, of a real function $f$ from $C^{0,1}(G)$, then $D$ is a Lipschitz domain. This follows from the fact that the transformation $\alpha\left(x^{\prime}, x_{k}\right)=\left(x^{\prime}, x_{k}-f\left(x^{\prime}\right)\right)$ of the set $G \times \mathbb{R}$ onto itself is a Lipschitz map. In particular, finite convex domains are Lipschitz domains. If the function $f$ from this definition is continuously differentiable, then we say that $D$ is a domain with a smooth boundary.

Theorem 2.3.1. Lipschitz domains are uniformly connected.

Proof. Each domain $D$ is linearly connected. To any pair $x, y$ of its points, one can assign the value

$$
l(x, y ; D)=\inf l\left(\Gamma_{x, y}\right),
$$

where the infimum is taken over all rectifiable $\operatorname{arcs} \Gamma_{x, y} \subseteq D$. It is easy to verify that this function of the variables $x$ and $y$ satisfies all three distance axioms. It is called the inner metric of the domain $D$. Inequality (2.1.9) defining the uniform connectedness is equivalent to the estimate $l(x, y ; D) \leq M|x-y|$ for this metric. This estimate and the triangle inequality imply that

$$
\left|l(x, y)-l\left(x_{0}, y\right)\right| \leq l\left(x, x_{0}\right) \leq M\left|x-x_{0}\right| .
$$

Therefore, the function $l(x, y)$ is continuous with respect to both variables.

From the definition given by (2.3.5), it follows that the domain $G=D \cap V(a)$ is uniformly connected for any point $a$ from $\partial D$. If $a \in D$, then there exists a ball $G \subseteq D$ centered at this point. Obviously, it is also uniformly connected.

Assume that the domain $D$ is not uniformly connected. Then, according to the definition given by (2.1.9), there exist sequences of points $x_{n}$ and $y_{n}$ from $D$ such that

$$
l\left(x_{n}, y_{n} ; D\right) \geq n\left|x_{n}-y_{n}\right|, \quad n=1,2, \ldots
$$

Without loss of generality, one can assume that there exist points $a$ and $b$ from $\bar{D} \cup \infty$ such that the sequences $x_{n}$ and $y_{n}$ converge to them respectively. Obviously, due to (2.3.6), the case where $a \neq b$ is impossible. However, the case where $a=b \in \bar{D}$ is also impossible. To prove that, we assume that such a case takes place. Then there exists a neighborhood $G$ such that the condition (2.3.6) is not satisfied in it, but the points $x_{n}$ and $y_{n}$ lie in it provided that $n$ is sufficiently large.

in [74], the notion of Lipschitz domains is considered in detail: it is used to construct extension operators from $D$ to $\mathbb{R}^{k}$ for functions in the whole scale of Sobolev spaces. For Lipschitz domains, it is easy to extend functions, preserving their Hölder property.

Theorem 2.3.2. Let $D$ be a Lipschitz domain. Then there exist bounded extension operators $p$ and $P$ acting from $C^{\mu}(\partial D)$ and $C^{\mu}(\bar{D})$ (respectively) to $C^{\mu}\left(\mathbb{R}^{k}\right)$, i.e., operators such that $(p \varphi)(x)=\varphi(x)$, $x \in \partial D$, and $(P \varphi)(x)=\varphi(x), x \in D$.

Proof. First, we assume that a function $\psi\left(x^{\prime}\right)$ of variables $x^{\prime}=\left(x_{1}, \ldots, x_{k-1}\right)$ belongs to the class $C^{\mu}$ in a ball $B_{0}=\left\{\left|x^{\prime}\right|<r\right\}$ and vanishes in a neighborhood of its boundary. Let $\chi(t)$ belong to $C_{0}^{\infty}(B)$, where $B=\{|x|<r\}$, and is identically equal to 1 in a neighborhood of the origin. Then the operator $(q \psi)(x)=\chi(x) \psi\left(x^{\prime}\right), x \in B$, boundedly maps $C^{\mu}\left(B^{\prime}\right)$ into $C^{\mu}(B)$ and $(q \psi)(x)=0$ in a neighborhood of $\partial B$.

Cover the boundary $\partial D$ of the domain $D$ by a finite set $V_{1}, \ldots, V_{n}$ of neighborhoods mentioned in the definition given by (2.3.5). Consider the corresponding Lipschitz maps $\alpha_{i}$ of the sets $V_{i}$ onto 
the map $B$ (or its exterior). Let the partition $\left(\chi_{i}\right)$ of unity be defined by this covering according to Lemma 1.8.2. Then the operator $p_{i}$ acting according to the relation

$$
\left(p_{i} \varphi\right) \circ \alpha_{i}^{-1}=q\left[\left(\chi_{i} \varphi\right) \circ \alpha_{i}^{-1}\right]
$$

boundedly maps $C^{\mu}(\partial D)$ into $C^{\mu}\left(V_{i}\right)$ and $p_{i} \varphi=0$ in $\partial V_{i}$. Therefore, extending $p_{i} \varphi$ by zero, one can treat $p_{i}$ as a bounded operator from $C^{\mu}(\partial D)$ to $C^{\mu}\left(\mathbb{R}^{k}\right)$. If $x \in V_{i} \cap \partial D, x=\alpha_{i}\left(y^{\prime}\right)$, and $y^{\prime} \in B_{0}$, then

$$
\left(p_{i} \varphi\right)(x)=\chi(y)\left(\chi_{i} \varphi\right)\left(y^{\prime}\right)=\chi\left[\alpha_{i}(x)\right] \chi_{i}(x) \varphi(x) .
$$

Obviously, the function $\chi$ from the definition of the operator $q$ can be selected such that the relation $\chi_{i}(x) \chi\left[\alpha_{i}(x)\right]=\chi_{i}(x), x \in V_{i} \cap \partial D$, is valid for any $i$. Since $\sum \chi_{i}=1$, it follows that the operator $p=\sum p_{i}$ is an extension operator.

Consider the extension of functions from a domain $D$. Let a function $\psi$ belong to $C^{\mu}$ in a semiball $G=\left\{|x| \leq r, x_{k}>0\right\}$ and vanish in a neighborhood of the manifold $|x|=r$. Then the operator $(Q \psi)\left(x^{\prime}, x_{k}\right)=\chi(x) \psi\left(x^{\prime},\left|x_{k}\right|\right)$ boundedly maps $C^{\mu}(G)$ into $C^{\mu}(B)$.

Further reasoning is the same as above. Consider an open covering $V_{1}, \ldots, V_{m}, m \geq n$, of a closed domain $D$, where the sets $V_{1}, \ldots, V_{n}$, cover the boundary and mean the same as above, while any other set $V_{j}$ is either a ball located in $D$ together with its closure or the exterior of a ball (in the case where the domain $D$ is infinite). Let the partition $\left(\chi_{i}\right)$ of unity be defined by this covering according to Lemma 1.8.2. Introduce the operators $P_{i}, 1 \leq i \leq m$, as follows: for $i \leq n$, they are defined similarly to (2.3.7), while $P_{i} \varphi=\chi_{i} \varphi$ otherwise. As above, we immediately verify that it is possible to select a function $\chi$ in the definition $Q$ such that the operator $P \varphi=\sum P_{i} \varphi$ satisfies the necessary requirements.

Theorem 2.3.2 is a special case of the general Whitney result about the extension of a function from any compact set such that its Hölder smoothness is preserved (see [46]).

In Lipschitz domains, Theorem 2.2.1 can be substantially strengthened.

Lemma 2.3.1. Let $D$ be a Lipschitz domain. Then, under the assumptions of Theorem 2.2.1, the claimed sequence $\left\{\varphi_{n}\right\}$ can be selected from the class of functions from $C_{0}^{\infty}\left(\mathbb{R}^{k}\right)$ vanishing in a neighborhood of $F$.

Proof. Let a function $\varphi(x)$ from $C^{\nu}(D)$ be equal to zero on a closed subset $F$ of $\bar{D}$. Then the function $P \varphi$ from $C^{\nu}\left(\mathbb{R}^{k}\right)$ possesses the same property. Fix $\nu_{1}$ such that $\nu_{1}<\nu$. Then fix $\mu$ such that $\mu<\nu_{1}$. Apply Theorem 2.2.1 with $\nu_{1}$ instead of $\mu$ and $\mathbb{R}^{k}$ instead of $G$ to the function $P \varphi$. Thus, for any fixed positive $\delta$ there exists a function $\psi$ from $C^{\nu_{1}}\left(\mathbb{R}^{k}\right)$ vanishing in a neighborhood $F$ and satisfying the inequality $|\varphi-\psi|_{\nu_{1}} \leq \delta$. Then apply Lemma 2.2.1 to the function $\psi$ to find a positive $\varepsilon$ such that $\left|\psi-T_{\varepsilon} \psi\right|_{\mu} \leq \delta$. Obviously, $T_{\varepsilon} \psi \in C_{0}^{\infty}\left(\mathbb{R}^{k}\right)$. Arguing as in the proof of Lemma 1.8.1, we find that the function $T_{\varepsilon} \psi$ vanishes in a neighborhood $F$ provided that $\varepsilon$ is sufficiently small. It remains to note that previous estimates combined with (2.2.3) yield the inequality $\left|\varphi-T_{\varepsilon} \psi\right|_{\mu} \leq 3 \delta$.

One more important property of Lipschitz domains is provided by the next assertion.

Lemma 2.3.2. Let $D$ be a Lipschitz domain and $\varphi \in C^{1}(\bar{D})$. Then for any positive $\varepsilon$ there exists a positive $\delta$ such that

$$
\left|\varphi(x)-\varphi(y)-\varphi^{\prime}(z)(x-y)\right| \leq \varepsilon|x-y|
$$

for any points $x, y$, and $z$ from $D$ such that $|x-z| \leq \delta$ and $|y-z| \leq \delta$.

Proof. It suffices to prove the local variant of the lemma, i.e., to prove that for any point $a$ from $\bar{D}$ there exists its neighborhood $V$ such that inequality (2.3.8) holds for any triple $x, y, z$ of points from $D \cap V$ such that $|x-y| \leq \delta$ and $|z-y| \leq \delta$.

Indeed, let this local variant takes place, but the claim of the theorem in the whole domain $D$ is not fulfilled. Then there exist a positive $\varepsilon$ and sequences $\left\{x_{n}\right\},\left\{y_{n}\right\}$, and $\left\{z_{n}\right\}$ in $D$ such that 
$\left|x_{n}-z_{n}\right| \leq 1 / n$ and $\left|y_{n}-z_{n}\right| \leq 1 / n$, but

$$
\left|\varphi\left(x_{n}\right)-\varphi\left(y_{n}\right)-\varphi^{\prime}\left(z_{n}\right)\left(x_{n}-y_{n}\right)\right| \geq \varepsilon\left|x_{n}-y_{n}\right| \text {. }
$$

By virtue of the compactness of $\bar{D}$, without loss of generality, one can assume that all the three sequences converge to a point $a$ from $\bar{D}$. Then, beginning from sufficiently large $n$, they belong to the neighborhood $V$ of this point, defined at the local variant of the lemma, and the distances $\left|x_{n}-z_{n}\right|$ and $\left|y_{n}-z_{n}\right|$ do not exceed $\delta$ corresponding to the neighborhood $V$. Thus, inequality (2.3.9) contradicts the claim of the local variant of the lemma.

Pass to the proof of the local variant of the lemma. For any point $a$ from $\bar{D}$ there exists its neighborhood $V$ such that there exists a Lipschitz map $\alpha$ of the the domain $G=D \cap V$ onto a convex domain $\widetilde{G}$. For points $a$ from $\partial D$, this follows from the definition of Lipschitz domains. If $a \in D$, then a ball of a sufficiently small radius centered at $a$ can be taken as $G$, while the identity map can be taken as $\alpha$. Let the considered map be an $M$-Lipschitz map, i.e., satisfy the condition (2.3.1) with the constant $M$. Since the vector-function $\alpha^{\prime}$ is uniformly continuous on the compact set $\bar{G}$, it follows that there exists a positive $\delta_{0}$ such that

$$
\left|\varphi^{\prime}(x)-\varphi^{\prime}(y)\right| \leq \varepsilon / M \quad \text { for } \quad|x-y| \leq \delta_{0} .
$$

Assuming that $\delta=\delta_{0} / M^{2}$, consider points $x, y$, and $z$ from $G$ such that $|x-y| \leq \delta$ and $|z-y| \leq \delta$. By virtue of the convexity, the segment $[\widetilde{x}, \widetilde{y}]$ with endpoints $\widetilde{x}=\alpha(x)$ and $\widetilde{y}=\alpha(y)$ is contained in $\widetilde{G}$. By virtue of (2.3.1), the distances $|\widetilde{x}-\widetilde{z}|$ and $|\widetilde{y}-\widetilde{z}|$ do not exceed $\delta_{0} / M$. Let a rectifiable arc $\Gamma_{x, y} \subseteq G$ be the preimage of this segment under the map $\alpha$. Then $|\alpha(t)-\alpha(z)| \leq \delta_{0} / M$ for any point $t$ from $\Gamma_{x, y}$. Then, taking into account (2.3.1), we deduce that

$$
|t-z| \leq \delta_{0} \quad \text { for any } t \text { from } \Gamma_{x, y} .
$$

Consider the function $\psi(x)=\varphi(x)-\varphi^{\prime}(z)(x-y)$, where $y$ and $z$ are fixed. For this function, we have the relation $\psi^{\prime}(x)=\varphi^{\prime}(x)-\varphi^{\prime}(z)$. Then, by virtue of Theorem 2.2.2, the following inequality holds:

$$
\left|\varphi(x)-\varphi(y)-\varphi^{\prime}(z)(x-y)\right|=|\psi(x)-\psi(y)| \leq \max _{t \in \Gamma_{x, y}}\left|\varphi^{\prime}(t)-\varphi^{\prime}(z)\right| l\left(\Gamma_{x, y}\right) .
$$

By virtue of (2.3.4), the length $l\left(\Gamma_{x, y}\right)$ does not exceed $M|x-y|$. Combining this with (2.3.10)-(2.3.11), we obtain inequality (2.3.8).

If a sequence of functions $\varphi_{n}$ from $C^{1}(\bar{D}), n=1,2, \ldots$, converges to $\varphi$ with respect to the norm of the space $C^{1}(\bar{D})$, then there exists a value of $\delta$ such that the lemma is valid for all $n$. This follows from the fact that all functions $\varphi_{n}$ can be selected to satisfy the condition (2.3.10).

Let a map $\alpha$ be a continuously differentiable homeomorphism $D \rightarrow \widetilde{D}$ of open subsets of $\mathbb{R}^{k}$ such that the inverse map is also continuously differentiable. Such maps are called diffeomorphisms. Due to the chain rule, its Jacobi matrix $\alpha^{\prime}=\mathcal{D} \alpha$ is linked with the similar matrix $\mathcal{D} \beta$ of the inverse map $\beta=\alpha^{-1}$ by the relation $[(\mathcal{D} \beta) \circ \alpha] \mathcal{D} \alpha=1$, where 1 denotes the unit $k \times k$-matrix. Therefore, $(\operatorname{det} \mathcal{D} \alpha)(x) \neq 0$ provided that $x \in D$. Then, from Theorem 2.2.2, we conclude that, for any compact subset $K$ of $D$, the map $\alpha$ treated as a map of $K$ onto the compact set $\widetilde{K}=\alpha(K) \subseteq \widetilde{D}$ is a Lipschitz map.

From the inverse map theorem, it follows that if a $k$-vector-function $\alpha$ from $C^{1}(D)$ satisfies the condition $(\operatorname{det} \mathcal{D} \alpha)(a) \neq 0$ at a fixed point $a$ of $D$, then there exists a subset $D_{0}$ of $D$ such that $D_{0}$ contains this point, there exists a domain $\widetilde{D}_{0}$ such that $\alpha$ is a homeomorphism of $D_{0}$ onto $\widetilde{D}_{0}$, and the inverse map is continuously differentiable in the domain $\widetilde{D}_{0}$. Thus, if the specified condition is satisfied everywhere in $D$, then $\alpha$ is a locally Lipschitz map. For $s \geq k$, this fact can be extended to continuously differentiable $s$-vector-functions in closed Lipschitz domains.

Lemma 2.3.3. Let $s \geq k$ and $\alpha$ be a continuously differential one-to-one s-vector-function in a closed Lipschitz domain $\bar{D} \subseteq \mathbb{R}^{k}$. Then, if

$$
\operatorname{rang}(\mathcal{D} \alpha)(x)=k, x \in \bar{D},
$$


where $\mathcal{D} \alpha$ is the Jacobi matrix, then $\alpha$ is a Lipschitz map. Conversely, if $\alpha$ is a Lipschitz map, then (2.3.12) holds.

Proof. By virtue of Theorems 2.3.1 and 2.2.2, the function $\alpha$ satisfies the Lipschitz condition. Therefore, under the assumption that the map $\alpha$ satisfies (2.3.5), it suffices to prove the left-hand side of the two-sided inequality (2.3.1). By condition, $(\mathcal{D} \alpha)(x) \xi \neq 0$ for any unit vector $\xi$ from $\mathbb{R}^{k}$. Let $\Omega$ denote the unit sphere in $\mathbb{R}^{k}$. Then the function $|(\mathcal{D} \alpha)(x) \xi|$ is continuous on $\bar{D} \times \Omega$ and vanishes at no point of this set. Therefore, there exists a positive constant $m$ such that

$$
\mid \mathcal{D} \alpha)(x) \xi|\geq 2 m| \xi \mid
$$

for any $\xi$ from $\mathbb{R}^{k}$ and any $x$ from $\bar{D}$.

According to Lemma 2.3.2, there exists a positive $\delta$ such that

$$
|\alpha(x)-\alpha(y)-(\mathcal{D} \alpha)(y)(x-y)| \leq m|x-y|
$$

provided that $|x-y| \leq \delta$. Taking into account (2.3.13), this implies that

$$
|\alpha(x)-\alpha(y)| \geq|(\mathcal{D} \alpha)(y)(x-y)|-m|x-y| \geq m|x-y|
$$

provided that $|x-y| \leq \delta$. It remains to note that the function $f(x, y)=|\alpha(x)-\alpha(y)| /|x-y|$ is continuous on the compact set $\{(x, y) \in \bar{D} \times \bar{D},|x-y| \geq r\}$ and, therefore, is bounded from below by a positive constant.

Conversely, let $\alpha$ from $C^{1}(\bar{D})$ be an $M$-Lipschitz map. By virtue of Theorem 2.3.2, one can assume that there exists an open subset $D^{1}$ of $\bar{D}$ such that the function $\alpha$ is continuously differentiable in $D^{1}$. Then, for any fixed $a$ from $\bar{D}$, the relation

$$
\alpha(x)-\alpha(a)-(\mathcal{D} \alpha)(a)(x-a)=|x-a| \sigma(x), \quad x \in D^{1},
$$

where $\sigma(x)$ is a vector-function such that $\sigma(x) \rightarrow 0$ as $x \rightarrow a$, holds. As above, we use the estimate

$$
|(\mathcal{D} \alpha)(a)(x-a)| \geq|\alpha(x)-\alpha(a)|-|\sigma(x)||x-a| .
$$

Here, we assume that $x=a+r \xi, r>0$. Then we fix $\xi$ from $\Omega$ and pass to the limit as $r \rightarrow 0$. Then, due to (2.3.1), we arrive at the estimate (2.3.13) with $2 m=1 / M$, which is equivalent to (2.3.12).

\subsection{Smooth Surfaces}

Consider main notions related to $(k-1)$-dimensional surfaces in $\mathbb{R}^{k}$ (curves in $\mathbb{R}^{2}$ ). Let $G$ be a finite Lipschitz domain in $\mathbb{R}^{k-1}$. Let $\gamma$ from $C^{1}(\bar{G})$ valued in $\mathbb{R}^{k}$ be a one-to-one vector-function such that its Jacobi matrix $(\mathcal{D} \gamma)(s)$ satisfies the following condition:

$$
\operatorname{rang}(\mathcal{D} \gamma)(s)=k-1, \quad s \in \bar{G} .
$$

The columns of this $k \times(k-1)$-matrix are vectors

$$
\frac{\partial \gamma}{\partial s_{i}}, \quad 1 \leq i \leq k-1
$$

and the condition (2.4.1) means that these vectors are linearly independent. The image $\Gamma=\gamma(G)$ is called a smooth surface (with boundary) and the map $\gamma$ itself is called its smooth parametrization. We say that a surface $\Gamma$ belongs to a class $C^{1, \mu}$ if it admits a parametrization $\gamma$ from this class. The points $\gamma(s), s \in \partial G$, form the boundary $\partial \Gamma$ of this surface. Other its points are called its interior points. Note that, by virtue of Lemma 2.3.3, the smooth parametrization $\gamma: \bar{G} \rightarrow \Gamma$ is a Lipschitz map. In particular, the operator $\varphi \rightarrow \varphi \circ \gamma$ is a bounded and invertible operator from $C^{\mu}(\Gamma)$ to $C^{\mu}(G)$.

In the two-dimensional case (i.e., for $k=2), \bar{G}$ is a segment of the line $\mathbb{R}$. Respectively, $\Gamma$ is called a smooth arc and the endpoints of the segment are mapped into the endpoints of this arc. The vector $\gamma^{\prime}(s)$ determines the line tangential to $\Gamma$ at the point $t=\gamma(s)$. In the general case (i.e., for $k \geq 2$ ), 
the vectors $\partial \gamma / \partial s_{j}$ form a base of the plane tangential to this surface at the point $\gamma(s)$. The normal to this plane can be described as follows. Consider the vector

$$
m=\left(m_{1}, \ldots, m_{k}\right), \quad m_{j}=(-1)^{j+k} \operatorname{det} M_{j},
$$

where the $(k-1) \times(k-1)$-matrix $M_{j}$ is the matrix $\mathcal{D} \gamma$ without its $j$ th row.

If we take the matrix $(\mathcal{D} \gamma)(s)$, add a vector $\xi$ from $\mathbb{R}^{k}$, treated as its $k$ th column, and expand the determinant of this matrix with respect to its $k$ th column, then we obtain the sum $\sum_{1}^{k} m_{j}(s) \xi_{j}$. If we substitute $\xi=\partial \gamma / \partial s_{i}$ in this matrix, then we obtain the zero determinant. Thus, the vector $m(s)=\left(m_{1}(s), \ldots, m_{k}(s)\right)$ is orthogonal to the plane tangential at the point $\gamma(s)$. Hence, the unit vector normal to $\Gamma$ at this point can be defined by the relation $n[\gamma(s)]=m(s) /|m(s)|$.

Applying the parametrization, one can introduce the surface integrating: the class of summable functions $\varphi$ is defined by the condition $\varphi \circ \gamma \in L(G)$ and, by definition,

$$
\int_{\Gamma} \varphi(y) d_{k-1} y=\int_{G} \varphi[\gamma(s)]|m(s)| d_{k-1} s,
$$

where the vector $m$ is defined by (2.4.2). Respectively, if $G_{0}$ is a measurable subset of $G$, then the relation

$$
\operatorname{mes}\left[\gamma\left(G_{0}\right)\right]=\int_{G_{0}}|m(s)| d s
$$

defines the surface Lebesgue measure of the set $\gamma\left(G_{0}\right) \subseteq \Gamma$. In other words, $d_{k-1} y=|m(s)| d_{k-1} s$ is the area element on the surface. It is easy to verify that all above definitions do not depend on the choice of the parametrization. All main properties of integrals, described in Sec. 1.8, are also extended to the case considered.

A basic example of a smooth parametrization is the vector-function $\gamma(s)=(s, f(s))$, where $f$ is a scalar function from $C^{1}(\bar{G})$. The corresponding surface $\Gamma=\gamma(G)$ is the graph of the function $f$. The next theorem shows that any smooth surface $\Gamma$ has a similar structure in a neighborhood of any its interior point $a$. Let $u=\left(\widetilde{u}, u_{k}\right)$ from $\mathbb{R}^{k}$ be a local system of Cartesian coordinates with the origin at the point $a$, such that its axis $u_{k}$ is directed along the normal $n(a)$. In this coordinate system, introduce neighborhoods of the point $a$ of the kind

$$
C_{\rho}(a)=\left\{|\widetilde{u}| \leq \rho,\left|u_{k}\right| \leq 2 \rho\right\} .
$$

Theorem 2.4.1. Let $\Gamma$ be a smooth surface with boundary, determined by a parametrization $\gamma: G \rightarrow$ $\Gamma$, and a compact set $K$ be a subset of $\Gamma \backslash \partial \Gamma$. Then there exists a positive $\rho_{0}=\rho_{0}(\Gamma, K)$ such that, for any point a from $K$, the intersection of $\Gamma$ with the neighborhood $C_{\rho}(a), \rho \leq \rho_{0}$, in the local coordinate system is described by the equation $u_{k}=f(\widetilde{u})$ in the ball $B_{\rho}=\{|\widetilde{u}| \leq \rho\}$, where $f$ is a continuously differentiable function such that

$$
f(0)=0, \quad f^{\prime}(0)=0, \quad\left|f^{\prime}\right|_{0} \leq 1 .
$$

If $\Gamma \in C^{1, \mu}$, then $f \in C^{1, \mu}\left(B_{\rho}\right)$ and the seminorm $\left[f^{\prime}\right]_{\mu}$ depends only on $\left[\gamma^{\prime}\right]_{\mu}$.

Proof. According to Lemma 2.3.3, the parametrization $\gamma$ is an $M$-Lipschitz map. Taking this into account, assign

$$
2 M r=d(K, \partial \Gamma),
$$

where $d$ is the distance between the compact set $K$ and the boundary $\partial \Gamma=\gamma(\partial G)$.

In notation (2.4.2), add the column $m(s)$ to the rectangular $k \times(k-1)$-matrix $(\mathcal{D} \gamma)(s)$ to obtain a square matrix and denote the latter one by $A(s)$. Obviously, its $\operatorname{determinant} \operatorname{det} A(s)$ is equal to 
$|m(s)|^{2}$. Therefore, the inverse matrix-function $A^{-1}(s)$ belongs to $C^{1}(\bar{G})$. By virtue of the uniform continuity of $\mathcal{D} \gamma$ and Lemma 2.3.2, there exists a positive $\delta$ such that

$$
\begin{gathered}
\left|A^{-1}\left(s_{0}\right)\left[(\mathcal{D} \gamma)\left(s_{1}\right)-(\mathcal{D} \gamma)\left(s_{2}\right)\right]\right| \leq 1 / 2 \quad \text { for } \quad\left|s_{1}-s_{2}\right| \leq 2 \delta, \\
\left|A^{-1}\left(s_{0}\right)\left[\gamma\left(s_{1}\right)-\gamma\left(s_{2}\right)-(\mathcal{D} \gamma)^{\prime}\left(s_{0}\right)\left(s_{1}-s_{2}\right)\right]\right| \leq 1 / 2 \text { for }\left|s_{j}-s_{0}\right| \leq \delta .
\end{gathered}
$$

Since the three-variable function $A^{-1}\left(s_{0}\right)\left[\gamma\left(s_{1}\right)-\gamma\left(s_{2}\right)\right]\left|s_{1}-s_{2}\right|^{-1}$ does not vanish on the compact subset $\bar{G} \times \bar{G} \times \bar{G}$ determined by the inequality $\left|s_{1}-s_{2}\right| \geq \delta$ and is continuous, one can introduce a positive constant $q$ such that

$$
0<q \leq 1, \quad 2 q \leq \min _{s_{0},} \frac{\mid A^{-1}\left(s_{0}\right)\left[\gamma\left(s_{1}\right)-\gamma\left(s_{2}\right) \mid\right.}{\left|s_{1}-s_{2}\right| \geq \delta}
$$

Assuming that $r, \delta$, and $q$ are given, define $\rho_{0}$ as follows:

$$
\rho_{0}=\min (r, \delta, q) \text {. }
$$

Applying the change of variables

$$
\left(\widetilde{u}, u_{k}\right)=A^{-1}\left(s_{0}\right)(x-a)
$$

in a neighborhood of the point $a=\gamma\left(s_{0}\right)$ belonging to $K$, we obtain the local Cartesian coordinate system mentioned above. Indeed, (2.4.9) is equivalent of the relation $x-a=\gamma^{\prime}\left(s_{0}\right) \widetilde{u}+m\left(s_{0}\right) u_{k}$ showing that the axis $u_{k}$ is directed along the normal $n(a)$. Taking this into account, consider the vector-function

$$
\left(\widetilde{\alpha}(s), \alpha_{k}(s)\right)=A^{-1}\left(s_{0}\right)\left[\gamma(s)-\gamma\left(s_{0}\right)\right]
$$

in the ball $B_{0}=\left\{\left|s-s_{0}\right| \leq 2 r\right\}$. According to (2.3.2) and (2.4.5), this ball is contained in $G$ and inequalities (2.4.6) are satisfied for all $s_{1}$ and $s_{2}$ from $B_{0}$. Note that

$$
\widetilde{\alpha}^{\prime}\left(s_{0}\right)=1, \quad \alpha_{k}^{\prime}\left(s_{0}\right)=0,
$$

where 1 denotes the unit $(k-1) \times(k-1)$-matrix. Hence,

$$
A^{-1}\left(s_{0}\right)\left[\gamma^{\prime}\left(s_{1}\right)-\gamma^{\prime}\left(s_{2}\right)\right]=\left[\widetilde{\alpha}^{\prime}\left(s_{1}\right)-\widetilde{\alpha}^{\prime}\left(s_{2}\right), \alpha_{k}^{\prime}\left(s_{1}\right)-\alpha_{k}^{\prime}\left(s_{2}\right)\right]
$$

and

$$
A^{-1}\left(s_{0}\right)\left[\gamma^{\prime}\left(s_{1}\right)-\gamma^{\prime}\left(s_{2}\right)\right]=\left[\widetilde{\alpha}\left(s_{1}\right)-\widetilde{\alpha}\left(s_{2}\right)-\left(s_{1}-s_{2}\right), \alpha_{k}\left(s_{1}\right)-\alpha_{k}\left(s_{2}\right)\right] .
$$

Then, by virtue of (2.4.6), we conclude that

$$
\left|s_{1}-s_{2}\right| / 2 \leq\left|\widetilde{\alpha}\left(s_{1}\right)-\widetilde{\alpha}\left(s_{2}\right)\right| \leq 2\left|s_{1}-s_{2}\right|
$$

and, in particular,

$$
1 / 2 \leq\left|\alpha_{k}^{\prime}(s)\right| \leq 2, \quad s \in B_{0} .
$$

Therefore, $\widetilde{\alpha}$ is a Lipschitz map taking the ball $B_{0}$ into a domain $\widetilde{B}_{0}$ containing the point $\widetilde{u}=0$. Due to Lemma 2.3.3, it diffeomorphically maps $B_{0}$ onto $\widetilde{B}_{0}$.

Assign

$$
\beta=\widetilde{\alpha}^{-1}, \quad f(\widetilde{u})=\alpha_{k}[\beta(\widetilde{u})] .
$$

By virtue of (2.4.12), the derivative $\beta^{\prime}$ satisfies the inequalities $|\xi| / 2 \leq\left|\beta^{\prime}(\widetilde{u}) \xi\right| \leq 2|\xi|$ and $\widetilde{u} \in \widetilde{B}_{0}$. Combining this with (2.4.13), we deduce the estimate $\left|f^{\prime}\right|_{0} \leq 1$ for the derivative of the function $f$. Then, taking into account (2.4.11), we see that the conditions (2.4.5) are satisfied for this function.

Thus, the vector-function $\widetilde{\gamma}(\widetilde{u})=(\widetilde{u}, f(\widetilde{u})), \widetilde{u} \in \widetilde{B}_{0}$, is a smooth parametrization of the surface $\gamma\left(B_{0}\right)$. In other words, this surface is the graph of the function $u_{k}=f(\widetilde{u}), \widetilde{u} \in \widetilde{B}_{0}$, in the local coordinate system. By virtue of (2.4.12), the distance between the point $\tilde{u}=0$ and the boundary of the domain $\widetilde{B}_{0}$ is not less than $r$. Thus, taking into account (2.4.8), we conclude that the ball $B_{\rho}=\{|\widetilde{u}| \leq \rho\}$ is contained in the domain $\widetilde{B}_{0}$. Thus, it remains to consider the function $f$ in this ball and verify that the intersection of the surface $\Gamma$ with neighborhood (2.4.4) is the graph of this function. 
Assume that, in addition to the point $(\widetilde{u}, f(\widetilde{u}))$, another point $\left(\widetilde{u}, u_{k}\right)$ of the surface $\Gamma$ lies inside $C_{\rho}(a)$. Then

$$
(\widetilde{u}, f(\widetilde{u}))=A^{-1}\left(s_{0}\right)\left[\gamma(s)-\gamma\left(s_{0}\right)\right], \quad\left(\widetilde{u}, u_{k}\right)=A^{-1}\left(s_{0}\right)\left[\gamma\left(s_{*}\right)-\gamma\left(s_{0}\right)\right]
$$

where $s \in B_{0}$ and $s_{*} \notin B_{0}$. Therefore, $\left|s_{*}-s_{0}\right|>2 r$. By virtue of (2.4.12), we have the inequality $\left|s-s_{0}\right| \leq \rho / 2$ implying the inequality $\left|s-s_{*}\right|>2 r-\rho / 2$. On the other hand, the relation

$$
\left(\widetilde{u}, f(\widetilde{u})-u_{k}\right)=A^{-1}\left(s_{0}\right)\left[\gamma(s)-\gamma\left(s_{*}\right)\right]
$$

combined with (2.4.4) and (2.4.7) shows that

$$
3 \rho \geq\left|f(\widetilde{u})-u_{k}\right|=\left|A^{-1}\left(s_{0}\right)\left[\gamma(s)-\gamma\left(s_{*}\right)\right]\right| \geq 2 q\left|s-s_{*}\right|
$$

(we take into account the fact that the function $f$ satisfies the inequality $|f(\widetilde{u})| \leq|\widetilde{u}|, \widetilde{u} \in B_{\rho}$, by virtue of (2.4.5)). Thus, $3 \rho>2 q(2 r-\rho / 2)$ and, therefore, $4 \rho \geq(3+q) \rho>4 q r$, which contradicts (2.4.8).

It remains to consider the last claim of the theorem. If the parametrization $\gamma$ belongs to $C^{1, \mu}(\bar{G})$, then it is obvious that the functions $\widetilde{\alpha}$ and $\alpha_{k}$ from (2.4.10) belong to the same class. Combining the relation $\beta^{\prime} \circ \widetilde{\alpha}=\left(\widetilde{\alpha}^{\prime}\right)^{-1}$ with $(2.4 .12)$, we conclude that the Hölder constant $\left[\beta^{\prime}\right]_{\mu}$ in the ball $|\widetilde{u}| \leq \rho$ is uniformly bounded with respect to $a$ from $K$. Hence, the derivative of the function $f=\alpha_{k} \circ \beta$ possesses the same property.

Let $\Gamma$ be a smooth surface with boundary. Then, due to Theorem 2.4.1, for any point $a$ from $\Gamma \backslash \partial \Gamma$ there exists a positive $\rho$ such that the surface $\Gamma$ decomposes the neighborhood $C_{\rho}(a)$ into two connected components $C_{\rho}^{ \pm}(a)$ defined by the condition $\pm\left[f(\widetilde{u})-u_{k}\right]>0$. They are called the left- and right-hand half-neighborhoods for the signs of minus and plus respectively. These signs depend on the choice of the normal $n(a)$. We say that a domain $D$ is located to the left (to the right) of $\Gamma$ if for any point $a$ of $\Gamma \backslash \partial \Gamma$, the domain $D$ does not intersect right-hand (left-hand) half-neighborhoods of this point provided that $\rho$ are sufficiently small. In this case, it is obvious that $D \cap \Gamma=\varnothing$ and the vector $n(a)$ is the inner (outer) normal with respect to $D$ for any point $a$ from $\bar{D} \cap \Gamma$.

Using Theorem 2.4.1, one can consider the following generalization of Lemma 2.2.2.

Theorem 2.4.2. Let the boundary of a finite domain $D$ contain a smooth surface $\Gamma$ with boundary such that $D$ is located from one side of $\Gamma$. Let a subdomain $D_{0}$ of $D$ be such that $\Gamma_{0}=\bar{D}_{0} \cap \partial D \subseteq \Gamma \backslash \partial \Gamma$. Let a function $\varphi$ be continuously differentiable in the domain $D$ and its gradient derivative $\varphi^{\prime}$ admit an estimate

$$
\left|\varphi^{\prime}(x)\right| \leq M d^{\mu-1}(x, \Gamma), \quad x \in D_{0},
$$

where $M>0$ and $0<\mu \leq 1$. Then $\varphi \in C^{0, \mu}\left(D_{0}\right)$ and $[\varphi]_{\mu} \leq C M$, where $C$ is a positive constant depending only on $\Gamma$ and the distance between the domain $D_{0}$ and $\partial D \backslash \Gamma$ (for any fixed $\mu$ ).

Proof. For definiteness, let the domain $D$ lie to the left of $\Gamma$. Introduce the notation $\Gamma^{\prime}=\partial D \backslash \Gamma$. By assumption, the number $2 r_{0}=d\left(D_{0}, \Gamma^{\prime}\right)$ is positive and one can introduce a compact set $K=\{a \in \Gamma$, $\left.d\left(a, \Gamma^{\prime}\right) \geq r_{0}\right\}$. Obviously, it contains $\Gamma_{0}$. Select the number $\rho_{0}=\rho_{0}(\Gamma, K)$ with respect to $K$ as it is done in Theorem 2.4.1.

Obviously, the number $2 \rho=\min \left(\rho_{0}, r_{0}\right)$ depends (as the set $K$ ) only on the distance between the domain $D_{0}$ and $\Gamma^{\prime}$. Fix such a number $2 \rho$ and consider the neighborhood $C_{\rho}(a)$ of the point $a$ from $K$, mentioned in Theorem 2.4.1. In addition to this neighborhood, introduce the neighborhood $D_{\rho}(a) \subseteq C_{\rho}(a)$ defined by the inequalities $\left|f(\widetilde{u})-u_{k}\right| \leq \rho$ and $|\widetilde{u}|<\rho$ in the local coordinates. As above, let one-sided half-neighborhoods $D_{\rho}^{ \pm}(a)$ be determined by the sign of $f(\widetilde{u})-u_{k}$.

Note that the ball of radius $\rho / \sqrt{2}$ centered at $a$ is contained in this neighborhood. Indeed, it suffices to verify that $|\widetilde{u}|^{2}+|f(\widetilde{u})+\rho|^{2} \leq \rho^{2} / 2$. Since $|f(\widetilde{u})| \leq|\widetilde{u}|$, it follows that this inequality is reduced to the inequality $s^{2}+(\rho-s)^{2} \geq \rho^{2} / 2$ for $0 \leq s \leq \rho$, which is obvious. Taking into account the fact that the domain $D$ is located to the left by assumption, this implies that the intersection of the domain $D$ with the disc $\{|x-a|<\rho / \sqrt{2}\}$ is contained in $D_{\rho}^{+}(a)$. 
Introduce the notation $\Gamma_{2}=\Gamma \cap C_{2 \rho}(a)$. Let us prove that

$$
d(x, \Gamma) \geq d\left(x, \Gamma_{2}\right) / 9, \quad x \in C_{\rho}(a) .
$$

Indeed, the neighborhood $C_{2 \rho}(a)$ is contained in the ball $\{|x-a| \leq 2 \sqrt{5} \rho\}$ and, therefore, $d\left(x, \Gamma_{2}\right) \leq$ $3 \sqrt{5} \rho<9 \rho$. Since the distance between $C_{\rho}(a)$ and $\Gamma \backslash \Gamma_{2}$ is not less than $\rho$, it follows that

$$
d(x, \Gamma)=\min \left[d\left(x, \Gamma_{2}\right), d\left(x, \Gamma \backslash \Gamma_{2}\right)\right] \geq \min \left[\frac{\rho}{d\left(x, \Gamma_{2}\right)}, 1\right] d\left(x, \Gamma_{2}\right),
$$

which yields the estimate (2.4.15).

In local coordinates, the transformation $\alpha(u)=\left(\widetilde{u}, f(\widetilde{u})-u_{k}\right)$ maps $D_{\rho}^{+}(a)$ to the cylinder $B_{\rho}=$ $\left\{|\widetilde{u}|<\rho,\left|u_{k}\right|<\rho\right\}$, while the boundary surface $\Gamma \cap C_{\rho}(a)$ is mapped to the base of this cylinder. Since $\alpha(u)-\alpha(v)=(0, s)$, where $s=f(\widetilde{u})-f(\widetilde{v})-\left(u_{k}-v_{k}\right)$, and $[f]_{1} \leq 1$, it follows that the vector-function $\alpha$ satisfies the Lipschitz condition, where the seminorm $[\alpha]_{1}$ does not exceed 2 . The inverse transformation $\beta=\alpha^{-1}$ acts according to the relation $\alpha(u)=\left(\widetilde{u}, u_{k}-f(\widetilde{u})\right)$ and, therefore, satisfies a similar Lipschitz condition. Thus, $\alpha$ is a Lipschitz map such that $M=2$ in (2.3.1).

By virtue of the choice of $\rho$, it can be replaced by $2 \rho$ in these assertions. Therefore, due to (2.3.2) and (2.4.15), we have the inequality $d(\beta(y), \Gamma) \geq y_{k} / 18, y \in B_{\rho}$. Combining it with (2.4.14), we conclude that Lemma 2.2.2 can be applied to the function $\psi(y)=\varphi[\beta(y)]$ in the cylinder $B_{\rho}$; then the function $\psi$ belongs to $C^{\mu}\left(B_{\rho}\right)$ and its seminorm is estimated accordingly. Hence, $\varphi$ also belongs to $C^{\mu}\left(D_{\rho}^{+}\right)$and its seminorm satisfies the estimate $[\varphi]_{\mu} \leq C_{0} M$, where $C_{0}$ is a positive constant depending only on $\mu$ and $\rho$.

Now, consider two arbitrary points $x$ and $y$ from $D_{0}$ such that $|x-y| \leq \rho / 8$. First, we assume that $d(x, \Gamma) \leq \rho / 2$. Let $a$ from $\Gamma$ be a point, where the distance $d(x, \Gamma)$ is achieved, i.e., $d(x, \Gamma)=|x-a|$. Since $\rho\left(a, \Gamma^{\prime}\right) \geq \rho\left(x, \Gamma^{\prime}\right)-|x-a| \geq 2 r_{0}-\rho / 2>r_{0}$, it follows that the point $a$ belongs to $K$. Since $|y-a| \leq|y-x|+|x-a|<\rho / \sqrt{2}$, it follows that both points $x$ and $y$ belong to $D_{\rho}^{+}(a)$. Then, as we proved above, the following estimate holds:

$$
|\varphi(x)-\varphi(y)| \leq C_{0} M|x-y|^{\mu} .
$$

Now, consider the case where $d(x, \Gamma) \geq \rho / 8$. In this case, the ball $B$ of radius $\rho / 8$ centered at $a$ contains the point $y$ and $d(B, \Gamma) \geq \rho / 4$. Since $d\left(B, \Gamma^{\prime}\right) \geq 2 r_{0}>\rho / 4$, it follows that the distance of the ball $B$ to the full boundary $\partial D$ is not greater than $\rho / 4$, Then, due to Theorem 2.2 .2 , we have the estimate

$$
|\varphi(x)-\varphi(y)| \leq C_{1} M|x-y|^{\mu}
$$

where $C_{1}$ is a positive constant depending only on $\rho$. Once this estimate is combined with (2.4.16), one can apply Theorem 2.1.3, where $\rho / 8$ and $D_{0}$ are taken instead of $r$ and $G$ respectively; this completes the proof of the theorem.

Investigate stability conditions for the constant $C$ from Theorem 2.4.2 under changes of the surface $\Gamma$. We say that a sequence $\Gamma_{n}, n=1,2, \ldots$, of surfaces with boundaries converges to $\Gamma$ as $n \rightarrow \infty$ in the class $C^{1}$ if there exist a Lipschitz domain $G$ and their parametrizations $\gamma_{n}: G \rightarrow \Gamma_{n}$ in $G$, converging to the parametrization $\gamma$ of the surface $\Gamma$ in the space $C^{1}(\bar{G})$. The convergence in the class $C^{1, \nu}$ with respect to the space $C^{1, \nu}(G)$ has a similar sense.

Lemma 2.4.1. Let sequences $\left\{\Gamma_{n}\right\},\left\{D_{n}\right\}$, and $\left\{D_{n}^{0}\right\}$ from $D_{n}$ satisfy the assumptions of Theorem 2.4.2, the diameters of the domains $D_{n}$ be uniformly bounded, the surfaces $\Gamma_{n}$ tend to $\Gamma$ as $n \rightarrow \infty$ in the class $C^{1}$, and

$$
\inf _{n} d\left(D_{n}^{0}, \partial D_{n} \backslash \Gamma_{n}\right)>0 .
$$

Then, if functions $\varphi_{n}$ from $C^{1}\left(D_{n}\right)$ admit estimates

$$
\left|\varphi_{n}^{\prime}(x)\right| \leq M_{n} d^{\mu-1}\left(x, \Gamma_{n}\right), \quad x \in D_{n}^{0},
$$


then the estimate

$$
[\varphi]_{\mu, D_{n}^{0}} \leq C M_{n},
$$

where $C$ is a positive constant independent of $n$, holds.

Proof. Let $2 r_{0}$ denote the infimum of (2.4.17). Similarly to the proof of Theorem 2.4.2, assign $K_{n}=$ $\left\{a \in \Gamma_{n}, d\left(a, \Gamma^{\prime}\right) \geq r_{0}\right\}$. Then the sequence $\left\{\rho_{0}\left(K_{n}, \Gamma_{n}\right)\right\}$ determined by Theorem 2.4.1 can be selected to satisfy the condition

$$
\inf _{n} d\left(K_{n}, \partial \Gamma_{n}\right)=\rho_{0}>0 .
$$

Indeed, the sequence of parametrizations $\gamma_{n}: \bar{G} \rightarrow \Gamma_{n}$ converges in the class $C^{1}(\bar{G})$ by condition. Using Lemma 2.3.3, one can easily verify that $\gamma_{n}$ are Lipschitz parametrizations uniformly with respect to $n$, i.e., there exists $M$ such that each $\gamma_{n}$ is an $M$-Lipschitz map. Let a positive $r$ be such that $2 M r \leq r_{0}$. This corresponds to the choice of $(2.4 .5)$ for $K_{n}$ and $\partial \Gamma_{n}$. By virtue of the remark to Lemma 2.3.2, a unique $\delta$ for all $\gamma_{n}$ can be selected in (2.4.6). Obviously, $q$ in (2.4.7) possesses the same property. Therefore, it remains to select $\rho_{0}$ according to (2.4.8).

Assign $2 \rho=\min \left(\rho_{0}, r_{0}\right)$ and take into account the fact that only $\rho$ and the diameter of the domain $D_{n}$ are involved in further arguments on Theorem 2.4.2. This yields the estimate (2.4.18), where the constant $C$ is independent of $n$.

Theorem 2.4.1 is consistent with the definition of domains with smooth boundaries from Sec. 2.3. This theorem allows one to provide the following general definition: a set $\Gamma$ from $\mathbb{R}^{k}$ is called an open smooth surface if each its point has a neighborhood of kind (2.4.4) such that the specified set is the graph of a continuously differentiable function $f(\widetilde{u}),|\widetilde{u}| \leq \rho$, inside the specified neighborhood. We say that the specified surface belongs to a class $C^{1, \mu}$ if the function $f$ belongs to this class. If the above takes place and the set $\Gamma$ is compact, then it is called a closed smooth surface and the number $\rho$ from the theorem can be selected to be unique for all points $a$ from $\Gamma$.

Indeed, the surface $\Gamma$ can be covered by a finite set of surfaces $\Gamma_{j}, 1 \leq j \leq m$, with boundaries such that open surfaces $\Gamma_{j} \backslash \partial \Gamma_{j}$ cover $\Gamma$. Moreover, there exist compact sets $K_{j} \subseteq \Gamma_{j} \backslash \partial \Gamma_{j}$ possessing a similar property. Therefore, if $\rho_{j}$ is defined by $\Gamma_{j}$ and $K_{j}$ as in Theorem 2.4.1, then it suffices to assign

$$
\rho=\min _{1 \leq j \leq m}\left(\rho_{j}, r_{j}\right)
$$

where $3 r_{j}$ denotes the distance between $K_{j}$ and $\Gamma \backslash \Gamma_{j}$, and to take into account the fact that neighborhood (2.4.3) is contained in the ball $|x-a| \leq 3 \rho$. The number $\rho$ defined this way is called the standard radius of the closed smooth surface $\Gamma$.

In the same way, Theorem 2.4.2 can be proved for the full domain $D$ bounded by the smooth closed surface $\Gamma$ : if a function $\varphi$ from $C^{1}(D)$ admits the estimate (2.4.14), then $\varphi \in C^{0, \mu}(D)$ and $[\varphi]_{\mu} \leq C M$, where $C$ is a positive constant depending only on the standard radius $\rho$ and the diameter of the domain $D$.

\subsection{Smooth and Piecewise-Smooth Curves}

In the two-dimensional case (i.e., for $k=2$ ), we deal with plane curves instead of surfaces and with smooth arcs instead of surfaces with boundaries. It is convenient to treat the two-dimensional space $\mathbb{R}^{2}$ as the complex plane $\mathbb{C}$. Respectively, a parametrization of a smooth arc $\Gamma$ is a one-to-one complex-valued function $\gamma$ from $C^{1}[0, l]$ such that its derivative is different from zero everywhere. Any segment other than $[0, l]$ can also be taken as the parametrization domain. The function $\gamma$ defines the unit tangential vector

$$
e(t)=\gamma^{\prime}(s) /\left|\gamma^{\prime}(s)\right|, \quad t=\gamma(s),
$$

at a point $t$ of the arc $\Gamma$ and determines its orientation, i.e., the natural order of points, determined by the parameter $s$. The endpoints and interior points of the arc correspond to the endpoints and 
interior points of the segment $I$ respectively. Taking into account Lemma 2.3.3 and using notation (2.5.1), one can define the class $C^{1, \mu}$ of smooth arcs by the condition $e \in C^{\mu}(\Gamma)$.

As in the case of surfaces, the expression $d_{1} t=\left|\gamma^{\prime}(s)\right| d s$ is the element of the arc length. The integral

$$
\int_{\Gamma} \varphi(t) d_{1} t=\int_{0}^{l} \varphi[\gamma(s)]\left|\gamma^{\prime}(s)\right| d s,
$$

which is independent of the choice of the parametrization, is understood in the same sense. In particular, the relation

$$
\operatorname{mes}_{1} \Gamma=\int_{0}^{l}\left|\gamma^{\prime}(s)\right| d s
$$

determines the length of the full curve $\Gamma$. Any smooth arc admits the so-called natural parametrization $\gamma_{0}$ such that $\left|\gamma_{0}^{\prime}(s)\right| \equiv 1$. In this case, $l$ coincides with curve length. For any smooth parametrization $\gamma:[0,1] \rightarrow \Gamma$, the natural parametrization can be defined by the relation $\gamma_{0}(s)=\gamma[\alpha(s)], 0 \leq s \leq l$, where the map $\alpha$ of the segment $[0, l]$ onto $[0,1]$ is inverse to the function

$$
\beta(r)=\int_{0}^{r}\left|\gamma^{\prime}(u)\right| d u, \quad 0 \leq r \leq 1
$$

Thus, the parameter $s$ of the natural parametrization plays the role of the arc length, counted from its end.

On an oriented arc, one can introduce the operation $\varphi \rightarrow \varphi_{s}^{\prime}$ of differentiation with respect to the natural parameter $s$. Using this operation, one can introduce the class $C^{1}(\Gamma)$. The relation between this operation and an arbitrary parametrization $\gamma$ coordinated with the orientation is as follows:

$$
\varphi^{\prime} \circ \gamma=(\varphi \circ \gamma)^{\prime}\left|\gamma^{\prime}\right|^{-1} \text {. }
$$

Obviously, if $\alpha$ from $C^{1}(\Gamma)$ is a one-to-one complex-valued function and $\alpha^{\prime}(t) \neq 0$ for any $t$ from $\Gamma$, then the image $\alpha(\Gamma)$ is a smooth arc with the parametrization $\alpha \circ \gamma$. Maps of such type are called translations of smooth arcs. It is clear that the translation $\alpha$ is a Lipschitz map since $\gamma$ and $\alpha \circ \gamma$ possess this property. In this sense, the map $\gamma^{-1}$ inverse to the parametrization $\gamma:[0, l] \rightarrow \Gamma$ is a translation acting from $\Gamma$ to $[0, l]$.

Using the unit function $e=e_{1}+i e_{2}$ from $C(\Gamma)$, defined by (2.5.1), one can introduce line integrals with complex differential $d z=d x+i d y$ on an orientable smooth $\operatorname{arc} \Gamma$ as follows:

$$
\int_{\Gamma} f_{1}(z) d x+f_{2}(z) d y=\int_{\Gamma}\left(f_{1} e_{1}+f_{2} e_{2}\right) d_{1} z, \quad e=e_{1}+i e_{2} .
$$

Note that the change of the orientation changes the sign of such an integral.

The union of a finite set of smooth arcs such that their pairwise intersections can contain only their endpoints is called a piecewise-smooth curve. The integral of a function $\varphi$ defined on such a curve is treated as the sum of the integrals (2.5.2) over the corresponding arcs. If all connected components of the considered curve are homeomorphic to a circle, then the curve is called a piecewise-smooth contour. For domains bounded by piecewise-smooth contours, the Green formula from Sec. 1.8 can be presented in terms of line integrals of kind (2.5.4).

Theorem (the Green formula). Let a plain domain $D$ be bounded by a piecewise-smooth contour $\Gamma$ positively oriented with respect to $D$ (i.e., the domain $D$ is located to the left under the moving along $\Gamma$ to the positive direction). Then, if functions $f$ and $g$ from $C(\bar{D})$ are continuously differentiable in 
the domain $D$, then

$$
\int_{D}\left(\frac{\partial f}{\partial x}+\frac{\partial g}{\partial y}\right) d_{2} z=\int_{\Gamma} f d y-g d x
$$

provided that the integrand at the left-hand side of the relation is integrable in the domain D.

To prove, it suffices to note that if the direction of the unit tangential vector $e$ is coordinated with the orientation of the contour, then $e=i n$, where $n=n_{1}+i n_{2}$ is the unit vector of the outer normal.

We say that $\Gamma$ is a radial arc with respect to its endpoint $a$ if it admits a parametrization of the kind

$$
\gamma(r)=a+r e^{i f(r)}, \quad 0 \leq r \leq \rho,
$$

where the real function $f$ is continuously differentiable on $(0, \rho]$, and there exists a constant $\theta$ such that $\lim _{r \rightarrow 0} f(r)=\theta$, and $\lim _{r \rightarrow 0} r f^{\prime}(r)=0$.

Obviously, the function $\gamma(r)$ defined above is a one-to-one function on $[0, \rho]$ and its derivative $\gamma^{\prime}(r)=\left[1+r \theta^{\prime}(r)\right] e^{i \theta(r)}$ is continuous on this segment and vanishes nowhere. Thus, the relation (2.5.5) defines a smooth parametrization indeed. Its parameter is $r=|t-a|, t \in \Gamma$; in particular, $\rho$ is the distance between the endpoints of the arc. If the unit tangential vector $e(t), t \in \Gamma$, is determined by this parametrization similarly to (2.5.1), then its value at the end of $a$ coincides with $e^{i \theta}$.

We say that (2.5.5) is a radial parametrization. The next lemma provides a simple criterion of the parametrization to be radial.

Lemma 2.5.1. Let $\Gamma$ be a smooth arc and the oscillation $m=[e]_{0}$ of its unit tangential vector $e(t)$ satisfy the condition

$$
m=\max _{t_{j} \in \Gamma}\left|e\left(t_{1}\right)-e\left(t_{2}\right)\right| \leq 1 / 4
$$

Then this is a radial arc with respect to any its endpoint a, the derivative of its radial parametrization $\gamma(r)$ admits the estimate

$$
1 / 3 \leq\left|\gamma^{\prime}(r)\right| \leq 3, \quad 0 \leq r \leq \rho,
$$

and the arc $\Gamma$ lies in the $\pi / 2$-sector such that its vertex is a and its bisectrix is directed along the vector $e(a)$.

Proof. Let $\gamma_{0}(s)$ and $0 \leq s \leq l$ be the natural parametrization of the arc $\Gamma$ with endpoint $a=\gamma_{0}(0)$. In the square $I=\left\{0 \leq s_{0}, s \leq l\right\}$, consider the function

$$
q\left(s_{0}, s\right)=\frac{\gamma_{0}(s)-\gamma_{0}\left(s_{0}\right)}{s-s_{0}}=\int_{0}^{1} \gamma_{0}^{\prime}\left[r s+(1-r) s_{0}\right] d r
$$

for $s=s_{0}$, its value is equal to $\gamma_{0}^{\prime}(s)$. Since $e\left[\gamma_{0}(s)\right]=\gamma_{0}^{\prime}(s)$, it follows that $m$ coincides with the oscillation of the function $\gamma_{0}^{\prime}(s)$. Obviously, this is valid for the oscillation of the function $q\left(s_{0}, s\right)$ in the square $I \times I$. Thus, ||$q(u)|-| q(v)|| \leq|q(u)-q(v)| \leq m$, where $u, v, \ldots$ denote points of the square. Since $|q(v)|=\left|\gamma^{\prime}\left(s_{0}\right)\right|=1$ for $v=\left(s_{0}, s_{0}\right)$, it follows that $|q(u)| \geq 1-m$. Hence, for all $u$ and $v$ from $I \times I$, we have the inequality

$$
\left|\frac{q(u)}{|q(u)|}-\frac{q(v)}{|q(v)|}\right| \leq \frac{|q(u)||q(u)|-|q(v)||+| q(v)|| q(u)-q(v)||}{|q(u)||q(v)|} \leq \frac{2 m}{1-m} .
$$

Taking into account (2.5.7), we deduce the inequality

$$
\left|\frac{q(u)}{|q(u)|}-\frac{q(v)}{|q(v)|}\right| \leq \frac{2}{3}
$$

This inequality implies that the angle $\varphi$ between the unit vectors $e_{1}=q(u) /|q(u)|$ and $e_{2}=q(v) /|q(v)|$ satisfies the inequality $1-\cos \varphi \leq 2 / 9$, whence $\varphi \leq \pi / 4$. For $u=(s, 0)$ and $v=(0,0)$, this angle 
coincides with the angle between the vector $\gamma_{0}(s)-\gamma_{0}\left(s_{0}\right)$ and the tangent to $\Gamma$ at the point $a=\gamma_{0}(0)$, which proves the last claim of the lemma.

Now, consider the functions $\alpha(s)=\left|\gamma_{0}(s)-\gamma_{0}(0)\right|$ and $a(s)=\arg \left[\gamma_{0}(s)-\gamma_{0}(0)\right]$. It is obvious that $a(s) \rightarrow \theta$ as $s \rightarrow 0$, where $e^{i \theta}=e(a)$. The derivatives of these functions are expressed as follows:

$$
\alpha^{\prime}(s)=\operatorname{Re} \frac{\left[\gamma_{0}(s)-\gamma_{0}(0)\right] \overline{\gamma_{0}^{\prime}(s)}}{\left|\gamma_{0}(s)-\gamma_{0}(0)\right|}=\operatorname{Re} \frac{[q(s, 0) \overline{q(s, s)}]}{|q(s, 0)|}, \quad a^{\prime}(s)=\operatorname{Im} \frac{\gamma_{0}^{\prime}(s)}{\gamma_{0}(s)-\gamma_{0}(0)} .
$$

In particular, $s a^{\prime}(s) \rightarrow 0$ as $s \rightarrow 0$. It is obvious that

$$
1-\alpha^{\prime}(s)=\operatorname{Re} Q, \quad Q=\left[q(s, s)-\frac{q(s, 0)}{|q(s, 0)|}\right] \overline{q(s, s)},
$$

and $|Q| \leq 2 / 3$ by virtue of (2.5.8). This yields the inequality

$$
1 / 3 \leq \alpha^{\prime}(s) \leq 3, \quad 0 \leq s \leq l .
$$

Therefore, there exists a function $s=\beta(r), 0 \leq r \leq \rho=\alpha(l)$, inverse to $\alpha$. It is obvious that $f(r)=a[\beta(r)] \rightarrow \theta$ and $r f^{\prime}(r) \rightarrow 0$ as $r \rightarrow 0$ and the relation $\gamma_{0}(s)-\gamma_{0}(0)=\alpha(s) e^{i a(s)}$ in the notation $\gamma(r)=\gamma_{0}[\beta(r)]$ can be expressed by (2.5.4). Then $\Gamma$ is a radial arc. On the segment $[0, \rho]$, the derivative $\beta^{\prime}$ satisfies an estimate similar to (2.5.9). Then, taking into account the relation $\gamma^{\prime}=\left(\gamma_{0}^{\prime} \circ \beta\right) \beta^{\prime}$, we obtain the estimate (2.5.7) completing the proof of the lemma.

Lemma 2.5.1 shows that any smooth arc $\Gamma$ is radial with respect to its endpoint in a sufficiently small neighborhood of this endpoint.

In the considered two-dimensional case, circle (2.4.3) is the rectangle $C_{\rho}(a)=\left\{\left|u_{1}\right| \leq \rho,\left|u_{2}\right| \leq 2 \rho\right\}$ containing the disc $\{|z-a| \leq \rho\}$. If $a$ is an interior point of a smooth arc $\Gamma$, then the local coordinate system $u_{1}, u_{2}$ with origin at the point $a$ is uniquely determined by the curve since the axis $u_{1}$ is directed along the tangential vector $e(a)$, while the axis $u_{2}$ is directed along the normal to the curve. In this case, Theorem 2.4.1 can be refined.

Lemma 2.5.2. Let $a$ be an interior point of a smooth arc $\Gamma$ and $\rho$ be selected with respect to $K=\{a\}$ as in Theorem 2.4.1. Then the point a decomposes the arc $\Gamma_{a}=\Gamma \cap C_{\rho}(a)$ into two arcs radial with respect to their common endpoint a. In particular, the intersection $\Gamma \cap\{|z-a| \leq \rho\}$ is an arc itself and $a$ is its interior point.

Proof. According to Theorem 2.4.1, the arc $\Gamma_{a}$ is the graph of the function $u_{2}=f\left(u_{1}\right)$, where the function $f$ from $C^{1}[-\rho, \rho]$ is such that $f(0)=f^{\prime}(0)=0$ and $\left|f^{\prime}\left(u_{1}\right)\right| \leq 1$ provided that $\left|u_{1}\right| \leq 1$.

We must show that the graph of the function $y=f(x), 0 \leq x \leq \rho$, is a radial arc with respect to the point $z=0$. As in the proof of Lemma 2.5.1, it suffices to verify that the derivative of the function $r=\sqrt{x^{2}+f^{2}(x)}$ is positive, i.e., $x+f(x) f^{\prime}(x)>0$ for $x>0$. This fact easily follows from the inequality $\left|f^{\prime}\right| \leq 1$.

Indeed, assume, to the contrary, that there exists a positive $c$ such that $f^{\prime}(c)[f(c) / c]=-1$. Since neither $|f(x)| / x$ nor $\left|f^{\prime}(x)\right|$ exceeds 1 , it follows that

$$
f^{\prime}(c)= \pm 1, \quad f(c) \pm c=0 .
$$

The last relation can be represented in the form

$$
\int_{0}^{1}\left[1 \pm f^{\prime}(t c)\right] d t=0,
$$

which is possible only if $f^{\prime}(x)=\mp 1,0 \leq x \leq c$. However, this property contradicts the first relation of $(2.5 .10)$. 
Consider the common piecewise-smooth curve $\Gamma$. We say that its point $\tau$ is interior if there exists a (sufficiently small) radius $\rho$ such that the intersection of the curve with the disc $\{|z-\tau| \leq \rho\}$ is a smooth curve such that $\tau$ is its interior point. Other points of the curve are called boundary points, their set is finite, and they form the boundary $\partial \Gamma$. In particular, the boundary of a smooth curve consists of two points that are its endpoints. The case where $\partial \Gamma=\varnothing$ is possible: $\Gamma$ is called a smooth contour in such a case. According to Lemma 2.5.1, the disc of radius $\rho$ centered at $\tau$ from $\partial \Gamma$ is decomposed by the curve into a finite set of radial $\operatorname{arcs} \Gamma_{\tau, j}, j=1, \ldots, n_{\tau}$, with the joint endpoint $\tau$ provided that $\rho$ is sufficiently small. Thus,

$$
\Gamma \cap\{|z-\tau| \leq \rho\}=\bigcup_{j=1}^{n_{\tau}} \Gamma_{\tau, j}, \quad \Gamma_{\tau, i} \cap \Gamma_{\tau, j}=\{\tau\}, \quad i \neq j .
$$

In this case, we say that arcs $\Gamma_{\tau, j}$ converge to $\tau$.

Obviously, if $\tau \in F \backslash \partial \Gamma$, i.e., $\tau$ is an interior point, then the angle between the $\operatorname{arcs} \Gamma_{\tau, 1}$ and $\Gamma_{\tau, 2}$ is equal to $\pi$, i.e., these arcs form a smooth curve. In the general case, if $n_{\tau} \geq 2$, then it is possible that the angle between the $\operatorname{arcs} \Gamma_{\tau, i}$ and $\Gamma_{\tau, j}$ is equal to zero, i.e., they touch each other at the point $\tau$ in the interior way. Then $\tau$ is called a cusp of the curve $\Gamma$.

For $n_{\tau}=1$, the point $\tau$ is called the endpoint of the curve $\Gamma$. If $n_{\tau}=2$ for any $\tau$, then the curve $\Gamma$ is a piecewise-smooth contour, i.e., its connected components are homeomorphic to a circle. If all boundary points are endpoints, i.e., $n_{\tau}=1$ for any $\tau$ from $\partial \Gamma$, then $\Gamma$ is a union of pairwise disjoint smooth arcs. If the boundary $\partial \Gamma$ consists of one point $\tau$, then all connected components are homeomorphic to an open interval of the line and are called open smooth arcs (with the common endpoint $\tau$ ).

The strict definition is as follows. An open smooth arc $\dot{\Gamma}$ is the image of the interval $(0,1)$ of a complex-valued function $\gamma$ from $C^{1}[0,1]$ if it is a one-to-one function on the semi-open intervals $(0,1]$ and $[0,1)$ and $\gamma^{\prime}(s) \neq 0$ provided that $0 \leq s \leq 1$. The above function $\gamma$ is called the parametrization. As above, the notation $\dot{\Gamma} \in C^{1, \mu}$ means that the parametrization $\gamma$ belongs to $C^{1, \mu}[0,1]$.

We say that an open arc $\dot{\Gamma}$ is disjoined if $\gamma(0) \neq \gamma(1)$ and joined otherwise. Thus, to obtain a disjoined open arc $\dot{\Gamma}$, it suffices to take a smooth arc $\Gamma$ and exclude its endpoints. Any joined open arc $\dot{\Gamma}$ united with the common endpoint $\tau=\gamma(0) \neq \gamma(1)$ forms a simple piecewise-smooth contour (its smoothness is not guaranteed).

The notion of a translation $\alpha: \dot{\Gamma} \rightarrow \dot{\Gamma}_{1}$ of open arcs is introduced in the same way as above: if $\gamma$ is a parametrization of $\dot{\Gamma}$, then $\alpha \circ \gamma$ has the same sense with respect to $\dot{\Gamma}_{1}$. Thus, the function $\alpha$ is continuously differentiable on $\dot{\Gamma}$, it and its derivative $\alpha^{\prime}$ has limits at the endpoints of the arc (if the arc is joined, then they are one-sided limits), and $\alpha^{\prime}$ is different from zero everywhere, including these limits at endpoints.

The convenience of the accepted terminology of open arcs is as follows: for any finite subset $F$ of a curve $\Gamma$, containing all boundary points of $\Gamma$, any connected component of the set $\Gamma \backslash F$ is either a simple smooth contour or an open (joined or disjoined) smooth arc. Thus,

$$
\Gamma \backslash F=\Gamma_{0} \cup \dot{\Gamma}_{1} \cup \ldots \cup \dot{\Gamma}_{m}, \quad \partial \Gamma \subseteq F,
$$

where $\Gamma_{0}$ is a smooth contour (in general, it is a composite one), $\dot{\Gamma}_{j}$ are open smooth arcs, and all these curves are pairwise disjoint. Usually, the set $F$ is contained in $\Gamma$; sometimes, it might contain points not belonging to this curve. It is easy to see that the number $2 m$ in the notation (2.5.11) is equal to the sum of all $n_{\tau}$ such that $\tau \in \Gamma \cap F$.

If a domain $D$ is bounded by a piecewise-smooth contour such that its corner points are not cusps, then $D$ is a Lipschitz domain. Indeed, let $a$ be a corner point such that two $\operatorname{arcs} \Gamma_{\tau, 1}$ and $\Gamma_{\tau, 2}$ converge to it. Consider a local system of Cartesian coordinates such that its origin is the point $a$ and its axis $y$ is directed along the line bisecting the interior angle of the domain $D$ at the point $a$. Then the 
$\operatorname{arcs} \Gamma_{\tau, 1}$ and $\Gamma_{\tau, 2}$ form the graph of a piecewise-smooth function $y=f(x)$ satisfying the Lipschitz condition. Then, as we know from Sec. 2.3, $D$ is a Lipschitz domain in a neighborhood of $a$.

Let the boundary $\partial D$ of an open set $D$ be a piecewise-smooth curve $\Gamma$ (we say that such set is piecewise-smooth). Consider a family of subdomains $D_{j} \subseteq D, 1 \leq j \leq n$, bounded by piecewisesmooth contours such that $\bar{D}=\bar{D}_{1} \cup \ldots \cup \bar{D}_{n}$. We say that a function $\varphi$ from $C(D)$ belongs to the class $C(\widehat{D}, F)$ if its restrictions to $D_{j}$ belong to $C\left(\bar{D}_{j}\right), 1 \leq j \leq n$. Obviously, this definition is independent of the choice of $D_{1}, \ldots, D_{n}$ and $C(\widehat{D})$ is a Banach space with respect to the norm

$$
|\varphi|=\max _{j}|\varphi|_{C\left(\bar{D}_{j}\right)} \cdot
$$

This definition is introduced to take into account possible one-sided limit values at boundary points $a$ from $\partial D$. The boundary of the closure $\bar{D}$ of the set $D$ is a piecewise-smooth contour $\Gamma_{1} \subseteq D$. Therefore, $\Gamma=\Gamma_{1} \cup \Gamma_{2}$, where the amount of common points of the piecewise-smooth curve $\Gamma_{2}$ and $\Gamma_{1}$ is finite. Other points of this curve are interior points of $\bar{D}$. Obviously, in a neighborhood of a point $a$ from $\Gamma_{1} \backslash \partial \Gamma\left(a \in \Gamma_{2} \backslash \partial \Gamma\right)$, the set $D$ lies from one side (from both sides) of $\Gamma$. The curve $\Gamma_{2}$ is called a cut of the set $D$. Respectively, the function $\varphi$ from $C(\widehat{D})$ has one or two limit values at the point $a$.

These boundary values can be described more strictly. Let a compact set $K$ be a subset of $\Gamma \backslash \partial \Gamma$. Then, by virtue of Theorem 2.4.1 and Lemma 2.5.2, there exists a positive $\rho$ such that, for any point $a$ of $K$, the intersection of $\Gamma$ with the $\operatorname{disc} B(a)=\{|z-a| \leq \rho\}$ is a smooth $\operatorname{arc} \Gamma_{\rho}(a)$ such that $a$ is its interior point. In particular, the complement of $B(a) \backslash \Gamma$ consists of two connected components $B^{ \pm}(a)$. Obviously, each one lies in a connected component of $\mathbb{C} \backslash \Gamma$. Hence, the two following cases are possible: either one of them is contained in $D$, while another one is contained in $\mathbb{C} \backslash \bar{D}$, or both ones are contained in $D$. In the former case, the point belongs to $\Gamma_{1}$ and any function $\varphi$ from $C(\widehat{D})$ has one limit value at this point, denoted by

$$
\varphi^{(+)}(a)=\lim _{x \rightarrow a} \varphi(x), \quad a \in K \cap \Gamma_{1} .
$$

In the latter case, it has two limit values

$$
\varphi^{ \pm}(a)=\lim _{\substack{x \rightarrow a \\ x \in B^{ \pm}(a)}} \varphi(x), \quad a \in K \cap \Gamma_{2} .
$$

It is convenient to fix the signs of the one-sided neighborhoods $B^{ \pm}(a)$ by means of the orientation of the arc $\Gamma(a)$ as follows: moving along this arc in the positive direction, we leave the set $B^{+}(a)$ from the left. Thus, the function $\varphi$ has one boundary value $\varphi^{(+)}$from $C\left(\Gamma_{1} \backslash \partial \Gamma\right)$ and two boundary values $\varphi^{ \pm}$from $C\left(\Gamma_{2} \backslash \partial \Gamma\right)$. At a point $a$ belonging to $\partial \Gamma$, there might be several such boundary values: in the notation (2.5.11), their amount is equal to $n_{\tau}$. Thus, $\widehat{D}$ can be treated as a compactification of the open set $D$, determined by the described one-sided neighborhoods.

The space $C^{\mu}(\widehat{D})$ is introduced as above: if the subdomains $D_{j} \subseteq D$ are bounded by piecewisesmooth contours and $\bar{D}=\bar{D}_{1} \cup \ldots \cup \bar{D}_{n}$, then this space consists of all functions $\varphi$ from $C(D)$, belonging to $C^{\mu}\left(\bar{D}_{j}\right), 1 \leq j \leq n$.

Lemma 2.5.3. The space $C^{\mu}(\widehat{D})$ is independent of the choice of the subdomains $D_{1}, \ldots, D_{n}$ and it is a Banach space with respect to the norm

$$
|\varphi|=\max _{j}|\varphi|_{C^{\mu}\left(\bar{D}_{j}\right)} .
$$

Proof. Similarly to Theorem 2.3.1, it is easy to show that any domain bounded by a piecewise-smooth contour is uniformly connected. Let $D_{j}^{\prime} \subseteq D, 1 \leq j \leq n^{\prime}$, be another family of subdomains such that the union of their closures coincides with $\bar{D}$ and let $|\varphi|^{\prime}$ be defined by this family similarly to (2.5.14). Then each $D_{j}^{\prime}$ is the union of the subsets $D \cap D_{i}, 1 \leq i \leq n$, and it follows from Theorem 2.1.2 that

$$
|\varphi|_{C^{\mu}\left(D_{j}^{\prime}\right)} \leq C|\varphi|,
$$


whence $|\varphi|^{\prime} \leq C|\varphi|$. The opposite inequality is proved in the same way. Hence, the norms $|\varphi|$ and $|\varphi|^{\prime}$ are equivalent.

\subsection{The Space $C_{*}^{\mu}(G)$ on the Riemann Sphere}

Let $G$ be an unbounded subset of $\mathbb{R}^{k}$. By $C_{*}(G)$, denote the class of functions $\varphi$ from $C(G)$, admitting a limit $\varphi(\infty)=\lim \varphi(x)$ as $|x| \rightarrow \infty$. It is convenient to introduce the one-point compactification $\overline{\mathbb{R}^{k}}=\mathbb{R}^{k} \cup\{\infty\}$ of the Euclidean space $\mathbb{R}^{k}$ by means of the element $\infty$ denoting the the point at infinity. In this compactification, its neighborhoods are defined as complements to balls. In particular, $C_{*}(G)$ can be treated as the class of functions continuous in $G \cup \infty$ with respect to the said topology. For $n=2$, the stereographic projection establishes a homeomorphism of the compact set $\overline{\mathbb{C}}$ onto the unit sphere $\Omega$ of the three-dimensional space; this is the reason to call it the Riemann sphere. For $k \geq 3$, a similar projection can also be introduced; therefore, the specified term can also be preserved in this case.

It is obvious that the inversion

$$
\delta(x)=\frac{x-a}{|x-a|^{2}}
$$

with respect to the sphere $|x-a|=1$ centered at point $a$ is a homeomorphic map of the compact set $\overline{\mathbb{R}^{k}}$ onto itself such that $\delta(a)=\infty$ and $\delta(\infty)=0$. For $a=0$, this map is denoted by $\delta(x)=x^{*}$. Obviously, it rearranges the points 0 and $\infty$ and is mutually inverse. In the general case, the inverse map to (2.6.1) is the map $y \rightarrow a+y^{*}$.

The compact set $\overline{\mathbb{R}^{k}}$ can be endowed with a natural metric space structure: to any pair $x, y$ of its points, we assign the nonnegative number $d(x, y)$ as follows:

$$
\begin{aligned}
d(x, y) & =(1+|x|)^{-1}(1+|y|)^{-1}|x-y|, \quad x \neq \infty, y \neq \infty, \\
d(x, \infty) & =d(\infty, x)=(1+|x|)^{-1}, \quad x \neq \infty ; \quad d(\infty, \infty)=0 .
\end{aligned}
$$

Then $d(x, y) \rightarrow d(x, \infty)$ as $y \rightarrow \infty$.

Lemma 2.6.1. The function $d(x, y)$ is a distance such that inversion (2.6.1) satisfies the two-side estimate

$$
(1+|a|)^{-2} d(x, y) \leq d[\delta(x), \delta(y)] \leq(1+|a|)^{2} d(x, y)
$$

with respect to it.

Proof. To prove the first assertion, we verify the triangle inequality for a triple $x, y, z$ of points from $\overline{\mathbb{R}^{k}}$. If one of these points coincides with $\infty$, then the said inequality is established immediately. Therefore, we must prove the inequality

$$
\frac{|x-z|}{(1+|x|)(1+|z|)} \leq \frac{|x-y|}{(1+|x|)(1+|y|)}+\frac{|y-z|}{(1+|y|)(1+|z|)}
$$

or, which is equivalent, the inequality

$$
(1+|y|)|x-z| \leq(1+|z|)|x-y|+(1+|x|)|y-z| .
$$

It suffices to verify that $|y||x-z| \leq|z||x-y|+|x||y-z|$. This inequality is obvious if one of the points $x, y$, and $z$ coincides with 0 . In the general case, dividing by $|x||y||z|$, we transform it into the inequality

$$
\left|\frac{\tilde{x}}{|z|}-\frac{\tilde{z}}{|x|}\right| \leq\left|\frac{\tilde{x}}{|y|}-\frac{\tilde{y}}{|x|}\right|+\left|\frac{\tilde{y}}{|z|}-\frac{\tilde{z}}{|y|}\right|
$$

where $\tilde{x}=x /|x|$ and $\tilde{y}$ and $\tilde{z}$ are defined in the same way. We have

$$
\left|\frac{\tilde{x}}{|z|}-\frac{\tilde{z}}{|x|}\right|^{2}=\frac{1}{|z|^{2}}+\frac{1}{|x|^{2}}-2 \frac{\tilde{x} \tilde{y}}{|x||z|}=\left|\frac{\tilde{x}}{|x|}-\frac{\tilde{z}}{|z|}\right|^{2}=\left|x^{*}-z^{*}\right|^{2}
$$


and similar relations for other point pairs. Therefore, the last inequality coincides with the triangle inequality with respect to the Euclidean metric.

The proof of the second assertion of the lemma is based on the relation

$$
\left|x^{*}-y^{*}\right|=\frac{|x-y|}{|x||y|}
$$

equivalent to the relation

$$
\left.|| y\right|^{2} x-\left.|x|^{2} y\right|^{2}=|x|^{2}|y|^{2}|x-y|^{2}
$$

Its left-hand side is equal to

$$
|y|^{4}|x|^{2}-2|x|^{2}|y|^{2} x y+|x|^{4}|y|^{2}=|x|^{2}|y|^{2}\left(|x|^{2}+|y|^{2}-2 x y\right),
$$

which coincides with its right-hand side.

By virtue of (2.6.1) and (2.6.4), the distance $d[\delta(x), \delta(y)]$ can be represented in the form

$$
\left(1+\frac{1}{|x-a|}\right)^{-1}\left(1+\frac{1}{|y-a|}\right)^{-1} \frac{|x-y|}{|x-a||y-a|}=\frac{|x-y|}{(1+|x-a|)(1+|y-a|)},
$$

whence

$$
d[\delta(x), \delta(y)]=q(x) q(y) d(x, y), \quad q(x)=\frac{1+|x|}{1+|x-a|} .
$$

It remains to note that the estimate $(1+|a|)^{-1} \leq q(x) \leq 1+|a|$ holds due to the obvious inequality $1+|x+b| \leq(1+|x|)(1+|b|)$.

For functions defined on an arbitrary metric space, one can introduce the Hölder condition with respect to its metric $d(x, y)$ as follows: at the right-hand side of $(2.1 .1),|x-y|^{\mu}$ is replaced by $[d(x, y)]^{\mu}$. The corresponding class is denoted by $C^{\mu}(G)=C^{\mu}(G ; d)$. This is a Banach space with respect to the norm

$$
|\varphi|_{\mu}=|\varphi|_{0}+[\varphi]_{\mu}, \quad[\varphi]_{\mu}=\sup _{x \neq y} \frac{|\varphi(x)-\varphi(y)|}{[d(x, y)]^{\mu}} .
$$

Using the Hölder condition $d_{2}[\alpha(x), \alpha(y)] \leq C\left[d_{1}(x, y)\right]^{\mu}$, one can introduce the class $C^{\mu}\left(G_{1}, G_{2}\right)$ of maps $\alpha$ from the metric space $G_{1}$ to $G_{2}$.

Relations (2.1.6), (2.1.7), and (2.2.3) as well as Theorem 2.1.1 also hold in the case considered since the properties of the Euclidean distance were not used in the proof.

In the sequel, in addition to the Euclidean distance, we use the metric (2.6.2). The space $C^{\mu}(G)$ with respect to this metric is denoted by the special symbol $C_{*}^{\mu}(G)$. The notation $C_{*}^{\mu}\left(G_{1}, G_{2}\right)$ has the same sense for maps from $G_{1}$ to $G_{2}$ with respect to this metric. For example, due to Lemma 2.6.1, inversion (2.6.1) belongs to the class $C_{*}^{1,0}\left(\overline{\mathbb{R}^{k}}, \overline{\mathbb{R}^{k}}\right)$.

Obviously, if a set $G$ is bounded and, e.g., is contained in the map $|x| \leq R$, then the space $C_{*}^{\mu}(G)$ and $C^{\mu}(G)$ coincide and the corresponding norms are equivalent. This follows from the equivalence of metric (2.6.2) and the Euclidean one in the specified ball:

$$
(1+R)^{-2}|x-y| \leq d(x, y) \leq|x-y|
$$

Similarly to Sec. 2.3, a homeomorphism $\alpha: G_{1} \rightarrow G_{2}$ of two sets $G_{j}$ from $\overline{\mathbb{R}^{k}}$ is called a Lipschitz map (in the generalized sense) if the following two-side estimate is satisfied:

$$
M^{-1} d(x, y) \leq d[\alpha(x), \alpha(y)] \leq M d(x, y) .
$$

For example, due to Lemma 2.6.1, the inversion is a map of the above type. The following lemma shows that, using compositions with inversions, one can reduce such maps to Lipschitz maps in the classical sense. 
Lemma 2.6.2. Let $\alpha$ be a homeomorphism from $G_{1}$ to $G_{2}$, both sets $G_{j}$ be unbounded, and

$$
\alpha(\infty)=\infty \text {. }
$$

Then $\alpha$ is a Lipschitz map with respect to the metric $d$ if and only if it is a Lipschitz map in the classical sense. In particular, any Lipschitz map with respect to the metric d can be represented as the superposition of a Lipschitz map in the classical sense and inversion (2.6.1).

Proof. By virtue of (2.6.7), on the set $E_{1}$, the function

$$
q(x)=\frac{1+|\alpha(x)|}{1+|x|}
$$

is bounded from above and from below by positive constants. Since

$$
\frac{d[\alpha(x), \alpha(y)]}{d(x, y)}=\frac{|\alpha(x)-\alpha(y)|}{|x-y|} q(x) q(y),
$$

it follows that the conditions (2.3.1) and (2.6.7) are equivalent.

An unbounded domain $D$ is called a Lipschitz domain if there exists its inversion to a bounded Lipschitz domain. An analog of Theorem 2.3.1 also holds in this case.

Theorem 2.6.1. Unbounded Lipschitz domains are uniformly connected.

Proof. Let $D$ be an unbounded Lipschitz domain. Then there exist a bounded Lipschitz domain $D_{0}$ and an inversion mapping it to $D$. Without loss of generality, one can assume that $0 \in \overline{D_{0}}$ and the inversion is $\delta(x)=x /|x|^{2}$. According to the definition of Lipschitz domains (see Sec. 2.3), one can assume that if $0 \in \partial D_{0}$, then there exists a neighborhood of the point $x=0$ such that its intersection with $D_{0}$ is a semiball $\left\{|x|<\rho, x_{k}>0\right\}$. Then the intersection $V_{\infty} \cap D$, where $V_{\infty}$ is the corresponding neighborhood of the point $\infty$, is the exterior of a semiball, i.e., a set of the kind $\left\{|x|>1 / \rho, x_{k}>0\right\}$. It is easy see that this set is uniformly connected. Obviously, if $0 \in D_{0}$, then such a neighborhood of $\infty$ is the exterior of a ball; also, it is contained in $D$ and is a uniformly connected set. Therefore, it remains to repeat the corresponding reasoning of Theorem 2.3.1, taking into account the fact that if sequences $\left\{x_{n}\right\}$ and $\left\{y_{n}\right\}$ possessing the property (2.3.9) converge to $\infty$, then their elements get to $V_{\infty} \cap D$ provided that $n$ is sufficiently large.

The space $C_{*}^{\mu}(G)$ can be describes without the distance (2.6.2) as follows. Cover the Riemann sphere by two intersecting neighborhoods

$$
U_{0}=\{|x|<2\}, \quad U_{1}=\{|x|>1\}
$$

of the points 0 and $\infty$. To any function $\varphi$ defined on the set $G \subseteq \mathbb{R}^{k}$ assign the function pair

$$
\varphi_{0}(x)=\varphi(x), x \in G_{0}=G \cap U_{0}, \quad \varphi_{1}(x)=\varphi\left(x^{*}\right), x \in G_{1}=\left(G \cap U_{1}\right)^{*},
$$

where $E^{*}$ is the image of the set $E$ under the involution $x \rightarrow x^{*}$. Then, by virtue of Theorem 2.1.1 and Lemma 2.6.1, the relation

$$
|\varphi|=\max _{k=0,1}\left|\varphi_{k}\right|_{C^{\mu}}
$$

defines an equivalent norm of the space $C_{*}^{\mu}(G)$. If one of the sets $G \cap U_{k}$ is empty, then the norm $\left|\varphi_{k}\right|$ at the right-hand side of (2.6.10) is assigned to be equal to zero.

For maps $\varphi: G \subseteq \mathbb{R}^{k} \rightarrow \mathbb{R}^{s}$, the reasoning is the same. Let notation (2.6.8) be preserved for both spaces $\mathbb{R}^{k}$ and $\mathbb{R}^{s}$. Then $\varphi \in C_{*}^{\mu}\left(G, \mathbb{R}^{s}\right)$ if and only if

$$
\varphi_{k r}(x) \in C^{\mu}\left(G_{k}\right), \quad k, r=0,1,
$$

where $\varphi_{k 0}$ and $\varphi_{k 1}$ are defined similarly to (2.6.9) with respect to the functions $\varphi(x)$ and $[\varphi(x)]^{*}$ respectively.

If the set $G$ is a domain $D$, then classes $C_{*}^{n}(D)$ are defined in the same way. The sphere $\overline{R^{k}}$ can be treated as a compact $k$-dimensional manifold of the class $C^{\infty}$ (see, e.g., [37]) defined by means of two 
charts (2.6.8). In this sense, the class $C_{*}^{n}(D)$ with respect to $D$ treated as a domain coincides with the class $C^{n}(D)$ on this manifold. Note that involution (2.6.1) can be treated as a $C^{\infty}$-diffeomorphism of the considered manifold $\overline{R^{k}}$ onto itself.

To conclude, consider the case where $k=2$ in detail. This is the planar case and, as in Sec. 2.5 , it is convenient to treat the plane as the complex plane. In this case, $\overline{\mathbb{C}}=\mathbb{C} \cup \infty$ is the classical Riemann sphere and inversion (2.6.1) is a linear-fractional function (up to a complex conjugation):

$$
\delta(z)=\frac{1}{\bar{z}-\bar{a}} .
$$

Conversely, any linear-fractional function can be expressed via involutions respectively. Therefore, the class $C_{*}^{\mu}$ can be defined by the condition of the invariance with respect to linear-fractional transformations of the plane. Smooth arcs and piecewise-smooth curves on the Riemann sphere are defined in the same way: we say that an infinite curve $\Gamma$ is piecewise-smooth if the image $\widetilde{\Gamma}$ of this curve under the linear-fractional transformation $z \rightarrow z /(z-a)$, where $a \notin \Gamma$, lying in a finite part of the plane, is a piecewise-smooth curve in the sense of the definition from Sec. 2.5. For any such curve, the point $\infty$ belongs to its boundary $\partial \Gamma$. All the corresponding terminology of Sec. 2.5 is preserved unchanged. In particular, classes $C_{*}^{\mu}(\widehat{D})$ are defined for domains with infinitely smooth boundaries.

Consider radial arcs with edge $\tau=\infty$ in detail. Recall their definition: we say that an arc $\Gamma$ is radial with respect to the said edge if the arc $\widetilde{\Gamma}$ is radial with respect to the edge $\tau=0$. If the second edge of the arc $\Gamma$ does not coincide with the point $z=0$, then, similarly to (2.5.5), this arc can be defined by the parametrization

$$
\gamma(r)=\frac{e^{i f(r)}}{r}, \quad 0<r \leq \rho
$$

where $f$ is a real function from $C[0, \rho]$ such that it is continuously differentiable on $(0, \rho]$ and $r f^{\prime}(r) \rightarrow 0$ as $r \rightarrow 0$. Indeed, under the inversion $z \rightarrow 1 / \bar{z}$, this relation passes to (2.5.5). Frequently, it is convenient to replace $f(r)$ by $f(1 / r)$ to define this arc in the form

$$
\gamma(r)=r e^{i f(r)}, \quad r \geq \rho,
$$

where $f(r)$ is a continuously differentiable on $[\rho, \infty)$ function such that

$$
\lim _{r \rightarrow \infty} \theta(r)=\theta_{\infty}, \quad \lim _{r \rightarrow \infty} r \theta^{\prime}(r)=0 .
$$

The limit case is an infinite arc with edges $\tau=0$ and $\tau=\infty$ such that it is radial with respect to both edges. It is defined by the radial parametrization (2.6.13) with respect to the interval $(0, \infty)$, where the function $\theta(r)$ satisfies not only (2.6.14), but similar conditions as $r \rightarrow 0$, i.e.,

$$
\lim _{r \rightarrow 0} \theta(r)=\theta_{0}, \quad \lim _{r \rightarrow 0} r \theta^{\prime}(r)=0 .
$$

\subsection{Homogeneous Spaces $C_{0}^{\mu}(G)$}

It is obvious that seminorm (2.1.2) is invariant with respect to translations $x \rightarrow x+a$. Hence, the space $C^{\mu}$ possesses the same property. The relation

$$
\{\varphi\}_{\mu}=\sup _{x, y \in G} \frac{|x|^{\mu}|\varphi(x)-\varphi(y)|}{|x-y|^{\mu}}
$$

defines a seminorm possessing the same property with respect to extensions $x \rightarrow r x, r>0$. Here, the point $x=0$ does not belong to the domain of the function $\varphi$, i.e., this function is defined on $G \backslash 0$. Since the points $x$ and $y$ can be interchanged, the factor $|x|^{\mu}$ under the supremum sign can be replaced by the symmetric expression $\max \left[|x|^{\mu},|y|^{\mu}\right]$.

As in Sec. 2.1, Definition (2.7.1) immediately imply the following relations similar to (2.1.6):

$$
\begin{gathered}
\text { (a) }\{\varphi \psi\}_{\mu} \leq|\varphi|_{0}\{\psi\}_{\mu}+\{\varphi\}_{\mu}|\psi|_{0}, \\
\text { (b) }\{f \circ \varphi\}_{\mu, G} \leq[f]_{1, D}\{\varphi\}_{\mu, G}, \quad \varphi(G) \subseteq D,
\end{gathered}
$$




$$
\text { (c) }\{\varphi \circ \alpha\}_{\mu, \widetilde{G}} \leq\left(M[\alpha]_{1, \widetilde{G}}\right)^{\mu}\{\varphi\}_{\mu, G}, \quad \alpha(\widetilde{G}) \subseteq G,
$$

where $M=\sup _{x \in \widetilde{G}}\left(|\alpha(x)|^{-1}|x|\right)$.

In the same way, the definition immediately implies the interpolational property

$$
\{\varphi\}_{\mu} \leq[\varphi]_{0}^{1-\mu / \nu}\{\varphi\}_{\nu}^{\mu / \nu}, \quad 0 \leq \mu \leq \nu,
$$

of the considered seminorm, similar to (2.1.7).

Introduce the space $C_{0}^{\mu}(G)$ of all bounded on $G \backslash 0$ functions $\varphi$ such that the seminorm $\{\varphi\}_{\mu}$ is finite. As in Sec. 2.2, we show that it is a Banach space with respect to the norm

$$
|\varphi|=|\varphi|_{0}+\{\varphi\}_{\mu} .
$$

For $\mu=1$, we denote this space by $C_{0}^{1,0}$, preserving the symbol $C_{0}^{1}$ for other purposes.

The points 0 and $\infty$, which are limit points for $G$, play a special role for functions $\varphi$ from $C_{0}^{\mu}(G)$. It is obvious that $\varphi$ satisfies the Hölder condition with power $\mu$ outside neighborhoods of these points and remains to be bounded, approaching $\tau$. If neither 0 nor $\infty$ is a limit point, i.e., if the set $G$ is contained in the spherical layer $\delta \leq|x| \leq \delta^{-1}, \delta>0$, then seminorms (2.1.2) and (2.7.1) are equivalent and, therefore, the spaces $C^{\mu}$ and $C_{0}^{\mu}$ coincide.

For example, verify the relation

$$
\sin (\ln |x|) \in C_{0}^{\mu}(B), \quad B=\{x,|x| \leq 1\} .
$$

Indeed, the function $\rho(x)=|x|^{\mu}$ can be taken instead of $\rho_{\mu}$ in (2.7.1) for this case. Hence, it suffices to estimate the difference relation

$$
\frac{|x|^{\mu}|\sin (\ln |x|)-\sin (\ln |y|)|}{|x-y|^{\mu}} \leq \frac{|x|^{\mu}|\ln | x|-\ln | y||}{\left.|| x|-| y\right|^{\mu}}=\frac{t^{\mu}|\ln t|}{|1-t|^{\mu}}, \quad t=\frac{|x|}{|y|} .
$$

Since the expression on the right-hand side of this inequality treated as a function of $t$ is bounded on the semiaxis $\{t>0\}$, it follows from Definition (2.7.4) that (2.7.5) holds.

As in Sec. 2.2, using the interpolational inequality (2.7.3), we show that the inequality

$$
|\varphi|_{C_{0}^{\mu}} \leq 2|\varphi|_{C_{0}^{\nu}}
$$

(similar to $(2.2 .3))$ holds provided that $\mu<\nu \leq 1$, which implies the embedding $C_{0}^{0, \nu} \subseteq C_{0}^{0, \mu}$ of Banach spaces.

From the relation $(2.7 .2)\left(\right.$ a) it follows that $C_{0}^{\mu}(G)$ is a Banach algebra with respect to pointwise operations. In the same way, the relation $(2.7 .2)(\mathrm{b})$ means that if a (vector) function $\varphi$ belongs to $C_{0}^{\mu}(G)$ and a function $f$ belongs to $C^{0,1}(\widetilde{G})$, where $\widetilde{G}$ contains the image $\varphi(G)$, then the superposition $f \circ \varphi$ belongs to $C_{0}^{\mu}(G, F)$. In particular, the inequality

$$
\inf _{G}|\varphi(x)|>0
$$

is a necessary and sufficient condition of the invertibility of the scalar complex function $\varphi$ in the Banach algebra $C_{0}^{\mu}$. Indeed, if this condition is satisfied, then there exists a positive (sufficiently small) $\delta$ such that the ring $\delta<|z|<1 / \delta$ of the complex plane contains the image $\varphi(G)$ of the function $\varphi$. It remains to note that the function $f(z)=1 / z$ satisfies the Lipschitz condition in this ring.

The next important lemma describes the relation between seminorms (2.1.2) and (2.7.1).

Lemma 2.7.1. The space $C_{0}^{\mu}(G)$ consists of all bounded functions $\varphi$ such that the function $\psi(x)=$ $|x|^{\mu} \varphi(x)$ satisfies the Hölder condition with power $\mu$ on $G$. The relation

$$
|\varphi|=|\psi(c)|+[\psi]_{\mu},
$$

where $c$ is a fixed point of the set $G$, defines an equivalent norm in $C_{0}^{\mu}(G)$. 
Proof. If $\varphi \in C_{0}^{\mu}(G)$, then

$$
|\psi(x)-\psi(y)| \leq|x|^{\mu}|\varphi(x)-\varphi(y)|+\left.|\varphi(y)||| x\right|^{\mu}-|y|^{\mu} \mid .
$$

Then, taking into account (2.1.3), we obtain the estimates

$$
[\psi]_{\mu} \leq\{\varphi\}_{\mu}+|\varphi|_{0}, \quad|\psi(c)| \leq|c|^{\mu}|\varphi|_{0} .
$$

Conversely, let $\varphi$ be a bounded function such that the function $\psi(x)=|x|^{\mu} \varphi(x)$ satisfies the Hölder condition with power $\mu$ on $G$. Then there exists a positive constant $C$ independent of $\varphi$ such that

$$
|\varphi(x)|=|\psi(x)||x|^{-\mu} \leq C\left(|\psi(c)|+[\psi]_{\mu}\right), \quad x \in G .
$$

Indeed, if $0 \in \bar{G}$, then $|\psi(x)| \leq[\psi]_{\mu}|x|^{\mu}$ and the claimed estimate is obvious. If $0 \notin \bar{G}$, then there exists a neighborhood of the point $\tau=0$ such that the set $G$ does not intersect it and, therefore, both functions $|x|^{-\mu}$ and $|x-c|^{\mu}|x|^{-\mu}$ are bounded on $G$. Therefore, the inequality

$$
|\psi(x)||x|^{-\mu} \leq\left(|\psi(c)|+[\psi]_{\mu}|x-c|^{\mu}\right)|x|^{-\mu}
$$

shows that the estimate (2.7.10) also holds in this case.

To estimate the seminorm $\{\varphi\}_{\mu}$, assume that $x \in G, y \in G$, and

$$
1 / 2 \leq|x|^{-1}|y| \leq 2
$$

Then, taking into account (2.7.10), we have the inequality

$$
\frac{|x|^{\mu}|\varphi(x)-\varphi(y)|}{|x-y|^{\mu}}=\frac{\left.|| y\right|^{\mu} \psi(x)-|x|^{\mu} \psi(y) \mid}{|y|^{\mu}|x-y|^{\mu}} \leq[\psi]_{\mu}+C\left(|\psi(c)|+[\psi]_{\mu}\right) M, \quad M=\frac{\left.|x|^{\mu}|| x\right|^{\mu}-|y|^{\mu} \mid}{|y|^{\mu}|x-y|^{\mu}} .
$$

It is obvious that

$$
M \leq \frac{\left.|x|^{\mu}|| x\right|^{\mu}-|y|^{\mu} \mid}{\left.|y|^{\mu}|| x|-| y\right|^{\mu}}=\frac{s^{\mu}\left|s^{\mu}-1\right|}{|s-1|^{\mu}}, \quad s=\frac{|x|}{|y|},
$$

and, by virtue of (2.7.11), the value of $M$ is bounded by a constant depending only on $\mu$.

If the condition (2.7.11) is violated, then, taking into account the fact that $s^{\mu}|1-s|^{-\mu} \leq 2^{\mu}$ for $0<s<1 / 2$ and for $s>2$, we conclude that

$$
\frac{|x|^{\mu}|\varphi(x)-\varphi(y)|}{|x-y|^{\mu}} \leq 2|\varphi|_{0} \frac{|x|^{\mu}}{\left.|| x|-| y\right|^{\mu}} \leq 2^{1+\mu}|\varphi|_{0} .
$$

Combining the obtained inequalities and taking into account (2.7.10), we obtain the estimate

$$
\{\varphi\}_{\mu} \leq \max \left(C+M, 2^{1+\mu} C\right)\left(|\psi(c)|+[\psi]_{\mu}\right) .
$$

Combining it with (2.7.9), we complete the proof of the lemma.

The next two theorem add two different descriptions of the space $C_{0}^{\mu}$ in terms of $C^{\mu}$.

Theorem 2.7.1. Let $0<\delta<1$ and $G_{j}=\left\{\delta<|y|<\delta^{-1}, \delta^{j} y \in G\right\}, j=0, \pm 1, \ldots$ Then the space $C_{0}^{\mu}(G)$ can be defined by the equivalent norm

$$
|\varphi|=|\varphi|_{0}+\sup _{j}\left[\varphi_{j}\right]_{\mu, G_{j}}, \quad \varphi_{j}(y)=\varphi\left(\delta^{j} y\right) .
$$

Note that norm (2.7.12) has a sense for any function $\varphi$ satisfying the Hölder condition with power $\mu$ on $G$ outside any neighborhood of the points 0 and $\infty$. Since the open sets $\delta^{j+1}<|y|<\delta^{j-1}$, $j=0, \pm 1, \ldots$, cover $\mathbb{R}^{k} \backslash\{0\}$, it follows from Theorem 2.1.1 that the function $\varphi$ possesses the specified property if and only if $\varphi_{j}(y)=\varphi\left(\delta^{j} y\right) \in C^{\mu}\left(G_{j}\right)$ for any $j$. If a set $G_{j}$ is empty, then $\left[\varphi_{j}\right]_{\mu, G_{j}}$ is assigned to be equal to zero. 
Proof. If $x \in G, y \in G$, and one of the inequalities $|y| \leq \delta|x|$ and $|x| \leq \delta|y|$ is satisfied, then $|x-y| \geq|x|-|y| \geq(1-\delta)|x|$ or $|x-y| \geq(1-\delta)|y|$ respectively. In both cases, we have the inequality

$$
|x|^{\mu} \frac{|\varphi(x)-\varphi(y)|}{|x-y|^{\mu}} \leq \frac{2|\varphi|_{0}}{1-\delta}
$$

Therefore, norm (2.7.4) is equivalent to the norm

$$
|\varphi|=|\varphi|_{0}+[\varphi]_{\mu}^{\prime}, \quad[\varphi]_{\mu}^{\prime}=\sup _{\delta|x| \leq|y| \leq \delta^{-1}|x|}|x|^{\mu} \frac{|\varphi(x)-\varphi(y)|}{|x-y|^{\mu}} .
$$

Let $x \in G, y \in G$, and $\left|y^{\prime}\right| \leq\left|x^{\prime}\right|$ (for definiteness). Then the points $x=\delta^{j} x^{\prime}$ and $y=\delta^{j} x^{\prime}$ belong to $G$ and $\delta^{2}|x| \leq|y| \leq|x|$. Therefore, the relation

$$
\frac{\left|\varphi_{j}\left(x^{\prime}\right)-\varphi_{j}\left(y^{\prime}\right)\right|}{\left|x^{\prime}-y^{\prime}\right|^{\mu}}=\delta^{j \mu} \frac{|\varphi(x)-\varphi(y)|}{|x-y|^{\mu}} \leq \frac{|x|^{\mu}}{\delta^{\mu}} \frac{|\varphi(x)-\varphi(y)|}{|x-y|^{\mu}}
$$

holds, which implies the estimate of norm (2.7.13) via norm (2.7.12), where $\delta$ is to be replaced by $\delta^{2}$.

Conversely, let $x \in G, y \in G$, and $\delta|x| \leq|y| \leq \delta^{-1}|x|$. Select an integer $j$ to satisfy the inequality $\delta^{j+1} \leq|y| \leq \delta^{j}$. Then $\delta^{j+1} \leq|x| \leq \delta^{j-1}$. Hence, the points $x^{\prime}=\delta^{-j} x$ and $y^{\prime}=\delta^{-j} x$ belong to $G_{j}$. Therefore, the relation

$$
|x|^{\mu} \frac{|\varphi(x)-\varphi(y)|}{|x-y|^{\mu}}=\left|x^{\prime}\right|^{\mu} \frac{\left|\varphi_{j}\left(x^{\prime}\right)-\varphi_{j}\left(y^{\prime}\right)\right|}{\left|x^{\prime}-y^{\prime}\right|^{\mu}} \leq \frac{1}{\delta^{\mu}} \frac{\left|\varphi\left(x^{\prime}\right)-\varphi\left(y^{\prime}\right)\right|}{\left|x^{\prime}-y^{\prime}\right|^{\mu}}
$$

holds, which yields the opposite estimate of norm (2.7.13) via norm (2.7.12).

The following assertion easily follows from the proved theorem.

Lemma 2.7.2. If a function $\varphi$ from $C^{1}\left(\mathbb{R}^{k} \backslash 0\right)$ is bounded and its gradient $\varphi^{\prime}$ admits an estimate

$$
\left|\varphi^{\prime}(x)\right| \leq C /|x|
$$

then $\varphi \in C_{0}^{0,1}\left(\mathbb{R}^{k}\right)$.

Proof. By virtue of (2.7.14), the sequence of functions $\varphi_{j}(x)=\varphi\left(\delta^{j} x\right), j=0, \pm 1, \ldots$, is uniformly bounded in the spherical layer $S=\{\delta<|x|<1 / \delta\}$ together with their derivatives. Since $S$ is a uniformly connected domain, it follows from Theorem 2.2.2 that these functions are also uniformly bounded with respect to the norm of the space $C^{0,1}(S)$. Therefore, by virtue of Theorem 2.7.1, the function $\varphi$ belongs to $C_{0}^{0,1}\left(\mathbb{R}^{k}\right)$.

In particular, it follows from the lemma that for any real $\alpha$, any positive $\varepsilon$, and any nonnegative integer $n$, the following relations hold:

$$
\begin{array}{ll}
|x|^{i \alpha+\varepsilon} \ln ^{n}|x| \in C_{0}^{0,1}(G), & G=\{|x| \leq R\}, \\
|x|^{i \alpha-\varepsilon} \ln ^{n}|x| \in C_{0}^{0,1}(G), & G=\{|x| \geq R\} .
\end{array}
$$

Let $\Omega$ denote the unit sphere of the space $\mathbb{R}^{k}$. It is obvious that the transformation

$$
\omega(s, u)=e^{s} u, \quad(s, u) \in \mathbb{R} \times \Omega,
$$

is a homeomorphism of $\mathbb{R} \times \Omega$ onto $\mathbb{R}^{k} \backslash 0$. The inverse map is $x \rightarrow(\ln |x|, x /|x|)$.

Theorem 2.7.2. Let a set $\widetilde{G}$ from $\mathbb{R} \times \Omega$ be the image of $G \backslash 0$ under the map $\omega^{-1}(x)=(\ln |x|, x /|x|)$. Then the operator $\psi \rightarrow \psi \circ \omega$ isomorphically maps the Banach space $C^{\mu}(\widetilde{G})$ to the Banach space $C_{0}^{\mu}(G)$. 
Proof. For the group of translations $(s, u) \rightarrow\left(s+s_{0}, u\right)$ of the set $\mathbb{R} \times \Omega$ onto itself, the corresponding analog of Theorem 2.1.1 is valid in the space $C^{\mu}(\widetilde{G})$. Namely, fix a positive $r$ and consider a sequence of sets

$$
\widetilde{G}_{j}=\{(s, u) \in(-r, r) \times \Omega \mid(s+j r, u) \in \widetilde{G}\}, \quad j=0, \pm 1, \ldots
$$

Further, define a function $\psi$ on $\widetilde{G}$ and assign the following function sequence to it:

$$
\psi_{j}(s, u)=\psi(s+j r, u), \quad(s, u) \in \widetilde{G}_{j} .
$$

Then the space $C^{\mu}(\widetilde{G})$ can be defined by the equivalent norm

$$
|\psi|=\sup _{j}\left|\psi_{j}\right|_{C^{\mu}}
$$

Assign $r=|\ln \delta|$, where $\delta$ is from Theorem 2.7.1. In the notation of this theorem, substitution (2.7.15) homeomorphically maps $\widetilde{G}_{j}$ onto $G_{j}$ and the relation $\varphi=\psi \circ \omega$ is equivalent to $\varphi_{j}=\psi_{j} \circ \omega$ for any $j$. It is immediately verified that the vector-functions $\omega$ and $\omega^{-1}$ satisfy the Lipschitz condition on the sets $[-r, r] \times \Omega$ and $S=\left\{x \in \mathbb{R}^{k}, \delta^{-1}<|x|<\delta\right\}$ respectively. Therefore, the operator $\psi \rightarrow \psi \circ \omega$ isomorphically maps the Banach space $C^{0, \mu}([-r, r] \times \Omega)$ to the Banach space $C_{0}^{n, \mu}(\bar{S})$. Therefore, norms (2.7.13) and (2.7.17) are equivalent.

An intermediate application of Theorem 2.7.2 is as follows: the space $C_{0}^{\mu}$ is invariant with respect to the involution $x^{*}=x /|x|^{2}$. Moreover, the superposition operator $\varphi(x) \rightarrow \varphi\left(x^{*}\right)$ is invertible from $C_{0}^{\mu}(G)$ to $C_{0}^{\mu}\left(G^{*}\right)$, where $G^{*}=\left\{x, x^{*} \in G\right\}$.

To prove that, it suffices to note that the involution $x \rightarrow x^{*}$ passes to the transformation $(s, u) \rightarrow$ $(-s, u)$ under substitution $(2.7 .16)$.

\subsection{Weight Spaces $C_{\lambda}^{\mu}(G, F)$}

In this and the previous sections, we treat the closure $\bar{G}$ of a set $G$ from $\mathbb{R}^{k}$ as the closure with respect to the Riemann sphere, i.e., we assume that the closure contains the point $\infty$ if the set $G$ is unbounded.

Let $F$ be a finite subset of $\bar{G}$ such that it contains $\infty$ if $G$ is unbounded. Consider the neighborhoods

$$
B_{\rho}(\tau)=\{|x-\tau| \leq \rho\}, \tau \neq \infty, \quad B_{\rho}(\tau)=\{|x| \geq 1 / \rho\}, \tau=\infty,
$$

of points of $F$, where the positive $\rho$ is sufficiently small to guarantee that these neighborhoods are pairwise disjoint.

To any function $\varphi$ defined on $G \backslash F$, assign the function family

$$
\begin{array}{ll}
\varphi_{\tau}(x)=\varphi(x+\tau), & x \in G_{\tau}=G \cap B_{\rho}(\tau)-\tau, \tau \neq \infty, \\
\varphi_{\tau}(x)=\varphi(x), & x \in G_{\tau}=G \cap B_{\rho}(\tau), \tau=\infty, \\
\widetilde{\varphi}(x)=\varphi(x), & x \in \widetilde{G}=G \backslash \bigcup_{\tau} B_{\rho / 2}(\tau) .
\end{array}
$$

Let $\lambda=\left(\lambda_{\tau}, \tau \in F\right)$ be a family of real numbers. By $C_{\lambda}^{\mu}(G, F)$ denote the class of all functions $\varphi$ such that $\widetilde{\varphi}_{\tau}(x)=|x|^{-\lambda_{\tau}} \varphi_{\tau}(x) \in C_{0}^{\mu}\left(G_{\tau}\right), \tau \in F$, and $\widetilde{\varphi} \in C^{\mu}(\widetilde{G})$. It is obvious that this is a Banach space with respect to the norm

$$
|\varphi|=\max _{\tau}\left|\widetilde{\varphi}_{\tau}\right|_{C_{0}^{\mu}\left(G_{\tau}\right)}+|\widetilde{\varphi}|_{C^{\mu}(\widetilde{G})} .
$$

If $\mu=1$, then this space is denoted by $C_{\mu}^{0,1}(G, F)$. Sometimes, it is convenient to include points $\tau$ to the set $F$ such that $\tau \notin \bar{G}$. In this case, (2.8.2) and (2.8.3) are treated with respect to $\tau$ from $F \cap \bar{G}$. For consistency, it is also convenient to consider the space $C_{\lambda}^{\mu}(G, F)$ for the empty set $F$; in this case, this space is identified with $C^{\mu}(G)$. 
In this notation, the space $C_{0}^{\mu}(G)$ (see the previous section) can be represented in the form

$$
C_{0}^{\mu}(G)= \begin{cases}C_{0}^{\mu}(G, 0), & 0 \in \bar{G}, G \subseteq\{|x| \leq R\}, \\ C_{0}^{\mu}(G, \infty), & \infty \in \bar{G}, G \subseteq\{|x| \geq R\}, \\ C_{0}^{\mu}(G ; 0, \infty), & 0, \infty \in \bar{G}\end{cases}
$$

From the definition, we see that the space $C_{0}^{\mu}(G, F)$ consists of bounded functions and is a Banach algebra with respect to multiplication (since this is valid for the spaces $C^{\mu}$ and $C_{0}^{\mu}$ introduced in Secs. 2.2 and 2.7). In the same way, we conclude that if $\varphi \in C_{0}^{\mu}(G, F)$ and a function $f$ satisfies the Lipschitz condition on the image $\varphi(G)$, then $f \circ \varphi$ also belongs to $C_{0}^{\mu}(G, F)$. In particular, for complex functions $\varphi$, the condition (2.7.7) is necessary and sufficient for the invertibility of $\varphi$ in the algebra $C_{0}^{\mu}$ (cf. Sec. 2.7).

Arguing in the same way and taking into account (2.2.3), (2.7.6), and (2.7.15), we conclude that the family $C_{\lambda}^{\mu}(G, F)$ of Banach spaces monotonously decreases (in the embedding sense) with respect to both parameters $\mu$ and $\lambda_{\tau}, \tau \neq \infty$, and monotonously increases with respect to $\lambda_{\infty}$. From Definition (2.8.2)-(2.8.3), it immediately follows that the product of functions, treated as a bilinear map $\left(\varphi_{1}, \varphi_{2}\right) \rightarrow \varphi_{1} \varphi_{2}$ from $C_{\lambda_{1}}^{\mu} \times C_{\lambda_{2}}^{\mu}$ to $C_{\lambda_{1}+\lambda_{2}}^{\mu}$, is bounded. This is the reason to call $C_{\lambda}^{\mu}$ the weight space and to call the family $\lambda=\left(\lambda_{\tau}, \tau \in F\right)$ the weight order. If $\lambda_{\tau}$ is independent of $\tau$, then the weight order is identified with a real number.

If a function $\rho$ does not vanish on $G \backslash F$, then we call it a weight function for the space $C_{\lambda}^{\mu}$ provided that $\rho^{ \pm 1} \in C_{ \pm \lambda}^{\mu}$. It is obvious that the operator $\varphi \rightarrow \rho \varphi$ of the multiplication by this weight function isomorphically maps the Banach space $C_{0}^{\mu}$ to the Banach space $C_{\lambda}^{\mu}$ and $C_{\lambda^{\prime}}^{\mu}$ to $C_{\lambda+\lambda^{\prime}}^{\mu}$.

A simple example of a weight function is the function

$$
\rho_{\lambda}(x, F)=\prod_{\tau \in F} \rho_{\lambda_{\tau}}(x, \tau)
$$

where

$$
\rho_{\delta}(x, \tau)= \begin{cases}|x-\tau|^{\delta}(1+|x|)^{-\delta}, & \tau \neq \infty, \\ (1+|x|)^{\delta}, & \tau=\infty .\end{cases}
$$

It is easy to see that this function belongs to $C_{\lambda}^{0,1}(G, F)$ for any $\lambda$. Indeed, by virtue of Lemma 2.7.2, the function

$$
a_{\tau}(x)= \begin{cases}|x|^{-\lambda_{\tau}} \rho_{\lambda}(x+\tau), & \tau \neq \infty, \\ |x|^{-\lambda_{\tau}} \rho_{\lambda}(x), & \tau=\infty,\end{cases}
$$

belongs to $C_{0}^{0,1}(B)$, where $B=\{|x| \leq \rho\}$ for $\tau \neq \infty$ and $B=\{|x| \geq \rho\}$ for $\tau=\infty$.

If there exist points $\tau$ from $F$, lying outside $\bar{G}$, then the function $\rho_{\lambda}(x, F)$ still belongs to the space $C_{\lambda}^{0,1}(G, F)$. This follows from the fact that if $F_{0} \cap \bar{G}=\varnothing$, then the function $\rho_{\lambda}\left(x, F_{0}\right)$ belongs to $C_{0}^{0,1}(G, F)$ for any weight order $\lambda$ on $F_{0}$.

In the considered case, the following analog of Lemma 2.7.1 holds.

Lemma 2.8.1. The space $C_{\mu}^{\mu}(G, F)$ consists of functions $\psi(x)$ such that they satisfy the Hölder condition with power $\mu$ and vanish at finite points $\tau$ of $F$. The relation

$$
|\psi|=|\psi(c)|+[\psi]_{\mu},
$$

where $c$ is a fixed point of the set $G$, defines an equivalent norm in $C_{\mu}^{\mu}(G, F)$.

Proof. Let $\psi$ belong to $C_{\mu}^{\mu}(G, F)$. Then, form Lemma 2.7.1 and Definition (2.8.2)-(2.8.3), we conclude that, on both sets $G \cap B(\tau), \tau \in F$, and $\widetilde{G}$, the function $\psi$ satisfies the Hölder condition with power $\mu$ and the corresponding norm estimates

$$
[\psi]_{\mu, G \cap B(\tau)}+\left|\psi\left(c_{\tau}\right)\right| \leq C|\psi|_{C_{\mu}^{\mu}}, \quad[\psi]_{\mu, \widetilde{G}}+|\psi(\widetilde{c})| \leq C|\psi|_{C_{\mu}^{\mu}}
$$


are valid for any fixed point $c_{\tau}$ from $G \cap B(\tau)$ and any fixed point $\widetilde{c}$ from $\widetilde{G}$. If $\tau \neq \infty$, then one can assign $c_{\tau}=\tau$. Thus, the term $\left|\psi\left(c_{\tau}\right)\right|$ can be excluded from the above estimate.

By virtue of Theorem 2.1.1, this implies that $\psi$ satisfies the Hölder condition with power $\mu$ on the whole set $G$ and norm (2.8.5) is estimated via the norm $|\psi|$ in $C_{\mu}^{\mu}$. One must take into account the fact that the point $c_{\tau}$ with $\tau=\infty$ and the point $\tilde{c}$ are allowed to coincide with the point $c$.

Conversely, let a function $\psi$ satisfy the Hölder condition with power $\mu$ and vanish at finite points $\tau$ from $F$. Then, by virtue of Lemma 2.7.1, the functions $|x|^{-\lambda_{\tau}} \psi_{\tau}(x)$ belong to $C_{0}^{\mu}\left(G_{\tau}\right)$. Hence, $\psi \in C_{\mu}^{\mu}(G, F)$ and the claimed norm estimates are valid by definition.

Using Lemma 2.8.1, one can extend Theorems 2.1.1 and 2.1.2 and Lemma 2.1.2 to the spaces $C_{\lambda}^{\mu}$.

\section{Theorem 2.8.1.}

(a) Let open sets $V_{j}, 1 \leq j \leq m$, cover $\bar{G}$. Let one of these sets be a neighborhood of $\infty$ in the case where $G$ is unbounded. Then the relation

$$
|\varphi|=\max _{1 \leq j \leq m}|\varphi|_{C_{\lambda}^{\mu}\left(G \cap V_{j}, F\right)}
$$

defines an equivalent norm in the space $C_{\lambda}^{\mu}(G, F)$.

(b) Let a set $G$ be a uniformly connected union $G_{1} \cup \ldots \cup G_{m}$. Let $\varphi$ belong to $C(G \backslash F)$. Then the relation

$$
|\varphi|=\max _{1 \leq j \leq m}|\varphi|_{C_{\lambda}^{\mu}\left(G_{j}, F\right)}
$$

defines an equivalent norm in the space $C_{\lambda}^{\mu}(G, F)$.

(c) If the assumptions of Lemma 2.1.2 are satisfied, then the relation

$$
|\varphi|=\max _{j=1,2}|\varphi|_{C_{\lambda}^{\mu}\left(G_{j}, \tau\right)}
$$

defines an equivalent norm in the space $C_{\lambda}^{\mu}(G, \tau)$.

Recall that the spaces $C^{\mu}(G)$ and $C^{\mu}(\bar{G})$ coincide. If $\mu=0$ (this case is stated explicitly each time), then these spaces are different. Obviously, the same is also valid for weight spaces. The only exception is the space $C^{\mu}(\widehat{D})$ in two-dimensional domains $D$ with piecewise-smooth boundaries (see Sec. 2.5) such that one-sided boundary values of its elements are treated as different ones. Theorem 2.8.1(b) allows one to introduce weighted spaces $C_{\lambda}^{\mu}(\widehat{D}, F)$ in the same way. Namely, if subdomains $D_{j} \subseteq D$ are bounded by piecewise-smooth contours and $\bar{D}=\bar{D}_{1} \cup \ldots \cup \bar{D}_{n}$, then $C_{\lambda}^{\mu}(\widehat{D}, F)$ consists of all functions $\varphi$ from $C(D)$ such that their restrictions to $D_{j}$ belong to $C_{\lambda}^{\mu}\left(D_{j}, F\right), 1 \leq j \leq n$. This space is endowed with the norm

$$
|\varphi|=\max _{j}|\varphi|_{C_{\lambda}^{\mu}\left(D_{j}\right), F}
$$

it is a Banach space with respect to this norm. This definition is independent of the choice of subdomains $D_{1}, \ldots, D_{n}$, which is proved in the same way as Lemma 2.5.3 (if we take Theorem 2.8.1(b) into account). Another way to the proof is as follows. For $\lambda=\mu$, the claimed fact follows from Lemma 2.5.3 and Lemma 2.8.1; for other weight orders, it suffices to use the multiplication by an appropriate weight function.

The following assertion is easily deduced from Lemma 2.8.1: if the set $G$ is bounded and the inequalities $\mu<\nu$ and $\lambda<\lambda^{\prime}$ are strict, then the embedding $C_{\lambda^{\prime}}^{\nu} \subseteq C_{\lambda}^{\mu}$ is compact.

Indeed, select a positive (sufficiently small) $\varepsilon$ such that the inequalities $\nu-\mu \geq \varepsilon$ and $\lambda^{\prime}-\lambda \geq \varepsilon$ are satisfied for all values of $\tau$. Then it suffices to prove the compactness of the embedding $C_{\lambda+\varepsilon}^{\mu+\varepsilon} \subseteq C_{\lambda}^{\mu}$. Multiplying these spaces by the weight function $\rho_{\mu-\lambda}$, one can assume (without loss of generality) that $\lambda=\mu$. In this case, it remains to use Lemma 2.8.1 and the compactness of the embedding $C^{\mu+\varepsilon} \subseteq C^{\mu}$ proved in Sec. 2.2.

Investigate the boundedness of the superposition operator $T(\alpha) \varphi=\varphi \circ \alpha$ in weight spaces, where $\alpha: G \rightarrow G_{1}$ is a continuous map. 
Theorem 2.8.2. Let $G \subseteq \mathbb{R}^{k}, G_{1} \subseteq \mathbb{R}^{s}$, and a map $\alpha: G \rightarrow G_{1}$ satisfy the Lipschitz condition. Let $\alpha(\infty)=\infty$ provided that $G$ is an unbounded set. Let the image $\alpha(F)$ be contained in a finite subset $F_{1}$ of $\bar{G}_{1}$ and there exist pairwise disjoint neighborhoods $U_{\tau}$ of points $\tau$ from $F$ such that the inequality

$$
|\alpha(x)-\alpha(\tau)| \geq q|x-\tau|, \quad x \in G \cap U_{\tau},
$$

where $0<q<1$, is satisfied for $\tau \neq \infty$, while the inequality

$$
|\alpha(x)| \geq q|x|, \quad x \in G \cap U_{\infty},
$$

is satisfied for $\tau=\infty$.

Then the operator $T(\alpha) \varphi=\varphi \circ \alpha$ boundedly maps $C_{\lambda_{1}}^{\mu}\left(G_{1}, F_{1}\right)$ to $C_{\lambda}^{\mu}(G, F)$, where the weight orders $\lambda$ and $\lambda_{1}$ on $F$ and $F_{1}$ (respectively) are such that $\lambda_{1}[\alpha(\tau)]=\lambda(\tau), \tau \in F$.

Note that the conditions (2.8.6) and (2.8.7) are satisfied for any Lipschitz map $\alpha$. Only (2.8.7) is to be justified. By virtue of the Lipschitz condition satisfied for $\alpha$, we have the inequality $|\alpha(x)| \leq$ $|\alpha(c)|+[\alpha]_{1}|x-c|$, where $c$ is a fixed point of $G$. Therefore, $|\alpha(x)| \leq\left(1+[\alpha]_{1}\right)|x|$ provided that $|x| \geq|\alpha(c)|+[\alpha]_{1}|c|$. Applying this arguing to the inverse map $\beta=\alpha^{-1}$, we obtain the validity of the condition (2.8.7).

Proof. We use the weight function notation (2.8.4) both with respect to the sets $G$ and $G_{1}$. If this weight function is treated as a multiplication operator, then it suffices to verify that the operator $A=\rho_{\lambda-\mu}^{-1} T(\alpha) \rho_{\lambda-\mu}$ from $C_{\mu}^{\mu}\left(G_{1}, F_{1}\right)$ to $C_{\mu}^{\mu}(G, F)$ is bounded. This operator acts as follows:

$$
(A \varphi)(x)=a(x) \varphi[\alpha(x)], \quad a(x)=\frac{\rho_{\lambda_{1}-\mu}[\alpha(x)]}{\rho_{\lambda-\mu}(x)} .
$$

Therefore, due to Lemma 2.8.1 and (2.1.6)(c), it suffices to verify that the function $a$ belongs to $C_{0}^{\mu}(G, F)$. If there exists a neighborhood of $F$ such that a subset $K$ of $G$ lies outside this neighborhood, then the image $K_{1}=\alpha(K)$ possesses the same property with respect to $F_{1}$. Therefore, $\rho_{\mu-\lambda} \in$ $C^{\mu}(K), \rho_{\lambda_{1}-\mu} \in C^{\mu}\left(K_{1}\right)$, and, due to (2.1.6)(c), the function $a$ belongs to $C^{\mu}(K)$. Thus, according to Theorem 2.8.1, it suffices to verify that $a \in C_{0}^{\mu}\left(G_{\tau}, \tau\right)$ for any point $\tau$ of $F$, where $G_{\tau}=G \cap V_{\tau}$ and $V_{\tau}$ is an appropriate neighborhood of $\tau$. More exactly, let $V_{\tau}=\{|x-\tau| \leq \delta\}$ for finite points $\tau$ and $V_{\tau}=\{|x| \geq 1 / \delta\}$ for $\tau=\infty$, where $\delta$ is positive and such that $V_{\tau} \subseteq U_{\tau}$.

First, consider the case where $\tau$ is finite. In this case, we have the relation

$$
a(x)=a_{0}(x)[b(x)]^{\lambda(\tau)-\mu}, \quad b(x)=\frac{|\alpha(x)-\alpha(\tau)|}{|x-\tau|},
$$

where $a_{0} \in C^{\mu}\left(G_{\tau}\right)$. It is obvious that the function $c(x)=|\alpha(x)-\alpha(\tau)|$ satisfies the Lipschitz condition and vanishes at the point $\tau$. Therefore, due to Lemma 2.8.1, it belongs to $C_{1}^{0,1}\left(G_{\tau}, \tau\right)$. Then $b(x)=|x-\tau|^{-1} c(x) \in C_{0}^{0,1}\left(G_{\tau}, \tau\right)$. By virtue of (2.8.6), this is valid for the function $b^{\lambda(\tau)-\mu}$. Hence, $a$ also belongs to $C_{0}^{\mu}\left(G_{\tau}, \tau\right)$.

Now, consider the case where $\tau=\infty$. In this case, the relation

$$
a(x)=f(x) g[\alpha(x)][b(x)]^{\lambda_{\tau}-\mu}, \quad b(x)=\frac{|\alpha(x)|}{|x|}, \quad x \in G_{\tau},
$$

holds, where the function $f(x)$ is continuously differentiable in the domain $V=\{|x| \geq 1 / \delta\}$ and its derivative satisfies the estimate $\left|f^{\prime}(x)\right| \leq C|x|^{-1}$, while the function $g(y)$ is defined in the domain $V_{1}=\{|y| \geq q / \delta\}$ (taking into account (2.8.7)) and possesses a similar property in this domain. Therefore, due to Lemma 2.7.2, the function $f$ belongs to $C_{0}^{0,1}(V, \tau)$ and, in the same way, the function $g$ belongs to $C_{0}^{0,1}\left(V_{1}, \tau\right)$. In terms of these functions, the relation (2.8.8) takes the form

$$
a(x)=f(x) h(x)[b(x)]^{\lambda_{\tau}},
$$

where $h(x)=|x|^{-\mu} g_{1}[\alpha(x)]$ and $g_{1}(y)=|y|^{\mu} g(y)$. By virtue of Lemma 2.7.1, the function $g_{1}(y)$ satisfies the Lipschitz condition on $V_{1}$. Hence, the function $g_{1}[\alpha(x)]$ satisfies this condition on $G_{\tau}$. Applying 
Lemma 2.7.1 to $g_{1} \circ \alpha$ and $\alpha$, we conclude that the functions $h(x)$ and $b(x)$ belong to $C_{0}^{0,1}\left(G_{\tau}, \tau\right)$. The values of the function $b$ lie outside the neighborhood of zero, i.e., they lie in the set, where the function $|t|^{\lambda_{\tau}}$ satisfies the Lipschitz condition. Therefore, $b^{\lambda}$ belongs to the class $C_{0}^{0,1}$ together with $b$. Thus, all the three factors of product (2.8.9) belong to this class. Hence, $a$ also belongs to $C_{0}^{0,1}\left(G_{\tau}, \tau\right)$.

Theorem 2.8.2 can be complemented by superposition operators defined by the inversion. Since any inversion can be expanded as a superposition of the inversion $x \rightarrow x^{*}$ and a translation satisfying the assumption of Theorem 2.8.2, it follows that it suffices to consider the case where $\alpha(x)=x^{*}$.

Lemma 2.8.2. Let a map $\alpha(x)$ be the inversion $x^{*}=x /|x|^{2}$ and the point $x=0$ either belong to $F$ or be located outside $\bar{G}$. Then $T(\alpha)$ is a bounded and invertible operator from $C_{\lambda^{*}}^{\mu}\left(G^{*}, F^{*}\right)$ to $C_{\lambda}^{\mu}(G, F)$ with respect to $G^{*}=\alpha(G), F^{*}=\alpha(F)$, and the weight order

$$
\lambda_{\tau}^{*}=\left\{\begin{aligned}
\lambda_{\tau^{*}}, & \tau \neq 0, \infty, \\
-\lambda_{\tau^{*}}, & \tau=0, \infty .
\end{aligned}\right.
$$

Proof. Let $F_{0}=F \cap\{0, \infty\}, F_{1}=F \backslash F_{0}$, and $G_{k}=G \cap U_{k}, k=0,1$, in notation (2.8.1), where

$$
U_{0}=\bigcup_{\tau \in F_{0}} B_{\rho}(\tau), \quad U_{1}=\mathbb{R}^{k} \backslash \bigcup_{\tau \in F_{0}} B_{\rho / 2}(\tau) .
$$

According to Theorem 2.8.2, T( $\alpha)$ is a bounded and invertible operator from $C_{\lambda^{*}}^{\mu}\left(G_{1}^{*}, F_{1}^{*}\right)$ to $C_{\lambda}^{\mu}\left(G_{1}, F_{1}\right)$. Since $\left[B_{\rho}(\tau) \cap G\right]^{*}=B_{\rho}\left(\tau^{*}\right) \cap G^{*}$, it follows that the same assertion for $G_{0}$ follows from the remark to Theorem 2.7.2 and Definition (2.8.2)-(2.8.3). Hence, it remains to use Theorem 2.8.1(a).

According to Lemma 2.6.2, Theorem 2.8.2 together with Lemma 2.8.2 cover all maps Lipschitz with respect to the distance on the Riemann sphere. Applying the same arguing to Lemma 2.8.1, we see that, in the notation of Sec. 2.6, the space $C_{-\mu}^{\mu}(G, \infty)$ coincides with the subspace $C_{*}^{\mu}(G)$ of functions vanishing at the point $\tau=\infty$. In particular, for $\lambda^{\prime}>\lambda$ and $0<\mu<\nu \leq 1$, the embedding $C_{\lambda}^{\nu}(G, \infty) \subseteq C_{\lambda^{\prime}}^{\mu}(G, \infty)$ is compact.

The following standard way to introduce weight spaces is broadly propagated. If $X(G)$ is a main Banach space of functions defined on a set $G$ and $\rho$ is a weight function positive on $G$, then the weight space $X(G, \rho)$ is defined by the condition $\rho \varphi \in X(G)$. This is a Banach space with respect to the corresponding norm

$$
|\varphi|=|\rho \varphi|_{X(G)}
$$

For Hölder spaces, $X=C^{\mu}$ or any its subspace $\widetilde{C}^{\mu}(G)$ of a finite codimension is usually selected. For example, let the latter subspace be the class of all functions $\varphi$ satisfying the Hölder condition with power $\mu$ and vanishing at the endpoints $\tau$ from $F$, endowed with norm (2.8.5). Then, due to Lemma 2.8.1, the space $\widetilde{C}^{\mu}\left(G, \rho_{\lambda-\mu)}\right.$ coincides with $C_{\lambda}^{\mu}(G, F)$. This leads (see Chap. 5 below) to the following phenomenon: for classical Fredholm operators on a piecewise-smooth curve, considered in the weight space $\widetilde{C}^{\mu}\left(G, \rho_{\delta}\right)$, the Fredholm property criterion depends not only on $\delta$, but also on $\mu$.

The weight spaces $C_{\lambda}^{\mu}$ in the above form are introduced in [65], where their main properties are described; they are contained in this section and two following ones.

\subsection{Hölder Spaces of Differentiable Functions}

Consider the class $C^{n}(D)$ of functions $n$ times differentiable in a domain $D$. Recall that $C^{0}(\bar{D})=$ $C^{0,0}(\bar{D})$ is the Banach space of functions continuous and bounded in the closed domain $\bar{D}$. According to definitions of Sec. 2.2, gradient (2.2.11) is called the derivative of the function $\varphi$ from $C^{1}$ though (2.2.11) is a vector, while the derivative at a point $a$ from $D$ is usually treated as a linear map 
$\xi \rightarrow \varphi^{\prime}(a) \xi$ in $\mathbb{R}^{k}$. The notation $C^{1}$ covers also vector-functions: in this case, $\varphi^{\prime}$ is a matrix with the columns $\partial \varphi / \partial x_{i}$. Similarly to (2.2.11), an ordered (in any way) collection

$$
\varphi^{(m)}=\left(\frac{\partial^{\alpha} \varphi}{\partial x^{\alpha}}, \quad|\alpha|=m\right)
$$

of $m$-order partial derivatives can be introduced for functions $\varphi$ from $C^{n}(D)$; the obtained vector $\varphi^{(m)}$ is also called the derivative of order $m$. For $m=0$, we assign $\varphi^{(0)}=\varphi$.

By $C^{n, \mu}(\bar{D}), 0 \leq \mu \leq 1$, denote the space of functions $\varphi$ from $C^{n}(D)$ such that all their partial derivatives $\varphi^{(m)}, m \leq n$, are bounded, can be extended to the boundary of the domain $D$ as continuous functions, and belong to the class $C^{0, \mu}(\bar{D})$. The notation of these derivatives is preserved for their limit values at boundary points. The continuous extendability requirement has a sense only for $\mu=0$. If $0<\mu \leq 1$, then it is already satisfied (due to the requirement for $\mu$ ) and $C^{n, \mu}(D)=C^{n, \mu}(\bar{D})$. This is a Banach space with respect to the norm

$$
|\varphi|=\sum_{m \leq n}\left|\varphi^{(m)}\right|_{C^{0, \mu}}
$$

Indeed, if $\left\{\varphi_{s}\right\}$ is a fundamental sequence in $C^{n, 0}(\bar{D})$ and $\alpha$ is a multi-index such that its order $|\alpha|$ does not exceed $n$, then the sequence $\partial^{\alpha} \varphi_{s} / \partial x^{\alpha}$ converges as with respect to the sup-norm as $s \rightarrow \infty$. Denote its limit by $\varphi^{\alpha}$. Then (as is well known from the general course of calculus) the function $\varphi=\varphi^{0}$ belongs to the class $C^{n}$ and its corresponding partial derivatives coincide with $\varphi^{\alpha}$. Hence, $\varphi$ belongs to the space $C^{n, \mu}(\bar{D})$ and $\varphi_{s} \rightarrow \varphi$ in this space.

In the sequel, unless otherwise stated, it is assumed that $\mu>0$ and, therefore, we may write $D$ instead of $\bar{D}$. The only exception is the space $C^{n, \mu}(\widehat{D})$ for two-dimensional piecewise-smooth open sets $D$, defined from $C^{\mu}(\widehat{D})$ as above.

Similarly to $(2.2 .18)$, the space $C^{n, \mu}(D)$ can be introduced inductively with respect to $n$ by means of the conditions that $\varphi$ and $\varphi^{\prime}$ belong to $C^{n-1, \mu}(\bar{D})$. Its norm is defined as follows:

$$
|\varphi|=|\varphi|_{C^{n-1, \mu}}+\left|\varphi^{\prime}\right|_{C^{n-1, \mu}}
$$

Sequentially applying this relation, we obtain expression (2.9.1).

According to the remark at the end of Sec. 2.6, the sphere $\overline{\mathbb{R}^{k}}$ can be treated as a $C^{\infty}$-manifold and, respectively, the space $C_{*}^{n, \mu}(D)$ can be introduced by means of charts (2.6.8). If the set $D$ is bounded, then the notation $C^{n, \mu}(D)$ can be used instead of $C_{*}^{n, \mu}(D)$.

It is convenient to combine all properties of the introduced space in one theorem, assuming (according to Sec. 2.2) that $0<\mu \leq 1$. If $\mu=0$, then this result is also valid for spaces considered in the closure of the domain.

\section{Theorem 2.9.1.}

(a) If $\mu<\nu \leq 1$, then the embedding $C^{n, \mu}(D) \subseteq C^{n, \nu}(D)$ of Banach spaces takes place. If the domain $D$ is uniformly connected, then the embedding $C^{n, 0}(\bar{D}) \subseteq C^{n-1,1}(\bar{D})$ also holds.

(b) The product of functions is bounded as the bilinear map $\left(\varphi_{1}, \varphi_{2}\right) \rightarrow \varphi_{1} \varphi_{2}$ from $C^{n, \mu} \times C^{n, \mu}$ to $C^{n, \mu}$; hence, the space $C^{n, \mu}(D)$ is a Banach algebra with respect to multiplication. If an s-vectorfunction $\varphi$ belongs to $C_{0}^{n, \mu}(D, F)$, a function $f$ belongs to $C^{n, 1}(G), G \subseteq \mathbb{R}^{s}$, and $\varphi(D) \subseteq \bar{G}$, then the superposition $f \circ \varphi$ belongs to $C^{n, \mu}(D)$. In particular, (2.7.7) is a necessary and sufficient invertibility condition for its elements.

(c) Let a vector-function $\alpha$ from $C^{n}(D)$ satisfy the Lipschitz condition, $\alpha(D) \subseteq \bar{D}_{1}$, and $\mathcal{D} \alpha \in$ $C^{n-1, \mu}(\bar{D})$ (provided that $\left.n \geq 1\right)$. Then $\varphi \rightarrow \varphi \circ \alpha$ is a bounded operator from $C^{n, \mu}\left(\bar{D}_{1}\right)$ to $C^{n, \mu}(\bar{D})$.

If the above assumption is satisfied, $\alpha$ is a Lipschitz map, and $D_{1}=\alpha(D)$ is a domain of $\mathbb{R}^{k}$, then the inverse map $\beta=\alpha^{-1}$ belongs to the class $C^{n}\left(D_{1}\right)$ and its derivative $\mathcal{D} \beta$ belongs to the class $C^{n-1, \mu}\left(D_{1}\right)$. 
Proof. The first part of assertion (a) follows from (2.2.3) and the inductive definition (2.9.2) of the norm of the space $C^{n, \mu}$. For $n=0$, the second part of assertion (a) is covered by Theorem 2.2.2. Assume that it is valid in the case where the order of the space does not exceed $n-1$ and the function $\varphi$ belongs to $C^{n, 0}, n \geq 2$. Then $\varphi^{\prime} \in C^{n-1,0} \subseteq C^{n-2,1}$ due to the induction assumption. In the same way, we prove that $\varphi \in C^{n-1,0} \subseteq C^{n-2,1}$. Hence, $\varphi$ and $\varphi^{\prime}$ belong to $C^{n-2,1}$. Then due to the inductive definition of the spaces, $\varphi \in C^{n-1,1}$ and the corresponding estimate of norms holds.

For $n=0$, assertion (b) and the first part of (c) follow from the relations (2.1.6). In the general case, as above, we use induction with respect to $n$ and assume that the specified assertions hold with respect to $C^{n-1, \mu}$. Then the inequality $|\varphi \psi| \leq C|\varphi||\psi|$ holds for the norm in $C^{n-1, \mu}$, where $C$ is a positive constant independent of $\varphi$ and $\psi$. Differentiating the product, we obtain the relation $(\varphi \psi)^{\prime}=\varphi^{\prime} \psi+\varphi \psi^{\prime}$. From this, taking into account (2.9.1), we deduce a similar estimate for the norm in $C^{n, \mu}$. In the same way, differentiating the superposition of functions, we obtain the relation $\mathcal{D}(f \circ \varphi)=(\mathcal{D} f \circ \varphi) \mathcal{D} \varphi$, where the right-hand side is the product of Jacobi matrices. Due to the induction assumption, this implies that $\mathcal{D}(f \circ \varphi) \in C^{n-1, \mu}$ and, therefore, $f \circ \varphi \in C^{n, \mu}$. The first part of assertion (c) is proved in the same way.

Pass to the second part of (c). From Lemma 2.3.1, it follows that $\beta$ is a continuously differentiable map and its derivative treated as a Jacobi matrix is related to $\mathcal{D} \alpha$ as follows:

$$
\mathcal{D} \beta=(\mathcal{D} \alpha \circ \beta)^{-1} \text {. }
$$

Since $\beta$ is a Lipschitz map and the matrix-function $\mathcal{D} \alpha$ and its inverse belong to the class $C^{\mu}(D)$, it follows from (2.9.3) that the function $\mathcal{D}$ belongs to $C^{\mu}\left(D_{1}\right)$. Further, we use induction and assume that there exists $m \in[1, n)$ such that $\mathcal{D} \beta \in C^{m-1, \mu}\left(D_{1}\right)$. Then, by virtue of assertion (c) applied to $\beta$, the function $\mathcal{D} \alpha \circ \beta$ belongs to $C^{m, \mu}\left(D_{1}\right)$; hence, by virtue of $(2.9 .3), \mathcal{D} \beta$ also belongs to $C^{m, \mu}\left(D_{1}\right)$. Thus, after a finite number of steps, we obtain that $\mathcal{D} \beta \in C^{n-1, \mu}\left(D_{1}\right)$.

Similarly to (2.2.4), it is convenient to introduce the class

$$
C^{n, \mu+0}=\bigcup_{\varepsilon>0} C^{n, \mu+\varepsilon}, \quad 0 \leq \mu<1 .
$$

For $\mu=0$, it is denoted by $C^{n,+0}$. Obviously, this class is an algebra with respect to multiplication and all assertions (b) and (c) of the theorem are still valid (apart from the boundedness of the operator in the last assertion).

In the notation of Theorem 2.4.1, the class $C^{n, \mu}$ of smooth surfaces (or curves) can be introduced by the following condition: $f(\tilde{u}) \in C^{n, \mu}$ in the ball $|\tilde{u}| \leq \rho$ with respect to any point $a$ from $\Gamma$. For surfaces with boundaries, defined by the parametrization $\gamma: \bar{G} \rightarrow \Gamma$, this class is introduced by the following condition: $\gamma \in C^{n, \mu}(\bar{G})$.

In the same way, on surfaces $\Gamma$ from $C^{n, \mu}, n \geq 1$, one can use a parametrization $\gamma$ from $C^{n, \mu}(G)$ to introduce the class $C^{n, \mu}(\Gamma)$ of differentiable functions; this is done by the following condition: $\varphi \circ \gamma \in C^{n, \mu}(G)$. This definition is coordinated with the similar class for $k$-dimensional regions in the following sense: if $\Gamma$ is contained in a closed subdomain $\bar{D}$ of $\mathbb{R}^{k}$, then the restriction operator $\left.\varphi \rightarrow \varphi\right|_{\Gamma}$ boundedly maps $C^{n, \mu}(D)$ to $C^{n, \mu}(\Gamma)$. To prove this, one can use induction with respect to $n$ and the following differentiation rule for superpositions of functions: $\mathcal{D}(\varphi \circ \gamma)=[(\mathcal{D}) \varphi \circ \gamma] \mathcal{D} \gamma$.

Similarly to (2.9.2), inductively define the homogeneous space $C_{0}^{n, \mu}(D)$ by the conditions

$$
\varphi(x), \psi(x)=|x| \varphi^{\prime}(x) \in C_{0}^{n-1, \mu}(D) .
$$

It is a Banach space with respect to the norm

$$
|\varphi|=|\varphi|_{C_{0}^{n-1, \mu}}+|\psi|_{C_{0}^{n-1, \mu}} .
$$

Theorem 2.9.2. In the claims of Theorem 2.7 .1 and Theorem 2.7.2, the symbol $C^{\mu}$ can be replaced by the symbol $C^{n, \mu}$. 
Proof. According to the inductive definition (2.9.5), assume that the claim of Theorem 2.7.1 is valid for the space $C_{0}^{n-1, \mu}(D)$. Let $|\varphi|_{(n)}$ denote norm (2.9.6) and let

$$
|\varphi|_{(n)}^{1}=\sup _{j}\left|\varphi_{j}\right|_{C^{n, \mu}}
$$

with respect to the sequence $\left\{\varphi_{j}\right\}$ from (2.7.12). Then, according to the specified inductive definition of norms, we have the relations

$$
|\varphi|_{(n)}=|\varphi|_{(n-1)}+|\psi|_{(n-1)}, \quad|\varphi|_{(n)}^{1}=|\varphi|_{(n-1)}^{1}+\left|\varphi^{\prime}\right|_{(n-1)}^{1} .
$$

It is obvious that the corresponding sequence $\left\{\psi_{j}\right\}$ is related to $\left\{\varphi_{j}\right\}$ as follows:

$$
\psi_{j}(x)=|x| \varphi_{j}^{\prime}(x), \quad x \in D_{j} .
$$

For any $m$, the functions $|x|^{ \pm 1}$ belong to $C^{m, \mu}$ in the spherical layer $\left\{\delta<|x|<\delta^{-1}\right\}$. From the proof of Theorem 2.9.1, we see that the constant $C_{m}$ from the estimate

$$
|a \varphi|_{C^{m, \mu}} \leq C_{m}|a|_{C^{m, \mu}}|\varphi|_{C^{m, \mu}}
$$

where $a$ and $\psi$ belong to $C^{n, \mu}(D)$, is independent of the set $D$. Assigning $a(x)=|x|^{ \pm 1}$ and applying (2.9.8), we obtain the two-side estimates

$$
|\psi|_{n-1}^{1} \leq C\left|\varphi^{\prime}\right|_{n-1}^{1}, \quad\left|\varphi^{\prime}\right|_{n-1}^{1} \leq C|\psi|_{n-1}^{1}
$$

This, (2.9.7) and the induction assumption imply the validity of the lemma for the space $C_{0}^{n, \mu}(D)$.

Pass to Theorem 2.7.2. It is obvious that the transformation $\omega$ in (2.7.16) homeomorphically maps $\mathbb{R} \times \Omega$ onto $\mathbb{R}^{k} \backslash 0$ and the inverse map is $x \rightarrow(\ln |x|, x /|x|)$. Spaces $C^{n, \mu}$ can be naturally introduced on domains of the unit sphere $\Omega$ of the space $\mathbb{R}^{k}$. To do this, it is not necessary to use the structure of $\Omega$ as a differentiable manifold. For any function $\varphi$ defined in a neighborhood of a point $a$ of the sphere $\Omega$, assign

$$
\varphi^{\prime}(a)=\left(\frac{\partial \widetilde{\varphi}}{\partial x_{1}}, \ldots, \frac{\partial \widetilde{\varphi}}{\partial x_{k}}\right)(a)
$$

where $\widetilde{\varphi}$ is the following extension of $\varphi$ to a neighborhood of the point $a$ of the space $\mathbb{R}^{k}: \widetilde{\varphi}(x)=$ $\varphi(x /|x|)$. Then, for any open subset $G$ of $\Omega$, the space $C^{n, \mu}(G)$ still can be obtained by induction. If $G \subseteq \mathbb{R} \times \Omega$, then this space has a similar sense.

Arguing as in the proof of Theorem 2.7.2, we see that it suffices to verify that the operator $\psi \rightarrow \psi \circ \omega$ is an isomorphism of the Banach spaces

$$
C^{n, \mu}([-r, r] \times \Omega) \rightarrow C_{0}^{n, \mu}(S) .
$$

As we note above, the vector-functions $\omega$ from $C^{\infty}([-r, r] \times \Omega)$ and $\alpha=\omega^{-1}$ from $C^{\infty}(\bar{S})$ satisfy the Lipschitz condition on $[-r, r] \times \Omega$ and $S$ respectively. Therefore, the specified assertion follows from Theorem 2.9.1(c). For the manifold $[-r, r] \times \Omega$, it is proved in the same way; just the differentiation relations

$$
\frac{\partial \varphi}{\partial x_{i}}=\frac{\partial \widetilde{\psi}}{\partial s} \frac{x_{i}}{|x|^{2}}+\frac{\partial \widetilde{\psi}}{\partial u_{i}} \frac{|x|^{2}-x_{i}^{2}}{|x|^{3}}
$$

for the function $\varphi(x)=\psi(\ln |x|, x /|x|)$, following from Definition (2.9.9), are to be taken into account.

Let $\mathcal{H}_{\lambda}, \lambda \in \mathbb{R}$, denote the class of all functions $Q(\xi)$ from $C^{\infty}\left(\mathbb{R}^{k} \backslash 0\right)$ such that they are homogeneous functions of power $\lambda$, i.e., they are such that $Q(r \xi)=r^{\lambda} Q(\xi), r>0$. It is easy to see that the differentiation operation $Q \rightarrow Q^{\prime}$ acts from $\mathcal{H}_{\lambda}$ to $\mathcal{H}_{\lambda-1}$ and, therefore, the weight operation $Q(\xi) \rightarrow$ $|\xi| Q^{\prime}(\xi)$ is invariant in $\mathcal{H}_{\lambda}$. Since $\mathcal{H}_{0} \subseteq C_{0}^{0,1}\left(\mathbb{R}^{k}\right)$ due to Theorem 2.7.2, it follows from the inductive definition that

for any positive integer $n$.

$$
\mathcal{H}_{0} \subseteq C_{0}^{0,1}\left(\mathbb{R}^{k}\right)
$$


A simple example of functions from the class $\mathcal{H}_{1}$ is the function $Q(\xi)=|\xi|$. All its derivatives $Q^{(m)}$ of order $m$ belong to $\mathcal{H}_{1-m}$. Therefore, similarly to (2.9.1), instead of the inductive definition of the norm of the space $C_{0}^{n, \mu}$, given by (2.9.6), one can select the equivalent norm

$$
|\varphi|=\sum_{m \leq n}\left|\psi_{m}\right|_{C_{0}^{\mu}}, \quad \psi_{m}(x)=|x|^{m} \varphi^{(m)}(x) .
$$

Let a finite set $F$ be contained in $\bar{D}$ and $\infty$ belong to $F$ if the open set $D$ is unbounded. If a point belongs to $D$, then it is assumed to be an isolated boundary point for the set $D \backslash F$. For example, if the boundary $\partial D$ is compact, then $\infty$ is treated as an isolated boundary point of $D$ (on the Riemann sphere).

For functions $\varphi$ from $C^{n}(D \backslash F)$, the space $C_{\lambda}^{n, \mu}(D, F)$ can be defined by two equivalent ways. One way is similar to Sec. 2.8 and is based on the spaces $C^{n, \mu}(D)$ and $C_{0}^{n, \mu}(D)$. Another way is the inductive definition by the conditions

$$
\varphi \in C_{\lambda}^{n-1, \mu}, \quad \varphi^{\prime} \in C_{\lambda-1}^{n-1, \mu} .
$$

One can use the last definition to introduce the norm in this space by induction. Another way to introduce this norm is to use the relation

$$
|\varphi|=\sum_{m \leq n}\left|\varphi^{(m)}\right|_{C_{\lambda-m}^{0, \mu}}
$$

The introduced space is a Banach space with respect to this norm.

From the inductive definition (2.9.11), it follows that Theorem 2.8.1 also holds for the space $C_{\lambda}^{n, \mu}$. Also, this definition implies that if the domain $D$ is bounded, i.e., $\infty$ does not belong to $F$, then the embedding

$$
C_{n+\mu}^{n, \mu}(D, F) \subseteq C^{n, \mu}(D)
$$

of Banach spaces holds and all derivatives $\varphi^{(m)}, 0 \leq m \leq n$, of functions $\varphi$ from $C_{\mu+n}^{n, \mu}$ vanish at points $\tau$ from $F$.

All main properties of this space are provided by the next theorem.

\section{Theorem 2.9.3.}

(a) The family of Banach spaces $C_{\lambda}^{n, \mu}$ monotonously decreases with respect to the parameters $\mu$ and $\lambda_{\tau}$, where $\tau \neq \infty$, and monotonously increases with respect to $\lambda_{\infty}$. If $\mu<\nu$, then $\lambda_{\tau}<\lambda_{\tau}^{\prime}, \tau \neq \infty$. If $\lambda_{\tau}>\lambda_{\tau}^{\prime}, \tau=\infty$, then the embedding $C_{\lambda^{\prime}}^{n, \nu} \subseteq C_{\lambda^{\prime \prime}}^{n, \mu}$ is compact.

If the domain $D$ is uniformly connected, then the embedding $C_{\lambda}^{n, 0}(\bar{D}, F) \subseteq C_{\lambda}^{n-1,1}(D, F)$ also holds.

(b) The product of functions treated as bilinear map $\left(\varphi_{1}, \varphi_{2}\right) \rightarrow \varphi_{1} \varphi_{2}$ boundedly maps $C_{\lambda_{1}}^{n, \mu} \times C_{\lambda_{2}}^{n, \mu}$ to $C_{\lambda_{1}+\lambda_{2}}^{n, \mu}$, and, therefore, the space $C_{0}^{n, \mu}(D, F)$ is a Banach algebra with respect to multiplication. If an s-vector-function $\varphi$ belongs to $C_{0}^{n, \mu}(D, F)$, a function $f$ belongs to $C^{n, 1}(G), G \subseteq \mathbb{R}^{s}$, and $\varphi(D) \subseteq \bar{G}$, then the superposition $f \circ \varphi$ belongs to $C_{0}^{n, \mu}(D, F)$. In particular, the condition (2.7.7) is necessary and sufficient for the invertibility of elements of this superposition.

Proof. Assertion (b) and the first part of assertion (a) are proved by means of induction with respect to $n$, i.e., in the same way as Theorem 2.9.1. Regarding the second part of assertion (a), it suffices to prove it for $n=1$ and use induction with respect to $n$ then. Thus, we assume that the domain $D$ is uniformly connected and a function $\varphi$ from $C^{1}(D)$ admits the estimates

$$
|\varphi(x)| \leq C_{0} \rho_{\lambda}(x), \quad\left|\varphi^{\prime}(x)\right| \leq C_{1} \rho_{\lambda-1}(x)
$$

with positive constants $C_{j}$.

From Definition (2.8.4), we see that the function $\rho_{1-\lambda}$ is continuously differentiable and its gradient is representable in the form $\rho_{1-\lambda}^{\prime}=a \rho_{-\lambda}$, where the vector-function is bounded and its sup-norm $|a|_{0}$ depends only on $\lambda$. Consider the function $\psi=\rho_{1-\lambda} \varphi$ with the "derivative" $\psi^{\prime}=a \rho_{-\lambda} \varphi+\rho_{1-\lambda} \varphi^{\prime}$. By 
virtue of (2.9.10), this function belongs to $C^{1,0}(D)$ and has a one-order zero at finite points $\tau$ of $F$. More exactly, the inequality

$$
|\psi|_{0}+\left|\psi^{\prime}\right|_{0} \leq\left(1+|a|_{0}\right) C_{0}+C_{1}
$$

holds. Therefore, Theorem 2.2.2 is applicable to the function $\psi$. Then $\psi$ satisfies the Lipschitz condition with constant $[\psi]_{1}$ not exceeding $M\left|\psi^{\prime}\right|_{0}$. Then, by virtue of Lemma 2.8.1, the function $\rho_{-\lambda} \varphi=\rho_{-1} \psi$ belongs to $C_{0}^{0,1}(D, F)$ and, therefore, the function $\varphi$ belongs to $C_{0}^{0,1}(D, F)$ and the corresponding estimate of its norm holds.

It is obvious that the weight function $\rho_{\lambda}$ from (2.8.4) belongs to the class $C_{\lambda}^{n+1,0}\left(\mathbb{R}^{k}, F\right)$ for any $n$ (the set $D=\mathbb{R}^{k}$ is unbounded in the considered case, which means that $\infty \in F$ ). Therefore, by virtue of the last assertion of Theorem 2.9.3(a), this function belongs to $C_{\lambda}^{n, 1}\left(\mathbb{R}^{k}, F\right)$. In particular, the operator of the multiplication by $\rho_{\lambda}$ isomorphically maps $C_{\lambda^{\prime}}^{n, \mu}(D, F)$ onto $C_{\lambda+\lambda^{\prime}}^{n, \mu}(D, F)$.

Similarly to Theorem 2.9.1, one can use induction with respect to $n$ to extend Theorem 2.8.2 to the case of spaces $C_{\lambda}^{n, \mu}$.

Theorem 2.9.4. Let $n \geq 1$ and $\mathcal{D} \alpha \in C_{0}^{n-1, \mu}(D, F)$ under the assumptions of Theorem 2.8.2. Then the operator $\varphi \rightarrow \varphi \circ \alpha$ boundedly maps $C_{\lambda_{1}}^{n, \mu}\left(D_{1}, F_{1}\right)$ to $C_{\lambda}^{n, \mu}(D, F)$.

If the above is satisfied, $s=k, \alpha$ is a Lipschitz map, and $D_{1}=\alpha(D)$, then the inverse map $\beta=\alpha^{-1}$ belongs to the class $C^{n}\left(D_{1}\right)$ and $\mathcal{D} \beta \in C_{0}^{n-1, \mu}\left(D_{1}, F_{1}\right)$.

Lemma 2.8.2 admits the corresponding analog for the considered spaces, i.e., the symbol $C^{\mu}$ can be replaced by $C^{n, \mu}$ in this lemma. To prove that, we take into account the fact that, due to Lemma 2.9.1, the remark to Theorem 2.7.2 concerning the operation $\varphi(x) \rightarrow \varphi\left(x^{*}\right)$ still holds with respect to $C^{n, \mu}$.

Applying Lemma 2.8.2 to $C_{\lambda}^{n, \mu}$, one can obtain an embedding of Banach spaces, similar to (2.9.12), in the case where the domain $D$ is unbounded. Let this domain lie outside a neighborhood of the point $\tau=0$ and $D^{*}$ be the image of $D$ under the map $x \rightarrow x^{*}$. Due to the definition from Sec. 2.6, this map takes the space $C_{*}^{n, \mu}(D)$ to $C^{n, \mu}\left(D^{*}\right)$. Therefore,

$$
C_{-n-\mu}^{n, \mu}(D, \infty) \subseteq C_{*}^{n, \mu}(D) .
$$

From Theorem 2.9.3(a), it follows that the following classes are well defined:

$$
C_{\lambda+0}^{n, \mu}=\bigcup C_{\lambda+\delta}^{n, \mu}, \quad C_{\lambda-0}^{n, \mu}=\bigcap C_{\lambda-\delta}^{n, \mu} .
$$

Here the union and the intersection are taken with respect to weight orders $\delta$ such that $\delta_{\tau}>0$ for $\tau \neq \infty$ and $\delta_{\tau}<0$ for $\tau=\infty$. If $\lambda=0$, then the symbol $\lambda$ in the notation of these classes is omitted.

In particular, similarly to $(2.7 .15)$, the relation

$$
|x|^{\zeta_{0}}(1+|x|)^{\zeta_{1}} \ln ^{n}|x| Q(x) \in C_{\lambda-0}^{n, 1}\left(\mathbb{R}^{k} ; 0, \infty\right),
$$

where $\lambda_{0}=m+\operatorname{Re} \zeta_{0}$ and $\lambda_{\infty}=m+\operatorname{Re}\left(\zeta_{0}+\zeta_{1}\right)$, holds provided that $Q(x) \in \mathcal{H}_{m}, \zeta_{0} \in \mathbb{C}$, and $\zeta_{1} \in \mathbb{C}$, $n=1,2, \ldots$

The following properties of these classes are immediately deduced from Definition (2.9.15).

Lemma 2.9.1. The space $C_{\lambda}^{n, \mu}$ is located between the classes (2.9.15), i.e.,

$$
C_{\lambda+0}^{n, \mu} \subseteq C_{\lambda}^{n, \mu} \subseteq C_{\lambda-0}^{n, \mu} .
$$

The multiplication operation $\left(\varphi_{1}, \varphi\right) \rightarrow \varphi_{1} \varphi_{2}$ acts from $C_{\lambda_{1}-0}^{n, \mu} \times C_{\lambda_{2}+0}^{n, \mu}$ to $C_{\lambda_{1}+\lambda_{2}+0}^{n, \mu}$.

More complicated functions can also be constructed by means of (2.9.16). Let the support of a $C^{\infty}$-function $\chi_{\tau}(x)$ lie inside the domain $B_{\rho}(\tau)$ defined by (2.8.1) and $\chi(x) \equiv 1$ in a neighborhood of $\tau$. Let complex functions $\zeta_{\tau, j}$ belong to the line $\operatorname{Re} \zeta=\lambda_{\tau}$, homogeneous functions $Q_{\tau, j}(\xi)$ belong to 
$\mathcal{H}_{0}$, and $p_{\tau, j}(\xi), 1 \leq j \leq m_{\tau}$, be polynomials. Let $\dot{C}_{\lambda}^{n, \mu}(D, F)$ denote the class of all functions of the kind

$$
\varphi(x)=\sum_{\tau \in F} \chi_{\tau}(x) \sum_{1 \leq j \leq m_{\tau}}|x-\tau|^{\zeta_{\tau, j}} Q_{\tau, j}(x-\tau) p_{\tau, j}(\ln |x-\tau|)+\varphi_{0}(x),
$$

where $x-\tau$ is to be replaced by $x$ for $\tau=\infty$, and $\varphi_{0} \in C_{\lambda+0}^{n, \mu}(G, F)$.

It is clear that this class is also located between the classes (2.9.15), i.e., it satisfies the relations (2.9.17). It is useful for the selection of the asymptotics of functions $\varphi$ from $C_{\lambda-0}^{n, \mu}$ in neighborhoods of singular points $\tau$ in the investigation of singular integral equations (see [61]) and elliptic boundary-value problems (see [62]).

\subsection{Modified Spaces $C_{(\lambda)}^{n, \mu}$}

According to Sec. 2.9, the space $C_{\lambda}^{n, \mu}$ is defined by the conditions $\varphi^{(m)} \in C_{\lambda-m}^{\mu}$ provided that $0 \leq m \leq n$. This is the motivation to investigate properties of a function $\varphi$ satisfying only the last condition, i.e., the condition $\varphi^{(n)} \in C_{\lambda-n}^{\mu}$. For example, what conditions guarantee that such a function belongs to $C_{\lambda}^{n, \mu}(D, F)$ (up to a smooth term)?

It is convenient to introduce the following definition. A domain $D$ satisfies the cone condition at its boundary point $\tau$ if there exist an open connected cone $K$ with vertex at the origin, a neighborhood $V_{\tau}$ of the point $\tau$, a positive $\delta$, and a Lipschitz map $\alpha$ of the set $D_{\tau}=D \cap V_{\tau}$ onto

$$
\alpha\left(D_{\tau}\right)=G, \quad G= \begin{cases}K \cap\{|x|<\delta\}, & \tau \neq \infty, \\ K \cap\{|x|>1 / \delta\}, & \tau=\infty,\end{cases}
$$

such that $\alpha(\tau)=0, \tau \neq \infty$, and $\alpha(\infty)=\infty$.

For example, it follows from the definitions of Secs. 2.3 and 2.6 that any bounded or unbounded Lipschitz domain satisfies the cone condition at each its point. Another example is any isolated boundary point of a domain $D$ : the whole space can be treated as the cone $K$ and the selected point $\tau$ can be treated as its vertex.

Theorem 2.10.1. Let $D$ be a uniformly connected domain satisfying the cone condition at all points $\tau$ from $F$ and let the derivative $\varphi^{\prime}$ of a function $\varphi$ from $C^{1}(D)$ belong to $C_{\lambda-1}^{0}(\bar{D}, F)$, where $\lambda_{\tau} \neq$ $0, \tau \in F$. Then there exists a function $\varphi_{0}$ from $C^{1}(D)$ such that it is constant in a neighborhood of any point $\tau$ from $F$ and $\varphi-\varphi_{0} \in C_{\lambda}^{0,1}(D, F)$.

Proof. Let $V_{\tau}$ be the neighborhood of the point $\tau$ from $F$, mentioned in the cone condition, and $\alpha_{\tau}$ be the corresponding Lipschitz map of the domain $D_{\tau}$. Without loss of generality, one can assume that the closed sets $\bar{D}_{\tau}, \tau \in F$, are pairwise disjoint (otherwise, $\delta$ in (2.10.1) can be appropriately decreased). Let $D^{0}$ be the complement to the union of such sets in $D$. Let us prove that the function $\varphi$ is bounded in the domain $D^{0}$ :

$$
|\varphi(x)| \leq C^{0}, \quad x \in D^{0} .
$$

Assume the inverse. Then, since the set $D^{0}$ is bounded, it follows that there exists a sequence of its points $x_{n}$, converging to a point $a$ from $\partial D \cap \partial D^{0}$ such that

$$
\lim _{x_{n} \rightarrow a}\left|\varphi\left(x_{n}\right)\right|=+\infty .
$$

Let $B$ denote the ball of radius $r$ centered at $a$ such that

$$
B_{1} \cap F=\varnothing, \quad B_{1}=\{|z-a| \leq(M+1) r\},
$$

where $M$ is the uniform connectedness constant of the domain $D$. Let $x$ and $y$ belong to $B \cap D$. Then there exists a rectifiable arc $\Gamma$ from $D$ with endpoints $x$ and $y$ such that its length $l(\Gamma)$ does not exceed $M|x-y| \leq 2 M r$. Therefore, for any point $z$ of $\Gamma$, either $|z-x|$ or $|z-y|$ does not exceed $l(\Gamma) / 2 \leq M r$. Hence, $|z-a| \leq(M+1) r$, i.e., $\Gamma$ is contained in the ball $B_{1}$. By virtue of $(2.10 .4)$, the derivative $\varphi^{\prime}$ 
is bounded in $B_{1} \cap D$. Let $\left|\varphi^{\prime}(x)\right| \leq C_{1}$ and $x \in B_{1} \cap D$. Then, by virtue of Theorem 2.2 .2 , we have the inequality

$$
|\varphi(x)-\varphi(y)| \leq C_{1} l(\Gamma) \leq C_{1} M|x-y|,
$$

i.e., the function $\varphi$ satisfies the Lipschitz condition on $B_{1} \cap D$. Then it is bounded, which contradicts (2.10.3).

By the condition, in the domain $D_{\tau}$, the derivative of the function $\varphi$ satisfies the estimate

$$
\left|\varphi^{\prime}(x)\right| \leq C_{\tau}|x-\tau|^{\lambda_{\tau}-1}, \quad x \in D_{\tau},
$$

where $x-\tau$ is to be replaced by $x$ if $\tau=\infty$.

Let us show that there exists a constant $c_{\tau}$ such that $\varphi(x)-c_{\tau} \in C_{\lambda_{\tau}}^{0}\left(D_{\tau}, \tau\right)$ or, which is the same, there exists a positive constant $C_{\tau}^{0}$ such that

$$
\left|\varphi(x)-c_{\tau}\right| \leq C_{\tau}^{0}|x-\tau|^{\lambda_{\tau}}, \quad x \in D_{\tau},
$$

where $x-\tau$ is to be replaced by $x$ if $\tau=\infty$.

It suffices to prove this estimate in the case of finite points $\tau$ from $F$ since the case $\tau=\infty$ is reduced to the case $\tau=0$ by means of the inversion $x^{*}=x /|x|^{2}$ mapping the domain $D_{\infty}$ onto $D_{\infty}^{*}=D_{0}$. Indeed, consider the function $\varphi_{0}(x)=\varphi\left(x^{*}\right)$ in the domain $D_{0}$. Due to Lemma 2.6.2, it is described similarly to (2.10.1) with respect to $\tau=0$ and the Lipschitz map $\alpha_{0}(x)=\left[\alpha_{\infty}\left(x^{*}\right)\right]^{*}$. It is obvious that its derivative $\varphi_{0}^{\prime}(x)$ is equal to $\left(x^{*}\right)^{\prime} \varphi^{\prime}\left(x^{*}\right)$, where the Jacobi matrix $\left(x^{*}\right)^{\prime}$ is a homogeneous function of power -2 of the independent variable $x$. Then $\left|\left(x^{*}\right)^{\prime}\right| \leq M|x|^{-2}$, where the positive constant $M$ is independent of $x$. Therefore, for $\tau=\infty$, the estimate (2.10.5) passes to the estimate

$$
\left|\varphi_{0}^{\prime}(x)\right| \leq M C_{\infty}|x|^{-\lambda_{\infty}-1}, \quad x \in D_{0},
$$

coinciding with (2.10.5) in the case where $\tau=0$. Finally, for $\varphi_{0}$, we obtain the estimate (2.10.6) with $\lambda_{0}=-\lambda_{\infty}$. With respect to $\varphi(x)=\varphi_{0}\left(x^{*}\right)$, this estimate passes to $(2.10 .6)$ with respect to $\tau=\infty$.

Thus, let the estimate $(2.10 .5)$ be satisfied with $\tau \neq \infty$. By the definition of the Lipschitz map $\alpha=\alpha_{\tau}$ in (2.10.1), we have the two-side inequality (2.3.1). Then

$$
M^{-1}|x| \leq|\alpha(x)-\alpha(\tau)| \leq M|x|, \quad x \in D_{\tau} .
$$

Therefore, it suffices to verify that the function $\psi(x)=\varphi\left[\alpha^{-1}(x)\right]$ satisfies a similar to (2.10.6) estimate in the domain $G$, i.e., to verify that

$$
\left|\psi(x)-c_{\tau}\right| \leq C|x|^{\lambda_{\tau}}, \quad x \in G .
$$

Fix $r$ from $(0, \delta)$ and consider the segment $I=[x, y]$ with endpoints $x$ and $y$, located in the spherical layer $S_{r}=\{r \leq|z| \leq \delta\}$. It is known from Sec. 2.3 that its image $\Gamma=\alpha^{-1}(I)$ under the map $\alpha^{-1}$ is a rectifiable map of length $l(\Gamma)$ not exceeding $M|x-y|$. By virtue of (2.10.7), this arc lies in $D_{\tau} \cap\{|z-\tau|>r / M\}$. Therefore, due to Lemma 2.2.2 applied to $\varphi$, we arrive at the estimate

$$
|\psi(x)-\psi(y)| \leq \max _{z \in \Gamma}\left|\varphi^{\prime}(z)\right| l(\Gamma) \leq C_{\tau}\left(\frac{r}{M}\right)^{\lambda-1} M|x-y| .
$$

Thus, there exists a positive constant $C_{0}$ depending only on $C_{\tau}, \lambda_{\tau}$, and the domain $D_{\tau}$ such that

$$
|\psi(x)-\psi(y)| \leq C_{0} r^{\lambda_{\tau}-1}|x-y|
$$

for any point pair $x, y$ lying in the spherical layer $S_{r}$ together with the segment $[x, y]$.

Consider the sequence $x_{k}=\delta^{k} x, k=s, s+1, \ldots$, where the nonnegative integer $s$ is determined by the condition

$$
\delta^{2}<\left|x_{s}\right| \leq \delta .
$$

By virtue of (2.10.9), we have the inequality

$$
\mid \psi\left(x_{k}\right)-\psi\left(\left.x_{k+1}\left|\leq C_{0}\right| x_{k+1}\right|^{\lambda_{\tau}-1}\left|x_{k}-x_{k+1}\right|=C_{1} \delta^{k \lambda_{\tau}}|x|^{\lambda_{\tau}}\right.
$$


with constant $C_{1}=C_{0} \delta^{\lambda_{\tau}-1}(1-\delta)$. Hence, if $\lambda_{\tau}>0$, then there exists $c_{x}$ such that the sequence $\psi\left(x_{k}\right)$ converges to $c_{x}$ as $k \rightarrow+\infty$. This limit is independent of the choice of the point $x$. Indeed, let $y_{k}=\delta^{k} y$ and, for definiteness, let $|x| \leq|y|$. Then, by virtue of (2.10.9), we obtain the inequality

$$
\mid \psi\left(x_{k}\right)-\psi\left(\left.y_{k}\left|\leq C_{0}\right| x_{k}\right|^{\lambda_{\tau}-1}\left|x_{k}-y_{k}\right|=C_{0} \delta^{k \lambda_{\tau}}|x|^{\lambda_{\tau}}|x-y|,\right.
$$

which means that $c_{x}=c_{y}$.

Now, let $x$ and $y$ be arbitrary points of the spherical layer $S_{r}$. By the condition, the cone $K$ is connected. Hence, the set $S_{r}$ is also connected. Therefore, there exists a broken line $L \subseteq S_{r}$ connecting the points $x$ and $y$ and such that its tops are $z_{0}=x, z_{1}, \ldots, z_{n}=y$. Since $c_{x_{i-1}}=c_{x_{i}}$ (which is proved above), it also follows that $c_{x}=c_{y}$ in the general case.

Assigning $c_{\tau}=c_{x}, x \in S_{r}$, we deduce the following estimate from (2.10.11):

$$
\left|\psi(x)-c_{\tau}\right| \leq C_{1} \sum_{k \geq 0} \delta^{k \lambda_{\tau}}|x|^{\lambda_{\tau}} \leq C|x|^{\lambda_{\tau}},
$$

where $C=C_{1} /\left(1-\delta^{\lambda_{\tau}}\right)$.

If $\lambda_{\tau}<0$, then the following estimate is obtained in the same way:

$$
\left|\psi(x)-\psi\left(x_{s}\right)\right| \leq C_{1} \sum_{s \leq k \leq 0} \delta^{k \lambda_{\tau}}|x|^{\lambda_{\tau}} \leq \frac{C_{1}}{1-\delta^{-\lambda_{\tau}}}|x|^{\lambda_{\tau}} .
$$

By virtue of (2.10.9), function $\psi$ is bounded in the layer $\left\{x \in K, \delta^{2} \leq|x| \leq \delta\right\}$. Then the previous inequality combined with (2.10.10) yields the estimate $|\psi(x)| \leq C|x|^{\overline{\lambda_{\tau}}}$ with a positive constant $C$. Assigning $c_{\tau}=0$ for $\lambda_{\tau}<0$, we can join this estimate and (2.10.12) to obtain (2.10.8).

Now, let a function $\varphi_{0}$ from $C^{1}(\bar{D})$ be such that it is identically equal to $c_{\tau}$ in the domain $D_{\tau}$. Then, due to (2.10.2) and (2.10.8), the difference $\varphi-\varphi_{0}$ belongs to $C_{\lambda}^{0}(D, F)$. Then the derivative of this function and $\varphi^{\prime}$ belong to $C_{\lambda-1}^{0}(D, F)$. Therefore, due to Theorem 2.9.1(a), the function $\varphi-\varphi_{0}$ belongs to $C_{\lambda}^{0,1}(D, F)$, which completes the proof of the theorem.

For any real $\lambda$, denote by $P_{\lambda}$ the finite-dimensional class of polynomials

$$
p(x)=\sum_{|\alpha|<\lambda} a_{\alpha} x^{\alpha}
$$

such that their powers are strictly less than $\lambda$. Here $x^{\alpha}$ denotes the monomial $x_{1}^{\alpha_{1}} \ldots x_{k}^{\alpha_{k}}$ determined by the multi-index $\alpha=\left(\alpha_{1}, \ldots, \alpha_{k}\right)$. For $\lambda \leq 0$, this class is assigned to be equal to zero. To describe functions on the Riemann sphere such that they are smooth in a neighborhood of $\infty$, introduce the class

$$
P_{\lambda}^{*}=\left\{p\left(x^{*}\right), p \in P_{-\lambda}\right\}
$$

according to Sec. 2.6. Obviously, any polynomial $p(x)$ from $P_{\lambda}$ and the related function $p\left(x^{*}\right)$ from $P_{-\lambda}$ can be represented in the form

$$
p(x)=\sum_{0 \leq k<\lambda} Q_{k}(x)|x|^{k}, \quad p\left(x^{*}\right)=\sum_{0 \leq k<\lambda} Q_{k}(x)|x|^{-k},
$$

where

$$
Q_{k}(x)=\sum_{|\alpha|=k} a_{\alpha}\left(\frac{x}{|x|}\right)^{\alpha} .
$$

It is obvious that $Q_{k}$ is a homogeneous function of power zero and it belongs to the class $\mathcal{H}_{0}$ introduced at the end of Sec. 2.9. 
As above, let $F$ be a finite subset of a closed domain $\bar{D}$ and the notation $\chi_{\tau}(x) \in C^{\infty}, \tau \in F$, mean the same as it means in definition (2.9.18) of the class $\dot{C}_{\lambda}^{n, \mu}$. By $C_{(\lambda)}^{n, \mu}(D, F)$ denote the class of all functions of the kind

$$
\varphi=\sum_{\tau} p_{\tau} \chi_{\tau}+\varphi_{0}, \quad \varphi_{0} \in C_{\lambda}^{n, \mu}
$$

where $p_{\tau} \in P_{\lambda_{\tau}}$ for $\tau \neq \infty$ and $p_{\tau} \in P_{-\lambda_{\tau}}^{*}$ for $\tau=\infty$. This class is considered only for weight orders $\lambda$ related to $n$ as follows:

$$
\lambda_{\tau} \leq n+1, \tau \neq \infty, \quad \lambda_{\tau} \geq-n-1, \tau=\infty .
$$

Obviously, this class is a finite-dimensional extension of $C_{\lambda}^{n, \mu}$ (and it coincides with the last class if $\lambda_{\tau} \leq 0$ for $\tau \neq \infty$ and $\lambda_{\tau} \geq 0$ for $\tau=\infty$ ). In particular, due to Sec. 1.1, the norm of the space $C_{\lambda}^{n, \mu}$ induces a norm of this class such that it is a Banach space with respect to it.

Note that Theorem 2.8.1(a) applied to $C_{\lambda}^{n, \mu}$ is also valid for the Banach space $C_{(\lambda)}^{n, \mu}(D, F)$ since if $\rho$ is sufficiently small, then any set $B_{\rho}(\tau)$ is contained in one of elements $V_{j}$. The same assertion is valid for Lemma 2.8.2. Let us provide the following application of the above assertions.

Lemma 2.10.1. In the notation of Sec. 2.6, assume that a function $\varphi(x)$ belongs to the class $C_{*}^{n+1}$ in a neighborhood of a set $\bar{D} \cup \infty$ on the Riemann sphere. Then $\varphi \in C_{(\lambda)}^{n, 1}(D, F)$, where $\lambda_{\tau}=n+1$ for $\tau \neq \infty$ and $\lambda_{\tau}=-n-1$ for $\tau=\infty$.

Proof. By virtue of Theorem 2.8.1 applied to $C_{(\lambda)}^{n, \mu}$, one can assume (without loss of generality) that $F$ consists of the unique point $\tau=0$ or $\tau=\infty$ and the domain $D$ is bounded in the former case, while the domain $D$ is unbounded and lies outside a neighborhood of zero in the latter case. Then the latter case is reduced to the former one by means of the involution $x^{*}=x /|x|^{2}$.

Thus, without loss of generality, we can assume that $D$ is a bounded domain, $0 \in \partial D$, and there exists a domain $\widetilde{D}$ such that $\widetilde{D} \supseteq \bar{D}$ and $\varphi \in C^{n+1}(\widetilde{D})$. We must prove that $\varphi \in C_{(n+1)}^{n, 1}(D, 0)$. Cover the compact set $\widetilde{D}$ by a finite set of closed balls $B_{0}, B_{1}, \ldots, B_{m}$ contained in $\widetilde{D}$ such that $B_{0}$ is centered at the point $\tau=0$, while other balls do not contain this point. Then, due to Theorem 2.8.1 applied to $C_{\lambda}^{n, \mu}$, it suffices to verify that $\varphi \in C_{(n+1)}^{n, 1}\left(B_{0}, 0\right)$. Taking onto account Theorem 2.2.2, one can use induction with respect to $n$ to verify that $\varphi \in C^{n, \mu}\left(B_{k}\right), k \geq 1$. In the ball $B_{0}$, the relation

$$
\varphi(x)=\sum_{|\alpha| \leq n} \frac{1}{\alpha !} \frac{\partial^{\alpha} \varphi}{\partial x^{\alpha}}(0)+\varphi_{0}(x),
$$

where $\alpha !=\alpha_{1} ! \ldots \alpha_{k} !$, is valid. Using the Taylor expansion, we verify that the function $\varphi_{0}$ admits the estimate

$$
\left|\varphi_{0}(x)\right| \leq C|x|^{n+1}, \quad x \in B_{0} .
$$

Differentiating the relation (2.10.18), we obtain a similar relation (where $n$ is replaced by $n-|\beta|$, $|\beta| \leq n+1)$ for the functions $\psi=\partial^{\beta} \varphi / \partial x^{\beta}$ and $\psi_{0}=\partial^{\beta} \varphi_{0} / \partial x^{\beta}$. Therefore, arguing as above, we obtain that

$$
\left|\frac{\partial^{\beta} \varphi_{0}}{\partial x^{\beta}}(x)\right| \leq C|x|^{n+1-|\beta|}, \quad x \in B_{0},
$$

provided that $|\beta| \leq n+1$. Hence, the function $\varphi_{0}$ belongs to $C_{n+1}^{n+1,0}\left(B_{0}, 0\right)$. Then, due to Theorem 2.9.1(a), the function $\varphi_{0}$ belongs to $C_{n+1}^{n, 1}\left(B_{0}, 0\right)$. By virtue of Theorem 2.8.1 applied to the space $C_{n+1}^{n, 1}$, this means that $\varphi_{0} \in C_{n+1}^{n, 1}(D, 0)$, which completes the proof of lemma (take (2.10.18) into account).

In terms of the space $C_{(\lambda)}^{n, \mu}$, Theorem 2.10.1 provides the following answer to the question posed at the beginning of this section. 
Theorem 2.10.2. Let a domain $D$ be bounded and uniformly connected. Let it satisfy the cone condition at all points $\tau$ of $F$. Let there exist $m$ such that $m \leq n$ and the derivative $\varphi^{(m)}$ of a function $\varphi$ from $C^{n}(D)$ belongs to $C_{(\lambda-m)}^{n-m, \mu}(D, F), \lambda \leq n+1$. Then $\varphi \in C_{(\lambda)}^{n, \mu}(D, F)$ provided that

$$
\lambda_{\tau} \neq 0, \ldots, m-1, \quad \tau \in F .
$$

Proof. First, we prove the theorem in the case where $m=1$. Fix $\tau$ from $F$ and consider the domain $D_{\tau}$ from Definition (2.10.1). By the assumption of the theorem, we have the relation

$$
\frac{\partial \varphi}{\partial x_{j}}(x)=p_{j}(x)+\varphi_{j}(x), \quad \varphi_{j} \in C_{\lambda_{\tau}-1}^{n-1, \mu}\left(D_{\tau}, 0\right),
$$

where the powers of the polynomials $p_{j}$ are strictly less than $\lambda_{\tau}-1$. Let us prove that there exists a polynomial $p_{\tau}(x)$ such that its power is less than $\lambda_{\tau}$ and

$$
\frac{\partial p_{\tau}}{\partial x_{j}}=p_{j}, \quad 1 \leq j \leq k .
$$

If $n=1$, then, due to (2.10.17), we have the inequality $\lambda_{\tau} \leq 2$ and, therefore, the polynomials $p_{j}$ are constants; then the claimed assertion is obvious. Let $n \geq 2$. Then the relation (2.10.20) can be differentiated; then

$$
p_{i j}=\frac{\partial p_{i}}{\partial x_{j}}-\frac{\partial p_{j}}{\partial x_{i}}=-\frac{\partial \varphi_{i}}{\partial x_{j}}+\frac{\partial \varphi_{j}}{\partial x_{i}} \in C_{\lambda_{\tau}-2}^{0}\left(D_{\tau}, \tau\right), \quad i \neq j .
$$

It is obvious that the power of the polynomial $p_{i j}$ is less than $\lambda_{\tau}-2$; then the previous representation is possible only if $p_{i j}=0$. This proves the existence of a polynomial $p_{\tau}$ possessing the property (2.10.21).

Thus, changing $\varphi$ for the function $\varphi-\sum_{\tau} p_{\tau} \chi_{\tau}$, one can assume (without loss of generality) that $\varphi^{\prime} \in C_{\lambda-1}^{n-1, \mu}(D, F)$. In particular, due to Theorem 2.10.1, this implies the existence of constants $c_{\tau}$ such that

$$
\varphi_{0}=\varphi-\sum_{\tau} c_{\tau} \chi_{\tau} \in C_{\lambda}^{0}(D, F) .
$$

It is clear that the derivatives $\varphi_{0}^{\prime}$ and $\varphi^{\prime}$ belong to the class $C_{\lambda-1}^{n-1, \mu}(D, F)$. Therefore, due to Theorem 2.9.1(a), the function $\varphi_{0}$ belongs to $C_{\lambda}^{\mu}(D, F)$ and $\varphi_{0}^{(k)} \in C_{\lambda-k}^{\mu}(D, F)$ provided that $0 \leq k \leq n$, i.e., $\varphi_{0} \in C_{\lambda}^{n \mu}(D, F)$.

Thus, the theorem is proved for $m=1$. In the general case, consider a partial derivative $\psi=$ $\partial^{\alpha} \varphi / \partial x^{\alpha}$ of order $|\alpha|=m-1$. Then $\psi^{\prime} \in C_{(\lambda-m)}^{n-m}$ by condition. From the proved assertion, where $\lambda$ is replaced by $\lambda-m+1$, we take into account the fact that the condition of Theorem 2.10 .1 for the weight order $\lambda-m+1$ is satisfied by virtue of (2.10.19) and conclude that the function $\psi$ belongs to $C_{(\lambda-m+1)}^{n-m+1}$. Thus, $\varphi^{(m-1)}$ belongs to $C_{(\lambda-m+1)}^{n-m+1}$. Repeating this procedure, we obtain (after a finite number of steps) that $\varphi$ belongs to $C_{(\lambda)}^{n}$.

Recall that, according to Sec. 1.1, we assume that $0<\mu \leq 1$. However, it is clear that the last theorem also holds for $\mu=0$ provided that the weight classes $C_{\lambda-m}^{n-m, 0}$ and $C_{\lambda}^{n, 0}$ are considered in a closed domain $\bar{D}$.

From the last theorem, it follows that, under its assumptions, the space $C_{(\lambda)}^{n, \mu}$ can be inductively defined by the conditions that $\varphi$ and $\varphi^{\prime}$ belong to $C_{(\lambda-1)}^{n-1}$. Due to Lemma 2.8.1, the spaces $C^{\mu}(G)$ and $C_{(\mu)}^{\mu}(G, F)$ coincide provided that the set $G$ is bounded. Therefore, if $D$ is a domain satisfying the conditions of Theorem 2.10.2, then one can inductively verify that the relation

$$
C^{n, \mu}(D)=C_{(n+\mu)}^{n, \mu}(D, F)
$$


holds for any $n$. This fact complements embedding (2.9.12) for the specified domains. In particular, the space $C^{n, \mu}(D)$ can be defined as follows: only the highest derivatives belong to the class $C^{\mu}$, i.e., $\varphi^{(n)} \in C^{\mu}(D)$. Arguing in the same way, we obtain that if $\varphi \in C^{n, \mu}(D)$, then the function

$$
\varphi_{0}(x)=\varphi(x)-\sum_{|\alpha| \leq n} a_{\alpha} x^{\alpha}, \quad a_{\alpha}=\frac{1}{\alpha !} \frac{\partial^{\alpha} \varphi}{\partial x^{\alpha}}(\tau),
$$

belongs to the space $C_{n+\mu}^{\mu}(D, \tau)$.

If $D$ is a Lipschitz domain, then all these properties hold for any point $\tau$ from $\bar{D}$. Indeed, all boundary points of $D$ satisfy the cone condition. All interior points $\tau$ are boundary points for the domain $D \backslash\{\tau\}$; obviously, they satisfy the cone condition with respect to this domain.

From Definition (2.10.17), it follows that Theorem 2.9.2(a) remains valid for the space $C_{(\lambda)}^{n, \mu}$. Assuming that $D$ is a bounded domain, consider the following analogs of Theorems 2.9.2(b) and 2.9.3 for this space.

\section{Theorem 2.10.3.}

(a) Let the weight order $\lambda_{1}$ be nonnegative. Then the product of functions treated as the bilinear map $\left(\varphi_{1}, \varphi_{2}\right) \rightarrow \varphi_{1} \varphi_{2}$ boundedly maps $C_{\left(\lambda_{1}\right)}^{n, \mu} \times C_{\left(\lambda_{2}\right)}^{n, \mu}$ to $C_{\left(\lambda_{1}+\lambda_{2}\right)}^{n, \mu}$ and the space $C_{(\lambda)}^{n, \mu}(D, F)$ is a Banach algebra with respect to multiplication provided that $\lambda \geq 0$.

(b) Let a vector-function $\varphi$ belong to the class $C_{(\lambda)}^{n, \mu}(D, F), \lambda \geq 0$, and an open set $G$ contain the compact $\overline{\varphi(D)}$. Then the superposition $f \circ \alpha$ belongs to $C_{(\lambda)}^{n, \mu}(D, F)$ provided that $f \in C^{n+1}(G)$. In particular, if $\varphi$ is a scalar function, then the condition (2.7.7) is necessary and sufficient for its invertibility in the Banach algebra $C_{(\lambda)}^{n, \mu}$.

(c) Let the conditions of Theorem 2.8 .2 be satisfied and the map $\alpha$ belong to the class $C_{\left(\lambda^{1}\right)}^{n, \mu}(D, F)$, where $\lambda^{1}=\max (\lambda, 1)$. Then the superposition operator $T(\alpha) \varphi=\varphi \circ \alpha$ boundedly maps $C_{\left(\lambda_{1}\right)}^{n, \mu}\left(D_{1}, F_{1}\right)$ to $C_{(\lambda)}^{n, \mu}(D, F)$.

Under the additional assumptions that $s=k, \alpha$ is a Lipschitz map, and $D_{1}=\alpha(D)$, the inverse map $\beta=\alpha^{-1}$ belongs to the class $C_{\left(\lambda^{1}\right)}^{n, \mu}\left(D_{1}, F_{1}\right)$, where the weight order $\lambda_{\alpha(\tau)}, \tau \in F$, is denoted by $\lambda$.

Proof. (a) By virtue of Lemma 2.10.1, the condition for the power of the polynomial $p$ in Definition (2.10.16) of the class $C_{(\lambda)}^{n, \mu}$ can be omitted. Therefore, the claimed assertion immediately follows from Theorem 2.9.3(b).

(b) By virtue of Theorem 2.8.1 applied to $C_{(\lambda)}^{n, \mu}$, one can assume (without loss of generality) that $F=\{0\}$. Consider a function $\chi$ from $C_{0}^{\infty}(G)$ such that it is identically equal to 1 on the compact set $\overline{\varphi(D)}$. Replacing $f$ by $\chi f$, one can assume (without loss of generality) that $f \in C^{n+1}\left(\mathbb{R}^{s}\right)$.

By definition, there exists a vector-polynomial $p=\left(p_{1}, \ldots, p_{s}\right)$ such that the difference $\varphi-p$ belongs to $C_{\lambda}^{n, \mu}(D, 0)$. By the condition of the theorem, the function $f \circ p$ belongs to the class $C^{n+1}$ in a neighborhood of then compact set $\bar{D}$. Then

$$
f \circ p \in C_{(\lambda)}^{n, \mu}(D, 0)
$$

due to Lemma 2.10.1. Therefore, it remains to verify that

$$
f \circ \varphi-f \circ p \in C_{\lambda}^{n, \mu}(D, 0) .
$$

Let a segment with endpoints $z$ and $y$ be contained in $G$. Then, applying the Newton-Leibnitz relation to the function $f_{0}(t)=f[y+t(z-y)], 0 \leq t \leq 1$, we obtain that

$$
f(z)-f(y)=\sum_{j=1}^{k} a_{j}(z, y)\left(z_{j}-y_{j}\right), \quad a_{j}(z, y)=\int_{0}^{1} \frac{\partial f}{\partial z_{j}}[y+t(z-y)] d t .
$$


Then

$$
f[\varphi(x)]-f[p(x)]=\sum_{j=1}^{m} a_{j}(x)\left[\varphi_{j}(x)-p_{j}(x)\right], \quad a_{j}(x)=\int_{0}^{1} \frac{\partial f}{\partial x_{j}}[p(x)+t \varphi(x)-t p(x)] d t .
$$

Since $\varphi_{j}-p_{j} \in C_{\lambda}^{n, \mu}(D, 0)$, it suffices to verify that the functions $a_{j}$ belong to the space $C_{0}^{n, \mu}(D, 0)$. For this, we use Theorem 2.7.2. In its notation, the function $p[\omega(s, u)]+t \varphi[\omega(s, u)]-t p[\omega(s, u)]$ belongs to the space $C^{n, \mu}$ with respect to the total variable $(s, u, t)$ on the corresponding set. Due to Theorem 2.9.1(b), the superposition of its superposition with $f$ possesses the same property. Hence, the integral with respect to $t$ of the obtained function possesses the same property. Using Theorem 2.7.2 again, we arrive at the validity of (2.10.23).

(c) Let us agree that the notation for the functions $\chi_{\tau}$ from (2.10.16) is also used with respect to the set $F_{1}$. Represent $\varphi$ from $C_{\left(\lambda_{1}\right)}^{n, \mu}\left(D_{1}, F_{1}\right)$ similarly to $(2.10 .16)$ and note that if $n \geq 1$, then the condition $\alpha \in C_{\left(\lambda^{1}\right)}^{n, \mu}$ of the theorem implies that $\alpha^{\prime} \in C_{0}^{n-1, \mu}$. Therefore, due to Theorem 2.9.3, it suffices to verify that

$$
\sum_{\tau \in F_{1}}\left(\chi_{\tau} p_{\tau}\right) \circ \alpha \in C_{(\lambda)}^{n, \mu}(D, F) .
$$

By virtue of Theorem 2.8.1 applied to $C_{(\lambda)}^{n, \mu}$, one can assume (without loss of generality) that the set $F$ consists of the only point $\tau$. Then $F_{1}=\left\{\tau_{1}\right\}$ with $\tau_{1}=\alpha(\tau)$. Since the polynomial $p_{\tau}$ is equal to zero for $\lambda \leq 0$, it follows that $\lambda$ can be assumed to be positive. It is obvious that the function $\chi_{\tau_{1}} \circ \alpha$ belongs to $C^{n, \mu}(D)$ and is identically equal to 1 in a neighborhood of $\tau$. Then it belongs to the class $C_{(\lambda)}^{n, \mu}(D, \tau)$. On the other hand, due to Lemma 2.10.1 and assertion (a) of the theorem, the function $p_{\tau} \circ \alpha$ belongs to the same class, which completes the proof of (2.10.24) and the first assertion of (c). The second assertion is proved (by induction with respect to $n$ ) in the same way as the similar assertion of Theorem 2.9.1.

Note that Lemma 2.8.2 applied to the space $C_{(\lambda)}^{n, \mu}$ and combined with Theorem 2.10.3 allows one to include the case of unbounded domains $D$ and $D_{1}$ in the consideration. Indeed, let inversions $\delta$ and $\delta_{1}$ maps $D$ and $D_{1}$ (respectively) to bounded domains. Then we must apply Theorem 2.10 .3 to the map $\widetilde{\alpha}=\delta_{1} \circ \alpha \circ \delta$, where the map $\alpha$ is such that $\widetilde{\alpha}$ satisfies the condition of Theorem 2.10.3.

To illustrate Theorems 2.10.2-2.10.3, we find conditions providing that the radial parametrization (2.5.5) belongs to the class $C^{1, \mu}[0, \rho]$, i.e., the radial arc $\Gamma$ belongs to $C^{1, \mu}$. It is obvious that any line segment satisfies conditions of Theorem 2.10 .2 and the class $C^{1, \mu}[0, \rho]$ coincides with $C_{(1+\mu)}^{1, \mu}([0, \rho], 0)$. Therefore, if $\gamma \in C^{1, \mu}[0, \rho]$, then there exist $\gamma_{0}$ from $C_{1+\mu}^{1, \mu}([0, \rho], 0)$ and a constant $a$ such that $\gamma(r)=$ $a r+\gamma_{0}(r)$. Hence, $e^{i \theta(r)}$ belongs to $C_{(\mu)}^{1+\mu}([0, \rho], 0)$. Then, taking into account Theorem 2.10.3(b), we conclude that this is also valid for the real function $\theta(r)$. Due to Theorem 2.10.2, this fact is equivalent to the assertion that $\theta^{\prime}(r)$ belongs to $C_{\mu-1}^{\mu}([0, \rho], 0)$, which is the claimed necessary and sufficient condition of the belonging of the radial $\operatorname{arc} \Gamma$ to the class $C^{1, \mu}$.

\section{Chapter 3}

\section{INTEGRALS WITH HOMOGENEOUS DIFFERENCE KERNELS}

\subsection{Homogeneous Functions}

Investigate the class $\mathcal{H}_{\lambda}$ of homogeneous functions introduced at the end of Sec. 2.7. Recall that it consists of functions $Q(\xi)$ belonging to $C^{\infty}\left(\mathbb{R}^{k} \backslash 0\right)$ and satisfying the homogeneity condition

$$
Q(r \xi)=r^{\lambda} Q(\xi), \quad r>0
$$


of power $\lambda \in \mathbb{R}$. By virtue of the homogeneity, the function $Q$ is entirely determined by its restriction to the unit sphere $\Omega$. In the one-dimensional case, the set $\Omega$ consists of the two points \pm 1 . Therefore, any homogeneous function of power $\lambda$ is a linear combination of the functions $Q_{1}(\xi)=|\xi|^{\lambda}$ and $Q_{2}(\xi)=(\operatorname{sgn} \xi)|\xi|^{\lambda}$.

Differentiating the relation (3.1.1) with respect to $\xi_{i}$, we see that the partial derivative $\partial Q / \partial \xi_{i}$ belongs to $\mathcal{H}_{\lambda-1}$. In the same way, the multiplication operation $Q(\xi) \rightarrow|\xi|^{\delta} Q(\xi)$ acts from $\mathcal{H}_{\lambda}$ to $\mathcal{H}_{\lambda+\delta}$.

Recall (see Sec. 2.8) that $Q^{(s)}$ denotes the ordered collection of all partial derivatives of order $s$. Considering it on the sphere $\Omega$, introduce the following norm in the class $\mathcal{H}_{\lambda}$ :

$$
|Q|_{(m)}=\sum_{0 \leq s \leq m}\left|Q^{(s)}\right|_{0, \Omega}
$$

It is obvious that it satisfies the estimates

$$
\left|Q^{\prime}\right|_{(m)} \leq C|Q|_{(m-1)},\left.\left.\quad|| \xi\right|^{\delta} Q(\xi)\right|_{(m)} \leq C|Q|_{(m)},
$$

where $C$ is a positive constant depending only on $\lambda$ and $\delta$.

Lemma 3.1.1. If $Q \in \mathcal{H}_{\lambda}$, then the inequality

$$
|Q(\xi)-Q(\eta)| \leq M|Q|_{(1)}\left(|\xi|^{\lambda-1}+|\eta|^{\lambda-1}\right)|\xi-\eta|,
$$

where $M$ is a positive constant depending only on $\lambda$, holds for all $\xi$ and $\eta$.

Proof. By virtue of the homogeneity of (3.1.1), one can assume (without loss of generality) that $|\xi|=1$. Then

$$
|Q(\xi)-Q(\eta)|=\left.|Q(\xi)-| \eta\right|^{\lambda}\left|Q\left(\frac{\eta}{|\eta|}\right)\right| \leq[Q]_{1, \Omega}\left|\xi-\frac{\eta}{|\eta|}\right|+\left.|Q|_{(0)}|1-| \eta\right|^{\lambda} \mid .
$$

Taking into account the fact that $|1-| \eta||=|| \xi|-| \eta|| \leq|\xi-\eta|$, we conclude that

$$
\left|\xi-\frac{\eta}{|\eta|}\right| \leq|\xi-\eta|+\left|1-\frac{1}{|\eta|}\right||\eta| \leq 2|\xi-\eta|
$$

and

$$
\left.|1-| \eta\right|^{\lambda}|\leq| \lambda\left|\max \left(1,|\eta|^{\lambda-1}\right)\right| 1-|\eta||\leq| \lambda\left|\left(1+|\eta|^{m-1}\right)\right| \xi-\eta \mid .
$$

Combining these inequalities, we arrive at the estimate

$$
|Q(\xi)-Q(\eta)| \leq\left(2[Q]_{1, \Omega}+|\lambda||Q|_{(0)}\right)\left(1+|\eta|^{\lambda-1}\right)|\xi-\eta| .
$$

It is obvious that the spherical layer $S=\{1 / 2<|\xi|<2\}$ is a uniformly connected domain. Then, by virtue of Theorem 2.2.2, the following estimate holds:

$$
[Q]_{1, S} \leq M_{1}\left|Q^{\prime}\right|_{0, S}
$$

On the other hand, the inequality

$$
\left|Q^{\prime}\right|_{0, S} \leq M_{2}|Q|^{(1)}
$$

where $M_{2}$ is a constant depending only on $\lambda$, holds due to the homogeneity of (3.1.1). Combining this with (3.1.5), we obtain (3.1.4).

Due to (3.1.5), the estimate (3.1.4) holds for any homogeneous function of power $\lambda$, satisfying the Lipschitz condition on the unit sphere $\Omega$. Here, the norm $|Q|_{C^{0,1}(\Omega)}$ plays the role of the norm $|Q|_{(1)}$.

Consider the case where a function $Q(\xi)=Q(x, \xi)$ from $\mathcal{H}_{\lambda}$ depends on the point $x$ from $G \subseteq \mathbb{R}^{l}$ as on a parameter. As above, let $Q_{\xi}^{(s)}$ denote the ordered collection of all partial derivatives of order $s$ with respect to $\xi$. By $C^{\nu(m)}(G)=C^{\nu(m)}\left(G, \mathcal{H}_{\lambda}\right)$ denote the space of all functions $Q(x, \xi)$ such that 
they and their derivatives $Q_{\xi}^{(s)}, s \leq m$, belong to $C^{\nu}(G)$ uniformly with respect to $\xi$ from $\Omega$. This is a Banach space with respect to the norm

$$
|Q|_{C^{\nu(m)}}=\sum_{s \leq m} \sup _{\xi \in \Omega}\left|Q_{\xi}^{(s)}(x, \xi)\right|_{C^{\nu}(G)} .
$$

Obviously, for $\nu=0$ and $s=0$, this norm coincides with the sup-norm of the function $Q(x, \xi)$ on $G \times \Omega$.

If $G$ is a domain $D$, then the space $C^{n, \nu(m)}(D)=C^{n, \nu(m)}\left(D, \mathcal{H}_{\lambda}\right)$ is defined similarly: it consists of functions $Q(x, \xi)$ such that $Q_{\xi}^{(s)}(x, \xi), 0 \leq s \leq m$, belong to $C^{n, \nu}(D)$ uniformly with respect to $\xi$ from $\Omega$. The norm in this space is defined by the relation (3.1.6), where the symbol $C^{\nu}$ is replaced by $C^{n, \nu}$.

From Definitions (3.1.2) and (3.1.6), it follows that if $Q \in C^{\nu(m)}(G), x, y \in G$, and $x \neq y$, then

$$
|\tilde{Q}|_{(m)} \leq|Q|_{C^{\nu(m)}}, \quad \tilde{Q}(\xi)=\frac{Q(x, \xi)-Q(y, \xi)}{|x-y|^{\nu}} .
$$

The following assertion holds.

Lemma 3.1.2. Let $Q(u, \xi) \in C^{\nu(1)}\left(G, \mathcal{H}_{\lambda}\right), \lambda \leq 0$, and maps $\alpha: K \rightarrow G$ and $\beta: K \rightarrow \mathbb{R}^{k}$ be such that $\alpha$ satisfies the Lipschitz condition, $\beta \in C^{\nu}(K)$, and there exists a positive $\delta$ such that $|\beta(x)| \geq \delta$ provided that $x \in K$. Then the function $q(x)=Q[\alpha(x), \beta(x)]$ belongs to $C^{\nu}(K)$.

Proof. Obviously, the absolute value of the function $q$ does not exceed $|Q|_{C^{\nu(0)}} \delta^{\lambda}$. Then, taking into account Lemma 3.1.1, the estimates

$$
|Q(u, \xi)-Q(v, \xi)| \leq \delta^{\lambda}|Q|_{C^{\nu(0)}}|u-v|^{\nu}, \quad \mid Q(u, \xi)-Q\left(u,\left.\eta\left|\leq 2 M \delta^{\lambda-1}\right| Q\right|_{C^{0(1)}}|\xi-\eta| .\right.
$$

hold provided that $|\xi| \geq \delta,|\eta| \geq \delta, u \in G$, and $v \in G$. It remains to apply the corresponding estimates from (3.1.8) to the right-hand side of the inequality

$$
|q(x)-q(y)| \leq|Q[\alpha(x), \beta(x)]-Q[\alpha(y), \beta(x)]|+|Q[\alpha(y), \beta(x)]-Q[\alpha(y), \beta(y)]| .
$$

Treating $\xi$ as a parameter, one can also apply Theorem 2.3.2 (the extension theorem) to the function $Q(x, \xi)$. More exactly, let $G$ be a Lipschitz domain and $\Omega$ denote the unit sphere in $\mathbb{R}^{k}$. Consider the bounded extension operator $P: C^{\nu}(G) \rightarrow C^{\nu}\left(\mathbb{R}^{k}\right.$ from Theorem 2.3.2. For $Q(x, \xi)$ from $C^{\nu(m)}(G)$, assign

$$
Q^{1}(x, \xi)=[P Q(\cdot, \xi)](x), \quad x \in \mathbb{R}^{k}, \xi \in \Omega,
$$

where the operation $P$ acts for a fixed $\xi$. Further, similarly to (3.1.1), extend the function $Q^{1}$ defined on $\Omega$ such that the extension is homogeneous:

$$
Q^{1}(x, r \xi)=r^{\lambda} Q^{1}(x, \xi) .
$$

Lemma 3.1.3. The operator $P^{1}$ acting according to the relations (3.1.9)-(3.1.10) is bounded as an operator from $P^{1}: C^{\nu(m)}\left(G, \mathcal{H}_{\lambda}\right)$ to $C^{\nu(m)}\left(\mathbb{R}^{k}, \mathcal{H}_{\lambda}\right)$.

Proof. For $m=0$, the assertion of the lemma immediately follows from the definition of the operator $P^{1}$. In the general case, we show that it preserves the property to be infinitely differentiable with respect to $\xi$. More exactly, the following relations hold:

$$
\frac{\partial Q^{1}}{\partial \xi_{j}}=P^{1}\left[\frac{\partial Q}{\partial \xi_{j}}\right], \quad 1 \leq j \leq k .
$$

Indeed, let $e_{j}$ be the unit vector of the axis $\xi_{j}$. Assign $\tilde{\xi}=(\xi+s e) /|\xi+s e|, \xi \in \Omega$, where the real $s$ varies in a neighborhood of the origin. Since $P$ is a linear operator and (3.1.10) holds, the difference $Q^{1}(x, \tilde{\xi})-Q^{1}(x, \xi)$ can be represented in the form

$$
|\xi+s e|^{\lambda} Q^{1}(x, \tilde{\xi})-Q^{1}(x, \xi)=P\left[|\xi+s e|^{\lambda} Q(\cdot, \tilde{\xi})-Q(\cdot, \xi)\right](x) .
$$


Dividing this relation by $s$ and passing to the limit as $s \rightarrow 0$, we arrive at the relations (3.1.11). Justifying the passage to the limit, we argue as in Lemma 1.8.3. Due to Definition (3.1.6) of the space $C^{\nu(m)}$, these relations lead to the validity of the lemma for positive values of $m$.

For example, the function

$$
Q(x, y, \xi)=q(x, y)|\xi|^{\lambda}, \quad q \in C^{\nu}(G \times G),
$$

belongs to the class $C^{\nu(0)}\left(G \times G, \mathcal{H}_{\lambda}\right)$.

In the general case, to represent $Q$ in the same way, one must assign $q(x, y)=Q_{0}(x, y, y-x)$, where $Q_{0}(x, y, \xi)=|\xi|^{-\lambda} Q(x, y, \xi)$ is a homogeneous function of power zero with respect to $\xi$. The next theorem finds additional conditions guaranteeing the belonging of this function $q$ to the class $C^{\nu}$.

Theorem 3.1.1. Let $G$ be a compact subset of $\mathbb{R}^{k}$ and a function $Q(x, y, \xi)$ from $C^{\nu(1)}\left(G \times G, \mathcal{H}_{0}\right)$ be identically equal to zero for $x=y$. Then the function $a(x, y)=Q(x, y, y-x)$ extended as zero to the manifold $x=y$ belongs to the class $C^{\nu}(G \times G)$ and the corresponding norm estimate $|a|_{C^{\nu}} \leq C|Q|_{C^{\nu(1)}}$ holds, where $C$ is a positive constant depending only on the diameter $R$ of the set $G$.

Proof. By virtue of (3.1.7), we have the estimate

$$
|a(x, y)| \leq|Q|_{C^{\nu(0)}}|x-y|^{\nu} \leq R^{\nu}|Q|_{C^{\nu(0)}} .
$$

Thus, only the differences $\Delta=a\left(x_{1}, y\right)-a\left(x_{2}, y\right)$ and $\Delta=a\left(x, y_{1}\right)-a\left(x, y_{2}\right)$ are to be estimated. It suffices to consider only the former one. Assign $\delta=\left|x_{1}-x_{2}\right|$ and separately consider the case where $\left|x_{1}-y\right| \leq 2 \delta$ and the case where $\left|x_{1}-y\right| \geq 2 \delta$. In the former case, we have $\left|x_{2}-y\right| \leq 3 \delta$ and, therefore,

$$
|\Delta| \leq|Q|_{C^{\nu(0)}}\left(\left|x_{1}-y\right|^{\nu}+\left|x_{2}-y\right|^{\nu}\right) \leq\left(2^{\nu}+3^{\nu}\right)|Q|_{C^{\nu(0)}} \delta^{\nu}
$$

In the former case, taking into account the triangle inequalities $\left|y-x_{1}\right|-\delta \leq\left|y-x_{2}\right| \leq\left|y-x_{1}\right|+\delta$, we obtain that

$$
\delta \leq\left|y-x_{2}\right| \leq 2\left|y-x_{1}\right|
$$

Consider the inequality

$$
|\Delta| \leq\left|Q\left(x_{1}, y, y-x_{1}\right)-Q\left(x_{2}, y, y-x_{1}\right)\right|+\left|Q\left(x_{2}, y, y-x_{1}\right)-Q\left(x_{2}, y, y-x_{2}\right)\right|
$$

and apply the estimates (3.1.7) and (3.1.4) to the corresponding $\mathcal{H}_{0}$-functions

$$
\tilde{Q}_{1}(\xi)=\frac{Q\left(x_{1}, y, \xi\right)-Q\left(x_{2}, y, \xi\right)}{\left|x_{1}-x_{2}\right|^{\nu}}, \quad \tilde{Q}_{2}(\xi)=\frac{Q\left(x_{2}, y, \xi\right)-Q(y, y, \xi)}{\left|x_{2}-y\right|^{\nu}}
$$

at its right-hand side. We obtain that

$$
|\Delta| \leq|Q|_{C^{\nu(0)}} \delta^{\nu}+M|Q|_{C^{\nu(1)}} \delta\left|x_{2}-y\right|^{\nu}\left(\left|x_{1}-y\right|^{-1}+\left|x_{2}-y\right|^{-1}\right) .
$$

By virtue of (3.1.13), we have the inequality

$$
\delta\left|x_{2}-y\right|^{\nu}\left(\left|x_{1}-y\right|^{-1}+\left|x_{2}-y\right|^{-1}\right) \leq 3 \delta\left|x_{2}-y\right|^{\nu-1} \leq 3 \delta^{\nu} .
$$

Substituting this inequality to the previous estimate and combining it with (3.1.12), we complete the proof of the theorem.

It follows from this theorem that if $Q(x, y, \xi) \in C^{\nu(1)}\left(G \times G, \mathcal{H}_{0}\right)$ and a function $a_{0}(x, y)$ from $C^{\nu}(G \times G)$ vanishes for $x=y$, then the product $a(x, y)=a_{0}(x, y) Q(x, y, y-x)$ also belongs to the class $C^{\nu}(G \times G)$. For example, the functions $|x-y| \ln ^{n}|x-y|, n=1,2 \ldots$, can be taken as $a(x, y)$ : they belong to the class $C^{\nu}, 0<\nu<1$, on any bounded subset of $\mathbb{R}^{k} \times \mathbb{R}^{k}$.

If $G$ is a smooth contour on a complex plane, then Theorem 3.1.1 can be complemented by the following assertion. 
Lemma 3.1.4. Let a smooth contour $\Gamma$ belong to $C^{1, \nu}$ with the unit tangential vector $e(t), t \in \Gamma$, and a kernel $Q_{0}\left(t_{0}, t ; \xi\right)$ from $C^{\nu(1)}\left(\Gamma \times \Gamma, \mathcal{H}_{0}\right)$ be even with respect to the variable $\xi$. Then the function $a\left(t_{0}, t\right)=Q_{0}\left(t_{0}, t ; t-t_{0}\right)$ extended by the value $Q\left(t_{0}, t_{0} ; e\left(t_{0}\right)\right)$ for $t=t_{0}$ belongs to $C^{\nu}(\Gamma \times \Gamma)$ and the estimate $|k|_{C^{\nu}} \leq C\left|Q_{0}\right|_{C^{\nu(0)}}$ holds for the corresponding norms.

Proof. It suffices to prove the claim of the lemma for any arc $\Gamma_{0}$ from $\Gamma$. Let $\gamma:[0,1] \rightarrow \Gamma_{0}$ be a parametrization of this arc, belonging to the class $C^{1, \nu}[0,1]$. Since this parametrization is a Lipschitz map, it follows that it suffices to prove this lemma for the function $b\left(s_{0}, s\right)=a\left[\gamma\left(s_{0}\right), \gamma(s)\right]$ in the square $0 \leq s, s_{0} \leq 1$. By virtue of the homogeneity and evennes of the kernel $Q_{0}$, this function can be represented in the form

$$
b\left(s_{0}, s\right)=Q_{0}\left[\gamma\left(s_{0}\right), \gamma(s) ; q\left(s_{0}, s\right)\right], \quad q\left(s_{0}, s\right)=\frac{\gamma(s)-\gamma\left(s_{0}\right)}{s-s_{0}} .
$$

Similarly to the proof of Lemma 2.4.1, we verify that the function $q$ belongs to $C^{\nu}([0,1] \times[0,1])$ and its absolute value is separated from zero. Therefore, it remains to apply Lemma 3.1.2 to the function $Q_{0}\left[\gamma\left(s_{0}\right), \gamma(s) ; q\left(s_{0}, s\right)\right]$.

Below, we use the space $C_{0}^{\nu(m)}\left(G, F ; \mathcal{H}_{\lambda}\right)$; as above, it is introduced with respect to the zero-order space $C_{0}^{\nu}(G, F)$. Another way to introduce it is to act similarly to Sec. 2.8 , based on $C^{\nu(m)}\left(G, \mathcal{H}_{\lambda}\right)$ and the space $C_{0}^{\nu(m)}\left(G, \mathcal{H}_{\lambda}\right)$; as above, the latter one is introduced with respect to the homogeneous space $C_{0}^{\nu}(G)$ of Sec. 2.7. It is easy to see that both ways are equivalent and yield the same space.

Note that Theorem 2.7.1 is also valid for the homogeneous space $C_{0}^{\nu(m)}\left(G, \mathcal{H}_{\lambda}\right)$. To verify that, we follow the proof of the specified theorem and obtain the norm inequalities for a fixed value of $\xi$. Then we take the supremum with respect to $\xi$ from $\Omega$ at the right-hand sides of these inequalities. Finally, we take the supremum with respect to $\xi$ from $\Omega$ at their left-hand sides.

\subsection{Integrals with Weak Singularities}

It is natural to use homogeneous functions as special-type kernels of integrals. We illustrate this by integrals with weak singularities, considered in Sec. 1.9.

Let $G$ be a compact subset of $\mathbb{R}^{k}$ and let a function $q(x, y)$ satisfy the conditions

$$
q(x, y) \in C^{\nu}(G \times G), \quad q(x, x) \equiv 0,
$$

where $0<\nu<1$. Consider the integral

$$
\psi(x)=\int_{G} \frac{q(x, y)}{|y-x|^{k}} \varphi(y) d y, \quad x \in G .
$$

Since $|q(x, y)| \leq[q]_{\nu}|x-y|^{\nu}$, it follows that the kernel $q(x, y)|x-y|^{-k}$ is summable with respect to $y$. It is obvious that the function $Q(x, y, \xi)=q(x, y)|\xi|^{-k}$ belongs to the class $C^{\nu(1)}\left(G \times G, \mathcal{H}_{-k}\right)$ and is identically equal to zero for $x=y$. By virtue of Theorem 3.1.1, the inverse is also valid: if $Q(x, y, \xi) \in C^{\nu(1)}\left(G \times G, \mathcal{H}_{-k}\right)$ and

$$
Q(x, x, \xi)=0, \quad x \in G,
$$

for any $\xi$ from $\mathbb{R}^{k}$, then $Q(x, y, \xi)=q(x, y)|\xi|^{-k}$, where the function $q(x, y)=Q(x, y ; y-x)|y-x|^{-k}$ satisfies (3.2.1). Thus, under the admitted assumption, the relation

$$
\psi(x)=\int_{G} Q(x, y, y-x) \varphi(y) d y, \quad x \in G,
$$

can be represented in the form (3.2.2).

In Sec. 1.9, it is found that any integral operator acting according to the relation (3.2.2) is compact in the space $C(G)$ of continuous functions. A stronger assertion is also valid. 
Theorem 3.2.1. Let a kernel $Q$ belong to $C^{\nu(1)}\left(G \times G, \mathcal{H}_{-k}\right)$ and satisfy the condition (3.2.3). Then, for any bounded function $\varphi$, the relation (3.2.4) defines a function $\psi$ from $C^{\mu}(G), 0<\mu<\nu$, and the norm estimate

$$
|\psi|_{C^{\mu}} \leq C|Q|_{C^{\nu(1)}}|\varphi|_{0}
$$

holds, where $C$ is a positive constant depending only on the diameter $R$ of the compact set $G$ provided that $\mu$ and $\nu$ are fixed.

Proof. As we note above, by virtue of Theorem 3.1.1, we have the estimate

$$
|Q(x, y ; y-x)| \leq C_{0}|y-x|^{\nu-k}
$$

whence

$$
|\psi|_{0} \leq C|Q|_{C^{\nu(0)}}|\varphi|_{0}
$$

where

$$
C=C_{0} \int_{|z| \leq R}|z|^{\nu-k} d z
$$

Thus, it remains to estimate the seminorm $[\psi]_{\mu}$. Due to (3.1.7) and Lemma 3.1.1, for any function $Q$ from $C^{\nu}\left(G \times G, \mathcal{H}_{k}\right)$, the following inequalities hold:

$$
\begin{gathered}
\left|Q_{0}(\xi)\right| \leq|Q|_{C^{\nu(0)}}\left|x_{1}-x_{2}\right|^{\nu}|\xi|^{-k}, \quad Q_{0}(\xi)=Q\left(x_{1}, y ; \xi\right)-Q\left(x_{2}, y ; \xi\right), \\
\left|Q_{0}\left(\xi_{1}\right)-Q_{0}\left(\xi_{2}\right)\right| \leq M|Q|_{C^{\nu(1)}}\left|x_{1}-x_{2}\right|^{\nu}\left|\xi_{1}-\xi_{2}\right|\left(\left|\xi_{1}\right|^{-k-1}+\left|\xi_{2}\right|^{-k-1}\right) .
\end{gathered}
$$

Then, assigning $x_{1}=x$ and $x_{2}=y$ and taking into account (3.2.3), we deduce the inequalities

$$
\begin{gathered}
|Q(x, y, \xi)| \leq|Q|_{C^{\nu(0)}}|x-y|^{\nu}|\xi|^{-k} \\
\left|Q\left(x, y, \xi_{1}\right)-Q\left(x, y, \xi_{2}\right)\right| \leq M|Q|_{C^{\nu(1)}}|x-y|^{\nu}\left|\xi_{1}-\xi_{2}\right|\left(\left|\xi_{1}\right|^{-k-1}+\left|\xi_{2}\right|^{-k-1}\right) .
\end{gathered}
$$

Further, we arguing in the same way as in Theorem 3.1.1. Fix two different points $x_{1}$ and $x_{2}$ from $G$, denote $\left|x_{1}-x_{2}\right|$ by $\delta$, and assign $G_{\delta}=\left\{y \in G,\left|y-x_{1}\right| \leq 2 \delta\right\}$. Then

$$
\psi\left(x_{1}\right)-\psi\left(x_{2}\right)=\int_{G}\left[Q\left(x_{1}, y, y-x_{1}\right)-Q\left(x_{2}, y, y-x_{2}\right)\right] \varphi(y) d y=\Delta_{1}+\Delta_{2},
$$

where $\Delta_{1}$ corresponds to the integral with respect to $G_{\delta}$. By virtue of (3.2.7)-(3.2.8), we have the estimates

$$
\left|\Delta_{1}\right| \leq|\varphi|_{0}|Q|_{C^{\nu(0)}} I_{1}(\delta), \quad\left|\Delta_{2}\right| \leq|\varphi|_{0}|Q|_{C^{\nu(0)}} I_{2}^{\prime}(\delta)+M|\varphi|_{0}|Q|_{C^{\nu(1)}} I_{2}^{\prime \prime}(\delta),
$$

where

$$
\begin{gathered}
I_{1}(\delta)=\int_{G_{\delta}}\left(\left|y-x_{1}\right|^{\nu-k}+\left|y-x_{2}\right|^{\nu-k}\right) d_{k} y, \quad I_{2}^{\prime}(\delta)=\delta^{\nu} \int_{G \backslash G_{\delta}}\left|y-x_{1}\right|^{-k} d_{k} y, \\
\left.I_{2}^{\prime \prime}(\delta)=\delta \int_{G \backslash G_{\delta}}\left|y-x_{2}\right|^{\nu}\left(\left|y-x_{1}\right|^{-k-1}+\left|y-x_{2}\right|^{-k-1}\right)\right] d_{k} y .
\end{gathered}
$$

Since the inequality $\left|y-x_{1}\right| \leq 2 \delta$ implies the inequality $\left|y-x_{2}\right| \leq 3 \delta$, we have the obvious inequalities

$$
I_{1}(\delta) \leq \int_{\left|y-x_{1}\right| \leq 3 \delta}\left|y-x_{1}\right|^{\nu-k} d_{k} y+\int_{\left|y-x_{2}\right| \leq 3 \delta}\left|y-x_{2}\right|^{\nu-k} d_{k} y \leq C_{1} \delta^{\nu},
$$

where $C_{1}$ is a positive constant depending only on $k$ and $\nu$.

For $\left|y-x_{1}\right| \geq 2 \delta$, one can use inequalities (3.1.13), which imply

$$
I_{2}^{\prime}(\delta)+I_{2}^{\prime \prime}(\delta) \leq \delta^{\nu} \int_{2 \delta \leq\left|y-x_{1}\right| \leq R}\left|y-x_{1}\right|^{-k} d_{k} y+\delta M 2^{k+2} \int_{\delta \leq\left|y-x_{2}\right|}\left|y-x_{2}\right|^{\nu-k-1} d_{k} y .
$$


This yields the estimate $I_{2}^{\prime}(\delta)+I_{2}^{\prime \prime}(\delta) \leq C_{2} \delta^{\nu}(1+|\ln \delta|)$, where the positive constant $C_{2}$ depends only on $k$ and $\nu$ as above. Substituting these estimates into (3.2.10) and taking into account (3.2.9), we obtain the inequality

$$
[\psi]_{\mu} \leq C|Q|_{C^{\nu(1)}}|\varphi|_{0}
$$

Combining it with (3.2.6), we complete the proof of (3.2.5).

Note that if $\alpha<k$, then the integral (3.2.4) with kernel $Q$ from $C^{\nu(1)}\left(G \times G, \mathcal{H}_{-\alpha}\right)$ is covered by Theorem 3.2.1. Indeed, the function $Q_{1}(x, y, \xi)=|x-y|^{k-\alpha}|\xi|^{\alpha-k} Q(x, y, \xi)$ belongs to the class $C^{\nu_{1}(1)}\left(G \times G, \mathcal{H}_{-k}\right)$ with exponent $\nu_{1}=\min (k-\alpha, \nu)$ and it is obvious that $Q(x, y, y-x)=Q_{1}(x, y, y-$ $x)$. Therefore, it remains to use the estimate (3.2.5) with $\mu<\nu_{1}$.

From Theorem 3.2.1, it follows that the operator $T(q)$ defined by the integral (3.2.2) boundedly maps $C^{0}$ to $C^{\nu}$. Since the embedding $C^{\nu} \subseteq C^{\mu}$ is compact for $\mu<\nu$, it follows that this is a compact operator from $C^{0}$ to $C^{\mu}$. In particular, it is a compact operator in the space $C^{\mu}(G)$. Thus, if $\lambda \in \mathbb{C}$, then $1-\lambda T(q)$ is a Fredholm operator in this space and any $C(G)$-solution $\varphi$ of the equation $\varphi+\lambda T(q) \varphi=f$ with a right-hand side $f$ from $C^{\mu}(G)$ also belongs to $C^{\mu}(G)$.

As in Sec. 1.9, we verify that $T(p) T(q)=T(p * q)$, where the functions

$$
(p * q)(x, y)=|x-y|^{k} \int_{G} \frac{p(x, z) q(z, y)}{|x-z|^{k}|y-z|^{k}} d z, \quad x \neq y,
$$

$p$, and $q$ satisfy the conditions (3.2.3). For the considered bilinear map, Lemma 1.9.2 is still valid in class (3.2.1). Finally, similarly to Sec. 1.9, we arrive at the following analog of Theorem 1.9.1.

Theorem 3.2.2. There exists at most a denumerable set $\Lambda$ from $\mathbb{C}$ such that the operator $1-\lambda T(q)$ is invertible for any $\lambda$ located outside $\Lambda$. Also, $[1-\lambda T(q)]^{-1}=1-T\left(r_{\lambda}\right)$, where the function $r_{z}(x, y)$ belongs to $C^{\nu}(G \times G)$, satisfies the condition (3.2.1), is analytic with respect to $z$ in the open set $\mathbb{C} \backslash \Lambda$, and admits poles at points $\lambda$ from $\Lambda$.

Similarly to (3.2.4), consider the integral

$$
\psi(x)=\int_{\Gamma} Q(x, y, y-x) \varphi(y) d_{k-1} y, \quad x \in \Gamma,
$$

on a smooth $(k-1)$-dimensional surface $\Gamma$, where the kernel $Q(x, y ; \xi)$ with respect to the variable $\xi$ belongs to $\mathcal{H}_{1-k}$.

Theorem 3.2.3. Let $\Gamma$ from $\mathbb{R}^{k}$ be a smooth surface with boundary and a kernel $Q$ from $C^{\nu(1)}(\Gamma \times$ $\left.\Gamma, \mathcal{H}_{1-k}\right)$ be such that $Q(y, y ; \xi)=0, y \in \Gamma$ (cf. (3.2.3)). Then, for any bounded on $\Gamma$ function $\varphi$, the relation (3.2.13) defines a function $\psi$ from $C^{\mu}(\Gamma), 0<\mu<\nu$, such that its norm satisfies the estimate (3.2.5), where $C$ is a positive constant depending only on $\Gamma$.

Proof. Let $\gamma: \bar{G} \rightarrow \Gamma$ be a smooth parametrization of the surface, where $G \subseteq \mathbb{R}^{k-1}$ is a Lipschitz domain. Due to Lemma 2.3.3, this parametrization is an $M$-Lipschitz map, i.e., there exists a constant $M$ such that $M \geq 1$ and

$$
|s-t| / M \leq|\gamma(s)-\gamma(t)| \leq M|s-t| .
$$

Using this parametrization, continue the proof, arguing as in the proof of Theorem 3.2.1.

By virtue of Theorem 3.1.1, we have the inequality $|Q(x, y ; y-x)| \leq C_{0}|y-x|^{\nu-k+1}$, which yields the estimate

$$
|\psi(x)| \leq|\varphi|_{0} C_{0} \int_{\Gamma}|x-y|^{\nu-k+1} d y .
$$


The last integral is estimated by a constant depending only on $\gamma$ and the diameter $R$ of the domain $G$ :

$$
\int_{\Gamma}|x-y|^{\nu-k+1} d y \leq M^{k-1-\nu} \int_{G}|s-t|^{\nu-k+1}|m(t)| d t, \quad x=\gamma(s),
$$

where the bounded function $|m(s)|$ is determined by $\gamma$ in the same way as is done in Sec. 2.4.

Fix two different points $x_{j}=\gamma\left(s_{j}\right)$ from $G, j=1,2$, and assign $\delta=\left|s_{1}-s_{2}\right|$ and $G_{\delta}=\{t \in$ $\left.G,\left|t-s_{1}\right| \leq 2 \delta\right\}$. Then the relation (3.2.9) holds, where $G$ and $G_{\delta}$ are replaced by $\gamma(G)$ and $\gamma\left(G_{\delta}\right)$, respectively. From inequalities (3.2.7)-(3.2.8), where $k$ is replaced by $k-1$, deduce the estimates (3.2.10) with the integrals (3.2.11), where $k$ is replaced by $k-1, G_{\delta}$ is replaced by $\gamma\left(G_{\delta}\right)$, and $G \backslash G_{\delta}$ is replaced by $\gamma\left(G \backslash G_{\delta}\right)$. For these integrals, we have the estimates

$$
I_{1}(\delta) \leq \int_{\left|t-s_{1}\right| \leq 3 \delta}\left|\gamma(t)-\gamma\left(s_{1}\right)\right|^{\nu-k+1}|m(t)| d t+\int_{\left|t-s_{2}\right| \leq 3 \delta}\left|\gamma(t)-\gamma\left(s_{2}\right)\right|^{\nu-k+1}|m(t)| d t
$$

and

$$
I_{2}^{\prime}(\delta)+I_{2}^{\prime \prime}(\delta) \leq \delta^{\nu} \int_{2 \delta \leq\left|t-s_{1}\right| \leq R}\left|\gamma(t)-\gamma\left(s_{1}\right)\right|^{-k+1}|m(t)| d t+\delta M 2^{k+2} \int_{\delta \leq\left|t-s_{2}\right|}\left|\gamma(t)-\gamma\left(s_{2}\right)\right|^{\nu-k}|m(t)| d t .
$$

As above, combining this with (3.2.14), we obtain the estimates

$$
I_{1}(\delta) \leq C_{1} \delta^{\nu} \quad \text { and } \quad I_{2}^{\prime}(\delta)+I_{2}^{\prime \prime}(\delta) \leq C_{2} \delta^{\nu}(1+|\ln \delta|),
$$

where the constants $C_{j}$ depend only on $\gamma$ and the diameter $R$ of the domain $G$. In the considered case, substituting them in the relations (3.2.9)-(3.2.10), we arrive at the estimate (3.2.12). The said estimate combined with (3.2.14) completes the proof of the theorem.

Note that Theorem 3.2.3 remains valid for the integrals (3.2.12) considered on smooth closed surfaces. To prove that, it suffices to select a finite set of surfaces $\Gamma_{j}, 1 \leq j \leq m$, with boundaries such that the open surfaces $\Gamma_{j} \backslash \partial \Gamma_{j}$ cover $\Gamma$; then Theorems 2.1.1 and 3.2.3 are applied to $\Gamma_{j}$.

\subsection{The Notion of Singular Integrals}

Assume that $D$ is an open subset of $\mathbb{R}^{k}$ (no boundedness of $D$ is required), $a_{1}, \ldots, a_{n-1}$ are its points, and $f(x) \in L(D)$. To any collection $\varepsilon=\left(\varepsilon_{1}, \ldots, \varepsilon_{n}\right)$ of positive numbers, assign the bounded set $D_{\varepsilon}=\left\{x \in D,\left|x-a_{j}\right|>\varepsilon_{j}, j=1, \ldots, n-1,|x|<1 / \varepsilon_{n}\right\}$. Then, due to Sec. 1.8, there exists a limit

$$
\int_{D} f(x) d x=\lim _{\varepsilon \rightarrow 0} \int_{D_{\varepsilon}} f(x) d x .
$$

If the function $f$ is only locally summable on the set $\bar{D} \backslash\left\{a_{1}, \ldots, a_{n-1}\right\}$, but the above limit still exists, then it is called the singular integral (in the principal value sense) at the singular points $a_{j}$ and at the singular point $a_{n}=\infty$ (the last one is added if there exists a ball such that the domain $D$ contains its exterior).

It is obvious that singular integrals preserve the basic linearity and additivity properties. Here, the last property is treated as follows: if $D_{1}$ and $D_{2}$ are disjoint open sets such that they cover all points $a_{j}$ and $\bar{D}_{1} \cup \bar{D}_{2}=\bar{D}$, then the singular integral over $D$ is equal to the sum of the singular integrals over $D_{1}$ and $D_{2}$.

If $x=a$ is a singular point, then the image of the set $\{|x-a|>\varepsilon\}$, i.e., the set $\{y,|\alpha(y)-\alpha(a)|>\varepsilon\}$, is not guaranteed to be the complement to a ball. Therefore, one must be careful, performing the change of variables $x=\alpha(y)$ to a singular integral. Due to the additivity of singular integrals, it suffices to consider the case where $a=0$ and this point is unique. Let us describe the situation where such a change is possible in the multi-dimensional case. 
Theorem 3.3.1. Let a finite domain $D$ contain the point $x=0$ and let $\alpha$ be a diffeomorphism of this domain onto $G$ such that the Jacobi matrix $\alpha^{\prime}(0)$ is orthogonal up to a constant factor. Let a function $f(x)$ be continuous for nonnegative $x$ and let it admit the estimate

$$
|f(x)| \leq C|x|^{-k} \text {. }
$$

Then the singular integral over the domain $D$ with the singular point $x=0$ admits the change of variables

$$
\int_{D} f(x) d x=\int_{G} f[\alpha(y)]\left|\operatorname{det} \alpha^{\prime}(y)\right| d y
$$

in the following sense: any of these integrals exists if and only if the other one exists and if they exist, then they coincide.

Proof. If $\alpha(x)$ is a linear transformation, $\alpha(x)=\lambda A x$, such that $\lambda>0$ and $A$ is an orthogonal matrix, then the claim of the lemma is obvious. Therefore, without loss of generality, one can assume that $\alpha(0)=0$ and $\alpha^{\prime}(0)$ is the identity matrix. Then $|\alpha(y)| /|y| \rightarrow 1$ as $y \rightarrow 0$ and the functions

$$
\sigma_{1}(r)=\min _{|y|=r}|\alpha(y)|, \quad \sigma_{2}(r)=\max _{|y|=r}|\alpha(y)|
$$

possess a similar property, i.e.,

$$
\lim _{r \rightarrow 0} \frac{\sigma_{j}(r)}{r}=1
$$

For brevity, introduce the notation $g(y)=f[\alpha(y)]\left|\operatorname{det} \alpha^{\prime}(y)\right|$. Then

$$
\int_{|x| \geq \varepsilon} f(x) d x=\int_{|\alpha(y)| \geq \varepsilon} g(y) d y .
$$

Assign $G_{\varepsilon, j}=\left\{y \in G, \sigma_{j}(|y|) \geq \varepsilon\right\}, j=1,2$, and take into account the fact that

$$
G_{\varepsilon, 1} \subseteq\{y \in G,|\alpha(y)| \geq \varepsilon\} \subseteq G_{\varepsilon, 2}
$$

by virtue of (3.3.3). Therefore, it suffices to verify that

$$
\lim _{\varepsilon \rightarrow 0} \int_{G_{\varepsilon, 2} \backslash G_{\varepsilon, 1}} g(y) d y=0 .
$$

It is obvious that the function $g(y)$ admits an estimate similar to (3.3.2). Therefore, the function $g(y)$ can be replaced by the function $|y|^{-k}$; then it remains to prove the relation

$$
\lim _{\varepsilon \rightarrow 0} \int_{\Delta(\varepsilon)} \frac{d r}{r}=0,
$$

where $\Delta(\varepsilon)=\left\{r \mid \sigma_{1}(r) \leq \varepsilon \leq \sigma_{2}(r)\right\}$. In the notation

$$
\delta_{j}^{-}(\varepsilon)=\min \left\{r \mid \sigma_{j}(r)=\varepsilon\right\}, \quad \delta_{j}^{+}(\varepsilon)=\max \left\{r \mid \sigma_{j}(r)=\varepsilon\right\},
$$

the inclusion $\Delta(\varepsilon) \subseteq\left[\delta_{2}^{-}(\varepsilon), \delta_{1}^{+}(\varepsilon)\right]$ hods. Hence,

$$
\int_{\Delta(\varepsilon)} \frac{d r}{r} \leq \int_{\delta_{2}^{-}(\varepsilon)}^{\delta_{1}^{+}(\varepsilon)} \frac{d r}{r}
$$

and it remains to verify that

$$
\lim _{\varepsilon \rightarrow 0} \frac{\delta_{1}^{+}(\varepsilon)}{\delta_{2}^{-}(\varepsilon)}=1 .
$$


Omit the index $j$ in the notation for Definition (3.3.5) for $\sigma\left(r^{ \pm}\right)=\varepsilon$. Then

$$
\frac{\delta^{ \pm}(\varepsilon)}{\varepsilon}=\frac{r^{ \pm}}{\sigma\left(r^{ \pm}\right)}
$$

Takin into account (3.3.4), we deduce that $\delta^{ \pm}(\varepsilon) / \varepsilon \rightarrow 0$ as $\varepsilon \rightarrow 0$, which completes the proof of (3.3.6) and of the theorem.

Using the inversion $x^{*}=x /|x|^{2}$, one can also apply Theorem 3.3.1 to cover the case of the infinity point $\infty$. Indeed, let a domain $D$ be a neighborhood of $\infty$ and be separated from the point $x=0$. Since this inversion maps the domain $\{x \in D,|x| \geq 1 / \varepsilon\}$ to $\left.y \in D^{*},|y| \geq \varepsilon\right\}$, it follows that the relation

$$
\int_{D} f(x) d x=\int_{D^{*}} f\left(y^{*}\right)\left|\operatorname{det}\left(y^{*}\right)^{\prime}\right| d y
$$

also holds for singular operators. Elements of the Jacobi matrix $\left(y^{*}\right)^{\prime}$ are partial derivatives

$$
\frac{\partial\left(y_{i}|y|^{-2}\right)}{\partial y_{j}}=|y|^{-2} M_{i j}(y), \quad M_{i j}(y)=\delta_{i j}-2 \frac{y_{i} y_{j}}{|y|^{2}} .
$$

It is obvious that the matrix $M$ is symmetric and homogeneous of power 0 and its second power coincides with the identity matrix. In particular, $\operatorname{det}\left(y^{*}\right)^{\prime}=|y|^{-2 k}$ and the condition (3.3.2) for the function $f$ in a neighborhood of $\infty$ passes to a similar condition for the function $f\left(y^{*}\right) \operatorname{det}\left(y^{*}\right)^{\prime}$ in a neighborhood of the origin.

This allows one to reformulate Theorem 3.3.1 with respect to the singular point $x=\infty$. We omit this, but note that if (3.3.2), then the singular integral admits changes of variables with respect to translations $x \rightarrow x-a$ and extensions $x \rightarrow \lambda x, \lambda>0$, leaving the point $\infty$ fixed.

Consider sufficient conditions for the function $f$, providing the existence of the singular integral. It is obvious that it suffices to consider the case of one point $a=0$ belonging to the domain $D=\{|x|<1\}$. Assume that the function $f(x)$ can be represented in the form

$$
f(x)=Q(x)+f_{0}(x), \quad f_{0} \in L(D),
$$

where the homogeneous function $Q(x)$ belongs to $\mathcal{H}_{-k}$.

By virtue of the homogeneity of the function $Q$, we have the relation

$$
\int_{\varepsilon<|x|<1} Q(x) d x=\left(\int_{\Omega} Q(\xi) d \xi\right) \int_{\varepsilon}^{1} r^{-1} d r .
$$

Therefore, in this case, the existence of the singular integral (3.3.1) is equivalent to the relation

$$
\int_{\Omega} Q(\xi) d_{k-1} \xi=0
$$

and if the singular integral of $f$ exists, then it coincides with the classical integral of $f_{0}$. In the sequel, if integrals on sets of different dimensions are considered, then the dimension might be marked explicitly.

For the two-dimensional case, the notion of singular integrals is introduced in [77]; for the general case (i.e., for $k>2$ ), it is introduced in [42].

If a function $\varphi$ belongs to $C_{0}^{\infty}(D)$, then, similarly to (3.3.7), the product $f \varphi$ can be expanded to the sum $\varphi(0) Q(x)+f_{1}(x)$, where the function $f_{1}(x)=\varphi(x) f_{0}(x)+[\varphi(x)-\varphi(0)] Q(x)$ is summable. Hence, the singular integral

$$
(f, \varphi)=\int_{D} f(x) \varphi(x) d x
$$

coincides with the integral of the function $f_{1}$. This immediately implies that the linear functional $u(\varphi)=(f, \varphi)$ is continuous with respect to the $C_{0}^{\infty}(D)$-convergence introduced in Sec. 1.8, i.e., is a 
generalized function. As in the case of regular generalized functions, this functional is identified with $f$.

If the domain $D$ in (3.3.7) coincides with the space $\mathbb{R}^{k}$, then, arguing as above, one can show that the singular integral of the function $Q(x)$ with singular points $x=0$ and $x=\infty$ exists and equals to zero. Taking into account the remark to Theorem 3.3.1, we conclude that the similar relation

$$
\int_{\mathbb{R}^{k}} Q(x-a) d x=0
$$

also holds in the case where the singular points are $x=a$ and $x=\infty$.

Obviously, in the one-dimensional case (i.e., for $k=1$ ), the condition (3.3.8) is to be replaced by $Q(-1)=-Q(1)$, which means that $Q(x)=Q(1) / x$.

Below, several examples of the case where the condition (3.3.8) is satisfied are provided.

Lemma 3.3.1. The condition (3.3.8) is satisfied for any odd function $Q(\xi)$ from $\mathcal{H}_{1-k}$. If $Q$ is an even function satisfying (3.3.8), then the similar condition

$$
\int_{\Omega^{+}} Q(\xi) d_{k-1} \xi=0
$$

is satisfied for any hemisphere $\Omega^{+}=\{\xi \in \Omega, \xi n>0\}$, where $n$ is a unit vector and $\xi n$ denotes the scalar product.

The condition (3.3.8) is satisfied for any partial derivative $Q=\partial Q^{0} / \partial \xi_{i}$ of any function $Q^{0}(\xi)$ from $\mathcal{H}_{1-k}, k \geq 2$.

Proof. The first assertion of the lemma is obvious since, by virtue of the oddness, the change $\xi=-\xi^{\prime}$ alternates the sign of the integral (3.3.8). If the function $Q$ is even, then

$$
\int_{\Omega} Q(\xi) d \xi=\int_{\Omega^{+}} Q(\xi) d_{k-1} \xi+\int_{\Omega^{-}} Q(\xi) d_{k-1} \xi=2 \int_{\Omega^{+}} Q(\xi) d_{k-1} \xi,
$$

which proves the relation (3.3.10).

Pass to the last assertion of the theorem. Using the Green relation from Sec. 1.8, we obtain that

$$
\int_{1<|\xi|<2} Q(\xi) d \xi=\int_{\Omega_{1}} Q^{0}(\xi) \frac{\xi_{i}}{|\xi|} d_{k-1} \xi-\int_{\Omega} Q^{0}(\xi) \frac{\xi_{i}}{|\xi|} d_{k-1} \xi,
$$

where $\Omega_{1}$ denotes the sphere $|\xi|=2$. Apply the change $\xi=2 \xi^{\prime}, \xi^{\prime} \in \Omega$. Taking into account relations $d_{k-1} \xi=2^{k-1} \xi$ and $Q^{0}\left(2 \xi^{\prime}\right)=2^{1-k} Q^{0}\left(\xi^{\prime}\right)$, we see that the integral with respect to $\Omega_{1}$ passes to the corresponding integral with respect to $\Omega$. Therefore, the right-hand side of the previous relation is equal to zero. It remains to note that its left-hand side is transformed to the form

$$
\left(\int_{\Omega} Q(\xi) d \xi\right) \int_{1}^{2} r^{-1} d r
$$

Consider functions $Q$ with the property (3.3.10) in detail.

Lemma 3.3.2. Let there exist a vector $n$ such that a function $Q$ from $\mathcal{H}_{-k}$ satisfy the condition (3.3.10) with respect to $n$ and $P^{ \pm}(n)=\left\{x \in \mathbb{R}^{k}, \pm x n>0\right\}$. Then, for any $x$ from $P^{-}$, the singular integral

$$
H(x)=\int_{P^{+}} Q(y-x) d y, \quad x \in P^{-},
$$


with the singular point $\infty$ exists and is independent of $x$ and

$$
\int_{P^{+}} \frac{\partial Q}{\partial \xi_{i}}(y-x) d y=0, \quad x \in P^{-} .
$$

Proof. As above, using the condition (3.3.10), we verify that the singular integral of the function $Q$ over the domain $P^{+}$with the singular points 0 and $\infty$ exists and is equal to zero. This is also valid for the singular integral (3.3.11). The subspace $P^{+}$is invariant with respect to the transformations $x \rightarrow x-a$, an $=0$, and $x \rightarrow \lambda x, \lambda>0$. Therefore, arguing as above, we deduce the relations

$$
H(x)=H(x-a), \quad H(x)=H(\lambda x),
$$

which hold only in the case where $H$ is a constant function (denote its constant value by $H$ ). Fix a point $a$ from $P_{-}$. Then, by virtue of Lemma 3.1.1, the function $Q(y-x)-Q(y-a)$ is integrable over $P^{+}$and the constant function

$$
\int_{P_{+}}[Q(y-x)-Q(y-a)] d y, \quad x \in P^{-}, x \neq a,
$$

can be differentiated under the integral sign. This yields relation (3.3.12).

Singular integrals can also be considered on a smooth surface $\Gamma$ from $\mathbb{R}^{k}$ (if $k=2$, then it is a surface).

Lemma 3.3.3. Let a smooth $(k-1)$-dimensional surface $\Gamma$ from $\mathbb{R}^{k}$ belong to the class $C^{1, \nu}$ and a kernel $Q(y ; \xi)$ from $C^{\nu(1)}\left(\Gamma, \mathcal{H}_{1-k}\right)$ be odd with respect to the variable $\xi$. Then the singular integral

$$
\psi(a)=\int_{\Gamma} Q(y, y-a) d_{k-1} y, \quad a \in \Gamma,
$$

treated as the limit of integrals over $\Gamma \cap\{|y-a| \leq \varepsilon\}$ as $\varepsilon \rightarrow 0$, exists.

Proof. Without loss of generality, one can assume that $\Gamma$ is a smooth surface with boundary and $a$ is its interior point. Let a positive $\rho$ satisfy conditions of Theorem 2.4.1 with respect to this point. Due to this theorem, the intersection of $\Gamma$ with the neighborhood $C_{\rho}(a)$ is described (in the local coordinates) by the equation $u_{k}=f(\tilde{u}),|\tilde{u}| \leq \rho$, where $f$ is continuously differentiable in the $(k-1)$-dimensional ball $B_{\rho}=\{|s| \leq \rho\}$,

$$
f(0)=f^{\prime}(0)=0, \quad\left|f^{\prime}\right|_{0} \leq 1 .
$$

Recall that the axis $u_{k}$ of the local coordinate system with the origin at the point $a$ is directed along the normal to $\Gamma$ at this point. One can use any orthogonal matrix $U$ from $\mathbb{R}^{k \times k}$ to pass to this coordinate system as follows: $x-a=U\left(\tilde{u}, u_{k}\right)$. Respectively, the surface $\Gamma(a)=\Gamma \cap C_{\rho}(a)$ is described by the parametrical equation $\gamma(s)=a+U(s, f(s))$, where $s$ varies in the ball $B_{\rho}$.

Let us show that one can change variables in the singular integrals using the relation

$$
\int_{\Gamma(a)} Q(a, y-a) d y=\int_{|s| \leq \rho} \widetilde{Q}[s, f(s)]|m(s)| d s,
$$

where $\widetilde{Q}(\xi)=Q(a, U \xi)$ and $|m(s)|=\sqrt{1+\left|f^{\prime}(s)\right|^{2}}$. Theorem 3.3.1 is not directly applicable for the justification of this change since the map $\gamma(s)=a+U[s, f(s)]$ acts from $\mathbb{R}^{k-1}$ to $\mathbb{R}^{k}$. However, this map possesses the property

$$
\lim _{s \rightarrow 0} \frac{|\gamma(s)-a|}{|s|}=1
$$

which is the only property used to prove the said theorem. For this reason, this theorem is still applicable for the justification of (3.3.14). 
Pass to the singular integral at the right-hand side of (3.3.14). According to Theorem 2.4.1, the condition that $\Gamma$ belongs to $C^{1, \nu}$ implies that $f$ belongs to $C^{1, \nu}\left(B_{\rho}\right)$. Combining this with (3.3.13) and Lemma 3.1.1, we easily conclude that the function $\widetilde{Q}[s, f(s)]-\widetilde{Q}(s, 0)$ is summable in the ball $B_{\rho}$. Hence, it remains to use the oddness of the function $\widetilde{Q}$ and Lemma 3.3.1.

\section{4. $C^{\mu}$-Estimates of Singular Integrals}

In a finite subdomain $D$ of $\mathbb{R}^{k}$, consider the singular integral

$$
\psi(x)=\int_{D} Q(y, y-x) \varphi(y) d y, \quad x \in D,
$$

such that its kernel $Q(y, \xi)$ belongs to $\mathcal{H}_{-k}$ with respect to the variable $\xi$ and satisfies the condition

$$
\int_{\Omega} Q(x, \xi) d \xi=0, \quad x \in D .
$$

Let us start from the case where $\varphi=1$.

Lemma 3.4.1. Let a kernel $Q(y, \xi)$ belong to the class $C^{\nu(1)}\left(D, \mathcal{H}_{-k}\right)$ and satisfy the condition (3.3.8) with respect to the variable $\xi$. Then, for any closed subdomain $\bar{D}_{0}$ of $D$, the singular integral

$$
q(x)=\int_{D} Q(y, y-x) d y, \quad x \in D_{0},
$$

defines a function $q(x)$ from $C^{\mu}\left(D_{0}\right), 0<\mu<\nu$, such that the estimate

$$
|q|_{C^{\mu}\left(D_{0}\right)} \leq C|Q|_{C^{\nu(1)}}
$$

of its norm holds, where $C$ is a positive constant depending only on the distance from $D_{0}$ to $\partial D$.

Proof. First, we prove the lemma in the case where the function $Q(y, \xi)$ does not depend on $y$. According to (3.3.9), the function $q$ can be represented in the form

$$
q(x)=-\int_{\mathbb{R}^{k} \backslash D} Q(y-x)-Q(y-a) d y+\int_{D} Q(y-a) d y, \quad x \in D_{0},
$$

where $a$ is a fixed point of $D \backslash \bar{D}_{0}$. By virtue of Lemma 3.1.1, the function $Q(y-x)-Q(y-a)$ is integrable on $\mathbb{R}^{k} \backslash D$ and, therefore, the function $q(x)$ in this relation can be differentiated under the integral sign. Due to Theorem 2.2.2, this yields the estimate

$$
|q|_{0, D_{0}}+|q|_{1, D_{0}} \leq C|Q|_{(1)} .
$$

Pass to the general case. For any $x_{1}$ and $x_{2}$ from $D$, the following relation holds:

$q\left(x_{1}\right)-q\left(x_{2}\right)=\int_{D}\left[Q\left(x_{1}, y-x_{1}\right)-Q\left(x_{2}, y-x_{1}\right)\right] d y+\int_{D}\left[Q\left(x_{2}, y-x_{1}\right)-Q\left(x_{2}, y-x_{2}\right)\right] d y=\Delta_{0}+\Delta_{1}$.

Applying the estimate (3.4.5) to the functions $Q_{0}(\xi)=Q\left(x_{1}, y-x_{1}\right)-Q\left(x_{2}, y-x_{1}\right)$ and $Q_{1}(\xi)=$ $Q\left(x_{2}, \xi\right)$, we obtain the inequalities

$$
\left|\Delta_{0}\right| \leq C\left|Q_{0}\right|_{(1)}, \quad\left|\Delta_{1}\right| \leq C\left|Q_{1}\right|_{(1)}\left|x_{1}-x_{2}\right| .
$$

By virtue of (3.1.7), we have the inequality $\left|Q_{0}\right|_{(1)} \leq|Q|_{C^{\nu(1)}}\left|x_{1}-x_{2}\right|^{\nu}$. Obviously, this implies that $\left|Q_{1}\right|_{(1)} \leq|Q|_{C^{0(1)}}$. This immediately yields the estimate (3.4.4). 
Theorem 3.4.1. Let a kernel $Q(y, \xi)$ from $C^{\nu(1)}\left(D, \mathcal{H}_{-k}\right)$ satisfy the condition (3.4.2) and $\varphi$ belong to $C^{\mu}(D), 0<\mu<\nu$. Then, for any closed subset $\bar{D}_{0}$ of $D$, the singular integral (3.4.1) defines a function $\phi(x)$ from $C^{\mu}\left(D_{0}\right)$, satisfying the norm estimate

$$
|\psi|_{C^{\mu}\left(D_{0}\right)} \leq C|Q|_{C^{\nu(1)}}|\varphi|_{C^{\mu}}
$$

where $C$ is a positive constant depending only the distance between $D_{0}$ and $\partial D$ provided that $\mu$ and $\nu$ are fixed.

If the above is satisfied and another kernel $Q(u, y, \xi)$ depends on a parameter $u$ from $G$, belongs to the space $C^{\nu(1)}\left(G \times D, \mathcal{H}_{-k}\right)$, and satisfies the condition (3.4.2) for any $u$ and $y$, then the corresponding function

$$
\psi(u, x)=\int_{D} Q(u, y, y-x) \varphi(y) d y, \quad x \in D_{0},
$$

belongs to $C^{\mu}\left(G \times D_{0}\right)$ and a similar (3.4.6) estimate of norms holds.

Proof. Represent the integral (3.4.1) by the sum $\psi_{0}(x)+q(x) \varphi(x)$, where $q$ is defined by (3.4.3), while $\psi_{0}$ is defined by a classical integral as follows:

$$
\psi_{0}(x)=\int_{D} Q(y, y-x)[\varphi(y)-\varphi(x)] d y, \quad x \in D_{0} .
$$

According to Lemma 3.4.1, it suffices to prove the estimate (3.4.6) only for $\psi_{0}$. Since $|\varphi(y)-\varphi(x)| \leq$ $[\varphi]_{\mu}|x-y|^{\mu}$, we have the following estimate for the sup-norm of this function:

$$
\left|\psi_{0}\right|_{0, D_{0}} \leq C|Q|_{C^{\nu(0)}}[\varphi]_{\mu}
$$

Fir $x_{1}$ and $x_{2}$ from $D_{0}$, assign $\delta=\left|x_{1}-x_{2}\right|$. Then

$$
\psi_{0}\left(x_{1}\right)-\psi_{0}\left(x_{2}\right)=q\left(x_{1}\right)\left[\varphi\left(x_{2}\right)-\varphi\left(x_{1}\right)\right]+\Delta
$$

and

$$
\Delta=\int_{D}\left[Q\left(x_{1}, y-x_{1}\right)-Q\left(x_{2}, y-x_{2}\right)\right]\left[\varphi(y)-\varphi\left(x_{2}\right)\right] d y=\Delta_{1}+\Delta_{2},
$$

where $\Delta_{j}$ have the same sense as in the proof of Theorem 3.2.1. For the terms $\Delta_{j}$, one can repeat the corresponding arguing of the proof of this theorem. In particular, the following estimates (similar to (3.2.10)) hold for these terms:

$$
\left|\Delta_{1}\right| \leq[\varphi]_{\mu}|Q|_{C^{\nu(0)}} I_{1}(\delta), \quad\left|\Delta_{2}\right| \leq|\varphi|_{0}|Q|_{C^{\nu(0)}} I_{2}^{\prime}(\delta)+M|\varphi|_{0}|Q|_{C^{\nu(1)}} I_{2}^{\prime \prime}(\delta),
$$

where

$$
\begin{gathered}
I_{1}(\delta)=\int_{D_{\delta}}\left|y-x_{2}\right|^{\mu}\left(\left|y-x_{1}\right|^{-k}+\left|y-x_{2}\right|^{-k}\right) d_{k} y, \quad I_{2}^{\prime}(\delta)=\delta^{\nu} \int_{D \backslash D_{\delta}}\left|y-x_{1}\right|^{-k} d_{k} y, \\
\left.I_{2}^{\prime \prime}(\delta)=\delta \int_{D \backslash D_{\delta}}\left|y-x_{2}\right|^{\mu}\left(\left|y-x_{1}\right|^{-k-1}+\left|y-x_{2}\right|^{-k-1}\right)\right] d_{k} y .
\end{gathered}
$$

As in the proof of Theorem 3.2.1, this implies the estimate

$$
\left[\psi_{0}\right]_{\mu} \leq C|Q|_{C^{\nu(1)}}[\varphi]_{\mu}
$$

Combining this estimate with (3.4.8), we complete the proof of (3.4.6).

Actually, the second assertion of the theorem follows from the estimate (3.4.6). Indeed, if $u$ is fixed, then this estimate means that

$$
\left|\psi\left(u, x_{1}\right)-\psi\left(u, x_{2}\right)\right| \leq C|Q|_{C^{\nu(1)}}|\varphi|_{C^{\mu}}\left|x_{1}-x_{2}\right|^{\mu} .
$$


On the other hand, if $u_{1}$ and $u_{2}$ are different points of $G$, then, due to Lemma 2.1.2, the function

$$
\tilde{Q}(y, \xi)=\left|u_{1}-u_{2}\right|^{-\mu}\left[Q\left(u_{1}, y, \xi\right)-Q\left(u_{2}, y, \xi\right)\right]
$$

belongs to the class $C^{\nu-\mu,(1)}$. Let $\tilde{\psi}(x)$ be defined by $\tilde{Q}$ similarly to (3.4.1). Then

$$
\tilde{\psi}(x)=\left|u_{1}-u_{2}\right|^{-\mu}\left[\psi\left(u_{1}, x\right)-\psi\left(u_{2}, x\right)\right]
$$

and the first part of Theorem 3.4.1, where $\nu$ and $\mu$ are replaced by $\tilde{\nu}=\nu-\mu$ and $\tilde{\mu}$ exceeded by $\min (\nu-\mu, \mu)$ respectively, can be applied to this function. In particular, the corresponding estimate of its sup-norm, uniform with respect to $u_{1}$ and $u_{2}$, holds, which completes the proof of the theorem.

Let us describe the case where the function (3.4.7) can be differentiated under the integral sign.

Lemma 3.4.2. Let the assumptions of Theorem 3.4 .1 be satisfied, $G$ be a subdomain of $\mathbb{R}^{s}$, and a kernel $Q(u, y, \xi)$ be continuously differentiable with respect to the variable $u$ and belong to $C^{\nu(1)}(G \times D)$ together with its partial derivatives $Q_{u}^{\prime}$. Then, for any $\varphi$ from $C^{\mu}(D), 0<\mu<\nu$, the relation (3.4.7) can be differentiated under the integral sign:

$$
\frac{\partial \psi}{\partial u_{i}}(u, x)=\int_{D} \frac{\partial Q}{\partial u_{i}}(u, y, y-x) \varphi(y) d y, \quad x \in D_{0} .
$$

In particular, the function $\partial \psi / \partial u_{i}$ belongs to $C^{\mu}\left(G \times D_{0}\right)$ and an estimate of norms, similar to (3.4.6), holds.

Proof. Without loss of generality, $G$ can be assumed to be an interval of the real line. Let $\varepsilon_{n} \rightarrow 0$. For a fixed $u$, assign

$$
Q_{n}(y, \xi)=\varepsilon_{n}^{-1}\left[Q\left(u+\varepsilon_{n}, y, \xi\right)-Q(u, y, \xi)\right]-Q_{u}^{\prime}(u, y, \xi)=\int_{0}^{1}\left[Q_{u}^{\prime}\left(u+t \varepsilon_{n}, y, \xi\right)-Q_{u}^{\prime}(u, y, \xi)\right] d t
$$

For any fixed $\xi$ from $\Omega$, the integrand function is bounded in $C^{\nu}(D)$. It tends to zero as $n \rightarrow \infty$ uniformly with respect to $\xi$. The derivatives of this sequence with respect to $\xi_{i}$ possess the same property. Therefore, due to Theorem 2.1.1, the sequence $Q_{n}$ tends to zero in $C^{\mu(1)}(D)$ provided that $\mu<\nu$. In particular, due to Theorem 3.4.1, we have the relation

$$
\int_{D} Q_{n}(y, y-x) \varphi(y) d y \rightarrow 0 \quad \text { as } n \rightarrow \infty .
$$

Then the above differentiating is justified. By the condition, the function $Q$ satisfies the relation

$$
\int_{\Omega} Q(u, y, \xi) d \xi=0
$$

identically with respect to $u$ and $y$. This relation can be differentiated with respect to $u$. Then a condition similar to (3.4.2) is also satisfied for the kernel $Q_{u}^{\prime}$. By virtue of Theorem 3.4.1, this implies that the derivative $\psi_{u}^{\prime}$ belongs to $C^{\mu}\left(D_{0}\right)$.

In the one-dimensional case, Theorem 3.4 .1 is proved in [52]. In the multidimensional one, it is proved in [21]. Usually, the kernel of the singular integral (3.3.1) is represented in the form

$$
Q(x, y ; \xi)=\frac{Q_{0}(x, y, \xi)}{|\xi|^{k}}
$$

where the function $Q_{0}$ is homogeneous of power zero with respect to the variable $\xi$. The last function is called the characteristic of the singular integral (see [44]).

For the whole domain $D$, Theorem 3.4.1 is not valid in the one-dimensional case. To prove this, consider the function (3.4.3). Here the domain $D$ is an interval $(a, b)$ of the real line, while the kernel $Q(x ; \xi)$ can be represented in the form $c(x) / \xi$, where $c(x)=Q(x ; 1) \in C^{\nu}([a, b] \times[a, b]$. 
Indeed, we have the two-point set $\{ \pm 1\}$ instead of the unit sphere $\Omega$ here and the condition (3.4.2) passes to the relation $Q(x ; 1)+Q(x ;-1)=0$. In other words, the function $Q(x ; \xi)$ is odd with respect to $\xi$ and, therefore, has the Cauchy kernel form $Q(x ; \xi)=Q(x ; 1) \xi^{-1}$. Thus, the following relation holds:

$$
q(x)=c(x) \int_{a}^{b} \frac{d y}{y-x}, \quad a<x<b .
$$

Due to the definition of singular integrals, we have the relation

$$
\int_{a}^{b} \frac{d y}{y-x}=\lim _{\varepsilon \rightarrow 0}\left(\int_{a}^{x-\varepsilon}+\int_{x+\varepsilon}^{b}\right) \frac{d y}{y-x}=\ln \frac{b-x}{x-a} .
$$

Hence, for $c(a) \neq 0$, the function $q$ has a logarithmic singularity at the point $a$.

Below (see Theorem 3.5.1), we show that if $k>1$, then there are assumptions regarding the kernel $Q$ and the smoothness of the boundary of the domain $D$, providing the extension of Theorem 3.4.1 to the whole domain $D_{0}=D$. If $D=\mathbb{R}^{k}$, then it is easily deduced from Theorem 3.4.1: the integrand of (3.4.1) is multiplied by $\chi(y-x)$, where $\chi \in C_{0}^{\infty}\left(\mathbb{R}^{k}\right)$.

Theorem 3.4.2. Let $\chi(x) \in C_{0}^{\infty}\left(\mathbb{R}^{k}\right)$ and a kernel $Q(y, \xi)$ belong to $C^{\nu(1)}\left(\mathbb{R}^{k}, \mathcal{H}_{-k}\right)$ and satisfy the condition (3.4.2). Then the singular operator

$$
(R \varphi)(x)=\int_{\mathbb{R}^{k}} \chi(y-x) Q(y, y-x) \varphi(y) d y, \quad x \in \mathbb{R}^{k},
$$

is bounded in the space $C^{\mu}\left(\mathbb{R}^{k}\right), 0<\mu<\nu$.

Proof. Let the support of the function $\chi(x)$ be contained in a ball $|x| \leq R$. Fix a point $a$ from $\mathbb{R}^{k}$. Then, for $|x-a| \leq R$, the integral $(R \varphi)(x)$ can be represented by the sum

$$
\int_{|y-a| \leq 2 R}[\chi(y-x)-\chi(0)] Q(y, y-x) \varphi(y) d y+\chi(0) \int_{|y-a| \leq 2 R} Q(y, y-x) \varphi(y) d y .
$$

From Theorems 3.2.1 and 3.4.1, we obtain the estimate

$$
|R \varphi|_{C^{\mu}\left(B_{1}\right)} \leq C|R \varphi|_{C^{\mu}\left(B_{2}\right)},
$$

where $B_{1}$ and $B_{2}$ are the balls $\{|x-a| \leq R\}$ and $\{|x-a| \leq 2 R\}$ respectively, while $C$ is a positive constant independent of $a$. This immediately implies the claim of the theorem.

The obtained result is frequently called the Korn-Giraud theorem [3].

\subsection{Estimates up to the Boundary}

Let a subdomain $D_{0}$ of $D$ abut a smooth part $\Gamma$ of the boundary of the domain $D$ and lie from one side of this part in terms of Sec. 2.4. Recall that this notion is defined with respect to a selected unit normal $n(y)$ from $C(\Gamma)$ of the surface $\Gamma$. In the same way, the normal vector $n(y)$ decomposes the unit sphere $\Omega$ from $\mathbb{R}^{k}$ into the hemispheres $\Omega^{ \pm}(y)$ consisting all $\xi$ from $\Omega$ such that $\pm \xi n(y) \geq 0$.

Theorem 3.5.1. Let the boundary of a finite domain $D$ contain a smooth surface with boundary $\Gamma$ of the class $C^{1, \nu}$ such that $D$ lies from one side of it and the subdomain $D_{0}$ of $D$ be such that $\Gamma_{0}=\bar{D}_{0} \cap \partial D \subseteq \Gamma \backslash \partial \Gamma$. Let a kernel $Q(y, \xi)$ belong to $C^{\nu(2)}\left(D, \mathcal{H}_{-k}\right)$ and satisfy (3.4.2) and the following condition:

$$
\int_{\Omega^{+}(y)} Q(y, \xi) d_{k-1} \xi=0, \quad y \in \Gamma .
$$


Then, for any $\varphi$ from $C^{\mu}(D), 0<\mu<\nu$, the function $\psi$ defined by (3.4.1) belongs to the class $C^{\mu}\left(D_{0}\right)$ and admits the estimate

$$
|\psi|_{C^{\mu}} \leq C|Q|_{C^{\nu(2)}}|\varphi|_{C^{\mu}}
$$

where $C$ is a positive constant depending only on the distance between the domain $D_{0}$ and $\partial D \backslash \Gamma$.

Proof. Due to Theorem 2.3.2, there exists a bounded extension operator $P: C^{\mu}(D) \rightarrow C^{\mu}\left(\mathbb{R}^{k}\right)$. Due to Lemma 3.1.3, there exists a bounded extension operator $P^{1}: C^{\nu(1)}(D) \rightarrow C^{\nu(1)}\left(\mathbb{R}^{k}\right)$. Consider these extensions in a finite domain $D^{1}$ containing the domain $D$ and its closure. Assigning $\varphi^{1}=P \varphi$ and $Q^{1}=P^{1} Q$, we have relations $\varphi^{1}(y)=\varphi(y)$ and $Q^{1}(y ; \xi)=Q(y ; \xi)$ (for any $y$ from $D$ ) and the corresponding estimates

$$
\left|\varphi^{1}\right|_{C^{\mu}} \leq C^{1}|\varphi|_{C^{\mu}}, \quad\left|Q^{1}\right|_{C^{\nu(2)}} \leq C^{1}|Q|_{C^{\nu(2)}} .
$$

It is obvious that the function $\psi$ in the domain $D_{0}$ can be represented by the difference of the two integrals

$$
\psi^{0}(x)=\int_{D^{1}} Q^{1}(y, y-x) \varphi^{1}(y) d y, \quad \psi^{1}(x)=\int_{D^{1} \backslash D} Q^{1}(y, y-x) \varphi^{1}(y) d y, \quad x \in D_{0},
$$

such that the former is understood in the classical sense. The conditions of Theorem 3.4.1 are satisfied for $\psi^{0}$ and the pair $D_{0}, D^{1}$. Therefore, the following inequality holds:

$$
\left|\psi^{0}\right|_{C^{\mu}\left(D_{0}\right)} \leq C\left|Q^{1}\right|_{C^{\nu(1)}}\left|\varphi^{1}\right|_{C^{\mu}\left(D^{1}\right)} .
$$

Hence, taking into account (3.5.2), we see that it remains to prove the similar estimate

$$
\left|\psi^{1}\right|_{C^{\mu}\left(D_{0}\right)} \leq C\left|Q^{1}\right|_{C^{\nu(2)}}\left|\varphi^{1}\right|_{C^{\mu}\left(D^{1} \backslash D\right)}
$$

for the function $\psi^{1}$.

It is obvious that the function $\psi^{1}(x)$ can be differentiated under the integral sign:

$$
\frac{\partial \psi^{1}}{\partial x_{i}}(x)=-\int_{D^{1} \backslash D} Q_{i}(y, y-x) \varphi^{1}(y) d y, \quad 1 \leq i \leq k,
$$

where $Q_{i}(y, \xi)=\partial Q^{1} / \partial \xi_{i} \in C^{\nu(1)}\left(D^{1}, \mathcal{H}_{-k-1}\right)$.

Let us show that partial derivatives of the function $\psi^{1}$ admit the estimate

$$
\left|\frac{\partial \psi^{1}}{\partial x_{i}}(x)\right| \leq M\left|Q_{i}\right|_{C^{\nu(1)}}\left|\varphi^{1}\right|_{C^{\mu}} d^{\mu-1}(x, \Gamma), \quad x \in D_{0},
$$

where $M$ is a positive constant depending only on the distance between the domain $D_{0}$ and $\partial D \backslash \Gamma$. Then, taking into account the obvious inequality

$$
\left.\left|Q_{i}\right|_{C^{\nu(1)}}|\leq| Q\right|_{C^{\nu(2)}} \mid
$$

we immediately deduce the estimate (3.5.4) from Theorem 2.4.2.

Similarly to the proof of Theorem 2.4.2, we assign $\Gamma^{\prime}=\partial D \backslash \Gamma$ (for brevity). Then, by condition, $\Gamma \cap \overline{\Gamma^{\prime}}=\partial \Gamma$. Hence, the number $2 r_{0}=d\left(D_{0}, \Gamma^{\prime}\right)$ is positive. Define a compact set $K=\{a \in \Gamma$, $\left.d\left(a, \Gamma^{\prime}\right) \geq r_{0}\right\}$. Obviously, it contains $\Gamma_{0}$. For this compact set, select $\rho_{0}$ as is done in Theorem 2.4.1. Assign

$$
3 \rho=\min \left(r_{0}, \rho_{0}, r_{1}\right),
$$

where $r_{1}$ is the distance between $\partial D$ and $\partial D^{1} \backslash \partial D$, entirely defined by the choice of $D^{1}$. From the definition of $K$, we see that, in fact, $\rho_{0}$ (and, therefore, $\rho$ ) depend only on the distance $2 r_{0}=d\left(D_{0}, \Gamma^{\prime}\right)$.

If $x \in D_{0}$ and $d(x, \Gamma) \geq \rho$, then $d\left(x, D^{1}\right)=\min \left[d(x, \Gamma), d\left(x, \Gamma^{\prime}\right)\right] \geq \rho$ and, therefore, $\left|Q_{i}(y ; y-x)\right| \leq$ $\left|Q_{i}\right|_{C^{\nu(1)}} \mid \rho^{-k-1}$. Hence, no proof for inequality (3.5.5) is required in this case and, therefore, it suffices to consider the case where $d(x, \Gamma) \leq \rho$. Consider a point $a$ from $\Gamma$ such that $d(x, \Gamma)=|x-a|$. Thus, we have the inequality

$$
|x-a|=d(x, \Gamma) \leq \rho .
$$


The inequalities $d\left(a, \Gamma^{\prime}\right) \geq d\left(x, \Gamma^{\prime}\right)-|x-a| \geq 2 r_{0}-\rho \geq r_{0}$ show that $a \in K$. Hence, one can use Theorem 2.4.1, guaranteeing that the intersection $\Gamma(a)=\Gamma \cap C_{\rho}(a)$, where

$$
C_{\rho}(a)=\left\{|\widetilde{u}| \leq \rho,\left|u_{k}\right| \leq 2 \rho\right\},
$$

is described (in the local coordinate system) by the equation $u_{k}=f(\widetilde{u})$ in the ball $B_{\rho}=\{|\widetilde{u}| \leq \rho\}$, where $f$ is a continuously differentiable function such that

$$
f(0)=0, \quad f^{\prime}(0)=0, \quad\left|f^{\prime}\right|_{0} \leq 1, \quad\left[f^{\prime}\right]_{\nu} \leq M_{0},
$$

and $M_{0}$ is a constant depending only on $\Gamma$. In particular, this implies that $|f(s)| \leq|s|$ and, therefore,

$$
\{|y-a| \leq \rho\} \subseteq C_{\rho}(a) \subseteq\{|y-a| \leq 3 \rho\} .
$$

Then, according to (3.5.7), the point $x$ belongs to $C_{\rho}(a)$.

For definiteness, let $n(a)$ be the unit vector of the inner (with respect $D$ ) normal. Then, taking into account (3.5.6)-(3.5.7), we have the relations

$D \cap C_{\rho}(a)=C_{\rho}^{+}(a)=\left\{|\tilde{u}| \leq \rho, f(\tilde{u})<u_{k}<2 \rho\right\}, \quad D^{1} \cap C_{\rho}(a)=C_{\rho}^{-}(a)=\left\{|\tilde{u}| \leq \rho,-2 \rho<u_{k}<f(\tilde{u})\right\}$.

Let us prove that

$$
2|x-y| \geq \begin{cases}|x-a|+|y-a|, & y \in C_{\rho}^{-}(a), \\ \rho, & y \notin C_{\rho}(a) .\end{cases}
$$

Indeed, let a cone $K_{1}$ with vertex at the origin consists of all $z$ such that the angle between $z$ and $n(a)$ is not less $\pi / 4$. Let $K_{2}$ be the angle $\{z=\operatorname{tn}(a), t \geq 0\}$. Since $\left|f^{\prime}\right|_{0} \leq 1$, it follows that the function $f(s)$ admits the estimate $|f(s)| \leq|s|$ and, therefore, the vector $y-a$ belongs to $K_{1}$ provided that $y \in C^{-}(a)$. By virtue of Lemma 2.1.2, if $z_{j} \in K_{j}, j=1,2$, then

$$
\left|z_{1}-z_{2}\right| \geq r_{0}\left(\left|z_{1}\right|+\left|z_{2}\right|\right), \quad r_{0}=\min \left[d\left(K_{1} \cap \Omega, K_{2}\right), d\left(K_{2} \cap \Omega, K_{1}\right)\right] .
$$

In the considered case, it is easy to see that $r_{0}=1 / \sqrt{2}$, which proves the first part of the estimate (3.5.12). Its second part is obvious.

Now, represent $\partial \psi^{1} / \partial x_{i}$ by the sum

$$
\frac{\partial \psi^{1}}{\partial x_{i}}=\psi_{0}+\psi_{1}
$$

assigning

$$
\psi_{0}(x)=\int_{D^{1} \backslash D}\left[Q_{i}(y, y-x) \varphi^{1}(y)-Q_{i}(a, y-x) \varphi^{1}(a)\right] d y, \quad \psi_{1}(x)=\varphi^{1}(a) \int_{D^{1} \backslash D} Q_{i}(a, y-x) d y .
$$

For the former term, we have the obvious estimate

$$
\left|\psi_{0}(x)\right| \leq\left|Q_{i}\right|_{C^{\mu(0)}}\left[\varphi^{1}\right]_{\mu} I_{0}, \quad I_{0}=\int_{D^{1} \backslash D}|y-a|^{\mu}|y-x|^{-k-1} d y .
$$

By virtue of (3.5.10) and (3.5.12), we have the inequality

$$
I_{0} \leq 2^{k+1} \int_{|y-a| \leq 3 \rho} \frac{|y-a|^{\mu} d y}{(|x-a|+|y-a|)^{k+1}}+2^{k+1} \rho^{-k-1} \int_{D^{1} \backslash D}|y-a|^{\mu} d y .
$$

Since

$$
\int_{|y-a| \leq 3 \rho} \frac{|y-a|^{\mu} d y}{(|x-a|+|y-a|)^{k+1}} \leq|x-a|^{\mu-1} \int_{\mathbb{R}^{k}} \frac{|z|^{\mu} d z}{(1+|z|)^{k+1}}
$$

it follows that the estimate (3.5.5) holds for $\psi_{i}^{0}$. 
Consider the function $\psi_{1}$ in (3.5.13). Let $P^{-}(a)$ denote the half-space $\{x,(x-a) n(a)<0\}$. Inequality (3.5.12) is still valid if $D^{1}$ is replaced by $P^{-}(a)$; the proof is the same.

By virtue of (3.5.1), Lemma 3.3.2 is applicable to the kernel $Q^{1}(a, \xi)$. This yields the relation

$$
\int_{P^{-}(a)} Q_{i}(a, y-x) d y=0, \quad x \in P^{-}(a) .
$$

Therefore,

$$
\psi_{1}(x)=\varphi^{1}(a) I_{1}, \quad I_{1}=\left(\int_{D^{1} \backslash D}-\int_{P^{-}(a)}\right) Q_{i}(a, y-x) d y=I_{1}^{\prime}+I_{1}^{\prime \prime},
$$

where

$$
I_{1}^{\prime}=\left(\int_{C^{-}(a)}-\int_{P^{-}(a) \cap C(a)}\right) Q_{i} d y, \quad I_{1}^{\prime \prime}=\left(\int_{D^{1} \backslash D \backslash C(a)}-\int_{P^{-}(a) \backslash C(a)}\right) Q_{i} d y
$$

according to (3.5.11).

It is obvious that

$$
\left|I_{1}^{\prime \prime}\right| \leq\left|Q_{i}\right|_{C^{0(0)}}\left(\int_{D^{1} \backslash D \backslash C(a)}+\int_{P_{-}(a) \backslash C(a)}\right) \frac{d y}{|y-x|^{k+1}} .
$$

Note that $|x-y| \geq|a-y|$ provided that $y \in P_{-}(a)$ and $|y-a| \geq \rho / 2$ provided that $y \in D^{1} \backslash D \backslash C(a)$. Thus, taking into account (3.5.16), we conclude that

$$
\left|I_{1}^{\prime \prime}\right| \leq C\left|Q_{i}\right|_{C^{0(0)}}, \quad C=2^{k+1} \rho^{-k-1} \operatorname{mes}\left(D^{1} \backslash D\right)+\int_{|y-a| \geq \rho} \frac{d y}{|y-a|^{k+1}} .
$$

Pass to $I_{1}^{\prime}$. As we note above, inequality (3.5.12) is preserved under the change of $D^{1}$ by $P^{-}(a)$. Therefore, the inequality

$$
\left|I_{1}^{\prime}\right| \leq 2^{k+1}\left|Q_{i}\right|_{C^{0(0)}} \int_{E(a)} \frac{d y}{(|x-a|+|y-a|)^{k+1}},
$$

holds, where $E(a)$ denotes the symmetric difference of the sets $C_{-}(a)$ and $P_{-}(a) \cap C(a)$. By virtue of (3.5.9), the function $f$ admits the estimate $|f(s)| \leq M_{0}|s|^{\nu+1}$. Therefore, in terms of the local coordinates $u$ from (3.5.8), the set $E(a)$ is contained in $\left\{\left(\tilde{u}, u_{k}\right),|\tilde{u}| \leq \rho,\left|u_{k}\right| \leq M|\tilde{u}|^{\nu+1}\right\}$. Hence, the integral in the last estimate does not exceed

$$
\int_{|s| \leq \rho} \frac{2 M|s|^{\nu+1} d_{k-1} s}{(|x-a|+|s|)^{k+1}} \leq 2 M|x-a|^{\nu-1} \int_{\mathbb{R}^{k-1}} \frac{|z|^{\nu+1} d z}{(1+|z|)^{k+1}} .
$$

Combining this with (3.5.14)-(3.5.16), we arrive at the validity of the estimate (3.5.5) for the function $\psi_{1}$ in (3.5.13), which completes the proof of the theorem.

Hölder estimates up to the boundary for singular integrals are considered in [1]; this paper contains the condition (3.5.10). Regarding the approach applied in the present work, see [64, 66].

Taking into account the remark to Theorem 3.4.1, it is easy to describe conditions providing the stability of the estimate (3.5.2) with respect to the varying of $\Gamma$ and $D_{0}$. 
Lemma 3.5.1. Let domains $D_{n} \subseteq D^{1}, n=1,2, \ldots$, be such that

$$
\inf _{n} d\left(\partial D_{n}, \partial D^{1} \backslash \partial D_{n}\right)>0 \text {. }
$$

Let smooth surfaces $\Gamma_{n} \subseteq \partial D_{n}$ be such that $D_{n}$ lies from one side of $\Gamma_{n}$ for each $n$. Assume that $\Gamma_{n}$ admits a parametrization $\gamma_{n}$ from $C^{1, \nu}(G)$, where $G \subseteq \mathbb{R}^{k-1}$ is a Lipschitz domain such that $\gamma_{n} \rightarrow \gamma$ with respect to the norm of the space $C^{1, \nu}(G)$. Let kernels $Q_{n}(y, \xi)$ from $C^{\nu(2)}\left(D^{1}\right)$ satisfy the conditions (3.4.2) and (3.5.1) with respect to $D_{n}$ and $\Gamma_{n}$ respectively and functions $\varphi_{n}$ belong to $C^{\mu}\left(D^{1}\right), 0<\mu<\nu$. Let subdomains $D_{n}^{0}$ of $D_{n}$ be such that $\overline{D_{n}^{0}} \cap \partial D_{n} \subseteq \Gamma_{n} \backslash \partial \Gamma_{n}$ and

$$
\inf _{n} d\left(D_{n}^{0}, \Gamma_{n}^{\prime}\right)>0, \quad \Gamma_{n}^{\prime}=\partial D_{n} \backslash \Gamma_{n} .
$$

The the functions $\psi_{n}$ defined by the singular integrals

$$
\psi_{n}(x)=\int_{D_{n}} Q_{n}(y, y-x) \varphi_{n}(y) d y, \quad x \in D_{n}^{0},
$$

admit the following estimates uniform with respect to $n$ :

$$
\left|\psi_{n}\right|_{C^{\mu}\left(D_{n}^{0}\right)} \leq C\left|Q_{n}\right|_{C^{\nu(2)}}\left|\varphi_{n}\right|_{C^{\mu}}
$$

Proof. By virtue of (3.5.17), a similar to (3.5.19) estimate for

$$
\psi_{n}^{0}(x)=\int_{D^{1}} Q_{n}(y, y-x) \varphi_{n}(y) d y, \quad x \in D_{n}^{0},
$$

follows from Theorem 3.4.1. Regarding the functions

$$
\psi_{n}^{1}(x)=\int_{D^{1} \backslash D_{n}} Q_{n}(y, y-x) \varphi_{n}(y) d y, \quad x \in D_{n}^{0},
$$

it suffices to prove a uniform with respect to $n$ estimate

$$
\left|\frac{\partial \psi_{n}^{1}}{\partial x_{i}}(x)\right| \leq M\left|Q_{n}^{1}\right|_{C^{\nu(1)}}|\varphi|_{C^{\mu}} d^{\mu-1}(x, \Gamma), \quad x \in D_{n}^{0},
$$

for their partial derivatives and use Lemma 2.4.1 (its conditions are satisfied by virtue of (3.5.18)). Let $2 r_{0}$ be the lower bound of (3.5.18) and $K_{n}=\left\{a \in \Gamma_{n}, d\left(a, \Gamma_{n}^{\prime}\right) \geq r_{0}\right\}$. Proving Lemma 2.4.1, we found that there exists such a $\rho_{0}$ independent of pairs $K_{n}, \Gamma_{n}$. The remaining part of the proof of Theorem 3.5.1 is applicable to the function $\psi_{n}^{1}(x)$ in the domain $D_{n}^{0}$ with a fixed $n$; it leads to the claimed estimate (3.5.20).

The corresponding result for the whole domain $D_{0}=D$ follows directly from 3.5.1.

Theorem 3.5.2. Let the boundary of a finite domain $D$ belong to the class $C^{1, \nu}$ and a kernel $Q(y, \xi)$ belong to $C^{\nu(2)}\left(D, \mathcal{H}_{-k}\right)$ and satisfy the conditions (3.4.2) and (3.5.1).

Then the singular operator $R \varphi=\psi$ acting according to the relation (3.4.1) is bounded in $C^{\mu}(D)$, $0<\mu<\nu$.

In a natural way, singular integrals arise at the differentiating of functions of the kind

$$
\psi^{0}(x)=\int_{D} Q^{0}(y, y-x) \varphi(y) d y, \quad x \in D,
$$

with kernels $Q^{0}(x, y, \xi)$ belonging to $\mathcal{H}_{1-k}$ with respect to the variable $\xi$. To differentiate this integral, denote the product $Q^{0}(y, \xi) \varphi(y)$ by $Q(y, \xi)$. 
Lemma 3.5.2. If $Q \in C^{\nu(1)}\left(D, \mathcal{H}_{1-k}\right)$, then the function

$$
\psi(x)=\int_{D} Q(y, y-x) d y, \quad x \in D,
$$

is continuously differentiable and its partial derivatives are defined by the relation

$$
\frac{\partial \psi}{\partial x_{i}}(x)=-\sigma_{i}(x)-\int_{D} \frac{\partial Q}{\partial \xi_{i}}(y, y-x) d y, \quad \sigma_{i}(x)=\int_{\Omega} \xi_{i} Q(x, \xi) d \xi .
$$

Note that, by virtue of Lemma 3.3.1, the kernel $Q_{i}$ satisfies the necessary condition given by (3.4.2), i.e., the singular integral in the relation (3.5.23) is well defined.

Proof. Let $x$ vary in a neighborhood of a fixed point $a$ from $D$. Multiply $Q(t, \xi)$ by a suitable cut-off function $\chi(t)$. It suffices to consider the following two cases separately: the case where $Q(t) \equiv 0$ in a neighborhood of this point and the case where $Q(t) \equiv 0$ in a neighborhood of the boundary $\partial D$. In the former case, the direct differentiation of (3.5.22) under the integral sign yields the relation (3.5.23) (we take into account the fact that in the considered case, $\sigma_{i}(x)=0$ in a neighborhood of $a$ ).

Thus, without loss of generality, one can assume that the kernel $Q(y, \xi)$ is defined for any $y$ from the space $\mathbb{R}^{k}$ and there exists a compact set $K$ such that it vanishes outside this compact set:

$$
Q(y ; \xi)=0, \quad y \in \mathbb{R}^{k} \backslash K .
$$

Moreover, it suffices to consider the case where $Q(t, \xi) \in C^{1, \mu(1)}$. Indeed, assume that the relation (3.5.23) is already proved for such functions. Use the approximation operator $T_{\varepsilon}$ introduced in Sec. 1.8. Consider the sequence of functions $Q_{n}(t, \xi)=\left(T_{1 / n} Q\right)(t, \xi)$, where the operation $T$ is applied with respect to the variable $t$. Obviously, this function belongs to $C_{0}^{\infty}$ with respect to the variable $y$ and satisfies the condition (3.5.24) with respect to the compact set $K_{n}=\{y, d(y, K) \leq 1 / n\}$. By virtue of Lemma 2.2.1, this sequence converges to $Q$ with respect to the norm of the space $C^{\mu(1)}(D)$ provided that $\mu<\nu$.

If the function $\phi(x)$ is defined by the singular integral at the right-hand side of (3.5.23) and $\phi_{n}$ has a similar sense with respect to $Q_{n}$, then, by virtue of Theorem 3.4.1, the sequence $\phi_{n}(x)$ converges to $\phi(x)$ in the space $C^{\mu}(G)$ provided that $G$ is a compact set. Therefore, it remains to use the relation (3.5.23) for $Q_{n}$, pass to the limit as $n \rightarrow \infty$, and use the theorem on the differentiation under the integral sign (see Sec. 1.8).

Thus, let a kernel $Q(y, \xi)$ belong to $C^{1, \mu(1)}$ and satisfy the condition (3.5.24). Then

$$
\psi(x)=\int Q(y, y-x) d y=\int Q(x+y, y) d y
$$

(in this notation, the integration domain is $\mathbb{R}^{k}$ ). The function

$$
\psi_{\varepsilon}(x)=\int_{|y| \geq \varepsilon} Q(x+y, y) d y
$$

uniformly converges to $\psi$ as $\varepsilon \rightarrow 0$. For any fixed $\varepsilon$, it can be differentiated under the integral sign:

$$
\frac{\partial \psi_{\varepsilon}}{\partial x_{i}}(x)=\int_{|y| \geq \varepsilon} \frac{\partial Q}{\partial x_{i}}(x+y, y) d y \text {. }
$$

Since

$$
\frac{\partial Q}{\partial x_{i}}(x+y, y)=\frac{\partial}{\partial y_{i}}[Q(x+y, y)]-\frac{\partial Q}{\partial \xi_{i}}(x+y, y)
$$


it follows from the Green relation (see Sec. 1.8) that

$$
\int_{|z| \geq \varepsilon} \frac{\partial Q}{\partial x_{i}}(x+y, y) d y=-\int_{|y|=\varepsilon} Q(x+y, y) \frac{y_{i}}{|y|} d_{k-1} y-\int_{|y| \geq \varepsilon} \frac{\partial Q}{\partial \xi_{i}}(x+y, y) d y .
$$

By the homogeneity of $Q(y, \xi)$ with respect to $\xi$, the last surface integral is equal to

$$
\int_{\Omega} Q(x+\varepsilon \xi, \xi) \xi_{i} d \xi
$$

and tends to $\sigma_{i}(x)$ as $\varepsilon \rightarrow 0$.

On the other hand, the second term on the right-hand side of the relation (3.5.26) tends to the corresponding singular integral. Thus, substituting (3.5.26) in (3.5.25) and passing to the limit as $\varepsilon \rightarrow 0$, we conclude that the function $\psi$ is continuously differentiable and its partial derivatives are given by the relation $(3.5 .23)$.

Applying Lemma 3.5.2 to the function $\psi^{0}$ defined by the integral (3.5.21), we obtain the relation

$$
\frac{\partial \psi^{0}}{\partial x_{i}}(x)=-\sigma_{i}(x) \varphi(x)-\int_{D} \frac{\partial Q}{\partial \xi_{i}}(y, y-x) \varphi(y) d y, \quad \sigma_{i}(x)=\int_{\Omega} \xi_{i} Q^{0}(x, \xi) d \xi .
$$

Combining it with Theorem 3.5.2, we obtain the following result.

Theorem 3.5.3. Let the boundary of a domain $D$ belong to the class $C^{1, \nu}$, the kernel $Q^{0}$ belong to $C^{1, \nu(3)}\left(D, \mathcal{H}_{1-k}\right)$, and its partial derivatives $\partial Q / \partial \xi_{i}$ satisfy the conditions (3.5.1) at boundary points $y$ of $\partial D$.

Then the operator $R^{0} \varphi=\psi^{0}$ boundedly maps $C^{\mu}(D)$ to $C^{1, \mu}(D), 0<\mu<\nu$.

Note that if a kernel $Q^{0}(y, \xi)$ is odd with respect to the variable $\xi$, then its derivatives $\partial Q / \partial \xi_{i}$ are even with respect to this variable and, therefore, the condition (3.5.1) is satisfied (we take (3.4.2) into account).

\subsection{Generalized Cauchy-Type Integrals}

Let $\Gamma$ be a smooth $(k-1)$-dimensional surface with boundary. Let a kernel $Q(y ; \xi)$ from $C^{\nu(1)}\left(\Gamma, \mathcal{H}_{1-k}\right)$ be even with respect to the variable $\xi$. Consider the integral

$$
\phi(x)=\int_{\Gamma} Q(y ; y-x) \varphi(y) d_{k-1} y, \quad x \notin \Gamma,
$$

generalizing (in a way) the classical integral of the Cauchy type for analytic functions. It turns out to be especially useful for the investigation of multidimensional first-order elliptic systems (see $[5,51]$ ).

Let $x$ vary in a domain $D$ such that the distance between it and $\Gamma$ is positive, i.e., the difference $x-y$ is bounded from below by a positive constant provided that $x \in D$ and $y \in \Gamma$. Then, due to Lemma 3.1.2, the function $q(x, y)=Q(y ; y-x)$ belongs to the class $C^{\nu}(D \times \Gamma)$. Then $\phi \in C^{\nu}(D)$. Moreover, the function (3.6.1) can be differentiated infinitely many times under the integral sign. Thus, it belongs to the class $C^{\infty}(\bar{D})$. It and all its derivatives tend to zero as $x \rightarrow \infty$.

Our main concern refers to boundary properties of the function $\phi(x)$, i.e., its behavior under the tending of the point $x$ to an interior point $y_{0}$ of the surface $\Gamma$. As in Sec. 3.3, we start our investigation from the integral (3.6.1) on a $(k-1)$-dimensional plane.

Lemma 3.6.1. Let $Q(\xi)$ be an odd function from $\mathcal{H}_{1-k}, n$ be a unit vector, and $L$ be a $(k-1)$ dimensional plane in $\mathbb{R}^{k}, k \geq 2$, such that it is parallel to $n$, goes through the origin, and decomposes 
$\mathbb{R}^{k}$ into the half-spaces $P_{ \pm}=\left\{\eta \in \mathbb{R}^{k}, \pm \eta n>0\right\}$. If $\eta \notin L$, then the singular integral

$$
h(\eta)=\int_{L} Q(\xi-\eta) d_{k-1} \xi
$$

(with a singular point at infinity) exists and defines an odd function such that it is constant in each of the half-spaces $P_{ \pm}$, i.e., $h(\eta)= \pm c, \eta \in P_{ \pm}$,

$$
\int_{L} \frac{\partial Q}{\partial \xi_{i}}(\xi-\eta) d_{k-1} \xi=0, \quad \eta \in P_{ \pm}
$$

and

where $M$ is a positive constant independent of $Q$.

$$
|c|=|h(\eta)| \leq M|Q|_{(1)}
$$

If the above is satisfied and the function $Q$ vanishes on $L$, then the integral

$$
h_{0}(\eta)=\int_{L}|Q(\xi-\eta)| d_{k-1} \xi, \quad \eta n \neq 0,
$$

exists in the classical sense, does not depend on $\eta$, and satisfies an estimate similar to (3.6.4).

Proof. By virtue of the oddness of the kernel $Q$, the condition (3.3.8) is satisfied with respect to the $(k-2)$-dimensional unit sphere in $\mathbb{R}^{k-1}$, and the existence of the integral (3.6.2) is justified similarly to Sec. 3.3. Indeed, this integral is represented in the form

$$
h(\eta)=\int_{|\xi| \leq 1} Q(\xi-\eta) d \xi+\int_{|\xi| \geq 1}[Q(\xi-\eta)-Q(\xi)] d \xi
$$

where the integral at the right-hand side is treated in the classical sense by virtue of Lemma 3.1.1.

If a vector $a$ belongs to $L$ and $r \neq 0$, then the changes of variables $\xi=\xi^{\prime}-a, a \in L$, and $\xi=r \xi^{\prime}, \pm r>0$, in the singular integral (3.6.2) are justified similarly to Sec. 3.3. This leads to the relations

$$
h(x)=h(x-a), \quad h(x)=(\operatorname{sgn} r) h(r x) .
$$

It is obvious that they hold only in the case where the function $h$ is constant in the half-spaces $P_{ \pm}$ and is odd. Due to Lemma 3.1.1, the integral

$$
\int_{L}[Q(\xi-\eta)-Q(\xi-n)] d_{k-1} y
$$

exists in the classical sense. Therefore, the function $h(\eta)-h(n)$ can be differentiated under the integral sign, which yields the relation (3.6.3).

As in Sec. 3.3, we have

$$
h(\eta)=\int_{L \cap\{|\xi| \leq 1\}} Q(\xi-\eta) d_{k-1} \xi+\int_{L \cap\{|\xi| \geq 1\}}[Q(\xi-\eta)-Q(\xi)] d_{k-1} \xi ;
$$

taking into account Lemma 3.1.1, this implies the estimate (3.6.4).

Assume that $Q$ vanishes at the plane $L$. Then the condition (3.3.8) is still satisfied for the function $Q_{0}(\xi)=|Q(\xi)|$ with respect to the $(k-2)$-dimensional unit sphere in $\mathbb{R}^{k-1}$ since it is identically equal to zero on this sphere. Therefore, the last relation also holds for $Q_{0}$. According to the remark to Lemma 3.1.1, the norm $|Q|_{(1)}$ in the estimate (3.1.4) can be replaced by the norm in the space $C^{0,1}(\Omega)$. Therefore, the previous relation shows that the corresponding integral of the function $|Q|$ exists in the classical sense and the estimate

$$
\left|h_{0}(\eta)\right| \leq M\left|Q_{0}\right|_{C^{0,1}(\Omega)}
$$


holds. Since the norm of the function $\left|Q_{0}\right|$ in the space $C^{0,1}(\Omega)$ is estimated via the norm $|Q|_{(1)}$, it follows that the estimate (3.6.4) holds for $h_{0}$. As above, we prove that the function $h_{0}(\eta)$ does not depend on $\eta$.

Take a domain located from one side of $\Gamma$ (in terms of Sec. 2.4) and consider the surface integral (3.6.1) in this domain.

Theorem 3.6.1. Let a domain $D$ lie from one side of a $C^{1, \nu}$-smooth surface $\Gamma$ with boundary and a subdomain $D_{0}$ of $D$ be such that $\Gamma_{0}=\bar{D}_{0} \cap \partial D \subseteq \Gamma \backslash \partial \Gamma$. Let a kernel $Q(y ; \xi)$ from $C^{\nu(2)}\left(\Gamma, \mathcal{H}_{1-k}\right)$ be odd with respect to the variable $\xi$ and a function $\varphi$ belong to $C^{\mu}(\Gamma), 0<\mu<\nu$.

Then the integral (3.6.1) defines a function $\phi$ from $C^{\mu}(D)$, satisfying the norm estimate

$$
|\phi|_{C^{\mu}\left(D_{0}\right)} \leq C|Q|_{C^{\nu(2)}}|\varphi|_{C^{\mu}(\Gamma)}
$$

where $C$ is a positive constant depending only on the distance between $D_{0}$ and $\partial \Gamma$.

If the above holds and $Q(u, y, \xi)$ depends on a parameter $u$ from $G$ and belongs to $C^{\nu(2)}\left(G \times \Gamma, \mathcal{H}_{1-k}\right)$, then the corresponding function $\phi(u, x)$ belongs to $C^{\mu}\left(G \times D_{0}\right)$ and satisfies the norm estimate similar to $(3.6 .5)$.

Proof. The same argument as in Theorem 3.5.1 is applied. Relation (3.6.1) can be differentiated under the integral sign. Therefore,

$$
\frac{\partial \phi}{\partial x_{i}}(x)=-\int_{\Gamma} Q_{i}(y, y-x) \varphi(y) d y, \quad 1 \leq i \leq k,
$$

where the kernel $Q_{i}(y, \xi)$ is equal to $\partial Q / \partial \xi_{i}$ and belongs to $C^{\nu(1)}\left(\Gamma, \mathcal{H}_{-k}\right)$. As in the proof of Theorem 3.5.1, it suffices to justify the estimate

$$
\left|\frac{\partial \phi}{\partial x_{i}}(x)\right| \leq C\left|Q_{i}\right|_{C^{\nu(1)}}|\varphi|_{C^{\mu}} d^{\mu-1}(x, \Gamma), \quad x \in D,
$$

with a constant depending only on the distance $2 r_{0}=d\left(D_{0}, \partial D \backslash \partial \Gamma\right)$, for partial derivatives of the function $\phi$ and use Theorem 2.4.2.

Consider the set $K=\left\{y \in \Gamma, d(y, \partial \Gamma) \geq r_{0}\right\}$. Obviously, it contains $\Gamma \cap \bar{D}_{0}$. Let $\rho_{0}$ be defined with respect to $K$ and $\Gamma$ as in Theorem 2.4.1 and let $\rho=\min \left(r_{0}, \rho_{0}\right)$. It is obvious that it suffices to prove the estimate (3.6.6) for $x$ from $D_{0}, d(x, \Gamma) \leq \rho$. Then there exists a point $a$ from $K$ such that $d(x, \Gamma)=|x-a|$. Due to Theorem 2.4.1, in the local coordinate system, the intersection $\Gamma(a)=$ $\Gamma \cap C_{\rho}(a)$, where the neighborhood $C_{\rho}(a)$ is defined by $(3.5 .8)$, is described by the equation $u_{k}=f(\widetilde{u})$ in the ball $B_{\rho}=\{|\widetilde{u}| \leq \rho\}$ such that $f$ belongs to $C^{1, \nu}\left(B_{\rho}\right)$ and satisfies the conditions (3.5.9), where $M_{0}$ is a constant depending only on $\Gamma$.

We have the relation

$$
\frac{\partial \phi}{\partial x_{i}}=\phi_{0}+\phi_{1}
$$

where

$$
\phi_{0}(x)=\int_{\Gamma}\left[Q_{i}(y, y-x) \varphi(y)-Q_{i}(a, y-x) \varphi(a)\right] d y, \quad \phi_{1}(x)=\varphi(a) \int_{\Gamma} Q_{i}(a, y-x) d y .
$$

For the first term, we have the estimate

$$
\left|\phi_{0}(x)\right| \leq\left|Q_{i}\right|_{C^{\mu(0)}}[\varphi]_{\mu} I_{0}, \quad I_{0}=\int_{\Gamma}|y-a|^{\mu}|y-x|^{-k} d y .
$$


It is obvious that inequality (3.5.12) is still valid for $y$ from $\Gamma \cap C_{\rho}(a)$. Therefore, the following inequality holds:

$$
I_{0} \leq 2^{k} \int_{\Gamma \cap C_{\rho}(a)} \frac{|y-a|^{\mu} d y}{(|x-a|+|y-a|)^{k}}+2^{k} \rho^{-k} \int_{\Gamma \backslash C_{\rho}(a)}|y-a|^{\mu} d y .
$$

Similarly to the proof of Lemma 3.3.3, the surface $\Gamma \cap C_{\rho}(a)$ can be given by the parametric equation

$$
y-a=U[s, f(s)], \quad|s| \leq \rho,
$$

where $U$ is the corresponding orthogonal matrix from $\mathbb{R}^{k \times k}$. Taking into account (3.5.9), we have the following relations for the specified change of variables:

$$
|s| \leq|y-a| \leq 2|s|, d_{k-1} y=|m(s)| d s, \quad|m(s)|=\sqrt{1+\left|f^{\prime}(s)\right|^{2}} \leq 2 .
$$

Therefore, the first integral at the right-hand side of (3.6.8) does not exceed

$$
2^{1+\mu} \int_{|s| \leq \rho} \frac{|s|^{\mu} d s}{(|x-a|+|s|)^{k}} \leq|x-a|^{\mu-1} 2^{1+\mu} \int_{\mathbb{R}^{k-1}} \frac{|s|^{\mu} d s}{(1+|s|)^{k}} .
$$

Taking into account the relation $|x-a|=d(x, \Gamma)$, this leads to the validity of the estimate (3.6.6) for $\phi_{0}$.

Pass to the function $\phi_{1}$ from (3.6.7). By virtue of Lemma 3.6.1, we have the relation

$$
\int_{L(a)} Q_{i}(a, y-x) d y=0,
$$

where $L(a)$ denotes the plane $L(a)$ tangential to the surface $\Gamma$ at the point $a$. Therefore, the function $\phi_{1}$ can be represented in the form

$$
\phi_{1}(x)=\varphi(a)\left(\int_{\Gamma}-\int_{L(a)}\right) Q_{i}(a, y-x) d y=\varphi(a)\left(I_{1}^{\prime}+I_{2}^{\prime \prime}\right),
$$

where $I_{1}^{\prime}$ corresponds to the integrals over $\Gamma \cap C_{\rho}(a)$ and $L(a) \cap C_{\rho}(a)$. Since the segment with endpoints $a$ and $x$ is orthogonal to the plane $L(a)$, we have the obvious inequality $|y-x| \geq|y-a|$ for any $y$ from $L(a) \backslash C_{\rho}(a)$. Taking into account (3.5.12), this implies the inequality

$$
\left|I_{2}^{\prime \prime}\right| \leq\left|Q_{i}\right|_{C^{0(0)}}\left[2^{k} \rho^{-k} \int_{\Gamma \backslash C_{\rho}(a)} d y+\int_{L(a) \backslash C_{\rho}(a)}|y-a|^{-k} d y\right] \leq C^{\prime}\left|Q_{i}\right|_{C^{0(0)}},
$$

where $C^{\prime}$ is a constant depending only on $\rho$.

For definiteness, we assume that the unit normal $n(a)$ determining the direction of the axis $u_{k}$ of the local coordinate system is selected such that the local coordinates of $x$ are $\tilde{u}=0$ and $u_{k}=|x-a|$ or, which is the same, $x-a=U(0,|x-a|)$. Therefore,

$$
\begin{aligned}
& y-a=U[s, f(s)], \quad y-x=U[s, f(s)-r], \quad y \in \Gamma \cap C_{\rho}(a), \\
& y-a=U[s, 0)], \quad y-x=U[s,-r], \quad y \in L(a) \cap C_{\rho}(a),
\end{aligned}
$$

where $r=|x-a|$. In particular, inequality (3.5.12) combined with the estimate $|s| \leq|y-a|$ in (3.6.9) means that

$$
2 \sqrt{|s|^{2}+[f(s)-r]^{2}} \geq|s|+r, \quad|s| \leq \rho .
$$


In the notation of (3.6.9) and (3.6.11), the term $I_{1}^{\prime}$ is expressed as follows:

$$
I_{1}^{\prime}=\int_{|s| \leq \rho}[\widetilde{Q}(s, f(s)-r)|m(s)|-\widetilde{Q}(s,-r)] d s,
$$

where $\widetilde{Q}(\xi)=Q(a, U \xi)$. Using Lemma 3.1.1, (3.6.12), and the obvious inequality $2 \sqrt{|s|^{2}+r^{2}} \geq|s|+r$, we arrive at the following estimate of the integrand:

$$
|\widetilde{Q}(s, f(s)-r)| m(s)|-\widetilde{Q}(s,-r)| \leq 2 M|\widetilde{Q}|_{(1)} \frac{2^{k+1}|f(s)||m(s)|}{(|s|+r)^{k+1}}+2|\widetilde{Q}|_{(0)} \mid \frac{2^{k}|| m(s)|-1|}{(|s|+r)^{k}} .
$$

Thus, we obtain the inequality

$$
\left|I_{1}^{\prime}\right| \leq 2^{k+3} M|\widetilde{Q}|_{(1)} I_{2}+2^{k}|\widetilde{Q}|_{(0)} I_{3},
$$

where

$$
I_{2}=\int_{|s| \leq \rho} \frac{|f(s)|}{(|s|+r)^{k+1}} d s, \quad I_{3}=\int_{|s| \leq \rho} \frac{\sqrt{1+\left|f^{\prime}(s)\right|^{2}}-1}{(|s|+r)^{k}} d s .
$$

By virtue of (3.5.9), we have the obvious inequalities $\left|f^{\prime}(s)\right| \leq M_{0}|s|^{\nu}$ and $|f(s)| \leq M_{0}|s|^{\nu+1}$ for the function $f$. Therefore, the following estimate holds:

$$
I_{2}+I_{3} \leq M_{0} r^{\nu-1}\left[\int_{\mathbb{R}^{k-1}} \frac{|s|^{\nu+1} d s}{(|s|+1)^{k+1}}+\int_{\mathbb{R}^{k-1}} \frac{|s|^{\nu} d s}{(|s|+1)^{k}}\right] .
$$

Taking into account the fact that $r=|x-a|=d(x, \Gamma)$, we combine the last estimate with (3.6.10) and obtain the validity of the estimate (3.6.6) for the function $\phi_{1}$ in (3.6.7). Thus, the estimate (3.6.6) and, therefore, the first assertion of the theorem are proved.

Its second assertion is proved similarly to Theorem 3.4.1.

For classical integrals of the Cauchy type, Hölder estimates obtained via estimates of their derivatives near the boundary are well known (see, e.g., [12]). In [63, 64], this approach is used to investigate boundary properties of generalized Cauchy-type integrals related to elliptic systems.

An analog of Lemma 3.5.1 on variations of the surface $\Gamma$ also holds for the integrals (3.6.1).

Lemma 3.6.2. Let sequences $D_{n}, \Gamma_{n}, Q_{n}$, and $\varphi_{n}$ satisfy the conditions of Theorem 3.6.1 and

$$
\inf _{n} d\left(D_{n}, \partial \Gamma_{n}\right)>0 \text {. }
$$

Let the surfaces $\Gamma_{n}$ admit parametrizations $\gamma_{n}$ from $C^{1, \nu}$, converging to $\gamma$ in $C^{1, \nu}(G)$.

Then the functions

$$
\phi_{n}(x)=\int_{\Gamma_{n}} Q(x, y, y-x) \varphi_{n}(y) d y, \quad x \in D_{n},
$$

satisfy the estimate

uniform with respect to $n$.

$$
\left|\phi_{n}\right|_{C^{\mu}\left(D_{n}\right)} \leq C|Q|_{C^{\nu(1)}}\left|\varphi_{n}\right|_{C^{\mu}\left(\Gamma_{n}\right)}
$$

Proof. Using Lemma 2.4.1 and the scheme of the proof of Theorem 3.6.1, we argue in the same way as in the proof of Lemma 3.5.1.

Imposing additional assumptions on the kernel $Q$, one can also consider boundary properties of the integral (3.6.1) for functions $\varphi$ from $C(\Gamma)$. A classical example is the double-layer potential for the Laplace operator in a domain $D$. In our notation, it is defined by the kernel

$$
Q(y, \xi)=\frac{1}{\pi} \frac{\xi n(y)}{|\xi|^{k}}, \quad y \in \Gamma
$$


where $n(y)$ denotes the inner normal. It is well known that the function defined by the integral with this kernel and the density $\varphi \in C(\Gamma)$ is continuous up to $\Gamma$ from each side of the surface. The considered kernel is such that $Q(y, \xi)=0$ for $\xi n(y)=0$. It turns out that this is the key property also in the general case.

Theorem 3.6.2. Let conditions of Theorem 3.6.1 be satisfied and

$$
Q(y, \xi)=0 \quad \text { for } \xi n(y)=0, y \in \Gamma .
$$

Then, for any $\varphi$ from $C(\Gamma)$, the integral (3.6.1) defines a function $\phi$ from $C(\bar{D})$ such that

$$
|\phi|_{0} \leq C|Q|_{C^{\nu(1)}}|\varphi|_{0},
$$

where $C$ is a positive constant depending only on the distance between $D$ and $\partial \Gamma$.

Proof. We use the estimate

$$
\int_{\Gamma}|Q(y, y-x) d y| \leq C|Q|_{C^{\nu(2)}}, \quad x \in D,
$$

proved in the same way as Theorem 3.6.1.

Let $K \subseteq \Gamma$ and $\rho$ be defined in the same way as in the proof of Theorem 3.6.1. Let $x \in D$ and $d(x, \Gamma) \leq \rho$. Let $d(x, \Gamma)=|x-a|, a \in K$, and $x$ lie inside the neighborhood $C_{\rho}(a)$.

Since ||$Q(y, \xi)|-| Q(a, \xi)|| \leq|Q(y, \xi)-Q(a, \xi)|$, we have the following estimate for the difference $\Delta(x, y)=|Q(y, y-x)|-|Q(a, y-x)|:$

$$
|\Delta(x, y)| \leq|Q|_{C^{\nu(0)}}|y-a|^{\nu}|y-x|^{1-k} .
$$

Then, taking into account (3.5.12), we deduce the inequality

$$
|\Delta(x, y)| \leq 2^{1-k}|Q|_{C^{\nu(0)}}|y-a|^{\nu+1-k} . \quad y \in \Gamma \cap C_{\rho}(a),
$$

Then, in addition to the first inequality of (3.6.9), we have the inequality

$$
\int_{\Gamma \cap C_{\rho}(a)}|\Delta(x, y)| d y \leq 2^{1-k}|Q|_{C^{\nu(0)}} \int_{|s| \leq \rho}|s|^{\mu+1-k} d s .
$$

Thus, it suffice to prove (3.6.16) for $Q(a, \xi)$.

It is obvious that

$$
\int_{\Gamma \backslash C \rho(a)}|Q(a, y-x) d y| \leq C_{1}|Q|_{C^{0(0)}},
$$

where $C_{1}$ is a constant depending only on $\rho$.

On the other hand, by virtue of the second assertion of Lemma 3.6.1, we have the inequality

$$
\int_{L(a) \cap C(a)}|Q(a, y-x)| d y \leq \int_{L(a)}|Q(a, y-x)| d y \leq C_{1}|Q|_{C^{0(1)}} .
$$

Finally, similarly to the proof of Theorem 3.6.1, we have the relation

$$
\left(\int_{\Gamma \cap C(a)}-\int_{L(a) \cap C(a)}\right)|Q(a, y-x)| d y=\int_{|s| \leq \rho}[|\widetilde{Q}[s, f(s)-r]||m(s)|-|\widetilde{Q}(s,-r)|] d s,
$$

where $\widetilde{Q}(\xi)=|Q(a, B \xi)|,|m(s)|=\sqrt{1+\left|f^{\prime}(s)\right|^{2}}$, and $r=|x-a|$.

Taking into account the remark to Lemma 3.1.1, one can estimate the last integral in the same way as in the proof of Theorem 3.6.1. Combining this with (3.6.18) and (3.6.19), we complete the proof of the estimate (3.6.16). 
It remains to verify the continuity of the function $\phi$ in the closed domain $\bar{D}$. To do this, select a sequence of functions $\varphi_{n}$ from $C^{\mu}(\Gamma)$, converging to $\varphi$ with respect to the sup-norm. Due to Theorem 3.6.1, the functions $\phi_{n}$ defined by the integral (3.6.1) via $\varphi_{n}$ are continuous in $\bar{D}$. On the other hand, by virtue of (3.6.15), the sequence $\phi_{n}$ uniformly converges to $\phi$. Hence, the last function also belongs to $C(\bar{D})$.

In $[72,73]$, Theorem 3.6.2 was proved for the two-dimensional case.

\subsection{Relations for Boundary Values}

Due to Theorem 3.6.1, the function $\phi(x)$ defined in a domain $D$ by a Cauchy-type generalized integral (3.6.1) is continuous in its closure. Hence, its boundary values are defined on $\bar{D} \cap \Gamma$. It is possible that this domain adjoins $\Gamma$ from both sides. Therefore, two one-sided limit values $\phi^{ \pm}(a)$, $a \in \Gamma$, are defined. More exactly, by virtue of Theorem 2.4.1, if $\Gamma$ is a smooth surface with boundary, $a$ is its interior point, and $\rho$ is sufficiently small, then neighborhood (3.5.8) can be decomposed into two half-neighborhoods $C_{\rho}^{ \pm}(a)$ that are connected components selected by the condition $\pm\left[f(\widetilde{u})-u_{k}\right]>0$. The signs depend on the choice of the normal $n(a)$ such that the axis $u_{k}$ of the local coordinate system is directed along it. Respectively, one-sided boundary values of the function $\phi$ are defined as the following limits:

$$
\phi^{ \pm}(a)=\lim _{\substack{x \rightarrow a \\ x \in C^{ \pm}(a)}} \phi(x) .
$$

The description of these boundary values for the function (3.6.1) is closely related to the singular integral

$$
\phi^{*}(a)=\int_{\Gamma} Q(y, y-a) \varphi(y) d_{k-1} y
$$

from Lemma 3.3.3.

Let $L(a)$ be the tangential plane to $\Gamma$ at a point $a$. According to 3.6.1, a coefficient $\sigma(a)=h(\eta)$ is associated with this plane, where the function $h$ is defined by the kernel $Q(\xi)=Q(a, \xi)$ and the scalar product $\eta n(a)$ is positive. Its explicit expression is as follows:

$$
\sigma(a)=\int_{L(a)} Q\left(y-x_{0}\right) d_{k-1} y, \quad\left(x_{0}-a\right) n(a)>0 .
$$

This function $\sigma$ has the following continuity character on $\Gamma$.

Lemma 3.7.1. Let $\Gamma$ from $C^{1, \nu}$ be a surface with boundary. Let an odd (with respect to $\xi$ ) kernel $Q(y, \xi)$ belong to $C^{\nu(1)}\left(\Gamma, \mathcal{H}_{1-k}\right)$. Then, for any compact subset $K$ of $\Gamma \backslash \partial \Gamma$, the function $\sigma$ defined by (3.7.3) belongs to $C^{\mu}(K), 0<\mu<\nu$, and its norm satisfies the estimate

$$
|\sigma|_{C^{\mu}(K)} \leq C|Q|_{C^{\nu(1)}},
$$

where $C$ is a positive constant depending only on the distance between $K$ and $\partial \Gamma$.

Proof. It suffices to prove the lemma in any neighborhood $\Gamma_{a}$ of a fixed point $a$ from $\Gamma$. Let one component of the vector $n(a)$ be different from zero; denote it by $n_{s}(a)$. Without loss of generality, we assume that $n_{s}(y) \neq 0$ for any $y$ from $\Gamma_{a}$. Let $B(y)$ be an invertible $k \times k$-matrix from $C^{\nu}\left(\Gamma_{a}\right)$ such that for any $y_{0}$ from $\Gamma_{a}$, the linear transformation $u \rightarrow x=y_{0}+B\left(y_{0}\right) u$ maps the plane $u_{k}=0$ onto $L\left(y_{0}\right)$ and, respectively, maps the half-space $u_{k}>0$ onto $\left\{x,\left(x-y_{0}\right) n(y)>0\right\}$.

Let us prove the existence of such a matrix. Indeed, if $1 \leq i \leq k, i \neq s$, then by $b_{i}(y)$ denote the vector such that its $s$ th and $i$ th components coincide with $-n_{i}(y)$ and $n_{s}(y)$ respectively, while other its components are equal to zero. Obviously, the obtained $k-1$ vectors are linearly independent and orthogonal to $n(y)$. Now, we take the matrix such that its initial $k-1$ columns are formed by the 
vectors $b_{i}, i \neq s$, while the last one coincides with the vector $n$. This matrix satisfies all requirements for the matrix $B$.

Let $e$ be the vector $(0, \ldots, 0,1)$ from $\mathbb{R}^{k}$. We change the variables as follows: $y-y_{0}=B\left(y_{0}\right)(s, 0)$, $s \in \mathbb{R}^{k-1}$, and $x-y_{0}=B\left(y_{0}\right) e$. Under such a change, the area element $d_{k-1} y$ of the plane $L\left(y_{0}\right)$ is linked with $d s$ by the relation $d y=\left|m\left(y_{0}\right)\right| d s$, where the components of the vector $m\left(y_{0}\right)$ are the cofactors of the last column of the matrix $B\left(y_{0}\right)$ (according to Sec. 2.4). In particular, $m(y) \in C^{\nu}\left(\Gamma_{0}\right)$. Under the change specified, the relation (3.7.3) for $a=y_{0}$ passes to the relation

$$
\sigma\left(y_{0}\right)=\left|m\left(y_{0}\right)\right| \int_{\mathbb{R}^{k-1}} \widetilde{Q}\left(y_{0} ; s,-1\right) d s,
$$

where $\widetilde{Q}(y ; \xi)=Q[y ; B(y) \xi]$. As in the proof of Lemma 3.6.1, this singular integral (with a singular point at infinity) can be represented by the sum $\sigma_{1}\left(y_{0}\right)+\sigma_{2}\left(y_{0}\right)$ of the classical integrals

$$
\sigma_{1}\left(y_{0}\right)=\int_{|s| \leq 1} \widetilde{Q}\left(y_{0} ; s,-1\right) d s, \quad \sigma_{2}\left(y_{0}\right)=\int_{|s| \geq 1}\left[\widetilde{Q}\left(y_{0} ; s,-1\right)-\widetilde{Q}\left(y_{0} ; s, 0\right)\right] d s .
$$

It is obvious that the function $\sigma_{1}$ belongs to $C^{\nu}\left(\Gamma_{a}\right)$ and the estimate (3.7.4) with respect to $K=\Gamma_{a}$ is also obvious for this function.

For the integrand of the function $\sigma_{2}$, perform the change of variables $s=r \xi$, where $r>0, \xi \in \Omega$, and $\Omega$ denotes the unit sphere in $\mathbb{R}^{k-1}$. Due to Sec. 1.8, $d_{k-1} s=r^{k-2} d r d_{k-2} \xi$ under this change and, therefore, taking into account the oddness and homogeneity of the function $\widetilde{Q}$, we obtain the following expression for $\sigma_{2}$ :

$$
\sigma_{2}\left(y_{0}\right)=\int_{|r| \geq 1} \frac{q\left(y_{0}, r\right) d r}{r}, \quad q\left(y_{0}, r\right)=\int_{\Omega}\left[\widetilde{Q}\left(y_{0} ; \xi,-1 / r\right)-\widetilde{Q}\left(y_{0} ; \xi, 0\right)\right] d \xi .
$$

By virtue of Lemma 3.1.2, the function $\widetilde{Q}\left(y_{0} ; \xi, t\right)$ belongs to the class $C^{\nu}\left(\Gamma_{a} \times \Omega \times[-1,1]\right)$. Hence, by virtue of Lemma 2.1.1, the difference $\widetilde{Q}\left(y_{0} ; \xi, t\right)-\widetilde{Q}\left(y_{0} ; \xi, 0\right)$ can be represented in the form $|t|^{\nu-\mu} a\left(y_{0}, \xi, t\right)$, where the function $a\left(y_{0}, \xi, t\right)$ belongs to $C^{\mu}\left(\Gamma_{a} \times \Omega \times[0,1]\right)$ and its norm satisfies the estimate $|a|_{C^{\mu}} \leq C|Q|_{C^{\nu(1)}}$.

Then the function

$$
\sigma_{2}\left(y_{0}\right)=\int_{|r| \geq 1} r^{\mu-\nu-1} \int_{\Omega} a\left(y_{0}, \xi,-1 / r\right) d \xi
$$

also belongs to $C^{\mu}\left(\Gamma_{a}\right)$ and a similar to (3.7.4) estimate of its norm with respect to $K=\Gamma_{a}$ is valid.

Theorem 3.7.1. Under the assumptions of Theorem 3.6.1, boundary values of the function (3.6.1) at interior points $y_{0}$ of $\Gamma$ are given by the relation

$$
\phi^{ \pm}\left(y_{0}\right)= \pm \sigma\left(y_{0}\right) \varphi\left(y_{0}\right)+\phi^{*}\left(y_{0}\right)
$$

where the singular integral $\phi^{*}\left(y_{0}\right)$ and the coefficient $\sigma\left(y_{0}\right)$ are defined by (3.7.2) and (3.7.3) respectively.

Proof. If the normal $n\left(y_{0}\right)$ to the surface $\Gamma$ at a point $y_{0}$ is replaced by the opposite one, then, due to the definition (3.7.1), the boundary values $\phi^{ \pm}\left(y_{0}\right)$ change places, while the coefficient $\sigma\left(y_{0}\right)$ in $(3.7 .3)$ changes its sign due to Lemma 3.6.1. Thus, it suffices to prove the relation (3.7.6) for the upper sign.

Let $x$ from $C^{+}(a)$ tend to a boundary point $a$ from $\Gamma$ along the normal $n(a)$, i.e., $x-a=r n(a)$, $r>0$. As in the proof of Theorem 3.6.1, one can assume that $r \leq \rho$. Since

$$
\lim _{x \rightarrow a} \int_{\Gamma \backslash C(a)} Q(y, y-x) \varphi(y) d y=\int_{\Gamma \backslash C(a)} Q(y, y-a) \varphi(y) d y,
$$


it follows that it suffices to prove the relation (3.7.6) for $\Gamma \cap C(a)$. For the difference $\Delta(x, y)=$ $Q(y, y-x) \varphi(y)-Q(a, y-x) \varphi(a)$, we have the following estimate similar to (3.6.17):

$$
|\Delta(x, y)| \leq 2^{1-k}|Q|_{C^{\mu(0)}}|\varphi|_{C^{\mu}}|y-a|^{\mu+1-k}, \quad y \in \Gamma \cap C_{\rho}(a) .
$$

Since the function $g(y)=|y-a|^{\mu-k+1}$ is summable over $\Gamma \cap C(a)$, it follows from the Lebesgue majorized convergence theorem (see Sec. 1.8) that

$$
\lim _{x \rightarrow a} \int_{\Gamma \cap C(a)}[Q(y, y-x) \varphi(y)-Q(a, y-x) \varphi(a)] d y=0 .
$$

Thus, it suffices to prove the relation

$$
\lim _{x \rightarrow a} \int_{\Gamma \cap C(a)} Q(a, y-x) d y=\sigma(a)+\int_{\Gamma \cap C(a)} Q(a, y-a) d y .
$$

Similarly to the proof of Theorem 3.6.1, we use the parametric equation

$$
y-a=U[s, f(s)], \quad|s| \leq \rho,
$$

of the surface $\Gamma \cap C(a)$ to obtain the relation

$$
\int_{\Gamma \cap C(a)} Q(a, y-x) d y=\int_{|s| \leq \rho} \widetilde{Q}[s, f(s)-r]|m(s)| d s,
$$

where $\widetilde{Q}(\xi)=Q(a, U \xi),|m(s)|=\sqrt{1+\left|f^{\prime}(s)\right|^{2}}$, and $r=|x-a|$. If $y_{0}=a$ is fixed, then the orthogonal matrix $U$ from (3.7.8) can be taken as the matrix $B\left(y_{0}\right)$ in (3.7.5). In this case, the coefficient $\left|m\left(y_{0}\right)\right|$ is equal to 1 and the relation (3.7.5) takes the form

$$
\sigma(a)=\int_{\mathbb{R}^{k-1}} \widetilde{Q}\left(y_{0}, s,-1\right) d s .
$$

As we found in the proof of Lemma 3.3.3, the change of variables $y-a=B[s, f(s)]$ can also be performed for the singular integral on the right-hand side of the relation (3.7.7):

$$
\int_{\Gamma \cap C(a)} Q(a, y-a) d y=\int_{|s| \leq r} \widetilde{Q}[s, f(s)]|m(s)| d s .
$$

Thus, the relation (3.7.7) can be represented in the form

$$
\lim _{r \rightarrow 0} \int_{|s| \leq \rho} \widetilde{Q}[s, f(s)-r]|m(s)| d s=\sigma(a)+\int_{|s| \leq \rho} \widetilde{Q}[s, f(s)]|m(s)| d s,
$$

where $\widetilde{Q}(\xi) \in \mathcal{H}_{1-k}$. For this kernel, one can use the estimates (3.6.12), where $k$ is to be replaced by $k-1$. In particular, we have the inequality

$$
|\widetilde{Q}[s, f(s)-r]| m(s)|-\widetilde{Q}[s, f(s)-r]| \leq 4^{k-1} M|\widetilde{Q}|_{(0)}(r+s)^{\nu-k+1} .
$$

Therefore, as above, due to the Lebesgue majorized convergence theorem, it suffices to prove the relation

$$
\lim _{r \rightarrow 0} \int_{|s| \leq \rho} \widetilde{Q}[s, f(s)-r] d s=\sigma(a)+\int_{|s| \leq \rho} \widetilde{Q}[s, f(s)] d s .
$$

By Definition (3.7.9), we have the relation

$$
\sigma(a)=\lim _{r \rightarrow 0} \int_{|s| \leq \rho / r} \widetilde{Q}(s,-1) d s .
$$


By virtue of the homogeneity, we have the relation $\widetilde{Q}(s,-1)=r^{k-1} \widetilde{Q}(r s,-r)$; hence, the change of variables $t=r s$ yields the relation

$$
\sigma(a)=\lim _{r \rightarrow 0} \int_{|s| \leq \rho} \widetilde{Q}(s,-r) d s
$$

Since

$$
\int_{|s| \leq \rho} \widetilde{Q}(s, 0) d s=0,
$$

it follows the proof of the relation (3.7.11) is reduced to the justification of the limit passage

$$
\lim _{r \rightarrow 0} \int_{|s| \leq \rho}[\widetilde{Q}[s, f(s)-r]-\widetilde{Q}(s,-r)] d s=\int_{|s| \leq \rho}[\widetilde{Q}[s, f(s)]-\widetilde{Q}(s, 0)] d s .
$$

An estimate similar to (3.6.13) can also be obtained for the difference $\widetilde{Q}[s, f(s)-r]-\widetilde{Q}(s,-r)$ : one must take into account the fact that $\widetilde{Q}(\xi)$ is a homogeneous kernel of power $1-k$ in thiss case. Thus, we have the inequality

$$
|\widetilde{Q}[s, f(s)-r]-\widetilde{Q}(s,-r)| \leq 2 M|\widetilde{Q}|_{(1)} \frac{2^{k}|f(s)|}{(|s|+r)^{k}} \leq C|s|^{\nu-k+1} .
$$

Therefore, the validity of the limit relation (3.7.12) follows from the Lebesgue majorized convergence theorem, which completes the proof of the relation (3.7.6).

Theorem 3.7.1 combined with Lemma 3.6.1 immediately implies the corresponding result for the singular operator defined by the relation (3.7.2) on a closed smooth surface.

Theorem 3.7.2. Let $\Gamma$ be a closed surface from the class $C^{1, \nu}$. Let a kernel $Q(y ; \xi)$ from $C^{\nu(2)}\left(\Gamma, \mathcal{H}_{1-k}\right)$ be odd with respect to the variable $\xi$. Then the singular operator $R \varphi=\phi^{*}$ defined by the singular integral (3.7.2) is bounded in the space $C^{\mu}(\Gamma), 0<\mu<\nu$, and its norm admits the estimate $|R|_{\mathcal{L}\left(C^{\mu}\right)} \leq C|Q|_{C^{\mu(2)}}$.

Proof. Cover $\Gamma$ by open sets $V_{j}, 1 \leq j \leq m$, such that $\Gamma \cap V_{j}$ is a surface with boundary, while the set $V_{j} \backslash \Gamma$ consists of two connected components $V_{j}^{ \pm}$lying from different sides of $\Gamma$. Then, by virtue of Theorem 3.7.1, the singular operators

$$
\left.\varphi \rightarrow \phi^{*}\right|_{V_{j} \cap \Gamma}
$$

boundedly maps $C^{\mu}(\Gamma)$ to $C^{\mu}\left(V_{j} \cap \Gamma\right)$ and the corresponding estimates of its norm hold. This and Theorem 2.1.1 imply the boundedness of the operator $R$ in $C^{\mu}(\Gamma)$.

\subsection{Line Cauchy-Type Integral}

All the above considerations refer to the case of the Euclidean space $\mathbb{R}^{k}$. Below, we mainly deal with the case where $k=2$. In this case, it is convenient to treat $\mathbb{R}^{2}$ as the complex plane $\mathbb{C}$. Therefore, for points of the plane, we use the complex notation $z=x+i y, t=t_{1}+i t_{2}$, etc. In the plane case, roles of surfaces are played by curves (in Sec. 2.5, they are considered in detail). Hence, on a smooth surface with boundary, the generalized Cauchy-type integrals (3.6.1) pass to line integrals

$$
\phi(z)=\int_{\Gamma} Q(t ; t-z) \varphi(t) d_{1} t, \quad z \notin \Gamma,
$$

over a smooth arc $\Gamma$, where the kernel $Q$ belongs to $\mathcal{H}_{-1}$ and is odd with respect to $\xi$. The pair of endpoints of the arc plays the role of the boundary $\partial \Gamma$. 
For a point $a$ of $\Gamma$, denote the direction vector by $e(a)=e_{1}+i e_{2}$. Then the normal at this point is $n(a)=i e(a)$. Consider the tangent line $L(a)$ at this point. The choice of the vector-function $e(t)$ from $C(\Gamma)$ determines the orientation of the curve $\Gamma$. The half-neighborhoods $C^{+}(a)$ and $C^{-}(a)$ (see (3.7.1) above) lie from the left and from the right (respectively) of the curve $\Gamma$ with respect to this orientation. In Definition (3.7.3) of the coefficient $\sigma(a)$, select the normal vector $n(a)=i e(a)$ to satisfy the relation

$$
\sigma\left(t_{0}\right)=\int_{L\left(t_{0}\right)} Q\left(t_{0} ; t-z_{0}\right) d_{1} t
$$

where the point $z_{0}$ lies from the left of $L\left(t_{0}\right)$, i.e., $\operatorname{Im}\left[\left(z_{0}-t_{0}\right) / e\left(t_{0}\right)\right]>0$. Note that the condition (3.6.14) is equivalent to the condition

$$
Q\left[t_{0}, e\left(t_{0}\right)\right]=0
$$

in the considered case.

As it is noted in Sec. 3.7, the notion of generalized Cauchy-type integrals (3.8.1) covers not only classical Cauchy-type integrals

$$
\phi(z)=\frac{1}{2 \pi i} \int_{\Gamma} \frac{\varphi(t) d t}{t-z}, \quad z \notin \Gamma,
$$

where $d t=e(t) d_{1} t=d t_{1}+i d t_{2}$ denotes the complex differential on a curve; it also covers the classical double-layer potential

$$
u(z)=\frac{1}{\pi} \int_{\Gamma} \frac{\operatorname{Im}[(t-z) \overline{e(t)}]}{|t-z|^{2}} \varphi(t) d_{1} t, \quad z \in D,
$$

for the Laplace equation. It is obvious that the integrals (3.8.4) and (3.8.5) can be represented in the form (3.8.1) with respect to the kernels $Q(t, \xi)=e(t) /(\pi i \xi)$ and $Q(t, \xi)=\operatorname{Im}[\overline{\xi(t)}] /\left(\pi|\xi|^{2}\right)$ respectively.

For the integrals (3.8.4) and (3.8.5), Theorems 3.6.1,3.6.2, and 3.7.1 are well known. In the classical monograph [45], boundary properties of the Cauchy-type integrals (3.8.4) are presented in detail with $\sigma=1 / 2$ in the relation for boundary values. For the first time, this relation is discovered [58]. in [50], it is discovered again. Nowadays, it is called the Sochocki-Plemelj relation. in [52], this relation is considered under more general assumptions. Moreover, Theorem 3.8.1 for this integral holds for any smooth contour (no requirement it to be a Lyapunov contour is imposed). Thus, it is reasonable to select a class of kernels $Q$ possessing a similar property.

Similarly to (3.8.4), assume that the kernel of the integral (3.8.1) explicitly depends on the unit tangential vector $e(t)=e_{1}(t)+i e_{2}(t)$ on the contour $\Gamma$. More exactly, assume that this integral has the form

$$
\int_{\Gamma} Q(t ; t-z, d t) \varphi(t)=\int_{\Gamma} Q[z, t ; t-z, e(t)] \varphi(t) d_{1} t, \quad z \in D,
$$

where $Q(t ; \xi, \eta)=Q_{1}(z, t ; \xi) \eta_{1}+Q_{2}(z, t ; \xi) \eta_{2}, \xi, \eta \in \mathbb{C}$, and $Q_{j}$ are odd $\mathcal{H}_{-1}$-functions with respect to the variable $\xi$. A function $Q$ of such type is called a Cauchy kernel if $Q(t ; \xi, \xi)$ does not depend on $\xi$, i.e.,

$$
Q(t ; \xi, \xi)=c(t), \quad \xi \in \mathbb{C} .
$$

In such a case, the integral (3.8.6) is called a Cauchy-type integral.

Since the integral (3.8.4) can be represented in the form (3.8.6), where $\pi i Q(\xi, \eta)=\eta / \xi$, it is obvious that the condition (3.8.7) is satisfied for it. The integral (3.8.6) depends on the orientation of the curve $\Gamma$. If we pass to the opposite orientation, i.e., replace $e$ by $-e$, then the integral alternates it sign. As above, the notation $Q(t ; \xi, \eta) \in C^{n(m)}(G)$ is treated with respect to the functions $Q_{j}$ constituting $Q$. 
The following lemma illustrates an important property of the Cauchy kernel. Let a Cauchy kernel $Q(\xi, \eta)$ not depend on $t$ and

$$
2 \sigma_{0}=\int_{\mathbb{T}} Q(\xi, d \xi)
$$

where $\mathbb{T}$ is a unit circle oriented counterclockwise. Note that the similar interval $\Gamma_{1}$ along the semicircle $\mathbb{T}$ is equal to $\sigma_{0}$ since the transformation $\xi \rightarrow-\xi$ mapping $\Gamma_{1}$ onto the opposite semicircle $\Gamma_{2}$ changes the orientation of the curve and, therefore, does not change the expression $Q(\xi, d \xi)$ (by virtue of the oddness of $Q(\xi, \eta)$ with respect to $\xi)$.

Lemma 3.8.1. Let $Q(\xi, \eta)$ be a Cauchy kernel and $D$ be a finite domain bounded by a piecewisesmooth contour $\Gamma$ positively oriented with respect to $D$ (i.e., the domain $D$ is left from the left under the movement along the contour towards the positive direction). Then

$$
\int_{\Gamma} Q(t-z, d t)=2 \sigma_{0}, \quad z \in D
$$

in terms of (3.8.8) and

$$
\int_{\Gamma} Q\left(t-t_{0}, d t\right)=\sigma_{0}
$$

if $t_{0}$ from $\Gamma$ is an interior point.

In the same way, if a line $L$ divides the plane $\mathbb{C}$ into half-planes $P_{ \pm}$and is oriented positively with respect to $P_{+}$, then

$$
\int_{L} Q(t-z, d t)= \pm \sigma_{0}, \quad z \in P_{ \pm}
$$

where the last singular integral has a singular point at infinity.

Proof. By condition, the function $Q(\xi, \xi)=Q_{1}(\xi) \xi_{1}+Q_{2}(\xi) \xi_{2}$ is identically equal to a constant and, therefore, the following relations hold:

$$
\frac{\partial Q_{1}}{\partial \xi_{i}}+\frac{\partial Q_{2}}{\partial \xi_{i}}+Q_{i}=0, \quad i=1,2
$$

On the other hand, $Q_{i}(\xi)$ is a homogeneous function of power -1, i.e., $r Q_{i}(r \xi)=Q_{i}(\xi)$. Differentiating this identity with respect to $r$, we arrive at the Euler relation

$$
\frac{\partial Q_{i}}{\partial \xi_{1}}+\frac{\partial Q_{i}}{\partial \xi_{2}}+Q_{i}=0, \quad i=1,2,
$$

for homogeneous functions. Combining this with the previous relation, we conclude that

$$
\frac{\partial Q_{1}}{\partial \xi_{2}}=\frac{\partial Q_{2}}{\partial \xi_{1}}
$$

To prove the first assertion of the lemma, fix a point $z_{0}$ from $D$ and assume that a positive $\varepsilon$ is less than the distance $d\left(z_{0}, \Gamma\right)$. Now, consider the domain $D_{\varepsilon}=D \cap\left\{\left|z-z_{0}\right|>\varepsilon\right\}$ bounded by the composite contour $\partial D_{\varepsilon}=\Gamma \cap \Gamma_{\varepsilon}$, where $\Gamma_{\varepsilon}$ is the corresponding circle. Orient this contour clockwise with respect to $D_{\varepsilon}$. Then $\Gamma_{\varepsilon}$ is oriented clockwise. By virtue of (3.8.11) and the Green relation provided at Sec. 2.5, we have the relation

$$
\int_{\partial D_{\varepsilon}} Q_{1}\left(z-z_{0}\right) d x+Q_{2}\left(z-z_{0}\right) d y=\int_{D_{\varepsilon}}\left(\frac{\partial Q_{2}}{\partial x}-\frac{\partial Q_{1}}{\partial y}\right)\left(z-z_{0}\right) d_{2} z=0 .
$$

Thus,

$$
\int_{\Gamma} Q\left(t-z_{0}, d t\right)=\int_{\Gamma_{\varepsilon}} Q\left(t-z_{0}, d t\right)
$$


where the circle on the right-hand side is oriented counterclockwise. Since this integral passes to the integral (3.8.8) under the change of variables $z-z_{0}=\varepsilon \xi$, we arrive at the relation (3.8.9).

To prove the relation (3.8.10), we assume that $t_{0}$ is an interior point of the contour. Then, due to Sec. 2.5, there exists a positive $\rho$ such that if $\varepsilon \leq \rho$, then the intersection $\Gamma \cap\left\{\left|z-t_{0}\right| \leq \varepsilon\right\}$ is a smooth arc containing $t_{0}$ inside. Let $\Gamma_{\varepsilon}$ denote the part of the circle $\left|z-t_{0}\right|=\varepsilon$, lying outside the domain $D$. The contour formed by it and the curve $\Gamma \cap\left\{\left|z-t_{0}\right| \geq \varepsilon\right\}$ envelopes the point $t_{0}$. By virtue of (3.8.9), this implies that

$$
\int_{\Gamma} Q\left(t-t_{0}, d t\right)=\int_{\Gamma_{\varepsilon}} Q\left(t-z_{0}, d t\right) .
$$

If we apply the change $t-t_{0}=\varepsilon \xi$, then the integral on the right-hand side passes to an integral of kind (3.8.8); its integration domain is an arc $\mathbb{T}_{\varepsilon}$ of the circle, such that its radian measure tends to $\pi$ as $\varepsilon \rightarrow 0$. More exactly, the tangent to $\Gamma$ at point $t_{0}$ decomposes $\mathbb{T}$ into two semicircles and the $\operatorname{arc} \mathbb{T}_{\varepsilon}$ tends to one of these semicircles. Since the left-hand side of the relation (3.8.13) tends to the corresponding singular integral as $\varepsilon \rightarrow 0$, it follows that the relation (3.8.10) holds.

The proof of the last assertion of the lemma is the same. Without loss of generality, one can assume that the line $L$ goes through the origin. Let $z_{0} \in P_{+}$and the half-disk $P_{+} \cap\left\{\left|z-z_{0}\right| \geq R\right\}$ contain the point $z_{0}$ inside. Applying the relation (3.8.9) to this half-disk and passing to the limit as $R \rightarrow \infty$, we obtain the relation (3.8.11).

If the Cauchy kernel has the form $Q(t ; \xi, \eta)$, then Theorems 3.6.1 and 3.7.1 are still valid for any smooth arc. More exactly, the following assertion holds.

Theorem 3.8.1. Let a domain $D$ not intersect a smooth arc $\Gamma$ and lie from one side of it. Let its endpoints not belong to $\bar{D}$.

Then the function

$$
\phi(z)=\int_{\Gamma} Q(t ; t-z, d t) \varphi(t), \quad z \in D,
$$

determined by a Cauchy kernel $Q(t ; \xi, \eta)$ from $C^{\nu(1)}\left(\Gamma, \mathcal{H}_{-1}\right)$ and a density $\varphi$ from $C^{\mu}(\Gamma), 0<\mu<\nu$, belongs to $C^{\mu}(D)$ and satisfies the norm estimate

$$
|\phi|_{C^{\mu}(D)} \leq C|Q|_{C^{\nu(2)}}|\varphi|_{C^{\mu}(\Gamma)},
$$

where $C$ is a positive constant depending only on the difference between $D$ and the endpoints of the arc $\Gamma$. Its boundary values are linked with the corresponding singular integral by the relation (3.7.6), where

$$
\sigma\left(t_{0}\right)=\frac{1}{2} \int_{\mathbb{T}} Q\left(t_{0} ; \xi, d \xi\right) .
$$

If the Cauchy kernel $Q(u, t ; \xi, \eta)$ depends on the parameter $u$ from $G$ and belongs to $C^{\nu(1)}\left(G \times \Gamma, \mathcal{H}_{-1}\right)$, then the corresponding function $\phi(u, z)$ belongs to $C^{\mu}(G \times D)$ and satisfies the corresponding norm estimate.

The assertion of Lemma 3.6.2 about Cauchy-type integrals is valid for any sequence of smooth arcs converging in the class $C^{1}$.

Proof. The function $\phi$ can be differentiated under the integral sign with respect to the variables $x_{j}$ $\left(x_{1}+i x_{2}=z\right)$ :

$$
\frac{\partial \phi}{\partial x_{j}}(z)=-\int_{\Gamma} Q_{j}(t ; t-z, d t) \varphi(t), \quad j=1,2,
$$


where $Q_{j}(t ; \xi, \eta)=\partial Q / \partial \xi_{j} \in C^{\nu(0)}\left(\Gamma, \mathcal{H}_{-2}\right)$. As in the case of Theorem 3.6.1, the first assertion is reduced to the proof of the estimate

$$
\left|\frac{\partial \phi}{\partial x_{j}}(z)\right| \leq C\left|Q_{j}\right|_{C^{\nu(0)}}|\varphi|_{C^{\mu}} d^{\mu-1}(z, \Gamma), \quad z \in D .
$$

Similarly to (3.6.7), we have

where

$$
\frac{\partial \phi}{\partial x_{j}}=\phi_{0}+\phi_{1}
$$

$$
\phi_{0}(z)=\int_{\Gamma}\left[Q_{j}(t ; t-z, d t) \varphi(t)-Q_{j}(a ; t-z, d t) \varphi(a)\right], \quad \phi_{1}(z)=\varphi(a) \int_{\Gamma} Q_{j}(a ; t-z, d t) .
$$

The validity of the estimate (3.8.16) for $\phi_{0}$ is proved in the same way as Theorem 3.6.1. To investigate $\phi_{1}$, complement $\Gamma$ by an arc $\widetilde{\Gamma}$ such that $\Gamma \cup \widetilde{\Gamma}$ is a piecewise-smooth contour enveloping the domain $D$. Supply this contour with an orientation coinciding with the orientation of the $\operatorname{arc} \Gamma$. Then, by virtue of Lemma 3.8.1, we have the relation

$$
\int_{\Gamma \cup \widetilde{\Gamma}} Q(a ; t-z, d t)=\int_{\mathbb{T}} Q(a ; \xi, d \xi, \quad z \in D .
$$

Hence, the differentiation of this relation leads to the relation $\phi_{1}(z)+\widetilde{\phi}_{1}(z), z \in D$, where $\widetilde{\phi}_{1}$ is defined similarly to $\phi_{1}$ with respect to $\widetilde{\Gamma}$. It is possible to select the arc $\widetilde{\Gamma}$ such that the distance between it and the domain $D$ is positive. Hence, the estimate (3.8.18) for the function $\widetilde{\phi}_{1}$ and, therefore, for the function $\phi_{1}$ is obvious.

The fact that the coefficient $\sigma$ in the relation (3.7.6) is given by the relation (3.8.15) follows from the last assertion of Lemma 3.8.1. The last assertion of the theorem is proved in the same way as Lemma 3.6.2 (we take into account the fact that Lemma 2.4.1 holds for any sequence of smooth arcs converging in the class $C^{1}$ ).

To investigate the differentiability of Cauchy-type integrals, take a smooth orientable arc $\Gamma$ with endpoints $a$ and $b$ and introduce the operation $\varphi^{\prime}=\varphi_{s}^{\prime}$ of the differentiation with respect to the arc length on $\Gamma$. Obviously, this operation acts from $C^{1}(\Gamma)$ to $C(\Gamma)$. If $\gamma:[0,1] \rightarrow \Gamma$ is an arbitrary parametrization, then this operation can be defined by the relation $\varphi^{\prime} \circ \gamma=(\varphi \circ \gamma)^{\prime}\left|\gamma^{\prime}\right|^{-1}$. For example, for an arbitrary function $\varphi(t)=t$, we have the relation $t^{\prime}=e(t)$. Arguing in the same way, we obtain the following form of the Newton-Leibnitz relation for the considered case:

$$
\int_{\Gamma} \varphi^{\prime}(t) d_{1} t=\varphi(b)-\varphi(a)
$$

where the arc is assumed to be oriented from $b$ to $a$.

If there exists an arc $\Gamma_{0}$ from $\Gamma \backslash \partial \Gamma$ such that the domain $D$ abuts it and lies from the left of it, then the boundary value $\varphi^{+}$of any function $\varphi$ from $C^{1}(\bar{D})$ is continuously differentiable on $\Gamma_{0}$ and

$$
\left(\varphi^{+}\right)^{\prime}=\left(\frac{\partial \varphi}{\partial x_{1}}\right)^{+} e_{1}+\left(\frac{\partial \varphi}{\partial x_{2}}\right)^{+} e_{2}, \quad z=x_{1}+i x_{2},
$$

where $e$ is the unit tangential vector belonging to $C(\Gamma)$ and such that its direction is coordinated with the selected orientation.

Now, consider the case where $\psi\left(t_{0}, t\right)$ belongs to $C^{1}(\Gamma \times \Gamma)$, while $\partial \psi / \partial t_{0}$ and $\partial \psi / \partial t$ denote its partial derivatives with respect to the corresponding variables. Then

$$
[\psi(t, t)]^{\prime}=\frac{\partial \psi}{\partial t_{0}}(t, t)+\frac{\partial \psi}{\partial t}(t, t)
$$


Consider the function $\phi$ defined by the integral (3.8.14).

Lemma 3.8.2. Let a smooth arc $\Gamma$ with endpoints $a$ and $b$ be oriented from a to $b$ and be disjoint with the domain D. Let the Cauchy kernel $Q(t ; \xi, \eta)$ belong to $C^{1(0)}(\Gamma)$ and a function $\varphi$ belong to $C^{1}(\Gamma)$.

Then, for the function $\phi(z)$, defined by the relation (3.8.14), the following differentiation relation holds:

$$
\begin{aligned}
\left(\eta_{1} \frac{\partial \phi}{\partial x_{1}}+\eta_{2} \frac{\partial \phi}{\partial x_{2}}\right)(z)=Q(a ; a-z, \eta) \varphi(a)-Q(b ; b-z, \eta) \varphi(b) & \\
& +\int_{\Gamma} Q_{0}(t, t-z, \eta) \varphi(t) d_{1} t+\int_{\Gamma} Q(t, t-z, \eta) \varphi^{\prime}(t) d_{1} t
\end{aligned}
$$

where $Q_{0}(t ; \xi, \eta)=(\partial Q / \partial t)(t ; \xi, \eta)$.

Proof. Differentiating the function (3.8.14) under the integral sign, we obtain the relation

$$
\frac{\partial \phi}{\partial x_{j}}(z)=-\int_{\Gamma} \frac{\partial Q}{\partial \xi_{j}}(t ; t-z, d t) \varphi(t), \quad j=1,2 .
$$

Applying the relations (3.8.12) to the terms $Q(t ; \xi, d t)=Q_{1}(t, \xi) e_{1}(t)+Q_{2}(t, \xi) e_{2}(t)$, we see that

$$
\frac{\partial \phi}{\partial x_{j}}(z)=-\int_{\Gamma}\left[\frac{\partial Q_{j}}{\partial \xi_{1}}(t ; t-z) e_{1}(t)+\frac{\partial Q_{j}}{\partial \xi_{2}}(t ; t-z) e_{2}(t)\right] \varphi(t) d_{1} t .
$$

By virtue of (3.8.18), the expression in the square brackets is the derivative of the function $Q_{j}\left(t_{0}, t-z\right)$ with respect to $t$ for $t_{0}=t$. Hence, we have the relation

$$
\int_{\Gamma} \frac{\partial Q}{\partial \xi_{j}}(t ; t-z, d t) \varphi(t)=\left.\int_{\Gamma}\left[\frac{d}{d t} Q_{j}\left(t_{0}, t-z\right)\right]\right|_{t_{0}=t} \varphi(t) d_{1} t .
$$

Applying (3.8.19) to the function $\psi\left(t_{0}, t\right)=Q_{j}\left(t_{0} ; t-z\right) \varphi(t)$, one can represent the expression in the square brackets as follows:

$$
\frac{d}{d t}\left[Q_{j}(t, t-z)\right]-\frac{\partial Q_{j}}{\partial t}(t ; t-z)
$$

Taking into account (3.8.17), we obtain the relation

$$
\begin{aligned}
& \int_{\Gamma} \frac{\partial Q}{\partial \xi_{j}}(t ; t-z, d t) \varphi(t)=Q_{j}(b ; b-z) \varphi(b)-Q_{j}(a ; a-z) \varphi(a) \\
&-\int_{\Gamma} \frac{\partial Q_{j}}{\partial t}(t ; t-z) \varphi(t) d_{1} t-\int_{\Gamma} Q_{j}(t ; t-z) \varphi^{\prime}(t) d_{1} t . \quad j=1,2 .
\end{aligned}
$$

Substituting this expression to (3.8.21), we obtain relations passing to (3.8.20) (as a linear combination of partial derivative).

Note that the lemma is also valid for smooth contours: in this case, there are no integrated terms on the right-hand side of (3.8.20).

Applying Theorems 3.6.1 and 3.8.1 to the first and second integral (respectively) on the right-hand side of (3.8.20), we arrive at the corresponding analog of Theorem 3.6.1 for the space $C^{1, \mu}$.

Theorem 3.8.2. Let the assumptions of Theorem 3.8 .1 be satisfied, the arc $\Gamma$ belong to $C^{1, \nu}$, the Cauchy kernel $Q(t ; \xi, \eta)$ belong to $C^{1, \nu(2)}\left(\Gamma, \mathcal{H}_{-1}\right)$, and the density $\varphi$ belong to $C^{1, \mu}(\Gamma), 0<\mu<1$.

Then the function $\phi$ defined by the integral (3.8.14) belongs to $C^{1, \mu}(D)$ and its norm satisfies the estimate

$$
|\phi|_{C^{1, \mu}(D)} \leq C|Q|_{C^{\nu(2)}}|\varphi|_{C^{1, \mu}(\Gamma)},
$$


where $C$ is a positive constant depending only on the distance between $D$ and the endpoints of the $\operatorname{arc} \Gamma$.

\subsection{Line Singular Integrals}

On a smooth contour, consider the singular Cauchy integral

$$
\psi\left(t_{0}\right)=\int_{\Gamma} Q\left(t ; t-t_{0}, d t\right) \varphi(t), \quad t_{0} \in \Gamma,
$$

corresponding to the Cauchy-type integral (3.8.14). The general case of piecewise-smooth curves is studied in Sec. 3.10 in the framework of weight Hölder spaces.

In the considered case, Theorem 3.8.1 leads to the corresponding result in the same way as Theorem 3.7.2 above.

Theorem 3.9.1. Let $\Gamma$ be an orientable smooth contour and $Q(t ; \xi, \eta) \in C^{\nu(1)}\left(\Gamma, \mathcal{H}_{-1}\right)$. Then the singular operator $K$ defined by the relation (3.9.1) is bounded in the space $C^{\mu}(\Gamma), 0<\mu<\nu$, and its norm satisfies the estimate $|K|_{\mathcal{L}} \leq C|Q|_{C^{\nu(1)}}$.

If the kernel $Q(u, t ; \xi, \eta)$ depends on the parameter $u$ from $G$ and belongs to $C^{\nu(2)}\left(G \times \Gamma, \mathcal{H}_{-1}\right)$, then the function $\psi(u, z)$ belongs to $C^{\mu}(G \times D)$ and satisfies the corresponding norm estimate.

Using Lemma 3.8.2, one can complement the last theorem by a similar result for the space $C^{1, \mu}(\Gamma)$.

Theorem 3.9.2. If the assumptions of Theorem 3.9.1 are satisfied and

$$
\Gamma \in C^{1, \nu}, \quad Q(t ; \xi, \eta) \in C^{1, \nu(2)}\left(\Gamma, \mathcal{H}_{-1}\right),
$$

then the singular operator $K$ is bounded in the space $C^{1, \mu}(\Gamma)$, its norm satisfies the estimate $|K|_{\mathcal{L}} \leq$ $C|Q|_{C^{1, \nu(2)}}$, and

$$
\psi^{\prime}\left(t_{0}\right)=\int_{\Gamma} Q_{0}\left[t, t-t_{0}, e\left(t_{0}\right)\right] \varphi(t) d_{1} t+\int_{\Gamma} Q\left[t, t-t_{0}, e\left(t_{0}\right)\right] \varphi^{\prime}(t) d_{1} t,
$$

where $\psi=K \varphi$ and $Q_{0}(t ; \xi, \eta)=(\partial Q / \partial t)(t ; \xi, \eta)$.

Proof. Without loss of generality, one can assume that $\Gamma$ is a simple contour bounding a domain $D$. Let $\phi$ be defined by the integral (3.8.14) in this domain. Then, due to Theorem 3.8.2, the function $\phi^{+}$ belongs to $C^{1, \mu}(\Gamma)$. Taking into account (3.8.18), we conclude that

$$
\left(\phi^{+}\right)^{\prime}=\left(\frac{\partial \phi}{\partial x_{1}}\right)^{+} e_{1}+\left(\frac{\partial \phi}{\partial x_{2}}\right)^{+} e_{2}, \quad z=x_{1}+i x_{2} .
$$

To the partial derivatives on the right-hand side of this relation, one can apply the relation (3.8.20) with $\eta=e\left(t_{0}\right)$ (recall that it contains no integrated terms provided that the contour is smooth). Thus, the following relation holds:

$$
\left[e_{1}\left(t_{0}\right) \frac{\partial \phi}{\partial x_{i}}+e_{2}\left(t_{0}\right) \frac{\partial \phi}{\partial x_{i}}\right](z)=\int_{\Gamma} Q_{0}(z, t, t-z) \varphi(t) d_{1} t+\int_{\Gamma} Q\left[z, t, t-z, e\left(t_{0}\right)\right] \varphi^{\prime}(t) d_{1} t .
$$

Applying the relation (3.8.2) to the integrals of the last relation, we see that

$$
\begin{aligned}
\left(\phi^{+}\right)^{\prime}\left(t_{0}\right)=\sigma^{0}\left(t_{0}\right) \varphi\left(t_{0}\right)+\sigma\left(t_{0}\right) \varphi^{\prime}\left(t_{0}\right)+ & \int_{\Gamma} Q_{0}\left[t ; t-t_{0}, e\left(t_{0}\right)\right] \varphi(t) d_{1} t \\
& +\int_{\Gamma} Q\left[t, t-t_{0}, e\left(t_{0}\right)\right] \varphi^{\prime}(t) d_{1} t
\end{aligned}
$$


where

$$
\sigma_{0}\left(t_{0}\right)=\int_{L\left(t_{0}\right)} Q_{0}\left[t_{0}, t-z_{0}, e\left(t_{0}\right)\right] d_{1} t, \quad \sigma\left(t_{0}\right)=\int_{L\left(t_{0}\right)} Q\left[t_{0}, t-z_{0}, e\left(t_{0}\right)\right] d_{1} t .
$$

Recall that $L\left(t_{0}\right)$ is the tangent line to $\Gamma$ at the point $t_{0}$ such that its unit direction vector is $e\left(t_{0}\right)=$ $e_{1}+i e_{2}$, the neighborhood $C^{+}\left(t_{0}\right)$ is left from the left of the line, and the point $z_{0}$ lies from the left of $L\left(t_{0}\right)$, i.e., $\operatorname{Im}\left[\left(z_{0}-t_{0}\right) / e\left(t_{0}\right)\right]>0$.

Both $Q_{0}$ and $Q$ are Cauchy kernels. Indeed, differentiation of the relation $c(t)=Q(t ; \xi, \xi)$ with respect to $t$ yields the relation $c^{\prime}(t)=Q_{0}(t ; \xi, \xi)$. Therefore, due to the last assertion of Lemma 3.8.1, we arrive ar the relations

$$
\sigma_{0}(t)=\frac{1}{2} \int_{\mathbb{T}} Q_{0}(t ; \xi, d \xi), \quad \sigma(t)=\frac{1}{2} \int_{\mathbb{T}} Q(t ; \xi, d \xi)
$$

implying (as above) the relation $\sigma^{\prime}=\sigma_{0}$. Hence, the initial two terms on the right-hand side of (3.9.4) can be represented as $(\sigma \varphi)^{\prime}\left(t_{0}\right)$. Then, differentiating the relation (3.8.3) with the upper sign and combining the obtained result with (3.9.4), we obtain the relation (3.9.3).

Applying Theorem 3.8.1 to the relation (3.9.3), we arrive at the boundedness of the singular operator $K$ in $C^{1, \mu}(\Gamma)$ with the corresponding estimate of its norm.

It frequently occurs that the Cauchy kernel $Q\left(t_{0}, t ; \xi, \eta\right)$ depends on the two variables $t_{0}$ and $t$ varying in $\Gamma$. The variable $t_{0}=u$ can be treated as an independent parameter. Then, in the case where $Q \in C^{1, \nu(2)}(\Gamma \times \Gamma)$, the singular integral

$$
\psi\left(u, t_{0}\right)=\int_{\Gamma} Q\left(u, t ; t-t_{0}, d t\right) \varphi(t), \quad t_{0} \in \Gamma,
$$

can be differentiated with respect to the parameter $u$, which is justified in the same way as in Lemma 3.4.2. Thus, we have the relation

$$
\frac{\partial \psi}{\partial u}\left(u, t_{0}\right)=\int_{\Gamma} \frac{\partial Q}{\partial u}\left(u, t ; t-t_{0}, d t\right) \varphi(t), \quad t_{0} \in \Gamma .
$$

In the case where $u=t_{0}$, the integral (3.9.5) passes to

$$
\psi\left(t_{0}\right)=\int_{\Gamma} Q\left(t_{0}, t ; t-t_{0}, d t\right) \varphi(t), \quad t_{0} \in \Gamma .
$$

Then, taking into account (3.8.19), we arrive at the following modification of the differentiation relation given by (3.9.4): in the first integral, the expression $Q_{0}\left[t_{0} ; t-t_{0}, e\left(t_{0}\right)\right]$ is to be replaced by $Q_{0}\left(t_{0}, t ; t-t_{0}\right)$ with the kernel

$$
Q_{0}\left(t_{0}, t ; \xi\right)=\frac{\partial Q}{\partial t_{0}}\left[t_{0}, t ; \xi, e(t)\right]+\frac{\partial Q}{\partial t}\left[t_{0}, t ; \xi, e\left(t_{0}\right)\right]
$$

The function

$$
Q\left(t_{0}, t ; \xi, \eta\right)=\frac{k\left(t_{0}, t\right) \eta}{\pi i \xi},
$$

is a simple example of Cauchy kernels. Obviously, it satisfies the necessary condition given by (3.8.7). Also, it is clear that the conditions $k \in C^{\nu}(\Gamma \times \Gamma)$ and $Q \in C^{\nu(m)}$ are equivalent for any $m$. For the considered kernel, we take into account the relation $d t=e(t) d_{1} t$ and see that the singular operator (3.9.1) takes the form of the classical Cauchy operator

$$
(K \varphi)\left(t_{0}\right)=\frac{1}{\pi i} \int_{\Gamma} \frac{k\left(t_{0}, t\right) \varphi(t) d t}{t-t_{0}}, \quad t_{0} \in \Gamma .
$$


In the considered case, the differentiation relation given by (3.9.3) and (3.9.6) takes the form

$$
(K \varphi)^{\prime}\left(t_{0}\right)=\frac{1}{\pi i} \int_{\Gamma}\left[\frac{\partial k}{\partial t_{0}}\left(t_{0}, t\right) e(t)+\frac{1}{\pi i} \frac{\partial k}{\partial t}\left(t_{0}, t\right) e\left(t_{0}\right)\right]+\frac{\varphi(t) d_{1} t}{t-t_{0}}+\int_{\Gamma} \frac{k\left(t_{0}, t\right) \varphi^{\prime}(t) e\left(t_{0}\right) d_{1} t}{t-t_{0}} .
$$

Using the modified differentiation operation

$$
D \varphi=e^{-1} \varphi^{\prime}
$$

and taking into account the relation $d t=e(t) d_{1} t$, one can represent the above differentiation relation in the operator form

$$
D K=K_{0}+K D,
$$

where $K_{0}$ is defined similarly to (3.9.5) with respect to the function $k_{0}=\left(D_{t_{0}}+D_{t}\right) k$.

The next lemma shows that any Cauchy kernel can be represented by (3.9.8).

Lemma 3.9.1. Let $\Gamma$ be a smooth contour from $C^{1, \nu}$ and a kernel $Q_{0}\left(t_{0}, t ; \xi\right)$ belong to $C^{\nu(1)}\left(\Gamma \times \Gamma, \mathcal{H}_{0}\right)$ and be even with respect to the variable $\xi$. Then the function $k\left(t_{0}, t\right)=Q_{0}\left(t_{0}, t ; t-t_{0}\right)$ belongs to $C^{\nu}(\Gamma \times \Gamma)$ and the corresponding norms satisfy the estimate $|k|_{C^{\nu}} \leq C\left|Q_{0}\right|_{C^{\nu(0)}}$.

Proof. It suffices to prove the lemma for any arc $\Gamma_{0}$ from $\Gamma$. Let $\gamma:[0,1] \rightarrow \Gamma_{0}$ be a $C^{1, \mu}[0,1]$ parametrization of this arc. Since this parametrization is a Lipschitz map, it suffices to prove this lemma for the function $k_{0}\left(s_{0}, s\right)=k\left[\gamma\left(s_{0}\right), \gamma(s)\right]$ in the square $0 \leq s, s_{0} \leq 1$. By virtue of the homogeneity and evenness of the kernel $Q_{0}$, this function can be represented as follows:

$$
k_{0}\left(s_{0}, s\right)=Q_{0}\left[\gamma\left(s_{0}\right), \gamma(s) ; q\left(s_{0}, s\right)\right], \quad q\left(s_{0}, s\right)=\frac{\gamma(s)-\gamma\left(s_{0}\right)}{s-s_{0}} .
$$

As in the proof of Lemma 2.4.1, we verify that $q$ belongs to $C^{\nu}([0,1] \times[0,1])$ and its absolute value is separated from zero. Therefore, it remains to apply Lemma 3.1.2 to the function $Q_{0}\left[\gamma\left(s_{0}\right), \gamma(s) ; q\left(s_{0}, s\right)\right]$.

Thus, if a Cauchy kernel $Q\left(t_{0}, t ; \xi, \eta\right)$ belongs to $C^{\nu(1)}\left(\Gamma \times \Gamma, \mathcal{H}_{0}\right)$, then Lemma 3.9.1 is applicable to the function $Q_{0}\left(t_{0}, t ; \xi, \eta\right)=\xi Q\left(t_{0}, t ; \xi, \eta\right)$. Hence, the operator $K$ defined by the relation (3.9.1) can be represent in the form (3.9.9), where the kernel $k\left(t_{0}, t\right)$ belongs to $C^{\nu}(\Gamma \times \Gamma)$.

If $\pi i k\left(t_{0}, t\right)=1$, then the following special notation is used for the operator (3.9.9):

$$
(S \varphi)\left(t_{0}\right)=\frac{1}{\pi i} \int_{\Gamma} \frac{\varphi(t) d t}{t-t_{0}}, \quad t_{0} \in \Gamma .
$$

The classical Cauchy-type integral

$$
\phi(z)=\frac{1}{2 \pi i} \int_{\Gamma} \frac{\varphi(t) d t}{t-z}, \quad z \notin \Gamma,
$$

corresponds to this operator: the Cauchy kernel $2 \pi i Q(\xi, \eta)=\eta / \xi$ is similar to (3.9.8) and it defines a function analytical outside $\Gamma$, i.e., a function analytical in each connected component of the open set $\mathbb{C} \backslash \Gamma$. For the specified kernel, the relation (3.8.8) passes to the relation

$$
2 \sigma=\frac{1}{2 \pi i} \int_{\mathbb{T}} \frac{d \xi}{\xi}
$$

Hence, due to the Cauchy relation for analytical functions, we have $\sigma=1 / 2$ and (3.8.3) passes to the classical Sochocki-Plemelj relation

$$
2 \phi^{ \pm}= \pm \varphi+S \varphi .
$$


If $\varphi=1$, i.e., the density is constant, then the function

$$
\chi\left(t_{0}\right)=\frac{1}{\pi i} \int_{\Gamma} \frac{d t}{t-t_{0}}, \quad t_{0} \in \Gamma
$$

is constant on any connected component $\Gamma$. More exactly, it is described by the following assertion.

Lemma 3.9.2. Let $\Gamma$ be a smooth orientable contour consisting of components $\Gamma_{k}, 1 \leq k \leq n$, and $\sigma_{k}=1\left(\sigma_{k}=-1\right)$ if the contour $\Gamma_{k}$ is oriented counterclockwise (clockwise). Then

$$
\chi\left(t_{0}\right)=\sigma_{k}+2 \sum_{j}^{\prime} \sigma_{j}, \quad t_{0} \in \Gamma_{k},
$$

where the prime means that summing cover all values of $j$ such that the contour $\Gamma_{j}$ envelopes $\Gamma_{k}$.

Proof. First, assume that $n=1$. In this case, without loss of generality, one can assume that $\sigma=1$. If $\phi(z)$ is defined by (3.9.13) with $\varphi=1$, then, due to the Cauchy relation, $\phi(z)=1$ if the point $z$ is located inside $\Gamma$ and $\phi(z)=0$ if the point $z$ is located outside the contour. By virtue of (3.9.14), this implies that $\chi\left(t_{0}\right)=1$.

In the general case, we have the relation

$$
\chi\left(t_{0}\right)=\sigma_{k}+\sum_{j \neq k} \frac{1}{\pi i} \int_{\Gamma_{j}} \frac{d t}{t-t_{0}}, \quad t_{0} \in \Gamma_{k},
$$

and it remains to apply the Cauchy relation to the terms on the right-hand side.

In particular, this lemma implies that the function $\chi$ is constant provided that there exists a domain such that the contour $\Gamma$ is its boundary and is oriented positively with respect to it.

To consider compositions of singular integrals, we start from the following auxiliary assertion on permutations of special-kind integrals on smooth contours.

\section{Lemma 3.9.3.}

(a) For any function $f$ from $C^{\mu}(\Gamma \times \Gamma)$, the following change of the integration order is legal:

$$
\int_{\Gamma} d t_{0} \int_{\Gamma} \frac{f\left(t_{0}, t\right) d t}{t-t_{0}}=\int_{\Gamma} d t \int_{\Gamma} \frac{f\left(t_{0}, t\right) d t_{0}}{t-t_{0}} .
$$

(b) If $f \in C^{\mu}\left(\Gamma_{1} \times \Gamma_{2}\right)$, then

$$
\int_{\Gamma_{1}} d t_{0} \int_{\Gamma_{2}} \frac{f\left(t_{0}, t\right) d t}{t-t_{1}}=\int_{\Gamma_{2}} d t \int_{\Gamma_{1}} \frac{f\left(t_{0}, t\right) d t_{0}}{t-t_{1}}
$$

for any point $t_{1}$ from $\mathbb{C}$.

Proof. (a) Without loss of generality, one can assume that $\Gamma$ is a simple contour oriented counterclockwise. If $f(t, t) \equiv 0$, then the function $\left(t-t_{0}\right)^{-1} f\left(t_{0}, t\right)$ has a weak singularity and the relation (3.9.16) is the assertion of the Fubini theorem from Sec. 1.8. Therefore, it suffices to prove it for the function $f(t, t)=\varphi(t)$. Then, taking into account Lemma 3.9.2, one can represent this relation as follows:

$$
\int_{\Gamma}(S \varphi)\left(t_{0}\right) d t_{0}=-\int_{\Gamma} \varphi(t) d t .
$$

Consider the function $\phi(z)$ defined by the integral (3.9.13); it is analytic outside $\Gamma$. By virtue of (3.9.14) and the Cauchy relation applied to $\phi$ in the domain enveloped by $\Gamma$, we have the relation

$$
\int_{\Gamma}(S \varphi)\left(t_{0}\right) d t_{0}=\int_{\Gamma}\left[\phi^{+}\left(t_{0}\right)+\phi^{-}\left(t_{0}\right)\right] d t_{0}=\int_{\Gamma} \phi^{-}\left(t_{0}\right) d t_{0} .
$$


Let $\Gamma$ be contained inside a counterclockwise oriented circle $\Gamma_{0}$ and $D$ be the domain contained between these contours. Then, due to the Cauchy theorem applied to the function $\phi$ in the domain $D$, we obtain that

$$
\int_{\Gamma} \phi^{-}\left(t_{0}\right) d t_{0}=\int_{\Gamma_{0}} \phi\left(t_{0}\right) d t_{0}=\frac{1}{2 \pi i} \int_{\Gamma_{0}} d t_{0} \int_{\Gamma} \frac{\varphi(t) d t}{t-t_{0}} .
$$

Since the contours $\Gamma$ and $\Gamma_{0}$ do not intersect, it follows that the integration order on the right-hand side of this relation can be changed. Since

$$
\frac{1}{2 \pi i} \int_{\Gamma} \varphi(t) d t \int_{\Gamma_{0}} \frac{d t_{0}}{t-t_{0}}=-\int_{\Gamma} \varphi(t) d t
$$

due to the Cauchy relation, it follows that the relation (3.9.18) holds.

(b) Without loss of generality, one can assume that $t_{1} \in \Gamma_{1} \cup \Gamma_{2}$. If $t_{1} \in \Gamma_{1}$, then, as in case (a), one can replace $f$ by the function $\varphi(t)=f\left(t_{1}, t\right)$ depending only on $t$. In this case, the left and the right-hand sides of (3.9.17) vanish. In the same way, if $t_{1} \in \Gamma_{2}$, then $f$ can be replaced by the function $\varphi\left(t_{0}\right)=f\left(t_{0}, t_{1}\right)$ depending only on $t_{0}$. Then the left- and right-hand sides of (3.9.17) also vanish.

Lemma 3.9.3 allows one to obtain the known Poincaré-Bertrand permutation relation (see [45]) for two singular integrals on a smooth contour. This relation is given by the following assertion.

Theorem 3.9.3. If $f\left(t_{1}, t_{0}, t\right) \in C^{\mu+0}(\Gamma \times \Gamma \times \Gamma)$, then

$$
\int_{\Gamma} \frac{d t_{0}}{t_{0}-t_{1}} \int_{\Gamma} \frac{f\left(t_{1}, t_{0}, t\right) d t}{t-t_{0}}=-\pi^{2} f\left(t_{1}, t_{1}, t_{1}\right)+\int_{\Gamma} d t \int_{\Gamma} \frac{f\left(t_{1}, t_{0}, t\right) d t_{0}}{\left(t_{0}-t_{1}\right)\left(t-t_{0}\right)}
$$

for any point $t_{1}$ from $\Gamma$.

Note that

$$
\int_{\Gamma} \frac{f\left(t_{1}, t_{0}, t\right) d t_{0}}{\left(t_{0}-t_{1}\right)\left(t-t_{0}\right)}=\frac{g\left(t_{1}, t, t_{1}\right)-g\left(t_{1}, t, t\right)}{t-t_{1}}
$$

where the function

$$
g\left(t_{1}, t, t_{2}\right)=\int_{\Gamma} \frac{f\left(t_{1}, t_{0}, t\right) d t_{0}}{t_{0}-t_{2}}
$$

belongs to $C^{\mu+0}(\Gamma \times \Gamma \times \Gamma)$ due to Theorem 3.9.1.

Proof. Assign $f^{0}\left(t_{1}, t_{0}, t\right)=f\left(t_{1}, t_{0}, t\right)-f\left(t_{1}, t, t\right)$ and $f^{1}\left(t_{1}, t_{0}, t\right)=f\left(t_{1}, t, t\right)-f(t, t, t)$. Then

$$
f\left(t_{1}, t_{0}, t\right)=f^{0}\left(t_{1}, t_{0}, t\right)+f^{1}\left(t_{1}, t_{0}, t\right)+f(t, t, t) .
$$

first, we prove the validity of the relation (3.9.14) for $f^{j}$, i.e., the relation

$$
\int_{\Gamma} \frac{d t_{0}}{t_{0}-t_{1}} \int_{\Gamma} \frac{f^{j}\left(t_{1}, t_{0}, t\right) d t}{t-t_{0}}=\int_{\Gamma} d t \int_{\Gamma} \frac{f^{j}\left(t_{1}, t_{0}, t\right) d t_{0}}{\left(t_{0}-t_{1}\right)\left(t-t_{0}\right)}, \quad j=1,2 .
$$

For brevity, let $G=\Gamma \times \Gamma \times \Gamma, G^{0}=\left\{\left(t_{1}, t_{0}, t\right) \in G, t=t_{0}\right\}$, and $G^{1}=\left\{\left(t_{1}, t_{0}, t\right) \in G, t=t_{1}\right\}$. By the condition, there exists $\nu$ exceeding $\mu$ such that $f^{j}$ belongs to $C^{\nu}(G)$ and, obviously, vanishes on $G^{j}$. If there exists a neighborhood of the set $G^{0}$ such that the function $f^{0}$ vanishes in this neighborhood, then the function $f\left(t_{0}, t\right)=f^{0}\left(t_{1}, t_{0}, t\right)\left(t-t_{0}\right)^{-1}$ belongs to $C^{\mu}(\Gamma \times \Gamma)$ and (3.9.21) follows from the relation (3.9.17) of Lemma 3.9.3. In the same way, if there exists a neighborhood of the set $G^{1}$ such that $f^{1}$ vanishes in this neighborhood, then the function $f\left(t_{0}, t\right)=f^{1}\left(t_{1}, t_{0}, t\right)\left(t-t_{1}\right)^{-1}$ belongs to $C^{\mu}(\Gamma \times \Gamma)$ and (3.9.22) follows from the relation (3.9.16) of this lemma.

In the general case, due to Theorem 2.2.1, for any fixed $\mu$ satisfying the inequality $\mu<\nu_{1}<\nu$ there exists a sequence of functions $f_{n}^{j}$ from $C^{\nu_{1}}(G), n=1,2, \ldots$, converging to $f^{j}$ in the space $C^{\nu_{1}}(G)$ and 
such that each of these functions vanishes in a neighborhood of $G^{j}$. Then, by virtue of Theorem 3.9.1, one can pass to the limit as $n \rightarrow \infty$ in the relation (3.9.22) applied to $f_{n}^{j}$. To do this, one must transform the left-hand side of the last relation (similarly to (3.9.20)) to the form

$$
\int_{\Gamma} \frac{f_{n}^{j}\left(t_{1}, t_{0}, t\right) d t_{0}}{\left(t_{0}-t_{1}\right)\left(t-t_{0}\right)}=\frac{g_{n}^{j}\left(t_{1}, t, t_{1}\right)-g_{n}^{j}\left(t_{1}, t, t\right)}{t-t_{1}}
$$

and apply Theorem 3.9.1 to the sequence $\left\{g_{n}^{j}\right\}$.

Thus, the theorem is proved for the functions $f^{0}$ and $f^{1}$. Then, according to (3.9.21), one can assume (without loss of generality) that the function $f$ depends only on the variable $t$. Denoting this function by $\varphi(t)$, we see that it remains to prove the relation

$$
\int_{\Gamma} \frac{d t_{0}}{t_{0}-t_{1}} \int_{\Gamma} \frac{\varphi(t) d t}{t-t_{0}}=-\pi^{2} \varphi\left(t_{1}\right)+\int_{\Gamma} \varphi(t) d t \int_{\Gamma} \frac{d t_{0}}{\left(t_{0}-t_{1}\right)\left(t-t_{0}\right)} .
$$

Without loss of generality, $\Gamma$ can be assumed to be a simple contour. Indeed, let (in the general case) the point $t_{1}$ belong to a connected component $\Gamma_{1}$ of the contour $\Gamma$ and $\Gamma_{2}=\Gamma \backslash \Gamma_{1}$. Then, for $i \neq j$, the function $f\left(t_{0}, t\right)=\varphi(t)\left(t-t_{0}\right)^{-1}$ belongs to $C^{\mu}\left(\Gamma_{i} \times \Gamma_{j}\right)$ and

$$
\int_{\Gamma_{i}} \frac{d t_{0}}{t_{0}-t_{1}} \int_{\Gamma_{j}} \frac{\varphi(t) d t}{t-t_{0}}=\int_{\Gamma_{j}} \varphi(t) d t \int_{\Gamma_{i}} \frac{d t_{0}}{\left(t_{0}-t_{1}\right)\left(t-t_{0}\right)}
$$

due to Lemma 3.9.3(b). Since $f\left(t_{0}, t\right)=\varphi(t)\left(t_{0}-t_{1}\right)^{-1}$ belongs to $C^{\mu}\left(\Gamma_{1} \times \Gamma_{1}\right)$, it also follows from Lemma 3.9.3(a) that the relation (3.9.24) holds for $i=j=2$.

Thus, let $\Gamma$ be a simple contour; without loss of generality, one can assume that it is counterclockwise oriented. In this case, due to Lemma 3.9.2, the function $g$ in (3.9.20) is equal to $\pi i$ and the relation (3.9.23) takes the form

$$
S(S \varphi)=\varphi
$$

Let $\phi(z)$ be an analytic function defined by the integral (3.9.13) and considered in a finite domain $D$ inside $\Gamma$. Then, due to the Cauchy relation, we have the relation

$$
\phi(z)=\frac{1}{2 \pi i} \int_{\Gamma} \frac{\phi^{+}(t) d t}{t-z}, \quad z \in D .
$$

Apply the relation (3.9.14) to the Cauchy-type integral with density $\phi^{+}$. We obtain that $\phi^{+}=$ $\left(\phi^{+}+S \phi^{+}\right) / 2$. Substituting expression (3.9.14) with the upper sign in the last relation, we obtain the relation $(\varphi+S \varphi)=(1+S)(\varphi+S \varphi) / 2$. It is equivalent to (3.9.25), which completes the proof of the theorem.

Let $\mathcal{K}\left(C^{\mu+0}\right)$ denote the class of all singular operators of kind $(3.9 .9)$ with kernel $k\left(t_{0}, t\right)$ from $C^{\mu+0}(\Gamma \times \Gamma)$. Let $\mathcal{K}_{0}\left(C^{\mu+0}\right)$ be its subclass selected by the condition $k(t, t) \equiv 0$. Operators of the last class are defined by integrals with weak singularities and boundedly map $C(\Gamma)$ to $C^{\mu}(\Gamma)$ (due to Theorem 3.2.3). In particular, they are compact in the space $C^{\mu}(\Gamma)$. If we additionally assume that $k \in C^{1, \mu+0}(\Gamma \times \Gamma)$, then, by virtue of the differentiation the relations (3.9.10)-(3.9.11), these operators are also compact in $C^{1, \mu}(\Gamma)$.

Obviously, if a function $a$ from ${ }^{\mu+0}(\Gamma)$ is treated as a multiplication operator $\varphi \rightarrow a \varphi$, then $a S-S a \in$ $\mathcal{K}_{0}\left(C^{\mu+0}\right)$. The same arguing yields $K-a S \in \mathcal{K}_{0}\left(C^{\mu+0}\right)$ with respect to the function $a(t)=k(t, t)$. It immediately follows from Theorem 3.9.2 that the product of two operators $K_{1}$ and $K_{2}$ from $\mathcal{K}\left(C^{\mu+0}\right)$ can be represented in the form

$$
K_{1} K_{2}=a+K_{0}, \quad K_{0} \in \mathcal{K}_{0}\left(C^{\mu+0}\right),
$$


where $a(t)=k_{1}(t, t) k_{2}(t, t)$. According to (3.9.20), the function $k_{0}\left(t_{0}, t\right)$ defining the operator $K_{0}$ is represented as follows:

$$
k_{0}\left(t_{1}, t\right)=g\left(t_{1}, t, t_{1}\right)-g\left(t_{1}, t, t\right), \quad g\left(t_{1}, t, t_{2}\right)=\frac{1}{\pi} \int_{\Gamma} \frac{k_{1}\left(t_{1}, t_{0}\right) k_{2}\left(t_{0}, t\right) d t_{0}}{t_{0}-t_{2}}
$$

Thus, the class $\mathcal{K}\left(C^{\mu+0}\right)$ is an algebra and $\mathcal{K}_{0}\left(C^{\mu+0}\right)$ is contained in it and is its two-sided ideal.

If we apply notation (3.9.15) to the case where $K_{1}=K_{2}=S$, then the relation (3.9.26) passes to the relation

$$
S^{2}=1+K_{0}, \quad K_{0}=\chi S-S \chi .
$$

In particular, according to the remark to Lemma 3.9.2, we have the relation $S^{2}=1$ in the case where the contour $\Gamma$ is the boundary of the domain $D$ and is positively oriented with respect to this domain.

It follows from Lemma 3.9.3(a) that the operator $K$ admits an associate operator $K^{\prime}$ with respect to the bilinear form

$$
\langle\varphi, \psi\rangle=\int_{\Gamma} \varphi(t) \psi(t) d t
$$

it has the same type in the sense of the definition from Sec. 1.3, i.e., in the sense of the identity $\langle S \varphi, \psi\rangle=-\langle\varphi, S \psi\rangle$, and this operator is determined by the function $k^{\prime}\left(t_{0}, t\right)=-k\left(t, t_{0}\right)$.

\subsection{Weight $C^{\mu}$-Estimates of Cauchy-Type Integrals}

Consider the generalized Cauchy-type integral

$$
\phi(z)=\int_{\Gamma} Q(t ; t-z) \varphi(t) d_{1} t, \quad z \notin \Gamma,
$$

and the Cauchy-type integral (i.e., its special case)

$$
\phi(z)=\int_{\Gamma} Q(t ; t-z, d t) \varphi(t), \quad z \notin \Gamma,
$$

on a piecewise-smooth curve $\Gamma$ such that the terminology introduced in Sec. 2.5 is preserved with respect to it. Let $F$ be a finite set of points containing all boundary points of the curve and the infinity point $\infty$. Let $F$ apart from the infinity point be contained in $\Gamma$. Thus, any connected component of the set $\Gamma \backslash F$ is either a simple smooth contour or an open smooth (connected or disconnected) arc. If the curve $\Gamma$ is unbounded, then it is treated as a piecewise-smooth curve on the Riemann sphere $\overline{\mathbb{C}}=\mathbb{C} \cup \infty$ and it contains the point $\infty$. If a linear-fractional transformation maps $\Gamma$ to a bounded curve, then the latter is piecewise smooth in the classical sense. If $\infty \in \bar{D} \backslash \Gamma$, then the curve $\Gamma$ is bounded and the set $D$ is a neighborhood of $\infty$.

Similarly to Sec. 2.5, the curve $\Gamma$ can be represented in the form

$$
\Gamma \backslash F=\Gamma_{0} \cup \dot{\Gamma}_{1} \cup \ldots \cup \dot{\Gamma}_{m},
$$

where $\Gamma_{0}$ is a smooth contour (in general, it is a composite one), $\dot{\Gamma}_{j}$ are open smooth arcs, and all these curves are mutually disjoint.

Recall (see Sec. 2.5) that the open smooth arc $\dot{\Gamma}_{j}$ is given by a parametrization $\gamma_{j}$ from $C^{1}[0,1]$; on the semi-open intervals $(0,1]$ and $[0,1)$, it is a one-to-one map and $\gamma_{j}^{\prime}(s) \neq 0,0 \leq s \leq 1$. By definition, the notation $\dot{\Gamma}_{j} \in C^{1, \nu}$ means that $\gamma_{j} \in C^{1, \nu}[0,1]$. This condition can be weakened. We know from Sec. 2.10 that Theorem 2.10.2 implies that the classes $C^{1, \nu}[0,1]$ and $C_{(1+\nu)}^{1, \nu}([0,1] ; 0,1)$ coincide (and their norms are equivalent) and the following embeddings of Banach spaces take place:

$$
C_{(1+\nu)}^{1, \nu}([0,1] ; 0,1) \subseteq C_{(1+\varepsilon)}^{1, \nu}([0,1] ; 0,1) \subseteq C_{(1+\varepsilon)}^{1, \varepsilon}([0,1] ; 0,1), \quad 0<\varepsilon \leq \nu .
$$


This is the reason to describe the class $C_{(1+0)}^{1, \nu}$ of smooth open $\operatorname{arcs} \dot{\Gamma}_{j}$ with respect to their parametrization as follows: we use the condition $\gamma_{j} \in C_{(1+\varepsilon)}^{1, \nu}([0,1] ; 0,1)$, where $\varepsilon>0$. It is clear that this class contains $C^{1, \nu}$ and is contained in the general class $C^{1,+0}$ of open Lyapunov arcs.

For any $\tau$ from $F$, let $B_{\tau}(\rho)$ denote the disk $\{|z-\tau| \leq \rho\}$ for $\tau \neq \infty$ and the exterior of the disk $\{|z| \geq 1 / \rho\}$ for $\tau=\infty$. If $\rho$ is sufficiently small, then the intersection of $\Gamma$ with $B_{\tau}(\rho), \tau \in \Gamma$, is decomposed into a finite set of $n_{\tau}$ smooth arcs with a common endpoint $\tau$, i.e.,

$$
\Gamma \cap B_{\tau}(\rho)=\bigcup_{j=1}^{n_{\tau}} \Gamma_{\tau, j},
$$

where smooth arcs $\Gamma_{\tau, j}$ are pairwise intersected at the point $\tau$. Note that the sum of all numbers $n_{\tau}$ coincides with $2 m$.

Select a small $\rho$ in (3.10.4) to ensure that all arcs $\Gamma_{\tau, j}, 1 \leq j \leq n_{\tau}$, are radial arcs with respect to the endpoint $\tau$. Then they are defined by the radial parametrizations

$$
\gamma_{\tau, j}(r)=\left\{\begin{array}{ll}
r e^{i f_{\tau, j}(r)}, & \tau \neq \infty, \\
r^{-1} e^{i f_{\tau, j}(r)}, & \tau=\infty,
\end{array} \quad 0<r \leq \rho .\right.
$$

As we know from Sec. 2.10, the condition $\dot{\Gamma}_{j} \in C^{1, \nu}, 1 \leq j \leq m$, can be expressed (in terms of functions $\left.f_{\tau, j}\right)$ as follows: $f_{\tau, j} \in C_{(\nu)}^{1, \nu}([0, \rho], 0)$. This is equivalent to the condition $f_{\tau, j}(r)-\theta_{\tau, j} \in C_{\nu}^{1, \nu}([0, \rho], 0)$, where $\theta_{\tau, j}=\lim f_{\tau, j}(r)$ as $r \rightarrow 0$. By virtue of Theorem 2.10.2, this condition is equivalent to the belonging of the derivative $f_{\tau, j}^{\prime}$ to $C_{\nu-1}^{1, \nu}([0, \rho], 0)$. In the same way, the condition $\dot{\Gamma}_{j} \in C_{(1+0)}^{1, \nu}$ means that $f_{\tau, j} \in C_{(+0)}^{1, \nu}([0, \rho], 0)$, i.e., there exists a positive $\varepsilon$ such that $f_{\tau, j} \in C_{(\varepsilon)}^{1, \nu}([0, \rho], 0)$.

Consider the integrals (3.10.1) and (3.10.2) under the assumption that the generalized Cauchy kernel $Q(t ; \xi)$ (and, respectively, the Cauchy kernel $Q(t ; \xi, \eta)$ ) belongs to the class $C_{0}^{\nu(m)}(\Gamma, F)$ introduced in Sec. 3.1, while the density $\varphi$ belongs to $C_{\lambda}^{\mu}(\Gamma, F)$, where the weight order satisfies the condition $-1<\lambda<0$, i.e., $-1<\lambda_{\tau}<0, \tau \in F$. This condition guarantees the summability of the function $Q(t ; t-z) \varphi(t)$ on the curve $\Gamma$ regardless its boundedness or unboundedness.

It is clear that the function is infinitely differentiable outside $\Gamma$. If the curve $\Gamma$ is bounded, then the following estimates hold in a boundary of $\infty$ :

$$
|\phi(z)| \leq C|z|^{-1}, \quad\left|\phi^{\prime}(z)\right| \leq C|z|^{-2}
$$

Thus, $\phi$ belongs to the class $C_{-1}^{0,1}(G, \infty)$, where $G=B_{\rho}(\infty)$.

In a neighborhood of the compact set $K \subseteq \Gamma \backslash F$, its behavior is described by Theorems 3.6.1, 3.7.1, and 3.8.1 for the cases (3.10.1) and (3.10.2) respectively. In particular, the function $\phi$ admits one-sided boundary values $\phi^{ \pm}$from $C^{\mu}(K)$ satisfying the relation (3.8.3) with coefficient $\sigma$ defined by the relations (3.8.2) or (3.8.15), respectively. Let us verify that this coefficient belongs to the same class as the kernel $Q$. For definiteness, consider the case of the Cauchy kernel.

Lemma 3.10.1. If a Cauchy kernel $Q$ belongs to $C_{0}^{\nu(0)}(\Gamma, F)$, then the function $\sigma$ defined by the relation (3.8.15) belongs to the class $C_{0}^{\nu}(\Gamma, F)$.

Proof. It suffices to prove the lemma in the case where $\Gamma$ is a bounded curve. Otherwise, it suffices to use the linear-fractional substitution mapping $\Gamma$ to a bounded curve (see Sec. 2.5). Thus, let $\Gamma$ be a bounded curve. Then, by virtue of Lemma 2.8.1, the multiplication operator $\varphi \rightarrow \rho_{\nu} \varphi$ maps the space $C_{0}^{\nu}(\Gamma, F)$ onto the class of functions belonging to $C^{\nu}(\Gamma)$ and vanishing for $\tau \in F$.

If the kernel $Q(t ; \xi, \eta)$ is represented as $Q_{1}(t, \xi) \eta_{1}+Q_{2}(t, \xi) \eta_{2}$, then the obtained fact means that the functions $\widetilde{Q}_{j}(t, \xi)=\rho_{\nu}(t) Q_{j}(t, \xi)$ of variable $t$ possess the specified property uniformly with respect 
to $|\xi|=1$. Therefore, a similar property holds for the function

$$
\widetilde{\sigma}(t)=\frac{1}{2} \int_{\mathbb{T}} \widetilde{Q}(t ; \xi, d \xi) .
$$

Hence, $\sigma=\rho_{-\nu} \widetilde{\sigma}$ belongs to $C_{0}^{\nu}(\Gamma, F)$.

The main concern of this section is to investigate the behavior of $\phi$ near points $\tau$ from $F$. This behavior is described in terms of the space $C_{\lambda}^{\mu}(\widehat{D}, F)$ introduced (with respect to weight spaces) in Sec. 2.8. Here $D$ is the open set $\mathbb{C} \backslash \Gamma$.

\section{Theorem 3.10.1.}

(a) Let a Cauchy kernel $Q(t ; \xi, \eta)$ be defined on a piecewise-smooth curve $\Gamma$ and belong to $C_{0}^{\nu(1)}(\Gamma, F)$. Let $\varphi \in C_{\lambda}^{\mu}(\Gamma, F),-1<\lambda<0$. Then the function $\phi$ defined by the Cauchy-type integral (3.10.2) belongs to the class $C_{\lambda}^{\mu}(\widehat{D}, F), D=\mathbb{C} \backslash \Gamma$, and satisfies the norm estimate

$$
|\phi|_{C_{\lambda}^{\mu}} \leq C|Q|_{C_{0}^{\nu(1)}}|\varphi|_{C_{\lambda}^{\mu}}
$$

The boundary values $\phi^{ \pm}$of this function are linked by the relation (3.7.6) with the corresponding singular integral $\phi^{*}$ over $\Gamma$.

(b) Let the smooth contour $\Gamma_{0}$ and the open smooth arcs $\dot{\Gamma}_{j}$ from the expansion (3.10.3) belong to the classes $C^{1, \nu}$ and $C_{(1+0)}^{1, \nu}$ respectively. Let a generalized Cauchy kernel $Q$ belong to $C_{0}^{\nu(2)}(\Gamma, F)$. Then assertion (a) also holds for the integral (3.10.1).

Proof. (a) First, we assume that the function $\varphi$ is identically equal to zero in a neighborhood of $F$. Then, it is obvious that there exists a neighborhood of $F$ such that the function $\phi$ is infinitely differentiable in this neighborhood. On the other hand, if a domain $D_{0}$ from $D$ is bounded by a piecewise-smooth contour and lies outside a neighborhood of the set $F$, then, due to Theorem 3.8.1, the function $\phi$ belongs to $C^{\mu}\left(D_{0}\right)$ and satisfies the corresponding estimate of its norm. If $\infty \in \bar{D} \backslash \Gamma$, then this function also satisfies the estimate (3.10.6), which means that it belongs to the class $C_{-1}^{0,1}(G, \infty)$, where $G=B_{\rho}(\infty)$. Since $\lambda_{\infty}>-1$ (by condition), it follows that the said class is contained in $C_{\lambda_{\infty}}^{\mu}(G, \infty)$. Thus, the function $\phi$ belongs to $C_{\lambda}^{\mu}(\widehat{D}, F)$.

It follows from above considerations that it suffices to prove the theorem in the case where the support of $\varphi$ is contained in one of radial $\operatorname{arcs} \Gamma_{\tau, j}$ from (3.10.3) and the function $\varphi$ is identically equal to zero in a neighborhood of the second endpoint of this arc (i.e., the endpoint different from $\tau$ ). It is obvious that it suffices to consider only the cases where $\tau=0$ and $\tau=\infty$. It is convenient to join both these cases, selecting a radial smooth arc with endpoints $\tau=0$ and $\tau=\infty$ as $\Gamma$ and assuming that the weight order $\lambda$ of the function $\varphi$ from $C_{\lambda}^{\mu}(\Gamma ; 0, \infty)$ does not depend on $\tau$, i.e., is real. Let $\Gamma^{\prime}$ be another arc of the same type decomposing the domain $D=\mathbb{C} \backslash \Gamma$ into subdomains $D_{1}$ and $D_{2}$ bounded (on the Riemann sphere) by a piecewise-smooth contour $\Gamma \cup \Gamma^{\prime}$. Due to the definition of the class $C_{\lambda}^{\mu}(\widehat{D}, F)$, it suffices to prove (by means of decompositions into subdomains) the claim of the theorem for the domain $D_{1}$. We redenote it by $D$ again.

According to Sec. 2.5, any radial parametrization of the $\operatorname{arc} \Gamma$ is given by a function

$$
\gamma(r)=r e^{i f(r)}, \quad 0 \leq r<\infty,
$$

where $f(r)$ is a real function continuously differentiable on the interval $(0, \infty)$, admitting finite limits at its endpoints, and such that

$$
\lim _{r \rightarrow 0} r f^{\prime}(r)=\lim _{r \rightarrow \infty} r f^{\prime}(r)=0 .
$$


Due to the definition form Sec. 2.8, the weight multiplication operator $\varphi_{0}(t) \rightarrow|t|^{\lambda} \varphi_{0}(t)$ isomorphically maps $C_{0}^{\mu}$ to $C_{\lambda}^{\mu}$. Therefore, passing to redefinitions, it suffices to prove the theorem for the function

$$
\phi(z)=|z|^{-\lambda} \int_{\Gamma}|t|^{\lambda} Q(t ; t-z, d t) \varphi(t), \quad z \in D
$$

in the space $C_{0}^{\mu}(D ; 0, \infty)$.

The homothetic transformation $t \rightarrow 2^{-j} t, j=0, \pm 1, \ldots$, maps $\Gamma$ onto the arc $2^{-j} \Gamma$ of the same type. This is a radial arc and, similarly to $(3.10 .7)$, it is described by the equation

$$
\gamma_{j}(r)=r e^{i f_{j}(r)}, \quad 0 \leq r<\infty,
$$

where the function $f_{j}(r)=f\left(2^{j} r\right)$ satisfies the relation (3.10.8) as well as $f$. Under this homothetic transformation, the relation (3.10.9) passes to the relation

$$
\phi\left(2^{j} z\right)=|z|^{-\lambda} \int_{2^{-j} \Gamma}|t|^{\lambda} Q\left(2^{j} t ; t-z, d t\right) \varphi\left(2^{j} t\right), \quad z \in 2^{-j} D,
$$

Assign

$$
\begin{array}{ll}
\varphi_{j}(t)=\varphi\left(2^{j} t\right), \quad t \in \Gamma_{j}=\left(2^{-j} \Gamma\right) \cap\{1 / 4 \leq|t| \leq 4\}, \\
\widetilde{\varphi}_{j}(t)=\varphi\left(2^{j} t\right), \quad t \in\left(2^{-j} \Gamma\right) \backslash \Gamma_{j} .
\end{array}
$$

Then

$$
\phi\left(2^{j} z\right)=\phi_{j}(z)+\widetilde{\phi}_{j}(z), \quad z \in D_{j}=\left(2^{-j} D\right) \cap\{1 / 2<|z|<2\},
$$

where $\phi_{j}$ is determined by means of the integration over $\Gamma_{k}$.

From Definition (3.10.12), we see that the arc $\Gamma_{j}$ is defined by the parametrization $t=r e^{i f_{j} r}$, $1 / 4 \leq r \leq 4$. By the definition of radial arcs, the function $f(r)$ tends to $\theta_{0}$ as $r \rightarrow 0$ and tends to $\theta_{1}$ as $r \rightarrow \infty$. Combining $f_{j}^{\prime}(r)=2^{j} r f\left(2^{j} r\right)$ and (3.10.8), we conclude that the sequence of functions $f_{j}(r)$ converges (in the norm of the space $C^{1}[1 / 4,4]$ ) to the constant function $\theta_{0}, 1 / 4 \leq r \leq 4$, as $r \rightarrow-\infty$ and to the constant function $\theta_{1}, 1 / 4 \leq r \leq 4$, as $r \rightarrow+\infty$. In other words, the sequence of arcs $\Gamma_{j}$ converges (in the class $C^{1}$ ) as $j \rightarrow \pm \infty$ to the corresponding constant segments.

Let us prove that the functions

$$
\widetilde{\phi}_{j}(z)=|z|^{-\lambda} \int_{\left(2^{-j} \Gamma\right) \backslash \Gamma_{j}}|t|^{\lambda} Q\left(2^{j} t ; t-z, d t\right) \varphi\left(2^{j} t\right), \quad z \in D_{j},
$$

satisfy the estimate

$$
\left|\widetilde{\phi}_{j}\right|_{C^{\mu}\left(D_{j}\right)} \leq C|Q|_{C_{0}^{0(1)}}|\varphi|_{0}
$$

where $|\varphi|_{0}$ denotes the sup-norm of the function $\varphi$, and this estimate is uniform with respect to $j=0, \pm 1, \ldots$

Using parametrization (3.10.10) of the $\operatorname{arc} 2^{-j} \Gamma$, we obtain the inequality

$$
\left|\widetilde{\phi}_{j}(z)\right| \leq 2^{\lambda}|Q|_{C_{0}^{0(0)}}|\varphi|_{0}\left(\int_{0}^{1 / 4}+\int_{4}^{\infty}\right) r^{\lambda}\left|\gamma_{j}(r)-z\right|^{-1}\left|\gamma_{j}^{\prime}(r)\right| d r, \quad z \in D_{j} .
$$

Since $\left|\gamma_{j}^{\prime}\right|=1+r \mid f_{j}^{\prime}(r) \leq M$, where $M$ is the sup-norm of the function $1+r \mid f^{\prime}(r)$,

$$
\left|\gamma_{j}(r)-z\right| \geq \begin{cases}1 / 4, & 0<r \leq 1 / 4 \\ r-2, & r \geq 4\end{cases}
$$

and $-1<\lambda<0$, it follows that

$$
\left|\widetilde{\phi}_{j}\right|_{0} \leq C_{0}|Q|_{C_{0}^{0(0)}}|\varphi|_{0}
$$


In the same way, differentiating the function $\widetilde{\phi}_{j}(z)$ under the integral sign, we obtain the inequality

$$
\left|\widetilde{\phi}_{j}^{\prime}\right|_{0} \leq C_{1}|Q|_{C_{0}^{0(1)}}|\varphi|_{0}
$$

Combining it with the previous estimate, we see that

$$
\left|\widetilde{\phi}_{j}\right|_{C^{1}\left(\bar{D}_{j}\right)} \leq C|Q|_{C_{0}^{0(1)}}|\varphi|_{0}, \quad k=0, \pm 1, \ldots ;
$$

it is obvious that the sequence of the domains $D_{j}$ in (3.10.13) converges as $j \rightarrow \pm \infty$ and the limit domain is bounded by arcs of the circles $|z|=1 / 2$ and $|z|=2$ and the segments of the corresponding rays with vertex at the origin, confined between these arcs. It is clear that there exists a positive constant $M$ from $(1,+\infty)$ such that all these domains are $M$-uniformly connected. Hence, due to Theorem 2.2.2, the estimate

$$
|\phi|_{C^{\mu}\left(D_{j}\right)} \leq C|\phi|_{C^{1}\left(\bar{D}_{j}\right)}, \quad j=0, \pm 1, \ldots,
$$

holds and is uniform with respect to $j$. Combining this with (3.10.15), we obtain that (3.10.14).

Theorem 3.8.1 can be applied to the sequence of functions

$$
\phi_{j}(z)=|z|^{-\lambda} \int_{\Gamma_{j}}|t|^{\lambda} Q_{j}(t ; t-z, d t) \varphi_{j}(t), \quad z \in D_{j},
$$

with the Cauchy kernel $Q_{j}(t ; \xi, \eta)=Q\left(2^{j} t ; \xi, \eta\right)$. Obviously, the conditions of Lemma 3.6.2 are satisfied in this case. Therefore, the function $\phi_{j}$ belongs to $C^{\mu}\left(D_{j}\right)$ and satisfies the estimate

$$
\left|\phi_{j}\right|_{C^{\mu}\left(D_{j}\right)} \leq C\left|Q_{j}\right|_{C^{\nu(1)}}\left|\varphi_{j}\right|_{C^{\mu}\left(\Gamma_{j}\right)}
$$

uniform with respect to $j$.

By virtue of Theorem 2.7.1 applied to the spaces $C_{0}^{\mu}(\Gamma ; 0, \infty)$ and $C_{0}^{\nu(1)}(\Gamma ; 0, \infty)$, this yields the inequality

$$
\left|\phi_{j}\right|_{C^{\mu}\left(D_{j}\right)} \leq C|Q|_{C_{0}^{\nu(1)}}|\varphi|_{C_{0}^{\mu}}
$$

Taking into account (3.10.14), we see that the sequence $\phi\left(2^{j} z\right)=\phi_{j}(z)+\widetilde{\phi}_{j}(z), z \in D_{k}$, defined by (3.10.13) also satisfies the above estimate. Hence, it remains to use Theorem 2.7.1 for the space $C_{0}^{\mu}(D ; 0, \infty)$.

(b) This assertion is proved in the same way as assertion (a). As above, it suffices to consider the case of a radial arc $\Gamma$ with parametrization (3.10.7). Then, by the condition, there exists a positive $\varepsilon$ such that the functions $f(r)$ and $f(1 / r)$ belong to the class $C_{(\varepsilon)}^{1, \nu}([0,2], 0)$. In other words,

$$
f(r)=\left\{\begin{array}{l}
\theta_{0}+r^{\varepsilon} g_{0}(r), \\
\theta_{1}+r^{-\varepsilon} g_{1}(r),
\end{array} \quad r f^{\prime}(r)= \begin{cases}r^{\varepsilon} h_{0}(r), & 0<r \leq 2, \\
r^{-\varepsilon} h_{1}(r), & r \geq 2,\end{cases}\right.
$$

where $g_{0} \in C_{0}^{\nu}([0,2], 0), h_{0} \in C_{0}^{\nu}([0,2], 0), g_{1} \in C_{0}^{\nu}([2, \infty], \infty)$, and $h_{1} \in C_{0}^{\nu}([2, \infty], \infty)$. Assigning

$$
\begin{aligned}
& f_{n}^{+}(r)=f\left(2^{n} r\right), \quad f^{+}(r)=\theta_{1}, \quad 2 \leq r \leq 4, \\
& f_{n}^{-}(r)=f\left(2^{-n} r\right), \quad f^{-}(r)=\theta_{0}, \quad 1 / 2 \leq r \leq 2,
\end{aligned}
$$

where $n=1,2, \ldots$, and taking into account Theorem 2.7.1, we conclude that the sequence of functions $f_{n}^{+}\left(f_{n}^{-}\right)$converges to a constant function $f^{+}\left(f^{-}\right)$in the norm of the space $C^{1, \nu}[2,4]\left(C^{1, \nu}[1 / 2,2]\right)$ as $n \rightarrow \infty$. Therefore, the conditions of Lemma 3.6.2 are satisfied for the generalized Cauchy-type integral, and the remaining argument is not changed.

Theorem 3.10.1 can be complemented by a similar result for the space $C_{\lambda}^{1, \mu}(\widehat{D}, F)$. 
Theorem 3.10.2. Let the smooth contour $\Gamma_{0}$ and the open smooth arcs $\dot{\Gamma}_{j}$ from the expansion (3.10.3) belong to the classes $C^{1, \nu}$ and $C_{(1+0)}^{1, \nu}$ respectively. Let a generalized Cauchy kernel $Q(t ; \xi, \eta)$ belong to $C_{0}^{1, \nu(3)}(\Gamma, F)$. Let $\varphi \in C_{\lambda}^{1, \mu}(\Gamma, F),-1<\lambda<0$.

Then the function $\phi$ defined by the integral (3.10.2) belongs to the class $C_{\lambda}^{1, \mu}(\widehat{D}, F)$ and its norm satisfies the estimate

$$
|\phi|_{C_{\lambda}^{1, \mu}} \leq C|Q|_{C_{0}^{1, \nu(3)}}|\varphi|_{C_{\lambda}^{1, \mu}}
$$

Proof. Taking into account Theorem 3.8.2, one can consider only the case where $\Gamma$ is a radial arc with parametrization (3.10.7) (cf. the proof of Theorem 3.10.1(a)). Then, by the condition of the theorem, the function $f(r)$ is described by $(3.10 .17)$, where $g_{0}, h_{0} \in C_{0}^{\nu}([0,2], 0)$ and $g_{1}, h_{1} \in C_{0}^{\nu}([2, \infty], \infty)$. Assume that a domain $D$ is bounded by two radial arcs $\Gamma$ and $\Gamma^{\prime}$ with common endpoints $\tau=0$ and $\tau=\infty$ and a function $\varphi$ belongs to $C_{\lambda}^{1, \mu}(\Gamma, F), \lambda \in \mathbb{R},-1<\lambda<0$.

Further, we must deduce a weight differentiation relation since Lemma 3.8.2 is not applicable in the considered case. To do this, we represent the relation (3.8.21) in the form

$$
z \frac{\partial \phi}{\partial x_{j}}(z)=-\int_{\Gamma}(z-t) \frac{\partial Q}{\partial \xi_{j}}(t ; t-z, d t) \varphi(t)-\int_{\Gamma} \frac{\partial Q}{\partial \xi_{j}}(t ; t-z, d t) t \varphi(t), \quad z \in D .
$$

Take the last integral from the right-hand side of the last relation, change the integration domain for the arc $\Gamma_{\varepsilon} \subseteq \Gamma$ with endpoints $a=\gamma(\varepsilon)$ and $b=\gamma\left(\varepsilon^{-1}\right)$, and apply the relation (3.8.22) to it; this yields

$$
\begin{aligned}
\int_{\Gamma_{\varepsilon}} \frac{\partial Q}{\partial \xi_{j}}(t ; t-z, d t) t \varphi(t)=Q_{j}(b ; b-z) b \varphi(b)-Q_{j}(a ; a-z) a \varphi(a) & \\
& -\int_{\Gamma_{\varepsilon}} \frac{\partial Q_{j}}{\partial t}(t ; t-z) t \varphi(t) d_{1} t-\int_{\Gamma_{\varepsilon}} Q_{j}(t ; t-z)[t \varphi(t)]^{\prime} d_{1} t, \quad j=1,2 .
\end{aligned}
$$

If $z$ is fixed, then the function $Q_{j}(t, t-z) t \varphi(t)$ on $\Gamma$ is $O(1)|t|^{1+\lambda}$ as $t \rightarrow 0$ and $O(1)|t|^{\lambda}$ as $t \rightarrow \infty$. Therefore, the integrated terms of this relation tend to zero as $\varepsilon \rightarrow 0$. Passing to the limit as $\varepsilon \rightarrow 0$ and substituting the obtained relation to (3.10.19), we obtain that

$$
z \frac{\partial \phi}{\partial x_{j}}(z)=\int_{\Gamma}(t-z) \frac{\partial Q}{\partial \xi_{j}}(t ; t-z, d t) \varphi(t)+\int_{\Gamma} \frac{\partial Q_{j}}{\partial t}(t ; t-z) t \varphi(t) d_{1} t+\int_{\Gamma} Q_{j}(t ; t-z)[t \varphi(t)]^{\prime} d_{1} t .
$$

Since $t^{\prime}=e(t)$, it follows that this relation can be represented in the form

$$
z \frac{\partial \phi}{\partial x_{j}}(z)=\int_{\Gamma} Q_{(j)}(t ; t-z) \varphi(t) d_{1} t+\int_{\Gamma} Q_{j}(t ; t-z) t \varphi^{\prime}(t) d_{1} t
$$

where

$$
Q_{(j)}(t, \xi)=\xi\left[\frac{\partial Q_{1}}{\partial \xi_{j}}(t, \xi) e_{1}(t)+\frac{\partial Q_{2}}{\partial \xi_{j}}(t, \xi) e_{2}(t)\right]+t \frac{\partial Q_{j}}{\partial t}(t, \xi)+Q_{j}(t, \xi) e(t) .
$$

It is obvious that the function $Q_{(j)}(t, \xi)$ is even with respect to the variable $\xi$ and is homogeneous of power -1, i.e., is a generalized Cauchy kernel. Since $e(t) \in C_{(+0)}^{\nu}(\Gamma ; 0, \infty)$, it follows that $Q_{(j)}$ belongs to the class $C_{0}^{\nu(2)}(\Gamma ; 0, \infty)$ with respect to the variable $t$ and satisfies the corresponding estimate

$$
\left|Q_{(j)}\right|_{C^{\nu(2)}} \leq C|Q|_{C^{\nu(3)}} \text {. }
$$

Therefore, combining Theorem 3.10.1(b) applied to the integral on the right-hand side of (3.10.20) with Theorem 3.10.1(b), we obtain the validity of the estimate (3.10.18). 


\subsection{Weight $C^{\mu}$-Estimates of Singular Integrals}

In a domain $D \subseteq \mathbb{C}$ bounded by a piecewise-smooth contour $\Gamma$, consider the singular integral

$$
\psi(z)=\int_{D} Q(t ; t-z) \varphi(t) d_{2} t, \quad z \in D,
$$

such that $Q(t, \xi)$ is homogeneous of power -2 with respect to the variable $\xi$ and satisfies the condition (3.4.2) inside the domain $D$. Let $F$ be a finite set of points of $\bar{D}$ containing all boundary points of the curve. As above, the infinite point $\infty$ is assumed to belong to $F$ if the domain $D$ is unbounded (if the curve $\Gamma$ is bounded, then the domain $D$ is a neighborhood of this point). Since $\Gamma$ is a contour, it follows that the number $n_{\tau}=2$ in (3.10.4) is equal to 2 for any $\tau$ from $F \cap \Gamma$. Hence, radial arcs $\Gamma_{\tau, 1}$ and $\Gamma_{\tau, 2}$ decompose $B_{\rho}(\tau)$ into two domains $S_{\tau, 1}$ and $S_{\tau, 2}$ called curvilinear sectors with vertex $\tau$ (including the case where $\tau=\infty$ ). The openings of these sectors are called the internal angles of the domain $D$. Note that the internal angle of this domain at the curve cusp is equal to 0 or to $2 \pi$.

If the disk $B_{\rho}(\tau)$ is considered for a finite point $\tau$ of $D$, then $\rho$ is selected to be sufficiently small to ensure that the disk does not intersect $\Gamma$. The same refers to the infinity point $\tau=\infty$ for the case where $D$ is its neighborhood. For any such domain there exist two rectilinear cuts $\Gamma_{\tau, 1}$ and $\Gamma_{\tau, 2}$ decomposing $B_{\rho}(\tau)$ into two sectors.

It is assumed that the kernel $Q(t ; \xi)$ belongs to the class $C_{0}^{\nu(2)}(D, F)$ introduced in Sec. 3.1, while the density $\varphi$ belongs to $C_{\lambda}^{\mu}(D, F)$, where the weight order satisfies the condition $-2<\lambda<0$, i.e., $-2<\lambda_{\tau}<0, \tau \in F$. This condition guarantees the summability of the function $Q(t ; t-z) \varphi(t)$ in the domain $D$ outside any neighborhood of $F$ regardless of the boundedness or unboundedness of $D$.

Theorem 3.11.1. Let a domain $D$ be bounded by a piecewise-smooth contour $\Gamma$ and have no cusps. Let the smooth contour $\Gamma_{0}$ and the open smooth arcs $\dot{\Gamma}_{j}$ of the expansion (3.10.3) belong to the classes $C^{1, \nu}$ and $C_{(1+0)}^{1, \nu}$ respectively. Let a kernel $Q(t ; \xi)$ belong to $C_{0}^{\nu(2)}(D, F)$, be homogeneous of power -2 with respect to the variable $\xi$, satisfy the condition (3.4.2) inside the domain, and satisfy the condition (3.5.1) at its boundary. Let $\varphi \in C_{\lambda}^{\mu}(D, F),-2<\lambda<0$.

Then the function $\phi$ defined by the integral (3.11.1) belongs to the class $C_{\lambda}^{\mu}(D, F)$ and its norm satisfies the estimate

$$
|\psi|_{C_{\lambda}^{\mu}} \leq C|Q|_{C_{0}^{\nu(2)}}|\varphi|_{C_{\lambda}^{\mu}}
$$

Proof. We use the same scheme of the proof as for Theorem 3.10.1. The only difference is that we apply Theorem 3.5.1 and Lemma 3.5.1 for two-dimensional singular integrals.

First, we assume that the function $\varphi$ is identically equal to zero in a neighborhood of $F$. Then it is obvious that there exists a neighborhood of $F$ such that the function $\psi$ is infinitely differentiable in this neighborhood. If $D$ is a neighborhood of $\infty$, then it admits the following estimates (similar to $(3.10 .6))$ in $D$ :

$$
|\psi(z)| \leq C|z|^{-2}, \quad\left|\psi^{\prime}(z)\right| \leq C|z|^{-3} .
$$

They hold since the kernel $Q(t, \xi)$ belongs to $\mathcal{H}_{-2}$ with respect to the variable $\xi$. These estimates show that $\psi$ belongs to the class $C_{-2}^{0,1}(G, \infty)$ in the domain $G=B_{\rho}(\infty)$; by virtue of the inequality $\lambda_{\infty}>-2$, this class is contained in $C_{\lambda_{\infty}}^{\mu}(G, \infty)$.

On the other hand, if the domain $D_{0} \subseteq D$ is bounded by a piecewise-smooth contour and lies outside a neighborhood of the set $F$, then, by virtue of Theorem 3.4.1, the function $\psi$ belongs to $C^{\mu}\left(D_{0}\right)$ and its norm satisfies the corresponding estimate. Hence, $\psi$ belongs to the class $C^{\mu}(\widehat{D}, F)$ and its norm satisfies the corresponding estimate.

Thus, it suffices to prove the theorem under the assumption that the support of the function $\varphi$ is contained in one of curvilinear sectors $S_{\tau, j}$ and the function $\varphi$ is identically equal to zero in a neighborhood of the boundary arc of this sector. As in Sec. 3.10, it suffices to consider the two cases of the sectors with vertexes $\tau=0$ and $\tau=\infty$. It is convenient to join both these cases, assigning $D$ 
to be the domain bounded by two radial smooth $\operatorname{arcs} \Gamma^{0}$ and $\Gamma^{1}$ with endpoints $\tau=0$ and $\tau=\infty$ and assuming that the weight order $\lambda$ of the function $\varphi$ from $C_{\lambda}^{\mu}(\Gamma ; 0, \infty)$ does not depend on $\tau$, i.e., real. For the $\operatorname{arcs} \Gamma^{k}$, we have parametrizations (3.10.7) with functions $f=f^{k}, k=0,1$, satisfying the conditions (3.10.8). As in the proof of Theorem 3.10.1(b), an expansion similar to (3.10.17) is valid for these functions, where $g_{0}=g_{0}^{k}$ and $h_{0}=h_{0}^{k}$ belong to $C_{0}^{\nu}([0,2], 0)$, while $g_{1}=g_{1}^{k}$ and $h_{1}=h_{1}^{k}$ belong to $C_{0}^{\nu}([1 / 2, \infty], \infty)$.

Redenoting, we see that it suffices to prove the estimate (3.11.2) for the function

$$
\psi(z)=|z|^{-\lambda} \int_{D}|t|^{\lambda} Q(t, t-z) \varphi(t) d_{2} t, \quad z \in D,
$$

with respect to the space $C_{0}^{\mu}$. Represent this relation in the form

$$
\psi\left(2^{j} z\right)=|z|^{-\lambda} \int_{2^{-j} D}|t|^{\lambda} Q\left(2^{j} t, t-z\right) \varphi\left(2^{j} t\right) d_{2} t, \quad z \in 2^{-j} D,
$$

where $j=0, \pm 1, \ldots$ Assuming that

$$
\begin{array}{ll}
\varphi_{j}(t)=\varphi\left(2^{j} t\right), \quad t \in D_{j}=\left(2^{-j} D\right) \cap\{1 / 4 \leq|t| \leq 4\}, \\
\widetilde{\varphi}_{j}(t)=\varphi\left(2^{j} t\right), \quad t \in\left(2^{-j} D\right) \backslash D_{j},
\end{array}
$$

consider the sequence of functions

$$
\psi\left(2^{j} z\right)=\psi_{j}(z)+\widetilde{\psi}_{j}(z), \quad z \in D_{j}^{0}=\left(2^{-j} D\right) \cap\{1 / 2<|z|<2\},
$$

where $\psi_{j}$ depending on $D_{j}$ is defined by the integral (3.11.3).

Note that the domain $D_{j}$ is surrounded by the two $\operatorname{arcs}\left(2^{-j} \Gamma^{k}\right) \cap\{1 / 4 \leq|t| \leq 4\}, k=0,1$, and the corresponding two arcs of the circles $\{|t|=1 / 4\}$ and $\{|t|=4\}$. Similarly to the proof of Theorem 3.10.1(b), we verify that the arc sequence $\Gamma_{j}^{k}$ converges to the corresponding line segments $I_{ \pm}^{k}$ in the class $C^{1, \nu}$ as $j \rightarrow \pm \infty$. Since the internal angles of the domain $D$ are different from 0 and $2 \pi$ (by condition), it follows that the segments $I_{ \pm}^{0}$ and $I_{ \pm}^{1}$ are different; joining them with arcs of the specified circles, we obtain the piecewise-smooth contour of the limit domain $D_{ \pm}$.

The functions

$$
\widetilde{\psi}_{j}(z)=|z|^{-\lambda}\left(\int_{\left(2^{-j} D\right) \cap\{|t|<1 / 4\}}+\int_{\left(2^{-j} D\right) \cap\{|t|>4\}}\right)|t|^{\lambda} Q\left(2^{j} t, t-z\right) \varphi\left(2^{j} t\right) d_{2} t, \quad z \in D_{j}^{0},
$$

satisfy the obvious inequality

$$
\left|\widetilde{\psi}_{j}(z)\right| \leq|Q|_{C_{0}^{0(1)}}|\varphi|_{0}|z|^{-\lambda}\left(\int_{|t|<1 / 4}+\int_{|t|>4}\right)|t|^{\lambda}|t-z|^{-2} d_{2} t, \quad z \in D_{j}^{0} .
$$

A similar inequality also holds for partial derivatives of the function $\widetilde{\psi}_{j}$. Arguing as in the proof of Theorem 3.10.1, we arrive at the following estimate similar to (3.10.14) and uniform with respect to $j$ :

$$
\left|\widetilde{\psi}_{j}\right|_{C^{\mu}\left(D_{j}\right)} \leq C|Q|_{C_{0}^{0(1)}}|\varphi|_{0}, \quad j=0, \pm 1, \ldots
$$

Theorem 3.5.1 and Lemma 3.5.1 are applicable to the function sequence

$$
\psi_{j}(z)=|z|^{-\lambda} \int_{D_{j}}|t|^{\lambda} Q_{j}(t ; t-z) \varphi\left(2^{j} t\right) d t, \quad z \in D_{j}^{0},
$$


with kernel $Q_{j}(t ; \xi)=Q\left(2^{j} t ; \xi\right)$, which leads to the following estimate uniform with respect to $j$ :

$$
\left|\phi_{j}\right|_{C^{\mu}\left(D_{j}^{0}\right)} \leq C\left|Q_{j}\right|_{C^{\nu(2)}}\left|\varphi_{j}\right|_{C^{\mu}\left(D_{j}\right)} .
$$

Combining this estimate with (3.11.5), we obtain a similar estimate for the function $\psi\left(2^{j} z\right)=$ $\psi_{j}(z)+\widetilde{\psi}_{j}(z), z \in D_{j}^{0}$, from (3.11.4). As in Sec. 3.10, by virtue of Theorem 2.7.1, this implies the estimate (3.11.2) of the theorem.

As in Sec. 3.5, apply Theorem 3.11.1 to the following integral with a weak singularity:

$$
\psi^{0}(z)=\int_{D} Q^{0}(t ; t-z) \varphi(t) d_{2} t, \quad z \in D .
$$

It is assumed that its kernel $Q^{0}(t, \xi)$ is a homogeneous function of power -1 and the density $\varphi$ belongs to $C_{\lambda}^{\mu}(D, F)$, where the weight order satisfies the previous condition $-2<\lambda<0$ and the additional condition $\lambda_{\tau}<-1$ provided that $\tau=\infty$. This additional condition guarantees the summability of the function $Q^{0}(t, t-z) \varphi(t)$ in the domain $D$.

Relation (3.5.27) also remains valid in the case considered. Indeed, for any fixed point $a$ from $D \backslash F$, the function $\varphi$ can be represented by the sum of two terms such that one of them is identically equal to zero in a sufficiently small neighborhood of this point. The integral defined by this term is differentiable under the integral sign, while the relation (3.5.27) is applicable to the second term.

Note that the coefficient

$$
\sigma_{i}(x)=\int_{\Omega} \xi_{i} Q^{0}(x, \xi) d \xi
$$

of this relation belongs to the class $C_{0}^{\nu}(D, F)$ under the assumption that $Q^{0} \in C_{0}^{\nu(1)}(D, F)$; this is proved in the same way as Lemma 3.10.1. Therefore, as in Lemma 3.5, the relation (3.5.27) combined with Theorem 3.11.1 and Theorem 2.10.2 leads to the following result.

Theorem 3.11.2. Let a domain $D$ and its boundary contour $\Gamma=\partial D$ satisfy the conditions of Theorem 3.11 .1 and a kernel $Q^{0}(t ; \xi)$ belong to $C_{0}^{\nu(3)}(D, F)$, be homogeneous of power -1 with respect to the variable $\xi$, and satisfy the condition (3.5.1) at the boundary points $t$ from $\Gamma \backslash F$. Let $\varphi \in C_{\lambda}^{\mu}(D, F)$, where the weight order satisfies the conditions $-2<\lambda_{\tau}<0$ and $\lambda_{\tau} \neq-1$ if $\tau \neq \infty$ and satisfies the inequality $-2<\lambda_{\tau}<-1$ if $\tau=\infty$.

Then the function $\psi_{0}$ defined by the integral (3.11.7) belongs to the class $C_{(\lambda+1)}^{1, \mu}(D, F)$ and its norm satisfies the estimate

$$
|\psi|_{C_{(\lambda+1)}^{1, \mu}} \leq C\left|Q_{0}\right|_{C_{0}^{\nu(2)}}|\varphi|_{C_{\lambda}^{\mu}}
$$

Remark to Theorem 3.5.3 also remains valid in the case considered, i.e., if the kernel $Q^{0}(y, \xi)$ is odd with respect to the variable $\xi$, then the condition (3.5.1) is satisfied for its partial derivatives $\partial Q / \partial \xi_{i}$.

The singular Cauchy integral

$$
\psi\left(t_{0}\right)=\int_{\Gamma} Q\left(t, t-t_{0}, d t\right) \varphi(t), \quad t_{0} \in \Gamma,
$$

on a piecewise-smooth curve $\Gamma$ can be considered in the same way. However, it is easier to use Theorems 3.10.1-3.10.2 and the boundary-value relation

$$
\phi^{ \pm}\left(t_{0}\right)= \pm \sigma\left(t_{0}\right) \varphi\left(t_{0}\right)+\psi\left(t_{0}\right), \quad \sigma\left(t_{0}\right)=\frac{1}{2} \int_{\mathbb{T}} Q\left(t_{0} ; \xi, d \xi\right),
$$

for the corresponding Cauchy-type integral given by (3.10.2). Recall that $\mathbb{T}$ denotes the unit circle oriented counterclockwise. Obviously, if the Cauchy kernel belongs to $C_{0}^{\nu(1)}(\Gamma, F)$, then the coefficient 
$\sigma$ from this relation belongs to the class $C_{0}^{\nu}(\Gamma, F)$. In the same way, the function $\sigma$ belongs to $C_{0}^{1, \nu}(\Gamma, F)$ under the assumptions of Theorem 3.10.2.

Therefore, Theorems 3.10.1-3.10.2 lead to the following result.

Theorem 3.11.3. Let a Cauchy kernel $Q(t ; \xi, \eta)$ be defined on a piecewise-smooth curve $\Gamma$ and belong to $C_{0}^{\nu(1)}(\Gamma, F)$. Let $\varphi$ belong to $C_{\lambda}^{\mu}(\Gamma, F),-1<\lambda<0$. Then the function $\psi$ defined by the integral (3.11.8) belongs to the class $C_{\lambda}^{\mu}(\Gamma, F)$ and its norm satisfies the estimate

$$
|\psi|_{C_{\lambda}^{\mu}} \leq C|Q|_{C_{0}^{\nu(1)}}|\varphi|_{C_{\lambda}^{\mu}}
$$

If the above holds, the smooth contour $\Gamma_{0}$ and the open smooth arcs $\dot{\Gamma}_{j}$ of the expansion (3.10.3) belong to the classes $C^{1, \nu}$ and $C_{(1+0)}^{1, \nu}$ respectively, and the generalized Cauchy kernel $Q(t ; \xi, \eta)$ belongs to $C_{0}^{1, \nu(3)}(\Gamma, F)$, then the same assertion holds for the space $C_{\lambda}^{1, \mu}(\Gamma, F)$.

For the classical Cauchy kernel $Q(\xi, \eta)=\eta / \xi$, this classical result is presented in [45] (the Hölder exponent is not refined there). In $[8,9]$, a refined case is provided.

\section{CHAPTER 4}

\section{LEBESGUE SPACES}

\subsection{Spaces $L^{p}$ and $L_{\lambda}^{p}$}

Let $G$ be a measurable (with respect to the classical Lebesgue measure) subset of $\mathbb{R}^{k}$. Let $L^{p}(G$ ), $1 \leq p<\infty$, be the class of all functions $\varphi(y), y \in G$, such that their $p$ th power is summable. For $p=1$, it coincides with the class $L=L^{1}$ of all functions summable on $G$. To any function $\varphi$ from $L^{p}$, assign the nonnegative number

$$
|\varphi|_{L^{p}}=\left(\int_{G}|\varphi(x)|^{p} d x\right)^{1 / p} .
$$

It is obvious that $|\varphi|=0$ if and only if $\varphi(x)=0$ a.e. in $G$. In the sequel, we identify functions that differ on a zero-measure set.

Let $L^{\infty}(G)$ be the set of measurable functions bounded outside a zero-measure set. In the sequel, we use only its subspace $C^{0}(G)$ of continuous and bounded functions, endowed with the sup-norm (see Sec. 2.2).

To any exponent $p$ exceeding 1 , assign any exponent $q$ exceeding 1 as follows: $1 / q=1-1 / p$; we say that $q$ is conjugate to $p$. The product $\varphi \psi$ of a function $\varphi$ from $L^{p}$ and a function $\psi$ from $L^{q}$ is summable on $G$, and the following Hölder inequality holds:

$$
|\varphi \psi|_{L^{1}} \leq|\varphi|_{L^{p}}|\psi|_{L^{q}}
$$

Using this inequality, one can easily verify that the relation (4.1.1) defines a norm in the space $L^{p}$ (recall that we identify functions that differ on a zero-measure set). Actually, the proof of the fact that $|\varphi|$ is a norm requires only the triangle inequality $\left|\varphi_{1}+\varphi_{2}\right| \leq\left|\varphi_{1}\right|+\left|\psi_{1}\right|$; in this case, this inequality is called the Minkowski inequality.

Theorem (Riesz-Fisher). The space $L^{p}$ is complete with respect to norm (4.1.1).

The space $L^{p}(G)$ possesses the following well-known properties presented at standard courses of analysis. If the Lebesgue norm mes $G$ of a set $G$ is finite, then the function $\varphi(x)=1$ belongs to $L^{p}(G)$ for any $p$. Taking into account (4.1.2), this implies that the Banach space $L^{p_{2}}(G)$ is embedded in $L^{p_{1}}(G)$ provided that $1 \leq p_{1} \leq p_{2}$. In other words, if mes $G<\infty$, then the family of Banach 
spaces $L^{p}(G)$ monotonously decreases (in the embedding sense) with respect to the parameter $p$ from $(1,+\infty)$.

From the Hölder inequality, it immediately follows that the bilinear form

$$
(\varphi, \psi)=\int_{G} \varphi(x) \psi(x) d x
$$

is bounded on the direct product $L^{p} \times L^{q}$ for any $p$ exceeding 1 and the relation

$$
|\varphi|_{L^{p}}=\sup (\varphi, \psi),
$$

where sup is taken over all functions $\psi$ from $L^{q}$ such that $|\psi|_{L^{q}} \leq 1$, holds. The inverse assertion is also valid: for any linear functional $f^{*}$ from $\left(L^{p}\right)^{*}$ there exists $\psi$ from $L^{q}$ such that $f^{*}(\varphi)=(\varphi, \psi)$ for any $\varphi$. In other words, the Banach space $L^{p}, p>1$, is reflexive (see Sec. 1.2) and its adjoint space can be identified with $L^{q}$.

We say that a sequence $\left\{\varphi_{n}\right\}$ from $L^{p}$ weakly converges if there exists a function $\varphi$ from $L^{p}$ such that $\left\langle\varphi_{n}, \psi\right\rangle \rightarrow\langle\varphi, \psi\rangle$ as $n \rightarrow \infty$ for any $\psi$ from $L^{q}$.

Theorem (on weak convergence, Banach). Sequences weakly converging in $L^{p}, p>1$, are bounded. Conversely, any sequence bounded in $L^{p}$ contains a weakly converging subsequence.

This theorem combines the following two known theorems of functional analysis. By virtue of the reflexivity of $L^{p}$, it can be treated as the adjoint space $X^{*}$ for $X=L^{q}$. Then any weakly converging sequence $\varphi_{n}$ is a sequence of functionals $\varphi_{n}$ from $X^{*}$ such that $\varphi_{n}(x)$ converges for any $x$ from $X$. In particular, any number sequence $\varphi_{n}(x)$ is bounded for any $x$ and, due to the known Banach-Steinhaus theorem, the sequence $\varphi_{n}$ is bounded in $X^{*}$. The second assertion of the theorem follows from the general Banach-Alaoglu theorem on the weak *-compactness of the unit ball in $X^{*}$.

The space $L^{p}(D)$ is homogeneous with respect to the group of translations $x \rightarrow x+a$. On the other hand, the measure $d t /|t|^{k}$ is invariant with respect to extensions $x \rightarrow r x, r>0$, in $\mathbb{R}^{k}$. Therefore, similarly to Sec. 2.7, it is convenient to introduce the homogeneous space $L_{0}^{p}(G)$ as the $L^{p}$-space with respect to this measure and define the following norm in it:

$$
|\varphi|=\left(\int_{G}|\varphi(x)|^{p} \frac{d x}{|x|^{k}}\right)^{1 / p}
$$

Similarly to Sec. 2.7, this definition has meaning only in the case where $\tau=0$ or $\infty$ is a limit point for $G$. If the set $G$ is bounded and lies outside a neighborhood of the origin, then the space $L_{0}^{p}(G)$ coincides with $L^{p}(G)$.

Note that the Hölder inequality holds for $L^{p}$-spaces with respect to any measure; in particular, the inequality

$$
|\varphi \psi|_{L^{1}} \leq|\varphi|_{L_{0}^{p}}|\psi|_{L_{0}^{q}}
$$

holds.

The following assertion is an analog of Theorems 2.7.1-2.7.2 for the space $L_{0}^{p}(G)$.

\section{Theorem 4.1.1.}

(a) Let $0<\delta<1$ and $G_{j}=\left\{\delta<|y|<\delta^{-1}, \delta^{j} y \in G\right\}, j=0, \pm 1, \ldots$ Then the space $L_{0}^{p}(G, F)$ can be defined by an equivalent norm

$$
|\varphi|=\left(\sum_{j}\left[\varphi_{j}\right]_{L^{p}\left(G_{j}\right)}^{p}\right)^{1 / p}, \quad \varphi_{j}(y)=\varphi\left(\delta^{j} y\right) .
$$


(b) Let a set $\widetilde{G} \subseteq \mathbb{R} \times \Omega$ be the image of $G$ under the map $x \rightarrow(\ln |x|, x /|x|)$ inverse to the map $\omega(s, u)=e^{s} u, u \in \mathbb{R}^{k}$. Then the operator $\psi \rightarrow \psi \circ \omega$ isomorphically maps the Banach space $L^{p}(\widetilde{G})$ to $L_{0}^{0}(G)$. In particular, the operator $\varphi(x) \rightarrow \varphi\left(x^{*}\right)$ defined by the inversion $x^{*}=x /|x|^{2}$ is an invertible operator from $L_{0}^{p}(D)$ to $L_{0}^{p}\left(D^{*}\right)$.

Proof. By virtue of the denumerable additivity of the integral, the relation (4.1.5) can be represented in the form

$$
|\varphi|_{L_{0}^{p}}^{p}=\sum_{j} \int_{\delta \leq|y| \leq 1}\left|\varphi\left(\delta^{j} y\right)\right|^{p} \frac{d y}{|y|^{k}} .
$$

For brevity, assign $x_{j}=\left|\varphi_{j}\right|_{L^{p}\left(G_{j}\right)}$. This implies the inequality

$$
2^{-1} \sum_{j}\left(x_{j-1}^{p}+x_{j}^{p}\right) \leq|\varphi|_{L_{0}^{p}}^{p} \leq 2 \delta^{-1} \sum_{j}\left(x_{j-1}^{p}+x_{j}^{p}\right),
$$

which implies the equivalence of norms (4.1.5) and (4.1.7).

As in the case of Theorem 2.7.2, the latter assertion is proved by means of the former one. However, it can also be proved directly since under the substitution $\omega$, the measure $d x /|x|^{k}$ passes to the direct product $d s d u$ of measures.

Similarly to Sec. 2.8, based on the spaces $L^{p}$ and $L_{0}^{p}$, introduce weight spaces $L_{\lambda}^{p}(G, F)$ corresponding to the weight order $\lambda=\left(\lambda_{\tau}, \tau \in F\right)$. Thus, assigning $B_{\rho}(\tau)=\{|y-\tau| \leq \rho\}, \tau \neq \infty$, and $B_{\rho}(\tau)=$ $\{|y| \geq \rho\}, \tau=\infty$, where a positive $\rho$ is sufficiently small, one can define this space by the finiteness condition for the integrals

$$
\int_{G \cap B_{\rho}(\tau)}\left[|y-\tau|^{-\lambda_{\tau}}|\varphi(y)|\right]^{p} \frac{d y}{|y-\tau|}, \tau \in F, \quad \int_{\widetilde{G}}|\varphi(y)|^{p} d y,
$$

where $\widetilde{G}=G \backslash \bigcup_{\tau} B_{\rho / 2}(\tau)$ and $|x-\tau|$ is replaced by $|x|$ for the case where $\tau=\infty$.

To define these spaces directly, one can use the weight function $\rho_{\lambda}(x)$. Recall that it is given by the relation

$$
\rho_{\lambda}(x, F)=\prod_{\tau \in F} \rho_{\lambda_{\tau}}(x, \tau), \quad \rho_{\delta}(x, \tau)= \begin{cases}|x-\tau|^{\delta}(1+|x|)^{-\delta}, & \tau \neq \infty, \\ (1+|x|)^{\delta}, & \tau=\infty .\end{cases}
$$

In this notation, the space $L_{0}^{p}(G, F)$ can be defined as the $L^{p}$-space with respect to the measure $\rho_{-k}(x) d x$. Respectively, the space $L_{\lambda}^{p}(F, F)$ can be given by the equivalent norm

$$
|\varphi|=\left(\int_{G}\left|\rho_{-\lambda}(x) \varphi(x)\right|^{p} \rho_{-k}(x) d x\right)^{1 / p}=\left|\rho_{-\lambda-1 / k} \varphi\right|_{L^{p}}
$$

In particular, we have the relation

$$
L_{-k / p}^{p}(D, F)=L^{p}(D)
$$

and the corresponding norms are equivalent. This is the analog of Lemma 2.7.1 for $L^{p}$-spaces.

In general, the most frequent notation for weight spaces is as follows:

$$
L^{p}\left(\Gamma, \rho_{\delta}\right)=\left\{\varphi, \rho_{\delta} \varphi \in L^{p}(\Gamma)\right\} .
$$

By virtue of (4.1.11), this space coincides with $L_{-1 / p-\delta}^{p}(\Gamma, F)$. Due to various reasons, we prefer notation $L_{\lambda}^{p}$ (see the remark at the end of Sec. 4.6).

As in Sec. 2.8, the choice of the weight function (4.1.9) is convenient since it is also acceptable for the case where $F$ contains points $\tau$ lying outside $\bar{G}$. In this case, $L_{\lambda}^{p}(G, F)$ coincides with $L_{\lambda}^{p}\left(G, F_{0}\right)$, where $F_{0}=F \cap \bar{G}$. If the set $G$ is bounded, then, e.g., $\rho_{\lambda}(x)=\prod_{\tau}|x-\tau|^{\lambda_{\tau}}$ can be taken as the weight function. 
Consider relations between weight spaces and the parameters $p$ and $\lambda_{\tau}$.

Lemma 4.1.1. The family $L_{\lambda}^{p}(G, F)$ monotonously decreases (in the sense of embeddings of Banach spaces) with respect to each parameter $\lambda_{\tau}$ for $\tau \neq \infty$ and decreases with respect to $\lambda_{\infty}$. Assume that $p>q$. Then $L_{\lambda}^{p} \subseteq L_{\lambda^{\prime}}^{q}$ provided that $\lambda_{\tau}>\lambda_{\tau}^{\prime}$ if $\tau$ is finite and $\lambda_{\tau}<\lambda_{\tau}^{\prime}$ if $\tau=\infty$. If $-k<\lambda<0$, then the space $L_{\lambda}^{p}(G, F)$ is embedded in $L^{1}(G)$.

Proof. According to the definition of weighted spaces $L_{\lambda}^{p}(G, F)$, it suffices to consider the two following cases of $L_{\lambda}^{p}\left(B_{j}, \tau_{j}\right), j=1,2$ : the case where $B_{1}=\{|x| \leq 1\}$ and $\tau_{1}=0$ and the case where $B_{2}=$ $\{|x| \geq 1\}$ and $\tau_{2}=\infty$. For definiteness, consider the former one. In this case, the first assertion of the theorem is reduced to the obvious embedding $L_{\lambda}^{p}(B, 0) \subseteq L_{0}^{p}(B, 0), \lambda \geq 0$, while the remaining two assertions are reduced to the embeddings

$$
L_{\lambda}^{p}(B, 0) \subseteq L_{0}^{q}(B, 0), \lambda>0, \quad L_{\lambda}^{p}(B, 0) \subseteq L^{1}(B),-k<\lambda<0,
$$

easily proved by means of the inequality (4.1.6).

Finally, we consider the one-dimensional case $(k=1)$ of functions defined on the real line $\mathbb{R}$. Consider the classical singular Cauchy integral

$$
\psi\left(t_{0}\right)=\frac{1}{\pi i} \int_{\mathbb{R}} \frac{\varphi(t) d t}{t-t_{0}}
$$

treated (as above) as the limit of integrals over the domain $\left|t-t_{0}\right| \geq \varepsilon$ as $\varepsilon \rightarrow 0$. For functions $\varphi$ from $L^{p}(\mathbb{R})$ and for $p>1$, the last integrals have meaning by virtue of the Hölder inequality and the following result takes place.

The operator defined by the specified singular Cauchy integral is called the Hilbert transformation.

Theorem (Riesz). If $\varphi \in L^{p}(\mathbb{R}), p>1$, then the singular Cauchy integral exists for a.e. $t_{0}$ and the estimate

$$
|\psi|_{L^{p}} \leq C|\varphi|_{L^{p}}
$$

holds.

The classical Riesz result can be complemented by $L^{p}$-estimates of the so-called Hardy-Littlewood maximal functions (see, e.g., [74]).

Theorem (Hardy-Littlewood). Let a function $\varphi$ belong to $L^{p}(\mathbb{R}), p>1$, and have a compact support. Then the upper bounds

$$
\left(M_{0} \varphi\right)\left(t_{0}\right)=\sup _{\varepsilon>0} \frac{1}{2 \varepsilon} \int_{\left|t-t_{0}\right| \leq \varepsilon}|\varphi(t)| d t, \quad\left(M_{1} \varphi\right)\left(t_{0}\right)=\sup _{\varepsilon>0}\left|\int_{\left|t_{0}-t\right| \geq \varepsilon} \frac{\varphi(t) d t}{t-t_{0}}\right|
$$

are finite for a.e. $t_{0}$ and they define $L^{p}$-functions such their norms satisfy the estimate

$$
\left|M_{0} \varphi\right|_{L^{p}}+\left|M_{1} \varphi\right|_{L^{p}} \leq C|\varphi|_{L^{p}}
$$

where $C$ is a positive constant independent of $\varphi$.

The following auxiliary assertion is also valid.

Lemma 4.1.2. Let $f$ from $L^{1}(\mathbb{R})$ be a nonnegative function admitting the estimate

$$
\int_{|s| \leq r} f(s) d s \leq C r
$$


for any positive $r$, where $C$ is a positive constant independent of $r$. Then

$$
r \int_{|s| \geq r} \frac{f(s)}{s^{2}} d s \leq 3 C .
$$

If the above is satisfied and

$$
\lim _{r \rightarrow 0} \frac{1}{r} \int_{|s| \leq r} f(s) d s=0
$$

then

$$
\lim _{r \rightarrow 0} r \int_{|s| \geq r} \frac{f(s)}{s^{2}} d s=0
$$

Proof. Assign

$$
g(r)=\int_{|s| \leq r} f(s) d s=\int_{0}^{r}[f(s)+f(-s)] d s .
$$

Integrating by parts, we see that

$$
\int_{|s| \geq r} \frac{f(s)}{s^{2}} d s=\int_{r}^{\infty} \frac{d g(s)}{s^{2}}=-\frac{g(r)}{r^{2}}+2 \int_{r}^{\infty} \frac{g(s)}{s^{3}} d s .
$$

Therefore, by virtue of (4.1.14), we have the inequality

$$
r \int_{|s| \geq r} \frac{f(s)}{s^{2}} d s \leq C+2 C r \int_{r}^{\infty} \frac{d s}{s^{2}}=3 C .
$$

Further, let the condition (4.1.15) be satisfied. Assigning $g_{0}(s)=g(s) / s$, represent (4.1.16) as follows:

$$
r \int_{|s| \geq r} \frac{f(s)}{s^{2}} d s=-g_{0}(r)+2 \int_{1}^{\infty} \frac{g_{0}(r s)}{s^{2}} d s .
$$

By condition, $g_{0}(s) \leq C$ and $g_{0}(s) \rightarrow 0$ as $s \rightarrow 0$. Therefore, due to the the Lebesgue majorized convergence theorem, the right-hand side of (4.1.16) also tends to zero as $r \rightarrow 0$.

\subsection{Convolution of Functions}

For functions $f$ and $g$ defined on $\mathbb{R}^{k}$, one can introduce the notion of the convolution:

$$
(f * g)(x)=\int_{\mathbb{R}^{k}} f(x-y) g(y) d y .
$$

If both functions $f$ and $g$ belong to $L=L^{1}\left(\mathbb{R}^{k}\right)$, then the integrand treated as a function of two variables is summable on $\mathbb{R}^{k} \times \mathbb{R}^{k}$. It is easily verified by means of the following linear change of variables: $x^{\prime}=x-y, y^{\prime}=y$. From the same considerations, taking into account the Fubini theorem from Sec. 1.8, we conclude that the integral (4.2.1) exists for a.e. $x$ and defines a summable function such that

$$
\int_{\mathbb{R}^{k}}(f * g)(x) d x=\int_{\mathbb{R}^{k}} f(y) d y \int_{\mathbb{R}^{k}} g(y) d y .
$$


In (4.2.1), pass to the inequality

$$
|(f * g)(x)| \leq \int_{\mathbb{R}^{k}}|f(x-y)||g(y)| d y .
$$

In (4.2.2), we replace the functions $f$ and $g$ by their absolute values. We obtain the following norm estimate:

$$
|f * g|_{L} \leq|f|_{L}|g|_{L}
$$

It is easy to see that the bilinear convolution operation is commutative and associative. Therefore, by virtue of (4.2.3), the space $L$ is a commutative Banach algebra such that the convolution is its multiplication operation.

Also, the convolution operation is defined if one of the functions (e.g., $f$ ) is summable while the other belongs to various classes.

Theorem 4.2.1.

(a) If $\varphi \in L^{p}, p \geq 1$, then integral (4.2.1) exists for a.e. $x$ and defines a function from $L^{p}$ satisfying the estimate

$$
|f * \varphi|_{L^{p}}<|f|_{L}|\varphi|_{L^{p}}
$$

for its norm.

(b) If $\varphi \in C^{\mu}, 0 \leq \mu \leq 1$, then integral (4.2.1) exists for all $x$ and defines a function from $C^{\mu}$ satisfying the estimate

$$
|f * \varphi|_{C^{\mu}}<|f|_{L}|\varphi|_{C^{\mu}}
$$

for its norm.

Proof. (a) By virtue of (4.2.3), it suffices to consider the case where $p>1$. In the integrand of (4.2.1), assign $g=\varphi$ and represent this integrand as the product

$$
|f(x-y)||\varphi(y)|=\left(|f(x-y)|^{1 / p}|\varphi(y)|\right)|f(x-y)|^{1 / q} .
$$

By virtue of inequality (4.1.2), this implies the estimate

$$
|(f * \varphi)(x)| \leq\left(\int_{\mathbb{R}^{k}}|f(x-y)||\varphi(y)|^{p} d t\right)^{1 / p}|f|_{L}^{1 / q} .
$$

Taking both parts to the power $p$, we obtain the estimate

$$
|(f * \varphi)(x)|^{p} \leq\left(\int_{\mathbb{R}^{k}}|f(x-y)||\varphi(y)|^{p} d t\right)|f|_{L}^{p / q} .
$$

Integrating and applying the estimate (4.2.3) with $g=|\varphi|^{p}$ from $L$ to the integral on the right-hand side, we obtain the inequality

$$
|f * \varphi|_{p}^{p} \leq|f|_{L}|\varphi|_{p}^{p}|f|_{L}^{p / q}
$$

Taking it to the power $1 / p$, we obtain (4.2.4).

(b) Represent the integral (4.2.1) in the form

$$
(f * \varphi)(x)=\int_{\mathbb{R}^{k}} f(y) \varphi(x-y) d y .
$$

If the function $\varphi$ belongs to $C^{0}$, i.e., is continuous and bounded, then $|f(y) \varphi(x-y)| \leq|\varphi|_{0}|f(y)|$. Therefore, due to Theorem 1.8.1 on integrals depending on parameters, the function $f * \varphi$ is continuous 
and the estimate (4.2.5) holds with $\mu=0$. From the same considerations, if $\varphi \in C^{\mu}, 0<\mu \leq 1$, then, in notation of $(4.2 .3)$, we have the inequality

$$
\left|(f * \varphi)\left(x^{\prime}\right)-(f * \varphi)\left(x^{\prime \prime}\right)\right| \leq|f|_{L}[\varphi]_{\mu}\left|x^{\prime}-x^{\prime \prime}\right|^{\mu} .
$$

This inequality combined with the definition of the norm in $C^{\mu}$ from Sec. 2.1 leads to (4.2.5).

Estimates of Theorem 4.2.1 mean that the convolution operator $R(f) \varphi=f * \varphi$ is bounded in any Banach space $X=L^{p}$ and $X=C^{\mu}$ and its norm does not exceed $|f|_{L}$.

An example of the convolution operator is the operator $T_{\varepsilon}=R\left(\chi_{\varepsilon}\right)$ with the averaging kernel

$$
\chi_{\varepsilon}(y)=\frac{1}{\varepsilon^{k}} \chi\left(\frac{y}{\varepsilon}\right), \quad 0<\varepsilon \leq 1,
$$

introduced in Sec. 1.8. Recall that $\chi(y)$ is a nonnegative function from $C_{0}^{\infty}\left(\mathbb{R}^{k}\right)$ such that

$$
\chi(y)=0 \text { for }|y| \geq 1, \int_{|y| \leq 1} \chi(y) d y=1 .
$$

Lemmas 1.8.1 and 2.2.1 can be complemented by a similar result for the space $L^{p}\left(\mathbb{R}^{k}\right)$.

Lemma 4.2.1. If $\varphi \in L^{p}\left(\mathbb{R}^{k}\right), 1 \leq p<\infty$, then $\chi_{\varepsilon} * \varphi \rightarrow \varphi$ in $L^{p}$ as $\varepsilon \rightarrow 0$. In particular, for any open subset $D$ of $\mathbb{R}^{k}$, the class $C_{0}^{\infty}(D)$ is dense in $L^{p}(D)$.

Proof. Let $\varphi \in L^{p}$. Since the $L$-norms of all functions $\chi_{\varepsilon}$ are equal to 1, it follows from (4.2.4) that the convolution operators $R\left(\chi_{\varepsilon}\right) \varphi=\chi_{\varepsilon} * \varphi$ are bounded in $\mathcal{L}\left(L^{p}\right)$ uniformly with respect to $\varepsilon$. Therefore, due to Lemma 1.2.1, it suffices to prove the claimed assertion for a subspace dense in $L^{p}$. Let us take the class of compactly supported continuous functions as such subspace. If a function $\varphi$ belongs to this class, then the supports of all $\chi_{\varepsilon} * \varphi$ are contained in a ball independent of $\varepsilon$. Therefore, taking into account Lemma 2.2.1, we conclude that the sequence $\chi_{\varepsilon} * \varphi$ also tends to $\varphi$ with respect to the $L^{p}$-norm as $\varepsilon \rightarrow 0$.

To prove the second assertion of the lemma, select a sequence of compacts $K_{1} \subseteq K_{2} \ldots$ in $D$ such that their union is equal to $D$. It is obvious that the sequence of functions $\varphi_{n}$ coinciding with $\varphi$ on $K_{n}$ and equal to zero outside $K_{n}$ converges to $\varphi$ in the $L^{p}$-norm. For any $n$ there exists a positive $\varepsilon_{n}$ such that the $\varepsilon_{n}$-neighborhood of the compact set $K_{n}$ is contained in $D$ and, therefore, the convolution $\chi_{\varepsilon} * \varphi_{n}$ belongs to $C_{0}^{\infty}(D)$ provided that $\varepsilon \leq \varepsilon_{n}$. Taking into account the fact that all norms $\left|\chi_{\varepsilon}\right|_{L}$ are equal to 1 , we apply (4.2.4) to the difference $\chi_{\varepsilon} * \varphi_{n}-\varphi=\chi_{\varepsilon} *\left(\varphi_{n}-\varphi\right)+\chi_{\varepsilon} * \varphi-\varphi$ to obtain the estimate

$$
\left|\chi_{\varepsilon} * \varphi_{n}-\varphi\right|_{L^{p}} \leq\left|\varphi_{n}-\varphi\right|_{L^{p}}+\left|\chi_{\varepsilon} * \varphi-\varphi\right|_{L^{p}}
$$

It remains to note that one can make the right-hand side of this estimate arbitrarily small, selecting appropriate $n$ and $\varepsilon$.

In the space $\left(C_{0}^{\infty}\right)^{\prime}=\left(C_{0}^{\infty}\right)^{\prime}\left(\mathbb{R}^{k}\right)$ of generalized functions considered on $\mathbb{R}^{k}$, the notion of the convolution can also be introduced. Recall that its subspace of compactly supported generalized functions is denoted by $\left(C^{\infty}\right)^{\prime}$. Similarly to $(4.2 .1)$, the convolution of a function $u$ from $\left(C_{0}^{\infty}\right)^{\prime}$ with a function $\varphi$ from $C_{0}^{\infty}$ is defined by the relation

$$
(\varphi * u)(x)=(u * \varphi)(x)=u[\varphi(x-t)] .
$$

By virtue of Lemma 1.8.3, the function $u * \varphi$ is infinitely differentiable and

$$
\frac{\partial^{\alpha}}{\partial x^{\alpha}}(u * \varphi)=u *\left(\frac{\partial^{\alpha} \varphi}{\partial x^{\alpha}}\right)
$$

It is easy to see that

$$
(u * \varphi)^{\vee}=u^{\vee} * \varphi^{\vee}, \quad u\left(\varphi^{\vee}\right)=(u * \varphi)(0),
$$

where $\varphi^{\vee}(t)=\varphi(-t)$ and the operation $\varphi \rightarrow \varphi^{\vee}$ is extended from $C_{0}^{\infty}$ to $\left(C_{0}^{\infty}\right)^{\prime}$ according to the relation $u^{\vee}(\varphi)=u\left(\varphi^{\vee}\right)$. 
Lemma 4.2.2. If $\varphi$ coincides with the averaging kernel defined by (4.2.6), then $u * \chi_{\varepsilon} \rightarrow u$ as $\varepsilon \rightarrow 0$ in the sense of the convergence in the space $\left(C_{0}^{\infty}\right)^{\prime}$.

Proof. By Definition (4.2.8), we have the relation

$$
\left(u * \chi_{\varepsilon}\right)(\varphi)=\int_{\mathbb{R}^{k}} u\left[\chi_{\varepsilon}(x-t)\right] \varphi(x) d x .
$$

Let $K$ be the support of a function $\varphi$ from $C_{0}^{\infty}$. Select a cut-off function $f$ from $C_{0}^{\infty}$ such that $f(t) u\left[\chi_{\varepsilon}(x-t)\right]=u\left[\chi_{\varepsilon}(x-t)\right]$ for any $t$ and any $x$ from $K$. Then, due to the second assertion of Lemma 1.8.3, the previous relation can be represented in the form

$$
\left(u * \chi_{\varepsilon}\right)(\varphi)=u\left[\int_{\mathbb{R}^{k}} \chi_{\varepsilon}(x-t) \varphi(x) d x\right] .
$$

It is obvious that the expression in the square brackets is the convolution $\left(\chi_{\varepsilon}\right)^{\vee} * \varphi=\left(\chi_{\varepsilon} * \varphi^{\vee}\right)^{\vee}$. It remains to note that if $\varphi \in C_{0}^{\infty}$, then the sequence $\varphi * \chi_{\varepsilon}$ tends to $\varphi$ as $\varepsilon \rightarrow 0$ in the sense of the convergence in the class $C_{0}^{\infty}$.

The convolution $u * \varphi=\varphi * u$ of a compactly supported generalized function $u$ from $\left(C^{\infty}\right)^{\prime}$ and an arbitrary function $\varphi$ from $C^{\infty}$ is defined similarly to (4.2.9). This allows one to introduce the operation $u_{1} *\left(u_{2} * \varphi\right)$, where at least two of three factors have compact supports. Using Lemma 4.2.2, one can easily show that $u_{1}$ and $u_{2}$ can be swapped; this allows one to introduce the convolution $u_{1} * u_{2}=u_{2} * u_{1}$ of two generalized functions such that one of them has a compact support as follows: $\left(u_{1} * u_{2}\right) * \varphi=u_{1} *\left(u_{2} * \varphi\right)$. Here, it is taken into account the fact that, by virtue of (4.2.10), the convolution $u * \varphi$ defines the generalized function $u$ uniquely.

An example of a compactly supported generalized function is the $\delta$-function $\delta_{a}$ concentrated at a point $a$ from $\mathbb{R}^{k}$ and acting as follows: $\delta_{a}(\varphi)=\varphi(a)$. According to (4.2.8), the convolution with it is expressed as follows:

$$
\left(\delta_{a} * \varphi\right)(x)=\varphi(x-a) .
$$

Thus, $\varphi \rightarrow \delta_{a} * \varphi$ is a translation operator. Since the convolution is a commutative operator and, in particular, $u * \delta_{a}=\delta_{a} * u$, it follows that the convolution operator $\varphi \rightarrow u * \varphi$ commutes with translation operators.

Consider the convolution $Q * \varphi$ with a function $Q(\xi)$ from $\mathcal{H}_{-k}$, satisfying the necessary condition

$$
\int_{\Omega} Q(x i) d_{k-1} \xi=0
$$

on the unit sphere $\Omega$. According to Sec. 3.3, it can be treated as a generalized function, while its convolution $Q * \varphi$ can be treated in the sense of generalized functions (as above) or defined by a singular integral (as in Sec. 3.3). If $\varphi \in C^{\mu}=C^{\mu}\left(\mathbb{R}^{k}\right)$, then no existence of the singular integral $(Q * \varphi)(x)$ is guaranteed since no integrability of the function $Q(x-y) \varphi(y)$ in the domain $\{y,|y-x| \geq \varepsilon\}$ is guaranteed. However, the product of $\chi Q$ and $\chi \in C_{0}^{\infty}$ is free from this disadvantage and, according to Theorem 3.4.2 (the Korn-Giraud theorem), the convolution operator $\varphi \rightarrow(\chi Q) * \varphi$ is bounded in $C^{\mu}$.

The $L^{p}$-case is opposite since, if $p>1$, then the function $Q(x-y)$ belongs to the class $L^{q}$ with the conjugate exponent $q=p /(p-1)$ with respect to the variable $y$ from the domain $|y-x| \geq \varepsilon$. Hence, due to the Hölder inequality, the function $Q(x-y) \varphi(y)$ is integrable in this domain. According to the known Calderón-Zygmund theorem, the singular convolution operator $\varphi \rightarrow Q * \varphi$ is bounded in $L^{p}, p>1$. This profound result requires much more refined tools comparing with the Korn-Giraud theorem. From the proof of this theorem, provided at [44], one can see that even a stronger assertion is valid. 
Theorem (Calderón-Zygmund). Let a function $Q(x, \xi)$ belong to $C^{0(0)}\left(\mathbb{R}^{k}, \mathcal{H}_{-k}\right)$ (in notation of Sec. 3.1) and satisfy the condition (4.3.7) with respect to $\xi$. Then the following assertions hold for any function $\varphi \in L^{p}, 1<p<\infty$.

(a) The singular integral

$$
\psi(x)=\int_{\mathbb{R}^{k}} Q(x, x-y) \varphi(y) d y
$$

exists a.e. and defines an $L^{p}$-function $\psi$ satisfying the estimate

$$
|\psi|_{L^{p}} \leq C|\varphi|_{L^{p}}
$$

(b) The function

$$
\widetilde{\psi}(x)=\sup _{\varepsilon>0}\left|\psi_{\varepsilon}(x)\right|, \quad \psi_{\varepsilon}(x)=\int_{|y| \geq \varepsilon} Q(x, x-y) \varphi(y) d y,
$$

belongs to $L^{p}$ and admits an estimate similar to (4.2.13) and $\lim _{\varepsilon \rightarrow 0}\left|\psi-\psi_{\varepsilon}\right|_{L^{p}}=0$.

Actually, the Calderón-Zygmund theorem is valid for much more general assumptions about the kernel $Q(x, \xi)$. For example, it suffices to require the function $Q$ to belong to $L^{q}(\Omega), q=p /(p-1)$, with respect to the variable $\xi$ on the unit sphere $\Omega$. Note that the relation (4.2.14) is an analog of (4.1.13) and the function $\widetilde{\psi}$ defined by it is called the Hardy-Littlewood maximal functions.

As we note in Sec. 4.1, in the one-dimensional case (i.e., the case where $k=1$ ), the function $Q$ coincides with the Cauchy kernel $K(t)=-1 /(\pi i t)$ up to a constant factor; the operator of the convolution with this kernel is called the Hilbert transformation. The corresponding result similar to the Calderón-Zygmund theorem is obtained by Riesz (by means of methods of complex analysis) much earlier (see [75] for details). The Riesz theorem can be also extended to the convolution with the truncated Cauchy problem

$$
s(t)=-\frac{\chi(t)}{\pi i t}, \quad \chi \in C_{0}^{\infty}(\mathbb{R}), \quad \chi(0)=1 .
$$

Similarly to Theorem 4.2.1, we also provide it for the $C^{\mu}$-case.

Theorem 4.2.2. The singular convolution operator $\varphi \rightarrow s * \varphi$ with the function (4.2.15) is bounded in each space $C^{\mu}(\mathbb{R}), 0<\nu<1$, and $L^{p}(\mathbb{R}), 1<p<\infty$.

Proof. As we note above, the $C^{\mu}$-case is covered by Theorem 3.4.2. Since any difference of functions of kind (4.2.15) belongs to $C_{0}^{\infty}$, it follows that one can assume (without loss of generality) that $\chi(t)=0$ for $|t| \geq 1$. In this case, if $\left|t_{0}\right| \leq 1$, then $\chi\left(t_{0}-t\right)=0$ for $|t| \geq 2$. Hence, assigning $\psi=s * \varphi$, we see that the following relation holds for any integer $i$ :

$$
\psi\left(t_{0}+i\right)=\frac{1}{\pi i} \int_{-2}^{2} \frac{\chi\left(t_{0}-t\right)}{t-t_{0}} \varphi(t+i) d t, \quad\left|t_{0}\right| \leq 1 .
$$

It is obvious that the function $\left[\chi\left(t_{0}-t\right)-1\right]\left(t-t_{0}\right)^{-1}$ is continuous in the square $\left\{-2 \leq t_{0}, t \leq 2\right\}$. Thus, due to the Riesz theorem, we have the estimate

$$
|\psi(t+i)|_{L^{p}(-1,1)} \leq C|\varphi(t+i)|_{L^{p}(-2,2)},
$$

where $C$ is a positive constant independent of $i$. Taking this inequality to the power $p$ and summing with respect to $i$, we arrive at the validity of the estimate (4.2.13).

For $p=1$, Theorem 4.2.2 does not hold. More exactly, if $f \in L^{1}$, then the singular integral $(s * f)\left(t_{0}\right)$ exists for a.e. $t_{0}$, but no local summability of the obtained function is guaranteed (see [74]). It is 
obvious that the convolution operation $\varphi \rightarrow s * \varphi$ is invariant in the class $C_{0}^{\infty}$, which is dense in $L^{1}$. Let $L^{(1)}$ denote the completion of this class with respect to the norm

$$
|\varphi|=|\varphi|_{L}+|s * \varphi|_{L} .
$$

We obtain a Banach space embedded in $L^{1}$; the convolution operator $f \rightarrow s * f$ is bounded in this Banach space by definition. It is obvious that if $f \in L^{(1)}$, then the convolution $f * s$ belongs to $L^{1}$ and is treated in the sense of generalized functions. The question whether it a.e. coincides with the singular integral mentioned above is left open. However, the relation $(f * s) * \varphi=f *(s * \varphi), \varphi \in X$, holds for $X=L^{p}, p>1$, and for $X=C^{\mu}$. For $f \in C_{0}^{\infty}$, this fact is obvious. In the general case, taking into account Theorem 4.2.2, we prove it by passage to the limit with respect to norm (4.2.16).

From the definition, we see that the space $L^{(1)}$ is a Banach algebra with respect to the convolution treated as the multiplication and is an ideal in the similar algebra $L$, i.e., $f * g \in L^{(1)}$ provided that $f \in L^{(1)}$ and $g \in L^{1}$.

Indeed, if $f \in L^{(1)}$ and $g \in L^{1}$, then, in addition to (4.2.3), we have the similar inequality $\mid(s * f) *$ $\left.g\right|_{L} \leq|s * f|_{L}|g|_{L}$, which provides that $f * g \in L^{(1)}$. Due to (4.2.16), this implies the inequality

$$
|f * g|_{L^{(1)}} \leq|f|_{L^{(1)}}|g|_{L^{1}}
$$

It is easy to describe (see [65]) simple sufficient conditions of the belonging to the class $L^{(1)}$. Let $L^{1, p}(\mathbb{R}), p>1$, denote the class of all functions $g$ from $L_{l o c}^{p}(\mathbb{R})$ such that the norm

$$
|g|=\sum_{i=-\infty}^{+\infty}|g|_{L^{q}[i, i+1]}
$$

is finite (we identify functions that differ on a zero-measure set). It is obvious that $L^{1, p}$ is a Banach space embedded in $L^{1}$ with respect to this norm. It is clear that the class $C_{0}^{\infty}$ is dense in this space.

Lemma 4.2.3. The space $L^{1, p}, p>1$, is embedded in $L^{(1)}$ and contains all functions $g$ from $L_{l o c}^{p}(\mathbb{R})$ such that

$$
\int_{\mathbb{R}}(1+|t|)^{\alpha}|g(t)|^{p} d t<\infty, \quad \alpha>p .
$$

Proof. Similarly to Theorem 4.2.2, we prove that the operator of the convolution with the function $s$ is bounded in $L^{1, p}$. Thus, for any $\varphi$ from $C_{0}^{\infty}$, an estimate similar to $(4.2 .13)$ holds with respect to the norm in $L^{1, p}$. Hence,

$$
|\varphi|_{L^{1}} \leq|\varphi|_{L^{1, p}}, \quad|s * \varphi|_{L^{1}} \leq|s * \varphi|_{L^{1, p}} \leq C|\varphi|_{L^{1, p}},
$$

which leads to the estimate

$$
|\varphi|_{L^{(1)}} \leq(1+C)|\varphi|_{L^{1, p}} .
$$

Taking into account the density of $C_{0}^{\infty}$ in $L^{1, p}$, we see that it means the embedding $L^{1, p} \subseteq L^{(1)}$.

To prove the second assertion of the lemma, we note that the obvious inequality

$$
\int_{i}^{i+1}|g(t)|^{p} d t \leq \delta_{i} \int_{i}^{i+1}(1+|t|)^{\alpha}|g(t)|^{p} d t, \quad \delta_{i}= \begin{cases}(1+i)^{-\alpha}, & i \geq 0, \\ (-i)^{-\alpha}, & i<0\end{cases}
$$

holds for any integer $i$. Therefore, norm (4.2.17) does not exceed

$$
|g| \leq C\left(\int_{\mathbb{R}}(1+|t|)^{\alpha}|g(t)|^{p} d t\right)^{1 / p}, \quad C=\sum_{i} \delta_{i}^{1 / p}<\infty .
$$




\subsection{Fourier Transformation}

The Fourier transform of a function $f$ from $L\left(\mathbb{R}^{k}\right)$ is defined by the integral

$$
\hat{f}(x)=\int_{\mathbb{R}^{k}} e^{-i x y} f(y) d y,
$$

where $x y=x_{1} y_{1}+\cdots+x_{k} y_{k}$. The operation $f \rightarrow \hat{f}$ itself is called the Fourier transformation. By virtue of Theorem 1.8.1, the function $\hat{f}$ is continuous. Also, it is clear that this function is bounded and its sup-norm admits the estimate

$$
|\hat{f}|_{0} \leq|f|_{L}
$$

From Theorem 1.8.1, one can easily deduce the relations

$$
\frac{\partial \hat{f}}{\partial x_{j}}=\left[\left(-i y_{j} f(y)\right]^{\wedge}, \quad x_{j} \hat{f}(x)=\left[i \frac{\partial f}{\partial y_{j}}\right]^{\wedge}(x)\right.
$$

under the assumption that $f$ and the functions in the square brackets belong to the class $L$. In the second relation, it is additionally assumed that $f \in C^{1}\left(\mathbb{R}^{k}\right)$ and $f(x) \rightarrow 0$ as $|x| \rightarrow \infty$. For a summable function, the notation $f \in C$ or $f \in C^{1}$ means that it can be changed on a zero-measure set such that the changed function belongs to the specified class.

In particular, if $f \in C_{0}^{\infty}\left(\mathbb{R}^{k}\right)$, then the function $\varphi=\hat{f}$ is infinitely differentiable and decays at $\infty$ faster than any power of $|x|$. In other words, the norms

$$
|\varphi|_{m, n}=\max _{|\alpha| \leq n}\left|(1+|x|)^{m} \frac{\partial^{\alpha} \varphi}{\partial x^{\alpha}}\right|_{0}, \quad m, n=0,1, \ldots,
$$

where $|\cdot|_{0}$ denotes the sup-norm, are finite for this function.

By virtue of (4.3.2) and the density of the class $C_{0}^{\infty}$ in $L$, we conclude that if $f \in L\left(\mathbb{R}^{k}\right)$, then $\hat{f}(x) \rightarrow 0$ as $|x| \rightarrow \infty$ (the Riemann-Lebesgue measure).

There are conditions allowing one to invert the Fourier transformation explicitly.

Theorem (the inversion formula). If the function $\hat{f}$ is summable, then the original function $f$ from $L$ is restored by the inverse Fourier transformation:

$$
f(y)=\frac{1}{(2 \pi)^{k}} \int_{\mathbb{R}^{k}} \hat{f}(x) e^{i x y} d x
$$

(this relation is a.e. valid).

In particular, this theorem implies that the Fourier transformation is one-to-one on $L$. Also, it possesses the following property (see, e.g., $[20,79]$ ).

Theorem (Wiener). If $f \in L$ and $1+\hat{f}(x) \neq 0$ for any $x$ from $\mathbb{R}^{k}$, then there exists a function $g$ from $L$ such that $(1+\hat{f})^{-1}=1+\hat{g}$.

The Fourier transformation is linked with convolution (4.2.1) by the relation

$$
(f * g)^{\wedge}=\hat{f} \hat{g}, \quad f, g \in L .
$$

Indeed, by the definition of the convolution, we have

$$
(f * g)^{\wedge}(x)=\int\left[\int f(y-t) g(t) d t\right] e^{-i x y} d y .
$$

Assigning $f_{1}(t)=e^{-i x t} f(t)$ and $g_{1}(t)=e^{-i x t} g(t)$, one can represent the right-hand side as the convolution $\left(f_{1} * g_{1}\right)(x)$; then the claimed result follows from (4.2.2). 
Recall that the space $L$ is a Banach algebra, where the multiplication operation is the convolution. By virtue of (4.3.2) and (4.3.5), the Fourier transformation embeds this algebra into the Banach algebra of continuous functions vanishing at $\infty$, defined by the pointwise operation and the sup-norm.

In addition to $C_{0}^{\infty}\left(\mathbb{R}^{k}\right)$, a wider $S$ chwartz class $\mathcal{S}$ is frequently considered; it consists of all functions $\varphi(x)$ from $C^{\infty}$ such that they and all their derivatives decay on $\infty$ faster than any power of $|x|$ or, which is equivalent, norms (4.3.4) are finite for them. It is obvious that the relations (4.3.3) also hold for functions $f$ from $\mathcal{S}$. Then the Fourier transformation is invariant in $\mathcal{S}$. The same is also valid for the inverse transformation (4.3.5). Taking into account the inversion formula, we conclude that the Fourier transformation isomorphically maps the class $\mathcal{S}$ onto itself.

Sometimes, it is convenient to supply the Fourier transformation (4.3.1) by the norming factor $(2 \pi)^{-k / 2}$. In this case, we denote it by the symbol $F$. Then the inverse transformation $F^{-1}$ in the class $\mathcal{S}$ is also defined with a similar factor:

$$
(F \varphi)(x)=\frac{1}{(2 \pi)^{k / 2}} \int_{\mathbb{R}^{k}} e^{-i x y} \varphi(y) d y, \quad\left(F^{-1} \varphi\right)(x)=\frac{1}{(2 \pi)^{k / 2}} \int_{\mathbb{R}^{k}} e^{i x y} \varphi(y) d y .
$$

In particular, $F^{-1} \varphi=(J F J) \varphi, \varphi \in \mathcal{S}$, where $J \varphi=\bar{\varphi}$ denotes the operators of the complex conjugating of functions. Thus, in operator involution terms (see Sec. 1.3), we have the relation $F^{-1}=\bar{F}$.

Let us verify that the operator $F$ preserves the norm of the space $L^{2}$, i.e., $|F \varphi|_{L^{2}}=|\varphi|_{L^{2}}, \varphi \in \mathcal{S}$.

Indeed, in notation of form (4.1.3) (with respect to $G=\mathbb{R}^{k}$ ), the second power of the norm of the function $\varphi$ in $L^{2}$ is equal to $(\varphi, J \varphi)$. On the other hand, from Definition (4.3.1), we see that

$$
\langle\varphi, \hat{\psi}\rangle=\langle\hat{\varphi}, \psi\rangle, \quad \varphi, \psi \in \mathcal{S}
$$

Therefore, $|F \varphi|_{L^{2}}^{2}=\langle F \varphi, J F \varphi\rangle=\langle\varphi, F J F \varphi\rangle=\langle\varphi, J \varphi\rangle$, which implies that the $L^{2}$-norms of the functions $\varphi$ and $F \varphi$ are equal.

The class $C_{0}^{\infty}$ is dense in the space $L^{2}\left(\mathbb{R}^{k}\right)$. Hence, the class $\mathcal{S}$ is dense in this space a fortiori. Therefore, the operator $F$ is extended as an isometric isomorphism of the space $L^{2}$ onto itself; this isomorphism is denoted by $F$ again. Taking into account the first relation of (4.3.3), we conclude that the Sobolev space $W^{n, 2}\left(\mathbb{R}^{k}\right)$ can be described by the equivalent norm

$$
|\varphi|=\left(\int_{\mathbb{R}^{k}}\left(1+|x|^{2}\right)^{n}(F \varphi)^{2}(x) d x\right)^{1 / 2} .
$$

In a natural way, the Fourier transformation is extended to the so-called tempered distributions. To do this, define the convergence in the Schwartz class $\mathcal{S}$ as follows: a sequence $\varphi_{j}$ converges to $\varphi$ in $\mathcal{S}$ as $j \rightarrow \infty$ if $\left|\varphi_{j}-\varphi\right|_{m, n} \rightarrow 0$ for any $m$ and $n$, where the norm is defined by (4.3.4). The class of linear functionals over $\mathcal{S}$ continuous with respect to the introduced convergence is denoted by $\mathcal{S}^{\prime}$. It is obvious that the convergence $\varphi_{j} \rightarrow \varphi$ in $C_{0}^{\infty}$ implies the corresponding convergence in $\mathcal{S}$. It is easy to show that for any $\varphi$ from $\mathcal{S}$ there exists a sequence $\varphi_{j}$ from $C_{0}^{\infty}$ converging to $\varphi$ in $\mathcal{S}$. Hence, the restriction of $u$ to $C_{0}^{\infty}$, where $u$ is an arbitrary functional from $S^{\prime}$, belongs to $\left(C_{0}^{\infty}\right)^{\prime}$ and the specified restriction is uniquely defined by $u$. Therefore, elements $u$ of $\mathcal{S}^{\prime}$ can be identified with generalized functions from $\left(C_{0}^{\infty}\right)^{\prime}$. They are called tempered distributions. This term is related to measured tempered functions $f(x)$ defined as functions admitting the estimate

$$
|f(x)| \leq C(1+|x|)^{m},
$$

where $m$ is a positive integer; they belong to $\mathcal{S}^{\prime}$ since they are regular generalized functions. Obviously, all compactly supported generalized functions also belong to the class $\mathcal{S}^{\prime}$. Thus, $\left(C^{\infty}\right)^{\prime} \subseteq \mathcal{S}^{\prime} \subseteq\left(C_{0}^{\infty}\right)^{\prime}$.

Also, it is clear from (4.3.3)-(4.3.4) that the convergence $\varphi_{n} \rightarrow \varphi$ in $S$ implies the convergence $\hat{\varphi}_{n} \rightarrow \hat{\varphi}$ in $\mathcal{S}$. Therefore, taking into account (4.3.7), one can "extend" the Fourier transformation from $\mathcal{S}$ to $\mathcal{S}^{\prime}$ as follows:

$$
\hat{u}(\varphi)=u(\hat{\varphi}), \quad \varphi \in \mathcal{S}
$$


Thus, we obtain an invertible transformation of $\mathcal{S}^{\prime}$ onto itself, also called the Fourier transformation. It is obvious that the relations (4.3.3) remain valid for $f$ from $\mathcal{S}^{\prime}$. If $f \in L \subseteq \mathcal{S}^{\prime}$, then the provided definition of the Fourier transformation coincides with (4.3.1).

Similarly to $\left(C_{0}^{\infty}\right)^{\prime}$, the class $\mathcal{S}^{\prime}$ is endowed with the pointwise convergence of its elements. The space $\mathcal{S}^{\prime}$ is complete with respect to this convergence and the Fourier transformation continuously maps $\mathcal{S}^{\prime}$ to $\mathcal{S}^{\prime}$. The isometric isomorphism $F$ of the space $L^{2}$, defined above, coincides (up to the factor $(2 \pi)^{-k / 2}$ ) with the restriction of the Fourier transformation acting in the space $\mathcal{S}^{\prime}$ to its subspace $L^{2}$.

Theorem 4.3.1. The Fourier transform of any compactly supported generalized function $u$ from $\left(C^{\infty}\right)^{\prime}$ is a regular generalized function, belongs to the class $C^{\infty}$, and is determined by the relation

$$
\hat{u}(x)=u_{t}\left(e^{-i x t}\right) \text {. }
$$

The relation

$$
(u * v)^{\wedge}=\hat{u} \hat{v}, \quad u, v \in\left(C^{\infty}\right)^{\prime}
$$

extending (4.3.6) to generalized functions with compact support holds.

Note that if $x=\left(x_{1}, \ldots, x_{k}\right) \in \mathbb{C}^{k}$, then the right-hand side of the relation (4.3.9) determines an entire function called the Fourier-Laplace transformation.

Proof. By virtue of Lemma 1.8.3, the function on the right-hand side of (4.3.3) belongs to the class $C^{\infty}$. Denote this function by $f(x)$. There exist a positive integer $m$ and a compact subset $K$ of $\mathbb{R}^{k}$ such that

$$
|f(x)| \leq C\left|e^{-i x t}\right|_{C^{m}(K)},
$$

where $x$ is a fixed point and the norm on the right-hand side is taken with respect to the variable $t$.

Indeed, let a function $\chi$ from $C_{0}^{\infty}$ be identically equal to zero in a neighborhood of $\operatorname{supp} u$. It suffices to show that $|u(\varphi)| \leq C|\varphi|_{C^{m}(K)}, \varphi \in C^{\infty}$.

If this estimate does not hold, then for any positive integer $m$ there exists a function $\varphi_{m}$ from $C^{\infty}(D)$ such that

$$
\left|u\left(\varphi_{m}\right)\right| \geq 1, \quad\left|\varphi_{m}\right|_{C^{m}(K)} \leq 1 / m .
$$

Then $\chi \varphi_{m} \rightarrow 0$ as $m \rightarrow \infty$ in $C_{0}^{\infty}(D)$, which contradicts the continuity of the functional $u$ with respect to this convergence.

From (4.3.11), it follows that the estimate (4.3.8) holds for the function $f(x)=u_{t}\left(e^{-i x t}\right)$, which means that it slowly grows at $\infty$. The function $f$ belongs to $\mathcal{S}^{\prime}$ since it is a regular generalized function.

On the other hand, if $\varphi \in C_{0}^{\infty}$, then, taking into account Lemma 1.8.3 and Definition (4.3.14), we have the relation

$$
\hat{u}(\varphi)=u\left(\int_{\mathbb{R}^{k}} e^{-i x t} \varphi(t) d t\right)=\int_{\mathbb{R}^{k}} f(t) \varphi(t) d t
$$

implying that $\hat{u}=f$. From the same considerations, we obtain the relation

$$
(u * \varphi)^{\wedge}(y)=\int_{\mathbb{R}^{k}} e^{-i x t} u_{t}[\varphi(x-t)] d t=u_{t}\left[\int_{\mathbb{R}^{k}} e^{-i x t} \varphi(x-t) d t\right] .
$$

Since the expression in the square brackets on the right-hand side coincides with $e^{-i y t} \hat{\varphi}(y)$, we have the relation

$$
(u * \varphi)^{\wedge}=\hat{u} \hat{\varphi}, \quad u \in\left(C^{\infty}\right)^{\prime}, \varphi \in C_{0}^{\infty},
$$

extending (4.3.6) to the case where one of the functions is a generalized function. In the general case, i.e., if $u$ and $v$ belong to $\left(C^{\infty}\right)^{\prime}$, the specified relation yields the relations

$$
(u * v * \varphi)^{\wedge}=(u * v)^{\wedge} \hat{\varphi}, \quad(u * v * \varphi)^{\wedge}=\hat{u}(v * \varphi)^{\wedge}=\hat{u} \hat{v} \hat{\varphi},
$$


implying the validity of (4.3.10) in the case of two compactly supported generalized functions.

Note that the Fourier transformation of a function $Q$ from $\mathcal{H}_{-k}$, possessing the property (4.2.12) is defined by a singular integral similarly to (4.3.1). It can be also treated in the sense of generalized functions. It is known that $\widehat{Q}$ is a homogeneous function of power 0 . In the one-dimensional case, the Fourier transform of this kernel is represented as follows:

$$
\hat{K}\left(t_{0}\right)=\operatorname{sgn} t_{0}, \quad K(t)=-\frac{1}{\pi i t} .
$$

Indeed, by definition, we have the relation

$$
K(\hat{\varphi})=-\frac{1}{\pi i} \lim _{n \rightarrow \infty} \int_{-n}^{n} \frac{d t}{t} \int_{\mathbb{R}} e^{-i t t_{0}} \varphi\left(t_{0}\right) d t_{0} .
$$

Changing the integration order, we obtain the relation

$$
-\frac{1}{\pi i} \int_{-n}^{n} \frac{e^{-i t t_{0}}}{t} d t=\frac{2 \operatorname{sgn} t_{0}}{\pi} \int_{0}^{n\left|t_{0}\right|} \frac{\sin t}{t} d t .
$$

The following property of the corresponding improper integral is well known:

$$
\lim _{s \rightarrow \infty} \int_{0}^{s} \frac{\sin t}{t} d t=\frac{\pi}{2} .
$$

Therefore, passing to the limit as $n \rightarrow \infty$ in the relation (4.3.14), we obtain (4.3.13).

In particular, the relation (4.3.13) shows that $\hat{K}^{2}=1$ and, therefore, the convolution $K *(K * \varphi)$ is equal to $\varphi$ if $\varphi \in C_{0}^{\infty}$. In terms of generalized functions, this fact is expressed by the relation $K * K=\delta_{0}$. The same is valid for the "truncated" Cauchy kernel defined by (4.2.15), which is a compactly supported generalized function.

Lemma 4.3.1. The function $\hat{s}(t)$ tends to \pm 1 as $t \rightarrow \pm \infty$, its derivative belongs to the class $C_{0}^{\infty}$, and $\chi_{0}=s * s-\delta_{0} \in C_{0}^{\infty} ;$ then

$$
(s * s) * \varphi=\varphi+\chi_{0} * \varphi, \quad \chi_{0} \in C_{0}^{\infty},
$$

for any locally integrable function $\varphi$ from $\mathcal{S}^{\prime}$.

Proof. Let $K_{0}(t)=K(t)$ for $|t| \leq 1$ and $K_{0}(t)=0$ for $|t| \geq 1$. Then, similarly to (4.3.13), we verify that $\widehat{K}_{0}\left(t_{0}\right)$ is determined by the expression on the right-hand side of (4.3.14) with $n$ assigned to be equal to 1 . Thus, $\widehat{K}_{0}\left(t_{0}\right) \rightarrow \pm 1$ as $t_{0} \rightarrow \pm \infty$. Comparing the functions $K_{0}(t)$ and $s(t)$ in $(4.2 .15)$, we see that the difference $K_{0}-s$ belongs to $L$ and, therefore, (4.3.15) implies that

$$
\lim _{x \rightarrow \pm \infty} \hat{s}(x)= \pm 1 \text {. }
$$

According to Lemma 1.8.3, the derivative $\hat{s}^{\prime}$ is computed under the sign of the singular integral and, therefore, coincides (up to a constant factor) with the Fourier transformation of the function $\chi$ from (4.2.15). In particular, $\hat{s}^{\prime} \in \mathcal{S}$. Then, taking into account (4.3.16), we conclude that the function $\hat{s}^{2}(t)-1$ also belongs to $\mathcal{S}$. Then, applying the relation (4.3.10) to $u=v=s$, we conclude that $\chi_{0}=u * u-\delta_{0} \in \mathcal{S}$. Since the function $\chi_{0}$ is compactly supported, it follows that it belongs to the class $C_{0}^{\infty}$.

As we note in Sec. 4.2, the space $L^{(1)}$ is a Banach algebra with respect to the convolution treated as the multiplication operation, it is embedded into $L^{1}$, and $f * g \in L^{(1)}$ provided that $f \in L^{(1)}$ and $g \in L^{1}$. The Wiener theorem is also valid for this algebra.

Indeed, let $f \in L^{(1)}$ and $1+\hat{f}(t) \neq 0$ for any real $t$. Then, due to the Wiener theorem, there exists a function $g$ from $L^{1}$ such that $1+\hat{g}=(1+\hat{f})^{-1}$, i.e., $\hat{f}+\hat{g}+\hat{f} \hat{g}=0$. Taking into account (4.3.6) and 
the injectivity of the Fourier transformation, this implies that $f+g+f * g=0$. Since the convolution $f * g$ belongs to $L^{(1)}$, it follows that $g=-f-f * g$ also belongs to $L^{(1)}$.

Recall that, according to Sec. 1.7, the symbol $C$ denotes the space of all continuous and bounded functions $x(t), t \in \mathbb{R}$. This space is a Banach algebra with respect to the pointwise operators and the sup-norm $|x|_{0}$. The symbol $C^{0}$ denotes the subalgebra of functions $x$ from $C$ vanishing at infinity. Let $M^{0}$ denote the image of $L^{(1)}$ under the Fourier transformation. By virtue of (4.3.6), the space $M^{0}$ is a Banach algebra with respect to multiplication, its norm is defined by the relation

$$
|y|=|g|_{L^{(1)}}, \quad y=\hat{g},
$$

and it is embedded into $C^{0}$.

The next lemma shows that $M^{0}$ is densely embedded in $C^{0}$.

Lemma 4.3.2. The class $\mathcal{R}$ of rational functions such that their restrictions to the real line belong to $C^{0}$ is dense in the Banach algebra $M^{0}$.

Proof. Consider the relations

$$
i \int_{0}^{\infty} e^{i(\zeta-s) t} d t=(s-\zeta)^{-1}, \operatorname{Im} \zeta>0 \quad \text { and } \quad i \int_{-\infty}^{0} e^{i(\zeta-s) t} d t=-(s-\zeta)^{-1}, \operatorname{Im} \zeta<0 .
$$

According to (4.3.1), they can be represented in the form

$$
\widehat{f}_{ \pm}(s)=(s-\zeta)^{-1}
$$

where

$$
f_{+}(t)=\left\{\begin{array}{ll}
i e^{i \zeta t}, & t>0, \\
0, & t<0,
\end{array} \quad \operatorname{Im} \zeta>0, \quad f_{-}(t)=\left\{\begin{array}{ll}
0, & t>0, \\
i e^{i \zeta t}, & t<0,
\end{array} \quad \operatorname{Im} \zeta<0 .\right.\right.
$$

Differentiating the relation (4.3.18), we arrive at the following description of the class of functions $f$ from $L^{(1)}$ such that their Fourier transforms belong to $\mathcal{R}$ : on each semiaxis $\pm t>0$, the function $f(t)$ is a finite sum of terms of kind $p(t) e^{i \zeta t}$, where $\pm \zeta>0$. It remains to note that, by virtue of Lemma 4.2 .3 , the class of functions of this kind is dense in $L^{(1)}$.

Let $M$ denote the space of all functions $x$ from $C$ such that the multiplication operator $y \rightarrow x y$ is bounded in $M^{0}$; it is endowed with the norm

$$
|x|=|x|_{\mathcal{L}\left(M^{0}\right)} .
$$

It is easy to see that the space $M$ is embedded into $C$ with respect to this norm, i.e., the estimate

$$
|x|_{0} \leq C_{0}|x|_{M}
$$

holds, where $C_{0}$ is a positive constant independent of $x$.

Indeed, in notation (4.3.17), we have the relation $x \hat{g}=\hat{f}$. Then, by definition, the following inequality is valid:

$$
|f|_{L^{(1)}} \leq|x|_{M}|g|_{L^{(1)}} \text {. }
$$

Therefore, for any real $a$, the following inequality is valid:

$$
|x(a) \hat{g}(a)| \leq|f|_{L^{1}} \leq|f|_{L^{(1)}} \leq|x|_{M}|g|_{L^{(1)}} .
$$

Let $g(t)=g_{a}(t)$ be the function coinciding with $e^{i a t}$ on the segment $[0,1]$ and equal to zero outside this segment. Then

$$
\hat{g}(a)=\int_{\mathbb{R}} e^{-i a t} g(t) d t=1 .
$$

Now, it suffices to verify that

$$
C_{0}=\sup _{a}\left|g_{a}\right|_{L^{(1)}}<\infty .
$$


It is obvious that one can assume (without loss of generality) that $s(t)=0$ provided that $|t| \geq 1$. Hence, the function $s * g_{a}$ vanishes outside the segment $[-1,2]$. It is obvious that $\left|g_{a}\right|_{L^{1}}=\left|g_{a}\right|_{L^{2}}=1$. Hence, due to Theorem 4.2.2, the function $s * g_{a}$ belongs to $L^{2}$ and satisfies the estimate

$$
\left|s * g_{a}\right|_{L^{2}} \leq C_{1}
$$

where $C_{1}$ is the $L^{2}$-norm of the operator of the convolution with $s$. By virtue of the Hölder inequality, this implies the inequality $\left|s * g_{a}\right|_{L^{1}} \leq \sqrt{3} A_{1}$. Thus, according to Definition (4.2.16) of the $L^{(1)}$-norm, we arrive at the estimate $(4.3 .21)$ with the constant $C_{0}=1+\sqrt{3} C_{1}$.

From the definition of $M$ and (4.3.20), it follows that the space $M$ is a Banach algebra with respect to multiplication. Its elements are called $L^{(1)}$-multipliers. They can be treated as the Fourier transforms of generalized functions $u$ from $\mathcal{S}^{\prime}$ such that the operator of the convolution with them is bounded in $L^{(1)}$. It is obvious that the functions $\hat{s}$ and $\hat{f}$, where $f \in L^{1}$, are $L^{(1)}$-multipliers. Another example of

$L^{(1)}$-multipliers are the Fourier transforms of $\delta$-functions $\widehat{\delta}_{a}(t)=e^{-a t}$ : due to (4.2.11), the operator of the convolution with each such function serves as a translation operator. Since these operators $f(t) \rightarrow f(t-a)$ are bounded in $L^{(1)}$ uniformly with respect to $a \in \mathbb{R}$, it follows that the function $e^{i a t}$ belongs to $M$ and their norms $\left|e^{i a t}\right|_{M}$ are uniformly bounded. The general theory of multipliers and related operators invariant with respect to translations is presented, e.g., in [27, 76].

Let $M^{1}$ denote the closure of the set of finite sums $\sum c_{k} e^{i a_{k} t}$ in $M$. By virtue of (4.3.20), almost periodic functions belong to the space $M^{1}$. In other words, in notation of Sec. 1.7, the space $M^{1}$ is a Banach algebra embedded into $C^{1}$. According to Lemma 4.3.1 and the terminology of Sec. 1.7, functions of the kind

$$
x(t)=x_{1}(t)+\hat{s}(t) x_{2}(t)+y(t), \quad x_{j} \in M^{1}, \quad y \in M^{0},
$$

are semi-almost periodic.

\subsection{Convolution-Type Operators on the Real Line}

An integral convolution-type operator with a function $f$ from $L(\mathbb{R})$ is defined by the relation

$$
[K(f) \varphi]\left(t_{0}\right)=\int_{\mathbb{R}} k\left(t_{0}, t\right) f\left(t_{0}-t\right) \varphi(t) d t, \quad t_{0} \in \mathbb{R} .
$$

We assume that $k\left(t_{0}, t\right)$ is a continuous and bound function, i.e., a function from the space $C^{0}(\mathbb{R} \times \mathbb{R})$. It is not included in the operator notation, but the operator does depend on it. If $k\left(t_{0}, t\right)=1$, then the operator passes to the convolution operator $R(f) \varphi=f * \varphi$, i.e., the symbol $K$ is replaced by $R$ in this case. It is easy to describe conditions for $k$, providing the boundedness or compactness of the operator $K(f)$ in the spaces $L^{p}(\mathbb{R}), 1 \leq p<\infty, C^{\mu}(\mathbb{R}), 0<\mu<1$, and $C^{1, \mu}(\mathbb{R})$.

Theorem 4.4.1.

(a) Let $k\left(t_{0}, t\right) \in C^{\mu+0}(\mathbb{R} \times \mathbb{R})$. Then the operator $K(f)$ is bounded in the spaces $L^{p}(\mathbb{R})$ and $C^{\mu}(\mathbb{R})$ and its norm admits the estimates

$$
|K(f)|_{\mathcal{L}\left(L^{p}\right)} \leq|k|_{C^{0}}|f|_{L}, \quad|K(f)|_{\mathcal{L}\left(C^{\mu}\right)} \leq|k|_{C^{\mu}}|f|_{L},
$$

where the norm in $C^{0}$ is the sup-norm.

If the above holds and

$$
\lim _{\substack{t \rightarrow \infty,\left|t_{0}-t\right| \leq n}} k\left(t_{0}, t\right)=0, \quad n=1,2, \ldots,
$$

then the operator $K(f)$ is compact in these spaces.

(b) Let $k \in C^{1, \mu+0}(\mathbb{R} \times \mathbb{R})$. Then the operator $K(f)$ is bounded in the space $C^{1, \mu}(\mathbb{R})$. If this holds and the condition (4.4.3) is satisfied for the function $k$ and for the function

$$
k^{1}=\frac{\partial k}{\partial t_{0}}+\frac{\partial k}{\partial t}
$$


then the operator $K(f)$ is compact in this space.

Proof. The boundedness of the operator $K(f)$ in $L^{p}$ and the estimate (4.4.2) are proved in the same way as Theorem 4.3.1(a).

Let the condition (4.4.3) be satisfied. Due to Theorem 1.2.3, the set of compact operators is closed with respect to the operator norm. Therefore, by virtue of the density of the class $C_{0}^{\infty}(\mathbb{R})$ and $L(\mathbb{R})$ and the estimates (4.4.2), without loss of generality one can assume that $f \in C_{0}^{\infty}$. Take a cut-off function $\chi$ from $C_{0}^{\infty}$ on supp $f$ and take into account the fact that the expression $k\left(t_{0}, t\right) f\left(t_{0}-t\right)$ is not changed if we multiply it by $\chi\left(t_{0}-t\right)$. Therefore, changing $k\left(t_{0}, t\right)$ for $k\left(t_{0}, t\right) \chi\left(t_{0}-t\right)$ and taking into account (4.4.3), one can assume (without loss of generality) that $k\left(t_{0}, t\right) \rightarrow 0$ as $|t|+\left|t_{0}\right| \rightarrow \infty$. Then the function $k$ can be approximated with respect to the sup-norm by continuous compactly supported functions. Taking into account Lemma 2.2.1, one can assume (without loss of generality) that $k \in C_{0}^{\infty}(\mathbb{R} \times \mathbb{R})$. Then the compactness of the operator $K(f)$ in the space $L^{p}$ is obvious.

Further, we represent the relation (4.4.1) in the form

$$
\left[(K(f) \varphi]\left(t_{0}\right)=\int_{\mathbb{R}} k\left(t_{0}, t_{0}-t\right) f(t) \varphi\left(t_{0}-t\right) d t .\right.
$$

This immediately yields the second estimate of (4.4.2).

Let $k \in C^{\nu}(\mathbb{R} \times \mathbb{R}), \nu>\mu$, and the condition (4.4.3) be satisfied. By virtue of Theorem 2.1.2 and Lemma 2.2.1, the sequence $\left\{k_{n}\left(t_{0}, t\right)\right\}$ belongs to $C_{0}^{\infty}(\mathbb{R} \times \mathbb{R})$ and converges to $k\left(t_{0}, t\right)$ with respect to the norm of the space $C^{\mu}$. Therefore, as above, taking into account the estimate (4.4.2), one can assume that $f \in C_{0}^{\infty}(\mathbb{R})$ and $k\left(t_{0}, t\right) \in C_{0}^{\infty}(\mathbb{R} \times \mathbb{R})$, which guarantees the compactness of the operator $K$ in the space $C^{\mu}$.

If $k \in C^{1, \mu}(\mathbb{R} \times \mathbb{R})$, then the relation (4.4.5) can be differentiated under the integral sign, which leads to the relation

$$
[K(f) \varphi]^{\prime}=K^{1}(f) \varphi+K(f) \varphi^{\prime}
$$

where the operator $K^{1}$ is defined similarly to (4.4.1) with respect to the function (4.4.4). This immediately yields the boundedness of the operator $K$ in $C^{1, \mu}$.

Further, let the condition (4.4.3) be satisfied for both functions $k$ and $k^{1}$. Then, by virtue of (a), both operators $K(f)$ and $K^{1}(f)$ are compact in the space $C^{\mu}$. Then, taking into account (4.4.6), we conclude that the operator $K(f)$ is compact in $C^{1, \mu}$.

Actually, the belonging of $k$ to $C^{\mu+0}$ is too strong for estimates (4.4.2). In the $L^{p}$-case, it suffices to require the function $k\left(t_{0}, t\right)$ to be just bounded. In the $C^{\mu}$-case, the said condition can be changed for the belonging of $k$ to $C^{\mu}$. We impose the said condition so that the assumptions of different assertions can be unified.

Apply Theorem 4.4.1 to the convolution operator $R(f) \varphi=f * \varphi$, assuming that the function $a(t)$ belongs to $C(\mathbb{R})$,

$$
a( \pm \infty)=\lim _{t \rightarrow \pm \infty} a(t)
$$

and the function $b(t)$ possesses the same property. Then the relations

$$
a R(f) \sim R(f) a, \quad a R(f) b R(g) \sim a b R(f * g)
$$

are valid for all functions $f$ and $g$ from $L(\mathbb{R})$, where $\sim$ means that the left- and right-hand sides are equal modulo $\mathcal{T}\left(L^{p}\right)$ of compact operators and $a$ and $b$ are treated as multiplication operators.

If we additionally assume that $a$ and $b$ belong to $C^{\mu+0}(\mathbb{R})$, then these relations also hold with respect to $\mathcal{T}\left(C^{\mu}\right)$. In the same way, if $a$ and $b$ belong to $C^{1, \mu+0}(\mathbb{R})$ and the derivatives $a^{\prime}$ and $b^{\prime}$ also possess the property (4.4.7), then the relations (4.4.8) also hold with respect to $\mathcal{T}\left(C^{\mu}\right)$.

Indeed, the first relation of (4.4.8) follows from Theorem 4.4.1 since the function $k\left(t_{0}, t\right)=a\left(t_{0}\right)-$ $a(t)$ satisfies the condition (4.4.6). The second relation follows from the first one: $a R(f) b R(g) \sim$ $a R(f * g) b \sim a b R(f * g)$. 
An analog of Theorem 4.4.1 holds in the case where the function $s$ from (4.2.15) plays the role of $f$ and (4.4.1) is treated as a singular integral.

\section{Theorem 4.4.2.}

(a) Let $k\left(t_{0}, t\right) \in C^{\mu+0}(\mathbb{R} \times \mathbb{R})$. Then the singular operator $K(s)$ is bounded in the spaces $L^{p}(\mathbb{R})$, $p>1$, and $C^{\mu}(\mathbb{R}), 0<\mu<1$. If the above holds, $k(t, t) \equiv 0$, and the condition (4.4.3) is satisfied, then the operator $K(s)$ is compact on these spaces.

(b) Let $k \in C^{1, \mu+0}(\mathbb{R} \times \mathbb{R})$. Then the operator $K(s)$ is bounded in the space $C^{1, \mu}(\mathbb{R})$. If the above holds, $k(t, t) \equiv 0$, and the condition (4.4.3) holds for both functions $k$ and $k^{1}$ in (4.4.5), then $K(s)$ is a compact operator.

Proof. By Lemma 2.1.1, if $\varepsilon>0$ is sufficiently small, then the function $k_{0}\left(t_{0}, t\right)=\left|t-t_{0}\right|^{-\varepsilon}\left[k\left(t_{0}, t\right)-\right.$ $\left.k\left(t_{0}, t_{0}\right)\right]$ also belongs to the class $C^{\mu+0}$. Therefore, by Theorem 4.4.1, the operator $K_{0}\left(f_{0}\right)$ defined by $k_{0}\left(t_{0}, t\right)$ and $f_{0}(t)=|t|^{\varepsilon} s(t)$ similarly to (4.4.1) is bounded in the spaces $L^{p}$ and $C^{\mu}$. If $k\left(t_{0}, t\right)$ satisfies the condition (4.4.3), then the function $k_{0}$ also satisfies it. Then the operator $K_{0}\left(f_{0}\right)$ is compact in these spaces. Since $K(s) \varphi=a(s * \varphi)+K_{0}\left(f_{0}\right) \varphi$, where $a(t)=k(t, t)$, it remains to use Theorem 4.2.2.

Representing the operator $K(s)$ in the form (4.4.5) and using Lemma 3.4.2, we obtain the differentiation relation $[K(s) \varphi]^{\prime}=K^{1}(s) \varphi+K(s) \varphi^{\prime}$ similar to (4.4.6). By virtue of this relation, we arrive at the validity of $(\mathrm{b})$.

From Theorem 4.4.2, similarly to (4.4.8), one can easily deduce the relations

$$
a R(s) \sim R(s) a, \quad a R(s) b R(g) \sim b R(g) a R(s) \sim a b R(s * g),
$$

where $\sim$ means the same as above, $g \in L^{(1)}$, and the functions $a$ and $b$ belong to $C^{+0}(\mathbb{R})$ and have limits (4.4.7) at infinity. If this holds and $a$ and $b$ belong to $C^{\mu+0}(\mathbb{R})$, then these relations also hold modulo $\mathcal{T}\left(C^{\mu}\right)$.

For the operator $a R(f)$, the compactness condition given by (4.4.3) is reduced to the condition $a(t) \rightarrow 0$ as $t \rightarrow \infty$. The next lemma shows that this is also a necessary compactness condition.

Lemma 4.4.1. Let a function $\chi(t)$ from $C^{\mu+0}(\mathbb{R})$ admit limits (4.4.7) and at least one of them be different from zero. Then the compactness of the operator aR $(f), f \in L^{1}$, in the space $X=L^{p}$ or $C^{\mu}$ implies the relation $f=0$.

Proof. For definiteness, assume that $a(+\infty) \neq 0$. Let a function $\chi$ from $C^{\infty}(\mathbb{R})$ be identically equal to 1 in a neighborhood of $+\infty$ and be identically equal to zero in a neighborhood of $-\infty$. Then $a R(f) \sim a(+\infty) \chi R(f)$ and one can assume (without loss of generality) that $a=\chi$.

First, we show that

$$
f * \varphi=0, \quad \varphi \in C_{0}^{\infty} .
$$

Fix $\varphi$ from $C_{0}^{\infty}$ and assign $\varphi_{n}(t)=\varphi\left(t-a_{n}\right)$, where $a_{n} \rightarrow+\infty$. The sequence $\varphi_{n}$ is uniformly bounded in $X$. Then, by the definition of compact operators, there exists a sequence $\chi R(f) \varphi_{n_{k}}$ converging in $X$; denote its limit by $\psi$. Redenoting $\varphi_{n_{k}}$ by $\varphi_{n}$, we have the relation

$$
\left.\mid \chi R(f) \varphi_{n}\right)-\left.\psi\right|_{X} \leq \alpha_{n}, \quad \lim _{n \rightarrow \infty} \alpha_{n}=0 .
$$

Let a sequence $f_{k}$ from $C_{0}^{\infty}$ converge to $f$ from $L^{1}$. Then the following inequality holds for this sequence:

$$
\left|\chi R\left(f-f_{k}\right) \varphi_{n}\right|_{X} \leq \beta_{k}, \quad \lim _{k \rightarrow \infty} \beta_{k}=0 .
$$

The operator $R(f)$ commutes with the translation operators $\left(T_{n} \varphi\right)(t)=\varphi\left(t-a_{n}\right)$. Hence $\chi R\left(f_{k}\right) \varphi_{n}=$ $\chi R\left(f_{k}\right) T_{n} \varphi=\chi T_{n}\left(f_{k} * \varphi\right)$. Since $f_{k} * \varphi \in C_{0}^{\infty}$, it follows that the support of the function $T_{n}\left(f_{k} * \varphi\right)$ lies in a fixed neighborhood of $+\infty$ provided that $n \geq n_{k}$ and $n$ is sufficiently large. One can assume 
that $\chi=1$ in this neighborhood. Then $\chi R\left(f_{k}\right) \varphi_{n}=T_{n}\left(f_{k} * \varphi\right), n \geq n_{k}$. Combining this with (4.4.11) and (4.4.12), we deduce the inequality

$$
\left|T_{n}\left(f_{k} * \varphi\right)-\psi\right|_{X} \leq \alpha_{n}+\beta_{k}, \quad n \geq n_{k} .
$$

For any segment $I$ there exists a number $n_{k}^{\prime}$ such that $n_{k}^{\prime} \geq n_{k}$ and the support of the function $T_{n}\left(f_{k} * \varphi\right)$ does not intersect $I$. Then, taking into account (4.4.13) and the norm definitions in the spaces $X=L^{p}$ and $C^{\mu}$, we deduce the inequality

$$
|\psi|_{X(I)} \leq C\left(\alpha_{n}+\beta_{k}\right), \quad n \geq n_{k}^{\prime}
$$

where $C$ is a positive constant depending only on $I$. Pass to the limit as $n \rightarrow \infty$. Then pass to the limit as $k \rightarrow \infty$. We arrive at the relation $\psi=0$.

Assuming that $\psi=0$ in (4.4.13) and taking into account the fact that $T_{n}^{-1}$ are uniformly bounded in $\mathcal{L}(X)$, we obtain the estimate $\left|f_{k} * \varphi\right|_{X} \leq C\left(\alpha_{n}+\beta_{k}\right), n \geq k$. Pass to the limit as $n \rightarrow \infty$. Then pass to the limit as $k \rightarrow \infty$. We arrive at the validity of (4.4.10).

From (4.4.10), it follows that $f * \chi_{\varepsilon}=0$, where $\chi_{\varepsilon}$ is introduced by (4.2.6). By virtue of Lemma 4.2.1, passing to the limit as $\varepsilon \rightarrow 0$, we obtain that $f=0$.

To conclude, we provide the criterion of the Fredholm property for the following operators of the Wiener-Hopf type:

$$
N=1+\chi(R f) \chi
$$

where $f \in L^{1}(\mathbb{R})$ and $\chi(t)$ is a smooth function identically equal to $1(0)$ in a neighborhood of $t=-\infty$ $(t=+\infty)$. By virtue of (4.4.8), one of the factors $\chi$ on the right-hand side of (4.4.14) can be omitted since it does not affect the Fredholm property and the index $N$.

The Fredholm property is provided in terms of the Fourier transform of the function $f$. The vector case is also covered: the operator $N$ acts in the space of $l$-vector-functions on the line and $f(t)$ is an $l \times l$-matrix-function.

Theorem 4.4.3. The operator $N$ has the Fredholm property in the spaces $L^{p}(\mathbb{R})$ and $C^{\mu}(\mathbb{R})$ if and only if

If this holds, then

$$
\operatorname{det}[1+\hat{f}(s)] \neq 0, \quad s \in \mathbb{R}
$$

$$
\text { ind } N=-\left.\frac{1}{2 \pi} \arg \operatorname{det}[1+\hat{f}(s)]\right|_{-\infty} ^{\infty}
$$

Proof. Let $X$ denote any of the Banach spaces $L^{p}(\mathbb{R})$ and $C^{\mu}(\mathbb{R})$. Let $A$ denote the class of all continuous $l \times l$-matrix-functions of the kind

$$
x(s)=c+\hat{f}(s), \quad s \in \mathbb{R},
$$

where $f \in L^{1}(\mathbb{R})$ and $c \in \mathbb{C}^{l \times l}$. According to Sec. 1.5, this is a Banach algebra with respect to the pointwise operations and the "transported" norm $|x|=|c|+|f|_{L^{1}}$. Obviously, it is densely embedded into the Banach algebra $C$ of all matrix-functions $x(s)$ possessing the limit $x(\infty)=\lim x(s)$ as $s \rightarrow \infty$, endowed with the sup-norm.

In terms of the algebra $A$, the Wiener theorem from Sec. 4.3 can be reworded as follows: if $x(s) \in A$ and $\operatorname{det} x(s) \neq 0, s \in \mathbb{R} \cup\{\infty\}$, then the inverse matrix-function $x^{-1}(s)$ belongs to $A$. Taking into account Lemma 4.3.2, we conclude that the embedding $A \rightarrow C$ satisfies the condition of Theorem 1.4.2. According to this theorem, the conditions $x \in G_{0}(A)$ and $x \in G_{0}(C)$ are equivalent provided that $x \in G(A)$. On the other hand, we know from Sec. 1.4 that the unit component $G_{0}(C)$ can be described by the condition Ind $x=0$, where

$$
\text { Ind } x=\left.\frac{1}{2 \pi} \arg \operatorname{det} x(s)\right|_{-\infty} ^{\infty}
$$

is the Cauchy index. Hence, this is also valid for $A$. 
Using notation (4.4.17), to any function $x$ from $A$, assign the operator $\Psi x=\lambda+\chi(R f) \chi$. In particular, in this notation, the operator (4.4.14) is equal to $\Psi(1+\hat{f})$. By virtue of (4.4.8), the relations $\chi R(f) \sim R(f) \chi \sim \chi R(f) \chi$ hold modulo $\mathcal{T}(X)$ of compact operators. This easily implies that $\Psi x \Psi y \sim \Psi(x y)$ for all $x$ and $y$ from $A$. In other words, the linear bounded map $\Psi: A \rightarrow \mathcal{L}(X)$ satisfies the condition of Theorem 1.4.3. From this theorem, it follows that the condition (4.4.15) is sufficient for the Fredholm property of the operator $N=\Psi x, x=1+\hat{f}$, and the relation Ind $x=0$ implies the relation ind $N=0$.

To verify the relation (4.4.16), take a diagonal matrix-function $a$ from $G(A)$ of the kind

$$
a(s)=\operatorname{diag}\left(\frac{s-i}{s+i}, 1, \ldots, 1\right)
$$

such that Ind $a=1$. From (4.3.18), it follows that

$$
\begin{gathered}
(s-i)(s+i)^{-1}=1-2 \hat{f}_{+}(s), \quad(s+i)(s-i)^{-1}=1-2 \hat{f}_{-}(s), \\
f_{+}(t)=\left\{\begin{array}{ll}
0, & t>0, \\
e^{t}, & t \geq 0,
\end{array} \quad f_{-}(t)= \begin{cases}e^{-t}, & t \leq 0, \\
0, & t<0 .\end{cases} \right.
\end{gathered}
$$

The integer-values function Ind from (4.4.18) possesses the group property Ind $x y=\operatorname{Ind} x+\operatorname{Ind} y$. Therefore, if $x \in G(A)$ and $m=\operatorname{Ind} x$, then $\operatorname{ind}\left(x a^{-m}\right)=0$ and, therefore, $x a^{-m} \in G_{0}(A)$. Thus, taking into account Theorem 1.4.3, we have the relation $0=$ ind $\Psi\left(x a^{-m}\right)=$ ind $\Psi x-m$ ind $\Psi a$. Substituting the value $m=$ Ind $x$ to the last relation, we obtain the relation ind $\Psi x=($ ind $\Psi a)$ Ind $x$. Hence, the proof of (4.4.16) is reduced to the proof of the relation

$$
\text { ind } \Psi a=-1 \text {. }
$$

For any diagonal operator matrix, its index is equal to the sum of indices of its diagonal elements. Hence, according to (4.4.19), without loss of generality, we can restrict the investigation to the scalar case, i.e., the case where $l=1$. Then $a(s)=(s-i) /(s+i)$ and, in notation (4.4.20), the operators $N_{ \pm}=\Psi\left(a^{ \pm 1}\right)$ are defined by the relation

$$
N_{ \pm}=1-2 \chi\left(R f_{ \pm}\right) \chi .
$$

In the same way, define $\tilde{N}_{ \pm}$for a step function $\tilde{\chi}(t)$ equal to $1(0)$ for $t \leq 0(t>0)$. Since $f_{ \pm}(t) \equiv 0$ for $\pm t \geq 0$, it follows that

$$
\tilde{\chi}\left(t_{0}\right) f_{-}\left(t_{0}-t\right)\left[( 1 - \tilde { \chi } ( t ) ] \equiv \left[\left(1-\tilde{\chi}\left(t_{0}\right)\right] f_{+}\left(t_{0}-t\right) \tilde{\chi}(t) \equiv 0\right.\right.
$$

on the whole plane. Hence, we have the relation

$$
\tilde{\chi}\left(R_{-} f\right)(1-\tilde{\chi})=(1-\tilde{\chi})\left(R_{+} f\right) \tilde{\chi}=0 .
$$

According to $(4.4 .20)$, the product $\left(1-2 \hat{f}_{+}\right)\left(1-2 \hat{f}_{-}\right)$is equal to 1 , whence $f_{+}+f_{-}=2 f_{+} * f_{-}$and, therefore,

$$
\tilde{N}_{-} \tilde{N}_{+}=1-4 \tilde{\chi}\left(R f_{-}\right)\left(R f_{+}\right) \tilde{\chi}+4 \tilde{\chi}\left(R f_{-}\right) \tilde{\chi}\left(R f_{+}\right) \tilde{\chi}
$$

By virtue of (4.4.23), this implies the relation

$$
\tilde{N}_{-} \tilde{N}_{+}=1 .
$$

In the space $X=L^{p}$, the difference between the operators $N_{ \pm}$and $\tilde{N}_{ \pm}$is compact. It follows from (4.4.24) that $\operatorname{Im} \tilde{N}_{-}=X$. On the other hand, the kernel ker $\tilde{N}_{-}$is described explicitly. If $\varphi-2 \tilde{\chi}\left(R f_{-}\right) \tilde{\chi} \varphi=0$, then $\varphi\left(t_{0}\right)=0$ for $t_{0}>0$, while

$$
\varphi\left(t_{0}\right)=2 \int_{-\infty}^{0} f_{-}\left(t_{0}-t\right) \varphi(t) d t=2 \int_{-\infty}^{t_{0}} e^{t-t_{0}} \varphi(t) d t
$$


for $t_{0} \leq 0$ due to (4.4.20). Hence, the function $y(t)=e^{t} \varphi(t)$ satisfies the differential equation $y^{\prime}=2 y$ on the semiaxis $(-\infty, 0)$, whence there exists a constant $c$ such that $\varphi(t)=c e^{t}$ for $t \leq 0$. Thus, the kernel ker $\tilde{N}_{-}$is one-dimensional and ind $\tilde{N}_{-}=1$. Thus, the relation (4.4.21) is proved for $X=L^{p}$.

In the case where $X=C^{\mu}(\mathbb{R})$, the above argument is to be changed since the operation of the multiplying by the function $\tilde{\chi}$ yields the product outside $X$ in this case. Let $\tilde{X}$ denote the extension of $X$ obtained as follows: we add functions constant on the semiaxes $\pm t \geq 0$; note that $\operatorname{dim} \tilde{X}=\operatorname{dim} X+1$. From (4.4.20), it is clear that the convolution $\left(R f_{ \pm}\right) \tilde{\chi}=f_{ \pm} * \tilde{\chi}$ belongs to $C^{0,1}(\mathbb{R})$. Therefore, the operators $R f_{ \pm}$boundedly map $\tilde{X}$ to $X$ and, therefore, the operator $N_{ \pm}$treated as an operator in $\tilde{X}$ has the same index as the operator $N_{ \pm}$treated as an operator in $X$. On the other hand, the operator $\tilde{N}_{ \pm}$belongs to $\mathcal{L}(\tilde{X})$ and the difference between it and $N_{ \pm}$from $\mathcal{L}(\tilde{X})$ is compact. Since ind $\tilde{N}_{ \pm}=\mp 1$ (as above), the validity of the relation (4.4.21) also for the space $X=C^{\mu}$ follows.

To complete the proof of the theorem, it remains to verify that the condition (4.4.15) is necessary for the Fredholm property of the operator (4.4.14). Assume the converse: there exists $x$ from $A$ such that $N=\Psi x$ is a Fredholm operator, but there exists a real $s_{0}$ such that $\operatorname{det} x\left(s_{0}\right)=0$.

Let $\mathcal{R}$ be the subalgebra of rational functions in $C$; according to 4.3 .2 , it is dense in $A$. Thus, there exists a sequence of real numbers $x_{n}$ converging to $x$ in $A$. Since the operator $\Psi: A \rightarrow \mathcal{L}(X)$ is continuous, it follows that $\Psi x_{n} \rightarrow \Psi x$ in $\mathcal{L}(X)$ and, by virtue of Theorem 1.3.1, the operators $\Psi x_{n}$ possess the Fredholm property provided that $n$ is sufficiently large. Since $x_{n}\left(s_{0}\right) \rightarrow 0$ as $n \rightarrow \infty$, it follows that there exists a sequence of eigenvalues $\lambda_{n}$ of the matrix $x_{n}\left(s_{0}\right)$ from $\mathbb{C}^{l \times l}$ converging to zero. Therefore, changing $x_{n}(s)$ for $x_{n}(s)-\lambda_{n}$, one can assume (without loss of generality) that the original function $x(s)$ belongs to $\mathcal{R}$.

For simplicity, assume that $s_{0}$ is the only $k$-multiple zero of the function $\operatorname{det} x(s)$. By the condition, there exists a nonzero vector $\xi$ from $\mathbb{C}^{l}$ such that $x\left(s_{0}\right) \xi=0$. Let the matrix $p$ from $\mathbb{C}^{l \times l}$ be the projector of $\mathbb{C}^{l}$ on the one-dimensional subspace spanned by the vector $\xi$. Then $x\left(s_{0}\right) p=0$ and, therefore, the function $x_{1}(s)=x(s)\left[r^{-1}(s) p+1-p\right]$, where $r(s)=\left(s-s_{0}\right) /(s+i)$, is analytic in a neighborhood of the point $s_{0}$. However, $\operatorname{det} x_{1}(s)=r^{-1}(s) \operatorname{det} x(s)$ and, therefore, the multiplicity of the zero of the function $x_{1}$ is equal to $k-1$. Repeating this procedure, we represent the matrix-function $x(s)$ by the product

$$
x(s)=x_{0}(s)\left[r(s) p_{1}+1-p_{1}\right] \cdots\left[r(s) p_{k}+1-p_{k}\right], r(s)=\frac{s-s_{0}}{s+i},
$$

where the factor $x_{0}$ is invertible in $A$, while $p_{j}$ from $\mathbb{C}^{l \times l}$ are one-dimensional projectors.

Replace $s_{0}$ by $s_{0} \pm i / n, n=1,2 \ldots$, in this expansion and denote the obtained value by $x_{n}^{ \pm}$. Then $x_{n}^{ \pm}$ are invertible in $A$ and $x_{n}^{ \pm} \rightarrow x$ in $A$ as $n \rightarrow \infty$. From Definition (4.4.18) and the argument principle for analytic functions, it follows that

$$
\operatorname{Ind} x_{n}^{+} \neq \operatorname{Ind} x_{n}^{-} \text {. }
$$

By virtue of the continuity of $\Psi$, the sequence of the operators $\Psi x_{n}^{ \pm}$tends to $\Psi x$ in $\mathcal{L}(X)$. Hence, due to Theorem 1.3.1, for sufficiently large $n$, they are Fredholm operators and their indices coincide with ind $\Psi x$. On the other hand, due to the index relation proved above, we have the relation ind $\Psi x_{n}^{ \pm}=-$Ind $x_{n}^{ \pm}$. Thus, we arrive at a contradiction with (4.4.25), which completes the proof of the theorem.

A detailed presentation of the theory of Wiener-Hopf operators can be found, e.g., in [23].

\subsection{Multiplicative Convolutions on the Semiaxis}

Consider the homogeneous spaces $C_{0}^{\mu}\left(\mathbb{R}_{+}\right), C_{0}^{1, \mu}\left(\mathbb{R}_{+}\right)$, and $L_{0}^{p}\left(\mathbb{R}_{+}\right)$on the semiaxis $\mathbb{R}_{+}=(0, \infty)$ of the real line and the corresponding weight spaces $X_{\lambda}\left(\mathbb{R}_{+} ; 0, \infty\right)$, where $X$ denotes any of the symbols $C^{\mu}, C^{1, \mu}$, and $L^{p}$, with the weight order $\lambda=\left(\lambda_{\tau}, \tau=0, \infty\right)$. Thus,

$$
X_{\lambda}\left(\mathbb{R}_{+} ; 0, \infty\right)=\rho_{\lambda} X_{0}\left(\mathbb{R}_{+}\right), \quad \rho_{\lambda}(t)=t^{\lambda_{0}}(1+t)^{\lambda_{\infty}-\lambda_{0}} .
$$


For $\lambda_{0}=\lambda_{\infty}$, i.e., for $\lambda \in \mathbb{R}$, this space is denoted by $X_{\lambda}=X_{\lambda}\left(\mathbb{R}_{+}\right)$and (4.5.1) passes to $X_{\lambda}=t^{\lambda} X_{0}$. Due to Lemma 2.8 .1 , the space $C_{\mu}^{\mu}\left(\mathbb{R}_{+}\right)$can be defined by the equivalent norm

$$
|\varphi|_{C_{\mu}^{\mu}}=\sup _{t>0} t^{-\mu}|\varphi(t)|+[\varphi]_{\mu} .
$$

According to (4.1.10), the norm of the space $L_{\lambda}^{p}$ can be defined by the relation

$$
|\varphi|_{L_{\lambda}^{p}}=\left(\int_{0}^{\infty} t^{-p \lambda-1}|\varphi(t)|^{p} d t\right)^{1 / p} .
$$

Using the next lemma, one can describe the general space given by (4.5.1) in terms of the spaces $X_{\lambda}$, $\lambda \in \mathbb{R}$.

Lemma 4.5.1. Let $X$ denote $C^{\mu}, L^{p}$, or $C^{1, \mu}$. Then

$$
X_{\lambda_{1}} \cap X_{\lambda_{2}}=X_{\lambda^{+}}\left(\mathbb{R}_{+} ; 0, \infty\right), \quad X_{\lambda_{1}}+X_{\lambda_{2}}=X_{\lambda^{-}}\left(\mathbb{R}_{+} ; 0, \infty\right),
$$

where

$$
\lambda_{\tau}^{+}=\left\{\begin{array}{ll}
\max \left(\lambda_{1}, \lambda_{2}\right), & \tau=0, \\
\min \left(\lambda_{1}, \lambda_{2}\right), & \tau=\infty,
\end{array} \lambda_{\tau}^{-}= \begin{cases}\min \left(\lambda_{1}, \lambda_{2}\right), & \tau=0, \\
\max \left(\lambda_{1}, \lambda_{2}\right), & \tau=\infty,\end{cases}\right.
$$

and the following embeddings of Banach spaces hold:

$$
X_{\lambda_{1}} \cap X_{\lambda_{2}} \subseteq X_{\lambda} \subseteq X_{\lambda_{1}}+X_{\lambda_{2}}, \quad \lambda_{1} \leq \lambda \leq \lambda_{2} .
$$

Proof. For definiteness, we prove the claim of the lemma for the case where $X=L^{p}$. For the given weight order $\lambda=\left(\lambda_{0}, \lambda_{\infty}\right)$, the space $L_{\lambda}^{p}\left(\mathbb{R}_{+} ; 0, \infty\right)$ can be described by means of the restrictions of its elements $\varphi$ to the intervals $J^{0}=(0,1)$ and $J^{1}=(1, \infty)$ as follows:

$$
\left.\varphi\right|_{J^{0}} \in L_{\lambda_{0}}^{p}\left(J^{0}, 0\right),\left.\quad \varphi\right|_{J^{1}} \in L_{\lambda_{\infty}}^{p}\left(J^{1}, \infty\right) .
$$

The norm equal to the sum of the norms of the functions $\left.\varphi\right|_{J^{k}}$ in the specified spaces is equivalent to the norm of $\varphi$ in $L_{\lambda}^{p}\left(\mathbb{R}_{+} ; 0, \infty\right)$. The family $\left\{L_{\nu_{0}}^{p}\left(J^{0}, 0\right)\right\}$ is a monotonously decreasing function of the variable $\lambda_{0}$ with respect to the embedding of Banach spaces. The family $\left\{L_{\lambda_{\infty}}^{p}\left(J^{1}, \infty\right)\right\}$ is a monotonously increasing function of the variable $\lambda_{\infty}$ with respect to the embedding of Banach spaces. Taking into account Lemma 1.1.2 on the definition of the norm in the space $X_{\lambda_{1}}+X_{\lambda_{2}}$, we immediately deduce both assertions of the lemma.

By virtue of Theorem 2.7.2, Theorem 2.9.2, and Theorem 4.1.1, the weight exponential transformation $E_{\lambda}$ acting according to the relation

$$
\left(E_{\lambda} \varphi\right)(-\ln t)=t^{\lambda} \varphi(t), \quad t>0,
$$

isomorphically maps the Banach space $X_{\lambda}\left(\mathbb{R}_{+}\right)$to the Banach space $X(\mathbb{R})$.

For functions defined on the semiaxis, the multiplicative convolution is defined by the integral

$$
(f \diamond \varphi)\left(t_{0}\right)=\int_{0}^{\infty} f\left(\frac{t_{0}}{t}\right) \varphi(t) \frac{d t}{t}, \quad t_{0}>0 .
$$

This convolution passes to the additive one under substitution (4.5.6), i.e.,

$$
E_{\lambda}(f \diamond g)=\left(E_{\lambda} f\right) *\left(E_{\lambda} g\right) .
$$

In particular, for the multiplicative convolution, the estimate (4.2.4) takes the form

$$
|f \diamond \varphi|_{X_{\lambda}} \leq|f|_{L_{\lambda}^{1}}|\varphi|_{X_{\lambda}}, \quad X=L^{p}, C^{\mu} .
$$

On the semiaxis, the analog of the singular function defined by (4.2.15) is as follows:

$$
s(t)=\frac{1}{\pi i} \frac{\chi(t)}{1-t}, \quad \chi \in C_{0}^{\infty}\left(\mathbb{R}_{+}\right), \quad \chi(1)=1 .
$$


Indeed, if we endow (temporarily) the functions $s$ and $\chi$ in (4.2.15) with the symbol $\mathbb{R}$ and apply the substitution $t^{-\lambda} s(t)=s_{\mathbb{R}}(\ln t)$, then the functions $\chi$ and $\chi_{\mathbb{R}}$ are related as follows:

$$
\chi(t)=\frac{t^{\lambda}(t-1)}{\ln t} \chi_{\mathbb{R}}(t)
$$

hence, $\chi$ satisfies the conditions (4.5.10).

Thus, taking to account (4.5.8), we see that Theorem 4.2.2 applied to the multiplicative convolution with the function (4.5.10) remains valid also for the spaces $C_{\lambda}^{\mu}$ and $L_{\lambda}^{p}(p>1)$.

The substitution (4.5.6) maps $L^{(1)}(\mathbb{R})$ onto the corresponding space $L_{\lambda}^{(1)}\left(\mathbb{R}_{+}\right)$and the space $L_{\lambda}^{1, p}\left(\mathbb{R}_{+}\right)$ has a similar meaning. It is obvious that $L_{\lambda}^{1, p}=t^{\lambda} L_{0}^{1, p}$ and the space $L_{0}^{1, p}$ can be described (similarly to $(4.2 .17))$ by the condition

$$
|\varphi|_{L_{\lambda}^{1, p}}=\sum_{i=-\infty}^{+\infty} 2^{i \lambda} \mid \varphi\left(\left.2^{-i} t\right|_{L^{p}(I)}, \quad I=[1 / 2,1] .\right.
$$

Respectively, Lemma 4.2.3 can also be reworded for the case considered, i.e., the space $L_{\lambda}^{1, p}, p>1$, is embedded into $L_{\lambda}^{(1)}$ and contains all functions $g$ from $L_{l o c}^{p}(\mathbb{R})$ such that

$$
\int_{0}^{\infty}(1+|\ln t|)^{\alpha} t^{-p \lambda-1}|f(t)|^{p} d t<\infty, \quad \alpha>p .
$$

Further, consider the multiplicative variant of the convolution-type integral operators

$$
[K(f) \varphi]\left(t_{0}\right)=\int_{0}^{\infty} k\left(t_{0}, t\right) f\left(\frac{t_{0}}{t}\right) \varphi(t) \frac{d t}{t}, \quad t_{0}>0,
$$

defined by an $L_{\lambda}^{1}$-function $f$, and the corresponding singular operator $K(s)$. As above, for these operators, Theorems 4.4.1 and 4.4.2 and Lemma 4.4.1 can be reworded as follows.

\section{Theorem 4.5.1.}

(a) Let a function $\widetilde{k}\left(s_{0}, s\right)=k\left(e^{s_{0}}, e^{s}\right)$ belong to $C^{\nu}(\mathbb{R} \times \mathbb{R})$, where $0<\nu<1$ and $\lambda \in \mathbb{R}$. Then the operators $K(f), f \in L_{\lambda}^{1}$, and $K(s)$ are bounded in the spaces $L_{\lambda}^{p}, 1<p<\infty$, and $C_{\lambda}^{\mu}, 0<\mu<\nu$, and their norms admit the estimates

$$
|K(f)|_{\mathcal{L}\left(X_{\lambda}\right)} \leq|k|_{C^{\mu}}|f|_{L_{\lambda}}, \quad|K(s)|_{\mathcal{L}\left(X_{\lambda}\right)} \leq|k|_{C^{\nu}} \quad \text { for } X=L^{p} \text { or } X=C^{\mu} .
$$

If the condition

$$
\lim _{\substack{t \rightarrow 0, 1 / n \leq t_{0} / t \leq n}} k\left(t_{0}, t\right)=\lim _{\substack{t \rightarrow \infty, 1 / n \leq t_{0} / t \leq n}} k\left(t_{0}, t\right)=0, \quad n=1,2, \ldots,
$$

is satisfied, then the operator $K(f)$ is compact in these spaces. If the said condition is satisfied and $k(t, t) \equiv 0$, then $K(s)$ possesses the same property.

(b) If $\widetilde{k}\left(s_{0}, s\right)=k\left(e^{s_{0}}, e^{s}\right) \in C^{1, \nu}(\mathbb{R} \times \mathbb{R})$, then the operators $K(f)$ and $K(s)$ are bounded in the space $C^{1, \mu}$. If the corresponding conditions of $(a)$ are satisfied for the functions $k\left(t_{0}, t\right)$ and

then these operators are compact.

$$
k^{1}\left(t_{0}, t\right)=t_{0} \frac{\partial k}{\partial t_{0}}+t \frac{\partial k}{\partial t}
$$

(c) Let $k\left(t_{0}, t\right)=a\left(t_{0}\right) a(t)$, where $a \in C_{0}^{\nu}\left(\mathbb{R}_{+}\right), \nu>\mu$, the limits

$$
a(0)=\lim _{t \rightarrow 0} a(t), \quad a(\infty)=\lim _{t \rightarrow \infty} a(t)
$$

exist, and at least one of these limits is different from zero. Then the compactness of the operator $K(f), f \in L_{\lambda}^{1}$, in the space $L_{\lambda}^{p}$ or $C_{\lambda}^{\mu}$ implies that $f=0$. 
Similarly to (4.5.11), the condition $\widetilde{k}\left(s_{0}, s\right)=k\left(e^{s_{0}}, e^{s}\right) \in C^{\nu}(\mathbb{R} \times \mathbb{R})$ of this theorem can be expressed in the form

$$
\sup _{i, j=0, \pm 1, \ldots}\left|k\left(2^{-i} t_{0}, 2^{-j} t\right)\right|_{C^{\nu}(I \times I)}<\infty, \quad I=[1 / 2,2]
$$

(no exponential substitution is used). According to Lemma 2.7.1, this condition is satisfied for any function $k\left(t_{0}, t\right)$ from $C_{0}^{\nu}\left(\mathbb{R}_{+} \times \mathbb{R}_{+}\right)$. In particular, as in the case of Lemma 2.7.1, we verify that the last class contains any bounded function $k\left(t_{0}, t\right)$ from $C^{1}\left(\mathbb{R}_{+} \times \mathbb{R}_{+}\right)$such that

$$
\left|\left(t_{0}+t\right) \frac{\partial k}{\partial t_{0}}\right|+\left|\left(t_{0}+t\right) \frac{\partial k}{\partial t}\right| \leq C, \quad t_{0}>0, t>0 .
$$

For any function $f$ from $L_{\nu}\left(\mathbb{R}_{+}\right)$, its Mellin transform is defined as follows:

$$
(\mathcal{M} f)(\zeta)=\int_{0}^{\infty} t^{-\zeta-1} f(t) d t, \quad \operatorname{Re} \zeta=\lambda .
$$

Comparing this relation with (4.3.1) and (4.5.6), we see that

$$
\left(P_{\lambda} f\right)^{\wedge}\left(i t_{0}\right)=(\mathcal{M} f)\left(\lambda+i t_{0}\right) .
$$

In the same way, in the considered case, the the inversion formula from Sec. 4.3 takes the form

$$
f(t)=\frac{1}{2 \pi i} \int_{\lambda-i \infty}^{\lambda+i \infty} t^{\zeta}(\mathcal{M} f)(\zeta) d \zeta, \quad t>0,
$$

where the function $\mathcal{M f}$ is assumed to be summable and the relation itself is assumed to be a.e. valid.

Relation (4.5.16) shows that the transformation $\mathcal{M}$ embeds the convolution algebra $L_{\lambda}^{(1)}\left(\mathbb{R}_{+}\right)$into the algebra of continuous on the line $\operatorname{Re} \zeta=\lambda$ functions vanishing at infinity. In Sec. 1.7, this algebra is denoted by $C^{0}[\lambda]$. The algebra $C[\lambda]$ of continuous bounded functions has a similar meaning. Using the transformation $x(\zeta) \rightarrow \tilde{x}(t)=x(\lambda+i t)$, one can "transport" the Banach algebras $M^{0}$ and $M$ from the real line to the line $\operatorname{Re} \zeta=\lambda$ such that their norms are preserved under this transport. These algebras are denoted by $M^{0}[\lambda]$ and $M[\lambda]$ respectively. Also, the relation (4.5.16) shows that $M^{0}[\lambda]$ is the image of $L_{\lambda}^{(1)}\left(\mathbb{R}_{+}\right)$under the transformation $\mathcal{M}$ with the transported norm and the norm in $M[\lambda]$ is defined by the relation

$$
|x|=\sup |x y|_{M^{0}[\lambda]},
$$

where sup is taken over all $y$ from the unit ball of $M^{0}[\lambda]$. It is obvious that an estimate similar to (4.3.20) is preserved for this norm. As in the case of the real line, elements of the Banach algebra $M[\lambda]$ are called $L_{\lambda}^{(1)}$-multipliers.

For the Mellin transformation, the analog of the relation $e^{-a s} \hat{f}(s)=[f(t-a)]^{\wedge}(s)$ is the relation

$$
\delta^{\zeta}(\mathcal{M} f)(\zeta)=\mathcal{M}[f(\delta t)](\zeta)
$$

meaning that the function $\delta^{\zeta}$ is a multiplier. The closure of the set of finite linear combinations of such functions with respect to norm (4.5.18) is the subalgebra $M^{1}[\lambda] \subseteq M[\lambda]$ of almost periodic functions.

For any $f$ from $C_{0}^{\infty}\left(\mathbb{R}_{+}\right)$, the relation (4.5.15) defines an entire function on the complex plane $\zeta$. Using Lemma 4.5.1, one can easily verify that the class $C_{0}^{\infty}\left(\mathbb{R}_{+}\right)$is dense in the space $L_{\lambda_{1}}^{(1)} \cap L_{\lambda_{2}}^{(1)}=$ $L_{\lambda^{+}}^{(1)}\left(\mathbb{R}_{+} ; 0, \infty\right)$. Therefore, if $\lambda_{1}<\lambda_{2}$, then the Mellin transformation embeds the convolution algebra $L_{\lambda^{+}}^{(1)}$ into the Banach algebra $C^{0}\left[\lambda_{1}, \lambda_{2}\right]$ of functions continuous in the band $\left[\lambda_{1}, \lambda_{2}\right]=\left\{\lambda_{1} \leq \operatorname{Re} \zeta \leq \lambda_{2}\right\}$, analytic inside this band, and vanishing at infinity. This algebra and the similar algebra $C\left[\lambda_{1}, \lambda_{2}\right]$ are introduced in Sec. 1.7. As in the case of the line, $M^{0}\left[\lambda_{1}, \lambda_{2}\right]$ denotes the image of $L_{\lambda^{+}}^{(1)}$ under the Mellin transformation and $M\left[\lambda_{1}, \lambda_{2}\right] \subseteq C\left[\lambda_{1}, \lambda_{2}\right]$ denotes the Banach algebra of multipliers with the 
norm similar to (4.5.18). The subalgebra $M^{1}\left[\lambda_{1}, \lambda_{2}\right] \subseteq M\left[\lambda_{1}, \lambda_{2}\right]$ of almost periodic functions has a similar meaning.

For example, the integral (4.5.15) is singular for the function (4.5.10), and the Mellin transform $(\mathcal{M s})(\zeta)$ of the function (4.5.10) belongs to $M\left[\lambda_{1}, \lambda_{2}\right]$. Similarly to Lemma 1.8.3, it is justified that the function $(\mathcal{M} s)(\zeta)$ is analytic on the whole plane and its derivative coincides with the Mellin transform of the function $f(t)=(-\ln t) s(t)$ belonging to $C_{0}^{\infty}\left(\mathbb{R}_{+}\right)$. According to Lemma 4.3.1, it belongs to $C\left[\lambda_{1}, \lambda_{2}\right]$ and tends to \pm 1 as $\operatorname{Im} \zeta \rightarrow \pm \infty$ uniformly in the band $\left[\lambda_{1}, \lambda_{2}\right]$. Theorem 4.2.2 for the multiplicative convolution, combined with Lemma 4.5.1, means that the function $\mathcal{M} s$ belongs to $M\left[\lambda_{1}, \lambda_{2}\right]$.

Find the Mellin transform for several basic examples of functions $f$ from $L_{\lambda}^{1}\left(\mathbb{R}_{+}\right)$. Assign

$$
f_{1}(t)=s(t)-\frac{1}{\pi i} \frac{1}{1-t}, \quad f_{2}(t)=\frac{1}{\pi i} \frac{1}{1+w t} \quad \text { for } t>0,
$$

where the complex number $w$ does not belong to $\mathbb{R} \backslash \mathbb{R}_{+}$. Taking into account (4.5.10), we conclude that the functions $f_{k}$ are infinitely differentiable on $\mathbb{R}_{+}$and

$$
\int_{0}^{\infty}(1+|\ln t|)^{\alpha} t^{-\lambda-1}\left|f_{k}(t)\right|^{q} d t<\infty, \quad k=0,1,
$$

provided that $\alpha>0, q>0$, and $-1<\lambda<0$. Hence, due to criterion (4.5.11), these functions belong to $L_{\lambda}^{q, 1}$ and, therefore, $\mathcal{M} f_{1} \in M^{0}\left[\lambda_{1}, \lambda_{2}\right]$ and $\mathcal{M} f_{2} \in M\left[\lambda_{1}, \lambda_{2}\right]$ for $-1<\lambda_{1}<\lambda_{2}$. The explicit representation is as follows (see [14]):

$$
\begin{array}{ll}
\mathcal{M}\left(\frac{1}{\pi i} \frac{1}{1-t}\right)(\zeta)=\frac{1}{i} \cot \pi \zeta, & -1<\operatorname{Re} \zeta<0, \\
\mathcal{M}\left(\frac{1}{\pi i} \frac{1}{1+w t}\right)(\zeta)=\frac{1}{i} \frac{w^{\zeta}}{\sin \pi \zeta}, & -1<\operatorname{Re} \zeta<0,
\end{array}
$$

where the branch of the power function $w^{\zeta}$ is fixed by the condition $|\arg w|<\pi$.

In the same way, we take into account relations

$$
\begin{array}{ll}
\frac{1}{n !} \int_{0}^{1}(\ln t)^{n} t^{\zeta_{0}-\zeta-1} d t=-\frac{1}{\left(\zeta-\zeta_{0}\right)^{n+1}}, \quad \operatorname{Re} \zeta>\operatorname{Re} \zeta_{0}, \\
\frac{1}{n !} \int_{1}^{\infty}(\ln t)^{n} t^{\zeta_{0}-\zeta-1} d t=\frac{1}{\left(\zeta-\zeta_{0}\right)^{n+1}}, \quad \operatorname{Re} \zeta<\operatorname{Re} \zeta_{0},
\end{array}
$$

where $n=0,1, \ldots$, to verify that the function $x(\zeta)=\left(\zeta-\zeta_{0}\right)^{-n-1}$ belongs to $M^{0}\left[\lambda_{1}, \lambda_{2}\right]$ provided that the band $\left[\lambda_{1}, \lambda_{2}\right]$ does not contain the point $\zeta_{0}$. Thus, any rational function vanishing at infinity and such that its poles are located outside the said band belongs to $M^{0}\left[\lambda_{1}, \lambda_{2}\right]$. Relations (4.5.21) show that, for any two points $\zeta_{1}$ and $\zeta_{2}$ satisfying the condition $\operatorname{Re} \zeta_{1}<\lambda_{1}<\lambda_{2}<\operatorname{Re} \zeta_{2}$, the class of functions $x(\zeta)$ of the kind $x(\zeta)=p(\zeta)\left(\zeta-\zeta_{1}\right)^{-n_{1}}\left(\zeta-\zeta_{2}\right)^{-n_{2}}$, where $n_{j}$ are arbitrary nonnegative integers and the power of the polynomial $p(\zeta)$ is less than $n_{1}+n_{2}$, is dense in the Banach algebra $M^{0}\left[\lambda_{1}, \lambda_{2}\right]$.

To conclude, we provide the following property of the Banach algebra $M^{0}\left[\lambda_{1}, \lambda_{2}\right]$.

Lemma 4.5.2. Let the restriction of a function $x(\zeta)$ from $C\left[\lambda_{1}, \lambda_{2}\right]$ to the boundary lines of the band belong to $M^{0}\left[\lambda_{j}\right], j=0,1$. Then $x \in M^{0}\left[\lambda_{1}, \lambda_{2}\right]$.

Proof. By the condition, there exist $f_{j}$ from $L_{\lambda_{j}}^{q, 1}, j=1,2$, such that

$$
x(\zeta)=\left(\mathcal{M} f_{j}\right)(\zeta), \quad \operatorname{Re} \zeta=\lambda_{j} .
$$


According to Lemma 4.5.1, it suffices to prove that $f_{1}=f_{2}$. Let us prove this for the case where $f_{j} \in L_{\lambda_{j}}$, i.e., for more general case.

First, we assume that $x \in C^{0}\left[\lambda_{1}, \lambda_{2}\right]$ and the functions $x(\zeta)$ are integrable on the lines $\operatorname{Re} \zeta=\lambda_{j}$. Then, for $f=f_{j}$ and $\lambda=\lambda_{j}$, the inversion formula (4.5.17) is valid:

$$
f_{j}(t)=\frac{1}{2 \pi i} \int_{\lambda_{j}-i \infty}^{\lambda_{j}+i \infty} t^{\zeta} x(\zeta) d \zeta, \quad t>0
$$

By the Cauchy theorem applied to the rectangle $P_{n}=\left\{\lambda_{\leq} \operatorname{Re} \zeta \leq \lambda_{2},|\operatorname{Im} \zeta| \leq n\right\}$, we have the relation

$$
\int_{\partial P_{n}} t^{\zeta} x(\zeta) d \zeta=0
$$

Since the integrals with respect to the horizontal segments tend to zero as $n \rightarrow \infty$, it follows that both integrals at the right-hand side of (4.5.23) are equal (after the passing to the limit).

In the general case, we use the construction of the convolution with the averaging kernel in the multiplicative variant. Namely, we take a nonnegative function $\chi$ from $C_{0}^{\infty}\left(\mathbb{R}_{+}\right)$such that

$$
\int_{0}^{\infty} \chi(t) \frac{d t}{t}=1
$$

It is obvious that the function $\chi_{\varepsilon}(t)=\varepsilon^{-1} \chi\left(t^{1 / \varepsilon}\right)$ possesses a similar property and its Mellin transform $\left(\mathcal{M} \chi_{\varepsilon}\right)(\zeta)$ is equal to $(\mathcal{M} \chi)(\varepsilon \zeta)$. Taking into account (4.5.22), we arrive at the relation

$$
(\mathcal{M} \chi)(\varepsilon \zeta) x(\zeta)=\left[\mathcal{M}\left(\chi_{\varepsilon} * f_{j}\right)\right](\zeta), \quad \operatorname{Re} \zeta=\lambda_{j} .
$$

Since the function at the left-hand side of this inequality belongs to $C^{0}\left[\lambda_{1}, \lambda_{2}\right]$ and is integrable on the lines $\operatorname{Re} \zeta=\lambda_{j}$, it follows from the assertions proved above that

$$
\chi_{\varepsilon} * f_{1}=\chi_{\varepsilon} * f_{2} .
$$

On the other hand, if $f \in L_{\lambda}\left(\mathbb{R}_{+}\right)$, then the convolution $\chi_{\varepsilon} * f$ tends to $f$ as $\varepsilon \rightarrow 0$ with respect to the norm of the space $L_{\lambda}$. Indeed, if $f \in C_{0}^{\infty}\left(\mathbb{R}_{+}\right)$, then the following relation holds:

$$
f\left(t_{0}\right)-\left(\chi_{\varepsilon} * f\right)\left(t_{0}\right)=\int_{0}^{\infty}\left[f\left(\frac{t_{0}}{t}\right)-f\left(t_{0}\right)\right] \chi_{\varepsilon}(t) \frac{d t}{t}=\int_{0}^{\infty}\left[f\left(\frac{t_{0}}{t^{\varepsilon}}\right)-f\left(t_{0}\right)\right] \chi(t) \frac{d t}{t} .
$$

Since there exists a segment $[\delta, 1 / \delta]$ such that the function $\chi$ vanishes outside it, it follows that the left-hand side of the last relation uniformly tends to zero. Therefore, it remains to verify that the convolution operators $R\left(\chi_{\varepsilon}\right)$ are uniformly bounded in $L_{\lambda}$. By virtue of (4.5.9), we have the estimate

$$
\left|R\left(\chi_{\varepsilon}\right)\right|_{\mathcal{L}\left(L_{\lambda}\right)} \leq\left|\chi_{\varepsilon}\right|_{L_{\lambda}}=\int_{0}^{\infty} t^{-\lambda-1} \chi_{\varepsilon}(t) d t=\int_{0}^{\infty} t^{-\varepsilon \lambda-1} \chi(t) d t
$$

which verifies that the claimed fact takes place indeed.

Thus, for both values $j=1,2$, the sequence of functions $\chi_{\varepsilon} * f_{j}$ from (4.5.24) converges to $f_{j}$ as $\varepsilon \rightarrow 0$ with respect to the norm of the space $L_{\lambda_{j}}$. According to Sec. 4.1, they contain subsequences a.e. converging to $f_{j}$, which yields the relation $f_{1}=f_{2}$ completing the proof of the lemma. 


\section{6. $\quad L^{p}$-Estimates of Integrals with Weak Singularities}

Assuming that $G$ is a measurable set of $\mathbb{R}^{k}, 0<\alpha<k$, and $a(x, y)$ is a bounded piecewise-continuous function, consider the following integral with a weak singularity:

$$
\psi(x)=\int_{G} \frac{a(x, y)}{|x-y|^{\alpha}} \varphi(y) d y .
$$

For $\varphi$ from $C(G)$ or from $C^{\mu}(G)$, this integral is considered in Sec. 1.9 and Sec. 3.2 respectively.

Theorem 4.6.1. Let $G$ be a bounded set and $\varphi \in L^{p}(G)$. Then the integral (4.6.1) defines a function $\psi$ from $L^{p}(G)$ such that

$$
|\psi|_{L^{p}} \leq|a|_{0}|\varphi|_{L^{p}}, \quad|a|_{0}=\sup _{x, y}|a(x, y)|,
$$

where $C$ is a positive constant depending only on $\alpha$ and the diameter $R$ of the set $G$.

If this holds and the function $a$ is continuous, then $K \varphi=\psi$ is a compact operator in $L^{p}$.

Proof. Consider the function

Obviously, it belongs to $L^{1}\left(\mathbb{R}^{k}\right)$ and

$$
f(x)= \begin{cases}|x|^{-\alpha}, & |x| \leq R \\ 0, & |x|>R .\end{cases}
$$

$$
|\psi(x)| \leq|a|_{0}|(f * \varphi)(x)|,
$$

i.e., the estimate (4.6.2) follows from (4.2.4).

To prove that the operator $K$ defined by (4.6.1) is compact under the assumption that $a \in C^{0}(G \times$ $G$ ), consider the sequence $\left\{f_{n}\right\}$ from $C_{0}^{\infty}\left(\mathbb{R}^{k}\right)$ converging to $f$ with respect to the norm of $L^{1}$. It is obvious that the operator $K_{n}$ acting according to the relation

$$
\left(K_{n} \varphi\right)(x)=\int_{G} a(x, y) f_{n}(x-y) \varphi(y) d y
$$

is compact in $L^{p}(G)$ and the difference $\left(K-K_{n}\right) \varphi$ satisfies the following estimate similar to (4.6.2):

$$
\left|\left(K-K_{n}\right) \varphi\right|_{L^{p}} \leq C|a|_{0}\left|f-f_{n}\right|_{L^{1}}|\varphi|_{L^{p}} .
$$

Therefore, the sequence $\left\{K_{n}\right\}$ tends to $K$ with respect to the operator norm, which means that the operator $K$ is also compact.

In [57] (see also [31]), the boundedness of the operators (4.6.1) in the space $L^{p}$ is investigated (for various values of $p$ ) in detail.

Consider the integral (4.6.1) with a weak singularity in the weight space $L_{\lambda}^{p}(G, F)$; the boundedness requirement for the measurable set $G$ is taken off now, but the function $a(x, y)$ is still assumed to be continuous and bounded on $G \times G$. Recall that if the set $G$ is unbounded, then the point $\infty$ is to be an element of $F$. According to Lemma 4.1.1, a sufficient condition of the summability of the function $|y-x|^{-\alpha} \varphi(y)$ with respect to $y$ in a small neighborhood of points $\tau$ from $F$ is as follows: $\lambda_{\tau}>-k$ for $\tau \neq \infty$ and $\lambda_{\infty}-\alpha<-k$ for $\tau=\infty$.

Theorem 4.6.2. Let

$$
\varphi \in L_{\lambda}^{p}(G, F), \quad-k<\lambda_{\tau}< \begin{cases}0, & \tau \neq \infty \\ \alpha-k, & \tau=\infty\end{cases}
$$

and the weight order $\nu$ satisfy the conditions

$$
\nu_{\tau}=\lambda_{\tau}, \tau \neq \infty, \quad \nu_{\infty}>k-\alpha+\lambda_{\infty} .
$$

Then the function $\psi$ defined by (4.6.1) belongs to the class $L_{\nu}^{p}(G, F)$ and the estimate

$$
|\psi|_{L_{\nu}^{p}} \leq C|a|_{0}|\varphi|_{L_{\lambda}^{p}}
$$


holds.

Proof. We use Theorem 4.6.1, which is also valid for bounded piecewise-continuous functions $a(x, y)$. Since the functions $\varphi(y)$ and $a(x, y)$ can be extended by zero to $\mathbb{R}^{k}$, it follows (without loss of generality) that $G=\mathbb{R}^{k}$ and $a=1$. Then

$$
\psi(x)=\int_{\mathbb{R}^{k}} \frac{\varphi(y) d y}{|x-y|^{\alpha}} .
$$

In this case, $\infty$ belongs to $F$.

Let $\chi_{\tau}$ be a continuous function identically equal to 1 in a neighborhood of $\tau$ and let its support be contained in $B_{\rho}(\tau)$ in notation (4.1.8). Then the function $\widetilde{\varphi}=\varphi-\sum_{\tau} \chi_{\tau} \varphi$ is identically equal to in a neighborhood of $F$. Therefore, if $\widetilde{\psi}$ is defined via $\widetilde{\varphi}$ similarly to (4.6.6), then, due to Theorem 4.6.1, the function $\widetilde{\psi}$ belongs to $L^{p}$ outside any neighborhood of the set $F$ and the corresponding norm estimate holds. This function is continuous in a neighborhood of the finite points $\tau$. Hence, taking into account (4.6.3), we conclude that it belongs to $L_{\lambda_{\tau}}^{p}\left(B_{\tau}, \tau\right)$. In a neighborhood of $\infty$, we have $\widetilde{\psi}(x)=O(1)|x|^{-\alpha}$. Hence, due to the inequality $-\alpha-\nu_{\infty}=-\left(\lambda_{\infty}+k\right)<0$ following from (4.6.3)-(4.6.4), the integral

$$
\int_{|x| \geq 1}|x|^{\left(-\alpha-\nu_{\infty}\right) p} \frac{d x}{|x|^{k}}
$$

is finite. Thus, $\widetilde{\psi} \in L_{\nu_{\tau}}^{p}\left(B_{\tau}, \tau\right)$ for $\tau=\infty$.

Thus, according to (4.1.8), the function $\widetilde{\psi}$ belongs to the space $L_{\lambda}^{p}\left(\mathbb{R}^{k}, F\right)$ and its norm satisfies the estimate (4.6.5). Therefore, our task is reduced to the consideration of the function $\chi_{\tau} \varphi$. Redenoting it by $\varphi$, one can assume that $\varphi=0$ outside $B_{\tau}$ and the function $\psi$ can be considered in $B_{\tau}$.

It is obvious that it suffices to consider the following two cases separately: the case where $\tau=0$ and the case where $\tau=\infty$. In the first case, $\lambda=\lambda_{0}$ according to (4.6.3) and, therefore, the condition $-k<\lambda<0$ is satisfied. Redenoting $|x|^{-\lambda} \varphi(x)$ by $\varphi(x)$ and doing the same with $\psi$, we reduce our task to the proof of the following assertion.

If $\varphi \in L_{0}^{p}(B, 0)$, then the integral

$$
\psi(x)=\int_{B}|x|^{-\lambda}|y|^{\lambda} \frac{\varphi(y) d y}{|y-x|^{\alpha}}, \quad x \in B
$$

belongs to $L_{0}^{p}(B, 0)$ in the domain $B=\{|y| \leq 1\}$ and the estimate

$$
|\psi|_{L_{0}^{p}} \leq C|\varphi|_{L_{0}^{p}}
$$

holds.

To prove this assertion, we argue as in Secs.3.10-3.11. Assigning $\varphi=0$ outside $B$, consider the sequence of functions

$$
\psi\left(2^{i} x\right)=2^{(k-\alpha) i}|x|^{-\lambda} \int_{|y| \leq 2^{-i}} \frac{|y|^{\lambda} \varphi\left(2^{i} y\right) d y}{|y-x|^{\alpha}}, \quad i=0,-1, \ldots,
$$

in the spherical layer $S=\{1 / 2 \leq|x| \leq 2\}$. Represent each such function as follows:

$$
\psi\left(2^{i} x\right)=2^{(k-\alpha) i}|x|^{-\lambda}\left[\psi_{i}^{0}(x)+\psi_{i}(x)+\psi_{i}^{1}(x)\right],
$$

where $\psi_{i}^{0}, \psi_{i}$, and $\psi_{i}^{1}$ are defined by the integrating over the domains $|y| \leq 1 / 4,1 / 4 \leq|y| \leq 4$, and $|y| \geq 4$ respectively.

By virtue of Theorem 4.6.1, we have the estimate

$$
\int_{1 / 2 \leq|x| \leq 2}\left|\psi_{i}(x)\right|^{p} d x \leq C \int_{1 / 2 \leq|x| \leq 2}\left|\varphi\left(2^{i} x\right)\right|^{p} d x,
$$


where $C$ is a positive constant independent of $i$. According to Theorem 4.1.1, this implies the inequality

$$
\left|\psi_{i}\right|_{L^{p}(S)} \leq C|\varphi|_{L_{0}^{p}}
$$

Since $|y-x| \geq 1 / 4$ for $|y| \leq 1 / 4$ and $|x| \geq 1 / 2$, we have the following obvious inequality for the function $\psi_{i}^{0}(x)$ :

$$
\left|\psi_{i}^{0}(x)\right| \leq 4^{\alpha} \int_{|y| \leq 1 / 4}|y|^{\lambda+k}\left|\varphi\left(2^{i} y\right)\right| \frac{d y}{|y|^{k}} .
$$

Combining it with the H'older inequality (4.1.6), we obtain the estimate

$$
\max _{S}\left|\psi_{i}^{0}(x)\right| \leq C|\varphi|_{L_{0}^{p}}
$$

Since $|y-x| \geq|y|-2 \geq|y| / 2$ for $x \in S$ and $|y| \geq 4$, we have (in the same way) the following inequality for the function $\psi_{i}^{1}(x)$ :

$$
\left|\psi_{i}^{1}(x)\right| \leq 2^{\alpha} \int_{4 \leq|y| \leq 2^{-i}}|y|^{\lambda+k-\alpha}\left|\varphi\left(2^{i} y\right)\right| \frac{d y}{|y|^{k}} .
$$

This yields the inequality

$$
\left|\psi_{i}^{1}(x)\right| \leq C\left(\int_{4 \leq|y| \leq 2^{-i}}|y|^{(\lambda+k-\alpha) q} \frac{d y}{|y|^{k}}\right)^{1 / q}|\varphi|_{L_{0}^{p}}
$$

where $q=p /(p-1)$ is the conjugate exponent. Since

$$
\int_{4 \leq|y| \leq 2^{-i}}|y|^{(\lambda+k-\alpha) q} \frac{d y}{|y|^{k}}=(\operatorname{mes} \Omega) \int_{4}^{2^{-i}} r^{(\lambda+k-\alpha) q-1} d r
$$

and the integral at the right-hand side of this relation does not exceed $\left(2^{-i(\lambda+k-\alpha) q}+4^{(\lambda+k-\alpha) q}\right)$, we have the following estimate for $\psi_{i}^{1}$ :

$$
\max _{S}\left|\psi_{i}^{1}(x)\right| \leq C 2^{-i(\lambda+k-\alpha)}|\varphi|_{L_{0}^{p}}
$$

Combining inequalities (4.6.10)-(4.6.12), we obtain the following estimate for sum (4.6.9):

$$
\left|\psi\left(2^{i} x\right)\right|_{L^{p}(S)} \leq C\left(2^{i(k-\alpha)}+2^{-i \lambda}\right)|\varphi|_{L_{0}^{p}}, \quad i \leq 0 .
$$

Since no term in the brackets exceeds 1 , it follows from Theorem 4.1.1 that the estimate (4.6.8) holds.

Consider the second case, i.e., assume that $\tau=\infty$. Again, denote $|x|^{-\lambda} \varphi(x)$ and $|x|^{-\nu} \psi(x)$ by $\varphi(x)$ and $\psi(x)$ respectively. We obtain the following (similar to (4.6.7)) integral in the domain $B=\{|y| \geq$ $1\}$ :

$$
\psi(x)=\int_{B}|x|^{-\nu}|y|^{\lambda} \frac{\varphi(y) d y}{|y-x|^{\alpha}}, \quad x \in B .
$$

According to (4.6.4), $\delta=\nu-(k-\alpha+\lambda)>0$ in the considered case. Hence, we have the following sequence in the spherical layer $S$ :

$$
\psi\left(2^{i} x\right)=2^{-\delta i}|x|^{-\nu} \int_{|y| \geq 2^{-i}} \frac{|y|^{\lambda} \varphi\left(2^{i} y\right) d y}{|y-x|^{\alpha}}, \quad i=0,1, \ldots
$$

As above, we decompose this expression into three terms as follows:

$$
\psi\left(2^{i} x\right)=2^{-\delta i}|x|^{-\nu}\left[\psi_{i}^{0}(x)+\psi_{i}(x)+\psi_{i}^{1}(x)\right]
$$


where $\psi_{i}^{0}, \psi_{i}$, and $\psi_{i}^{1}$ are defined by the integrating with respect to the domains $|y| \leq 1 / 4,1 / 4 \leq$ $|y| \leq 4$, and $|y| \geq 4$ respectively.

The terms $\psi_{i}$ and $\psi_{i}^{0}$ still satisfy the estimates (4.6.10)-(4.6.11). The third one satisfies the estimate

$$
\left|\psi_{i}^{1}(x)\right| \leq C\left(\int_{|y| \geq 4}|y|^{(\lambda+k-\alpha) q} \frac{d y}{|y|^{k}}\right)^{1 / q}|\varphi|_{L_{0}^{p}},
$$

where $q=p /(p-1)$ is the conjugate exponent. By the condition (4.6.3), the exponent $\lambda+k-\alpha$ is negative for $\tau=\infty$. Hence, the integral is well defined in the considered case. This yields the estimate

$$
\left|\psi\left(2^{i} x\right)\right|_{L^{p}(S)} \leq C\left(2^{-i \delta)}|\varphi|_{L_{0}^{p}}, \quad i \geq 0 .\right.
$$

Combining it with Theorem 4.1.1, we prove (4.6.8) for the second case.

It follows from Theorem 4.6.2 that if $G$ is a bounded set, then the integral operator defined by (4.6.1) is bounded in the space $L_{\lambda}^{p}(G, F)$ provided that

$$
-k<\lambda<0 .
$$

This is proved in [22]. Another proof if this result is provided in [44], where the result itself is presented in terms of the weight space defined by (4.1.12). Namely, the operator (4.6.1) is bounded in the space $L^{p}\left(G, \rho_{\delta}\right)$ if

$$
-\frac{k}{p}<\delta<\frac{k}{q}, \quad \frac{1}{q}=1-\frac{1}{p} .
$$

Taking into account (4.1.11) and passing to our notation, we reduce this condition to form (4.6.14), which is simpler (it does not include the summability order $p$ ). This explains the convenience of the use of weight spaces of the form $L_{\lambda}^{p}$.

\section{7. $\quad L^{p}$-Estimates of Singular Integrals}

Using Theorem 4.6.1 and the Calderón-Zygmund theorem from Sec. 4.2, one can easily extend results of Secs. 3.4, 3.5, and 3.11 to the $L^{p}$-case.

Theorem 4.7.1. Let a kernel $Q(y, \xi)$ from $C^{\nu(0)}\left(G, \mathcal{H}_{-k}\right)$, where $G$ is a measurable bounded set of $\mathbb{R}^{k}$, satisfy the condition (4.2.12) with respect to the variable $\xi$. Let $\varphi \in L^{p}(G), p>1$.

Then the singular integral

$$
\psi(x)=\int_{G} Q(y, y-x) \varphi(y) d y, \quad x \in G,
$$

exists for a.e. $x$ and defines a function $\psi$ from $L^{p}(G)$ such that

$$
|\psi|_{L^{p}} \leq C|Q|_{C^{\nu(0)}}|\varphi|_{L^{p}} .
$$

Proof. The relation

$$
\psi(x)=\int_{G} Q(x, y-x) \varphi(y) d y+\int_{G} \frac{a(x, y) \varphi(y) d y}{|y-x|^{k-\nu}},
$$

where $a(x, y)=|x-y|^{k-\nu}[Q(y, y-x)-Q(x, y-x)]$, is valid. By the definition of the class $C^{\nu(0)}$ from Sec. 3.1, we have the estimate

$$
|Q(y, \xi)-Q(x, \xi)| \leq|Q|_{C^{\nu(0)}}|\xi|^{-k}|x-y|^{\nu} .
$$

Therefore, the claim of the theorem immediately follows from Theorem 4.6.1 and the Calderón-Zygmund theorem from Sec. 4.2.

Now, let $G$ be an arbitrary measurable set and $F$ be a finite subset of its limit points containing the point $\infty$ if $G$ is unbounded. 
Theorem 4.7.2. Let a kernel $Q(y, \xi)$ belong to $C_{0}^{\nu(0)}\left(G, F ; \mathcal{H}_{-k}\right)$ and satisfy the condition (4.2.12) with respect to the variable $\xi$. Let $\varphi \in L_{\lambda}^{p}(G, F)$, where $p>1$ and $-k<\lambda<0$.

Then the singular integral given by (4.5.1) exists for a.e. $x$ and defines a function $\psi$ from $L_{\lambda}^{p}(G, F)$ such that

$$
|\psi|_{L_{\lambda}^{p}} \leq C|Q|_{C_{0}^{\nu(0)}}|\varphi|_{L_{\lambda}^{p}}
$$

Proof. Extending the function $\varphi$ to $\mathbb{R}^{k}$ by zero, one can assume (without loss of generality) that $G=\mathbb{R}^{k}$ (and, therefore, $\infty \in F$ ). Further considerations follow the scheme of the proof of Theorems 3.11.1 and 4.6.2.

First, we assume that the function $\varphi$ is identically equal to zero in a neighborhood of $F$. Then there exists a neighborhood of $F$ such that $\psi$ is continuously differentiable in this neighborhood. Then

$$
\psi \in L_{\lambda_{\tau}}^{p}\left(B_{\rho}(\tau), \tau\right)
$$

for finite points $\tau$ of $F$, where $B_{\rho}(\tau)=\{|x-\tau| \leq \rho\}$ and $\rho$ is sufficiently small. The estimate $|\psi(z)| \leq C|z|^{-k}$ is valid in a neighborhood of $\infty$. This means that if $\tau=\infty$, then (4.7.3) holds in the domain $B_{\rho}(\infty)=\{|y| \geq 1 / \rho\}$ since, by the condition, $\lambda_{\infty}+k>0$ and, therefore, the integral

$$
\int_{|y| \geq 1 / \rho}|y|^{-p\left(\lambda_{\infty}+k\right)} \frac{d y}{|y|^{k}}
$$

is finite. On the other hand, if the set $G_{0}$ is bounded and there exists a neighborhood of the set $F$ such that $G_{0}$ lies outside this neighborhood, then, due to Theorem 4.6.1, the function $\psi$ belongs to $L^{p}\left(G_{0}\right)$ and the corresponding estimate of its norm holds.

Thus, if a function $\varphi$ is identically equal to zero in a neighborhood of $F$, then $\psi$ belongs to the class $L_{\lambda}^{p}\left(\mathbb{R}^{k}, F\right)$ and the corresponding estimate of its norm holds. Hence, it suffices to prove the theorem under the assumption that there exists a domain $B_{\rho}(\tau)$ containing the support of the function $\varphi$. As in Sec. 3.10, it suffices to consider the two cases $\tau=0$ and $\tau=\infty$. It is convenient to combine these cases, considering $\varphi$ in $L_{\lambda}^{p}\left(\mathbb{R}^{k}, F\right)$ with respect to $F=\{0, \infty\}$ and $\lambda$ from $\mathbb{R}$.

Redenoting., we see that it suffices to prove the estimate (4.7.2) for the function

$$
\psi(x)=|x|^{-\lambda} \int_{\mathbb{R}^{k}}|y|^{\lambda} Q(y, y-x) \varphi(y) d y
$$

in the norm of the space $L_{0}^{p}$. Represent this relation in the form

$$
\psi\left(2^{i} x\right)=|x|^{-\lambda} \int_{\mathbb{R}^{k}}|y|^{\lambda} Q\left(2^{i} y, y-x\right) \varphi\left(2^{i} y\right) d y
$$

and assign

Since the relation

$$
\begin{array}{ll}
\varphi_{i}(y)=\varphi\left(2^{i} y\right), & y \in S=\{1 / 4 \leq|y| \leq 4\} \\
\psi_{i}(y)=\psi\left(2^{i} y\right), & y \in S^{0}=\{1 / 2 \leq|y| \leq 2\}
\end{array}
$$

$$
4 \int_{\mathbb{R}^{k}} f(y) d y=\sum_{j=-\infty}^{+\infty} \int_{\left(2^{-j}\right) / 4<|y|<4\left(2^{-j}\right)} f(y) d y=\sum_{j=-\infty}^{+\infty} 2^{-(k+\lambda) j} \int_{1 / 4<|y|<4} f\left(2^{-j} y\right) d y
$$

holds for any summable function, it follows that the relation (4.7.4) in the spherical layer $S^{0}$ can be represented in the form

$$
4 \psi_{i}(x)=\sum_{j=-\infty}^{+\infty} \psi_{i j}(x), \quad \psi_{i j}(x)=2^{-(k+\lambda) j} \int_{S}|y|^{\lambda} Q\left(2^{i-j} y, 2^{-j} y-x\right) \varphi_{i-j}(y) d y .
$$


In particular, the following inequality holds:

$$
4\left|\psi_{i}\right|_{L^{p}\left(S^{0}\right)} \leq \sum_{j=-\infty}^{+\infty}\left|\psi_{i j}\right|_{L^{p}(S)}, \quad i=0, \pm 1, \ldots
$$

By virtue of Theorem 4.7.1, we have the estimate

$$
\left|\psi_{i 0}\right|_{L^{p}\left(S^{0}\right)} \leq C\left|\varphi_{i}\right|_{L^{p}(S)},
$$

where $C$ is a positive constant independent of $i=0, \pm 1, \ldots$

On the other hand, for $|j| \geq 1$, the absolute values of the functions $\psi_{i j}$ are estimated as follows:

$$
\left|\psi_{i j}(x)\right| \leq 8^{-\lambda}|Q|_{C^{0(0)}} 2^{-(k+\lambda) j} \int_{S} \frac{\left|\varphi_{i-j}(y)\right| d y}{\left|2^{-j} y-x\right|^{k}} .
$$

Since the inequalities $\left|2^{-j} y-x\right| \geq 1 / 4$ (provided that $j \geq 1$ ) and $\left|2^{-j} y-x\right| \geq\left|2^{-j} y\right| / 2 \geq 2^{-j} / 8$ hold for any $x$ from $S^{0}$ and any $y$ from $S$, it follows that

$$
\left|\psi_{i j}(x)\right| \leq C \sigma_{j} \int_{S}\left|\varphi_{i-j}(y)\right| d y, \quad \sigma_{j}= \begin{cases}2^{-(k+\lambda) j}, & j \geq 1 \\ 2^{-\lambda j}, & j \leq 1 .\end{cases}
$$

Assigning $\sigma_{j}=1$ for $j=0$ and taking into account (4.7.7), we arrive at the inequality

$$
\left|\psi_{i j}\right|_{L^{p}\left(S^{0}\right)} \leq C \sigma_{j}\left|\varphi_{i-j}\right|_{L^{p}(S)}
$$

uniform with respect $i$. Substituting it in (4.7.6), we obtain the estimate

$$
\left|\psi_{i}\right|_{L^{p}\left(S^{0}\right)} \leq C|Q|_{C^{0(0)}} \sum_{j} \sigma_{j}\left|\varphi_{i-j}\right|_{L^{p}(S)}
$$

with a new constant $C$.

Consider the Banach space $l^{p}$ of two-sided sequences $\xi=\left(\xi_{i}, i=0, \pm 1, \ldots\right)$ summable with the $p$ th power and endowed with the corresponding norm

$$
|\xi|=\left(\sum_{i}\left|\xi_{i}\right|^{p}\right)^{1 / p}
$$

For such sequences, one can introduce the convolution $\eta=\sigma * \xi$ as follows:

$$
\eta_{i}=\sum_{j} \sigma_{i-j} \xi_{j}=\sum_{j} \sigma_{j} \xi_{i-j}
$$

This convolution satisfies the inequality $|\eta|_{l^{p}} \leq|\sigma|_{l^{1}}|\xi|_{l^{p}}$ similar to (4.2.3). Since the sequence $\sigma$ defined by (4.7.8) is summable, it follows from (4.7.9) and Theorem 4.1.1 that the estimate (4.7.2) holds in $L_{0}^{p}$ for the integral (4.7.4).

As in Secs. 3.5 and 3.11, the presented results are complemented by the differentiation relation for the integral

$$
\psi^{0}(x)=\int_{G} Q^{0}(y, y-x) \varphi(y) d y, \quad x \in G,
$$

with kernels $Q^{0}(y, \xi)$ from $C^{\nu(0)}\left(G, \mathcal{H}_{1-k}\right)$. To do this, we must introduce generalized derivatives and Sobolev spaces. 
Let $f$ be locally summable in $D$. Then, according to Sec. 1.8, it can be treated as a regular generalized function $\tilde{f}$ acting in the class $C_{0}^{\infty}(D)$ as follows: $\widetilde{f}(\varphi)=(f, \varphi)$. For any multi-index $\alpha$, its derivative $\widetilde{f}^{(\alpha)}$ can be defined as the generalized function acting as follows:

$$
\widetilde{f}^{(\alpha)}(\varphi)=(-1)^{|\alpha|}\left(f, \frac{\partial^{\alpha} \varphi}{\partial x^{\alpha}}\right), \quad \varphi \in C_{0}^{\infty}(D) .
$$

If a generalized function $\widetilde{f}^{(\alpha)}$ is regular, i.e., coincides with a function $g$ summable in the domain $D$, then $g$ is called the generalized derivative $\partial^{\alpha} f / \partial x^{\alpha}$ of the function $f$.

Define the Sobolev space $W^{n, p}(D)$ as the set of functions $\varphi$ from $L^{p}(D)$ such that all their partial derivatives up to the order $n$ (inclusively) exist and belong to $L^{p}(D)$. In this case, similarly to Sec. 2.9, one can introduce an ordered (in any way) collection

$$
\varphi^{(m)}=\left(\frac{\partial^{\alpha} \varphi}{\partial x^{\alpha}}, \quad|\alpha|=m\right)
$$

and endow the space $W^{n, p}(D)$ with the norm

$$
|\varphi|=\sum_{m \leq n}\left|\varphi^{(m)}\right|_{L^{p}}
$$

it is a Banach space with respect to this norm. To unify the notation, we assign $L^{p}=W^{0, p}$. It is easy to see that, similarly to Sec. 2.3, this space can be inductively introduced by the condition that $\varphi$ and $\varphi^{\prime}$ belong to $W^{n-1, p}$, where the gradient vector $\varphi^{\prime}$ consists of the generalized derivatives. In this case, the relation

$$
|\varphi|_{W^{n, \mu}}=|\varphi|_{W^{n-1, \mu}}+\left|\varphi^{\prime}\right|_{W^{n-1, \mu}}
$$

defines an equivalent norm. Also, it is clear that if a function $g$ from $C^{\infty}(D)$ is bounded together with all its derivatives such that their orders do not exceed $n$ (i.e., belong to $C^{n, 0}(D)$ in the notation of Sec. 2.9), then the multiplication operator $\varphi \rightarrow g \varphi$ is bounded in the space $W^{n, p}(D)$.

Let us describe the relations between spaces $W^{n, p}(D)$, where $D$ is a Lipschitz domain, for various $n$ and $p$.

Theorem (the embedding Sobolev theorem). Let $D$ be a finite Lipschitz domain. Then the class $C^{\infty}(\bar{D})$ is dense in $W^{n, p}(D), 1 \leq p<\infty$, and the estimates

$$
|\varphi|_{C^{\mu}(D)} \leq C|\varphi|_{W^{n, p}}, \quad n \geq \mu+k / p,
$$

and

$$
|\varphi|_{L^{p}(\partial D)} \leq C|\varphi|_{W^{1, p}}, \quad n \geq 1,
$$

where $0<\mu<1$ and $C$ is a positive constant independent of $\varphi$, hold for any $\varphi$ from $C^{\infty}(\bar{D})$.

Let us discuss the above estimates. Let $\varphi \in W^{n, p}(D)$ and $n \geq \mu+k / p$. Select a sequence of functions $\varphi_{i}$ from $C^{1}(\bar{D})$ converging to $\varphi$ in $W^{n, p}(D)$. Then, due to the estimate (4.7.11) applied to the difference $\varphi_{i}-\varphi_{j}$, the sequence $\varphi_{i}$ is fundamental in $C^{\mu}(D)$ and, therefore, there exists a function $\tilde{\varphi}$ from $C^{\mu}(D)$ such that it converges to $\tilde{\varphi}$ with respect to the $C^{\mu}$-norm. Since $\varphi_{i} \rightarrow \varphi$ with respect to the $L^{p}$-norm, it follows that $\tilde{\varphi}=\varphi$ a.e. in $D$. Thus, it is possible to change the values of the function $\varphi$ on a zero-measure set such that the changed function satisfies the Hölder condition with exponent $\mu$ and the estimate (4.7.11) holds. The embedding $W^{n, p} \subseteq C^{\mu}$ is understood in the above sense.

Applying the same argument as for (4.7.12), we obtain that the sequence of restrictions of $\varphi_{i}$ to $\Gamma=\partial D$ converges in $L^{p}(\Gamma)$ to a function $\psi$ from $L^{p}(\Gamma)$, depending only $\varphi$ and called the trace (or boundary value) of the function $\varphi$ on $\Gamma$. The embedding $W^{n, p}(D) \subseteq L^{p}(\Gamma)$ is understood in the above sense.

Let $W_{l o c}^{n, p}(D)$ denote the class of functions belonging to $W^{n, p}\left(D_{0}\right)$ for any finite domain $D_{0}$ such that it and its closure are contained in $D$. To unify the notation, we assign $L^{p}=W^{0, p}$ and, similarly 
to 2.9, introduce the homogeneous Sobolev space $W_{0}^{n, \mu}(D)$, using induction with respect to $n$ with the following conditions:

$$
\varphi(x),|x| \varphi^{\prime}(x) \in W_{0}^{n-1, \mu}(D) .
$$

This is a Banach space with respect to the corresponding norm. In the explicit form, the space $W_{0}^{n, p}(D)$ consists of all functions $\varphi$ from $W_{l o c}^{n, p}(D \backslash 0)$ such that

$$
|x|^{|\alpha|} \frac{\partial^{\alpha} \varphi}{\partial x^{\alpha}} \in L_{0}^{p}, \quad|\alpha| \leq n .
$$

If at least one of the points 0 and $\infty$ is a limit point of the set $D$, then the above definition introduces a new space. Otherwise, this space coincides with $W^{n, p}(D)$.

For the space $W_{0}^{n, p}$, the corresponding analog of Theorem 4.1.1, where the symbol $L^{p}$ is to be replaced by $W^{n, p}$, holds. The proof is similar to the proof of Theorem 2.9.2.

As in Sec. 2.9, for functions $\varphi$ from $W_{l o c}^{n, p}(D \backslash F)$, the space $W_{\lambda}^{n, p}(D, F)$ can be defined in two equivalent way. The first one is similar to Sec. 2.8 and is based on the spaces $W^{n, p}(D)$ and $W_{0}^{n, p}(D)$. Another way is the inductive definition by the conditions

$$
\varphi \in W_{\lambda}^{n-1, p}, \quad \varphi^{\prime} \in W_{\lambda-1}^{n-1, p} .
$$

Based on this definition, one can inductively introduce the norm in this space. Another way to introduce the norm is to use the relation

$$
|\varphi|=\sum_{m \leq n}\left|\varphi^{(m)}\right|_{C_{\lambda-m}^{0, \mu}} .
$$

It is clear that the introduced space is a Banach space with respect to the introduced norm. Both approaches are equivalent and lead to the same result.

Using the analog of Theorem 4.1.1 for the space $W_{0}^{n, p}$, one can easily extend the embedding Sobolev theorem to the space $W_{\lambda}^{n, p}$. For simplicity, we restrict our consideration by the two-dimensional case. Let a finite domain $D$ on the Riemann sphere be bounded by a piecewise-smooth contour and let a finite set $F$ contain all its corner points. It is assumed that this is a Lipschitz domain, which is equivalent to the absence of cusps on the curve $\Gamma$.

Theorem 4.7.3. Let $D$ be a Lipschitz domain with a piecewise-smooth boundary in $\mathbb{C}$. Then the following embeddings of Banach spaces take place:

$$
C_{\lambda}^{\mu}(D, F) \subseteq W_{\lambda}^{n, p}(D, F), n \geq \mu+k / p, \quad L_{\lambda}^{p}(\partial D, F) \subseteq W_{\lambda}^{1, p}(D, F) .
$$

They are understood in the same sense as in the Sobolev theorem above.

Proof. The scheme of the proof is the same as for Theorems 3.10.1 and 3.11.1. If $\varphi \in W^{n, p}$ and its support is compact in $D$, then the claim follows from the embedding Sobolev theorem. Therefore, it suffices to proof the claim of the theorem under the assumption that the support of the function $\varphi$ is contained in the domain $B_{\rho}(\tau)$ and is identically equal to zero in a neighborhood of a boundary circular arc of this domain. As in Secs. 3.10 and 3.11, it suffices to consider only the cases where the sector vertex is the point $\tau=0$ and the point $\tau=\infty$. It is convenient to combine both cases, taking the domain $D$ bounded by two radial smooth $\operatorname{arcs} \Gamma^{0}$ and $\Gamma^{1}$ with endpoints $\tau=0$ and $\tau=\infty$ and assuming that the weight order $\lambda$ of the function $\varphi$ from $W_{\lambda}^{n, p}(\Gamma ; 0, \infty)$ does not depend on $\tau$, i.e., is a real number.

In notation of Theorem 4.1.1(a), consider the sequence of domains $D_{j}$ in the spherical layer $S=$ $\left\{\delta<|x|<\delta^{-1}\right\}$. For sufficiently large $|j|$, they are bounded by circular $\operatorname{arcs} L^{ \pm}$from $\left\{|x|=\delta^{ \pm 1}\right\}$ and the $\operatorname{arcs} \Gamma_{j}^{0}$ and $\Gamma_{j}^{0}$. It is obvious that the arc $\Gamma_{j}^{k}$ tends to segments $L_{ \pm}^{k}$ of a line as $j \rightarrow \pm \infty$ in the metric of $C^{1}$. Hence, due to the Sobolev theorem, the following embeddings of Banach spaces uniform with respect to $j$ take place:

$$
C^{\mu}\left(\bar{D}_{j}\right) \subseteq W^{n, p}\left(D_{j}\right), n \geq \mu+k / p, \quad L^{p}\left(\partial D_{j}\right) \subseteq W^{1, p}\left(D_{j}\right) .
$$


Due to Theorem 4.1.1 formulated for $W_{0}^{n, p}$, this implies embeddings (4.7.15).

A detailed presentation of the theory of Sobolev spaces (including weigh ones) can be found in [35, 41, 47].

Pass to the integral (4.7.10). First, we assume that $G$ is a bounded domain. Let a bounded domain $D$ with a $C^{1, \nu}$-boundary contain $\bar{G}$ and a kernel $Q^{0}$ belong to $C^{\nu(1)}\left(D, \mathcal{H}_{1-k}\right)$. Extending the density $\varphi$ from $L^{p}(G), p>1$, by zero, one can assume (without loss of generality) that $\varphi \in L^{p}(D)$. Then $\psi^{0}$ belongs to the Sobolev space $W^{1, p}(D)$ and

$$
\frac{\partial \psi^{0}}{\partial x_{i}}(x)=-\sigma_{i}(x) \varphi_{n}(x)-\int_{D} \frac{\partial Q}{\partial \xi_{i}}(y, y-x) \varphi(y) d y, \quad \sigma_{i}(x)=\int_{\Omega} \xi_{i} Q^{0}(x, \xi) d \xi .
$$

Indeed, let $0<\mu<\nu$ and a sequence $\left\{\varphi_{n}\right\}$ from $C^{\mu}(D)$ converge to $\varphi$ in the norm of the space $L^{p}$. If $\psi_{n}^{0}$ is defined by $\varphi_{n}$ similarly to (4.7.10), then, due to Theorem 3.5.3, the function $\psi_{n}^{0}$ belongs to $C^{1, \mu}(D)$ and its partial derivatives are computed according to a relation similar to (4.7.16). Then, due to Theorem 4.7.1, we conclude that the sequence $\left\{\partial \psi_{n}^{0} / \partial x_{i}\right\}$ converges in the $L^{p}$-norm to the function $\psi_{i}^{0}$ defined by the right-hand side of (4.7.16) and the claimed assertion immediately follows from the definition of generalized derivatives.

Thus, under the admitted assumptions, the integral operator defined by (4.7.10) boundedly maps $L^{p}(G)$ to $W^{1, p}(D)$.

Now, let $G$ be an arbitrary measurable set and

$$
\varphi \in L_{\lambda}^{p}(G, F), \quad-k<\lambda_{\tau}<\left\{\begin{array}{rr}
0, & \tau \neq \infty \\
-1, & \tau=\infty
\end{array}\right.
$$

Extending $\varphi$ by zero, one can assume (without loss of generality) that $G=\mathbb{R}^{k}$. Let $Q^{0} \in C_{0}^{\nu(0)}\left(\mathbb{R}^{k}, F\right)$. Then $\psi^{0}$ is represented by (4.1.4) with $\alpha=k-1$. Then, by virtue of (4.7.17), the condition (4.6.3) of Theorem 4.6.2 is satisfied. Therefore, due to this Theorem, the function $\psi^{0}$ belongs to the space

$$
L_{\nu}^{p}\left(\mathbb{R}^{k}, F\right), \quad \nu_{\tau}= \begin{cases}\lambda_{\tau}, & \tau \neq \infty \\ \lambda_{\tau}+1, & \tau=\infty\end{cases}
$$

Decomposing the density $\varphi$ into the sum of two functions such that one of them is identically equal to zero in a neighborhood of a fixed point $a$ not belonging to $F$, we verify (similarly to Lemma 3.5.2) that the function $\psi^{0}$ belongs to the class $W_{l o c}^{1, p}\left(\mathbb{R}^{k} \backslash F\right)$ (i.e., to the space $W^{1, p}\left(D_{0}\right)$ for any bounded subdomain $D_{0}$ such that it and its closure lies in $\mathbb{R}^{k} \backslash F$ ) and the relation (4.7.16) holds.

Thus, the function $\psi^{0}$ belongs to space (4.7.18) and its generalized derivatives $\partial \psi^{0} / \partial x_{i}$ belong to the space $L_{\lambda}^{p}\left(\mathbb{R}^{k}, F\right)$. As in the $C^{\mu}$-case considered in Sec. 2.10, a problem of a refined description of the class of functions $\psi^{0}$ possessing this property arises, but we do not consider this problem in the present work.

\subsection{Singular Cauchy Integrals with $L^{p}$-Density}

Let $\Gamma$ be an orientable curve on $\mathbb{C}$. Consider the generalized singular Cauchy integral

$$
\psi\left(t_{0}\right)=\int_{\Gamma} Q\left(t ; t-t_{0}, d t\right) \varphi(t), \quad t_{0} \in \Gamma .
$$

In Sec. 3.11, these integrals are studied under the assumption that the density $\varphi$ belongs to the class $C^{\mu}$ on a smooth curve or the class $C^{\mu}$ with weight on a piecewise-smooth curve.

In the current section, we consider the case where $\Gamma$ is a smooth-Lyapunov curve and the density $\varphi$ belongs to the space $L_{\lambda}^{p}(\Gamma, F)$ defined with respect to the linear measure $d_{1} t$ similarly to Sec. 4.1. It is clear that Theorem 4.1.1 is still valid for the space $L_{0}^{p}(\Gamma, \tau)$, where $\Gamma$ is the radial arc with endpoint $\tau=0$ or $\tau=\infty$. 
First, we assume that $\Gamma$ is a bounded Lyapunov arc and $\varphi \in L^{p}(\Gamma)$. The Riesz theorem and HardyLittlewood theorem from Sec. 4.1 are easily extended to this case by means of the arc parametrization. Let a smooth arc $\Gamma$ belong to $C^{1, \nu}, 0<\nu<1$, and $l$ be its length. Consider the natural parametrization $\gamma:[0, l] \rightarrow \Gamma$ belonging to the class $C^{1, \nu}[0, l]$ by condition and the related function

$$
q\left(s_{0}, s\right)=\frac{\gamma(s)-\gamma\left(s_{0}\right)}{s-s_{0}}=\int_{0}^{1} \gamma^{\prime}\left[r s+(1-r) s_{0}\right] d r \quad \text { from } \quad C^{\nu}([0, l] \times[0, l])
$$

such that its value for $s=s_{0}$ is equal to $\gamma^{\prime}(s)$. This function is different from zero everywhere. Hence, there exists $m$ from $(0,1]$ such that the two-sided estimate

$$
m\left|s-s_{0}\right| \leq\left|\gamma(s)-\gamma\left(s_{0}\right)\right| \leq\left|s-s_{0}\right|
$$

holds.

Theorem 4.8.1. Let $\Gamma \in C^{1, \nu}, 0<\nu<1$, and $Q(t ; \xi, \eta) \in C^{\nu(1)}(\Gamma)$. Then, for any $\varphi$ from $L^{p}(\Gamma)$, $p>1$, the singular integral defined by (4.8.1) exists a.e. on $\Gamma$ and defines a function $\psi$ from $L^{p}(\Gamma)$ such that

$$
|\psi|_{L^{p}} \leq C|Q|_{C^{\nu(1)}}|\varphi|_{L^{p}}
$$

where $C$ is a positive constant depending only on the $C^{\nu}$-norm of the function $q$ and the constants $l$ and $m$ from (4.8.2) and (4.8.3).

The same estimate also holds for the maximal functions

$$
\begin{gathered}
\left(M_{0} \varphi\right)\left(t_{0}\right)=\sup _{r>0} \frac{1}{r} \int_{\left|t-t_{0}\right| \leq r}|\varphi(t)| d_{1} t, \quad\left(M_{0}^{\prime} \varphi\right)\left(t_{0}\right)=\sup _{r>0} r \int_{\left|t-t_{0}\right| \geq r} \frac{|\varphi(t)| d_{1} t}{\left|t-t_{0}\right|^{2}}, \\
\left(M_{1} \varphi\right)\left(t_{0}\right)=\sup _{r>0}\left|\int_{\left|t-t_{0}\right| \geq r} Q\left(t ; t-t_{0}, d t\right) \varphi(t)\right|, \quad t_{0} \in \Gamma .
\end{gathered}
$$

Proof. Consider the substitution $t=\gamma(s)$ mapping any $\varphi$ from $L^{p}(\Gamma)$ to the function $\widetilde{\varphi}(s)=\varphi[\gamma(s)]$, $0 \leq s \leq l$, such that

$$
|\varphi|_{L^{p}(\Gamma)}=|\widetilde{\varphi}|_{L^{p}[0, l]} \cdot
$$

It is convenient to extend the function $\widetilde{\varphi}$ to the whole line, preserving its notation.

Obviously, in the one-dimensional case, the condition of Theorem 3.3.1 for the singular integral given by (4.8.1) is satisfied for this substitution. Hence, as in the case of Lemma 2.3.3, we have the relation

$$
\psi\left[\gamma\left(s_{0}\right)\right]=\int_{0}^{l} Q\left[\gamma(s) ; \gamma(s)-\gamma\left(s_{0}\right), \gamma^{\prime}(s)\right] \varphi[\gamma(s)] d s, \quad 0<s_{0}<l .
$$

Since $Q(t ; \xi, \eta)$ is a homogeneous function of power -1 and it is odd with respect to $\xi$, it follows that

$$
Q\left[\gamma(s) ; \gamma(s)-\gamma\left(s_{0}\right), \gamma^{\prime}(s)\right]=\frac{k\left(s_{0}, s\right)}{s-s_{0}}, \quad k\left(s_{0}, s\right)=Q\left[\gamma(s) ; q\left(s_{0}, s\right), \gamma^{\prime}(s)\right] .
$$

By virtue of Lemma 3.1.2, the function $k\left(s_{0}, s\right)$ belongs to $C^{\nu}([0, l] \times[0, l])$ and there exists an absolute constant $C_{0}$ such that

$$
|k|_{C^{\nu}} \leq C_{0}|Q|_{C^{\nu(1)}}|q|_{C^{\nu}}
$$

Thus,

$$
\psi\left[\gamma\left(s_{0}\right)\right]=k\left(s_{0}, s_{0}\right) \int_{0}^{l} \frac{\widetilde{\varphi}(s) d s}{s-s_{0}}+\widetilde{\psi}(s), \quad \widetilde{\psi}(s)=\int_{0}^{l} \frac{k\left(s_{0}, s\right)-k\left(s_{0}, s_{0}\right)}{s-s_{0}} \widetilde{\varphi}(s) d s .
$$


It is obvious that $\widetilde{\psi}(s)$ is the integral with a weak singularity, considered in Sec. 4.6. Therefore, taking into account (4.8.6) and (4.8.8), we conclude that the first assertion is a corollary of the Riesz theorem from Sec. 4.1 and of Theorem 4.6.1.

Further, similarly to (4.8.9), we have the inequality

$$
\left|\int_{\left|t-t_{0}\right| \geq r} Q\left(t ; t-t_{0}, d t\right) \varphi(t)\right| \leq|k|_{C^{\nu}}\left(\left|\int_{\left|\gamma(s)-\gamma\left(s_{0}\right)\right| \geq r} \frac{\widetilde{\varphi}(s) d s}{s-s_{0}}\right|+\int_{0}^{l} \frac{|\widetilde{\varphi}(s)| d s}{\left|s-s_{0}\right|^{1-\nu}}\right) .
$$

By virtue of (4.8.3), the inequality $\mid \gamma_{0}(s)-\gamma_{0}\left(s_{0}\right) \geq r$ implies the inequality $\left|s-s_{0}\right| \geq r$. Therefore, arguing as above, we deduce the estimate (4.8.4) for the function $\psi=M_{1} \varphi$ from the Riesz theorem.

Pass to the estimate of the function $M_{0} \varphi$. By virtue of (4.8.3), the inequality $\left|\gamma(s)-\gamma\left(s_{0}\right)\right| \leq r$ implies the inequality $m\left|s-s_{0}\right| \leq r$, i.e.,

$$
\left(M_{0} \varphi\right)\left[\gamma\left(s_{0}\right)\right]=\frac{1}{r} \int_{|\gamma(s)| \leq r}|\widetilde{\varphi}(s)| d s \leq \frac{1}{r} \int_{m\left|s-s_{0}\right| \leq r}|\widetilde{\varphi}(s)| d s
$$

(we recall that the function $\widetilde{\varphi}$ is extended to the whole line by zero). Therefore, the estimate (4.8.4) for $\psi=M_{0} \varphi$ follows from the corresponding assertion of the Hardy-Littlewood theorem.

In the same way, we have the inequality

$$
\left(M_{0}^{\prime} \varphi\right)\left[\gamma\left(s_{0}\right)\right] \leq \frac{r}{m^{2}} \int_{|\gamma(s)| \geq r} \frac{|\widetilde{\varphi}(s)| d s}{\left|s-s_{0}\right|^{2}} \leq \frac{r}{m^{2}} \int_{m\left|s-s_{0}\right| \geq r} \frac{|\widetilde{\varphi}(s)| d s}{\left|s-s_{0}\right|^{2}} .
$$

According to Lemma 4.1.2, we have the inequality

$$
\frac{r}{m} \int_{m\left|s-s_{0}\right| \geq r} \frac{|\widetilde{\varphi}(s)| d s}{\left|s-s_{0}\right|^{2}} \leq 3 \sup _{r>0} \frac{1}{r} \int_{\left|s-s_{0}\right| \leq r}|\widetilde{\varphi}(s)| d s,
$$

i.e.,

$$
\left(M_{0}^{\prime} \varphi\right)\left[\gamma\left(s_{0}\right)\right] \leq \frac{3}{m} \sup _{r>0} \frac{1}{r} \int_{\left|s-s_{0}\right| \leq r}|\widetilde{\varphi}(s)| d s .
$$

Therefore, the estimate (4.8.3) for $\psi=M_{0}^{\prime} \varphi$ also follows from the Hardy-Littlewood theorem.

Complement Theorem 4.8.1 by the following assertion implied by the Lebesgue points theorem from Sec. 1.8 and by Lemma 4.1.2.

Lemma 4.8.1. Let a function $\varphi$ be summable on a smooth arc $\Gamma$. Then

$$
\lim _{r \rightarrow 0} \frac{1}{r} \int_{\left|t-t_{0}\right| \leq r}\left|\varphi(t)-\varphi\left(t_{0}\right)\right| d_{1} t=\lim _{r \rightarrow 0} r \int_{\left|t-t_{0}\right| \geq r} \frac{\left|\varphi(t)-\varphi\left(t_{0}\right)\right|}{\left|t-t_{0}\right|^{2}} d_{1} t=0
$$

for almost all points $t_{0}$ of $\Gamma$.

Proof. The first limit is equal to zero due to the Lebesgue points theorem from Sec. 1.8. Consider the second limit. Similarly to (4.8.10), we have the (double) inequality

$$
r \int_{\left|t-t_{0}\right| \geq r} \frac{\left|\varphi(t)-\varphi\left(t_{0}\right)\right|}{\left|t-t_{0}\right|^{2}} d_{1} t \leq \frac{r}{m^{2}} \int_{\left|\gamma(s)-\gamma\left(s_{0}\right)\right| \geq r} \frac{\left|\widetilde{\varphi}(s)-\widetilde{\varphi}\left(s_{0}\right)\right| d s}{\left|s-s_{0}\right|^{2}} \leq \frac{r}{m^{2}} \int_{m\left|s-s_{0}\right| \geq r} \frac{\left|\widetilde{\varphi}(s)-\widetilde{\varphi}\left(s_{0}\right)\right| d s}{\left|s-s_{0}\right|^{2}},
$$

where the inequality $m\left|s-s_{0}\right| \geq r$ in the last integral is considered on the segment $[0, l]$. Extending the function $f(s)=\left|\widetilde{\varphi}(s)-\widetilde{\varphi}\left(s_{0}\right)\right|$ by zero and using the second part of Lemma 4.1.2, we obtain that the second limit of (4.8.11) is also equal to zero. 
Now, consider a piecewise-smooth curve $\Gamma$ on the extended plane $\overline{\mathbb{C}}$. As in Sec. 3.10, we assume that a finite set $F$ contains all its boundary points (including $\infty$ if the curve is unbounded). Then $\Gamma$ can be represented in the form

$$
\Gamma \backslash F=\Gamma_{0} \cup \dot{\Gamma}_{1} \cup \ldots \cup \dot{\Gamma}_{m}
$$

where $\Gamma_{0}$ is a smooth contour (in general, it might be a composite contour), $\dot{\Gamma}_{j}$ are open smooth arcs, and all these curves are pairwise disjoint. As above, for any $\tau$ from $F, B_{\tau}(\rho)$ denotes the disk $\{|z-\tau| \leq \rho\}$ if $\tau \neq \infty$ and the exterior of the disk $\{|z| \geq 1 / \rho\}$ if $\tau=\infty$. If $\rho$ is sufficiently small, then the intersection $\Gamma_{\tau}=\Gamma \cap B_{\tau}(\rho)$ is decomposed into $n_{\tau}$ smooth $\operatorname{arcs} \Gamma_{\tau, j}$ with a common endpoint $\tau$. We select $\rho$ sufficiently small to ensure that all $\operatorname{arcs} \Gamma_{\tau, j}, 1 \leq j \leq n_{\tau}$, are radial with respect to the endpoint $\tau$.

First, we note that the following estimate holds:

$$
\sum_{\tau \neq \infty} \int_{\Gamma_{\tau}}|\varphi(t)| d_{1} t+\int_{\Gamma_{\infty}}|\varphi(t)||t|^{-1} d_{1} t \leq C|\varphi|_{L_{\lambda}^{p},} \quad-1<\lambda<0 .
$$

Indeed, if $\tau$ is a finite point of $F$, then, by virtue of the Hölder inequality, we have

$$
\int_{\Gamma_{\tau}}|\varphi(t)| d_{1} t \leq\left(\int_{\Gamma_{\tau}}|\varphi(t)|^{p}|t-\tau|^{-p \lambda_{\tau}-1} d_{1} t\right)^{1 / p}\left(\int_{\Gamma_{\tau}}|t-\tau|^{q \lambda_{\tau}-1} d_{1} t\right)^{1 / q},
$$

where $1 / q=1-1 / p$. It remains to note that the inequality $\lambda_{\tau}>-1$ implies the inequality $q \lambda_{\tau}+q / p>$ -1 and, therefore, the integrals on the right-hand side of this inequality are finite. If $\tau=\infty$, then $|t|^{-1}=|t|^{-\lambda_{\tau}-1 / p}|t|^{\lambda_{\tau}-1 / q}$ and, in the same way,

$$
\int_{\Gamma_{\tau}}|\varphi(t)||t|^{-1} d_{1} t \leq\left(\int_{\Gamma_{\tau}}|\varphi(t)|^{p}|t|^{-p \lambda_{\tau}-1} d_{1} t\right)^{1 / p}\left(\int_{\Gamma_{\tau}}|t|^{q \lambda_{\tau}+q / p} d_{1} t\right)^{1 / q},
$$

in this case, the inequality $\lambda_{\tau}<0$ implies the inequality $q \lambda_{\tau}-1<-1$.

The estimate (4.8.13) shows that the integrand of (4.8.1) is summable on $\Gamma$ outside any neighborhood of the point $t_{0}$ and, therefore, the singular integral is well defined.

Theorem 4.8.2. Let a piecewise-Lyapunov curve $\Gamma$ not contain cusps and a generalized Cauchy kernel $Q(t ; \xi, \eta)$ belong to $C_{0}^{\nu(1)}(\Gamma, F)$. Let $\varphi \in L_{\lambda}^{p}(\Gamma, F)$, where $p>1$ and $-1<\lambda<0$. Then the function $\psi$ defined by the integral (4.8.1) belongs to the class $L_{\lambda}^{p}(\Gamma, F)$ and the estimate

$$
|\psi|_{L_{\lambda}^{p}} \leq C|Q|_{C_{0}^{\nu(1)}}|\varphi|_{L_{\lambda}^{p}}
$$

of its norm holds.

Proof. We use the same scheme as for the proof of Theorem 4.7.2. It is possible to select $\nu$ sufficiently small to ensure that the smooth contour $\Gamma_{0}$ and the open smooth $\operatorname{arcs} \dot{\Gamma}_{j}$ of the expansion (4.8.12) belong to the classes $C^{1, \nu}$ and $C_{(1+0)}^{1, \nu}$ respectively.

If $\varphi$ vanishes in a neighborhood of $F$, then, by Theorem 4.8.1, the function $\psi$ belongs to $L^{p}\left(\Gamma_{0}\right)$ on any arc $\Gamma_{0}$ from $\Gamma \backslash F$. It is obvious that the function $\psi\left(t_{0}\right)$ is bounded in a neighborhood of finite points $\tau$ and behaves as $O(1)\left|t_{0}\right|^{-1}$ as $t_{0} \rightarrow \infty$. Taking into account the fact that $-1<\lambda<0$, we verify, similarly to the deduction of (4.8.13), that this function belongs to $L_{\lambda_{\tau}}^{p}\left(\Gamma_{\tau}, \tau\right)$ for any $\tau$ from $F$.

The above considerations show that it suffices to consider only the case where there exists $\tau$ from $F$ such that the function $\varphi$ is equal to zero outside $\Gamma_{\tau}$, the singular integral $\psi$ is considered on $\Gamma_{\tau}$, and $\tau=0$ or $\tau=\infty$. Both these cases can be combined for a curve $\Gamma$ composed by radial $\operatorname{arcs} \Gamma^{1}, \ldots, \Gamma^{n}$ with common endpoints $\tau=0$ and $\tau=\infty$ such that they are not cusps for this curve. 
Redenoting and assigning $\lambda_{\tau}=\lambda$, we see that it suffices to prove the estimate (4.8.14) for the function

$$
\psi\left(t_{0}\right)=\left|t_{0}\right|^{-\lambda} \int_{\Gamma}|t|^{\lambda} Q\left(t ; t-t_{0}, d t\right) \varphi(t), \quad t_{0} \in \Gamma,
$$

with respect to the space $L_{0}^{p}(\Gamma)$. Represent this relation in the form

$$
\psi\left(2^{i} t_{0}\right)=\left|t_{0}\right|^{-\lambda} \int_{2^{-i} \Gamma}|t|^{\lambda} Q\left(2^{i} t ; t-t_{0}, d t\right) \varphi\left(2^{i} t\right), \quad t_{0} \in 2^{-i} \Gamma, \quad i=0, \pm 1, \ldots,
$$

and assign (for brevity)

$$
\begin{array}{ll}
\varphi_{i}(t)=\varphi\left(2^{i} t\right), & t \in \Gamma_{i}=\left(2^{-i} \Gamma\right) \cap\{1 / 4 \leq|t| \leq 4\}, \\
\psi_{i}(t)=\psi\left(2^{i} t\right), & t \in \Gamma_{i}^{0}=\left(2^{-i} \Gamma\right) \cap\{1 / 2 \leq|t| \leq 2\} .
\end{array}
$$

Recall that $\Gamma$ consists of radial arcs $\Gamma^{k}, 1 \leq k \leq n$, with the common endpoints $\tau=0$ and $\tau=\infty$. Respectively, any curve $\Gamma_{i}$ consists of $n$ radial $\operatorname{arcs} \Gamma_{i}^{k}$. Proving Theorem 3.10.1(b), we found that the arc $\Gamma_{i}^{k}$ tends to the corresponding segment $I_{ \pm}^{k}$ as $i \rightarrow \pm \infty$ in the metric $C^{1, \nu}$. This segment is the intersection of the ring $\{1 / 4 \leq|t| \leq 4\}$ and the ray such that its vertex is the origin and it is parallel to the tangent to $\Gamma^{k}$ at the point $\tau=0$ (if $i \rightarrow+\infty$ ) or at the point $\tau=\infty$ (if $i \rightarrow-\infty$ ). In particular, the three parameters such that the constant $C$ in the estimate of Theorem 4.8.1 for the curves $\Gamma_{i}^{k}$ depends on them are uniformly bounded with respect to $i=0, \pm 1, \ldots$ Since, by condition, $\tau$ is not a cusp, it follows that the segments $I_{-}^{k}, 1 \leq k \leq n$, are pairwise different and the segments $I_{+}^{k}, 1 \leq k \leq n$, are pairwise different.

If a function $f$ is summable on $\Gamma$, then

$$
4 \int_{\Gamma} f(t) d_{1} t=\sum_{j=-\infty}^{+\infty} \int_{\Gamma \cap\left\{2^{-j-2}<|t|<2^{-j+2}\right\}} f(t) d_{1} t=\sum_{j=-\infty}^{+\infty} 2^{-j} \int_{2^{j} \Gamma \cap\{1 / 4<|t|<4\}} f(t) d_{1} t .
$$

Applying this fact to the curve $2^{-i} \Gamma$ and the integral (4.8.16), in notation of (4.8.17), we obtain the relation

$$
4 \psi\left(2^{i} t_{0}\right)=\sum_{j=-\infty}^{+\infty} \psi_{i j}\left(t_{0}\right), \quad \psi_{i j}\left(t_{0}\right)=2^{-(1+\lambda) j} \int_{\Gamma_{i-j}}|t|^{\lambda} Q\left(2^{i-j} t ; 2^{-j} t-t_{0}, d t\right) \varphi_{i-j}(t),
$$

whence

$$
4\left|\psi_{i}\right|_{L^{p}\left(\Gamma_{i}^{0}\right)} \leq \sum_{j=-\infty}^{+\infty}\left|\psi_{i j}\right|_{L^{p}\left(\Gamma_{i-j}\right)} .
$$

As we note above, it follows from Theorem 4.8.1 that

$$
\left|\psi_{i 0}\right|_{L^{p}\left(\Gamma_{i}^{0}\right)} \leq C|Q|_{C^{0(1)}}\left|\varphi_{i}\right|_{L^{p}\left(\Gamma_{i}\right)},
$$

where $C$ is a positive constant independent of $i$. For the functions $\psi_{i j}, j \neq 0$, we have the following estimate similar to (4.7.8):

$$
\sup _{t_{0} \in \Gamma_{i}^{0}}\left|\psi_{i j}\left(t_{0}\right)\right| \leq C \sigma_{j}|Q|_{C^{0(0)}} \int_{\Gamma_{i-j}}\left|\varphi_{i-j}(t)\right| d t, \quad \sigma_{j}= \begin{cases}2^{-(1+\lambda) j}, & j \geq 1, \\ 2^{-\lambda j}, & j \leq-1 .\end{cases}
$$

To obtain it, estimating the integrals over radial arcs contained in $2^{-j} \Gamma$, we take into account the fact that the absolute values of the derivatives of the radial parametrization of these arcs are bounded uniformly with respect to $j$. 
Indeed, let a radial arc $\Gamma^{k}$ contained in $\Gamma$ be given by the radial parametrization $\gamma(s)=s e^{i f(s)}$, $0 \leq s<\infty$, where $f$ is continuously differentiable for $0<s<\infty$ and has the following limits:

$$
\lim _{s \rightarrow 0} f(s)=c_{0}, \quad \lim _{s \rightarrow \infty} f(s)=c_{1}, \quad \lim _{s \rightarrow 0} s f^{\prime}(s)=\lim _{s \rightarrow \infty} s f^{\prime}(s)=0 .
$$

Then the curve $2^{-j} \Gamma^{k}$ has a radial parametrization $\gamma_{j}(s)=s e^{i f_{j}(s)}$, where the function $f_{j}(s)=f\left(2^{j} s\right)$ also possess the properties (4.8.21) and

$$
\left|\gamma_{j}^{\prime}(s)\right| \leq \max _{0<s<\infty}\left|\gamma^{\prime}(s)\right|
$$

which proves the claimed assertion.

Assigning $\sigma_{j}=1$ for $j=0$, we deduce the following estimate from (4.8.19)-(4.8.20):

$$
\left|\psi_{i j}\right|_{L^{p}\left(\Gamma_{i}^{0}\right)} \leq C|Q|_{C^{0(1)}}\left|\varphi_{i-j}\right|_{L^{p}\left(\Gamma_{i-j}\right)}, \quad|j| \geq 1 .
$$

Similarly to the proof of Theorem 4.7.2, we combine the last estimate with (4.8.18) and obtain that the estimate (4.8.14) with $\lambda=0$ is valid for the integral (4.8.15).

For the classical case of Cauchy-type integrals (where $Q(\xi, \eta)=\eta / \xi$ ), Theorem 4.8.2 is proved in [30] for the first time.

\subsection{Cauchy-Type Generalized Integrals with $L^{p}$-Density}

Let $\Gamma$ be an orientable piecewise-Lyapunov curve on the complex plane. Consider the Cauchy-type generalized integral

$$
\phi(z)=\int_{\Gamma} Q(t ; t-z, d t) \varphi(t), \quad t \notin \Gamma,
$$

where the density $\varphi$ belongs to $L_{\lambda}^{p}(\Gamma, F)$, and the corresponding singular integral defined by (4.8.1). In Chap. 3, this integral is studied in the case where $\varphi$ belongs to the class $C^{\mu}$ with weight. Recall that, under the corresponding assumptions about the kernel $Q$, the function $\phi(z)$ has one-sided limits $\phi^{ \pm}\left(t_{0}\right)$ at internal points $t_{0}$ of the curve $\Gamma$, linked with the value $\phi^{*}\left(t_{0}\right)$ of the integral (4.8.1) at these points by the relation

$$
\phi^{ \pm}\left(t_{0}\right)= \pm \sigma\left(t_{0}\right) \varphi\left(t_{0}\right)+\psi\left(t_{0}\right), \quad \sigma\left(t_{0}\right)=\frac{1}{2} \int_{\mathbb{T}} Q\left(t_{0} ; \xi, d \xi\right),
$$

where the unit circle $\mathbb{T}$ is oriented counterclockwise. Investigate the behavior of the function $\phi$ near the curve $\Gamma$ under the assumption that the density $\varphi$ is only summable. For classical Cauchy-type integrals with kernel $Q(\xi, \eta)=\eta / \xi$, this is investigated in [53] in detail. The case of general Cauchy kernels is studied in $[70,71]$.

First, we consider the case where $\Gamma$ is a bounded Lyapunov arc. It is convenient to assign to any point $t_{0}$ of $\Gamma$ and any $\theta$ from $[0, \pi]$ the centrally symmetric cone $K_{\theta}\left(t_{0}\right)$ such that its vertex is $t_{0}$, its angle of opening is equal to $\theta$, and it is defined by the inequality

$$
\left|\arg \left[\left(z-z_{0}\right) \overline{n\left(t_{0}\right)}\right]\right| \leq \theta,
$$

where $n\left(t_{0}\right)=i e\left(t_{0}\right)$ denotes the unit normal to $\Gamma$ at point $t_{0}$. It is obvious that the line containing $t_{0}$ and parallel to the vector $n\left(t_{0}\right)$ is the bisectrix of this cone. It is decomposed into the two cones $K_{\theta}^{+}\left(t_{0}\right)$ and $K_{\theta}^{-}\left(t_{0}\right)$ located from the left and from the right (respectively) of the orientable arc $\Gamma$.

Lemma 4.9.1. For any $\theta$ from $[0, \pi]$ there exist positive $\rho$ and $m$ such that

$$
|z-t| \geq m\left(\left|z-t_{0}\right|+\left|t-t_{0}\right|\right)
$$

for any $t$ and $t_{0}$ from $\Gamma$ and any $z$ from $K_{\theta}\left[t_{0} ; n\left(t_{0}\right)\right]$ such that $\left|z-t_{0}\right| \leq \rho$. 
Proof. Fix $\varepsilon$ from $(0, \pi-\theta)$ and consider the cone $K_{\varepsilon}\left[t_{0}, e\left(t_{0}\right)\right]$ of opening $\varepsilon$, defined in the same way with respect to the unit tangential vector $e\left(t_{0}\right)$. Obviously, the tangent at the point $t_{0}$ of $\Gamma$ is its bisectrix. As in the case of Lemma 2.5.1, it is easy to verify that there exists a positive $\rho$ such that

$$
\Gamma \cap\left\{\left|z-t_{0}\right| \leq 2 \rho\right\} \subseteq K_{\varepsilon}\left[t_{0}, e\left(t_{0}\right)\right]
$$

for any point $t_{0}$ of $\Gamma$. According to Lemma 2.1.2, there exists a positive $r_{0}$ depending only on $\theta$ and $\varepsilon$ such that inequality (4.9.4) with $r=r_{0}$ is satisfied for any $z$ from $K_{\theta}\left[t_{0} ; n\left(t_{0}\right)\right]$ and any $t$ from $K_{\varepsilon}\left[t_{0}, e\left(t_{0}\right)\right]$. Then, taking into account (4.9.5), we conclude that the said inequality holds for for any $t$ and $t_{0}$ from $\Gamma$ such that $\left|t-t_{0}\right| \leq 2 \rho$ and any $z$ from $K_{\theta}\left[t_{0} ; n\left(t_{0}\right)\right]$ such that $\left|z-t_{0}\right| \leq \rho$. On the other hand, if $t$ and $t_{0}$ belong to $\Gamma,\left|t-t_{0}\right| \geq 2 \rho$, and $\left|z-t_{0}\right| \leq \rho$, then we have the obvious inequality

$$
|z-t| \geq \rho \geq \frac{\rho}{r_{0}+R}\left(\left|z-t_{0}\right|+\left|t-t_{0}\right|\right)
$$

where $R$ denotes the diameter of the arc $\Gamma$. Thus, we arrive at the estimate (4.9.4), where $m$ is the least of the numbers $r_{0}$ and $\rho /\left(r_{0}+R\right)$.

Note that if a sequence of smooth $\operatorname{arcs} \Gamma_{n}$ converges to $\Gamma$ in the class $C^{1}$, then it is possible to select the numbers $\rho$ and $\delta$ in this lemma such that they do not depend on $n$, but the lemma holds for any $\operatorname{arc} \Gamma_{n}$.

Indeed, let $\Gamma_{n} \rightarrow \Gamma$ in the class $C^{1}$. Then there exists a number $\rho$ satisfying the condition (4.9.5) with respect to $\Gamma_{n}$ such that it does not depend on $n$; this is proved similarly to Lemma 4.9.1.

Consider the behavior of the Cauchy-type integral $\phi(z)$ near a smooth orientable arc $\Gamma$. For any $\theta$ from $(0, \pi)$, define $\rho=\rho(\theta, \Gamma)$ according to Lemma 4.9.1. Then it is possible to introduce the sectors

$$
S_{\theta}^{ \pm}\left(t_{0}, \Gamma\right)=K_{\theta}^{ \pm}\left(t_{0}\right) \cap\left\{z-t_{0} \mid \leq \rho(\theta, \Gamma)\right\}, \quad t_{0} \in \Gamma,
$$

such that the sector $S^{+}$lies from the left of $\Gamma$.

Theorem 4.9.1. Let a Cauchy-type integral be expressed by (4.9.1) such that $\Gamma$ is a $C^{1, \nu}$-arc, the Cauchy kernel $Q$ belongs to $C^{\nu(1)}(\Gamma)$, and $\varphi$ belongs to $L^{p}(\Gamma), p>1$. Let $0<\theta<\pi$. Then the functions

$$
\left(M_{\theta}^{ \pm} \varphi\right)\left(t_{0}\right)=\sup _{z \in S_{\theta}\left(t_{0}, \Gamma\right)}|\phi(z)|
$$

belong to $L^{p}(\Gamma),\left|M_{\theta}^{ \pm} \varphi\right|_{L^{p}} \leq C|\varphi|_{L^{p}}$, and for almost all $t_{0}$ from $\Gamma$ there exist one-sided limits $\phi^{ \pm}\left(t_{0}\right)=$ $\lim \phi(z)$ as $z \rightarrow t_{0}$ and $z \in S_{\theta}^{ \pm}\left(t_{0}, \Gamma\right)$, satisfying the relation (4.9.2).

The said one-sided limits are called the corner limit values.

Proof. Let $z \in S_{\theta}^{ \pm}\left(t_{0}, \Gamma\right)$ and $r=\left|z-t_{0}\right|$ be fixed. Represent the integral (4.9.1) by the sum $\phi(z)=$ $\phi_{r}\left(z, t_{0}\right)+\psi_{r}\left(t_{0}\right)$, where

$$
\begin{gathered}
\psi_{r}\left(t_{0}\right)=\int_{\left|t-t_{0}\right| \geq r} Q\left(t ; t-t_{0}, d t\right) \varphi(t), \\
\phi_{r}\left(z, t_{0}\right)=\int_{\left|t-t_{0}\right| \leq r} Q\left(t ; t-t_{0}, d t\right) \varphi(t)+\int_{\left|t-t_{0}\right| \geq r}\left[Q(t ; t-z, d t)-Q\left(t ; t-t_{0}, d t\right)\right] \varphi(t) .
\end{gathered}
$$

According to Lemma 3.1.1, the first term satisfies the inequality

$$
\left|\phi_{r}\right| \leq|Q|_{C^{0(1)}}\left(\int_{\left|t-t_{0}\right| \leq r} \frac{1}{|t-z|}|\varphi(t)| d_{1} t+r \int_{\left|t-t_{0}\right| \geq r}\left[\frac{1}{|t-z|^{2}}+\frac{1}{\left|t-t_{0}\right|^{2}}\right]|\varphi(t)| d_{1} t\right),
$$


Taking into account (4.9.4), this leads to the estimate

$$
\left|\phi_{r}\left(z, t_{0}\right)\right| \leq|Q|_{C^{0(1)}}\left(\frac{1}{m r} \int_{\left|t-t_{0}\right| \leq r}|\varphi(t)| d_{1} t+\frac{2 r}{m^{2}} \int_{\left|t-t_{0}\right| \geq r} \frac{|\varphi(t)| d_{1} t}{\left|t-t_{0}\right|^{2}}\right) .
$$

Hence, in notations (4.8.5), we have the inequality

$$
\left(M_{\theta}^{ \pm} \varphi\right)\left(t_{0}\right) \leq C\left[\left(M_{0} \varphi\right)\left(t_{0}\right)+\left(M_{0}^{\prime} \varphi\right)\left(t_{0}\right)+\left(M_{1} \varphi\right)\left(t_{0}\right)\right]
$$

and the first claim of the theorem is a corollary of Theorem 4.8.1.

It is obvious that the second claim holds in the case where the density is constant. Therefore, it suffices to prove that

$$
\lim _{z \rightarrow t_{0}} \int_{\Gamma} Q(t ; t-z, d t)\left[\varphi(t)-\varphi\left(t_{0}\right)\right]=\int_{\Gamma} Q\left(t ; t-t_{0}, d t\right)\left[\varphi(t)-\varphi\left(t_{0}\right)\right]
$$

provided that $z$ is located inside the sector $S_{\theta}\left(t_{0}, \Gamma\right)$.

To do this, we represent the function under the limit sign as the sum of the terms $\widetilde{\psi}_{r}\left(t_{0}\right)$ and $\widetilde{\phi}_{r}\left(z, t_{0}\right)$ defined similarly to $(4.9 .7)$ with respect to the density $\varphi(t)-\varphi\left(t_{0}\right)$. Then, according to the definition of singular integrals, to prove the relation (4.9.9), it suffices to verify that

$$
\lim _{r \rightarrow 0} \widetilde{\phi}_{r}\left(z, t_{0}\right)=0
$$

provided that $z$ is located inside the sector $K_{\theta}\left(t_{0}\right)$. In the considered case, similarly to (4.9.8), we have the estimate

$$
\left|\widetilde{\phi}_{r}\left(z, t_{0}\right)\right| \leq|Q|_{C^{0(1)}}\left(\frac{1}{m r} \int_{\left|t-t_{0}\right| \leq r}\left|\varphi(t)-\varphi\left(t_{0}\right)\right| d_{1} t+\frac{2 r}{m^{2}} \int_{\left|t-t_{0}\right| \geq r} \frac{\left|\varphi(t)-\varphi\left(t_{0}\right)\right| d_{1} t}{\left|t-t_{0}\right|^{2}}\right) ;
$$

combining it with Lemma 4.8.1, we prove (4.9.10).

Pass to the general case, i.e., assume that $\Gamma$ is a piecewise-Lyapunov curve and the function $\varphi$ belongs to $L_{\lambda}^{p}(\Gamma, F),-1<\lambda<0$. Then the behavior of the Cauchy-type integral $\phi(z)$ is described by Theorem 4.8.1 near any arc $\Gamma_{0}$ of $\Gamma \backslash F$. Consider the behavior of this integral in a neighborhood of the points $\tau$ of $F$. More exactly, consider it in the domain $B_{\rho}(\tau)$.

Recall that $B_{\rho}(\tau)=\{|z-\tau| \leq \rho\}$ provided that $\tau \neq \infty$ and $B_{\rho}(\tau)=\{|z| \geq 1 / \rho\}$ provided that $\tau=\infty$. By virtue of the choice of $\rho$, the circles $|z-\tau|=s, 0<s \leq \rho$ (the circles $|z|=1 / s$ for $\tau=\infty$ ) nontangentially intersect the radial $\operatorname{arcs} \Gamma_{\tau, k}, 1 \leq k \leq n_{\tau}$, composing the curve $\Gamma_{\tau}=\Gamma \cap B_{\rho}(\tau)$. Assign $J_{\tau}=(0, \rho]$ for $\tau \neq \infty$ and $J_{\tau}=[1 / \rho, \infty)$ for $\tau=\infty$ and introduce the following maximal function on $J_{\tau}$ :

$$
\left(M_{\tau} \phi\right)(s)=\sup _{|z-\tau|=s}|\phi(z)|,
$$

where $|z-\tau|$ is replaced by $|z|$ if $\tau=\infty$.

Theorem 4.9.2. Let a piecewise-Lyapunov curve $\Gamma$ not contain cusps, a generalized Cauchy problem $Q(t ; \xi, \eta)$ belong to $C_{0}^{\nu(1)}(\Gamma, F)$, and the density $\varphi$ from the integral (4.9.1) belong to $L_{\lambda}^{p}(\Gamma, F)$, where $p>1$ and $-1<\lambda<0$.

Then the function $M_{\tau} \phi$ from (4.9.11) belongs to $L_{\lambda_{\tau}}^{p}\left(J_{\tau}, \tau\right)$ and its norm satisfies the estimate

$$
\left|M_{\tau} \phi\right|_{L_{\lambda_{\tau}}^{p}} \leq C|\varphi|_{L_{\lambda}^{p}}
$$


Proof. It suffices to prove the theorem in the cases where $\tau=0$ or $\tau=\infty$. As in the proof of Theorem 4.8.2, both cases can be combined in the framework of a curve $\Gamma$ composed from radial arcs $\Gamma^{1}, \ldots, \Gamma^{n}$ with common endpoints $\tau=0$ and $\tau=\infty$ such that they are not cusps of this curve (by assumption).

Redenoting and assigning $\lambda_{\tau}=\lambda$, we see that it suffices to prove the theorem for the function

$$
\phi(z)=|z|^{-\lambda} \int_{\Gamma}|t|^{\lambda} Q(t ; t-z, d t) \varphi(t), \quad z \notin \Gamma,
$$

in the space $L_{0}^{p}$. More exactly, similarly to (4.9.11), this function is mapped to the maximal function

$$
(M \phi)(s)=\sup _{|z|=s}|\phi(z)|, \quad 0<s<\infty,
$$

and the estimate (4.9.12) with $\lambda=0$ is to be proved for it.

As in the proof of Theorem 4.8.2, from (4.9.13), pass to the relation

$$
\phi\left(2^{i} z\right)=|z|^{-\lambda} \int_{2^{-i} \Gamma}|t|^{\lambda} Q\left(2^{i} t ; t-z, d t\right) \varphi\left(2^{i} t\right), \quad|z|=s, \quad 1 / 2 \leq s \leq 2,
$$

and, for brevity, assign $\Gamma_{i}=\left(2^{-i} \Gamma\right) \cap\{1 / 4 \leq|t| \leq 4\}$ and $\varphi_{i}(t)=\varphi\left(2^{i} t\right), t \in \Gamma_{i}$. Then, as in Sec. 4.8, the previous relation can be represented as follows:

$$
4 \phi\left(2^{i} z\right)=\sum_{j=-\infty}^{+\infty} \phi_{i j}(z), \quad \phi_{i j}(z)=2^{-(1+\lambda) j} \int_{\Gamma_{i-j}}|t|^{\lambda} Q\left(2^{i-j} t ; 2^{-j} t-z, d t\right) \varphi_{i-j}(t) .
$$

Then

$$
4(M \phi)\left(2^{i} s\right) \leq \sum_{j=-\infty}^{+\infty}\left(M \phi_{i j}\right)(s), \quad 1 / 2 \leq s \leq 2
$$

with respect to the maximal functions

$$
\left(M \phi_{i j}\right)(s)=\sup _{|z|=s}\left|\phi_{i j}(z)\right|, \quad 1 / 2 \leq s \leq 2
$$

As we note above, if $1 / 2 \leq s \leq 2$, then the circle $|z|=s$ intersects the radial $\operatorname{arcs} \Gamma_{i}^{k}, 1 \leq k \leq n$, nontangentially. If $i \rightarrow \pm \infty$, then the arc $\Gamma_{i}^{k}$ tends to the segment $I_{ \pm}^{k}$ in the metric $C^{1, \nu}$. Therefore, as in the proof of Lemma 4.9.1, we verify the validity of inequality (4.9.4), where $z$ varies on the circle $|z|=s, 1 / 2 \leq s \leq 2, t$ belongs to $\Gamma_{i}$, and $t_{0}$ belongs to $\Gamma_{i},\left|t_{0}\right|=s$. Taking into account the remark to the said lemma, one can find a positive constant $m$ in this inequality such that it does not depend on $i=0, \pm 1, \ldots$ Therefore, one can use an analog of Theorem 4.9.1 to conclude that the maximal function $M \phi_{i 0}$ belongs to $L^{p}[1 / 2,2]$ and satisfies the estimate

$$
\left|M \phi_{i 0}\right|_{L^{p}} \leq C\left|\varphi_{i}\right|_{\Gamma_{i}}
$$

uniformly with respect to $i$. For the functions $\phi_{i j}, j \neq 0$, the following estimate similar to (4.8.20) is obtained:

$$
\sup _{1 / 2 \leq|z| \leq 2}\left|\phi_{i j}(z)\right| \leq C \sigma_{j} \int_{\Gamma_{i-j}}\left|\varphi_{i-j}(t)\right| d t, \quad \sigma_{j}= \begin{cases}2^{-(1+\lambda) j}, & j \geq 1, \\ 2^{-\lambda j}, & j \leq-1 .\end{cases}
$$

As in Sec. 4.8, these estimates combined with (4.9.15) imply the estimate (4.9.12) for the maximal function(4.9.14) in the space $L_{0}^{p}$.

Based on the properties of Cauchy-type integrals proved in Theorems 4.9.1-4.9.2, define HardyLittlewood classes $H^{p}$. Let a domain $D$ be bounded by a piecewise-smooth curve $\Gamma$ and a finite subset $F$ of $\Gamma$ contain all its boundary points. Then $\Gamma \backslash F$ is an open smooth curve. Let $H_{l o c}^{p}(D, F)$ denote 
the class of functions $\phi$ from $C(D)$ such that, in a neighborhood of each $\operatorname{arc} \Gamma_{0}$ of $\Gamma \backslash F$, they behave as the Cauchy-type integral from the first assertion of Theorem 4.9.1. Consider this definition in detail.

As we note in Sec. 2.5, there exists $\rho_{0}$ (called the standard radius of the arc $\Gamma_{0}$ ) such that the curve $\Gamma$ decomposes the disk $B\left(t_{0}, \rho_{0}\right)=\left\{\left|z-t_{0}\right| \leq \rho_{0}\right\}$ into two connected components $B^{ \pm}\left(t_{0}, \rho_{0}\right)$ provided that $t_{0} \in \Gamma_{0}$. If the arc $\Gamma_{0}$ is orientable, then the signs are selected such that $B^{+}\left(t_{0}, \rho_{0}\right)$ lies from the left of $\Gamma_{0}$ and $\rho_{0}$ can be selected sufficiently small to ensure that any of the components $B^{ \pm}\left(t_{0}, \rho_{0}\right)$ intersecting $D$ is contained in $D$. Then the domain $D$ lies from the left or from the right of $\Gamma_{0}$ if $B^{+}\left(t_{0}, \rho_{0}\right)\left(B^{-}\left(t_{0}, \rho_{0}\right)\right.$ respectively) is contained in $D$, but lies from both sides of $D$ otherwise (i.e., if $\Gamma_{0}$ is a cut for $\left.D\right)$.

Let the number $\rho=\rho\left(\theta, \Gamma_{0}\right)$ (see Definition (4.9.6) for sectors) for the arc $\Gamma_{0}$ not exceed the specified standard radius $\rho_{0}$. Assign $S\left(t_{0}, \Gamma_{0}\right)=S^{ \pm}\left(t_{0}, \Gamma_{0}\right)$ if the domain $D$ lies from one side of $\Gamma_{0}$ (with the corresponding choice of the sign) and assign $S\left(t_{0}, \Gamma_{0}\right)=S^{+}\left(t_{0}, \Gamma_{0}\right) \cup S^{-}\left(t_{0}, \Gamma_{0}\right)$ if $\Gamma_{0}$ is a cut for $D$. Then it is obvious that $S\left(t_{0}, \Gamma_{0}\right) \subseteq D$ and the following maximal function can be introduced for any $\phi$ from $C(D)$ :

$$
\left(M_{\theta} \phi\right)\left(t_{0}\right)=\sup _{z \in S_{\theta}\left(t_{0}, \Gamma_{0}\right)}|\phi(z)|, \quad t_{0} \in \Gamma_{0}
$$

Then the definition of the class $H_{l o c}^{p}(\Gamma, F), p \geq 1$, consists of the following requirement: for any arc $\Gamma_{0}$ from $\Gamma \backslash F$ and any $\theta$ from $(0, \pi)$, the introduced function $M_{\theta} \phi$ belongs to $L^{p}\left(\Gamma_{0}\right)$.

It is obvious that, multiplying elements of the class $H^{p}$ by bounded continuous functions, we do not leave the class. In the same way, it follows from the Hölder inequality that the product $\phi \psi$ of functions $\phi$ from $H^{p}$ and $\psi$ from $H^{q}$ belongs to the class $H^{1}$ provided that $p$ and $q$ are conjugate exponents. The next theorem combines several substantial properties of this class.

Let $\phi$ belong to $H^{p}(D, F)$, an arc $\Gamma_{0}$ be a subset of $\Gamma \backslash F$, and $\tau$ be an internal point of this arc.

\section{Theorem 4.9.3.}

(a) Let $\tau$ be an endpoint of a smooth arc $\Gamma_{1}$. Let $\Gamma_{1}$ without $\tau$ be contained in $D$. Then the restriction of the function $\phi$ to this arc belongs to $L^{p}\left(\Gamma_{1}\right)$.

(b) Let $L_{r}$ be the intersection of the circle $\{|z-\tau|=r\}$ with $D$ and

$$
\left(M_{\tau} \phi\right)(r)=\sup _{z \in L_{r}}|\phi(z)|, \quad 0<r \leq \varepsilon .
$$

Then the function $M_{\tau} \phi$ belongs to $L^{p}[0, \varepsilon]$ provided that $\varepsilon$ is positive and sufficiently small.

(c) Let a sequence of smooth arcs $\Gamma_{n}$ from $D, n=1,2, \ldots$, converge to $\Gamma_{0}$ in the metric of $C^{1}$, i.e., there exist their smooth parametrizations $\gamma_{n}:[0,1] \rightarrow L_{n}$ such that the sequence $\left\{\gamma_{n}\right\}$ converges to a smooth parametrization $\gamma_{0}$ of the arc $\Gamma_{0}$ in the space $C^{1}[0,1]$. Then

$$
\sup _{n}|\phi|_{L^{p}\left(\Gamma_{n}\right)}<\infty \text {. }
$$

Proof. (a) The point $\tau$ decomposes $\Gamma$ into two arcs $\Gamma_{0}^{ \pm}$. Without loss of generality, one can assume that $\Gamma_{1}$ and $\Gamma_{0}^{ \pm}$are radial arcs with respect to this point and the distance between it and the second endpoint of any of these arcs is equal to $\varepsilon$. Then it suffices to prove that the function $\phi\left[\gamma_{1}(r)\right]$ belongs to $L^{p}[0, \varepsilon]$ with respect to the radial parametrization $\gamma_{1}$ of the arc $\Gamma_{1}$. Since the absolute value of this function does not exceed $M_{\tau} \phi$ (in notation (4.9.16)), it follows that the considered assertion is a corollary of (b).

(b) Let $\Gamma_{1}$ satisfy conditions (a) and be orthogonal to $\Gamma_{0}$ at the point $\tau$. For definiteness, we assume that it lies from the left of $\Gamma_{0}$. In particular, one can assume that $S_{\theta}$ from (4.9.15) is the sector $S_{\theta}^{+}$. As in $(a)$, without loss of generality, one can assume that $\Gamma_{1}$ and $\Gamma_{0}^{ \pm}$are radial arcs with respect to the point $\tau$ and the distance between it and the second endpoint of any of these arcs is equal to $\varepsilon$. As above, the radial parametrizations of these arcs are denoted by $\gamma_{1}$ and $\gamma_{0}^{ \pm}$. If $\Gamma_{0}$ is a cut for $D$, then $L_{r}$ is assigned to be the circular arc intersecting $\Gamma_{1}$. Then the point $\gamma_{1}(r)$ decomposes $L_{r}$ into two $\operatorname{arcs} L_{r}^{ \pm}$with endpoints $\gamma_{1}(r)$ and $\gamma_{0}^{ \pm}(r)$ provided that $0<r \leq \varepsilon$. It is easy to see that there exists $\theta$ 
from $(0, \pi)$ such that the $\operatorname{arc} L_{r}^{ \pm}$is contained in the sector $S_{\theta}^{+}\left(t_{0}, \Gamma_{0}\right)$ with vertex $t_{0}=\gamma_{0}^{ \pm}(r)$ provided that $0<r \leq \varepsilon$. Therefore, the inequality

$$
\sup _{z \in L_{r}^{ \pm}}|\phi(z)| \leq\left(M_{\theta} \phi\right)\left[\gamma_{0}^{ \pm}(r)\right]
$$

holds. Hence, by the definition of the class $H^{p}$, the functions from the left-hand side of this inequality belong to $L^{p}[0, \varepsilon]$. Then this is also valid for the function (4.9.17).

(c) As in (b), without loss of generality, one can assume that the $\operatorname{arcs} \Gamma_{n}$ lie from the left of $\Gamma_{0}$. It suffices to prove that there exist $\theta$ from $(0, \pi)$, a sufficiently large $n_{0}$, and parametrizations $\gamma_{n}$ such that

$$
\gamma_{n}(s) \in S_{\theta}^{+}\left[\gamma(s), \Gamma_{0}\right], \quad 0 \leq s \leq 1 .
$$

Then, according to (4.9.15), the absolute values of the functions $\phi \circ \gamma_{n}$ are majorized by the function $M_{\theta} \circ \gamma$ belonging to $L^{p}[0,1]$.

It suffices to verify the condition locally. Therefore, one can assume that $\Gamma_{0}$ is given by the graph of a function $y=f(x), a \leq x \leq b$, in the Cartesian coordinate system $z=x+i y$, where $f$ is a real function from $C^{1}[a, b]$ and, respectively, $\Gamma_{n}$ are given by equations $y=f_{n}(x)$, where $f_{n}>f$ and $f_{n} \rightarrow f$ in $C^{1}[a, b]$. In such a case, the validity of the condition (4.9.18) is obvious.

In the definition of the class $H_{l o c}^{p}(D, F)$, the case where $F=\varnothing$ is not excluded. In this case, the curve $\Gamma$ contains no boundary points, i.e., it is a smooth contour, and it is natural to denote the Hardy-Littlewood class by $H^{p}(D)$. Then assertion $(c)$ of Theorem 4.9 .3 can be formulated with respect to a sequence of smooth contours $\Gamma_{n}$ from $D$ converging to the boundary contour $\Gamma$ in the metric $C^{1}$. In the same way, it follows from Theorem 4.9.3(a) that if $D_{0}$ is a subdomain of $D$ bounded by a smooth contour, then the restriction of any function $\phi$ from $H^{p}(D)$ to this subdomain belongs to $H^{p}\left(D_{0}\right)$. In particular, it follows from these properties that all $H^{p}(D)$-functions analytic in $D$ belong to the Hardy-Smirnov class $E^{p}(D)$ (see, e.g., [25]) generalizing the classical Hardy classes $H^{p}$ in the unit disk (see [26]). In the case of harmonic functions, the similar class is frequently denoted by $e^{p}(D)$ (see $[25])$.

Now, introduce the weight Hardy-Littlewood class $H_{\lambda}^{p}(D, F)$ with an arbitrary weight order $\lambda$ as follows: it consists of all functions $\phi$ from $H_{l o c}^{p}(D, F)$ such that, in a neighborhood of singular points $\tau$ from $F$, they behave as the Cauchy-type integrals in Theorem 4.9.2. In other words, in notation (4.9.11), for any $\tau$ from $F$, the function $M_{\tau} \phi$ belongs to $L_{\lambda_{\tau}}^{p}\left(I_{\tau}, \tau\right)$. According to Theorem 4.9.2, for $-1<\lambda<0$ and $p>1$, the Cauchy-type integral with density $\varphi$ from $L_{\lambda}^{p}(\Gamma, F)$ belongs to the class $H^{p}(D, F)$ provided that $\Gamma$ is a piecewise-Lyapunov curve without cusps. It is clear from the proof of this theorem that it suffices to require the positivity of internal angles of the domain $D$ at all points $\tau$ of $F$. In particular, the case where this angle is equal to $2 \pi$ at a point $\tau$ of $F$ (and this $\tau$ is a cusp of $\Gamma$ ), is not excluded.

From Theorem 4.9.3(b) combined with the property (4.1.11) of $L^{p}$-spaces, the next assertion immediately follows; it is an analog of the specified property for $H^{p}$-spaces.

Lemma 4.9.2. Let $\Gamma_{1}$ be a piecewise-smooth curve contained (except for several its endpoints) in the domain $D$. Let $D_{1}$ be a connected component of $D \backslash \Gamma_{1}$. Let $F_{1}$ be a finite subset of $\partial D_{1}$, containing $F \cup\left(\Gamma \cap \Gamma_{1}\right)$, and let the weight order $\lambda^{1}$ on $F_{1}$ be defined by the relation

$$
\lambda_{\tau}^{1}= \begin{cases}\lambda_{\tau}, & \tau \in F, \\ -1 / p, & \tau \in F_{1} \backslash F .\end{cases}
$$

Then the restriction of any $\phi$ from $H_{\lambda}^{p}(D, F)$ to $D_{1}$ belongs to $H_{\lambda^{1}}^{p}\left(D_{1}, \partial D_{1}\right)$.

\section{REFERENCES}

1. D. S. Anikonov, "On boundedness of a singular integral operator in the space $C^{\alpha}(\bar{G})$," $M a t$. $S b$., 104, No. 4, 516-534 (1977). 
2. J. Bergh and J. Löfström, Interpolation Spaces. An Introduction, Springer-Verlag, BerlinHeidelberg-New York (1976).

3. L. Bers, F. John, and M. Schechter, Partial Differential Equations, Interscience Publishers, New York-London-Sydney (1964).

4. O. V. Besov, V. P. Ilin, and S. M. Nikolskii, Integral Representation of Functions and Embedding Theorems [in Russian], Nauka, Moscow (1975).

5. A. V. Bitsadze, "Spatial analog of the Cauchy-type integral and some its applications," Dokl. AN SSSR, 93, No. 3, 389-392 (1953).

6. A. P. Calderon, "Cauchy integrals on Lipschitz curves and related operators," Proc. Natl. Acad. Sci. USA, 74, No. 4, 1324-1327 (1977).

7. M. Christ, Lectures on Singular Integral Operators, Am. Math. Soc., Providence (1990).

8. R. V. Duduchava, "On singular integral operators in weighted spaces of Hölder functions," Dokl. AN SSSR, 191, No. 1, 16-19 (1970).

9. R. V. Duduchava, "On the Noether theorems for singular integral equations in weighted spaces of Hölder functions," Proc. Symp. on Contin. Mech. and Related Probl. of Anal., Vol. 1, Tbilisi, $19^{71}$ [in Russian], Metsniereba, Tbilisi (1973), 89-102.

10. R. V. Duduchava, "On singular integral operators on piecewise smooth lines," Function Theoretic Methods in Differential Equations, Pitman, London-San Francisco-Melbourne (1976), 109-131.

11. R. V. Duduchava, "Integral convolution equations with discontinuous presymbols, singular integral equations with fixed singularities, and their applications to problems of mechanics," Tr. Tbil. Mat. Inst. Akad. Nauk GrSSR, 60, 1-135 (1979).

12. E. M. Dynkin, "Methods of the theory of singular integrals. I. Hilbert transformation and the Calderon-Zygmund theory," Itogi Nauki i Tekhniki, 15, 197-292 (1987).

13. E. M. Dynkin, "Methods of the theory of singular integrals. II. The Littlewood-Paley theory and its applications," Itogi Nauki i Tekhniki, 42, 105-198 (1989).

14. A. Erdélyi et. al., Tables of Integral Transforms. Vol. I, McGraw-Hill Book Co., New York (1954).

15. G. Fichera, "Linear elliptic equations of higher order in two independent variables and singular integral equations, with applications to anisotropic inhomogeneous elasticity," Proc. Int. Conf. Part. Differ. Equ. Contin. Mech., Univ. of Wisconsin Press, Madison, WI (1961), 55-80.

16. G. Fichera and P. E. Ricci, "The single layer potential approach in the theory of boundary value problems for elliptic equations," Lect. Notes Math., 561, 39-50 (1976).

17. F. D. Gakhov, Boundary-Value Problems [in Russian], Nauka, Moscow (1977).

18. T. Gamelin, Uniform Algebras [Russian translation], Mir, Moscow (1973).

19. F. R. Gantmacher, The Theory of Matrices [Russian translation], Nauka, Moscow (1988).

20. I. M. Gelfand, D. A. Raikov, and G. E. Shilov, Commutative Normed Rings [in Russian], Fizmatgiz, Moscow (1960).

21. G. Giraud, "Équations à intégrales principales; étude suivie d'une application," Ann. Sci. Éc. Norm. Supér (3), 51, 251-372 (1934).

22. V. P. Glushko, "On operators of potential type and certain embedding theorems," Dokl. AN SSSR, 126, No. 3, 467-470 (1959).

23. I. Ts. Gokhberg and I. A. Feldman, Convolutions Equations and Projection Methods of Their Solution [in Russian], Nauka, Moscow, (1970).

24. I. Ts. Gokhberg and N. I. Krupnik, Introduction to the Theory of One-Dimensional Singular Equations [in Russian], Shtiintsa, Kishinev (1973).

25. G. M. Goluzin, Geometric Theory of Functions of Complex Variable [in Russian], Nauka, Moscow, (1972).

26. K. Hoffman, Banach Spaces of Analytic Functions, Prentice-Hall, Englewood Cliffs (1962).

27. L. Hörmander, "Estimates for translation invariant operators in $L_{p}$ spaces," Acta Math., 104, 93-140 (1960). 
28. L. Hörmander, The Analysis of Linear Partial Differential Operators. I: Distribution Theory and Fourier Analysis, Springer-Verlag, Berlin-Heidelberg-New York-Tokyo (1983).

29. T. Kato, Perturbation Theory for Linear Operators, Springer-Verlag, Berlin-Heidelberg-New York (1966).

30. B. V. Khvedelidze, "Linear discontinuous problems of the theory of functions, singular integral equations, and some their applications," Tr. Tbil. Mat. Inst Akad. Nauk GrSSR., 23, 3-158 (1956).

31. M. A. Krasnosel'skii et. al., Integral Operators in the Space of Summable Functions [in Russian], Nauka, Moscow (1966).

32. S. G. Krein, Linear Equations in Banach Space [in Russian], Nauka, Moscow (1971).

33. S. G. Krein, Iu. I. Petunin, and E. M. Semenov, Interpolation of Linear Operators [in Russian], Nauka, Moscow (1978).

34. H. B. Krylov, Lectures on Elliptic and Parabolic Equations in Hölder Space [in Russian], Nauchnaia Kniga, Novosibirsk (1998).

35. A. Kufner, Weighted Sobolev Spaces, Teubner Texte zur Mathematik, Leipzig (1980).

36. O. A. Ladyzhenskaia and N. N. Uraltseva, Linear and Quasilinear Elliptic Equations [in Russian], Nauka, Moscow (1964).

37. S. Lang, Introduction to Differentiable Manifolds, Interscience Publishers, New York-London (1962).

38. B. M. Levitan, Almost Periodic Functions [in Russian], Gostekhizdat, Moscow (1953).

39. G. S. Litvinchuk, Boundary-Value Problems and Singular Integral Equations with Shift [in Russian], Nauka, Moscow (1977).

40. N. I. Maltsev, Essentials of Linear Algebra. 3d ed. [in Russian], Nauka, Moscow (1970).

41. V. G. Maz'ya, Sobolev Spaces [in Russian], LGU, Leningrad (1985).

42. S. G. Mikhlin, "Singular integral equations," Usp. Mat. Nauk, 3, No. 3, 29-112 (1948).

43. S. G. Mikhlin, Multidimensional Singular Integrals and Integral Equations [in Russian], Fizmatgiz, Moscow (1962).

44. S. G. Mikhlin and S. Prosdorf, Singular Integral Operators, Academie-Verlag, Berlin (1986).

45. N. I. Muskhelishvili, Singular Integral Equations [in Russian], Nauka, Moscow (1968).

46. R. Narasimkhan, Analysis on Real and Complex Manifolds [Russian translation], Mir, Moscow (1971).

47. S. M. Nikolskii, Approximation of Multivariable Functions and Embedding Theorems [in Russian], Nauka, Moscow (1969).

48. R. S. Palais, Seminar on the Atiyah-Singer Index Theorem [Russian translation], Mir, Moscow (1970).

49. A. Iu. Pirkovskii, Spectral Theory and Functional Calculi for Linear Operators [in Russian], MTsNMO, Moscow (2010).

50. J. Plemelj, "Ein Ergänzungssatz zur Cauchyschen Integraldarstellung analytischer Funktionen, Randwerte betreffend," Mon. Math. Phys., 19, 205-210 (1908).

51. V. A. Polunin and A. P. Soldatov, "Three-dimensional analog of the Cauchy-type integral," Differ. Uravn., 47, No. 3, 366-375 (2011).

52. I. I. Privalov, "The Cauchy integral," Izv. Fiz.-Mat. Fak. Saratov. Univ., 11, No. 1, 94-105 (1918).

53. I. I. Privalov, Boundary Properties of Analytic Functions. 2nd ed. [in Russian], Nauka, Moscow (1967).

54. S. Prössdorf, Some Classes of Singular Equations, North-Holland Publishing Co., AmsterdamNew York-Oxford (1978).

55. F. Riesz and B. Szokefalvi-Nagy, Functional Analysis, Blackie \& Son, London-Glasgow (1956).

56. U. Rudin, Functional Analysis [in Russian], Mir, Moscow (1991).

57. S. L. Sobolev, Some Applications of Functional Analysis in Mathematical Physics. 3d ed. [in Russian], Nauka, Moscow (1988). 
58. J. Sochocki, On Definite Integrals and Functions Used for Series Expansion [in Russian], Tipografiya M. M. Stasyulevicha, Saint-Petersburg (1873).

59. A. P. Soldatov, "To Noether theory of operators. Wiener embeddings of $B$-algebras," Differ. Uravn., 14, No. 1, 104-115 (1978).

60. A. P. Soldatov, "Category of duality in the theory of Noether operators," Differ. Uravn., 15, No. 2, 303-309 (1979).

61. A. P. Soldatov, "Asymptotics of solutions of singular integral equations," Differ. Uravn., 22, No. 1, 143-153 (1986).

62. A. P. Soldatov, "Asymptotics of solutions of boundary-value problems for elliptic systems near angular points," Dokl. AN SSSR, 315, No. 1, 34-36 (1990).

63. A. P. Soldatov, "Boundary properties of the Cauchy-type integrals," Differ. Uravn., 26, No. 1, 131-136 (1990).

64. A. P. Soldatov, "Generalized Cauchy-type integral," Differ. Uravn., 27, No. 2, 3-8 (1991).

65. A. P. Soldatov, One-Dimensional Singular Operators and Boundary-Value Problems of the Function Theory [in Russian], Vysshaya Shkola, Moscow (1991).

66. A. P. Soldatov, "Generalized Cauchy-type integral and singular integral in weighted Hölder space," Dokl. RAN, 330, 164-166 (1993).

67. A. P. Soldatov, "Algebra of singular operators with end symbol on a piecewise-smooth curve. I. Convolution-type operators on semiaxis," Differ. Uravn., 36, No. 9, 1209-1219 (2000).

68. A. P. Soldatov, "Algebra of singular operators with end symbol on a piecewise-smooth curve. II. Main constructions," Differ. Uravn., 37, No. 6, 825-838 (2001).

69. A. P. Soldatov, "Algebra of singular operators with end symbol on a piecewise-smooth curve. III. Normal type operators," Differ. Uravn., 37, No. 10, 1364-1376 (2001).

70. A. P. Soldatov, "Boundary properties of generalized Cauchy-type integrals with summable density," Dokl. Adygskoi (Cherkesskoi) Mezhd. Akad. Nauk, 10, No. 1, 62-66 (2008).

71. A. P. Soldatov and A. V. Aleksandrov, "Boundary properties of the Cauchy-type integrals. $L_{p^{-}}$ case," Differ. Uravn., 27, No. 1, 3-8 (1991).

72. T. A. Soldatova, "Generalized double layer potentials," Vestn. Mosk. Univ. Ser. I Mat. Mekh., 6, 8-17 (2009).

73. T. A. Soldatova, "Boundary properties of generalized Cauchy-type integrals in spaces of smooth functions," Izv. Saratov. Univ. Nov. Ser. Ser. Mat. Mekh. Inform., 11, No. 3(1), 95-109 (2011).

74. E. Stein, Singular Integrals and Differentiability Properties of Functions, Princeton University Press, Princeton (1970).

75. E. Stein and G. Weiss, Introduction to Fourier Analysis on Euclidean Spaces, Princeton University Press, Princeton (1971).

76. M. E. Taylor, Pseudodifferential operators, Princeton University Press, Princeton (1981).

77. F. Tricomi, "Formula d'inversione dell'ordine di due integrazioni doppie 'con asterisco'," Rend. Accad. d. L. Roma (6), 3, 535-539 (1926).

78. N. P. Vekua, Systems of Singular Integral Equations [in Russian], Nauka, Moscow (1970).

79. K. Yosida, Functional Analysis, Springer-Verlag, Berlin-Göttingen-Heidelberg (1965).

A. P. Soldatov

National Research University "Belgorod State University," Belgorod, Russia

E-mail: soldatov@bsu.edu.ru 